The Road to the Higgs

IN $p \bar{p}$ COLLisions AT $\sqrt{s}=1.96 \mathrm{TeV}$

A dissertation presented

by

Gabriel Facini

to

The Department of Physics

In partial fulfillment of the requirements for the degree of Doctor of Philosophy

in the field of

Physics

Northeastern University

Boston, Massachusetts

April, 2011 
(C)

Gabriel Facini, 2011

ALL RIGHTS RESERVED 
The Road to the Higgs

IN $p \bar{p}$ COllisions AT $\sqrt{s}=1.96 \mathrm{TeV}$

by

Gabriel Facini

\section{ABSTRACT OF DISSERTATION}

Submitted in partial fulfillment of the requirement for the degree of Doctor of Philosophy in Physics in the Graduate School of Arts and Sciences of Northeastern University, April, 2011. 
Abstract

Presented is a series of analyses which are central to the search for a low-mass Higgs boson. A search for $Z Z$ production in the $Z Z \rightarrow \ell^{-} \ell^{+} \nu \bar{\nu}$ [1] channel is introduced then the successful combination of this analysis with with the $Z Z \rightarrow \ell^{+} \ell^{-} \ell^{\prime+} \ell^{\prime-}$ search to produce the first observation of the $Z Z$ process at a hadron collider is then detailed [2]. The final analysis presented is the search for the Higgs in the $Z H \rightarrow \nu \bar{\nu} b \bar{b}$ channel [3] and the interpretation as a $Z Z \rightarrow \nu \bar{\nu} b \bar{b}$ search in order to validate the techniques. Common themes are discussed, such as multivariate techniques and instrumental backgrounds from energy measurement fluctuations and the tools used to combat them. The formalism of the statistical analysis of the final selected sample is introduced generally and demonstrated in the context of the above mentioned searches. The optimization of the selection through the identification of poorly reconstructed leptons is included as well as the utilization of b-quark identifying tools.

Some space is given to jet reconstruction/identification and the Level 1 Calorimeter Trigger. The efficient identification and calibration of jets is central to many physics analysis especially in the low mass higgs search. Another key component of the $Z H \rightarrow \nu \bar{\nu} b \bar{b}$ search is the proficient identification of jets and an imbalance of transverse energy in the first level of the triggering system. Therefore, the Level 1 Calorimeter Trigger, designed to achieve this, is a necessary component for a sensitive $Z H \rightarrow \nu \bar{\nu} b \bar{b}$ search.

My efforts were concentrated on but not completely restricted to the topics mentioned above. 


\section{ACKNOWLEDGEMENTS}

The Northeastern community has been welcoming, supportive, and encouraging. I cannot express enough gratitude toward my advisor, Professor Darien Wood, for his infinite patience to answer even the most absurd questions with the utmost seriousness. His ability to balance attentiveness without being overbearing and distance without seeming uninterested established an environment of support and freedom which I have enjoyed tremendously. On top of that, the guy is intimidatingly sharp which has made me hold myself to a higher standard. I would like to express gratitude toward my committee members Professor Emanuela Barberis and Professor Pran Nath. Finally, I thank Professors Haim Goldberg and Brent Nelson, as well as Daniel Feldman, Joe Haley, Baris Altunkaynak, Michael Holmes, Ari Adland, and Song Zhen.

I must thank Professor Ken Johns for conducting interesting lectures and giving me the opportunity to work in his lab. Without him, I would not have attended graduate school.

I would also like to take the time here to acknowledge the many people I met at D $\varnothing$ who have helped me these past three years. I am forever indebted and deeply appreciative for my time spent working with Jean-Francois Grivaz who embodies friendly cooperative scientific curiosity. I would also like to thank the following remarkable people I have been able to work alongside; Terry Wyatt, John Hobbs, Gianluca Cerminara, Gavin Hesketh, Emanuel Strauss, Krisztian Peters, Murilo Rangel, Thibault Guillemin, Tim Scanlon, and Theodoros Christoudias. I would also like to thank Wade Fisher, Aurelio Juste, Brendan Casey, Yuji Enari, Sebastien Greder and Mark Williams for the many fruitful conversations.

My parents, Mirjana and Ante, both started life in great hardship and continued in this fashion to give my siblings and I something they never had, a choice with what 
to do with our lives. You have have been my foundation, my solace, my guiding lights, and my determination. Everything I am today is because of you. You have been and continue to be wonderful parents. I want express gratitude to my best friends my brother, Adrian, and my sister, Marisa, for always being the silver lining. I would also like to thank my Arizona family who have encouraged me along the way.

Finally, I would like to thank the woman paramount to my happiness, Jaymi, for her understanding, support, and love. Your smile is all I need. 


\section{Contents}

$\begin{array}{ll}\text { Abstract } & 3\end{array}$

$\begin{array}{ll}\text { Acknowledgements } & 5\end{array}$

$\begin{array}{lll}1 & \text { Introduction } & 30\end{array}$

1.1 The Standard Model Particles . . . . . . . . . . . . . . . . . . . 32

1.2 The Standard Model . . . . . . . . . . . . . . . . . . . . . . . 34

1.2.1 The Theory of Quantum Electrodynamics . . . . . . . . . 34

1.2.2 The Theory of Quantum Chromodynamics . . . . . . . . 36

1.2.3 The Electroweak Theory . . . . . . . . . . . . . . . . . . . 38

1.2.4 The Higgs Mechanism . . . . . . . . . . . . . . . . . . 41

1.2.5 Constraints on the Higgs . . . . . . . . . . . . . . 45

2 Experimental Apparatus $\quad 53$

2.1 The Accelerator . . . . . . . . . . . . . . . . . . . . 54

2.1 .1 Proton Beam . . . . . . . . . . . . . . . . 56

2.1 .2 Antiproton Beam . . . . . . . . . . . . . . 58

2.1 .3 Tevatron . . . . . . . . . . . . . . . . . . 60

2.2 Detector . . . . . . . . . . . . . . . . . . . . 61

2.2 .1 Coordinates . . . . . . . . . . . . . . . . 63

2.2 .2 Tracking . . . . . . . . . . . . . . . . . . 64 
2.2.3 Preshower Detectors . . . . . . . . . . . . . . . . . 68

2.2.4 Calorimetery . . . . . . . . . . . . . 70

2.2.5 Muon Detection . . . . . . . . . . . . . . . . . 74

2.2.6 Luminosity Monitor . . . . . . . . . . . . . . . . 78

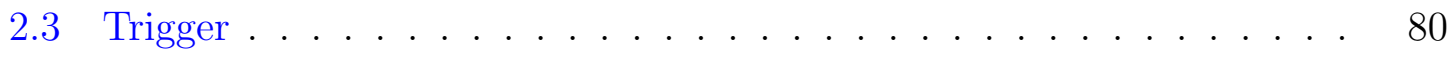

2.3.1 Level 1....................... . . 80

2.3.2 Level 2 . . . . . . . . . . . . . . . . . . . . . . 82

2.3.3 Level 3 . . . . . . . . . . . . . . . . . . . . . . . 83

3 Event Simulation $\quad 85$

3.1 Monte Carlo Generators . . . . . . . . . . . . . . 85

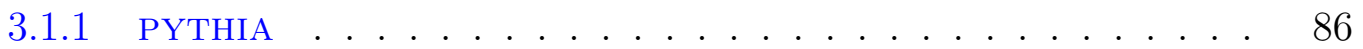

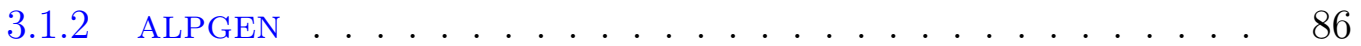

3.1.3 CompHEP ..................... 87

3.1.4 Monte Carlo for Femtobarn Processes . . . . . . . . . . . . . . 87

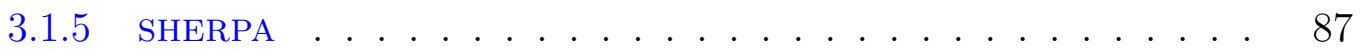

3.1.6 Mixing and Matching . . . . . . . . . . . . . 87

3.2 Detector Simulation . . . . . . . . . . . . . . . . . . . . 88

3.3 Monte Carlo Corrections . . . . . . . . . . . . . . . . . . . . 88

4 Object Reconstruction and Identification $\quad 91$

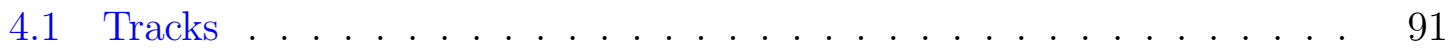

4.2 Primary Vertices . . . . . . . . . . . . . . . . . . 95

4.3 Muons ........................... . . 96

4.4 Electrons and Photons . . . . . . . . . . . . . . . . . . . . . . . 99

4.5 Taus ............................... 104

4.6 Jets . . . . . . . . . . . . . . . . . . . . . . 106 
4.6 .1 Creating Jet Objects . . . . . . . . . . . . . . . . . 106

4.6 .2 Good Jets . . . . . . . . . . . . . . . . . . . . . 107

4.6.3 The Jet Energy Scale . . . . . . . . . . . . . . . . . . . 108

4.6.4 Jet Smearing, Shifting, and Removal _ . . . . . . . . . 110

4.6.5 Higher Jet Quality Criteria _. . . . . . . . . . . . 112

4.7 Missing Transverse Energy . . . . . . . . . . . . . . . . . . . . . . . 114

5 Quality of the Measured $\not_{T} \quad 116$

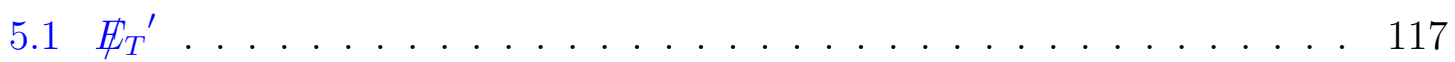

5.1 .1 Decomposition . . . . . . . . . . . . . . 117

5.1 .2 Object Resolution . . . . . . . . . . . . . . . . . . . 119

5.1 .3 Recoil Measured by the Calorimeter . . . . . . . . . . . . . 119

5.1 .4 Recoiling Tracks . . . . . . . . . . . . . . . . 120

5.1.5 Resultant Variable . . . . . . . . . . . . . . . . . . . . . 121

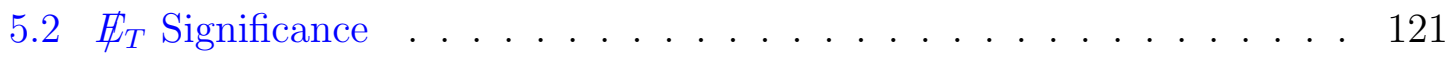

6 Multivariate Analysis $\quad 124$

6.1 Likelihood Discriminant . . . . . . . . . . . . . . . . 125

6.2 Decision Tree . . . . . . . . . . . . . . . . . . 126

6.3 Artificial Neural Network . . . . . . . . . . . . . . . . . . 130

$\begin{array}{lll}7 & \text { B-Quark Identification } & 133\end{array}$

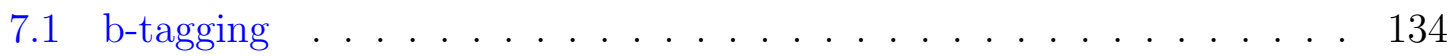

7.2 Multivariate b-Tagging . . . . . . . . . . . . . 135

7.3 Usage . . . . . . . . . . . . . . . . . . 136

$\begin{array}{lll}8 & \text { Statistics } & 139\end{array}$

8.1 Overview . . . . . . . . . . . . . . . . . . 140 
8.2 The Test Statistic . . . . . . . . . . . . . . . . . . . . . . 142

8.2.1 The Poisson Likelihood Ratio . . . . . . . . . . . . . . . . . . 142

8.2.2 The Profile Likelihood Ratio . . . . . . . . . . . . . . . . . . . 144

8.3 p-Values and Confidence Levels . . . . . . . . . . . . . . . . . 145

8.4 Cross Section Limits . . . . . . . . . . . . . . . . . . . . 147

8.5 Cross Section Measurements . . . . . . . . . . . . . . . . . 147

8.6 Evaluation of Systematic Uncertainties . . . . . . . . . . . . 147

8.6.1 Experimental Systematic Uncertainties . . . . . . . . . . . . 148

8.6.2 Theoretical Systematic Uncertainties . . . . . . . . . . . . 150

$9 \quad Z Z \rightarrow \ell^{-} \ell^{+} \nu \bar{\nu}$ Analysis 152

9.1 Dataset and Monte Carlo Samples . . . . . . . . . . . . . . . 154

9.1.1 Data .............................. 154

9.1.2 Monte Carlo . . . . . . . . . . . . . . . . . . . . 154

9.2 Preselection Requirements . . . . . . . . . . . . . . . 155

9.2.1 Electron Requirements . . . . . . . . . . . . . . 156

9.2.2 Muon Requirements . . . . . . . . . . . . . . . 156

9.2.3 Jet Requirements . . . . . . . . . . . . . . . . . . . 157

9.2.4 Additional Lepton Requirements . . . . . . . . . . . . . . . . 157

9.2.5 Track Requirements . . . . . . . . . . . . . . . . . . 159

9.3 Instrumental Backgrounds . . . . . . . . . . . . . . . . . 160

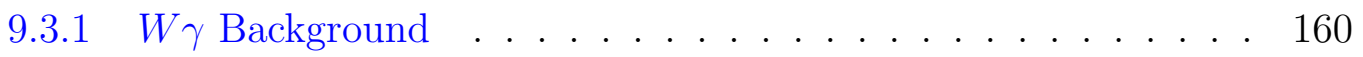

9.3.2 $W$ +jets Background ................ 161

9.4 Monte Carlo Normalization _. . . . . . . . . . . . . . . . . 162

9.5 Alternative $\not_{T}$ Selection . . . . . . . . . . . . . . 165

9.6 Yields . . . . . . . . . . . . . . . . . . . . . . . . 172 
9.7 Likelihood Discriminant . . . . . . . . . . . . . . . . . . . . . . . 174

9.8 Systematic Uncertainties . . . . . . . . . . . . . . . . . . . 182

9.8.1 Significance Systematics . . . . . . . . . . . . . . . . . 182

9.8.2 Cross Section Systematics . . . . . . . . . . . . . 188

9.9 Significance and Cross Section Measurement . . . . . . . . . . . . 194

9.10 First Observation of the $Z Z$ Production Process . . . . . . . . . . . 197

9.10.1 ZZ $\rightarrow \ell^{-} \ell^{+} \ell^{\prime-} \ell^{\prime+}$ Analysis . . . . . . . . . . . . . . 198

9.10.2 Combination . . . . . . . . . . . . . . . . 202

$10 Z H \rightarrow \nu \bar{\nu} b \bar{b}$ Analysis $\quad 207$

10.1 Dataset and Monte Carlo Samples . . . . . . . . . . . . . . . . . 208

10.1.1 Data . . . . . . . . . . . . . . . . 208

10.1.2 Monte Carlo . . . . . . . . . . . . . . . . . . . . . . 209

10.2 Trigger Description . . . . . . . . . . . . . . . . . . 210

10.3 Event Selection . . . . . . . . . . . . . . . . . . . . 211

10.3.1 Preselection . . . . . . . . . . . . . . . . 211

10.3.2 Lepton Identification . . . . . . . . . . . . . . . 216

10.3.3 Signal Selection . . . . . . . . . . . . . . . . . . 222

10.3.4 Sideband and MJ-model Sample . . . . . . . . . . . . . . . . . 222

10.3.5 Cut Flow . . . . . . . . . . . . . . . . 223

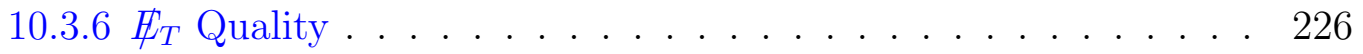

10.3 .7 b-tagging . . . . . . . . . . . . . . . . 228

10.4 Normalization . . . . . . . . . . . . . . . . . . . . . . 229

10.5 Electroweak Control Sample . . . . . . . . . . . . . . . . . . 230

10.6 Multijet Control Sample . . . . . . . . . . . . . . . . . . . 239

10.7 Signal Sample Sample . . . . . . . . . . . . . . . . . . . 248 
10.8 Multijet and Physics Decision Tree . . . . . . . . . . . . . . 256

10.9 Systematic Uncertainties . . . . . . . . . . . . . . . 274

10.10Upper Limits on $Z H \rightarrow \nu \bar{\nu} b \bar{b}$ and

$W H \rightarrow \ell^{ \pm} \nu b \bar{b}$ Production . . . . . . . . . . . . . . . . 275

10.10.1 Run IIb Results . . . . . . . . . . . . . . . . . . . . . . . . 281

10.10.2 Comments on the limit setting procedure . . . . . . . . . . . . 281

$10.11 Z Z \rightarrow \nu \bar{\nu} b \bar{b}$ Interpretation . . . . . . . . . . . . . . . . . 288

11 Conclusions and Outlook 293

$\begin{array}{lr}\text { Appendices } & 296\end{array}$

$\begin{array}{ll}\text { A Nomenclature } & 296\end{array}$

A.1 Units . . . . . . . . . . . . . . . . . . 296

A.2 List of Abbreviations . . . . . . . . . . . . . . . . . . . 297

B Level 1 Calorimeter Trigger $\quad 300$

B.1 Architecture . . . . . . . . . . . . . . . . 301

B.1.1 The ADF System . . . . . . . . . . . . . . . . . . . . 302

B.1.2 The TAB/GAB System . . . . . . . . . . . . . . . . 302

B.2 Sliding Windows Algorithms . . . . . . . . . . . . . . . . 303

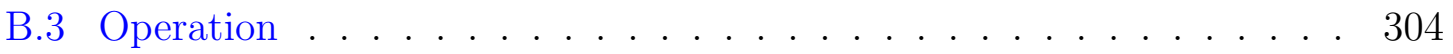

C ALPGEN Angular Reweightings 308

D b-tagging MVA Distributions $\quad 311$

E Decision Tree Training Input Variables 316

E.1 Overtraining Check for Decision Tree Training . . . . . . . . . . . . . 331 
F Physics Decision Trees

G Additional Run IIb1 Multijet Decision Tree Plots 342

G.1 Additional Run IIb1 Physics Decision Tree Plots . . . . . . . . . . . . 349

H Additional Run IIb2-3 Multijet Decision Tree Plots 353

H.1 Additional Run IIb2-3 Physics Decision Tree Plots . . . . . . . . . . . 360

I Shape Systematics with respect to the BDT for RunIIb1 single tag sample

J Shape Systematics with respect to the BDT for RunIIb1 double tag sample

K Shape Systematics with respect to the BDT for RunIIb23 single tag sample

L Shape Systematics with respect to the BDT for RunIIb23 double tag sample

Bibliography 


\section{List of Figures}

1.1 The simplest Higgs potential. . . . . . . . . . . . . . . . 42

1.2 The mexican hat potential. . . . . . . . . . . . . . . 44

1.3 Precision EW fits from LEP EWWG. . . . . . . . . . . . 47

1.4 Precision EW fits from the Gfitter Group. . . . . . . . . . . . 47

1.5 Standard Model Higgs branching ratio as a function of mass. . . . 49

1.6 Standard Model Higgs production cross section at the Tevatron. . 50

1.7 The latest Tevatron limits on the full Higgs mass range. . . . . . . 51

1.8 The latest Tevatron limits on the high mass Higgs production cross

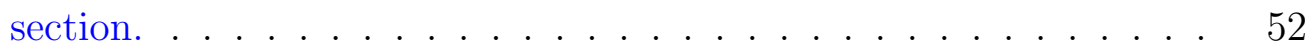

$2.1 \quad$ Fermilab National Accelerator Laboratory. . . . . . . . . . 55

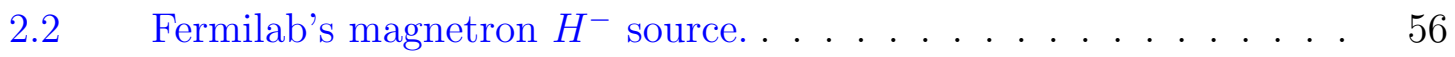

2.3 Cockroft-Walton generator at Fermilab. . . . . . . . . 57

$2.4 \quad$ Linear Accelerator of Fermilab. . . . . . . . . . . . . . 57

2.5 Fixed nickel target used for antiproton production. . . . . . . . . 59

2.6 The helical path of the proton and antiproton beams. . . . . . 60 
2.7 The $\mathrm{D} \emptyset$ Detector $\ldots \ldots \ldots \ldots$

2.8 The D $\varnothing$ Central Tracking System. . . . . . . . . . . . . . . 64

2.9 The D $\varnothing$ Silicon Microstrip Tracker (SMT) . . . . . . . . 66

2.10 The DØ Central Fiber Tracker $(\mathrm{CFT}) \ldots \ldots \ldots \ldots$

2.11 The magnetic field in the $\mathrm{D} \emptyset$ detector. . . . . . . . . 68

2.12 Dø Preshower Detector scintillator geometry . . . . . . . . . . 69

2.13 The $\mathrm{D} \varnothing$ Calorimeter . . . . . . . . . . . . . . . . . . 72

2.14 A typical unit cell of the $\mathrm{D} \varnothing$ Calorimeter . . . . . . . 73

2.15 Pseudo-projective read-out towers of the DØ Calorimeter . . . . 74

2.16 Exploded view of the $\mathrm{D} \emptyset$ muon drift tube system. . . . . . . . . 77

2.17 Exploded view of the $\mathrm{D} \varnothing$ muon scintillator system. . . . . . . 78

2.18 Position of the DØ Luminosity Monitor. . . . . . . . . . . . 79

2.19 Data flow in the $\mathrm{D} \emptyset$ three tiered trigger system. . . . . . . . 84

2.20 Sub-detector data flow in the $\mathrm{D} \emptyset$ three tiered trigger system. . . . 84

4.1 The HTF track finding algorithm. . . . . . . . . . . . 93

4.2 The AA track finding algorithm. . . . . . . . . . . . 94

4.3 Sigmoidal weight function of the Adaptive vertex fitter algorithm. . 96

4.4 Run IIb muon resolutions. . . . . . . . . . . . . . . . . . . . . . . . 99

$4.5 \quad$ Run IIb electron resolutions. . . . . . . . . . . . . . . . . . . 103 
$4.6 \quad$ Run IIb jet resolutions. . . . . . . . . . . . . . . . . . . . . . . . 112

$5.1 \quad$ Thrust axis for $E_{T}{ }^{\prime} \ldots \ldots \ldots \ldots \ldots \ldots \ldots$

$6.1 \quad$ Decision Tree Example . . . . . . . . . . . . . . . 130

$6.2 \quad$ Multilayer Perceptron Neural Net Example . . . . . . . . . . . . 132

7.1 Jet from the decay of a b-quark. . . . . . . . . . . 133

7.2 Comparisons of b-tagging algorithm performance. . . . . . . 138

8.1 Example LLR distributions. . . . . . . . . . . . . . . . . 144

8.2 Graphical representation of $\mathrm{CL}_{\mathrm{B}}$ and $\mathrm{CL}_{\mathrm{S}+\mathrm{B}} \ldots \ldots \ldots \ldots$

9.1 Feynman diagram of the leading order $Z Z \rightarrow \ell^{-} \ell^{+} \nu \bar{\nu}$ process $\ldots 153$

$9.2 \quad$ Di-lepton invariant mass before $\boldsymbol{H}_{T}{ }^{\prime}$ cut. . . . . . . . . . 163

$9.3 \quad$ Di-lepton $p_{T}$ before $E_{T}{ }^{\prime}$ cut. . . . . . . . . . . . . . . 164

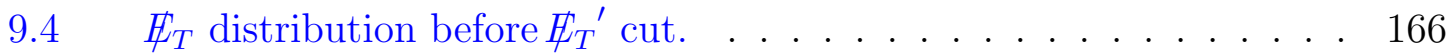

$9.5 \quad E_{T}{ }^{\prime}$ correction due to lepton resolution. . . . . . . . . 167

$9.6 \quad$ Calorimeter recoil correction for $E_{T}{ }^{\prime} \ldots \ldots \ldots \ldots \ldots$

9.7 Tracking activity correction for $\mathscr{H}_{T}{ }^{\prime}$. . . . . . . . . . . 169

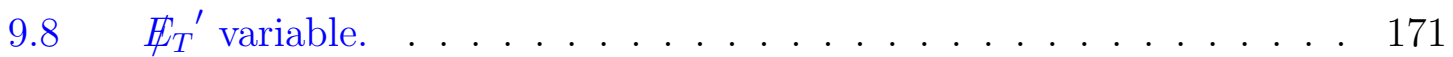

9.9 Likelihood input variables for the Run IIa di-electron channel. . . . 176

9.10 Likelihood input variables for the Run IIa di-muon channel. . . . . 177 
9.11 Likelihood input variables for the Run IIb di-electron channel. . . . 178

9.12 Likelihood input variables for the Run IIb di-muon channel. . . . . 179

9.13 Normalized likelihood distributions. . . . . . . . . . . . . . . . 180

9.14 Likelihood output. . . . . . . . . . . . . . . . . 181

9.15 Electron resolution systematic. . . . . . . . . . . . . 185

9.16 Muon resolution systematic. . . . . . . . . . . . . . 186

9.17 Event yield estimate for systematics of $\mathbb{E}_{T}{ }^{\prime}$ cut in the Run IIa dielectron sample. . . . . . . . . . . . . . . . . . . . . . . 190

9.18 Event yield estimate for systematics of $\mathbb{E}_{T}{ }^{\prime}$ cut in the Run IIa dimuon sample . . . . . . . . . . . . . . . . . . . 191

9.19 Event yield estimate for systematics of $\mathbb{F}_{T}{ }^{\prime}$ cut in the Run IIb dielectron sample. . . . . . . . . . . . . . . . . . . . . 192

9.20 Event yield estimate for systematics of $\mathbb{E}_{T}{ }^{\prime}$ cut in the Run IIb dimuon sample . . . . . . . . . . . . . . . . . . 193

9.21 LLR distribution of individual channels. . . . . . . . . . . . . . . . 194

9.22 LLR distribution for the full data set. . . . . . . . . . . . . . . 195

9.23 Cross section measurement cross check. . . . . . . . . . . . . 198

9.24 ZZ Observation: $Z Z \rightarrow \ell^{-} \ell^{+} \ell^{\prime-} \ell^{\prime+}$ final sample. . . . . . . . . 201

9.25 ZZ Observation: LLR plot for full combination. . . . . . . . . 205

10.1 Feynman diagram of the leading order $Z H \rightarrow \nu \nu b \bar{b}$ process . . . 208 
10.2 Distributions for jets failing the Good Jet criteria. . . . . . . . . 221

$10.3 \quad E_{T}{ }^{\prime}$ transverse component. . . . . . . . . . . . . . 227

$10.4 \quad \#_{T}$ Significance . . . . . . . . . . . . . . 228

10.5 ZH Analysis: Electroweak control sample with before b-tagging. . 233

10.6 ZH Analysis: Electroweak control sample with before $b$-tagging. . 234

10.7 ZH Analysis: Electroweak control sample with one b-tags. . . . . . 235

10.8 ZH Analysis: Electroweak control sample with one b-tags. . . . . . 236

10.9 ZH Analysis: Electroweak control sample with two b-tags. . . . . . 237

$10.10 Z H$ Analysis: Electroweak control sample with two b-tags. . . . . . 238

10.11 Data events with $\Delta \phi\left(\not p_{T}^{\mathrm{trk}}, E_{T}\right)>\pi / 2$ used to estimate the instrumental background . . . . . . . . . . . . . . . . . . 239

$10.12 \Delta \phi\left(\not p_{T}^{\mathrm{trk}}, \mathbb{E}_{T}\right)>\pi / 2$ in the electroweak control sample . . . . . . 240

10.13 Signal like and multijet events in the Leading Jet Pt vs .Leading Jet Track Pt plane . . . . . . . . . . . . . . . . . . . 241

10.14 ZH Analysis: Multijet control sample with before $b$-tagging. . . . . 242

10.15 ZH Analysis: Multijet control sample with before b-tagging. . . . . 243

10.16 ZH Analysis: Multijet control sample with one b-tag. . . . . . . . 244

10.17 ZH Analysis: Multijet control sample with one b-tag. . . . . . . . 245

$10.18 Z$ ZH Analysis: Multijet control sample with two b-tags. . . . . . . . 246

10.19 ZH Analysis: Multijet control sample with two b-tags. . . . . . . . 247 
10.20 Signal sample before $b$-tagging . . . . . . . . . . . . 250

10.21 Signal sample before $b$-tagging . . . . . . . . . . . . 251

10.22 Signal sample with one L6 b-tag . . . . . . . . . . . . . . 252

10.23 Signal sample with one L6 b-tag . . . . . . . . . . . . . . 253

10.24 Signal sample with two b-tags . . . . . . . . . . . . . 254

10.25 Signal sample with two b-tags . . . . . . . . . . . . 255

10.26 Multijet decision trees for a Higgs mass of $115 \mathrm{GeV} \ldots \ldots$. . . . 265

10.27 Single tag physics decision trees for a Higgs mass of $115 \mathrm{GeV}$. . 266

10.28 Double tag physics decision trees for a Higgs mass of $115 \mathrm{GeV}$. . 267

10.29 Signal Sample before b-tagging with MJ-DT cut . . . . . . . 268

10.30 Signal Sample before b-tagg and MJ-DT cut . . . . . . . . . 269

10.31 Signal sample with one b-tag after MJ-DT cut . . . . . . . . 270

10.32 Signal sample with one b-tag after MJ-DT cut . . . . . . . . 271

10.33 Signal sample with two L6 b-tags after MJ-DT cut . . . . . . . . 272

10.34 Signal sample with two L6 b-tags after MJ-DT cut . . . . . . . . 273

10.35 Expected and observed 95\% upper limits on the cross section ratios for Run IIb1. . . . . . . . . . . . . . . . . . . . . . . . . . . . 282

10.36 Expected and observed $95 \%$ upper limits on the cross section ratios for Run IIb2-3. . . . . . . . . . . . . . . . . . . . . . . . . . . 283

10.37 Expected and observed 95\% upper limits on the cross section ratios. 284 
10.38 Expected and observed $95 \%$ upper limits on the cross section ratios for the 2 tag sample. . . . . . . . . . . . . . . . 284

10.39 Expected and observed 95\% upper limits on the cross section ratios for the 1 tag sample. . . . . . . . . . . . . . . . . 285

10.40 Multijet decision trees for a the di-boson search. . . . . . . . . . . 289

10.41 Single tag physics decision trees for a di-boson search. . . . . . . . 290

10.42 Double tag physics decision trees for a di-boson search . . . . . . . 291

10.43 LLR distribution for di-boson search . . . . . . . . . . . . . . 292

11.1 Projected sensitivity to the SM Higgs. . . . . . . . . . . . 295

B.1 Main hardware components of the Level 1 Calorimeter Trigger. . . 306

B.2 The stages of the sliding windows algorithm. . . . . . . 306

B.3 Level 1 Calorimeter EM object definitions. . . . . . . . . . 307

B.4 Performance of the Run IIa and IIb L1Cal triggers. . . . . . . . . 307

C.1 ALPGEN angular reweighting function . . . . . . . . . 309

C.2 Electroweak control sample ALPGEN reweighting comparison plots 309

C.3 Signal sample ALPGEN reweighting comparison plots . . . . . . 310

D.1 MVA bl in the Run IIb1 electroweak control sample . . . . . . . . 311

D.2 MVA bl in the Run IIb2-3 electroweak control sample . . . . . . . 312

D.3 MVA bl operating points in the Run IIb1 electroweak control sample 312 
D.4 MVA bl operating points in the Run IIb2-3 electroweak control sample313

D.5 MVA bl in the Run IIb1 analysis sample . . . . . . . . . . . 313

D.6 MVA bl in the Run IIb2-3 analysis sample . . . . . . . . . . . 314

D.7 MVA bl operating points in the Run IIb1 analysis sample . . . . 314

D.8 MVA bl operating points in the Run IIb2-3 analysis sample . . . . 315

E.1 Run IIb1 MJ-DT input variable signal and background comparison. 319

E.2 Run IIb1 MJ-DT input variable signal and background comparison. 320

E.3 Run IIb23 MJ-DT input variable signal and background comparison. 321

E.4 Run IIb23 MJ-DT input variable signal and background comparison. 322

E.5 Run IIb1 single tag DT input variable signal and background comparison. . . . . . . . . . . . . . . . . 323

E.6 Run IIb1 single tag DT input variable signal and background comparison. . . . . . . . . . . . . . . . . . 324

E.7 Run IIb2-3 single tag DT input variable signal and background comparison. . . . . . . . . . . . . . . . . . 325

E.8 Run IIb2-3 single tag DT input variable signal and background com-

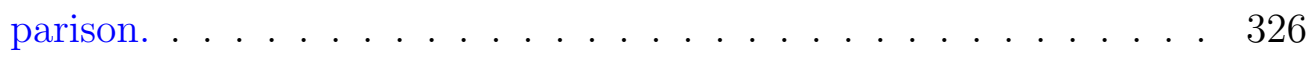

E.9 Run IIb1 double tag DT input variable signal and background comparison. . . . . . . . . . . . . . . . . 327 
E.10 Run IIb1 double tag DT input variable signal and background com-

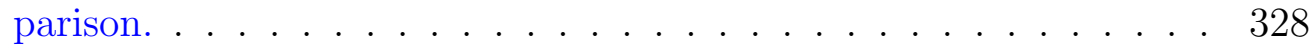

E.11 Run IIb2-3 double tag DT input variable signal and background comparison. . . . . . . . . . . . . . . . . . . . . . 329

E.12 Run IIb2-3 double tag DT input variable signal and background comparison. . . . . . . . . . . . . . . . . 330

E.13 Run IIb1 MJ-DT overtraining plots . . . . . . . . . . . . . . . 331

E.14 Run IIb2-3 MJ-DT overtraining plots . . . . . . . . . . . . . . 332

E.15 Run IIb1 1tag-DT overtraining plots . . . . . . . . . . . . . . 333

E.16 Run IIb2-3 1tag-DT overtraining plots . . . . . . . . . . . . . . 334

E.17 Run IIb1 2tag-DT overtraining plots . . . . . . . . . . . . . . . 335

E.18 Run IIb2-3 2tag-DT overtraining plots . . . . . . . . . . . . . . 336

F.1 Physics DTs (single tag) . . . . . . . . . . . . . 338

F.2 Physics DTs (double tag) . . . . . . . . . . . . . . 339

F.3 Physics DTs (single tag) . . . . . . . . . . . . . . . 340

F.4 Physics DTs (double tag) . . . . . . . . . . . . 341

G.1 Run IIb1 MJ-DT in pretag ew . . . . . . . . . . . . . . . 343

G.2 Run IIb1 MJ-DT in pretag qcd . . . . . . . . . . . . . . . . 344

G.3 Run IIb1 MJ-DT in pretag signal . . . . . . . . . . . . . . . 345 
G.4 Run IIb1 MJ-DT in single tag signal . . . . . . . . . . . . . . 346

G.5 Run IIb1 MJ-DT in single tag signal . . . . . . . . . . . . 347

G.6 Run IIb1 MJ-DT tree in double tag signal sample . . . . . . . . 348

G.7 Run IIb1 1tag Physics DT in single tag electroweak sample . . . . 349

G.8 Run IIb1 2tag Physics DT in double tag electroweak sample . . . . 350

G.9 Run IIb1 1tag Physics DT in single tag multi-jet sample . . . . . . 351

G.10 Run IIb1 2tag Physics DT in double tag multi-jet sample . . . . 352

H.1 Run IIb2-3 MJ-DT in pretag ew . . . . . . . . . . . . . . . . 354

H.2 Run IIb2-3 MJ-DT in pretag qcd . . . . . . . . . . . . . 355

H.3 Run IIb2-3 M.J-DT in pretag signal . . . . . . . . . . . . 356

H.4 Run IIb2-3 MJ-DT in single tag signal . . . . . . . . . . . . 357

H.5 Run IIb2-3 MJ-DT in single tag signal . . . . . . . . . . . . 358

H.6 Run IIb2-3 MJ-DT tree in double tag signal sample . . . . . . . . 359

H.7 Run IIb2-3 1tag Physics DT in single tag electroweak sample . . . 360

H.8 Run IIb2-3 2tag Physics DT in double tag electroweak sample . . . 361

H.9 Run IIb2-3 1tag Physics DT in single tag multi-jet sample . . . . . 362

H.10 Run IIb2-3 2tag Physics DT in double tag multi-jet sample . . . . 363

I.1 Single tag HF b-tagging relative DT shape systematic errors . . . 365

I.2 Single tag LF b-tagging relative DT shape systematic errors . . . 366 
I.3 Single tag JES relative DT shape systematic errors . . . . . . . 367

I.4 Single tag JER relative DT shape systematic errors . . . . . . . 368

I.5 Single tag Taggability/Vtx. Conf. relative DT shape systematic errors . . . . . . . . . . . . . . . . . . . 369

I.6 Single tag PDF relative DT shape systematic errors - $4 \ldots \ldots$

I.7 Single tag ALPGEN MLM relative DT shape systematic errors . . 370

I.8 Single tag ALPGEN Scale relative DT shape systematic errors . . 371

I.9 Single tag ALPGEN UE relative DT shape systematic errors _ . . 371

I.10 Single tag ALPGEN RW relative DT shape systematic errors . . . 372

I.11 All Single tag relative DT shape systematic errors . . . . . . . 372

J.1 double tag HF b-tagging relative DT shape systematic errors . . . 374

J.2 double tag LF b-tagging relative DT shape systematic errors _ . . 375

J.3 double tag JES relative DT shape systematic errors _ . . . . . 376

J.4 double tag JER relative DT shape systematic errors . . . . . . 377

J.5 double tag Taggability/Vtx. Conf. relative DT shape systematic

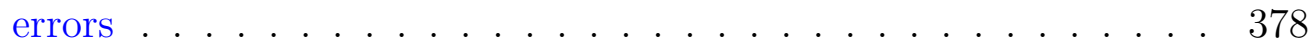

J.6 double tag PDF relative DT shape systematic errors - $4 \ldots \ldots$. . . 379

J.7 double tag ALPGEN MLM relative DT shape systematic errors . . 379

J.8 double tag ALPGEN Scale relative DT shape systematic errors . . 380 
J.9 double tag ALPGEN UE relative DT shape systematic errors . . . 380

J.10 double tag ALPGEN RW relative DT shape systematic errors . . . 381

J.11 All double tag relative DT shape systematic errors . . . . . . . . 381

K.1 Single tag HF b-tagging relative DT shape systematic errors . . . . 383

K.2 Single tag LF b-tagging relative DT shape systematic errors . . . 384

K.3 Single tag JES relative DT shape systematic errors . . . . . . . 385

K.4 Single tag JER relative DT shape systematic errors . . . . . . . . 386

K.5 Single tag Taggability/Vtx. Conf. relative DT shape systematic errors . . . . . . . . . . . . . . . . . 387

K.6 Single tag PDF relative DT shape systematic errors - $4 \ldots \ldots 388$

K.7 Single tag ALPGEN MLM relative DT shape systematic errors . . 388

K.8 Single tag ALPGEN Scale relative DT shape systematic errors . 389

K.9 Single tag ALPGEN UE relative DT shape systematic errors _ . . 389

K.10 Single tag ALPGEN RW relative DT shape systematic errors . . . 390

K.11 All Single tag relative DT shape systematic errors . . . . . . . 390

L.1 double tag HF b-tagging relative DT shape systematic errors . . . 392

L.2 double tag LF b-tagging relative DT shape systematic errors _ . . 393

L.3 double tag JES relative DT shape systematic errors _ . . . . . 394

L.4 double tag JER relative DT shape systematic errors _ . . . . . 395 
L.5 double tag Taggability/Vtx. Conf. relative DT shape systematic errors . . . . . . . . . . . . . . . . . 396

L.6 double tag PDF relative DT shape systematic errors - $4 \ldots \ldots 396$

L.7 double tag ALPGEN MLM relative DT shape systematic errors . . 397

L.8 double tag ALPGEN Scale relative DT shape systematic errors . . 397

L.9 double tag ALPGEN UE relative DT shape systematic errors . . . 398

L.10 double tag ALPGEN RW relative DT shape systematic errors . . . 398

L.11 All double tag relative DT shape systematic errors . . . . . . . . . 399 


\section{List of Tables}

1.1 Fermions . . . . . . . . . . . . . . . . . . . . . 33

1.2 Bosons . . . . . . . . . . . . . . . . . . . . . . 34

2.1 Tevatron operatering characteristics for Run I, IIa, and IIb . . . . . 61

4.1 Muon Types. . . . . . . . . . . . . . . . . . . . . . . . . . . 98

4.2 Electron identification operating points. . . . . . . . . . . . 102

$4.3 \tau$ decay modes. . . . . . . . . . . . . . . . . . 105

9.1 Run IIa signal and background Monte Carlo samples . . . . . . . . 154

9.2 Run IIb signal and background Monte Carlo samples . . . . . . . . 155

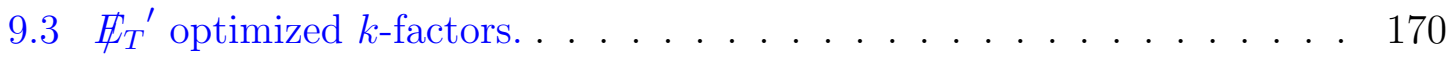

9.4 $\mathscr{H}_{T}{ }^{\prime}$ cut values for the di-electron and di-muon channels in RunIIa and RunIIb . . . . . . . . . . . . . . . . . 170

9.5 Run IIa di-electron cut-flow. . . . . . . . . . . . . . . . . . . 172

9.6 Run IIa di-muon cut-flow. . . . . . . . . . . . . . . . . . . 172

9.7 Run IIb di-electron cut-flow. . . . . . . . . . . . . . . . . . 173

9.8 Run IIb di-muon cut-flow. . . . . . . . . . . . . . . . . . 173

9.9 Jet related systematic uncertainties for the di-electron final state. . . 183

9.10 Jet related systematic uncertainties for the di-muon final state. . . . . 183

9.11 Uncertainties on the $W+$ jets normalization. . . . . . . . . 187

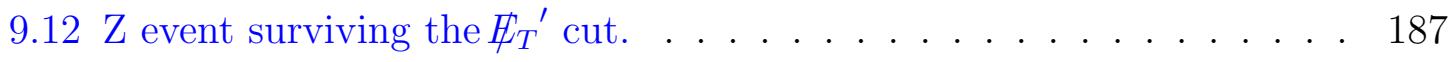


9.13 Pdf uncertainties on the $\frac{A_{Z}}{A_{Z Z}}$ ratio as estimated with the CTEQ prescription. . . . . . . . . . . . . . . . . . . 188

$9.14 Z Z \rightarrow \ell^{-} \ell^{+} \nu \bar{\nu}$ significance . . . . . . . . . . . . . . 196

9.15 Acceptances $\times$ efficiency and number of events after the extra activity cut for the $Z / \gamma^{*} \rightarrow \ell \ell\left(60<M_{\ell \ell}<130 \mathrm{GeV}\right)$ and the $Z Z \rightarrow \ell \ell \nu \nu$ samples. . . . . . . . . . . . . . . . . . . . 197

9.16 Sources of Systematic Uncertainties in the $Z Z \rightarrow \ell^{+} \ell^{-} \nu \bar{\nu}$ channel. All correlations noted refer to uncertainties within this channel alone. . . 204

9.17 Sources of Systematic Uncertainties in the $Z Z \rightarrow \ell^{+} \ell^{-} \ell^{\prime+} \ell^{\prime-}$ channel. All correlations noted refer to uncertainties within this channel alone. 204

9.18 Sources of systematic uncertainties present in both $Z Z$ analyses. . . . 205

9.19 Significance values estimated all $Z Z$ channels. . . . . . . . . . . . 206

10.2 Signal MC samples with cross sections . . . . . . . . . . . . 209

10.1 Data taking epochs. . . . . . . . . . . . . . . . . 209

10.3 Cut-flow for $1.2 \mathrm{fb}^{-1}$ RunIIb1 pre-selection. . . . . . . . . . . . . . . 214

10.4 Cut-flow for $4.9 \mathrm{fb}^{-1}$ RunIIb2-3 pre-selection. . . . . . . . . . . . . 215

10.5 Cut-flow for RunIIb1 selection . . . . . . . . . . . . . . . . . . 224

10.6 Cut-flow for RunIIb2-3 selection . . . . . . . . . . . . . . 225

10.7 Scale factors . . . . . . . . . . . . . . . . . . 231

10.8 Run IIb number of events in the electroweak control sample . . . . . 232

10.9 Run IIb number of events in the signal sample . . . . . . . . . . . . . 249

10.10Decision Tree Settings _. . . . . . . . . . . . . . 256

10.11Variables and rankings of inputs to the Run IIb1 multi-jet decision tree.257

10.12Variables and rankings of inputs to the Run IIb1 single tag physics decision tree. . . . . . . . . . . . . . . . . . . . . . 258 
10.13Variables and rankings of inputs to the Run IIb1 double tag physics decision tree. . . . . . . . . . . . . . . . . . . . 259

10.14Variables and rankings of inputs to the Run IIb2-3 multi-jet decision

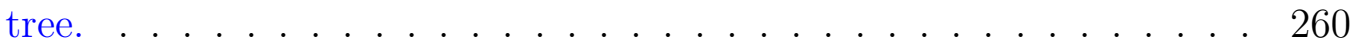

10.15Variables and rankings of inputs to the Run IIb2-3 single tag physics decision tree. . . . . . . . . . . . . . . . . 261

10.16Variables and rankings of inputs to the Run IIb2-3 double tag physics decision tree. . . . . . . . . . . . . . . . . 262

10.17Run IIb number of events in the signal sample after the multijet-DT cut263

10.18Systematic correlation table . . . . . . . . . . . . . 276

10.19Relative systematic uncertainties for the RunIIb1 single tag analysis . 277

10.20Relative systematic uncertainties for the RunIIb1 double tag analysis 278

10.21Relative systematic uncertainties for the RunIIb23 single tag analysis 279

10.22Relative systematic uncertainties for the RunIIb23 double tag analysis 280

10.23CLfast limits to the Standard Model Higgs production using BDT out-

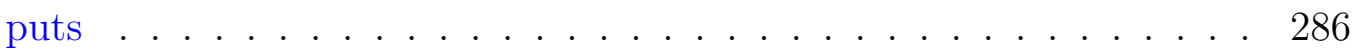

10.24CLfit2 limits to the Standard Model Higgs production using BDT outputs287 10.25Ratio of the expected (observed) limit to the Standard Model prediction for a di-boson search. . . . . . . . . . . . . . . . 288

E.1 Ten of the twenty kinematic variables used in the DTs . . . . . . . 317

E.2 Ten of the twenty kinematic variables used in the DTs . . . . . . . . 318 


\section{Chapter 1}

\section{Introduction}

Science is the acquisition and organization of knowledge in the form of testable explanations and predictions. At the most basic level known, where things cannot be further subdivided, the science is particle physics. The items of interest are fundamental particles which are not composite objects, but rather the most basic building blocks of the universe. The laws governing these particles are gathered in a Quantum Field Theory (QFT) known as the Standard Model (SM). We know that these laws are not the final statement, that there is physics beyond the SM, which is exactly where this field is headed. This work centers on the remaining element of the SM which is yet to be experimentally verified, the generation of mass.

This dissertation discusses work that began with the ancient Greek's idea of the atom. Since the size of this document should be somewhat reasonable, we skip ahead to just under 30 years ago when two multi-purpose detectors, D $\varnothing$ and Collider Detector at Fermilab (CDF), were constructed at Fermilab with the goal of continuing the research being conducted at European Organization for Nuclear Research (CERN). Countless hours have been dedicated to the design, construction, calibration, and understanding of the accelerator and detectors yielding ground breaking results such 
as the discovery of the top quark $[4,5]$ and hints as to why the universe is not equally composed of matter and antimatter but rather prefers the former [6]. Amongst the numerous physics analyses being conducted there has been one in particular that is of great interest to the community; the search for the Higgs boson.

This search resulting in a discovery or exclusion was thought out of the reach of the Tevatron but as the data began to pile up, it was moved to a long shot. With the ingenuity of the collaborations the problem was reconsidered and deemed possible. Finally, with an extension of the operations of the Tevatron through 2014, it was thought probable. But, being a time of financial strain, this extension was cancelled and the search once again is on uneasy ground at the Tevatron. Higgs production is rarest tree level SM process and before being sensitive it the more common processes must be observed. The final steps on this road to the Higgs is paved with the production of two gauge bosons. In this work we discuss the penultimate step, $Z Z$ production in Chapter 9, and the final step, the $Z H$ search in Chapter 10.

Before discussing the analyses themselves, the theory, experimental apparatus, and the general toolbox of the $\mathrm{D} \varnothing$ collaboration must be introduced. Chapter 2 described the accelerator and detector. The simulation of the theory and the detector are discussed in Chapter 3 while the reconstruction of objects from the information within the detector are reviewed in Chapter 4. At the analysis level several tools are need. Handling one of the more difficult signatures, missing transverse energy is discussed in Chapter 5. Chapter 6 involves multivariate techniques while bottom quark identification and the statistical interpretation of the final set of selected events are described in Chapter 7 and 8 respectively. With all the machinery in place, we continue down the road to the Higgs.

Let us begin with the Standard Model of particle interactions and the Higgs mechanism. For a more complete review, please refer to $[7,8,9,10]$. 


\subsection{The Standard Model Particles}

The standard model (SM) of particle interactions is a consistent amalgamation of the electromagnetic, chromodynamic, and weak interactions in terms of a quantum field theory. The model contains two basic types of particles, fermions and bosons which differ by the amount of inherent angular momentum, or spin, they possess in units of $\hbar$. Fermions have half-integer spin while bosons have integer spin. Within the ranks of fermions and bosons, particles are distinguished by the charge they carry. Quantum numbers, such as the spin and charge of a particle, are used to characterize the particle's state. The visible universe is made of two types of fermions, leptons and quarks. Of the 12 different fermions in the SM, the particles we interact with on a regular basis, are predominantly composed of three, the electron which is a lepton and the up and down quarks. The fermions, shown in Table 1.1, are grouped into three families, or generations, each containing two leptons, one with charge of -1 and

the other neutral, and two flavors of quarks, one of charge $+\frac{2}{3}$ and the other $-\frac{1}{3}$. Each particle has an associated anti-particle with the opposite charge i.e., the positron is the anti-electron and has charge +1 . Neutrinos, the neutral leptons, have very small masses and interact weakly with matter. Leptons exist freely in nature while quarks do not.

The three theories within the SM correspond to three forces which are mediated by bosons summarized in Table 1.2. That is, two particles do not collide, but rather a force carrying boson is passed between the two. The electromagnetic force governs the motion of electrons in circuits, the magnets sticking to the refrigerator, and all those annoying static shocks you get when reaching for the door. It is carried by the massless photon and the coupling strength is proportional to the electric charge. A consequence of having a massless force propagator is that this force has an infinite 
range. The strong force is responsible for holding the proton together and is mediated between quarks by eight massless gluons. The coupling is known as color and the quantum numbers associated with the three possible colors are usually called blue, green, and red. The increase in complexity comes with an increase in richness. Free quarks are never seen free in nature, a property known as confinement. Only states which are "colorless", such as quark-antiquark mesons or three quark baryons, are observed. Also at very short distances, or high energies, the quarks do not interact with each other very much. This behavior is referred to as asymptotic freedom. Even though the force mediating bosons are masseless, the range of the strong force is finite. Leptons do not carry color and therefore do not experience the color force.

The weak force is carried by the massive $W^{ \pm}$and $Z$ bosons therefore limiting it to a small range such as within an atomic nucleus. Despite this limitation, this force has a very interesting property. It is the only means by which the flavor of a particle can change and the weak interaction violates parity as well as CP symmetry. Flavor changing interactions allow neutrons to decay into protons $\left(n \rightarrow p+e^{-}+\bar{\nu}_{e}\right)$. Weak isospin is a name for the quantum number which relates to the strength by which a particle couples to the weak force. All fermions feel this force. There remains one boson, referred to as the Higgs boson, which has yet to be observed, if it does actually exist. Its place in the SM will be explained later, but in short, this boson is theorized to be a byproduct of a broken symmetry which gives rise to mass.

\begin{tabular}{|l|cc|cc|cc|}
\hline \multirow{2}{*}{ Type } & \multicolumn{2}{|c|}{$1^{\text {st }}$ Generation } & \multicolumn{2}{c|}{$2^{\text {nd }}$ Generation } & \multicolumn{2}{c|}{$3^{\text {rd }}$ Generation } \\
& Particle & $\mathrm{m}(\mathrm{MeV})$ & Particle & $\mathrm{m}(\mathrm{MeV})$ & Particle & $\mathrm{m}(\mathrm{MeV})$ \\
\hline Lepton $(-1 e)$ & Electron $(\mathrm{e})$ & 0.511 & Muon $(\mu)$ & 105.7 & Tau $(\tau)$ & 1776.8 \\
Neutrino & Electron $\left(\nu_{e}\right)$ & $<2 \times 10^{-6}$ & Muon $\left(\nu_{\mu}\right)$ & $<0.19$ & Tau $\left(\nu_{\tau}\right)$ & $<18.2$ \\
\hline Quark $\left(\frac{2}{3} e\right)$ & $\operatorname{up}(u)$ & $1.7-3.3$ & charm $(c)$ & $1.27 \times 10^{3}$ & top $(t)$ & $172.0 \times 10^{3}$ \\
Quark $\left(-\frac{1}{3} e\right)$ & down $(d)$ & $4.1-5.8$ & strange $(s)$ & 101 & bottom $(b)$ & $4.20 \times 10^{3}$ \\
\hline
\end{tabular}

Table 1.1: The three generations of fermions. 


\begin{tabular}{|l|c|c|c|}
\hline Particle & Mass $(\mathrm{GeV})$ & Spin & Force \\
\hline photon $(\gamma)$ & 0 & 1 & Electromagnetic \\
$Z^{0}$ & 91.1876 & 1 & Weak \\
$W^{ \pm}$ & 80.398 & 1 & Weak \\
gluon $(g)$ & 0 & 1 & Strong \\
\hline Higgs $(\mathrm{H})$ & $114.5<m_{H}<157$ or $m_{H}>173$ & 0 & - \\
\hline
\end{tabular}

Table 1.2: Bosons, the force carrying particles of the Standard Model.

\subsection{The Standard Model}

The SM is based on the gauge group $U(1)_{Y} \times S U(2)_{L} \times S U(3)_{C}$. The SM is highly successful but the missing piece is the Higgs boson. We discuss now the various elements of the SM.

\subsubsection{The Theory of Quantum Electrodynamics}

The simplest gauge theory is quantum electrodynamics (QED) described by the $U(1)$ gauge group. The Lagrangian is given by:

$$
\mathcal{L}_{Q E D} \equiv \bar{\psi}\left(i \gamma^{\mu} D_{\mu}-m\right) \psi-\frac{1}{4} F_{\mu \nu} F^{\mu \nu}
$$

where the field $\psi$ represents a spinor field of spin $1 / 2, \gamma^{\mu}$ are the Dirac matrices, $D_{\mu}$ is the covariant derivative, and $F_{\mu \nu}$ is the electromagnetic field tensor which describes the electromagnetic field strength. The covariant derivative is given by

$$
D_{\mu}=\partial_{\mu}+i e A_{\mu}
$$

with $A_{\mu}$ being the gauge field. We can identify this field as the photon and its coupling strength to any field is given by $e$ which we identify as the electromagnetic charge.

Let us perform the exercise of taking a simple theory and building a local gauge 
invariant model. Using the anti-commutating Dirac matrices, $\gamma^{\mu}$, where

$$
\left\{\gamma^{\mu}, \gamma^{\nu}\right\}=2 g^{\mu \nu}
$$

as the basis vectors for contravariant vectors in a Minkowski space, a relativistic theory for a free electron, the Dirac free electron theory is written as

$$
L=\bar{\psi}(x)\left(i \gamma^{m u} \partial_{\mu}-m\right) \psi(x)
$$

It can be easily seen that this equation is invariant under a global phase change

$$
\begin{aligned}
\psi(x) \rightarrow \psi^{\prime}(x) & =e^{-i \alpha} \psi(x) \\
\bar{\psi}(x) \rightarrow \bar{\psi}^{\prime}(x) & =e^{i \alpha} \bar{\psi}(x)
\end{aligned}
$$

but to have a gauge invariant theory, that is invariant under

$$
\begin{aligned}
& \psi(x) \rightarrow \psi^{\prime}(x)=e^{-i \alpha(x)} \psi(x) \\
& \bar{\psi}(x) \rightarrow \bar{\psi}^{\prime}(x)=e^{i \alpha(x)} \bar{\psi}(x)
\end{aligned}
$$

some alteration is needed. A gauge-covariant derivative, $D_{\mu}$, is needed to replace $\partial_{\mu}$ so that

$$
\bar{\psi}(x) D_{\mu} \psi(x) \rightarrow \bar{\psi}^{\prime}(x) D_{\mu} \psi^{\prime}(x)=\bar{\psi}(x) D_{\mu} \psi(x)
$$

This is achieved by introducing a vector field $A_{\mu}(x)$ in the covariant derivative as

$$
D_{\mu} \psi=\left(\partial_{\mu}+i e A_{\mu}\right) \psi
$$


and requiring that the gauge field transforms as

$$
A_{\mu} \rightarrow A_{\mu}^{\prime}=A_{\mu}+\frac{1}{e} \partial_{\mu} \alpha(x)
$$

Finally to make the gauge field truly dynamic a simple gauge invariant term needs to be added. For this, the field tensor, $F_{\mu \nu}$, defined as

$$
F_{\mu \nu}=\partial_{\mu} A_{\nu}-\partial_{\nu} A_{\mu}
$$

is used. This gives the gauge invariant QED Lagrangian as

$$
\mathcal{L}_{Q E D}=\bar{\psi}\left(i \gamma^{\mu} D_{\mu}-m\right) \psi-\frac{1}{4} F_{\mu \nu} F^{\mu \nu}
$$

The coupling, $e$, between the spinor field, $\psi$, and the gauge field, $A_{\mu}$, is identified as the electric change and contained in the covariant derivative whose form was constructed to maintain invariance after the electron field was transformed. The coupling of the photon to any matter field derives from the transformation property under the symmetry group. Expanding the covariant derivative gives

$$
\mathcal{L}_{Q E D}=\bar{\psi}\left(i \gamma^{\mu} \partial_{\mu}-m\right) \psi-\underbrace{e \bar{\psi} \gamma^{\mu} \psi A_{\mu}}_{\text {Interaction Term }}-\frac{1}{4} F_{\mu \nu} F^{\mu \nu}
$$

an explicit view of this coupling strength in the interaction term. Note a mass term for the photon such as $A_{\mu} A^{\mu}$ is not invariant and therefore not included.

\subsubsection{The Theory of Quantum Chromodynamics}

Quantum Chromodynamics (QCD), describes interactions via the strong force which controls the dynamics of quarks and gluons. By imposing invariance under $S U(3)_{C}$ 
local gauge transformations a QCD Lagrangian can be constructed. Such an exercise is done in an analogous way to what was show above to produce $\mathcal{L}_{Q E D}$. The QCD Lagrangian is give by

$$
\mathcal{L}_{Q C D}=\sum_{i}^{\text {flavors }} \bar{\psi}_{i}\left(i \gamma^{\mu} D_{\mu}-m_{i}\right) \psi_{i}-\frac{1}{4} G_{\mu \nu}^{a} G_{a}^{\mu \nu}
$$

where in the index $a$ runs over the eight color degrees of freedom, $i$ represents one of the six quark flavors, and $G_{\mu \nu}^{a}$, given by

$$
G_{\mu \nu}^{a}=\partial_{\mu} A_{\nu}^{a}-\partial_{\nu} A_{\mu}^{a}+g f^{a b c} A_{\mu}^{b} A_{\nu}^{c}
$$

is the gauge invariant gluon field strength tensor, analogous to the electromagnetic field strength tensor $F_{\mu \nu}$ of QED. Here the covariant derivative is given by

$$
D_{\mu}=\partial_{\mu}+i g_{s} A_{\mu}^{a} t^{a}
$$

where $A_{\mu}$ the vector field representing the gluons and $t^{a}$ are the generators of the $S U$ (3) group which obey the following commutation relation

$$
\left[t^{a}, t^{b}\right]=i f^{a b c} t^{c}
$$

where $f^{a b c}$ is called the structure constant. The forms are very similar to those seen in the QED Lagrangian but with more complexity due to the additional degrees of freedom. Expanding this Lagrangian into a kinetic term,

$$
\mathcal{L}_{Q C D}^{\text {kinetic }}=-\frac{1}{4}\left(\partial_{\mu} A_{\nu}^{a}-\partial_{\nu} A_{\mu}^{a}\right)\left(\partial^{\mu} A_{a}^{\nu}-\partial^{\nu} A_{a}^{\mu}\right)+\sum_{i}^{\text {flavors }} \bar{\psi}_{i}\left(i \gamma^{\mu} \partial_{\mu}-m_{i}\right) \psi_{i}
$$


an interaction term for quarks and gluons which shows all quarks and gluons couple with the same strength.

$$
\mathcal{L}_{Q C D}^{\mathrm{q}-\mathrm{g}}=-g_{s} \sum_{i}^{\text {flavors }} A_{\mu}^{a} \bar{\psi}_{i} \gamma^{\mu} A_{\mu}^{a} t^{a} \psi_{i}
$$

and a gluon triplet and quartic self-interaction term

$$
\mathcal{L}_{Q C D}^{\mathrm{g}-\mathrm{self}}=\frac{g_{s}}{2} f^{a b c}\left(\partial_{\mu} A_{\nu}^{a}-\partial_{\nu} A_{\mu}^{a}\right)\left(A_{b}^{\mu} A_{c}^{\nu}\right)-\frac{g_{s}}{2} f^{a b c} f_{a d e} A_{b}^{\mu} A_{c}^{\nu} A_{\mu}^{d} A_{\nu}^{e} .
$$

The interactions of Equation 1.19 term, not present in QED, arises because gluons themselves carry color quantum numbers.

\subsubsection{The Electroweak Theory}

When probing energies on the order of $100 \mathrm{GeV}$ the electromagnetic and weak forces are explicitly the same force, the electroweak force. This unification is achieved mathematically in a $S U(2)_{L} \times U(1)_{Y}$ gauge group. The $S U(2)_{L}$ and $U(1)_{Y}$ groups represent the weak isospin $(\mathbf{T})$ and hypercharge $(\mathbf{Y})$ space respectively. This representation conforms to the fact that only left-handed fermions participate in flavor-changing weak interactions and the lepton doublet contains a charged and neutral component. The three gauge field of $S U(2)_{L}$ are $W_{\mu}^{i=1,2,3}$ with coupling constant $g$ and $U(1)_{Y}$ has one gauge field $B_{\mu}$ and coupling constant $g^{\prime}$. The generators of the $S U(2)$ group obey the algebra

$$
\left[T_{a}, T_{b}\right]=i \epsilon_{a b c} T_{c}
$$

and the simplest matrix representations of $T_{i}$ are the Pauli matrices, $T_{i}=\left(\sigma_{i} / 2\right)$. 
From the previous two examples we know the gauge field kinetic terms will be

$$
\mathcal{L}_{E W}^{\text {gauge }}=-\frac{1}{4} W_{a}^{\mu \nu} W_{\mu \nu}^{a}-\frac{1}{4} B^{\mu \nu} B_{\mu \nu}
$$

with

$$
W_{\mu \nu}^{i}=\partial_{\mu} W_{\nu}^{i}-\partial_{\nu} W_{\mu}^{i}+g \epsilon^{i j k} A_{\mu}^{j} A_{\nu}^{k}
$$

and

$$
B_{\mu \nu}=\partial_{\mu} B_{\nu}-\partial_{\nu} B_{\mu}
$$

The purely right handed component involves a singlet of the lepton field, $R$, only interacts with the $B_{\mu}$ field as

$$
\mathcal{L}_{E W}^{\text {singlet }}=i \bar{R} \gamma^{\mu} D_{\mu} R=i \bar{R} \gamma^{\mu}\left(\partial_{\mu}+i g^{\prime} \frac{Y}{2} B_{\mu}\right) R
$$

and the lepton doublet interacts with gauge fields from both groups as

$$
\mathcal{L}_{E W}^{\text {doublet }}=i \bar{L} \gamma^{\mu}\left(\partial_{\mu}+i g^{\prime} \frac{Y}{2} B_{\mu}+i g T_{a} W_{\mu}^{a}\right) L
$$

where $\mathrm{L}$ is the lepton doublet

$$
L=\left(\begin{array}{c}
\nu \\
\ell^{-}
\end{array}\right) .
$$

This is a convenient representation but to connect back to experimental results, the 
mass eigenstates, $\gamma, Z$, and $W^{ \pm}$are written as superpositions of $B_{\mu} a n d W_{\mu}^{a}$ as follows

$$
\begin{aligned}
W_{\mu}^{ \pm} & =\frac{1}{\sqrt{2}}\left(W_{\mu}^{1} \pm W_{\mu}^{2}\right) \\
Z_{\mu} & =-\sin \left(\theta_{W}\right) B_{\mu}+\cos \left(\theta_{W}\right) W_{\mu}^{3} \\
A_{\mu} & =\cos \left(\theta_{W}\right) B_{\mu}+\sin \left(\theta_{W}\right) W_{\mu}^{3} \\
\cos \left(\theta_{W}\right) & =\frac{g}{\sqrt{g^{2}+g^{\prime 2}}}
\end{aligned}
$$

where $\cos \left(\theta_{W}\right)$ is the weak mixing angle or the Weinberg angle which mixes the $B_{\mu}$ and $W_{\mu}^{3}$ fields. In this scheme, the electromagnetic charge, $q$, is given by $T_{3}+\frac{Y}{2}$.

In light of these definitions, the portion of the Lagrangian involving gauge field self-interactions, $\mathcal{L}_{E W}^{\text {gauge }}$, can be rewritten to show explicitly three and four gauge boson interactions. The three point interaction is given by

$$
\begin{aligned}
\mathcal{L}_{E W}^{3-\text { gauge }}= & -i g\left(\partial_{\rho} W_{\mu}^{+}\right) W_{\nu}^{3} W_{\sigma}^{-}\left(g^{\rho \sigma} g^{\mu \nu}-g^{\rho \nu} g^{\mu \sigma}\right) \\
& -i g\left(\partial_{\rho} W_{\mu}^{-}\right) W_{\nu}^{3} W_{\sigma}^{+}\left(g^{\rho \nu} g^{\mu \sigma}-g^{\rho \mu} g^{\nu \sigma}\right) \\
& -i g\left(\partial_{\rho} W_{\nu}^{3}\right) W_{\mu}^{+} W_{\sigma}^{-}\left(g^{\rho \mu} g^{\nu \sigma}-g^{\rho \sigma} g^{\nu \mu}\right)
\end{aligned}
$$

and the four point is given by

$$
\mathcal{L}_{E W}^{4-\text { gauge }}=\frac{g^{2}}{4}\left(W_{\mu}^{+} W_{\nu}^{+} W_{\sigma}^{-} W_{\rho}^{-}-2 W_{\mu}^{+} W_{\nu}^{3} W_{\sigma}^{3} W_{\rho}^{-}\right) Q^{\mu \nu \rho \sigma}
$$

where

$$
Q^{\mu \nu \rho \sigma}=2 g^{\mu \nu} g^{\rho \sigma}-g^{\mu \rho} g^{\nu \sigma}-g^{\mu \sigma} g^{\nu \rho}
$$

Each term in both has a contribution from the charged $W$ fields and none include just a $Z$ or $A_{\mu}$ term meaning the SM does not have local $Z Z Z, Z Z \gamma$, or $Z \gamma \gamma$ vertex. Measuring the $W W$ and $Z Z$ cross sections is a direct test of the SM interactions and 
any excess could be a sign of physics beyond the standard model.

\subsubsection{The Higgs Mechanism}

As written above the SM, to some degree, is at odds with experimental results. Three of the electroweak force mediating bosons, $W^{ \pm}$and $Z$, are massive yet $\mathcal{L}_{E W}$ contains no mass terms for them. Explicitly adding such a term would alter the high energy behavior of the theory is a undesirable way. To obtain mass terms a symmetry can be spontaneously broken. In 1964, spontaneous symmetry breaking was formulated by Higgs, Brout, Englert, Guralnik, Hagen and Kibble [11] [12] [13] [14].

A straightforward way to show the phenomena is in the context of the Lagrangian for a real scalar field:

$$
\mathcal{L}_{\text {scalar }}=\frac{1}{2}\left(\partial_{\mu} \phi\right)^{2}-\left[\frac{1}{2} \mu^{2} \phi^{2}+\frac{1}{4} \lambda \phi^{4}\right]
$$

For the case of $\mu^{2}>0$ the mass of the particle is given by $\mu$ and the four point self interaction coupling strength is $\lambda$. The minimization of the potential gives the ground state, or the vacuum, which corresponds to $\phi=0$. A more interesting case is if $\mu^{2}<0$.

As shown in Figure 1.1, the ground state is either $\phi= \pm v$ with $v=\sqrt{-\mu^{2} / \lambda}$ and this is the point where perturbation calculations should be expanded around. Choosing the minimum as $+v$ or $-v$ is equivalent and making this decision spontaneously breaks the symmetry. Let us choose $+v$ as the minimum and write the field as $\phi(x)=v+\eta(x)$ in order to write the perturbative Lagrangian as

$$
\mathcal{L}_{\text {scalar }}^{\prime}=\frac{1}{2}\left(\partial_{\mu} \eta\right)^{2}-\lambda v^{2} \eta^{2}-\lambda v \eta^{3}-\frac{1}{4} \lambda \eta^{4}+\text { interaction terms. }
$$

Now we have a massive field $v$ with a mass of $\sqrt{-2 \mu^{2}}$. Solving $\mathcal{L}_{\text {scalar }}$ and $\mathcal{L}_{\text {scalar }}^{\prime}$ 
exactly will yield the same physics, but if solving perturbatively, only $\mathcal{L}_{\text {scalar }}^{\prime}$ will give the correct physics.
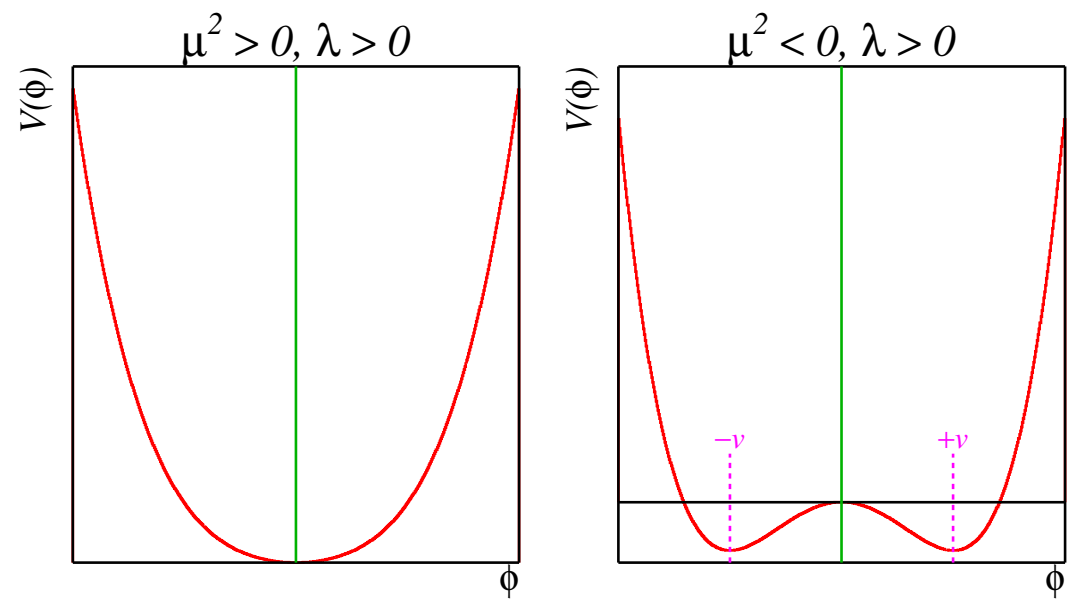

Figure 1.1: The minima of the potential for the case of $\mu^{2}>0$ (left) and $\mu^{2}<0$ (right) for a scalar field.

This is nice but what we want is a massive gauge field. Taking this one step further and using the Lagrangian for a complex scalar field which is locally gauge invariant under the $U(1)$ group,

$$
\mathcal{L}_{\text {complex }}=D^{\mu} \phi^{*} D_{\mu} \phi-\frac{1}{4} F^{\mu \nu} F_{\mu \nu}-\left[\mu^{2} \phi^{*} \phi+\lambda\left(\phi^{*} \phi\right)^{2}\right]
$$

and again looking at the case where $\mu^{2}<0$ we find exactly that. By writing the complex field as a combination of two real fields

$$
\phi=\frac{1}{\sqrt{2}}\left(\phi_{1}+i \phi_{2}\right)
$$

the minimum is mapped out by a circle:

$$
\phi_{1}^{2}+\phi_{2}^{2}=v^{2} \text { with } v=\sqrt{\frac{-\mu^{2}}{\lambda}}
$$


as shown in Figure 1.2. The choice of $\phi_{1}=v$ and $\phi_{2}=0$ spontaneously breaks the symmetry and again using a substitution we can write

$$
\phi=\frac{1}{\sqrt{2}}(v+\eta(x)+i \xi(x))
$$

Using Equation 1.40 in the Lagrangian gives

$$
\begin{aligned}
\mathcal{L}_{\text {complex }}^{\prime}= & \frac{1}{2}\left(\partial_{\mu} \eta\right)^{2}-v^{2} \lambda \eta^{2} \\
& +\frac{1}{2}\left(\partial_{\mu} \xi\right)^{2} \\
& -\frac{1}{4} F^{\mu \nu} F_{\mu \nu}+\frac{1}{2} q^{2} v^{2} A_{\mu} A^{\mu}-e v A_{\mu} \partial^{\mu} \xi+\text { interaction terms. }
\end{aligned}
$$

Similar to what was found for the scalar field above, the first line gives the kinetic term and mass, $\sqrt{2 v^{2} \lambda}=\sqrt{-2 \mu^{2}}$, of the scalar field $\eta$. The second line is the kinetic term for a massless scalar Nambu-Goldstone boson, $\xi$. One boson of this type originates for each generator of the global symmetry, in this case rotational symmetry, that is broken and can be thought of as excitations of the field in the direction of the broken symmetry. The final line gives the kinetic terms of the gauge field, $A_{\mu}$, its interaction term with the Goldstone boson, and the term we were looking for, a mass term. The once massless gauge field has acquired a mass of $q v$ which is proportional to the coupling strength. The fact that the Goldstone boson is massless derives from the fact that in the tangential direction there is an infinite degeneracy. Fixing the gauge by choosing a particular value for the polar angle would remove this degeneracy and give two massive fields. The choice of the unitary gauge 'absorbs' $\chi$ into the gauge 
field $A_{\mu}$ allowing us to write

$$
\begin{aligned}
\mathcal{L}_{\text {complex }}^{\prime \prime}= & \frac{1}{2}\left(\partial_{\mu} h\right)^{2}-v^{2} \lambda h^{2} \\
& -\frac{1}{4} F^{\mu \nu} F_{\mu \nu}+\frac{1}{2} q^{2} v^{2} A_{\mu} A^{\mu}+\text { interaction terms }
\end{aligned}
$$

a Lagrangian for two massive fields.

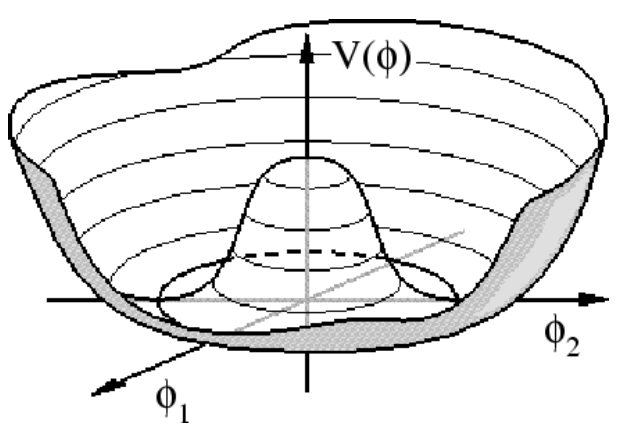

Figure 1.2: The minima of the potential for the case of $\mu^{2}<0$ for a complex scalar field.

We now extend the Higgs mechanism to a $U(1)_{Y} \times S U(2)_{L}$ invariant Lagrangian. With a doublet of complex scalar fields we can add four degrees of freedom to the Lagrangian which will create three Goldstone bosons if the symmetry is broken correctly. Three of the four gauge fields will absorb these extra degrees of freedom and obtain a mass. Adding an additional kinetic and potential term of the form

$$
\mathcal{L}_{H}=\left(D^{\mu} \Phi\right)^{\dagger}\left(D_{\mu} \Phi\right)-\left[\mu^{2} \Phi^{\dagger} \Phi+\lambda\left(\Phi^{\dagger} \Phi\right)^{2}\right]
$$

where

$$
\Phi=\left(\begin{array}{c}
\phi^{+} \\
\phi^{0}
\end{array}\right)
$$


and the covariant derivative is given by

$$
D_{\mu}=\partial_{\mu}+\frac{i g}{2} \sigma^{i} W_{\mu}^{i}+\frac{i g^{\prime}}{2} B^{\mu}
$$

with $\sigma^{i}$ again representing the Pauli matrices generates masses for the $W^{ \pm}$and $Z$ bosons. As before, for $\mu^{2}>0$ the minima are $\pm \mu^{2} / \lambda$ and in the unitary gauge we are left with three massive gauge fields and a new massive scalar field of the mass

$m_{H}=\sqrt{-2 \mu^{2}}=\sqrt{\lambda v^{2}}$. The residual massive scalar field is the Higgs boson which is the focus of this dissertation.

\subsubsection{Constraints on the Higgs}

Although this procedure works well in theory, it has not been verified experimentally through the observation of the Higgs boson itself. Constraints on the range of the Higgs mass can originate from a purely theoretical nature, indirectly from precision data, and most stringently by directly searching for the Higgs itself.

\section{Theoretical Constraints}

There are many free parameters in the SM including the masses of the particles and the coupling constants. By measuring these parameters precisely, a bound can be set on the parameter that has yet to be measured directly, the Higgs mass $\left(m_{H}\right)$. A constraint on the Higgs mass can be imposed from requiring the constant in the quartic term of the Higgs potential, $\lambda$, to remain finite positive up to an energy scale $\Lambda$ where the SM would be replaced by a more general theory. With this limit at the Planck scale $\left(\approx 10^{19} \mathrm{GeV}\right)$, where gravitational effects must be considered, the mass must be in the range $130<m_{H}<190 \mathrm{GeV}$. However if this scale is pushed down to $1 \mathrm{TeV}$ the range opens to $85<m_{H}<420 \mathrm{GeV}$. These constraints are well motivated, 
but not very powerful.

\section{Indirect Searches}

Radiative corrections to the mass of the $W$ boson, $m_{W}$, are logarithmically dependent on the $m_{H}$ and quadraticly dependent on the top quark mass, $m_{t}$. Through the precise measurement of both of these masses, the Higgs mass can be constrained. However the uncertainties are still too large to only consider these two parameters. The LEP Electroweak Working Group (LEP EWWG) expands on this idea, 18 EW parameters have been combined into a global fit with the Higgs mass using data from LEP, SLC, and the Tevatron [15]. The plot of the $\Delta \chi^{2}\left(\chi_{\min }^{2}\left(m_{H}\right)-\chi_{\min }^{2}\right)$ of this fit is shown in Figure 1.3. The preferred value is at $89_{-26}^{+35} \mathrm{GeV}$ at $68 \%$ confidence level (CL). An upper limit of $158 \mathrm{GeV}$ is set when including the experimental and theoretical error but when the direct search from LEP, discussed in the next section, is included, this upper limit moves to $185 \mathrm{GeV}$. Another collaboration, the Gfitter Group [16], performs a similar fit with and without the data from direct searches at LEP and the Tevatron shown in Figure 1.4. Without the direct searches the preferred value is $m_{h}=84_{-23}^{+30} \mathrm{GeV}$ at $68 \% \mathrm{CL}$ with a range of $42<m_{H}<159 \mathrm{GeV}$ at $95 \% \mathrm{CL}$. When including the direct searches, the minimum shifts to $m_{h}=120_{-5.2}^{+17} \mathrm{GeV}$ at $68 \% \mathrm{CL}$ with a range of $114<m_{H}<155 \mathrm{GeV}$ at $95 \% \mathrm{CL}$. 


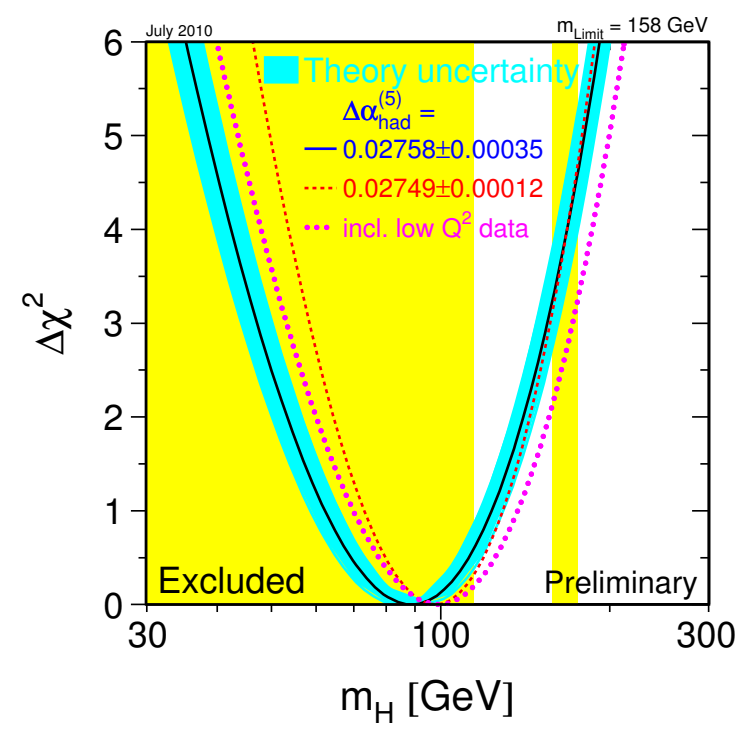

Figure 1.3: By fitting $18 \mathrm{EW}$ parameters to data from LEP, SLC, and the Tevatron, the Higgs mass is constrained to less than $185 \mathrm{GeV}$ at $95 \% \mathrm{CL}$.
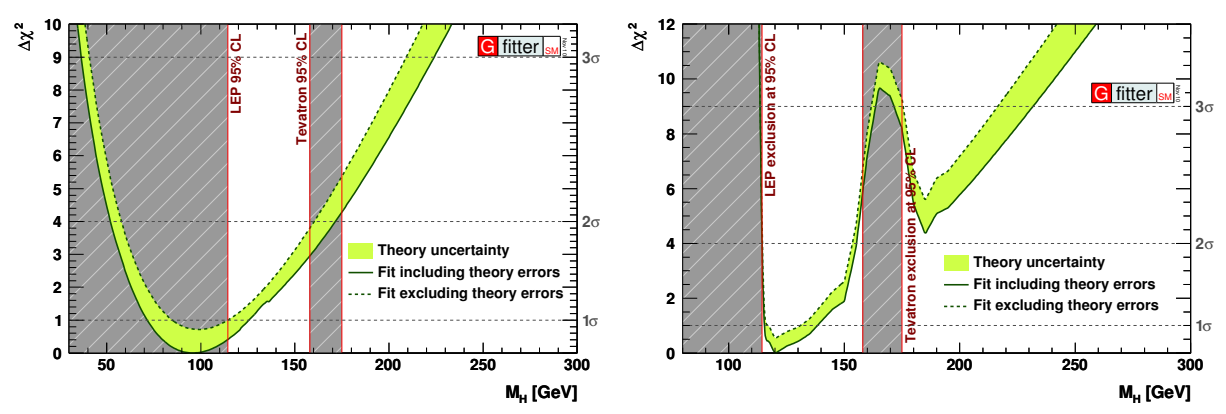

Figure 1.4: The Gfitter Group fits EW parameters to data from LEPand the Tevatron, the Higgs and finds a preferred value for the Higgs of $m_{h}=84_{-23}^{+30} \mathrm{GeV}$ at $68 \% \mathrm{CL}$ when ignoring direct searches and (left) $m_{h}=120_{-5.2}^{+17} \mathrm{GeV}$ at $68 \% \mathrm{CL}$ when using data from direct searches (right). 


\section{Direct Searches}

The direct search for the Higgs is divided into the low mass and high mass range divided approximately where the Higgs mass is equal to twice the $W$ mass (Figure 1.5). Below (above) this point, in the low (high) mass region, the Higgs decays to two b-quarks ( $W^{\prime}$ 's). Even though the largest production cross section is gluon fusion $(g g \rightarrow H)$, in the low mass search, the associated productions $(q \bar{q} \rightarrow Z H$ and $\left.q q^{\prime} \rightarrow W H\right)$ are preferred in order to have some a handle to pull this signal out of the QCD background (Figure 1.6). The LEP experiments set a lower limit of $114.4 \mathrm{GeV}$ at $95 \% \mathrm{CL}$ [17] using $2.461 \mathrm{fb}^{-1}$ of data collected from $e^{+} e^{-}$collisions at center of mass energies ranging from 189-209 GeV. Direct searches have also been carried out by both $\mathrm{D} \varnothing$ and $\mathrm{CDF}$ at the Tevatron. The results for the full mass range based on up to $6.7 \mathrm{fb}^{-1}$ of data [18] from both experiments can be seen in Figure 1.7 where a mass range of 158-175 GeV has been excluded. Most recently, the high mass search has been updated, shown in Figure 1.8, with up to $7.1 \mathrm{fb}^{-1}$ of data analyzed at CDF and $8.2 \mathrm{fb}^{-1}$ at $\mathrm{D} \varnothing$ excluding a mass range of $158-173 \mathrm{GeV}$ at $95 \%$ CL [19]. Chapter 10 discusses one of the major contributions to this search in the low mass region, the $Z H \rightarrow \nu \bar{\nu} b \bar{b}$ search conducted at $\mathrm{D} \varnothing$ detector. 


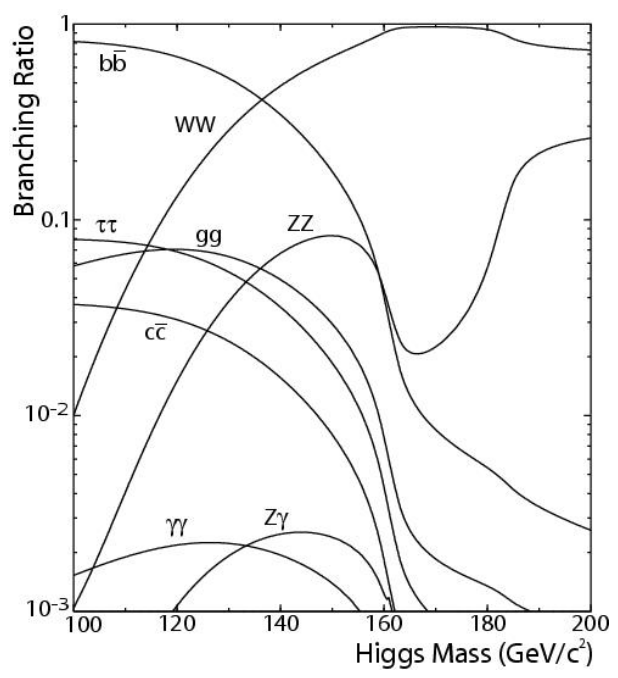

Figure 1.5: Standard Model Higgs branching ratio as a function of mass. Below the mass of two $W$ 's is the low mass region where the dominant decay channel is to two b-quarks. 


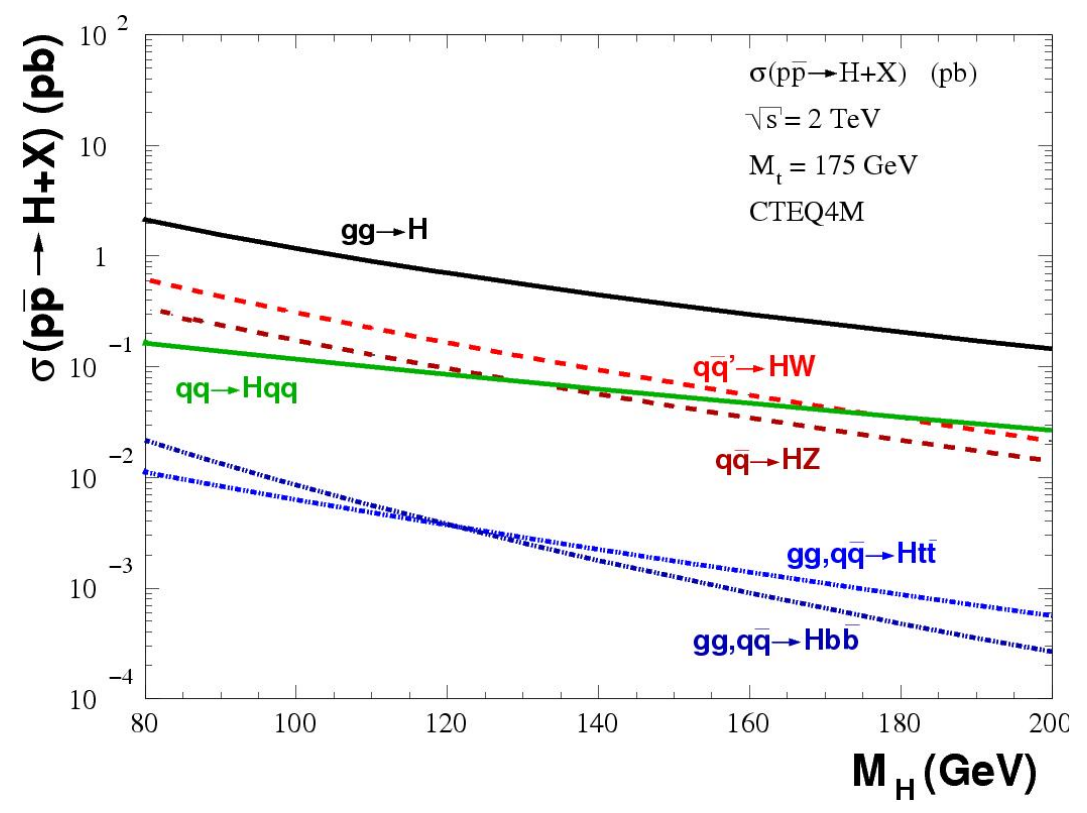

Figure 1.6: Standard Model Higgs production cross section at the Tevatron. In the low mass search the associated production channels are the only viable option since finding two b-quarks from the Higgs is impossible with all the QCD heavy-flavor production background with the handle of the gauge boson decay. 


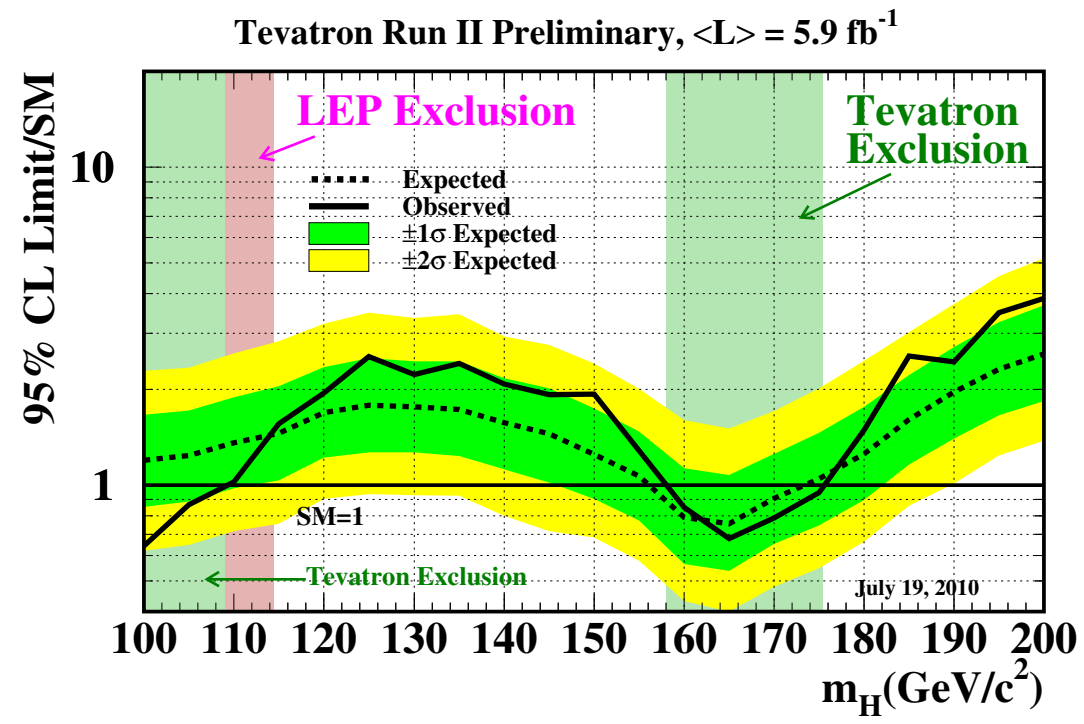

Figure 1.7: The latest Tevatron limits on the full Higgs mass range. A mass of 158-175 GeV has been excluded at 95\% CL. 


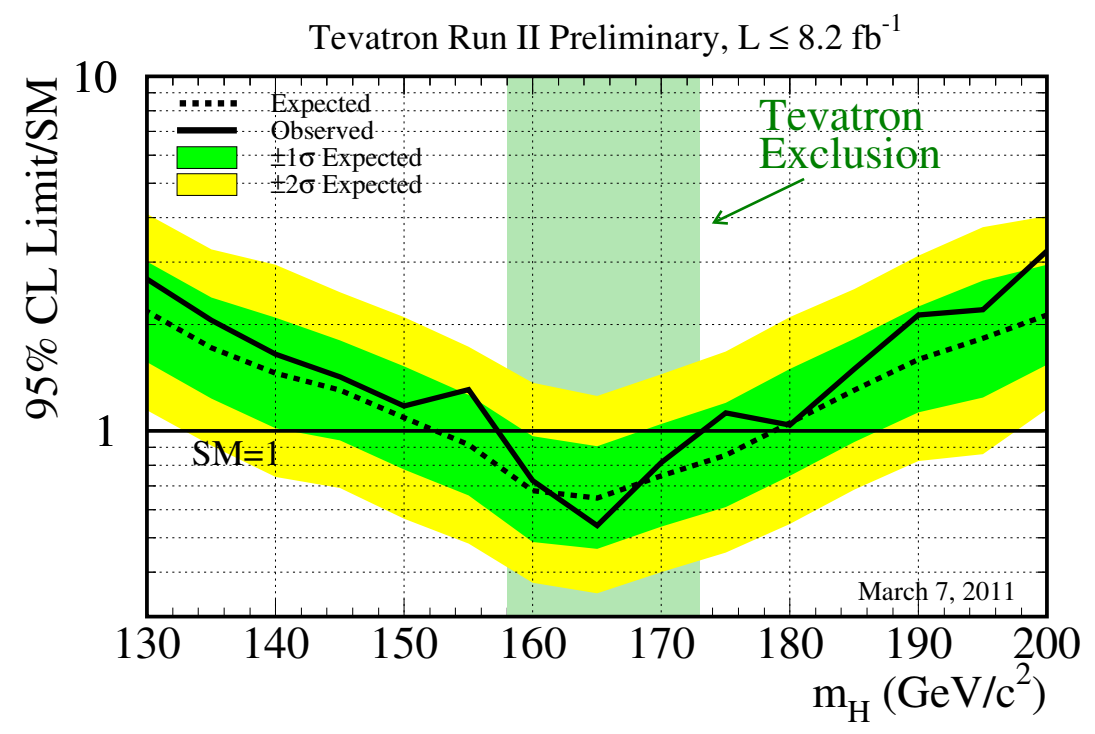

Figure 1.8: The latest Tevatron limits on the high mass Higgs production cross section. A mass of $158-173 \mathrm{GeV}$ has been excluded at $95 \% \mathrm{CL}$. 


\section{Chapter 2}

\section{Experimental Apparatus}

Fermilab National Accelerator Laboratory (Fermilab) is home to the proton-antiproton $(p \bar{p})$ collider known as the Tevatron. Operating at a center-of-mass energy of $\sqrt{s}=$ $1.96 \mathrm{TeV}$, this was the world's highest energy collider until November 30, 2010 when the Large Hadron Collider (LHC) housed in European Organization for Nuclear Research (CERN) successfully staged proton-proton collisions at $\sqrt{s}=2.36 \mathrm{TeV}$. There are three distinct operational periods of the Tevatron, Run I, IIa, and IIb. Run I took place between 1992-1996, Run IIa started in 2001, terminated in April 2006, and was followed by Run IIb which began in June of that same year. Run IIb is expected to end in September 2011, when funding for continued running is no longer available. Between Run I and IIa, the accelerator was upgraded to produce larger center of mass energies at higher instantaneous luminosities. In the summer of 2006, upgrades increased the instantaneous luminosity only. During these breaks both multi-purpose detectors, $\mathrm{CDF}$ and $\mathrm{D} \varnothing$ were upgraded to operate under the new conditions. The operating parameters of the accelerator are shown in Table 2.1.

This chapter introduces and gives a basic description of the various components and stages of the acceleration and detection processes. Section 2.1 discusses the 
accelerator complex followed by Section 2.2 which introduces the major components of the $\mathrm{D} \emptyset$ detector itself, followed by a passage, Section 2.3 on the trigger system used to select events of interest. This chapter is by no means an exhaustive description of the experimental apparatus. Please see the referenced documents for a more in-depth look into these systems.

\subsection{The Accelerator}

The Fermilab acceleration complex [20,21], shown in Figure 2.1, creates a $0.98 \mathrm{TeV}$ proton beam [22] by starting with hydrogen gas $\left(H_{2}\right)$ and uses the following hardware components:

- Magnetron

- Cockroft-Walton Generator

- LINear ACcelerator (LINAC)

- Three synchrotrons

Booster (75 m radius)

Main Injector (528 m radius)

Tevatron (1 km radius).

The antiprotons [23], once created, are manipulated and stored in the following synchrotrons:

- Debuncher (90 m radius)

- Accumulator (75 m radius)

- Recycler (528 m radius) 
followed by acceleration in the Main Injector and the Tevatron itself.
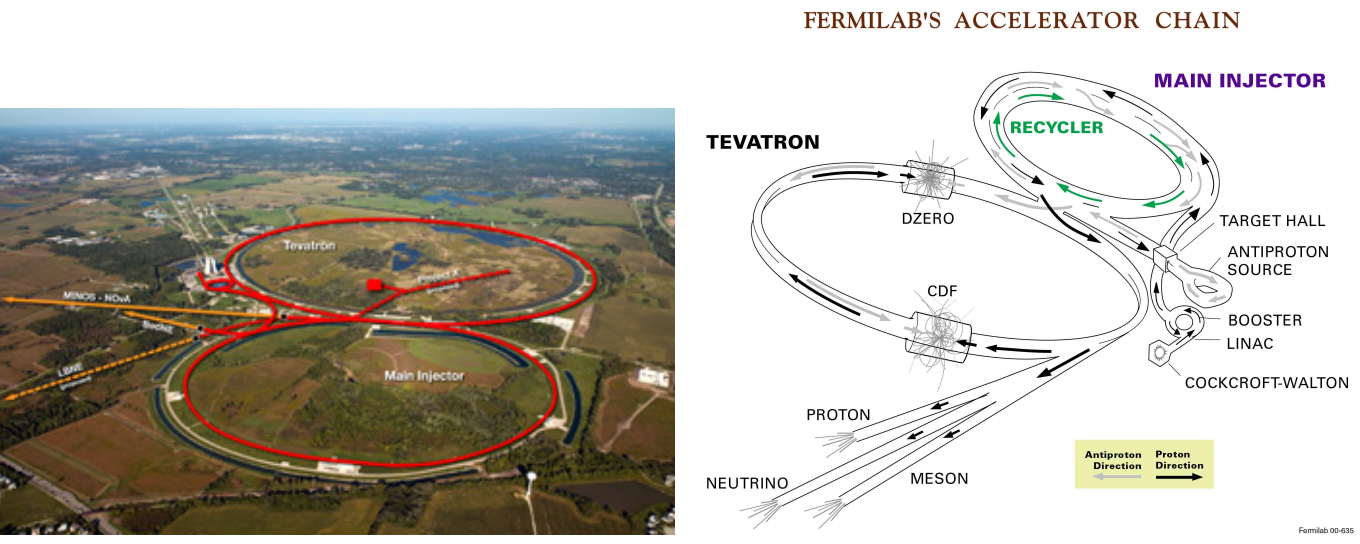

Figure 2.1: Schematic and aerial view of Fermilab's Tevatron complex.

Particle acceleration can be achieved by placing charged particles in a static electric field or within a series of radio-frequency (RF) cavities. In an RF cavity, a standing wave is formed in a metallic tube that has a series of separated drift tubes. The drift tubes shield ions from the standing wave while allowing them to experience an acceleration when in the gaps between drift tubes. Timing is such that particles arriving in the gap too early are slowed while those arriving late receive a larger kick. $\mathrm{RF}$ cavities force a beam structure of separate distinct bunches of particles rather than a continuous stream.

A synchrotron is a cyclic accelerator where particles are accelerated in a closed orbit. Magnetic fields are used to constrain the particles to this orbit and RF cavities accelerate the particles. Both fields must vary synchronous with the change in particle momentum. A turn is the amount of time necessary for a particle to complete one orbit. 


\subsubsection{Proton Beam}

Hydrogen gas is pumped into the magnetron ion source (Figure 2.2) that sits in a magnetic field. An electric pulse operating at $15 \mathrm{~Hz}$ and lasting $80 \mu$ sec causes a $40 \mathrm{~A}$ arc across the $1 \mathrm{~mm}$ gap between the cathode and the magnetron walls. The fields present in the gap separates the hydrogen into electrons and protons. While the former spiral in the gap to form a dense plasma the later strike the cathode creating $\mathrm{H}^{-}$ions nearly $10 \%$ of the time with the aid of Cesium vapor. These ions are accelerated through the extraction plate toward the Cockroft-Walton generator shown in Figure 2.3.

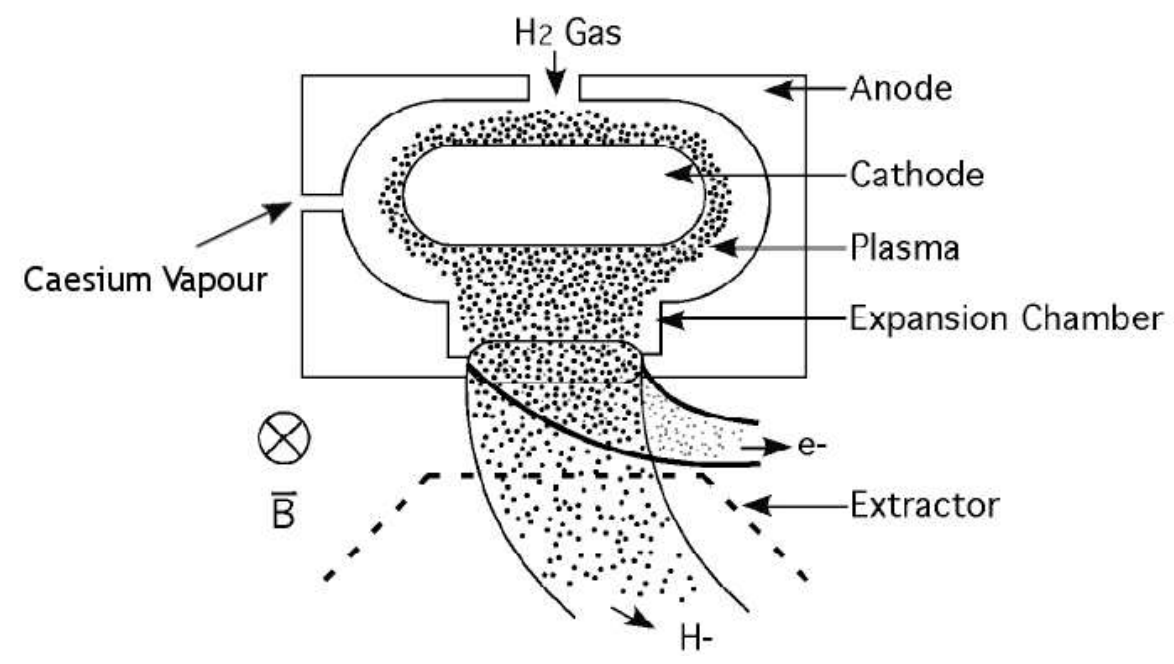

Figure 2.2: $\mathrm{H}_{2}$ molecules are injected into the magnetron chamber and leave as negatively charged $H^{-}$ions.

In a diode voltage multiplier circuit capacitors are charged in parallel by an $\mathrm{AC}$ voltage source and discharged in series to generate a larger voltage difference then the input. With this type of circuit the dome atop the Cockroft-Walton is charged to $-750 \mathrm{kV}$, a factor of ten increase of the input voltage. The dome is connected to a grounded resistive tunnel through which the $H^{-}$ions are accelerated to a speed of 
$0.04 c$, or $750 \mathrm{keV}$ of kinetic energy, by the static electric field within.

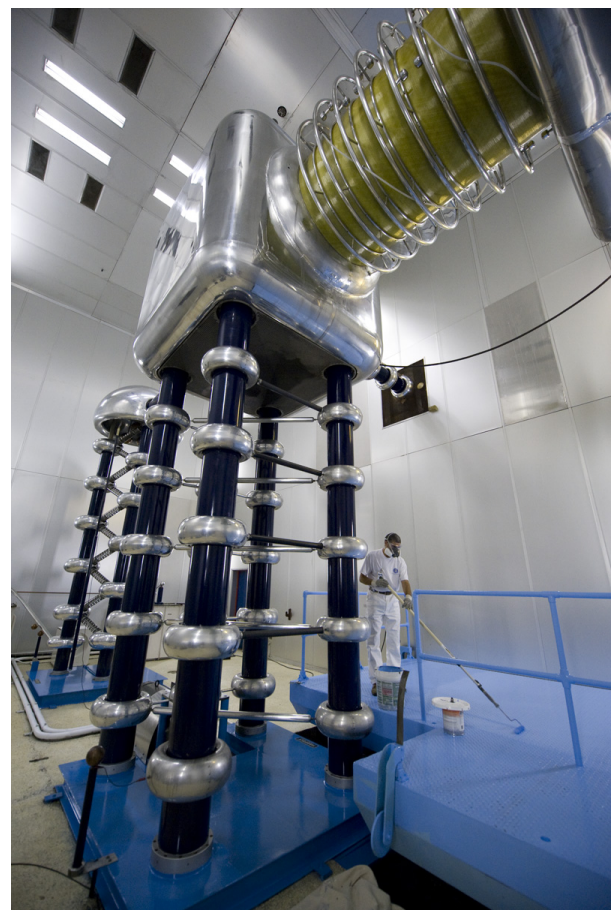

Figure 2.3: Fermilab's Cockroft-Walton generator.

The LINAC (Figure 2.4) accelerates the stream of $H^{-}$ions to relativistic energies in two stages with a series of RF cavities. Quadrupole magnets are embedded within the drift tubes to focus the beam and restrict the spread of the particles in the direction transverse to the path traveled. The ions have $116 \mathrm{MeV}$ of kinetic energy after the $79 \mathrm{~m}$ first stage and $400 \mathrm{MeV}$ after the second $67 \mathrm{~m}$ stage of acceleration.

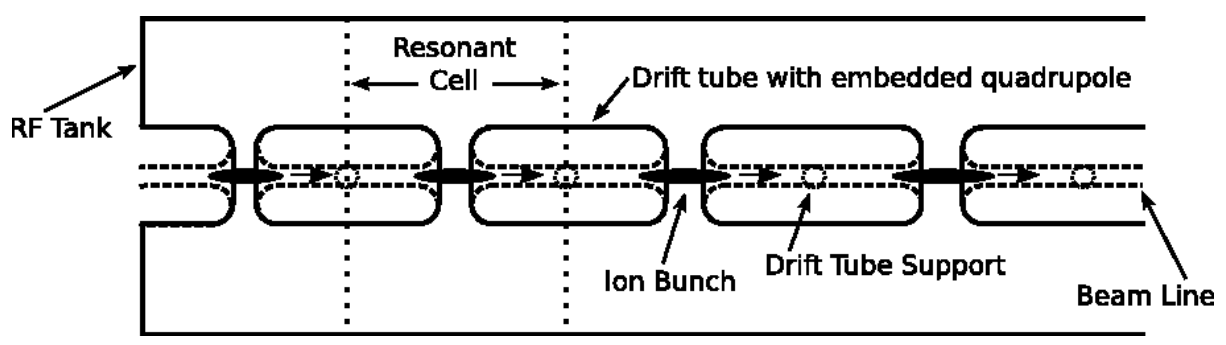

Figure 2.4: The LINAC accelerates particle bunches traveling through the RF cavities. 
Traveling at $0.71 c$, the hydrogen ions pass through a carbon foil that strips the electrons leaving the protons to be further accelerated by the Booster and the Main Injector synchrotrons. Operating with a $15 \mathrm{~Hz}$ cycle (66 ms), the Booster varies the $\mathrm{RF}$ frequency from 37.9 to $52.813 \mathrm{MHz}$ and the dipole magnetic fields from $740 \mathrm{G}$ to $7 \mathrm{kG}$ over a period of $33 \mathrm{~ms}$ to give the approximately $3 \times 10^{12}$ protons grouped in 84 bunches $8 \mathrm{GeV}$ of kinetic energy. Note that this equates the the protons traveling at a speed of $0.99 c$. Even though the following stages of acceleration will increase the kinetic energy of the protons significantly the speed with which they move will only slightly increase because of relativistic effects, therefore in the following, only the energy of the accelerating particles will be quoted.

Due to limitations in the size of the magnetic field, larger synchrotrons are needed for further acceleration. Protons transferred to the Main Injector are accelerated to either $150 \mathrm{GeV}$ and injected as 36 bunches into the Tevatron or $120 \mathrm{GeV}$ and used to create antiprotons at the Target Station.

\subsubsection{Antiproton Beam}

Protons leaving the Main Injector with $120 \mathrm{GeV}$ of energy collide with a fixed nickel target producing a shower of particles focused by a lithium lens. The antiprotons produced with a range of momentum around $8 \mathrm{GeV}$ and are redirected by a magnetic field as shown in Figure 2.5. These precious antiprotons, one of which is created for every $10^{5}$ incoming protons, are collected in the Debuncher. The Debuncher is a rounded triangular synchrotron used to manipulate and control the antiprotons. Reducing the emittance, or the longitudinal and transverse spread of the beam, is referred to as cooling. Experiencing the same magnetic field, the path particles traveling at different speeds will travel along different length paths around a synchrotron. The Debuncher takes advantage of this by varying the RF field such that the mo- 
mentum spread of the antiprotons decreases and the bunch structure is destroyed. A complex feedback system is used to further reduced the emittance. After $2.4 \mathrm{~s}$ the Debuncher is emptied into the Accumulator, located in the same tunnel, before the next bunch of protons are delivered to the Target Station. The Accumulator cools the antiprotons further through interactions with a low emittance electron beam in a process called electron cooling. This creates a stable beam which can reside in the Accumulator for several hours as the number of antiprotons increases during stacking. Eventually, the beam is accelerated and broken into a bunch structure and passed into the Main Injector where, in the same way the protons are accelerated, the antiprotons are pushed to $150 \mathrm{GeV}$ and injected into the Tevatron. As the antiproton density in the Accumulator grows, the stacking efficiency drops [24]. To reduce the resulting beam instabilities antiprotons are syphoned off the Accumulator into the Recycler. This collection of antiprotons, known as the stash, is transferred back to the Main Injector then injected into the Tevatron.

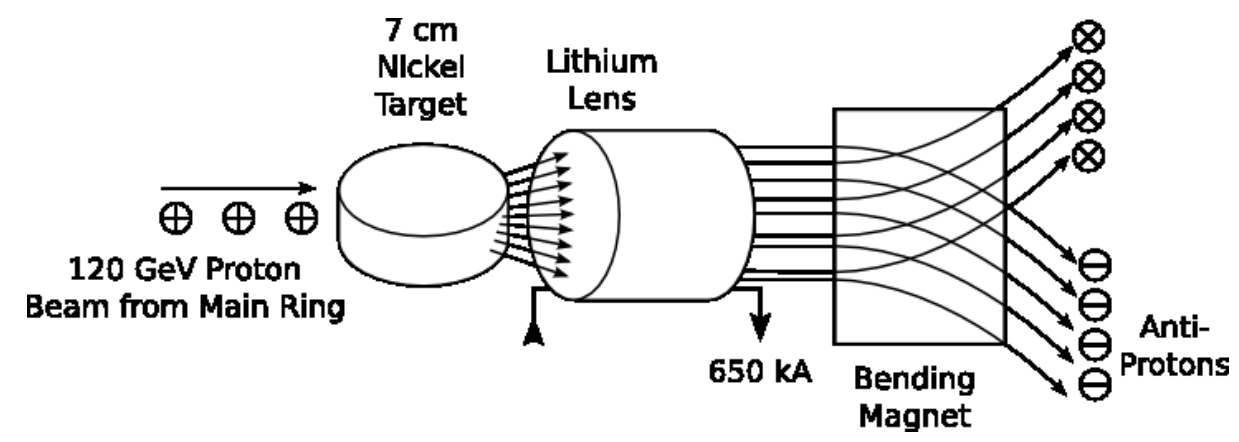

Figure 2.5: A stationary nickel taget is struck with $120 \mathrm{GeV}$ protons. Particles produced in the collision are focused by a lithium lens then the antiprotons are extracted by a magnetic field. 


\subsubsection{Tevatron}

Before high energy collisions can begin, the Tevatron is filled with 36 bunches of approximately $3 \times 10^{11}$ protons and 6 to $10 \times 10^{10}$ antiprotons each to denote the beginning of a store. These bunches are organized in three super-bunches separated by $2.64 \mu$ s with each super-bunch containing 12 bunches separated by 396 ns. A turn in the Tevatron takes $21 \mu$ sec.

The Tevatron must use superconducting electromagnets to bend and focus the $980 \mathrm{GeV}$ beam. The protons travel clockwise while the antiprotons travel counterclockwise in the same tunnel following a helical path to avoid undesired collisions (Figure 2.6). Collisions with a center-of-mass energy of $1.96 \mathrm{TeV}$ occur within the $\mathrm{CDF}$ and the $\mathrm{D} \varnothing$ detector in a region centered around the center of the detectors. This distribution of interactions in this luminous region conforms to a gaussian distribution with $\sigma_{z}=18 \mathrm{~cm}$.

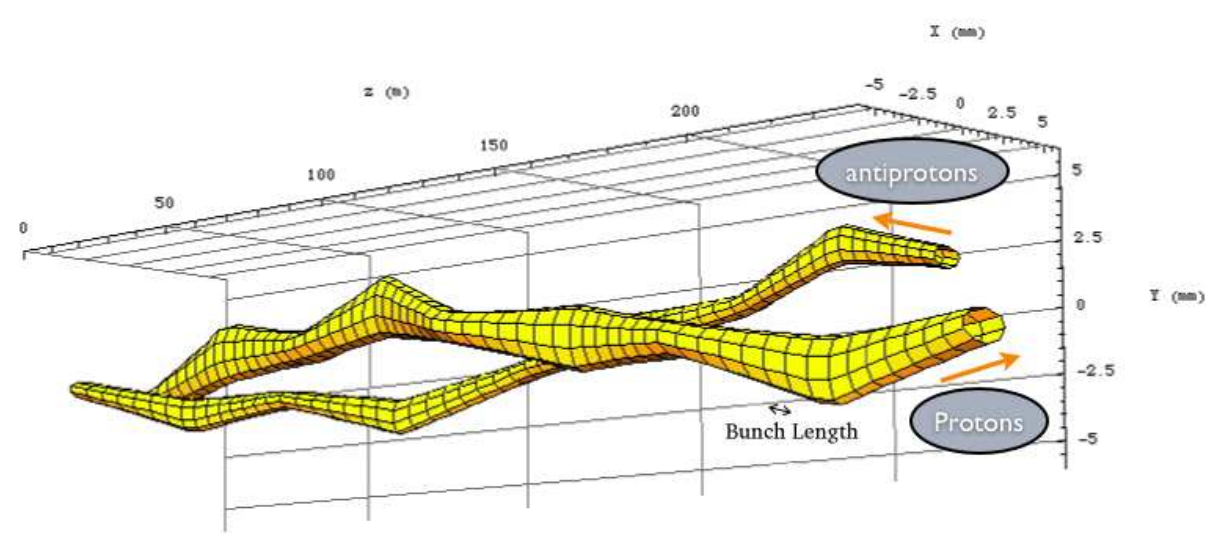

Figure 2.6: The proton and antiproton beams travel inside the same beam pipe and are forced into helical paths to avoid unwanted collisions. 


\begin{tabular}{|l|c|c|c|}
\hline Parameter & Run I & Run IIa & Run IIb \\
\hline Beam Energy $(\mathrm{GeV})$ & 900 & \multicolumn{2}{|c|}{980} \\
\hline Bunches & 6 & \multicolumn{2}{|c|}{36} \\
\hline$p /$ Bunch & $2.3 \times 10^{11}$ & $2.7 \times 10^{11}$ & $3.0 \times 10^{11}$ \\
\hline $\bar{p} /$ Bunch & $5.5 \times 10^{10}$ & $3.0 \times 10^{10}$ & $7.0 \times 10^{10}$ \\
\hline Bunch Spacing $(\mathrm{ns})$ & 3500 & \multicolumn{2}{|c|}{396} \\
\hline Peak Inst. Lumi $\left(\mathrm{cm}^{-2} \mathrm{~s}^{-1}\right)$ & $1.6 \times 10^{31}$ & $1.5 \times 10^{32}$ & $3.8 \times 10^{32}$ \\
\hline $\int \mathcal{L}\left(\mathrm{pb}^{-1} /\right.$ week $)$ & 3.2 & 17.3 & 70 \\
\hline
\end{tabular}

Table 2.1: Tevatron operatering characteristics for Run I, IIa, and IIb [25].

\subsection{Detector}

The 5,000 ton DØ detector [26] stands 30 x 30 x 50 feet. The design was first envisioned in a 1983 meeting at Stony Brook, the same year as the author's birth several miles from his birthplace. Construction was complete in February 1992 and data was taken from 1992 to 1996, the same year the author entered his final year of elementary school. During this last year and the author's four years of high school education, 1996 to 2001, the accelerator and D $\varnothing$ detector were upgraded. In the summer of 2005 , the author began to contribute to the D $\varnothing$ collaboration by working on the Run IIb upgrade, specifically the Level 1 Calorimeter-Track Trigger. The author and the detector met in the winter of 2005. Soon after, in 2006 accelerator upgrades to increase the instantaneous and integrated luminosity commenced and the detector was also modified to deal with these changes. Data taking resumed in June of 2006, a few months before the author started graduate school at Northeastern University. Five years later, the accelerator and detector are operating beyond expectations and this 
document is being prepared. Coincidently, this year, 2011, the author will obtain his final degree in this field and the detector will see its last collisions.

The $\mathrm{D} \varnothing$ detector, Figure 2.7, has a symmetrical design of concentric sub-detectors centered on the collision region. The central tracking system is the innermost layer, surrounded by a solenoid magnet, followed by the calorimeter and the muon system which contains a toroidal magnet. The following sections describe the detector in more detail.

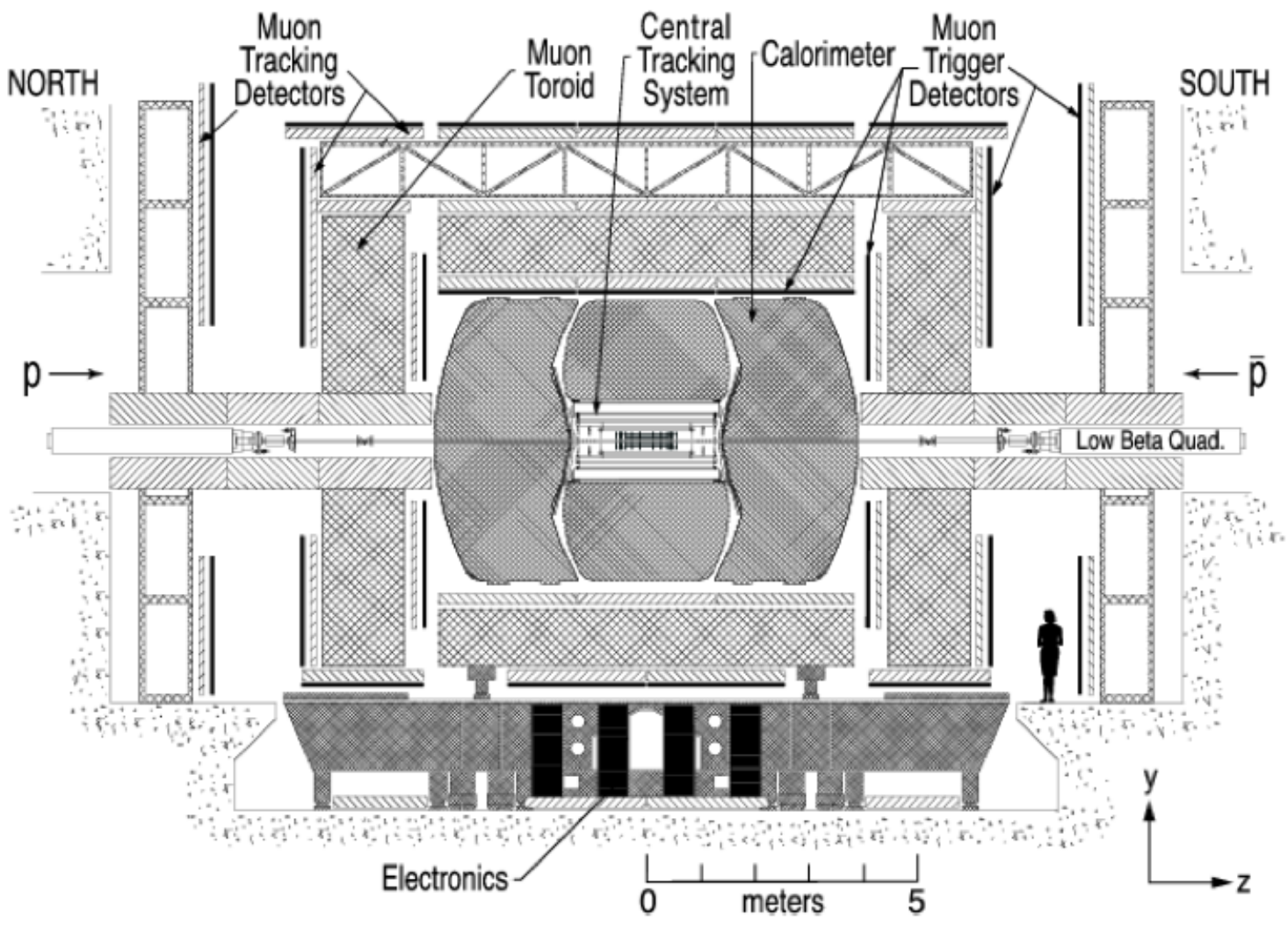

Figure 2.7: Schematic view of the DØ Detector 


\subsubsection{Coordinates}

The right-handed coordinate axis used throughout $\mathrm{D} \varnothing$ is orientated such that the z-axis points along the direction of the proton travel and the y-axis points away from the center of the Earth. The perpendicular distance from the z-axis is denoted by $r$. The polar and azimuthal angles are $\theta$ and $\phi$ respectively. Rapidity, which is invariant under Lorentz boosts along the beam line, is defined as

$$
y=\tanh ^{-1}\left(\frac{v}{c}\right)
$$

where $v$ is the particle speed and $c$ is the speed of light. The pseudo-rapidity of an object given by

$$
\eta=-\ln \left(\tan \frac{\theta}{2}\right)
$$

or in terms of momentum

$$
\eta=\frac{1}{2} \ln \left(\frac{|\vec{p}|+p_{L}}{|\vec{p}|-p_{L}}\right)
$$

where $p_{L}$ is the component of the momentum along the beam axis. In the relativistic limit, $\frac{m c^{2}}{E} \rightarrow 0$, pseudo-rapidity approximates the rapidity as

$$
\eta \approx \ln \left(\frac{E+p_{L}}{E-p_{L}}\right)=y
$$

The regions of high $\eta$ are described as the forward region. Distances between objects in $\eta-\phi$ space is calculated using the cone distance of

$$
\Delta R=\sqrt{\Delta \eta^{2}+\Delta \phi^{2}} .
$$




\subsubsection{Tracking}

Charged particle trajectories are determined by the Silicon Microstrip Tracker (SMT) and the Central Fiber Tracker (CFT). The SMT is located directly on the beam pipe with the CFT around it and both detectors experience a $1.92 \mathrm{~T}$ magnetic field created by the encompassing superconducting solenoid magnet (Figure 2.8). Momentum measurements are made by using the known magnetic field strength and the radius of curvature found by reconstructing the particles path through the detector. The central tracking system locates the primary interaction vertex (PV) with a resolution of $35 \mu \mathrm{m}$ along the beam-line and the impact parameter (IP) with resolution of better then $15 \mu \mathrm{m}$ in $r-\phi$ for particles with $p_{T}>10 \mathrm{GeV}$ at $|\eta|=0$.

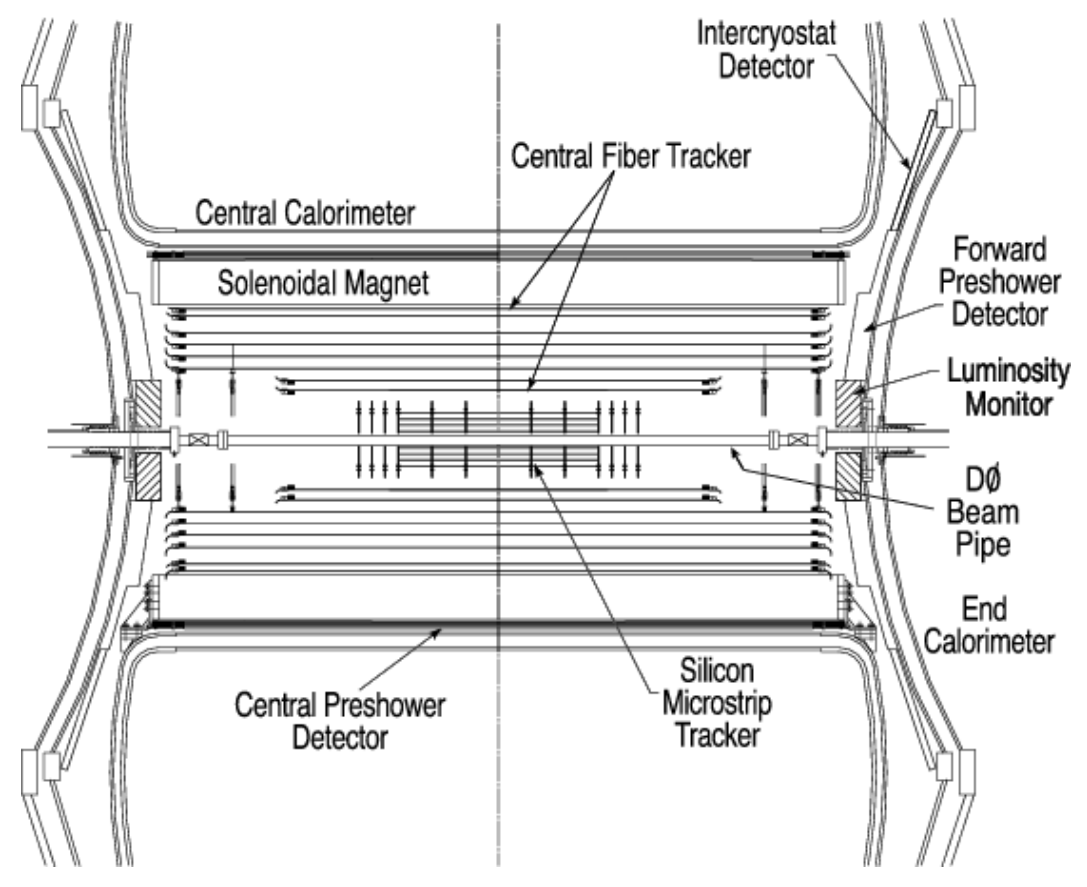

Figure 2.8: Cross-sectional view of the DØ Central Tracking System including the SMT, CFT and solenoid. 


\section{The Silicon Microstrip Tracker}

The SMT $[27,28]$ provides vertexing and tracking for almost the full $\eta$ converge of the calorimeter and muon systems. Mixed barrel and disk modules ensure that particle trajectories are mostly perpendicular to the detector surface for interactions anywhere in the luminous region (Figure 2.9). Both modules are constructed from a series of doped silicon semiconducting wafers. When a charged particle passes through the positive-negative (p-n) junction electron-hole pairs are produced. A voltage difference across the wafer causes the electrons and holes to drift to one side of the wafer where the charge is digitized and read-out. Hit resolution is around $10 \mu \mathrm{m}$ and the signal to noise ratio varies from $12: 1$ to $18: 1$.

A single barrel is made from five concentric layers of rectangular silicon wafers. Layer 0, the innermost layer, was installed before Run IIb to maintain high quality tracking and pattern recognition in spite of detector aging and the higher instantaneous luminosity. Six barrels in total, each $12 \mathrm{~cm}$ long with an inner (outer) radius of $2.7 \mathrm{~cm}(10.5 \mathrm{~cm})$ were installed symmetrically about $z=0$ with centers located at $|z|=6.2,19.0,31.8 \mathrm{~cm}$. A combination of double sided and single sided layers are used. The double sided wafers provide coordinates in the $r-\phi$ plane from the axial side, while the other side, by having an angle of $2^{\circ}$ or $90^{\circ}$ with respect to the beam line, gives a measurement in the $r-z$ plane. The single sided layers have an axial orientation.

The 12 disks, or F-disks, are constructed from 12 wedge shaped double sided silicon moduli. Each barrel end, except at $z=0$, is capped with an F-disk. The remaining six are placed at larger $|z|$ for forward tracks. The F-disks are located at $|z|=12.5,25.3,38.2,43.1$, and $53.1 \mathrm{~cm}$ and the larger H-disks were installed at $|z|=100.4$ and $121.0 \mathrm{~cm}$. When Layer 0 was add before Run IIb this outer pair of H-disks was removed. The disks provide a measure of the $r-z$ coordinate. 


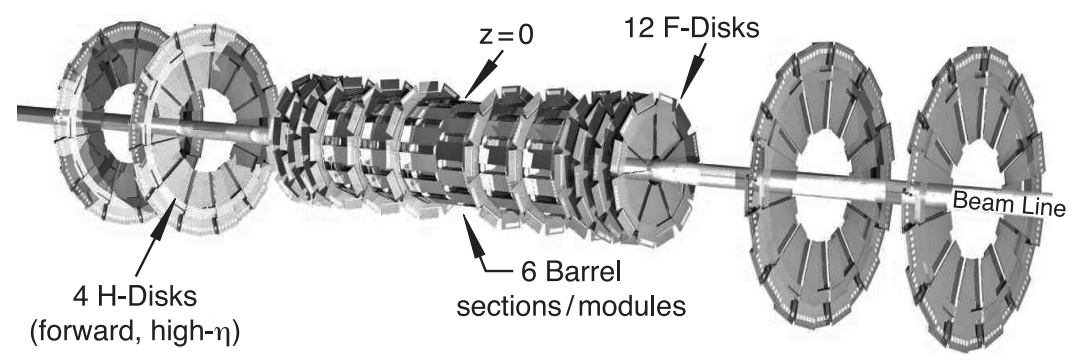

Figure 2.9: Layout of the SMT with the H-Disks prior to Run IIb

\section{Central Fiber Tracker}

The CFT provides tracking information in the region of $|\eta|<1.6$. It is comprised of concentric layers of scintillating fibbers constructed from polystyrene doped with an organic fluorescent dye, paraterphenyl (Figure 2.10). Eight concentric cylinders of four fibers arranged in an inner (axial) and outer (stereo) doublet layer are the active material of the system. The two innermost cylinders are $1.66 \mathrm{~m}$ long to accommodate the H-disks while the others stretch to $2.52 \mathrm{~m}$. Axial layers runs along the z-axis while stereo layers are orientated at a stereo angle of $\pm 3^{\circ}$ to the beam line. As long as the location of the fibers are know to better than $50 \mu \mathrm{m}$, the small fiber diameter of $835 \mu \mathrm{m}$ give an inherent resolution of $100 \mu \mathrm{m}$.

Charged particles excite the polystyrene core and the energy is transferred to the fluorescent paraterphenyl which emits photons with a wavelength around $340 \mathrm{~nm}$. Each fiber is almost totally internally reflective because the fiber has a refractive index of $n=1.59$ and a has a two layer sheath with an inner (outer) refractive index of 1.49 (1.42). The light is read out by visible light photon counters which can detect a single photon. 


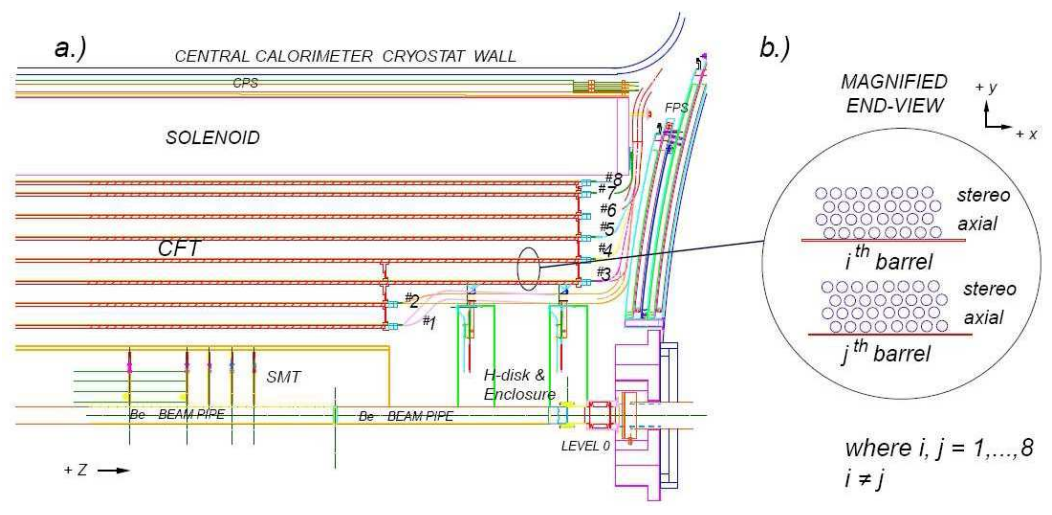

Figure 2.10: Layout of the CFT illustrating the layers of scintillating fibers

\section{Solenoid}

The solenoid, added between Run I and II to optimize the transverse momentum resolution $\left(\delta p_{T} / p_{T}\right)$ and track pattern recognition while conforming to the size constraints of the surrounding calorimeter, provides a $1.92 \mathrm{~T}$ field with two possible polarity configurations (Figure 2.11). It is $2.72 \mathrm{~m}$ in length with an inner (outer) radius of $1.07 \mathrm{~m}(1.42 \mathrm{~m})$ and is 1.1 radiation lengths thick. 


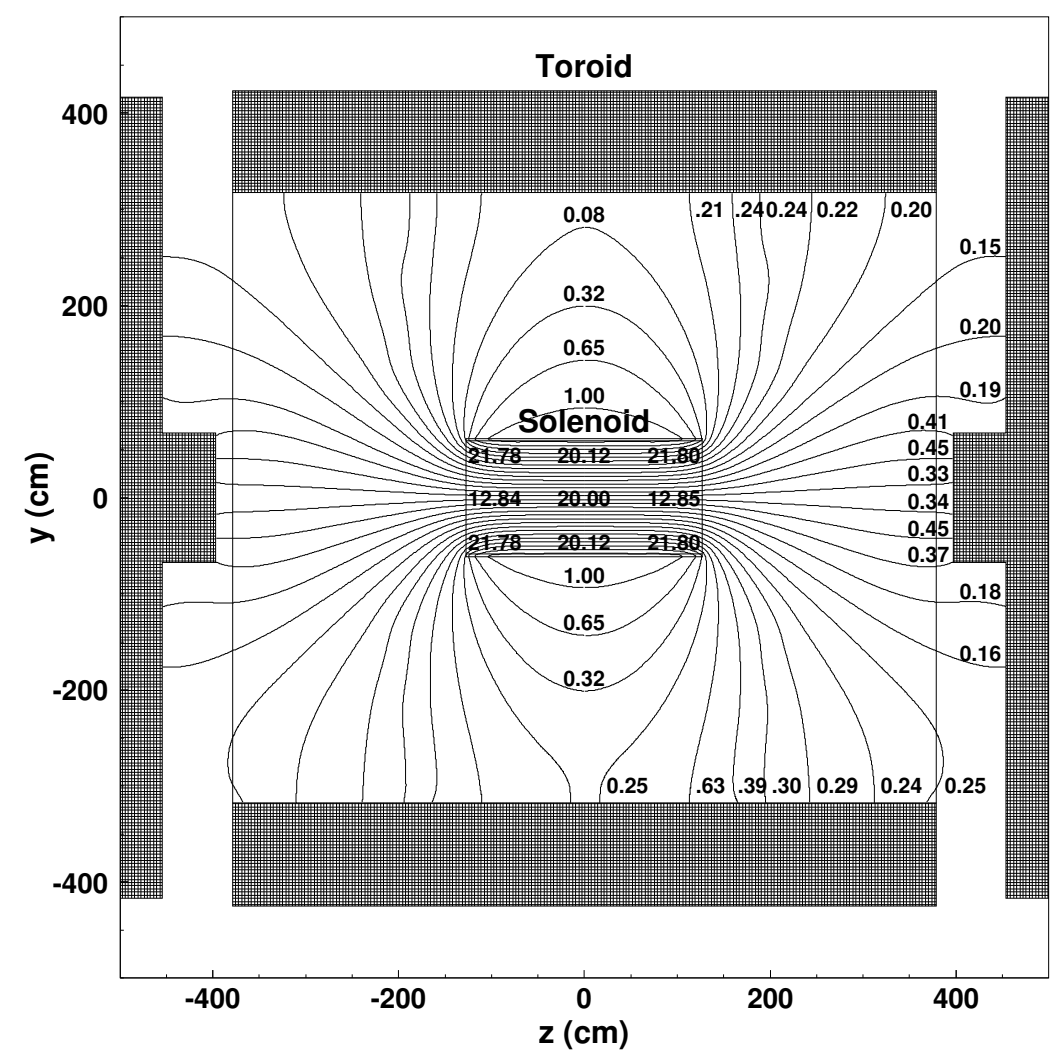

Figure 2.11: Magnetic filed lines in the $\mathrm{D} \varnothing$ detector with both the solenoidal and toroidal magnets at full current.

\subsubsection{Preshower Detectors}

Located between the solenoid and the central calorimeter covering an $|\eta|<1.3$ the Central Preshower Detectors (CPS) are a both calorimeters and tracking detectors which provide energy and position measurements fast enough to be used in the first level of the trigger system (see Section 2.3). The Forward Preshower Detectors (FPS), with the same characteristics, are located on the faces of the endcap calorimeters covering $1.5<|\eta|<2.5$. Both preshower detectors consist of interleaved triangular strips of scintillator made from polystyrene plastic doped with $1 \%$ p-terphenyl and 150 ppm diphenyl stilbene (Figure 2.12). The center of each strip hosts a wavelength- 
shifting fiber that collects and transports the light to the read-out end of the detector.

\section{Central Preshower Detector}

Located in the $5 \mathrm{~cm}$ gap between the solenoid and the central calorimeter is an approximately one radiation length $\left(X_{0}\right)$ thick lead radiator and three concentric cylindrical layers of triangular scintillator strips. The three layers are oriented such that there is one axial layer aligned with the z-axis and two stereo layers orientated at an angle of approximately $\pm 24^{\circ}$.

\section{Forward Preshower Detector}

Each FPS is made of two layers of scintillator strips with lead-stainless-steel absorber two $X_{0}$ thick in between.

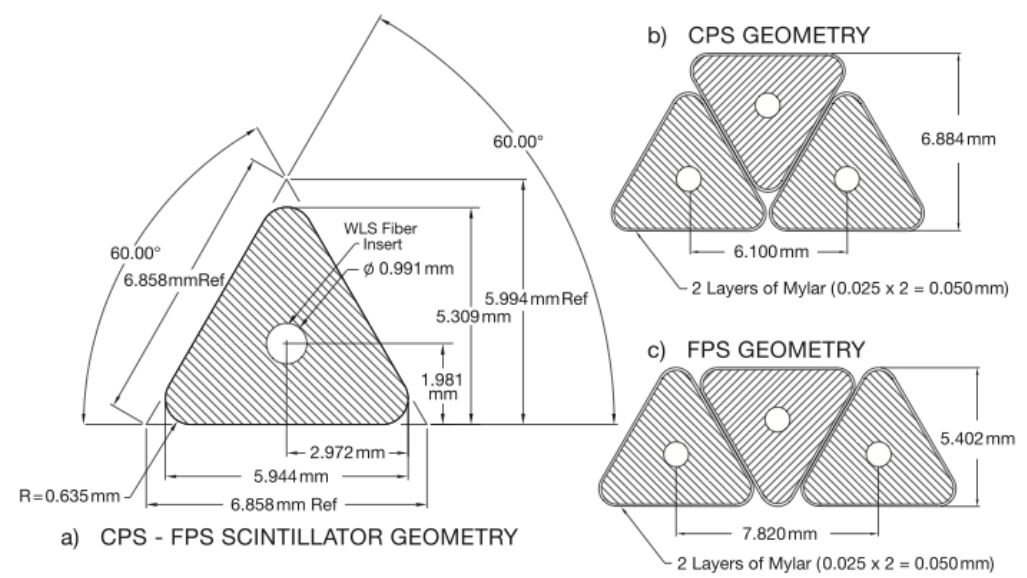

Figure 2.12: Cross section (a) and instillation geometry of the scintillator strips used in the CPS (b) and the FPS(c). The shaded area is the scintillating material while the circle in the center is the waveguide. 


\subsubsection{Calorimetery}

Most particles which are created in high energy collisions terminate in the calorimeter. This apparatus measures the energy deposited from electrons, photons, and hadrons as well as the imbalance in transverse energy which is indicative of the presence of a neutrino. To ease assembly of the tracking system which the calorimeter surrounds, there are actually three separate calorimeters (Figure 2.13), one central calorimeter (CC) covering $|\eta|<1$ and two endcap calorimeters (EC) extending the coverage to $|\eta| \approx 4$. Each calorimeter is located within a cryostat maintaining a temperature of $90 K$.

A unit cell of the DØ Uranium/Liquid-Argon sampling calorimeter, shown in Figure 2.14, consists of a grounded metal absorber plate and a signal board with the resistive surfaces connected to a high voltage source of typically 2.0 to $2.5 \mathrm{kV}$. Incoming high energy particles will react via the electromagnetic or strong force with the absorber plates resulting in showers of secondary particles. Liquid argon, being the active medium, is ionized by these secondary particles and the resultant electrons collect on the signal board where the magnitude of the charge is read out. Electron drift time across the $2.3 \mathrm{~mm}$ gap is $450 \mathrm{~ns}$, longer than the time between two consecutive bunch crossings. The calorimeter signal processing hardware, through fast signal shaping, is aptly capable of distinguishing and correctly disentangling these two signals.

To adequately sample the secondary particle shower shape and size for a range of particles and a range of energies the calorimeter is highly segmented and three distinct types of modules have been employed at different depths. The innermost or electromagnetic (EM) layers are where the electromagneticly interacting particles, such as photons, electrons, and some types of $\tau$ leptons, deposit most of their energy. The secondary particles are created from pair production $\left(\gamma \rightarrow e^{-} e^{+}\right)$and bremsstrahlung 
$\left(e^{ \pm} \rightarrow e^{ \pm} \gamma\right)$. The appropriate length scale for describing electromagnetic cascades is the radiation length, $X_{0}$ which is both $\frac{7}{9}$ the mean free path for pair production and the mean distance over which electrons lose all but 1/e (37.6\%) of its energy through bremsstrahlung. The radiation lengths of 2.0, 2.0,6.8, and $9.8 X_{0}$ in the $\mathrm{CC}$ and 0.3 , 2.6, 7.9 and $9.3 X_{0}$ in the EC for the four EM layers are achieved by the use of nearly pure depleted uranium $3 \mathrm{~mm}(4 \mathrm{~mm})$ thick in the CC (EC).

Interactions via the strong force deposit energy mostly in the deeper hadronic (HD) layers. The appropriate length scale for describing hadronic cascades is the absorption length $\left(\lambda_{A}\right)$, or the mean free path of a particle before it inelasticity interacts with a nucleus. This type of interaction produces more hadronic particles and some electromagnetic particles which in turn dispense energy into the active medium. Because of this longer showering process, the hadronic showers are typically wider and travel deeper into the detector's HD layers. Therefore $6 \mathrm{~mm}$ thick uraniumniobium (2\%) alloy was chosen for the fine hadronic $(\mathrm{FH})$ absorption plates while the coarse hadronic $(\mathrm{CH})$ cells contain $46.5 \mathrm{~mm}$ thick copper (stainless steel) in the CC (EC). In the $\mathrm{CC}$ the four EM layers total to $0.76 \lambda_{A}$ while the three $\mathrm{FH}$ layers are an additional 1.3, 1.0, and $0.76 \lambda_{A}$ respectively. The four FH layers in the EC each contribute $1.1 \lambda_{A}$ while the $\mathrm{CH}$ layer adds $4.1 \lambda_{A}$.

Pseudo-projective towers (Figure 2.15), with dimensions of 0.1 in $\eta, \frac{\pi}{32} \approx 0.1$ in $\phi$, and subdivided in depth are used to sample the energy deposits. The cell boundaries are aligned perpendicular to the absorber plates but the center of the cells lie on a ray projecting from the center of the luminous region. In the EM layers this corresponds to a smaller physical size then in the hadronic layers which matches the difference in the characteristic length scale of the respective showers. The level of segmentation is doubled in the third EM layer to better measure the centroid of EM showers. 


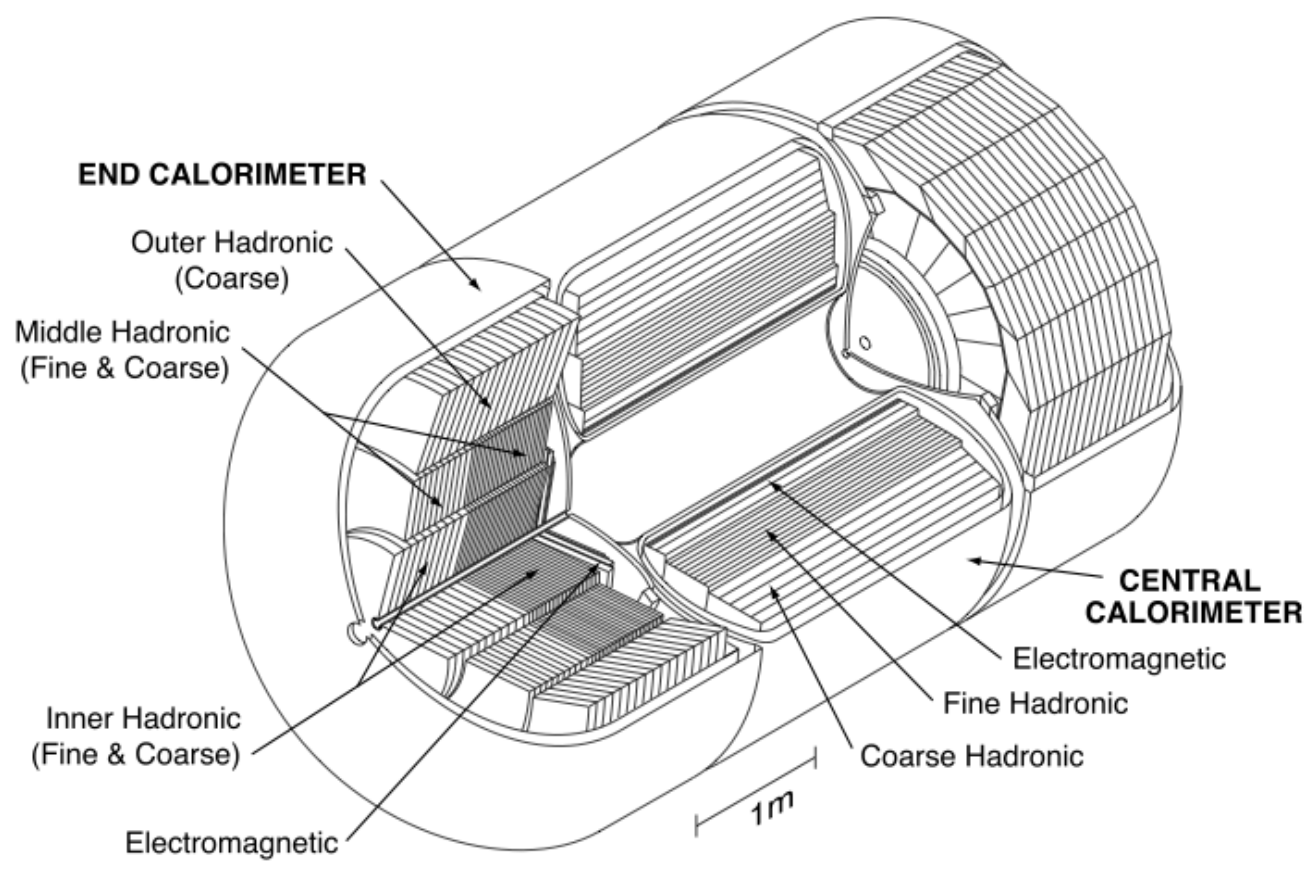

Figure 2.13: Schematic view of the different layers of the calorimeter showing the central and two endcap calorimeters. The electromagnetic layers as well as the fine and coarse hadronic layers can also be seen. 


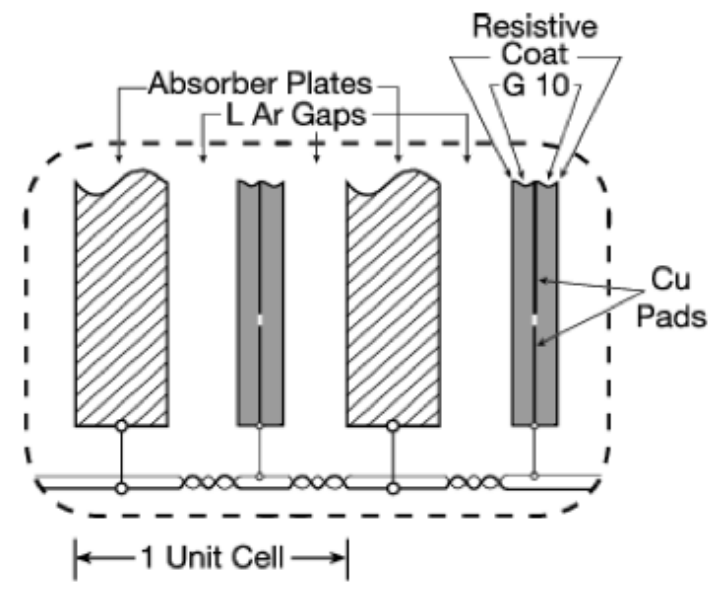

Figure 2.14: Schematic view of a typical unit cell of the DØ Calorimeter. The incoming particle(s) interact with the absorber plate showering into and ionizing the liquid Argon and the resultant charge is collected on the read-out plats. The $r$ axis in the $\mathrm{CC}$ runs horizontally across this figure. 


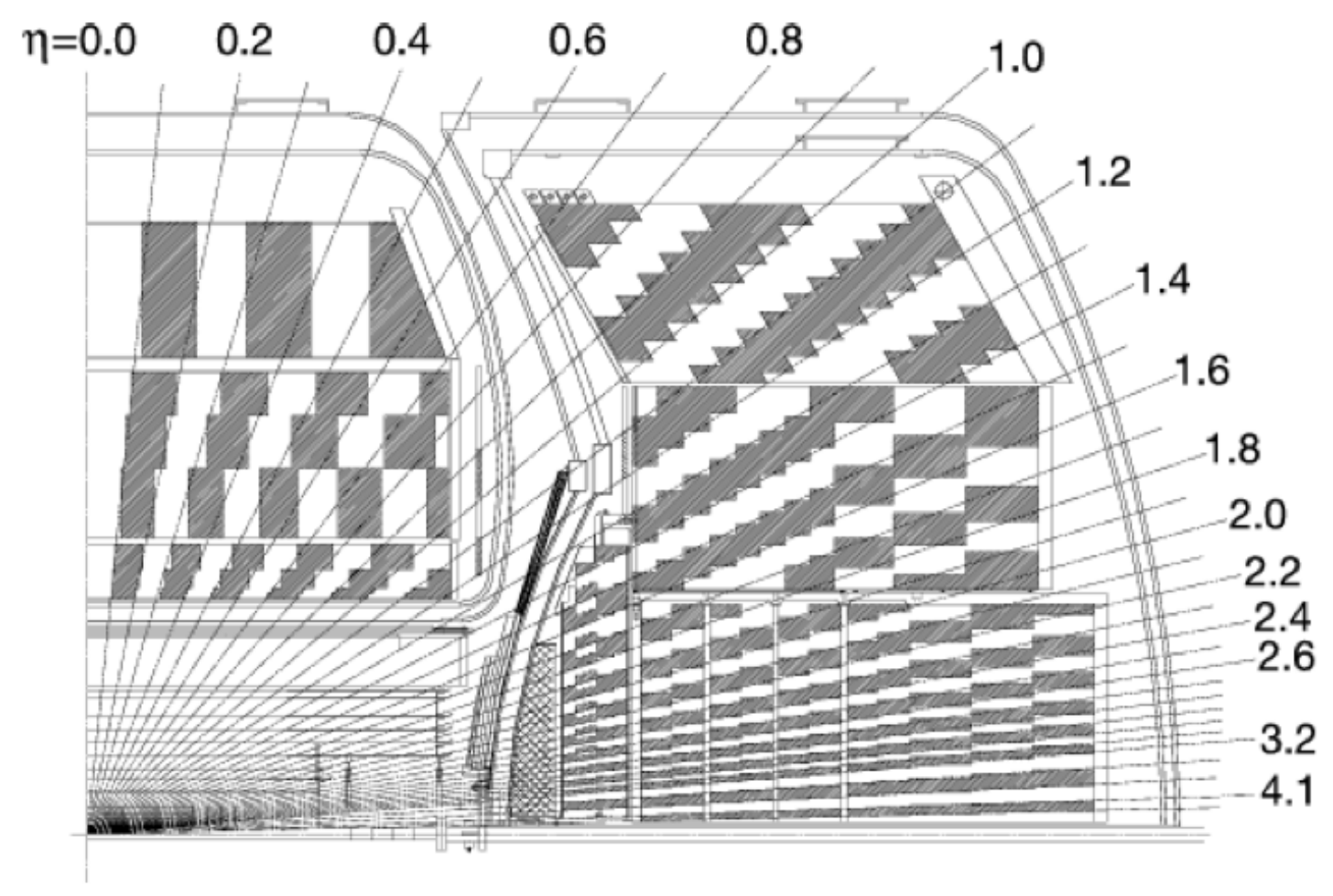

Figure 2.15: A view of the pseudo-projective read-out towers of the $\mathrm{D} \varnothing$ Calorimeter

\section{Inter-Cryostat Detectors (ICD)}

From $0.8<|\eta|<1.4$, because of the multiple cryostat design, a particle passes through less active material before reaching the end of the calorimeter. However there is a sufficient amount of passive material for showering to occur. To sample the showers in this region scintillating tile detectors of size in $0.1 \times 0.1$ in $\Delta \eta \times \Delta \phi$ were installed on the external walls of the endcap calorimeters.

\subsubsection{Muon Detection}

The presence of a muon is usually indicative of an event of interest. Compared to electrons, muons couple weakly to the detector material and usually passing completely 
through the entire calorimeter only depositing on average $0.25 \mathrm{GeV}$ per nuclear interaction length. The muons system employes two different types of detection methods; Scintillation counters provide a fast signal and thus can be used for triggering while drift tubes can provide a slower and more exact measure of the muon position which is important at the analysis level but can also contribute to the trigger decision.

The system itself is rectangular with a three-segment toroid dividing one layer of detection (A layer) from the remaining two layers (B and $\mathrm{C}$ ). For an muon momentum measurement independent of the tracking system, the central toroid supplies a magnetic field of $1.8 \mathrm{~T}$ while the forward toroids provide a $1.9 \mathrm{~T}$ magnetic field. An independent measurement allows for better matching with the central tracking system, improved background rejection, lower $p_{T}$ cutoff when selection muons, and increased resolution for high energy muons. Mapping the rectangular system to a coordinate meant for a more spherical segmentation, the central muon system, installed in Run I, covers approximately $|\eta|<1$ while the newer forward muon system extends coverage to about $|\eta|=2$.

A drift tube is a tube filled with an ionizing gas with an anode wire running through the center. When a charged particle passes through the gas, the ionized gas forms an avalanche of charge that falls to the anode wire where it is read out.

Proportional (PDT) and mini drift tubes (MDT) are located in the central and forward muon systems respectively (Figure 2.16). The A layer has four bands of drift tubes, while the $\mathrm{B}$ and $\mathrm{C}$ layers each have three. The PDTs, with a $10 \times 5.5 \mathrm{~cm}^{2}$ internal cross section, are filled with a gas mixture of $84 \%$ Argon, $64 \% C F_{4}$, and $8 \%$ methane and the anode wires are held at $4.7 \mathrm{kV}$ while the cathode walls are kept at $2.3 \mathrm{kV}$. Drift times in these tubes are maximally $500 \mathrm{~ns}$. The drift distance measurement resolution is approximately $1 \mathrm{~mm}$ and the resolution on the difference in the arrival time of the signal pulse at the end of the wire of the hit cell and its readout 
partner's wire relates to a $10 \mathrm{~cm}$ to $50 \mathrm{~cm}$ resolution depending on the distance of the hit from the electronics. Using charge division, the pad signal resolution is $5 \mathrm{~mm}$. The MDTs, with a $9.4 \times 9.4 \mathrm{~mm}^{2}$ internal cross section, are filled with a gas mixture of $90 \% C F_{4}$ and $10 \%$ methane and the anode wires are grounded while the cathode walls are kept at $3.2 \mathrm{kV}$. Drift times in these tubes range from 40 to $60 \mathrm{~ns}$. The signal arrival time is measured with respect to the $53 \mathrm{MHz}$ main accelerator clock to an accuracy of $18.8 \mathrm{~ns}(1.9 \mathrm{~mm})$.

The scintillators of the cosmic cap are located in the C layer of the top and sides of the central muon system while those of the cosmic bottom are in the $\mathrm{B}$ and $\mathrm{C}$ layers (Figure 2.17). By providing a fast timing signal, the information from these detectors is used to associate a muon detected in a PDT to the appropriate bunch crossing or reject the muon as from cosmic sources. The $A \phi$ scintillator counters covering the A layer PDTs provide a time stamp for low $p_{T}$ muons which do not fully penetrate the toroid and help reject out-of-time backscatter in the forward directions. Scintillator pixel detectors known as the trigger scintillation counters are mounted on each of the three layers of the forward muon system. The segmentation in $\phi$ matches that of the CFT and contribute to the trigger decision and vetoing cosmic muons. 


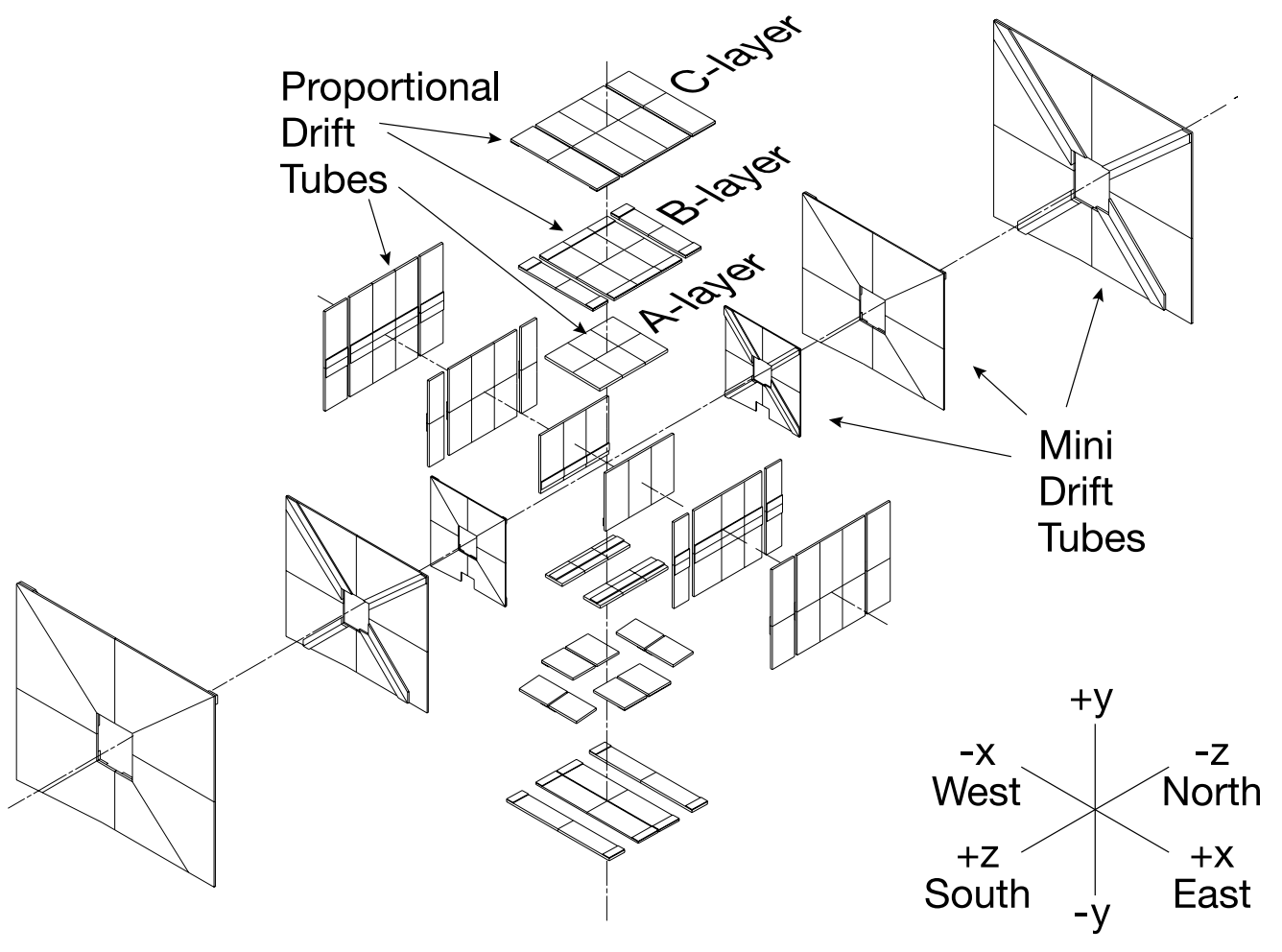

Figure 2.16: An exploded view of the $\mathrm{D} \varnothing$ muon drift tube system. 


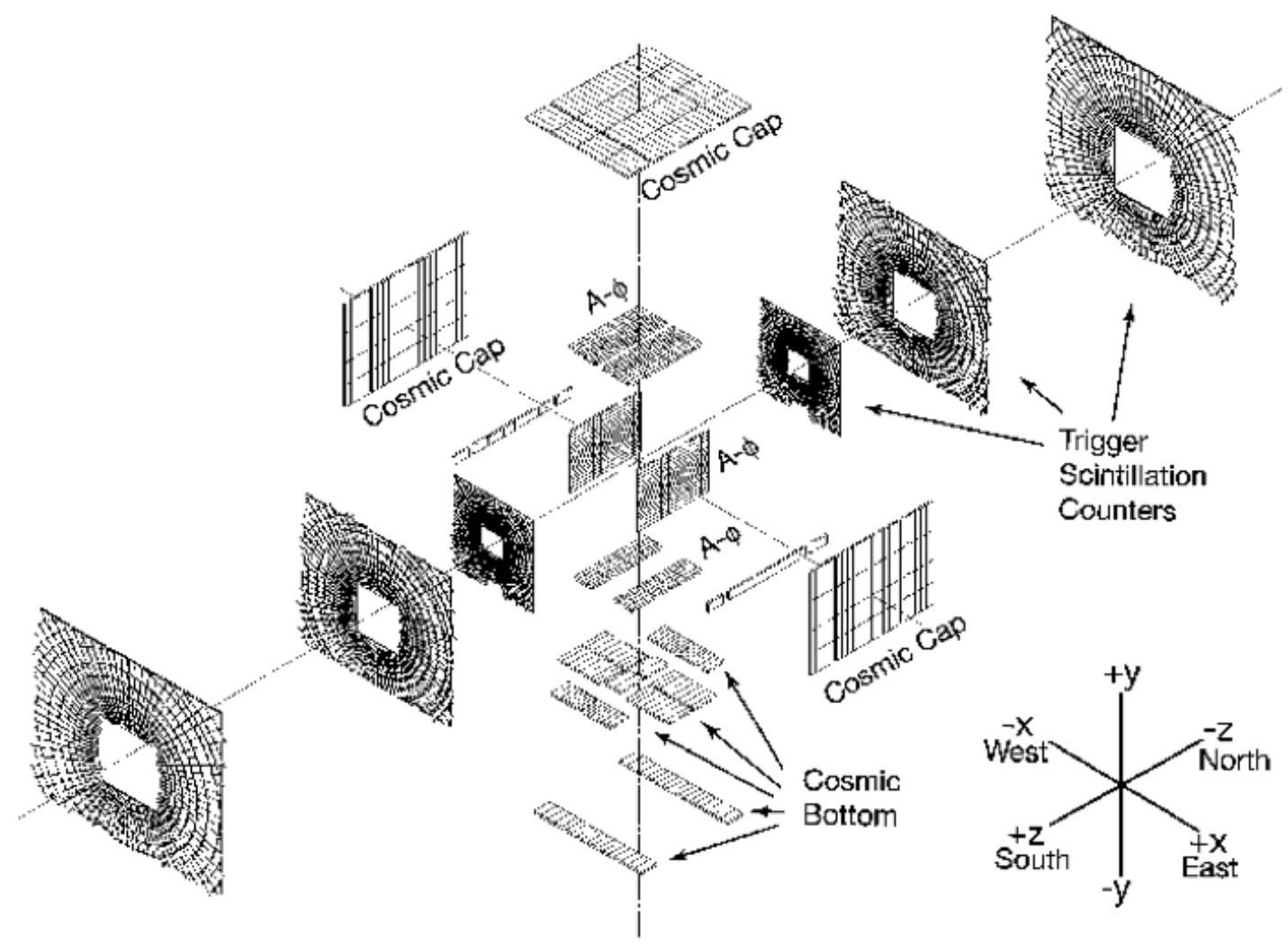

Figure 2.17: An exploded view of the $\mathrm{D} \varnothing$ muon scintillator system.

\subsubsection{Luminosity Monitor}

In high energy experiments we are interested in the rate and total number of inelastic collisions. Instantaneous luminosity is the rate of inelastic collisions per unit time and area while the integral of this quantity over time is the integrated luminosity. Since the distribution of particles within each bunch of protons and antiprotons is not known precisely at the Tevatron, the instantaneous luminosity cannot be calculated analytically and must be measured. For a fixed number of bunches, higher instantaneous luminosities correspond to denser bunches and therefore one can expect more interactions per beam crossing. Integrated luminosity is a necessary quantity to prop- 
erly compare the results of two different experiments and compare these results to the theoretical predictions.

The luminosity monitor (LM) at DØ consists an arrays of 24 plastic scintillation counters located around the beam pipe at $|z|=140 \mathrm{~cm}$ and so cover $2.7<\left|\eta_{\text {det }}\right|<4.2$ (Figure 2.18). Since the LM cannot distinguish multiple collisions in a single bunch crossing the number of crossings with no inelastic collisions is counted. Using this measurement and Poisson statistics the average number of inelastic collisions per beam crossing, $\bar{N}_{L M}$, can be determined. The instantaneous luminosity is then

$$
\mathcal{L}=\frac{f \bar{N}_{L M}}{\sigma_{L M}}
$$

where $f$ is the bunch crossing frequency and $\sigma_{L M}$ is the cross section of inelastic $p \bar{p}$ collisions taking into account the acceptance and efficiency of the LM itself [29]. Timing information is critical in order distinguish interactions with the LM and beam halo or the low $p_{T}$ particles produced by the majority of collisions.

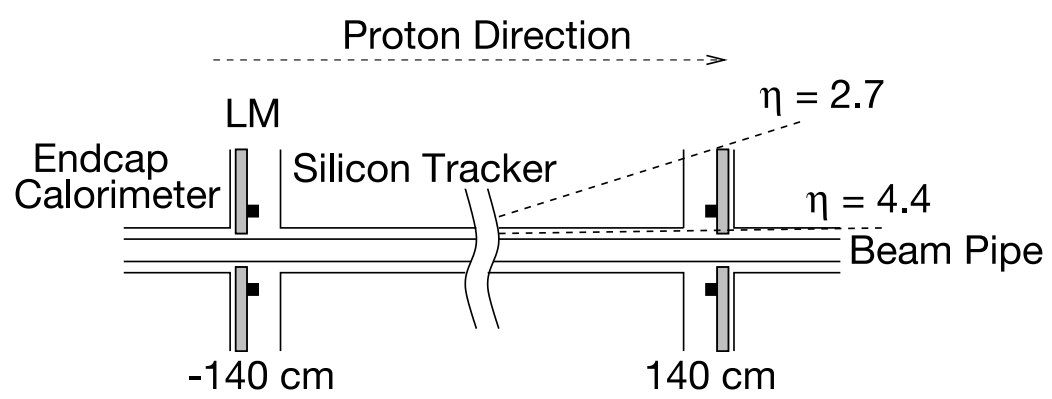

Figure 2.18: The LM detectors are installed $140 \mathrm{~cm}$ from the center of the detector near the beam pipe. 


\subsection{Trigger}

In one turn of the Tevatron, $21 \mu s, 36$ bunches of protons and antiprotons cross in the $\mathrm{D} \varnothing$ detector. That is over 1.7 million opportunities for there to be an inelastic collision of interest per second. In order to decide which events to record for analysis purposes a three tiered trigger system is employed. At each level, events are rejected in order to weed out all but the approximately 100 events per second which might be of interest. The three tiers (Figure 2.19 and 2.20), known as Level 1 (L1), Level 2 (L2), and Level 3 (L3), each have more time to analyze a given event then the previous stage and therefore do so in more sophisticated ways. To deal with the increased interaction rate, the trigger system was upgraded between Run I and Run II [26] and again between Run IIa and Run IIb [30].

\subsubsection{Level 1}

The L1 decision hardware issues accept/reject decisions every 132 ns through the use of fast, fixed-latency algorithms held in Field Programmable Gate Arrays (FPGAs). If an event passes the L1 criteria the detector is read out and collisions occurring at that time are ignored. Limiting this dead-time restricts the peak L1 rate to $5 \mathrm{kHz}$ but during normal operation it is approximately $2 \mathrm{kHz}$ which results in a dead-time of $5 \%$. Each of the six components of the L1 trigger generate a decision, know as an and/or term, and sends them to the trigger framework (TFW) where the decision to keep or reject the event is made. The components of the L1 trigger are as follows.

\section{- Level 1 Central Track Trigger (L1CTT)}

Only the axial layers of the CFT and CPS are used and each doublet layer is treated as a single layer to aid in rejection of fake high- $p_{T}$ tracks. Tracks reconstructed from these hits are matched to clusters in the axial layers of the 
CPS. This information is sent to the L1Mu and L1CalTrack systems.

\section{- Level 1 Preshower Trigger (L1PS)}

This trigger is based on clusters of energy found in the FPS detectors.

\section{- Level 1 Calorimeter Trigger (L1Cal)}

The pseudo-projective towers described in Section 2.2.4 are grouped in sets of four to create $1280 \Delta \eta \times \Delta \phi=0.2$ electromagnetic (EM) and hadronic (HD) trigger towers. A sliding windows algorithm is used to define objects in the $\eta$, $\phi$ grid [107]. In doing so, this algorithm identifies the optimal region of the calorimeter to maximize the transverse energy within the window of a given size with various threshold conditions depending on the object identified. This sub-system is explained in more detail in Appendix B.

\section{- Level 1 Calorimeter Track Trigger (L1CalTrack)}

By exploiting matches in the azimuthal positions of tracks from the L1CTT trigger with EM and jet objects from the L1Cal trigger, this system helps reduce the track and EM trigger rates. Since the muon system already does an azimuthal matching with the tracking system, the hardware is predominantly based on that used for L1Mu.

\section{- Level 1 Muon Trigger (L1Mu)}

This system looks for hits consistent with muons traveling through the wire chambers and scintillation counters and integrates track information from the L1CTT. In the central region, hits in the wire chambers are used to form tracks which are then confirmed with scintillator information. A subset of the highest $p_{T}$ tracks from L1CTT are matched to hits in the scintillator system. 
- Level 1 Forward Proton Detector is described in [26] and not discussed further here.

\subsubsection{Level 2}

The L2 decision must be made less that $100 \mu$ s which allows for the use of more sophisticated algorithms. The peak rate is restricted to $1 \mathrm{kHz}$ by the calorimeter digitization time which is the slightly larger than the normal operating rate. This level uses hardware to make rapid decisions like L1 but also uses microprocessors which are basically one-chip computers. Except for L1CalTrack, each L1 component has an analogous L2 component and a SMT module has been added. The components are:

\section{- Level 2 Silicon Track Trigger (L2STT)}

This system take the tracks found at L1 in the CFT and utilizes the much finer spatial resolution of the SMT by using all the hits lying within a road defined by the L1CTT track. With this better resolution impact parameter measurements are made to identify long-lived particles.

\section{- Level 2 Central Track Trigger (L2CTT)}

At Level 2 finer $p_{T}$ information is available as the tracks from the L1CTT are recalculated using additional hit information. Using this new information or tracks from L2STT, the azimuthal angle with respect to the beam-line and the third EM layer of the calorimeter as well as isolation information are all computed here.

\section{- Level 2 Preshower Trigger (L2PS)}

This system takes the clusters formed at L1 from the axial CPS layers and adds information from the stereo layers. Electron (photon) pre-showering is identified 
by clusters with (without) a L1CTT track match to clusters in both the CPS and FPS.

\section{- Level 2 Calorimeter Trigger (L2Cal)}

All 2560 trigger towers are used to in clustering algorithms. Jets are created from groups of $5 \times 5$ towers while EM and photon objets are created from two EMtowers with isolation criteria. $\mathbb{E}_{T}$ is also calculated from the vectorial sum of the towers using the center of the detector as the origin.

\section{- Level 2 Muon Trigger (L2Mu)}

More precise timing and calibration information is included at this level.

Information from the above sub-systems is passed to a global trigger, L2Global, which can test for correlations of signatures from several sub-detector and for events of interest transmits a L2 accept to the TFW.

\subsubsection{Level 3}

The L3 decision must be made less that $150 \mathrm{~ms}$. The peak rate at this final level is limited by the strain and cost of data storage and offline computing. During the trigger upgrades this rate was predicted to be $50 \mathrm{~Hz}$ but with over 400 nodes now in the L3 farm, this rate can be as high as $200 \mathrm{~Hz}$ but is usually around $100 \mathrm{~Hz}$. Since so much time has elapsed before L3 is aware of an event, the detector is fully digitized along the precision readout path. Farm nodes run a simplified reconstruction algorithm on the full detector readout and the information from all the sub-detectors is used to make the L3 decision. If an event passes all three levels it is written to permanent storage. 


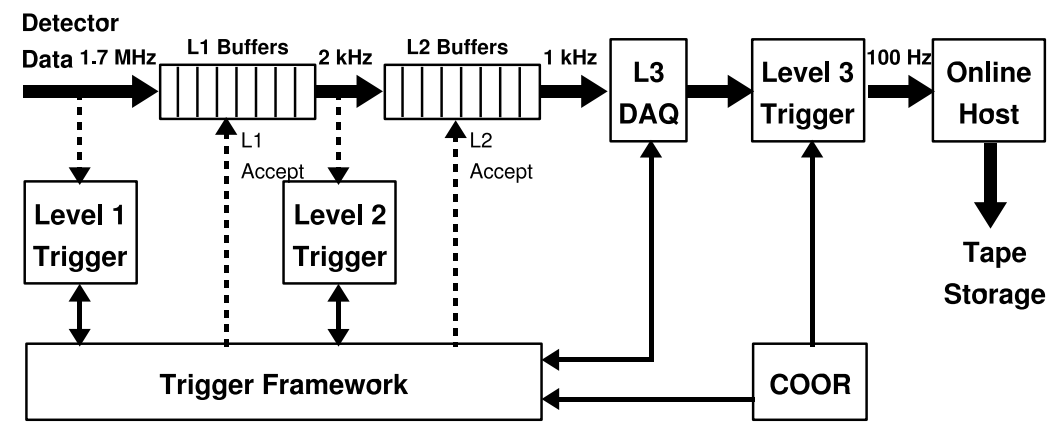

Figure 2.19: This flowchart shows how events pass through the D $\varnothing$ trigger.

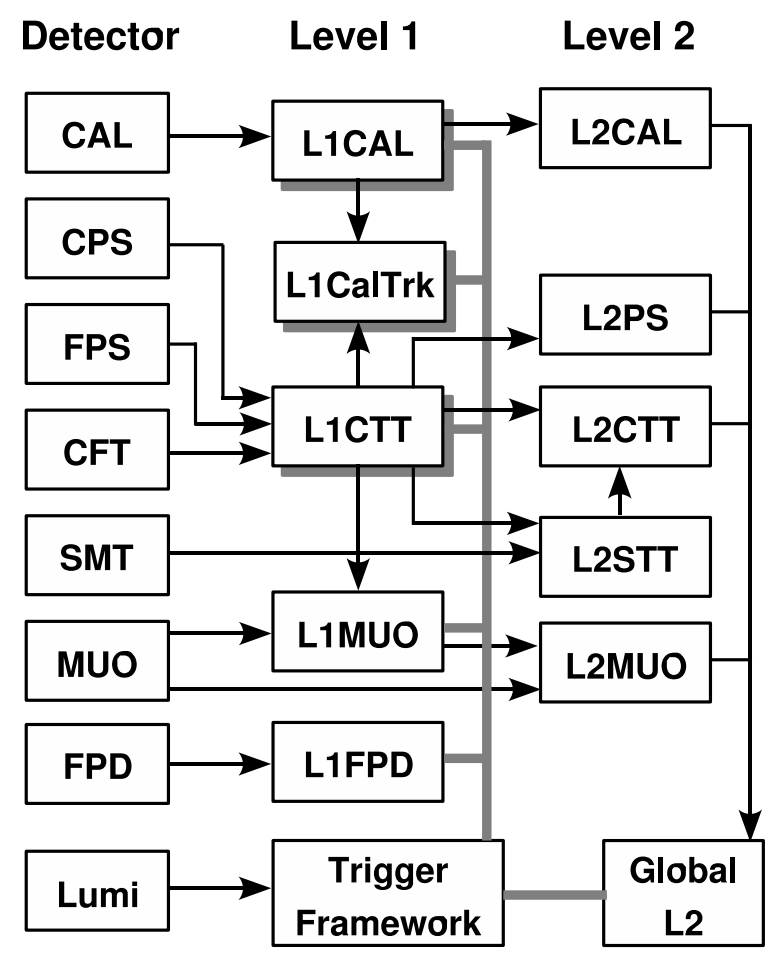

Figure 2.20: This flowchart shows how each sub-detector contributes to each of the three levels of the the $\mathrm{D} \varnothing$ trigger. 


\section{Chapter 3}

\section{Event Simulation}

The SM adheres to the laws of quantum mechanics where nature itself is probabilistic. A $p \bar{p}$ collision can result in a host of different particles and these particles decay again in a probabilistic manner to stable particles. The event topology in question can and usually does match the topology of several processes. When analyzing data, one cannot tell the difference between two different processes with the same topology with ease. The sophisticated techniques used to do this are discussed in Chapter 6, but before those techniques can be explored, one must estimate the relative contributions of the various productions and decays in the given sample. The way to do this is to simulate all possible outcomes from a $p \bar{p}$ collision and the detector's response to the particles created then compare this to the data. In this chapter, the programs used to simulate the $p \bar{p}$ collision and the detectors response is discussed.

\subsection{Monte Carlo Generators}

Quantum field theory is the framework in which the probabilistic interactions of the SM particles are calculated. These equations are not exactly solvable therefore a 
common practice is to expand about a minimum with a perturbation series in terms of the coupling strength. One can attempt expand as far as they like, but for practical purposes like a high energy experiment, one is mostly interested in the lowest order terms which the experiment can be sensitive to. At D $\varnothing$, the common cut off in leading order (LO) for kinematics and usually next-to-leading (NLO) order for the cross section. That is leading order generators are used but the resulting events are scaled to conform the to NLO cross section prediction.

\subsubsection{PYTHIA}

PYTHIA [31] is a LO event generator which at DØ is configured to use the CTEQ6L1 [32] parton distribution function (pdf) to model the quarks and gluons within the incoming proton and antiprotons. This generator, using the parton shower model method, does a good job of describing initial state (ISR) and final state radiation (FSR), the soft radiation of photons and gluons from the initial/final states. The hadronization of quarks attempting, but always failing, to escape the confinement of QCD is also well modeled. Hard radiation is not well modeled so other generators are employed for processes of this type.

\subsubsection{ALPGEN}

ALPGEN [33] in a LO Matrix Element event generator. The LO term is exactly calculated which provides a better description of hard radiation. This is necessary for events with many jets which do not all come from a particle decay. ALPGEN, however, does not describe hadronization very well and therefore has a inadequate model of the underlying jet structure. 


\subsubsection{CompHEP}

CompHEP [34] is also a LO Matrix Element event generator used to simulate single top events because it reproduces NLO distributions fairly well while maintaining the spin correlations between the top quarks and resulting $W$ boson.

\subsubsection{Monte Carlo for Femtobarn Processes}

Monte Carlo for FeMtobarn processes [35] (MCFM) is a program designed to calculate cross-sections for various femtobarn-level processes at hadron-hadron colliders. Most processes NLO matrix elements and incorporate full spin correlations. This program is used to verify/correct cross section predicted by other generators.

\subsubsection{SHERPA}

Sherpa [36] in a Matrix Element event generator shown to model boson+jet final states well [37] but does not model hadronization adequately.

\subsubsection{Mixing and Matching}

To get the best of both worlds, one can use ALPGEN or CompHEP to have a well modeled hard scatter and hard radiation then use PYTHIA to properly model the soft radiation and hadronization. This works well but one must be sure not to double count.

$W / Z+$ jets and $t \bar{t}$ processes are generated with ALPGEN interfaced with PYTHIA for the simulation of initial and final state radiation, and of jet hadronization. Theses processes are generated in exclusive parton multiplicities with the largest being inclusive. In the ALPGEN stage, the generated partons are required to have $p_{T}>8 \mathrm{GeV}$ and a separation $\Delta R>0.4$. After pythia showering, any particle jet with $p_{T}>8 \mathrm{GeV}$ 
is required to match an ALPGEN parton, except in the highest parton multiplicity (inclusive) sample, where additional (unmatched) particle jets are allowed. This is known as the MLM matching scheme [38].

All ALPGEN $W / Z+$ jets samples produced with the above described method undergo a process of heavy-flavor (HF) skimming; that is, events containing heavyflavored partons generated by PYTHIA in the region of phase space where they are also generated by ALPGEN in the hard process, have been removed in order to avoid double counting of heavy flavor production [39].

\subsection{Detector Simulation}

Millions of events containing a wide range of particles with various decays are produced and in order to be compared to the data the detectors response must also be simulated. To this end DØgstar, a detector simulation based on the GEometry ANd Tracking (GEANT) software package created at CERN is used. This program contains a full simulation of material, both active and passive, in the detector. Once these simulated particles interact with the detector material, another software package named DØsim simulates the electronic read-out including effects such as electronic noise and known inefficiencies. The result is a block of information which is of a form identical to the data itself. From this point on, the two can be treated in the same way, modulo some corrections applied to the simulation.

\subsection{Monte Carlo Corrections}

This simulation processes is nontrivial and therefore it does not exactly replicate the

collected data. In some cases, this differences are unavoidable. Some physics analyses 
use custom corrections to accurately model effects which are not simulated correctly but all analysis must correct the Monte Carlo for the following known differences.

\section{Luminosity profile}

As stated before, the higher the instantaneous luminosity the more collisions will occur at each bunch crossing. In the MC, only one collision occurs. To mimic the effects of different instantaneous luminosities, data events from randomly chosen unbiased beam crossings are overlaid to the simulation during DØsim. The luminosity distribution of these random events will not be exactly like the data so the simulated distribution is re-weighted to follow the trend seen in data.

\section{Beam Profile}

The position of the primary interaction is spread in a Gaussian distribution in the simulation. This is re-weighted to mimic the distribution seen in data which is not a perfect Gaussian.

\section{$\mathbf{W} / \mathbf{Z} p_{T}$}

Decays of a $Z / \gamma^{*}$ to two electrons have been used to test the validity of the simulated boson $p_{T}$ distribution. It was found that below $100 \mathrm{GeV}$ this spectrum is not well modeled by either PYTHIA or ALPGEN. A re-weighting has been derived to adjust the generator spectra to match the measured $Z / \gamma^{*} p_{T}$ distribution [40]. There is no measurement of the $W p_{T}$ distribution available. Here, the simulation is adjusted using the $Z / \gamma^{*} p_{T}$ correction and the ratio of $W$ to $Z$ differential cross sections [41]. 


\section{MLM Matching}

A reweighting recommended in Reference [92], designed to correct the MLM matching $p_{T}$ threshold, which was seen to be more appropriate at $13 \mathrm{GeV}$ than at the default $8 \mathrm{GeV}$ used in our MC sample generation is applied to the $(W / Z+)$ light-flavor-jets samples in more recent analysis.

\section{Energy Measurements}

The resolution of the simulated detector does not match that of the real detector. This is not due to lack of effort. The detector resolution is tricky thing to model correctly and changes over time. The corrections for individual objects are described in Chapter 4. Generally stated the energy measurements are convoluted with a gaussian so on average the simulation agrees with data.

\section{Identification Efficiencies}

The simulated detector is more efficient than the real detector so more objects are identified in the simulation then are observed. A function of the data and simulation efficiencies are used produce a correction factor. These efficiencies are determined in a dedicated sample using the tag and probe method. This is a method by which a relatively pure set of objects are selected by tight constraints on the tag object. Then the probe object, found usually within some spatial constraint from the tag object, is tested for a given set of criteria. The fraction of probe objects passing this test gives an efficiency. 


\section{Chapter 4}

\section{Object Reconstruction and}

\section{Identification}

Particles which interact with the detector material leave energy deposits in the subdetectors described in Section 2.2. In this chapter the algorithms used to interpret these deposits as physical objects is discussed. The first stage of this process is done in the $\mathrm{D} \varnothing$ reco package and objects are refined at the analysis level.

\subsection{Tracks}

Reconstructing tracks can be tricky business especially in high luminosity environments where many charged particles travel through the active material of the tracker. Inefficiencies of the detectors, noise which can fake a hit, and the unknown curvature of the tracks adds further complication. To optimize the performance of the track reconstruction, two complementary algorithms, the Histogramming Track Finder (HTF) which is better at high $p_{T}$, in the forward region, and at high instantaneous luminosity and the Alternative Algorithm (AA) which is better at low $p_{T}$, has a lower fake rate, 
and a higher efficiency for tracks having a large impact parameter. Both algorithms are based on a Kalman filter [42], a mathematical method that takes measurements containing random variations and produces values in a way which minimizes the variance of the estimation error.

The HTF is a pattern-recognition method [43]. In the transverse plane, the trajectory of a particle of charge $q$ moving through a magnetic field $B$ is completely described by the curvature

$$
\rho=\frac{q B}{p_{T}}
$$

the distance $\left(d_{0}\right)$ and direction $(\phi)$ of closest approach to the origin. With the assumption $d_{0} \approx 0$ and an unknown momentum, a series of paths can be drawn from the origin to a hit located at $\left(x_{i}, y_{i}\right)$ (Figure 4.1). Using a Hough transformation, this translates to a line in the $(\rho, \phi)$ parameter space, with each point along this corresponding to a possible path. All the hits produced by a given charged particle should cross at one point in the parameter space. The $(r, z)$ coordinate can also be translated to the $\left(z_{0}, C\right)$ parameter space where $C$ is the inclination $(d z / d r)$ of the track. This is done for the SMT and CFT separately producing a list of template tracks. Then the Kalman filter is given the hits associated with these templates and constructs tracks starting from a pair of hits in either detector and stepping through all the layers in a recursive manner. 


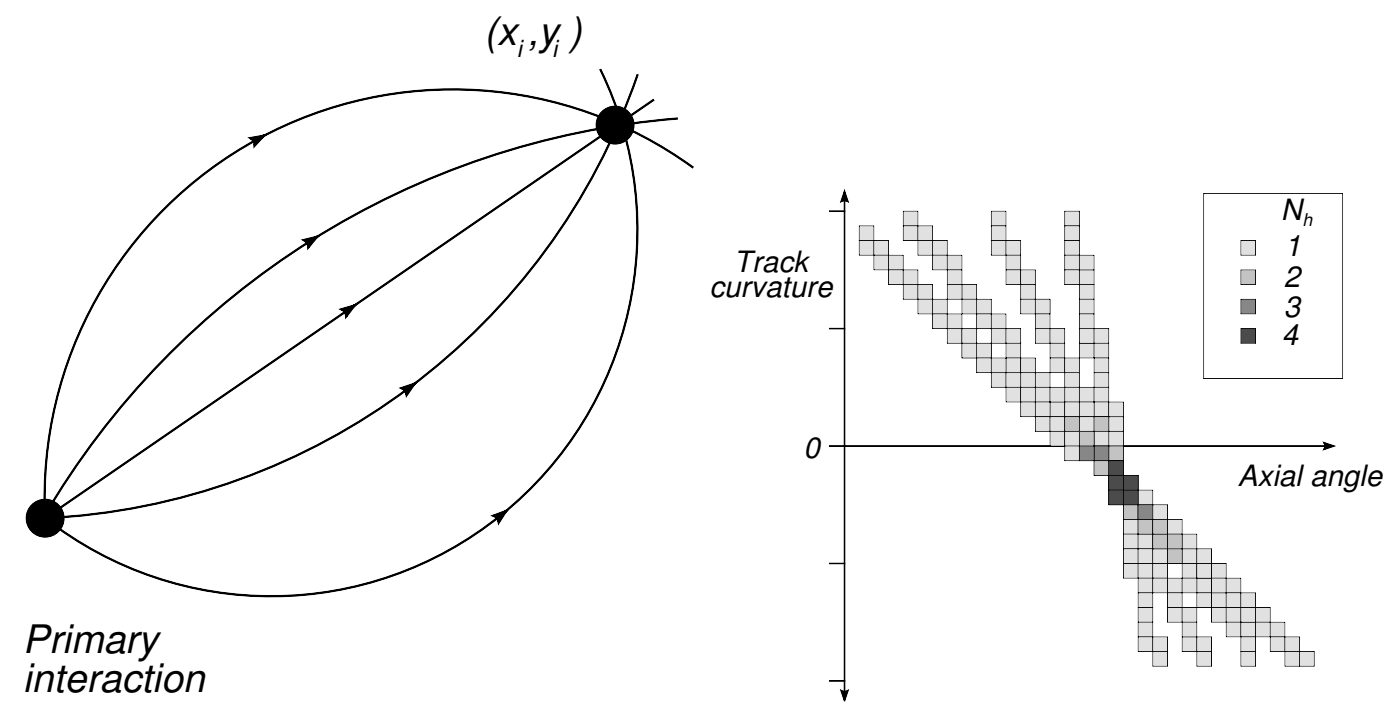

Figure 4.1: The HTF track finding algorithm. Multiple paths can be drawn between the origin and a hit in the tracker (left). Each possible path translates to a line in $(\rho, \phi)$ space (right). Figures from [44].

The AA starts with a road following method [45]. It begins with the innermost set of three hits in the SMT which satisfy conditions on their angular separation (Figure 4.2). Once track parameters are measured further constraints are put on the radius of curvature, transverse distance of closest approach, and the $\chi^{2}$ constructed from the uncertainties of the hit coordinates. The algorithm proceeds through each layer of the SMT and eventually the CFT adding hits that do not increase the $\chi^{2}$ by no more than 16 . If there are several candidates, all possible resulting tracks are considered. Limits on the number of misses, hits shared between tracks, and the ratio of hits to misses are imposed. Tracks can also being in the inner layers of the CFT and proceed into the SMT as long as they pass within $1.5 \mathrm{~cm}$ from the primary vertex reconstructed from the SMT seeded tracks. 


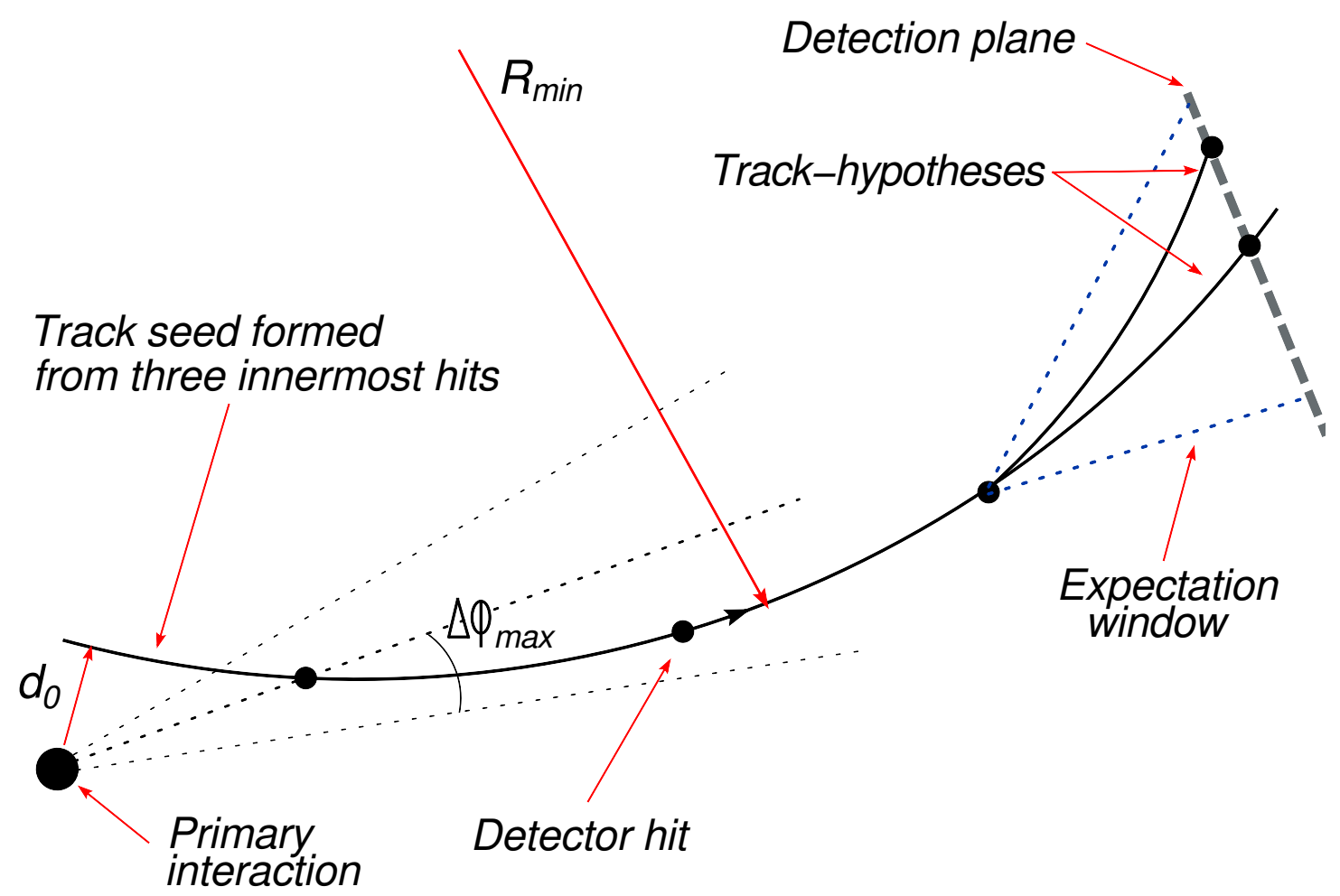

Figure 4.2: The AA track finding algorithm. The first two hits must have an angular separation less than $\Delta \phi_{\max }=0.08$ and a circle drawn through the first three hits must have a radius of curvature, $R>30 \mathrm{~cm}$ corresponding to $p_{T}=180 \mathrm{MeV}$. The expectation window is the region in which hits would not increase the $\chi^{2}$ by more than 16. Figure from [44].

Tracks from both methods are then give to the Global Tracking Refit [46,47]. This algorithm uses a Kalman filter in a more sophistication road finding technique. An effective multidimensional $\chi^{2}$ minimization is done while adding hits near the path predicted when solving for the path of a particle in a magnetic field and considering energy lost though the interaction with the detector material. Even though there is a large amount of detector material beyond the solenoid, the pre-shower and muon systems can be used to test the parameters of the final set of tracks. 


\subsection{Primary Vertices}

Primary vertices (PVs) are points in the luminous region of the detector where a proton has collided with an antiproton while a secondary vertex is a point where a long lived particle created in the initial interaction decays. Discriminating tracks from secondary vertices with short decay lengths and those from additional minimum bias interaction close to the PV in question is one of the main hurdles of this process. To overcome biases from displaced tracks an iterative, re-weighted Kalman Filter, the Adaptive vertex fitter algorithm, is used [48]. The difference is that instead of removing tracks which contribute more to the $\chi^{2}$ then some cut-off value, a sigmoidal weight, shown in Figure 4.3, is applied to the track error such that tracks from a secondary vertex may contribute to the vertex location measurement but with a weight smaller than unity.

Tracks with $p_{T}>0.5 \mathrm{GeV}$ and more than one SMT hit, for those inside the fiducial region of the SMT, are clustered if separated by less that $2 \mathrm{~cm}$ and within each cluster fit with a Kalman Filter to a common vertex thus determining the beam spot location and width. Tracks contributing the most to the $\chi^{2}$ are removed until the total $\chi^{2}$ per degree of freedom $\left(\chi^{2} / n d f\right)$ is less that ten. Tracks within five standard deviations of the measured beam spot are given to the Adaptive vertex fitter algorithm.

The list of PVs is sorted such that the first is most likely the hard-scatter vertex using a minimum bias probability selection algorithm [49]. 


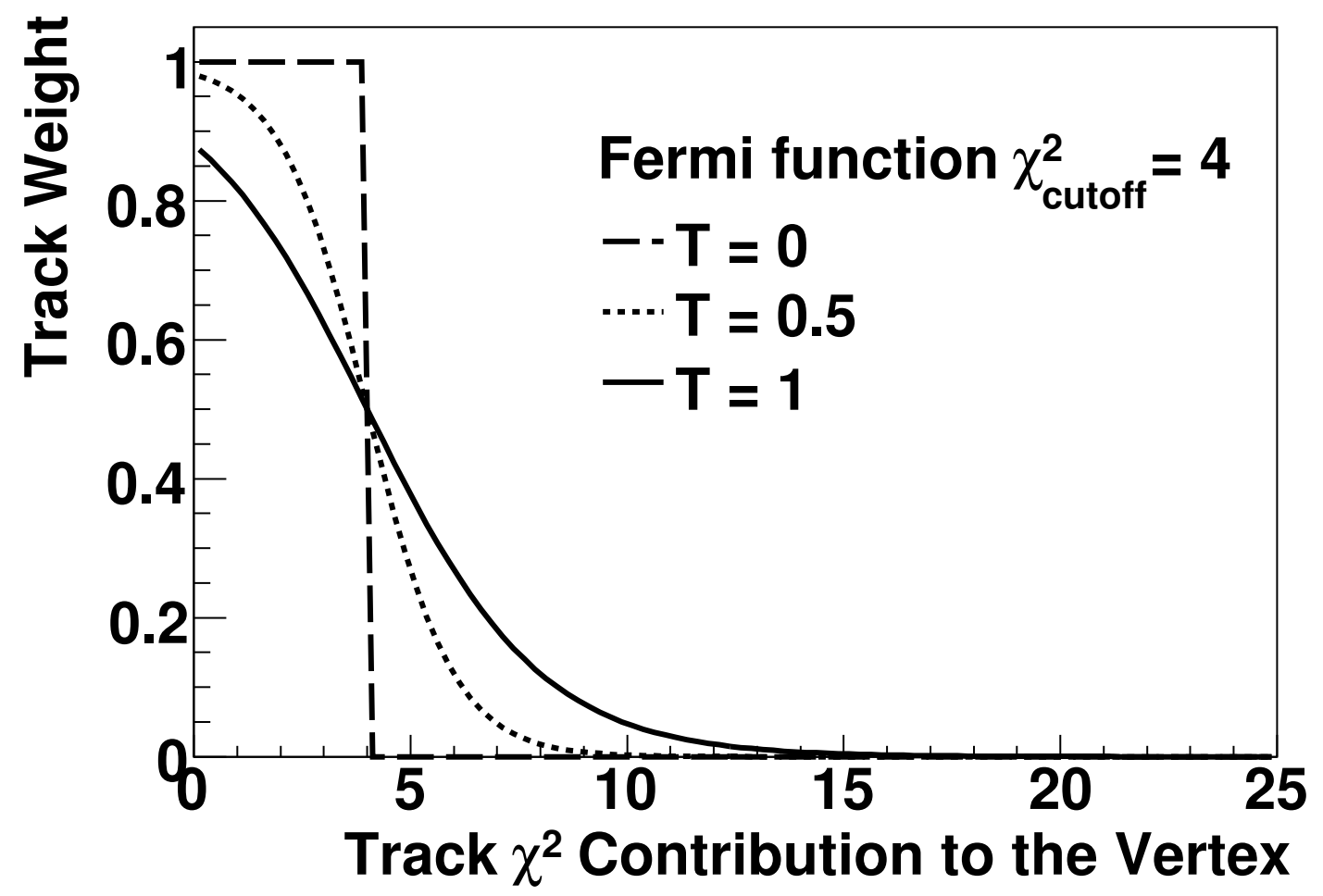

Figure 4.3: The sigmoidal weight function of the Adaptive vertex fitter algorithm $w_{i}=$ $\frac{1}{1+e^{\left(\chi_{i}^{2}-\chi_{\text {cutoff }}^{2}\right) / 2 T}}$ where $\chi_{i}^{2}$ is the $\chi^{2}$ contribution of track $i$ to the vertex and analogous to the Fermi function in statistical thermodynamics $T$, the temperature, controls the sharpness of the function.

\subsection{Muons}

Muons are identified in three different independent sub-detectors in the D $\varnothing$ detector $[50,51]$. Local muons are reconstructed in the three layer muon system itself covering $90 \%$ of the angular acceptance up to $|\eta|<2$. In the central tracking system which has angular coverage beyond that of the muon system is used to match tracks to local muons extrapolated back through the calorimeter and subsequently called central muons. Lastly, the calorimeter muons are identified via minimizing 
ionizing particle (MIP) signatures and these will not be further discussed here. Central muons, once identified, are refit using all available information to improve the momentum resolution. Central tracks are extrapolated outward to segments formed in only the A or BC layers.

Reconstructed muons are classified by a type, quality, an associated track quality, and an isolation. Type is given by the nseg parameter $(|n s e g| \leq 3)$ defined in Table 4.1. A muon's quality is either tight, medium or loose. These labels are based on the number of hits in the muon drift chambers and scintillators. The track quality of central muons are classified as either loose, medium, or tight also. Through the isolation, muons from semi-leptonicly decaying b-quark $(b, c \rightarrow \mu+X)$ are identified. The five isolation variables defined with respect to the tracks near the muon track or the calorimeter energy around the muon momentum vector are as follows:

- TrackHalo $=\left|\sum^{\text {tracks }} p_{T}\right|$ in $\Delta R$ (track, muon track $)<0.5$ cone.

- CalorimeterHalo $=\left|\sum^{\text {cells }} E_{T}\right|$ in $0.1<\Delta R$ (calorimeter-cells, muon calorimetertrack $)<0.4$.

- $\Delta R(\mu$, jet $)=$ Distance to closest jet in $\eta-\phi$ space.

- ScaledCalorimeterHalo $=\left|\sum^{\text {cells }} \frac{E_{T}}{p_{T}(\mu)}\right|$ in $0.1<\Delta R$ (calorimeter-cells, muon calorimeter-track) $<0.4$.

- ScaledTrackHalo $=\left|\sum^{\text {tracks }} \frac{p_{T}}{p_{T}^{(\mu)}}\right|$ in $\Delta R($ track, muon track $)<0.5$ cone.

To reduce muons from cosmic sources the distance of closest approach to the beam line and the difference between scintillator hits and the bunch crossing time is restricted while the acceptance of high $p_{T}$ muons from pion or kaon decays is controlled with the $\chi^{2} / n d f$ parameter. 


\begin{tabular}{|l|c|c|}
\multicolumn{3}{|c}{ Muon Types } \\
\hline NSeg & Muon Type & $\begin{array}{c}\text { Central Track } \\
\text { Matching Algorithm }\end{array}$ \\
\hline 3 & $\begin{array}{c}\text { Central track }+ \\
\text { local muon track } \\
\text { (A and BC layer) }\end{array}$ & $\begin{array}{c}\text { Muon to central if local } \\
\text { muon track fit converged. } \\
\text { Central to muon otherwise }\end{array}$ \\
\hline 2 & Central track + BC only & central to muon \\
\hline 1 & Central track + A only & central to muon \\
\hline 0 & $\begin{array}{c}\text { Central track }+ \text { muon hit } \\
\text { or central track }+ \text { MTC }\end{array}$ & $\begin{array}{c}\text { central to muon } \\
\text { central to calorimeter }\end{array}$ \\
\hline-1 & A segment only & no match \\
\hline-2 & BC segment only & no match \\
\hline-3 & $\begin{array}{c}\text { local muon track } \\
\text { (A }+ \text { BC) }\end{array}$ \\
\hline
\end{tabular}

Table 4.1: Muon Types.

\section{Muon Energy Resolution}

The muon momentum resolution was found by performing a fit to the $Z \rightarrow \mu^{+} \mu^{-}$ mass-peak in Run IIa data [50] and both the $Z \rightarrow \mu^{+} \mu^{-}$and $J / \psi \rightarrow \mu^{+} \mu^{-}$distributions in Run IIb [52] assuming the resolution of $1 / p_{T}$ is gaussian. The resolution is dependent on the presence of hits in the SMT and the detector $\eta$ measured at the first CFT layer $\eta_{C F T}$. For Run IIb the resolution is parameterized as

$$
\frac{\sigma\left(p_{T}\right)}{p_{T}}=\frac{\sigma\left(1 / p_{T}\right)}{1 / p_{T}}=\sqrt{A^{2} p_{T}^{2}+B^{2} \cosh \left(\eta_{\text {det }}\right)}
$$

and is shown in Figure 4.4. This function is used to smear the simulation to better agree with data. 


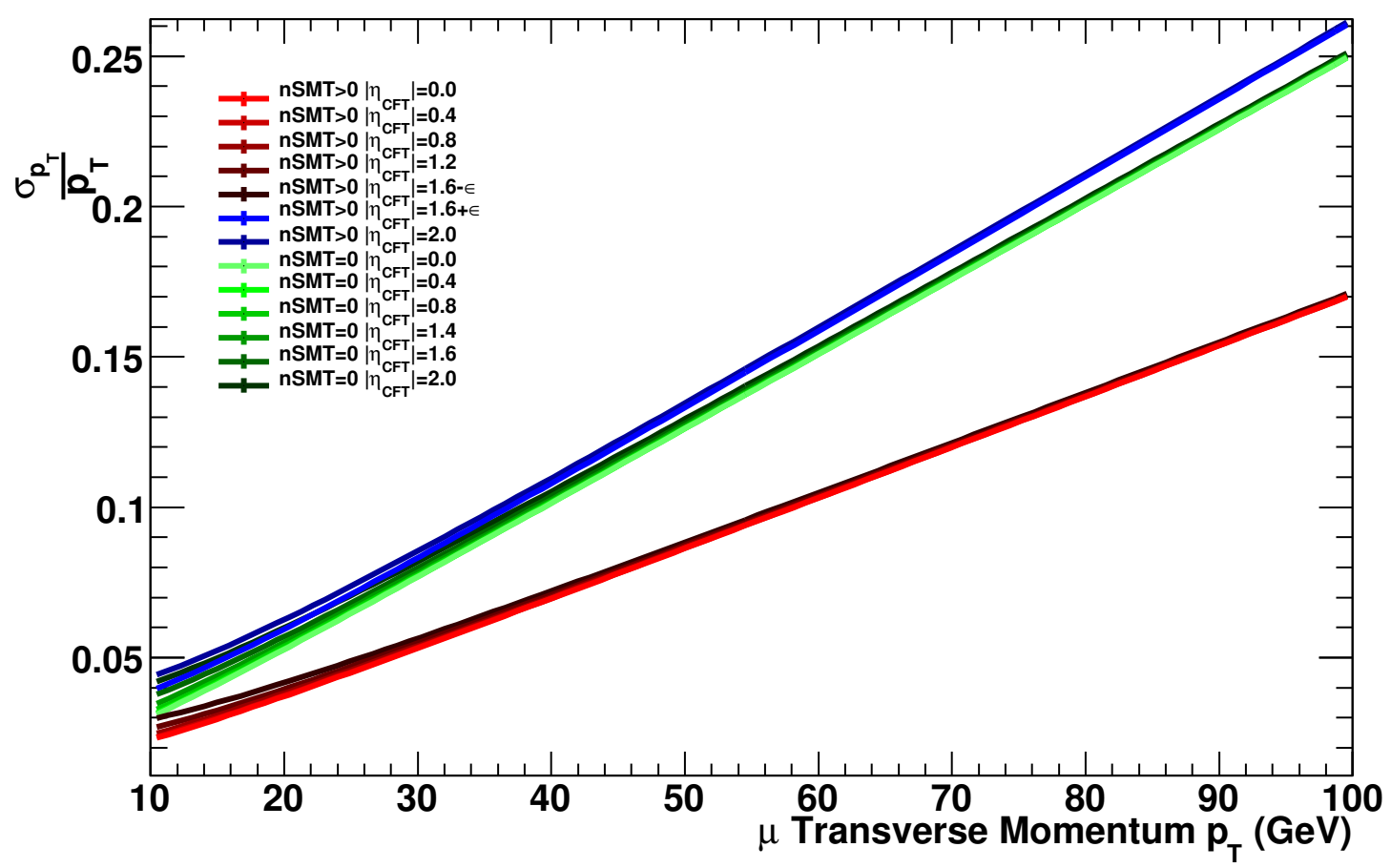

Figure 4.4: Comparison of the resolution of the three cases of muons in Run IIb data for a range of detector eta.

\subsection{Electrons and Photons}

Two types of EM objects are identified, which then seed a Simple Cone algorithm. Then, these objects are refined by matching to tracks and pre-shower hits $[53,54]$. In the first step, a list of preclusters is created from either groups of calorimeter towers seeded from a $0.5 \mathrm{GeV}$ tower, or soft electron candidates seeded from a track with $p_{T}>1 \mathrm{GeV}$ matched to CPS hits and EM towers with an EM fraction (EMF), defined in Equation 4.4, larger than 0.80. These objects are passed to the refinement stage if items of the two types match with $\Delta R<0.01$ or if a Simple Cone algorithm seeded 
from the list constructs an object with $\Delta R<0.4, p_{T}>1.5 \mathrm{GeV}, \mathrm{EMF}>0.90$, and isolation> 0.2 as defined in Equation 4.5 matched to tracks using the track-match criteria below.

At the analysis level, further requirements can be put on the electron objects. The full set of the criteria are listed below [55]:

- ID: 10 no track match, 11 track matched.

- Track Match: A $\chi^{2}$ probability, defined to match the CFT and SMT track coordinates and ensure that the $p_{T}$ of the track has a reasonable value compared to the energy in the calorimeter, is required to be greater than $10^{-2}$.

$$
\chi^{2}=\left(\frac{\Delta \phi}{\sigma_{\phi}}\right)^{2}+\left(\frac{\Delta z}{\sigma_{z}}\right)^{2}+\left(\frac{E_{T} / p_{T}-1}{\sigma_{E_{T} / p_{T}}}\right)^{2}
$$

- IsoHC4: Track isolation which is the total transverse momentum of tracks in the hollow cone with $0.05<R<0.4$ around the EM cluster.

- H-Matrix: Is the inverse of the covariance matrix of a set of variables meant to characterize the lateral and longitudinal shape of EM showers.

- Likelihood: The variable is built as described in Section 6.1. Seven variables are used to give a probability of the object being an electron or a jet.

- Neural Net: A neural net, similar to those described in Section 6.3, trained on seven variables including CPS information to distinguish electrons from jets and photons.

- Hits on the Road (HoR): Using the tracking information to test the probability of an EM object being an electron or photon. 
- EM fraction (EMF): Fraction of energy in the EM layers

$$
\frac{E_{E M}}{E_{E M+H D}}
$$

- Iso: Calorimeter Isolation

$$
\frac{E_{E M+H D}(R<0.4)-E_{E M}(R<0.2)}{E_{E M}(R<0.2)}
$$

For convenience, three qualities known as Point0, Point1, and Point2 defined for CC EM objects are defined in Table 4.2. These operating points yield $\sim 90 \%, 80 \%$, and $75 \%$ electron efficiency for a $\sim 5 \%, 0.9 \%$ and $0.2 \%$ fake rate respectively for $40 \mathrm{GeV}$ EM objects in the CC. These criteria are slightly tightened and the width of the EM object at the third EM layer of the calorimeter is used in the EC yielding similar performance for Point0 but Point1 and Point2 take a $20 \%$ hit in efficiency for the same fake rate. These operating points became available in Autumn of 2009. 


\begin{tabular}{|l|c|c|c|}
\hline Variable & Point0 & Point1 & Point2 \\
\hline \hline Iso & 0.09 & 0.08 & 0.08 \\
EMF & 0.9 & 0.9 & 0.9 \\
H-Matrix & - & 35 & 35 \\
IsoHC4 & 0.4 & 2.5 & 2.5 \\
NN & 0.4 & 0.9 & 0.9 \\
Likelihood & - & 0.2 & 0.6 \\
$E / p$ & - & 8.0 & 3.0 \\
Track Match & 0.0 & 0.0 & 0.0 \\
or HoR & 0.6 & - & - \\
\hline
\end{tabular}

Table 4.2: Electron identification operating points for CC electrons which became available in the Autumn of 2009.

\section{Electron and Photon Energy}

An energy scale correction modeled as

$$
E_{\text {measured }}=\alpha \cdot E_{\text {true }}+\beta
$$

is applied to the reconstructed EM objects in data and $\mathrm{MC}$ while the simulation energy is also smeared to match the data resolution modeled by

$$
\frac{\Delta E}{E}=1+C \cdot N(0,1)
$$

where $N(\mu, \sigma)$ is a gaussian distribution centered at $\mu$ with width $\sigma$. The parameters were all determined fitting the $Z$ mass-peak in $Z \rightarrow e^{+} e^{-}$events to as a function of $\eta_{\text {det }}$ and $\phi[56,57]$. An energy scale correction for non-fiducial effects in the CC is 
also applied in more recent analysis [58]. This correction, determined on $Z \rightarrow e^{+} e^{-}$ events, is a function of $\eta_{d e t}$, shower shape, and $\phi_{\text {mod }}$, where

$$
\phi_{\text {mod }}=\bmod (32 \phi / 2 \pi, 1)
$$

gives the location of the EM object with respect to the calorimeter cell boundaries. The simulation is also smeared by an additional factor to correct for non-gaussian tails arising from effects such as incomplete charge collection. The resolution of electrons is shown in Figure 4.5.

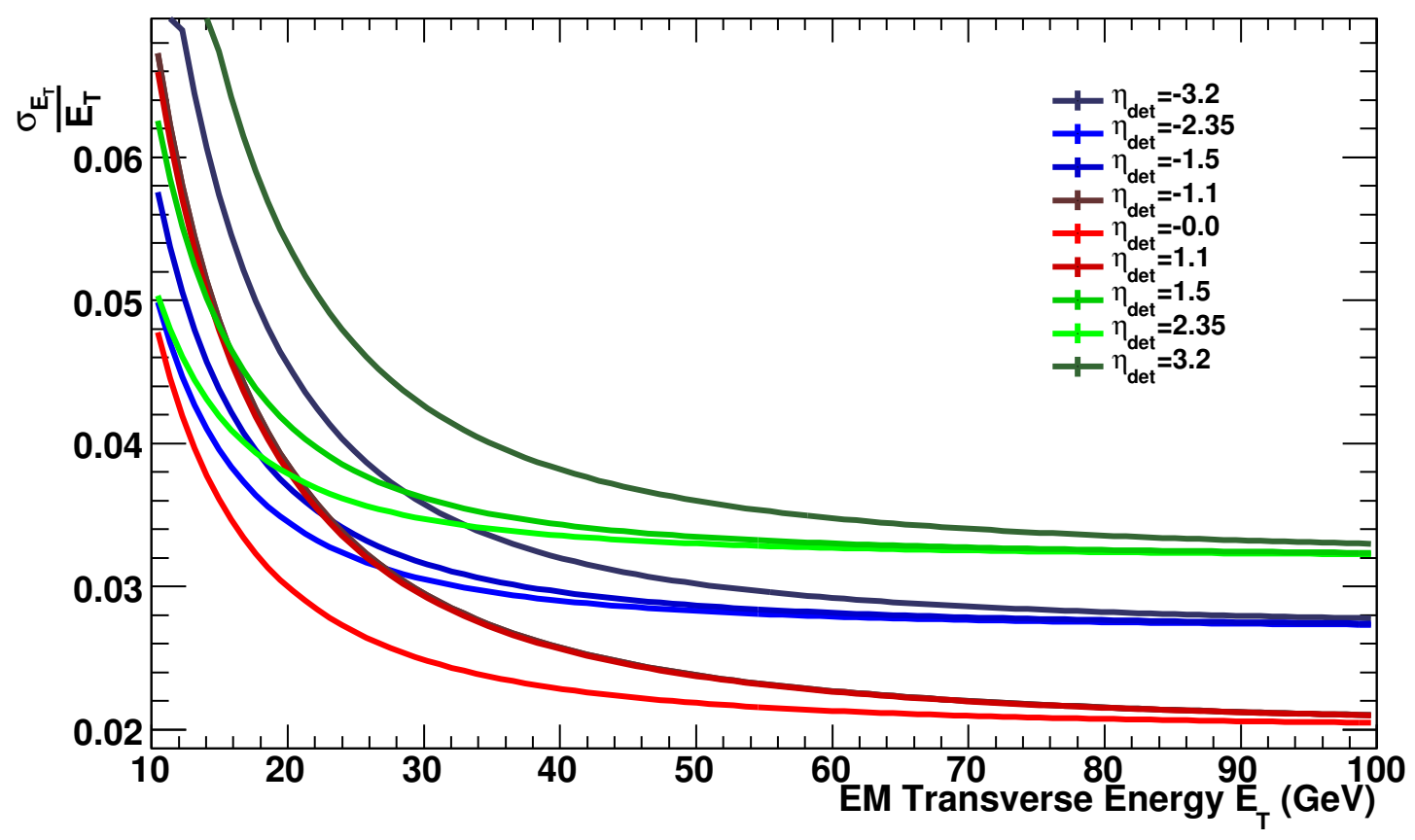

Figure 4.5: Comparison of the resolution of the three cases of electrons in Run IIb data. Note the asymmetry in the two endcap resolutions for a range of detector eta. 


\subsection{Taus}

Taus decay $100 \%$ of the time to a neutrino and a virtual $W$ which decays to another neutrino and a lepton $\sim 35 \%$ of the time. These taus must be identified by this lepton since the detector is oblivious to the neutrinos. The remaining $65 \%$ of taus are considered hadronic as they decay predominantly to pions through a virtual $W$ resulting in a relatively narrow shower. Tau decays are outlined in Table 4.3.

A reconstructed tau has three components [59], a calorimeter cluster, and EM subcluster, and associated tracks. The calorimeter cluster is formed using a simple cone algorithm with a cone radius of 0.5 and a width (RMS) lower than 0.25 . The RMS of a cluster is defined as

$$
R M S=\sqrt{\sum_{i}^{\text {cells }} \frac{E_{T}^{i}}{E_{T}}\left(\Delta \phi_{i}^{2}+\Delta \eta_{i}^{2}\right)} .
$$

The EM subcluster is formed using the Nearest Neighbor Algorithm using a seed cell in EM layer 3. This subcluster is meant to capture the electromagnetic part of the $\tau$ decay such as $\pi^{0} \rightarrow \gamma \gamma$. This is important for neutral decays products which will not create a track. The final component is from the tracking system. All tracks within a $\Delta R$ of 0.5 from the calorimeter cluster are considered and the leading track must have $p_{T} \geq 1.5 \mathrm{GeV}$. Up to two additional tracks can be considered if they are within $\Delta z \leq 2 \mathrm{~cm}$. The second track is added if its invariant mass of the two tracks is less that $1.1 \mathrm{GeV}$ and a third track is added if the total invariant mass of the three is less than $1.7 \mathrm{GeV}$. The objects which pass this far are considered tau candidates. However, the list must be further refined because jets can pass this criteria as well. To increase the purity of taus selected a neural network (TauNN v1) is employed [60] which was recently updated (TauNN v2) [61] in include more discriminating variables 
and bID information. This more recent NN uses 12 or 13 variables, depending on the tau type, and exploits isolation, shower composition and shape, and track-calorimeter correlations to exploit the differences. It was found that the preshower detectors did not add increased separation power but using b-quark identification terms did bring significant improvement. This is due to the fact that, like a b-quark, a $\tau$ will travel some detectable distance before decaying. A second $\mathrm{NN}$ is also available to distinguish type 2 taus from very similar electron signatures.

\begin{tabular}{|l|c|c|c|}
\hline Decay type & Tau Final State & Channel & $\mathrm{BR}(\%)$ \\
\hline Electron Mode & $e+\nu_{e}+\nu_{\tau}$ & Leptonic & 17.84 \\
Muon Mode & $\mu+\nu_{\mu}+\nu_{\tau}$ & 17.36 \\
\hline Hadronic Type-1 & $\pi(/ K)+\nu_{\tau}$ & Hadronic 1-prong (48.7\%) & 11.59 \\
Hadronic Type-2 & $\pi(/ K)+\geq 1 \pi^{0}+\nu_{\tau}$ & & 38.98 \\
\hline Hadronic Type-3 & $\pi \pi \pi+\geq \pi^{0}+\nu_{\tau}$ & Hadronic 3-prong & 14.23 \\
\hline
\end{tabular}

Table 4.3: The final state products of the main $\tau$ decay modes and the associated branching ratios.

\section{Tau Energy Scale}

The tau energy scale corrects the visible energy; no attempt is made to recover the energy carried by the neutrino. Since most hadronicly decaying taus decay to either one or more pions or kaons, this correction is based on the single pion response. For uncorrected energies under $70 \mathrm{GeV}$ for type 1 taus the best energy measurement comes from the tracking system. For uncorrected type 2 and 3 energies less than 100 and $120 \mathrm{GeV}$ respectively, the charged pion response correction is applied. Beyond these values, the calorimeter information, once corrected for the tau energy scale, is best $[62,63]$. The energy scale corrections have been determined on single tau events 
and a limited about of data was used to model the charged pion response as

$$
E_{\pi^{ \pm}}=p_{0}+p_{1} \cdot \tanh \left(\frac{p}{p_{2}}\right)+p_{3}+p \cdot p_{4}
$$

where $p$ is the momentum of the charged pion track and the constants, $p_{0,1,2,3,4}$, are a function of $\eta_{\text {det }}$. The effects of these corrections were tested on both data and MC in the Run IIa $Z \rightarrow \tau \tau$ cross section measurement base on $1.0 \mathrm{fb}^{-1}$ of data [64].

\subsection{Jets}

Jets are a ubiquitous feature of a hadron collider and so must be treated with care. A quark cannot exist in a free, colored state. If a quark is ejected from the proton or a particle such as a $Z$ boson decays into a pair of quarks, they do not stay in that state for long. The potential energy of QCD is such that it grows with the distance between quarks. When this energy reaches a large enough value a quark-antiquark pair pops into existence to reduce the potential energy. This continues and stable mesons and baryons are formed resulting is a jet of particles. The constituents of these jets are distributed randomly within the shower but we can take advantage of some general trends to help identify these objects.

\subsubsection{Creating Jet Objects}

The identification of a jet object is a four step process [65]. First jet towers are formed using the so called E-Scheme. The jet towers in turn seed preclusters which

are fed to the Run II Cone Algorithm followed by merging and splitting of jets to ensure stability and no double counting. The E-scheme calculates a four-momentum 
for each pseudo-projective tower of the calorimeter,

$$
p^{\text {tower }}=\sum_{i}^{\text {cells }} p_{i}
$$

The $0^{\text {th }}$ component of the four vector or the energy term is the energy of the cell, and the three vector component has a magnitude equal to that of the energy and a direction defined from the primary vertex. In this way the four vectors of the cells are massless. Each tower must have at least one cell.

Using a $p_{T}$ ordered list of jet towers with $p_{T}>0.1 \mathrm{GeV}$, preclusters seeded with the highest $p_{T}$ tower are formed by all towers within a $\Delta R<0.3$ and kept only if the precluster $p_{T}>1 \mathrm{GeV}$. During this process, if the highest $p_{T}$ tower has a cell in the $\mathrm{CH}$ or EC Massless Gap, the cell is removed and the tower's four vector is recalculated.

Run II Cone Algorithm constructs massive protojets with a radius of $R=(0.5,0.7)$ in $y-\phi$ space, seeded from preclusters separated from the nearest protojet by more than $R / 2$, and expanded with preclusters within $R$ of this seed until the centroid of the protojet changes less than $10^{-4}$ with the addition of a new precluster, or until the list of clusters has been iterated through 50 times. Protojets with $p_{T}>4 \mathrm{GeV}$ are kept and the midpoints between these objects are used as seeds to remove sensitivity to soft radiation.

In the final step, protojets which share more than $50 \%$ of their preclusters are merged, otherwise these shared preclusters are given to the closer protojet.

\subsubsection{Good Jets}

All the objects in this final list of jets are not used at the analysis level. It was found

that calorimeter noise can still mimic an object of this type so the following Good 
Jet criteria [66] was created:

- EMF $>0.5$ but allowed to be as low as $4 \%$ in the forward region and $3 \%$ in the $\eta$ region which has a gap in EM calorimetry. This cut is meant to remove noise jets which have a large amount of energy in the $\mathrm{CH}$ cells.

- $\mathrm{EMF}<0.95$ to remove electrons and photons.

- Charged Hadronic fraction $\mathrm{CHF}<0.4$ but a jet passing through the ICR does not have a lot of material to traverse before reaching the $\mathrm{CH}$ layers. In this region the cut is relaxed to $\mathrm{CHF}<0.6$ as long as the minimal number of towers that contain $90 \%$ of the jet's energy is less than 20. Forward jets are allowed a little leeway with a cut of 0.46 and very central jets can have a CHF up to 0.44.

- To reduce contamination from noise jets, a Level 1 confirmation for jets in data is done by requiring the jet participated in the Level 1 Calorimeter Trigger decision having a considerable L1 energy, $E_{L 1}>55 \mathrm{GeV}$. Less energetic jets can still be considered L1 confirmed if the ratio of Level $1 p_{T}$ over the precision $p_{T}$ using only those cells which participate in the trigger (EM and $\left.\mathrm{FH}\right)$ is greater than 0.5 or greater than 0.2 for very forward jets $(|\eta|>3)$. Jets may have fallen into a region that is not instrumented for the L1 Calorimeter Trigger. For these, the CHF must be less that 0.15 or the minimum number of towers that contain $90 \%$ of the energy must be less than half the total number of towers in the jet.

\subsubsection{The Jet Energy Scale}

Measured jet energies, $E_{j e t}^{M}$, need to be mapped back to the true energy $E_{j e t}^{T}$ in order for the results from $D \emptyset$ to be compared with theory or other experiments. The jet energy scale (JES) correction does exactly that for data and MC separately $[67,68,69]$. 
The equation used is

$$
E_{j e t}^{T}=\frac{E_{j e t}^{M}-E_{0}}{R_{j e t} \cdot S_{j e t}}
$$

where

- $E^{0}$ is the offset compensating for uranium decay, minimum-bias activity, pile-up (left overs from the previous bunch crossing), and noise in the electronics.

- $R_{j e t}$ is the calorimeter response. This is affected by energy deposited in the layers of the detector before the calorimeter or in region of the detector which are not instrumented, different responses of the various layers and non-linearities.

- $S_{j e t}$ is the correction to the limitations of the cone algorithm. Particles in the cone which showers exit the cone or vice-versa need to be considered.

The energy offset is measured in two parts. The activity from the calorimeter itself is measured by collecting data events at random, zero-bias events, and measuring the average energy. Effects from additional collisions and remnants from the previous collisions are estimated by taking a random set of data events with a inelastic collision, minimum-bias events, and measuring the average energy. The remaining quantities are a function of the location of the jet in the detector and the energy of the jet itself. The response correction is found from events where a photon and a jet are produced back to back $(\gamma+$ jet events $)$. If the photon is in the CC, its energy is measured to a high precision. With this value, and the fact that there should be no imbalance in the transverse plane, the correction to the jet energy can be derived. For the showering correction, MC studies were done to compare the energy in and out of the cone of various radii. This was used to create shower templates that are fit to $\gamma+j e t$ data to determine the ratio of measured energy inside the cone to the true jet energy inside the cone. Muons identified within the jet cone usually originate from semi-leptonic 
decays and therefore are indicative of a neutrino carrying off some of the jets energy. A separate correction known as JESMU has been derived on jets containing muons to account for this lose of information.

\subsubsection{Jet Smearing, Shifting, and Removal}

Known as JSSR, this procedure allows simulated jets to be re-calibrated, smeared and possibly discarded, all in a consistent way, so that the result matches the behavior observed in data $[70,71]$. This has been done with $\gamma+$ jet and $Z+j e t$ events, more recently the latter only. In either case the main tool is the $p_{T}$ imbalance given by

$$
\Delta S=\frac{p_{T}^{j e t}-p_{T}^{Z / \gamma}}{p_{T}^{Z / \gamma}}
$$

The derivation of the parameters of interest is a three step process all involving fitting the function

$$
f_{i}(\Delta S)=N_{i} \cdot \exp \left(-\frac{\left(\Delta S-<\Delta S>_{i}\right)^{2}}{\sigma_{i}^{2}}\right) \times\left[1+\operatorname{erf}\left(\frac{p_{T^{j e t}}-\alpha}{\sqrt{2} \beta}\right)\right]
$$

to the $\Delta S$ distribution in bins (i) of $p_{T}^{Z / \gamma}$. The second term, a turn-on needed to model the jet reconstruction threshold, is assumed to be independent of and therefore fit simultaneously over all $p_{T}^{Z / \gamma}$ bins. With $\alpha$ and $\beta$ fixed, two free parameters, a resolution, $\sigma_{i}$, and imbalance term, $\langle\Delta S\rangle_{i}$, remain. These resolutions are fit for each $p_{T}^{Z}$ bin, plotted as a function of $p_{Z}^{T}$, and fit with the standard sampling calorimeter resolution formula of

$$
\sigma=\sqrt{\frac{N^{2}}{\left(p_{T}^{Z}\right)^{2}}+\frac{E^{2}}{p_{T}^{Z}}+C^{2}}
$$

where the term, $S$, describes the fluctuations due to sampling and showering of the incident particles, $N$ describes noise fluctuations which are present in the low energy 
range, and the constant term $C$ is the limit on the resolution at high energies (Figure 4.6). With the tun-on term and resolutions fixed a final iteration of the fit is done to obtain the imbalance terms which are also plotted as a function $p_{Z}^{T}$ and fit with

$$
<\Delta S=A+B \cdot \exp \left(-C \cdot p_{T}^{Z}\right)+D \cdot \exp \left(-E \cdot\left(p_{T}^{Z}\right)^{2}\right)
$$

Simulation jets are smeared such that the resolution, $\sigma$, agrees on average with the data in the given region of the detector. Then the energy level of the jets is shifted to account for difference in the mean, $\Delta S$.

The efficiency for finding Good jets is compared in the simulation and in data. It has been found that on average, the ratio of the data to the simulation efficiency is 99\%. This value is found for various regions of the detector using the tag and probe method in a di-jet sample. For each simulation jet, a random number is drawn from a uniform distribution between 0 and 1 and if the random number is greater than this ratio, the jet is removed from the event. In this way, the jet multiplicity agrees in data and MC. 


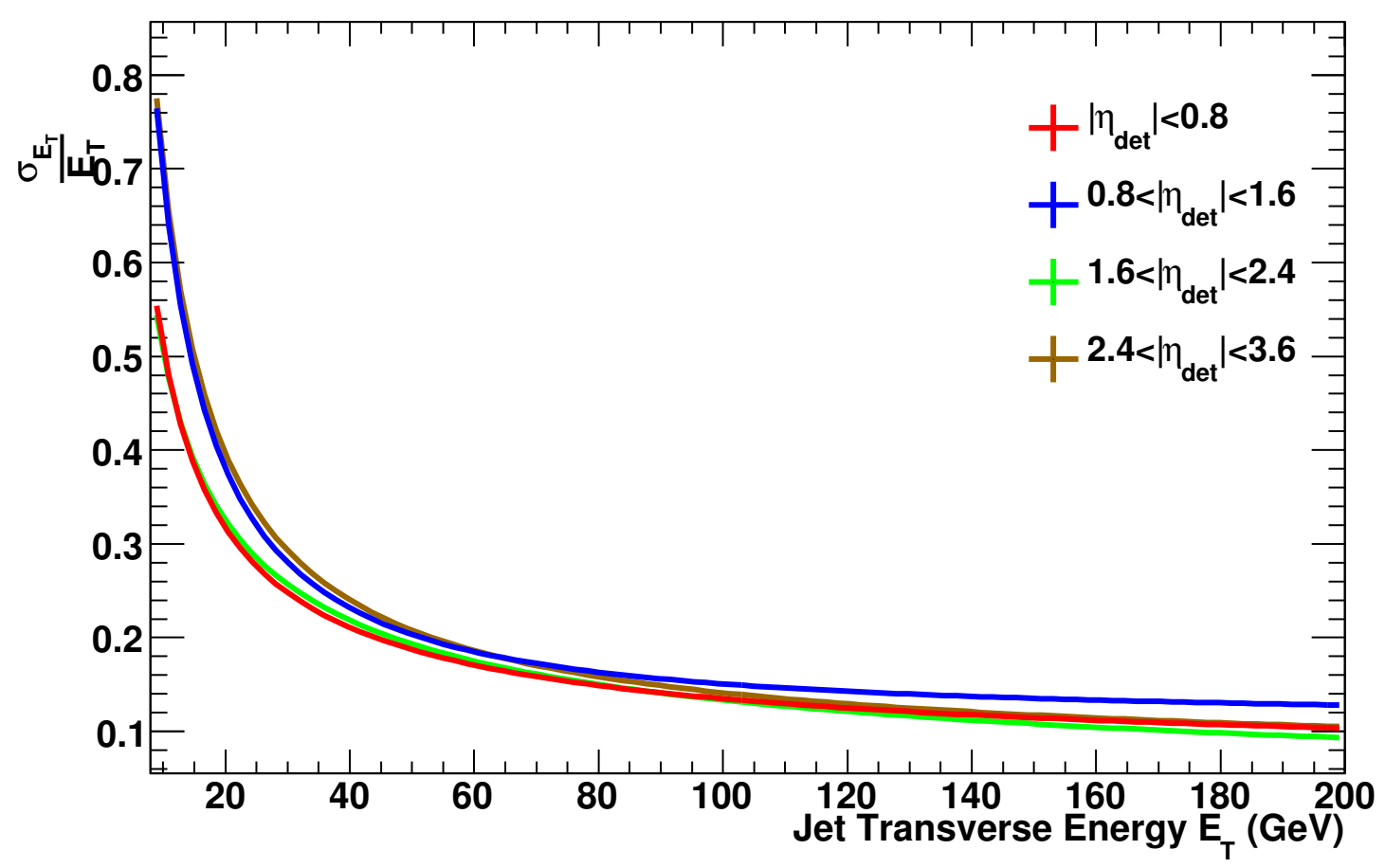

Figure 4.6: Comparison of the resolution of jets in four regions of the calorimeter in Run IIb data. These values were obtained from the JSSR procedure.

\subsubsection{Higher Jet Quality Criteria}

Beyond being classified as a good or bad jet, further criteria can be imposed to increase the likelihood that a jet truly originates from the primary interaction and/or has sufficient tracking information to apply the standard DØ b-quark identification tools discussed in Chapter 7. This is the first time in the jet reconstruction chain that tracks are used to make a statement on the quality of the jet object. 


\section{Vertex Confirmation}

A good jet can originate from the hard-scatter vertex or from another vertex in the event. Vertex Confirmation (VC) is a way of distinguishing the two. This is done simply by requiring that there be two tracks within the jet cone which have a $p_{T}>0.5 \mathrm{GeV}$, a distance of closest approach $(d c a)$ to the vertex is less than $0.5 \mathrm{~cm}$, and distance along the z-axis from the PV, $d c a_{z}$ is less than $0.1 \mathrm{~cm}$.

\section{Taggability}

This requirement must be satisfied before a jet can be evaluated by the b-quark identification tools. This requires that a jet be spatial matched to a track-jet. A track

jet is created from a list of tracks using the Snowmass jet algorithm [72] in a cone of radius 0.5. The tracks considered must have at least one SMT hit, a $d c a<2 \mathrm{~mm}$, and $d c a_{z}<4 \mathrm{~mm}$. The track jet is seeded with tracks having a minimum of $1 \mathrm{GeV}$.

\section{Monte Carlo Corrections}

Similar to the jet reconstruction efficiencies, the tag and probe method is used in a sample of di-jet events to measure the rate of which simulation and data jets are vertex confirmed, taggable, and both vertex confirmed and taggable. Since any efficiency difference is related to the modeling of the tracking systems they are parameterized in a way to separate object which pass through differ amounts of the tracking system. This is done by using bins of $\eta_{\text {det }}$ further divided into bins of the $P V_{z}$ position. Within each of these subsamples, the efficiency, $\epsilon$, is fit as function of the $p_{T}$ as

$$
\epsilon\left(p_{T}\right)=A+B \cdot e^{-C \cdot p_{T}}
$$


In some cases, it was found that the MC efficiency is greater than that of the data which makes the random removal procedure impossible to use. Instead, an event with $n$ good jets, $p$ of which pass the quality criteria, is given a weight

$$
w_{n}=\prod_{i=1}^{p} \frac{d_{i}}{m_{i}} \times \prod_{j=1}^{n-p} \frac{1-d_{j}}{1-m_{j}}
$$

where $d_{i}\left(m_{i}\right)$ is the data (MC) efficiency for the $i^{\text {th }}$ jet determined from the above parameterization.

\subsection{Missing Transverse Energy}

Through momentum conservation, the sum of the incoming and outgoing momentum of a collision is expected to be the same. In a hadron collider, because the proton and antiproton are composite objects and most of the particles from spectator parton interactions are directed undetected down the beam pipe, momentum conservation cannot be enforced in the direction of the beam. However, it is a reasonable assumption that the beam has no motion in the transverse direction which then should be true of the net transverse momentum of the particles resulting from a collision. Neutrinos are very weakly interacting particles and therefore do not deposit energy within the detector at all. The creation of this type of particle will result in an imbalance in the transverse momentum. There are also instrumental effects which can cause an apparent imbalance which is further discussed in Chapter 5. Because the momentum is approximated by the vectorial sum of the energy in each calorimeter cell, the miss-

ing transverse momentum as determined by the calorimeter is traditionally referred to as missing transverse energy, or $\mathscr{H}_{T}$.

The two components of the missing transverse energy are the negative of the sum 
of the components of the transverse energy calculated from the sum of the calorimeter cell energies as,

$$
E_{T}^{x, y}=-E_{T}^{x, y}=\sum_{i}^{c e l l s} E_{i}^{x, y} .
$$

This is known as the raw $\mathscr{E}_{T}$. The coarse hadronic cells are not included in the sum as they tend to be noisy and thus reduce the resolution of this already tricky variable. As stated previously the energy measured in the simulation usually needs to be corrected to match the data on average. This corrections must be propagated to the $\mathscr{H}_{T}$ by replacing the energy of the cells associated with the object with the corrected energy in the sum. Muons leave very little energy in the calorimeter. In fact, according to the calorimeter, muons are almost identical to neutrinos. Therefore the muon momentum is implicitly included in the above described $\not_{T}$. At the analysis level the $\mathscr{H}_{T}$ is usually corrected for the presence of muons and is referred to as the muon-corrected $\mathbb{H}_{T}$. 


\section{Chapter 5}

\section{Quality of the Measured $E_{T}$}

As stated in Section 4.7, momentum conservation not satisfied in the transverse plane is indicative of the presence of a neutrino. However, $\mathbb{E}_{T}$ is found in events where none is expected. Recall, as stated in Section 2.2.4, the $\mathrm{D} \varnothing$ calorimeter is a sampling calorimeter so the amount of energy deposited in the active material is statistical in nature and characterized by a resolution described by a gaussian distribution. In fact every sub-detectors's measurement is statistical in nature and is characterized in the same way. Events with large energy measurement fluctuations are those which are accounted for in the tails of these distribution and must be handled with care. Events of this type can be measured to have a significant imbalance in transverse energy when none is expected a priori. In effect, the $\mathscr{H}_{T}$ signature can be faked.

The analyses described in Chapters 9 and $10, Z Z \rightarrow \ell^{-} \ell^{+} \nu \bar{\nu}$ and $Z H \rightarrow \nu \bar{\nu} b \bar{b}$ respectively, both look for a signal characterized by $E_{T}$ consistent with the invisible decay of the $Z$ boson. In both cases, the pathology of significant fake $\not_{T}$ in processes with cross sections thousands of times larger than the signal must be surmounted. In the following, two tools meant to identify this class of events are described. 


\section{$5.1 \quad E_{T}^{\prime}$}

A way to approach this challenge is instead of making an unbiased or accurate estimate of the true $\not_{T}$, construct an experimental variable which loosely can be thought of as the minimum conceivable $\mathscr{F}_{T}$ by considering mismeasurements due to specific sources. In short, the measured objects are decomposed along specifically chosen axis and these components are reduced by a series of corrections. Finally the components are added in weighted quadrature to produce a variable which tends towards high values for events which have true $\mathbb{H}_{T}$.

This approach is inspired by searches at OPAL for the anomalous production of events containing a pair of charged leptons and missing $p_{T}[73]$. At D $\varnothing$, this method enabled the $Z Z \rightarrow \ell^{-} \ell^{+} \nu \bar{\nu}$ channel to contribute significantly to the first observation of $Z Z$ production at a hadron collider [74,75] and the basis of the recent measurement of the $Z / \gamma^{*}$ boson $p_{T}$ spectrum [76]. This method is presented in the context of the production of two bosons where one decays to measurable objects used to create the di-object system (noted by superscript do) and the other decays invisibly.

\subsubsection{Decomposition}

Inaccuracy in $\mathbb{F}_{T}$ can result from mismeasurement of boson decay products or from the recoil activity. These two sources are not easily disentangled. A thrust axis is defined as

$$
\begin{array}{ll}
\vec{t}=\vec{p}_{T}^{1}-\vec{p}_{T}^{2} \quad \text { for } \Delta \phi^{d o}>\frac{\pi}{2} \\
\vec{t}=\vec{p}_{T}^{1}+\vec{p}_{T}^{2} \text { for } \Delta \phi^{d o} \leq \frac{\pi}{2}
\end{array}
$$


where $\vec{p}_{T}^{1}$ and $\vec{p}_{T}^{2}$ are the transverse momenta of the leading and trailing objects respectively (Figure 5.1). This definition maximizes the scalar sum of the longitudinal component of the di-object $p_{T}\left(p_{T}^{d o}\right)$ and so the longitudinal direction, $\hat{a}_{l}$, is sensitive to the lepton transverse momentum while the transverse direction, $\hat{a}_{t}$, is sensitive to the recoil. Orientation of the axis is such that the decomposition of $p_{T}{ }^{d o}$ into a transverse (longitudinal) component $a_{t}\left(a_{l}\right)$ is always positive definite.

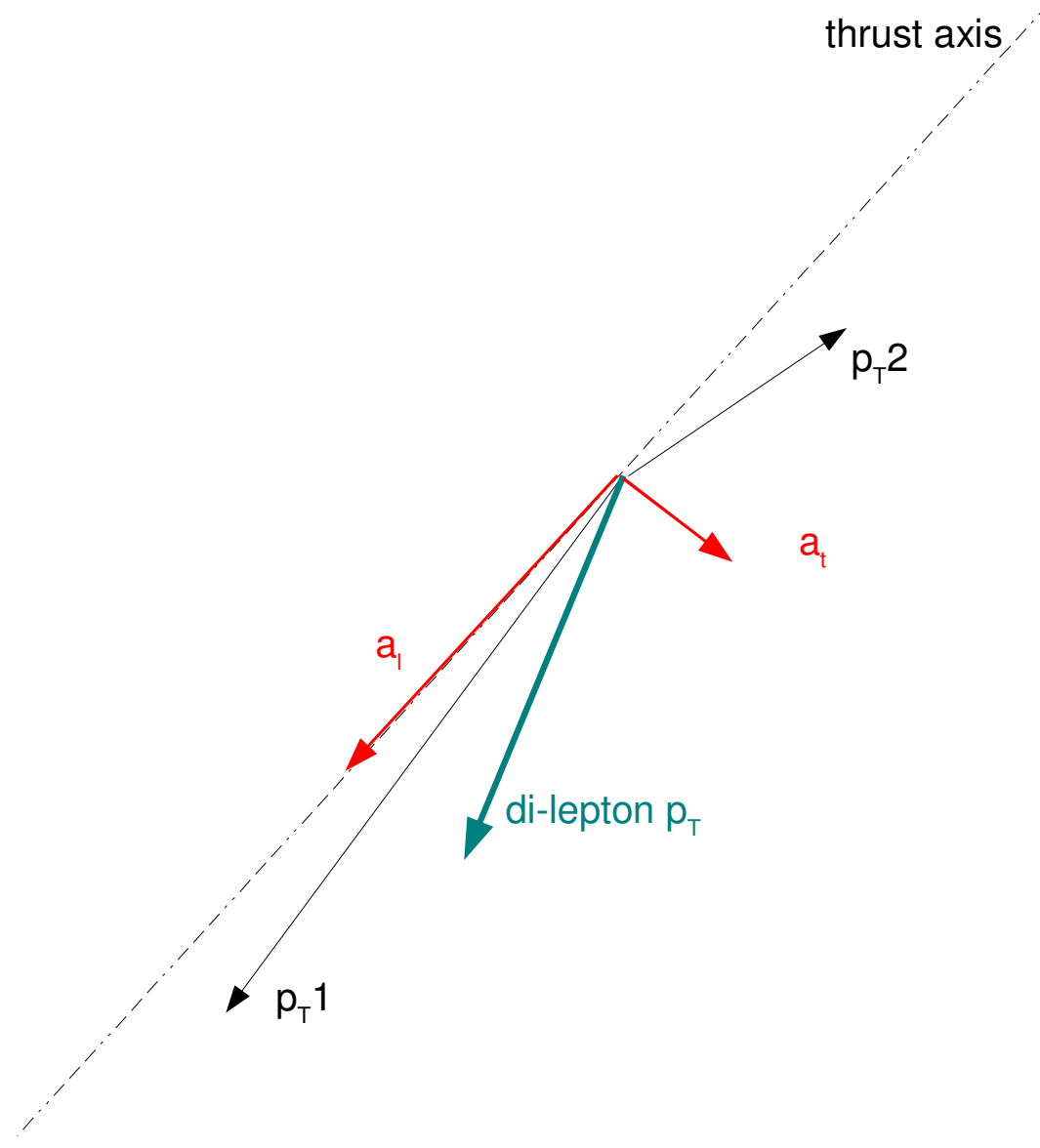

Figure 5.1: Sketched representation of the transverse plane decomposition decomposition of, in this case, the di-lepton transverse momentum along the thrust axis. 


\subsubsection{Object Resolution}

A correction with respect to the resolution of the objects is constructed from the difference of the original components and the decomposition after a minimization. A new longitudinal component, $a_{l}{ }^{\prime}$, is produced by decreasing (increasing) the leading (second) object $p_{T}$ by one standard deviation $\left(\sigma_{1}\right.$ and $\sigma_{2}$ respectively) and decomposing the resultant di-object $p_{T}$ along $\hat{a}_{l}$. The transverse component, $a_{t}$, is reduced by decreasing the transverse momenta of both objects one standard deviation. The thrust axis and di-object $p_{T}$ are then both redefined with the adjusted momenta, thus giving a new transverse decomposition

$$
a_{t}^{\prime}=\left(\vec{p}_{T}^{d o}\right)^{\prime} \cdot \hat{a}_{t}^{\prime}
$$

The resolution corrections are given by

$$
\begin{aligned}
& \delta a_{t}{ }^{\text {res }}=a_{t}{ }^{\prime}-a_{t} \\
& \delta a_{l}{ }^{\text {res }}=a_{l}{ }^{\prime}-a_{l}=\left(-\sigma_{1} \vec{p}_{T}^{1}+\sigma_{2} \vec{p}_{T}^{2}\right) \cdot \hat{a}_{l}
\end{aligned}
$$

This correction is especially important in the forward region where mismeasurements in the track curvature and minimum bias activity add to the measurement uncertainty.

\subsubsection{Recoil Measured by the Calorimeter}

Poorly reconstructed hadronic systems and calorimeter noise can affect the transverse momentum balance which can be unaccounted for even with the object corrections propagated to the $\mathbb{F}_{T}$ as described in Section 4.7. Both the jets in the event and the $E_{T}$, not including the di-object energy, are separately decomposed along the thrust 
axis. The one with the largest negative component,

$$
\begin{gathered}
a_{t}{ }^{c a l}=2 \min \left(a_{t}^{\not_{T}}, a_{t}{ }^{\text {jets }}\right), \\
a_{l}^{c a l}=2 \min \left(a_{l}^{\not_{T}}, a_{l}{ }^{\text {jets }}\right) .
\end{gathered}
$$

is used as a correction. A factor of two conservatively allows for only $50 \%$ of the actual recoil energy to be measured.

\subsubsection{Recoiling Tracks}

Low energy jets not propagating far enough into the calorimeter, jets traveling through the massless gaps, and the somewhat reduced resolution along the calorimeter modulus boundaries can cause some jets to have not yet been considered. To account for these objects, track-jets are built from tracks satisfying the following quality criteria:

- $\chi^{2} / N D F<4.0$

- DCA for tracks w/ SMT hits $<0.02 \mathrm{~cm}$

- DCA for tracks w/o SMT hits $<0.2 \mathrm{~cm}$

- $p_{T} \geq 1.0 \mathrm{GeV}$

- $\Delta\left(\operatorname{track}_{z}, P V_{z}\right) \leq 1.0$

- $\Delta R($ track, signal objects $) \geq 0.5$

- $\Delta R($ track, Calorimeter jet $) \geq 0.5$.

The highest $p_{T}$ tracks used to seed the object and all tracks within a cone of $\Delta R \leq 0.5$ are added to the track-jet object. When all tracks are associated with a track-jet and 
every track-jet has at least on track the transverse momentum of these objects is decomposed along the thrust axis to give

$$
\begin{aligned}
& a_{t}^{t^{t r k}}=\left(\sum p_{T}^{t r k-j e t}\right) \cdot \hat{a}_{t} \\
& a_{l}^{t r k}=\left(\sum p_{T}^{t r k-j e t}\right) \cdot \hat{a}_{l}
\end{aligned}
$$

\subsubsection{Resultant Variable}

In order to produce a discriminating experimental variable the two components $a_{t}^{d o}$ and $a_{l}^{d o}$ are combined giving more weight to the component which is less sensitive to the resolution of the objects used to define the thrust axis. To fine tune this sensitivity the final components are constructed as follows

$$
\begin{aligned}
& a_{t}^{c o r}=a_{t}+a_{t}^{c a l}+k \times a_{t}^{t r k-j e t}+k^{\prime} \times \delta a_{t}{ }^{\text {res }} \\
& a_{l}^{c o r}=a_{l}+a_{l}^{c a l}+k \times a_{l}^{t r k-j e t}+k^{\prime} \times \delta a_{l}{ }^{\text {res }}
\end{aligned}
$$

where only corrections which reduce the final sum are considered. The final variable is then a weighted quadrature sum of the two components

$$
\begin{aligned}
& \mathbb{E}_{T}{ }^{\prime}=\sqrt{\left(a_{l}{ }^{c o r}\right)^{2}+\left(1.5 a_{t}{ }^{c o r}\right)^{2}} \quad \text { for } \Delta \phi^{d o}>\frac{\pi}{2} \\
& E_{T}{ }^{\prime}=\sqrt{\left(1.5 a_{t}{ }^{\text {cor }}\right)^{2}} \quad \text { for } \Delta \phi^{d o} \leq \frac{\pi}{2} \text {. }
\end{aligned}
$$

\section{$5.2 \quad E_{T}$ Significance}

Another approach to discriminating events with real and fake $\mathbb{F}_{T}$ is to use a probabilistic approach. The $E_{T}$ significance algorithm takes into account the particular topology and measured physics objects of an event and assesses the likelihood of the 
$\mathbb{E}_{T}$ being consistent with energy measurement fluctuations in the direction of the $\mathbb{F}_{T}$. Recall the statistical significance is the relative amount a result deviates from its expected value considering random variations or sampling errors, thus the name $\mathbb{E}_{T}$ significance.

This method is based on the assumption that for an object measured with energy $\mathbf{E}_{i}$ there exists a gaussian probability distribution associated with the measurement $p\left(\mathbf{E}_{i}\right)=N\left(\mathbf{E}_{i}, \sigma_{i}\right)$ where $N\left(\mathbf{E}_{i}, \sigma_{i}\right)$ is a gaussian distribution centered at $\mathbf{E}_{i}$ with width $\sigma_{i}$. The width, $\sigma_{i}$ is exactly the resolution of the sub-detector(s) used to measure the energy discussed in Chapter 4. As shown in Section 4.7, $\not_{T}$ is defined as

$$
E_{T}=\sum_{i} \mathbf{E}_{i}
$$

and by convoluting all of the probability densities for the objects in the detector, an expression for the probability of the $\mathbb{E}_{T}$ is

$$
\begin{aligned}
P\left(E_{T}\right) & =\int \prod\left(P\left(E_{i}\right) d E_{i}\right) \delta\left(E_{T}+\sum_{i} E_{i}\right) \\
& =-N\left(E_{T}, \sqrt{\sum_{i} \sigma_{i}^{2}}\right)
\end{aligned}
$$

This probability needs to be evaluated in the direction of the $\mathbb{F}_{T}, \vec{m}$, in order to test how likely the measured $\mathbb{E}_{T}$ is due to a fluctuation in the direction of the measured energy imbalance. By projecting all of the individual object energies $\mathbf{E}_{\mathbf{i}}$ along the direction $\vec{m}$,

$$
E_{i}^{\vec{m}}=E_{i} \cdot \vec{m}=E_{i} \cos \left(\vec{i}, E_{T}\right)
$$


an expression for the probability density along a given direction is

$$
P\left(\ddot{E}_{T}^{\vec{m}}\right)=N\left(\ddot{E}_{T}, \sqrt{\sum_{i} \sigma_{i}^{2} \cos ^{2}(\vec{i}, \vec{m})}\right) .
$$

Based on this definition a likelihood of the measured $\mathscr{E}_{T}$ being due to a fluctuation is

$$
L=\log \frac{p\left(\not_{T}^{\vec{a}}\right)}{p\left(E_{T}=0\right)}=\frac{E_{T}^{2}}{2 \sigma^{2}}
$$

where $\sigma$ is the resolution of the $\not_{T}$ in the direction of $\vec{m}$ given by

$$
\sigma=\sum_{i} \sigma_{i}^{2} \cos ^{2}(\vec{i}, \vec{m})
$$

The jet resolution is derived from the JSSR procedure described in Section 4.6.4. The muon and electron resolutions are described in Sections 4.3 and 4.4 respectively. Unclustered energy $\left(U E_{T}\right)$ resolution energy tends to degrade with the number of jets in the event. Also soft jets which fall below the reconstruction threshold, warm calorimeter regions (noise), and energy deposits from parton remnants or the underlying event contribute to the unclustered energy. The probability distribution for the unclustered energy was still found to be modeled as a gaussian with the width given by a linear function

$$
\sigma_{U E}=a_{n}+b_{n} \cdot U E_{T}
$$

where the parameters $a_{n}$ and $b_{n}$ depend on the jet multiplicity $(n)$. 


\section{Chapter 6}

\section{Multivariate Analysis}

Searching for a very small signal in a large data set is a common problem in high energy physics today. The maximum available information from the data needs to be extracted and multivariate classification methods based on machine learning techniques have become a common way of doing so. In recent years statisticians have found new ways to tune and combine classifiers to further increase the performance of these methods. A package, Toolkit for Multivariate Data Analysis with ROOT (TMVA) [77] is a popular choice at D $\varnothing$ for training and evaluating multivariate discriminants. StatPatternRecognition [78] is also used.

All multivariate classification methods must be trained and evaluated on separate, independent samples to ensure an unbiased result. The training should be such that the general trends and correlations of a sample and not the random fluctuations

dictate the final algorithm. A supervised learning method is one in which the desired output for any input in the training set is known. In general there is no universal recipe concerning the usage, tuning, and training of multivariate tools and each case must be considered in the context in which it is needed. However techniques such as Decision Trees and Neural Networks based techniques generally outperform likelihood-based 
classifiers in the presence of strong non-linear correlations among the input variables.

\subsection{Likelihood Discriminant}

A maximum likelihood method is built from the product of probability density functions (PDF). The likelihood of an event being signal (background) is determined from the product the signal (background) PDFs of all the input variables normalized by the sum of the signal and background likelihoods. If the variables are correlated in some non-linear way, this is not the optimal choice as this method ignores correlations. This method is sometimes referred to as a "naive Bayes estimator" because of the strong (naive) independence assumption.

The likelihood ratio, $\mathrm{L}$, of a given event $i$ is given by

$$
L(i)=\frac{P_{S}(i)}{P_{S}(i)+P_{B}(i)}
$$

where

$$
P_{S(B)}(i)=\prod_{n}^{N_{v a r}} p_{S(B), n}\left(x_{n}(i)\right)
$$

where $p_{S(B), n}$ is the signal (background) PDF for the $n$th input variable $x_{n}$ usually determined in the form of a binned histogram as

$$
p_{S(B), n}=\frac{x_{n}^{S(B)}}{x_{n}^{S}+x_{n}^{B}}
$$

and fit by a function to reduce the impact of statistical noise in the tails. The PDFs are normalized such that

$$
\int p_{S(B), n}\left(x_{n}\right) d x_{n}=1 .
$$

Assuming there truly are no correlations between input variables, $L(i)$ will produce 
the optimal signal from background separation for the given set of input variables. A de-correlation of the input variables can be achieved, for example, by a linear transformation. However, in real-world cases this rarely completely removes all correlations. This method can handle large amounts of input variables without a large increase in training time or an increase in statistics. A multidimensional likelihood handles correlations but much more statistics are needed to properly train the discriminant.

\subsection{Decision Tree}

A decision tree (DT) is a logical extension to a simple cut-based analysis which divides the phase space by using many sets of cuts. This method is independent of monotonous variable transformations, immune against outliers, and weakly discriminating variables do not deteriorate the performance very much. A single DT, shown in Figure 6.1, is sensitive to statistical fluctuations in the training data. The idea of combining many, or a forest of DTs was proposed to overcome this stability prob$\operatorname{lem}[79,80]$.

A decision tree is a rooted binary tree. The root node is the entire training sample. The sample is split into two using a cut in a variable which gives the best separation gain. This splitting is continued at each node, dividing the training phase space into hypercubes, until a stop criteria is met. An example of stop criteria are the minimum number of events per node, maximum number of nodes, maximum depth, or when splitting the nodes further does not gain anymore separation. The final nodes are called leaves and are labeled signal (s) or background (b) depending on the purity, $p=s /(s+b)$, of the events in that leaf. The best separation gain can be measured

with various quantities but the most commonly used is the Gini-index, $p(1-p)$. Since a cut which selects predominantly background is as valuable as a cut which selects 
signal, the criterion is symmetric with respect to the event class. The maximum is when the samples are fully mixed, that is at a purity of 0.5. The sum of the Giniindex of the two daughter nodes, weighted by their relative fraction of events, is less than that of the parent when the separation of the two populations has increased. Sensitivity to fluctuations in the training data is more commonly know as overtraining in the forest application. Overtraining occurs when there are too few data points to properly set the model parameters; in other words, there are too many degrees of freedom with respect to the available statistics. The severity of this problem depends on the machine technique used. Boosted decision trees usually suffer from at least partial overtraining, owing to the large number of nodes in the constituent trees. If overtrained, the DT performance measured in the training sample and an independent test sample will differ considerably. The training (test) sample performance will be better (worse) than the objectively achievable performance. Therefore, a simple way to test for and measure the amount of overtraining is to compare the performance results between the independent training and test samples. In the cases where the tree is allowed to grow beyond a handful of nodes, pruning techniques such as removing subtrees which in total add less improvement than some cut-off can be employed to curb overtraining.

There are several ways to increase the performance of a forest of DTs. A very powerful technique to improve the performance of any weak classifier by sequentially applying an MVA algorithm to re-weighted versions of the training data and taking a weighted average to produce the final discriminant is known as boosting. The most popular boosting algorithm is adaptive boosting, known in the literature as AdaBoost. In this method, the original event weights are used for the training of the first tree 
while subsequent trees are multiplied by a common boost weight $\alpha$ give by

$$
\alpha=\frac{1-\chi}{\chi}
$$

where $\chi$ is the sum of weights of the misclassified events divided by the total sum of weights. The weights of the entire tree are then re-normalized such that the sum of weights remains constant.

Let us look at function estimation through boosting in another light, that is by considering a simple additive expansion approach. The function under consideration is assumed to be the weighted sum of parametrized base functions, $f\left(x ; a_{m}\right)$, or "weak learners", such as an individual decision tree or any machine learning technique, where $x$ represents the set of variables for the events in the tree and $a_{m}$ is the rule used to separate the signal and background populations, which would be the cut at each node for a decision tree. The function is therefore written as the following summation:

$$
F(\mathrm{x} ; P)=\sum_{m=0}^{M} \beta_{m} f\left(x ; a_{m}\right) ; P \in\left\{\beta_{m} ; a_{m}\right\}_{0}^{M}
$$

where $\beta_{m}$ is some multiplicative factor. Boosting adjusts the parameters, $P$, such that the difference between the response, $F(x ; P)$, and the true value $y$ is minimized. The figure of merit for this difference is the loss-function $L(F, y)$. This function fully determines the boosting procedure. AdaBoost is based on exponential loss, $L(F, y)=e^{-F(x ; P) y}$ which lacks robustness in the presence of outliers or mislabeled data points and thus performance degrades in noisy settings. By choosing a different loss function, such as a binomial log-likelihood,

$$
L(F, y)=\ln \left(1+e^{-2 F(x ; P) y}\right)
$$


one can improve on this performance. Since an analytical minimization of some functions is nontrivial the method of steepest-descent is employed. Thus the name, gradient boosting. Robustness is further enhanced by reducing the learning rate of the algorithm, or the step size of the minimization of the loss function. The smaller this number the slower the learning process and hence the more trees that must be grown.

Bagging is a resampling technique where a classifier is repeatedly trained using resampled training events such that the combination of these individual classifiers represents an average of the individual classifiers. This, in effect, smears over the statistical representation of the training sample. That is, if a subset of the training sample is selected for training, along with a subset of the input variables, it is possible for this subset to not fully represent the kinematics of the full training sample. The tree trained on this biased sample, will clearly have better performance for an equally biased evaluation sample when compared to a tree trained on the full training sample. A combination of many such biased tree can result in a more powerful discriminant referred to as a random forest $(\mathrm{RF})$. Gradient boosting benefits from a bagging-like resampling procedure using a random subsample of the training sample for growing trees, using the full list of variables for each tree. This is known as a Stochastic Gradient Boosted Decision Tree.

DTs in general perform well on the first iteration as this simple method only requires a one-dimensional cut optimization. Also the performance is not degraded by the inclusion of poorly discriminating variables as they are largely ignored in favor of the most discriminating variable available at each node. In theory, the given performance should be inferior to other techniques like neural networks described in the next section. In practice, either because of a lack of training statistics or difficulty in finding the optimal configuration for other classifiers, BDTs often outperform other 
techniques.

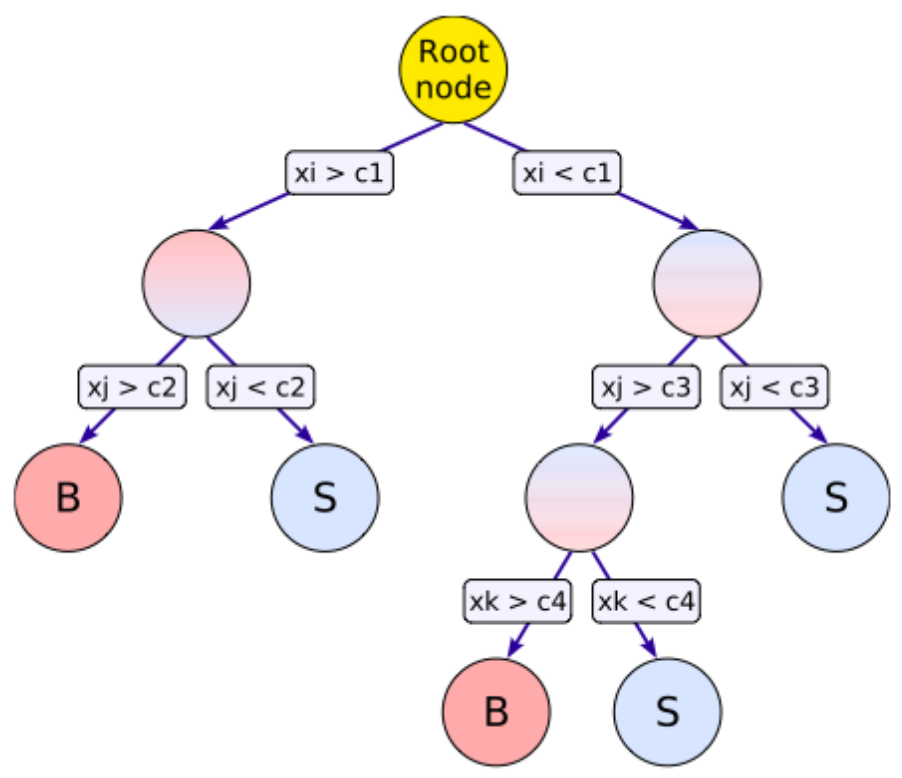

Figure 6.1: Schematic view of a decision tree. A tree is grown starting from the root node with a series of binary splits based on discriminating variables and ends with leaf nodes labeled 'S' for signal or 'B' for background depending on the majority of events contained within.

\subsection{Artificial Neural Network}

An artificial neural network $(\mathrm{NN})$, generally speaking, it is a simulated collection of interconnected neurons where each neuron produces a response for a set of input signals. An external signal is applied to one or more input neurons. This puts the network into a defined state which can be measured from the response of one or more output neurons. In effect, the network is a mapping from an input space to an output space using a model of the synaptic processes of the brain. This method takes into account correlations between input variables and therefore can be extremely powerful. 
A common type of $\mathrm{NN}$ is a multilayer perceptron (MLP). This is an artificial network model in which the data always moves from the input node through the neurons, called hidden layers, toward the output node and is never directed in loops or cycles (Figure 6.2). This is known as a feed forward network. Each neuron has a normalizable, differentiable, nonlinear activation function. A popular choice is the hyperbolic tangent. Supervised learning is achieved by back-propagation which is a means to send the error of the output neuron response back to the input neurons and adjusting the activation function such that the error is minimized for each event. To do so the activation functions but be differentiable.

NN produce excellent results for input variables with no, linear, and complex correlations. However this comes at a price. The optimal choice of training parameters is not intuitive and variables must be chosen carefully as weakly discriminating distributions can yield an overall degradation in performance. 


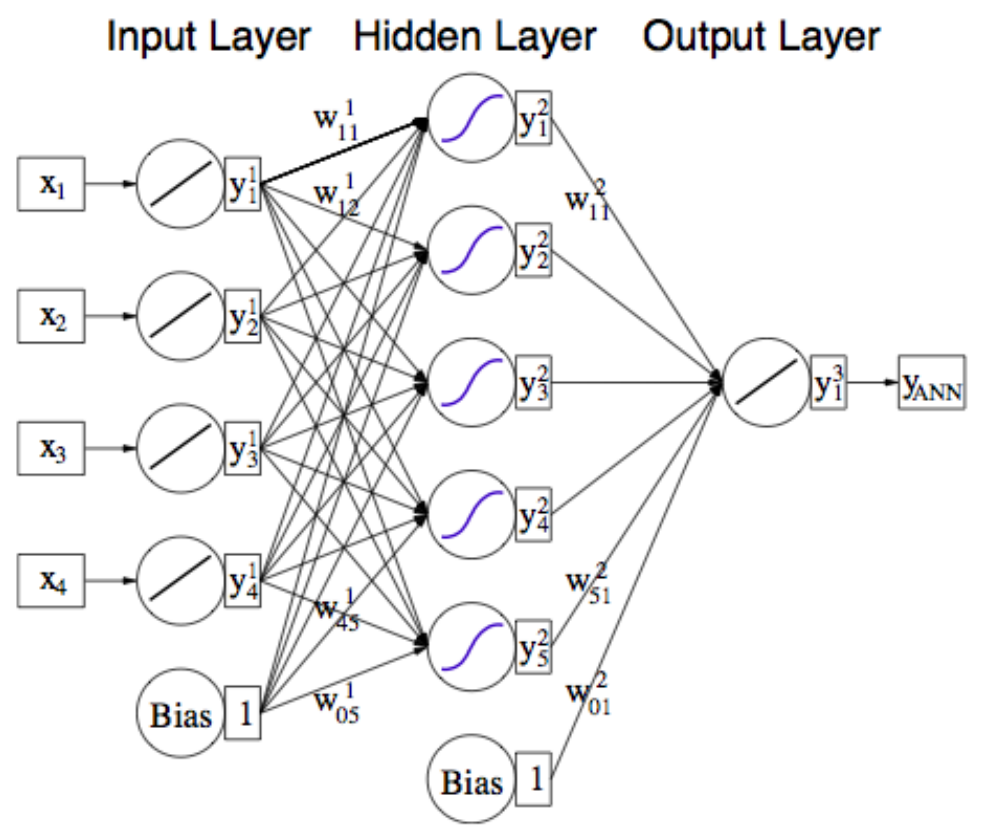

Figure 6.2: Schematic view of a Multilayer Perceptron Neural Net with four input variables and one hidden layer. 


\section{Chapter 7}

\section{B-Quark Identification}

Bottom quarks live for a relatively long period of time, $\approx 1.5 \mathrm{ps}$, because the mixing of flavor and weak eigenstates is such that their decay is suppressed. When produced in a hadron collider environment, this lifetime allows hadronization to begin before these quarks travel a few millimeters and decay to an off-shell $W(b \rightarrow W(u, c))$, producing a vertex displaced from the primary interaction vertex (Figure 7.1). This fact is the main ingredient to identifying, or tagging, jets originating from the hadronization of bottom quarks (b-jets).

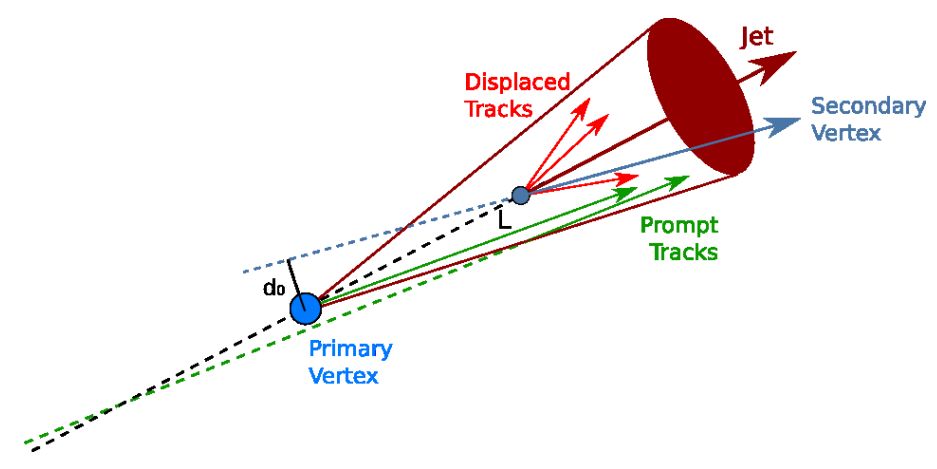

Figure 7.1: This is a sketch of a jet produced from the hadronization and decay of a b-quark. 
A b-tagging NN $[81,82]$ developed at $\mathrm{D} \varnothing$ is trained on seven input variables from three different and has become a cornerstone of many analysis including the low mass Higgs effort. This method, when introduced was a major step forward and a large improvement over the available taggers as show in Figure 7.2. Recently a new MLP NN [83], known as the MVA bl discriminant or MVA b-tagger, has been developed which is $\approx 13 \%$ more efficient at selecting b-jets while rejecting $\approx 50 \%$ more of the light jet background for benchmark jets of $p_{T}>40 \mathrm{GeV}$ and $0<\eta<0.8$ (Figure 7.2). In the following chapter this new tagger is introduced.

\section{$7.1 \quad$ b-tagging}

Both the NN and MVA taggers use information from two different impact parameter (IP) and one secondary vertex tagger trained on a sample of b-jet (signal) and light jets (background) such that when evaluated on the properties of a random jet higher values (1.0) are assigned to b-jets and lower values (0.0) to light jets. Traditional use of this information is to place a requirement on the number of jets above an optimally chosen value in order to select a heavy-flavor enriched sample.

The two IP taggers utilized are the Jet LIfetime Probability Tagger (JLIP) and Counting Signed Impact Parameters (CSIP) algorithms. JLIP computes the probability of a track originating from the primary vertex given the impact parameter. These probabilities for all tracks within a jet are combined into the JLIP Probability (JLIP Prob). Since b-jets create vertices displaced from the primary interaction point, the JLIP probability for these objects trends towards zero while light jets have a more uniform probability. CSIP calculates the signed impact parameter significance $\left(S_{d}=I P / \sigma_{I P}\right)$ for high quality tracks within the jet cone. A jet is tagged if there are at least two high quality tracks with $S_{d} / \alpha>3$ or at least three with $S_{d} / \alpha>2$ where 
$\alpha$ re-normalizing parameter.

The Secondary Vertex Tagger (SVT) uses tracks of a chosen quality to reconstruct secondary vertices with a Kalman filter and if one is found in the jet cone, the jet is tagged.

The NN b-tagger used six SVT variables calculated with tracks having $\left|S_{d}\right|>3$, two variables from the JLIP tagger, and one variable from the CSIP tagger.

\subsection{Multivariate b-Tagging}

The overall strategy of the MVA b-tagger is to combine information from the IP taggers in a RF, information from the SVT algorithm in a separate set of RFs, and combine these in a MLP NN to exploit the non-liner correlations. RFs were chosen due to their stability in terms of non-discriminating variables and relatively quick training time. Nine IP tagger-based variables, the three used in the NN, two additional track probability related variables, and four variables characterizing track-jets constructed from the set of tracks selected by the JLIP algorithm, are the inputs to one RF. Five more RFs are trained, each having 27 SVT related input variables corresponding to running the SVT with progressively higher quality tracks. These SVT related variables range from those involving track multiplicity, track momentum comparisons, decay lengths, and the dimensions of track-jets built from the selected tracks. These six RFs are then combined in a MLP NN. By replacing the light-flavor sample in the training with a c-quark sample, a discriminant, the bc-tagger, has been built with the aim of separating jets originating from b and c quark hadronization. A third discriminant, the bb-tagger, is trained to distinguish b-jets from gluon splitting and

those from a boson decay. Samples of inclusive, $b \bar{b}$, and $c \bar{c}$ taggable (see Section 4.6.5) QCD MC jets as well as hadronic decaying $Z \mathrm{MC}$ are used to train these taggers. 


\section{$7.3 \quad$ Usage}

Although the MVA b-tagger produces a continuous distribution, it can only be used in a pseudo-continuous way. The efficiency of the MC does not match that of data and correction factors are calculated at several distinct output values thus limiting the user to make requirements relative to these operating points. A jet is said to be tagged if it has an MVA output larger than the operating point of choice. The System $D$ method $(D=8)$ along with two samples enriched with heavy flavor and muonic jets are used to estimate the b, c, and light-flavor jet efficiency producing tag rate functions (TRFs) for data jets and scale factors to correct the simulated jet tagging efficiency to match these rates for each operating point. TRFs are calculated as a function of $p_{T}$ and $\eta_{\text {det }}$.

This information can be used in two ways when producing a simulated heavyflavor enriched sample. One way is to multiply the events weights by the probability that each jet could be tagged.This preserves the statistics but it has been seen that actually placing a cut at an operating point and applying a scale factor to correct the simulation efficiency has a more accurate sensitivity to detector performances and topological correlations. Analogous to Equation 4.18, the weight applied to an event with $p$ of $n$ jets passing the operating point cut is

$$
w_{n}=\prod_{i=1}^{p} \frac{T R F_{d}\left(p_{T_{i}}, \eta_{i}\right)}{T R F_{m}\left(p_{T i}, \eta_{i}\right)} \times \prod_{j=1}^{n-p} \frac{1-T R F_{d}\left(p_{T_{j}}, \eta_{j}\right)}{1-T R F_{m}\left(p_{T j}, \eta_{j}\right)}
$$

where $T R F_{d}\left(T R F_{m}\right)$ is the tag rate functions for data $(\mathrm{MC})$. If a relatively low operating point is chosen, the remainder of the distribution provides useful information that can still be exploited. In this case, the weights are applied in pseudo-continuous manner. That is a given jet weight is calculated based on the data and simulation 
efficiencies at the two adjacent operating points as

$$
w_{n}=\prod_{i=1}^{p} \frac{T R F_{d}^{b}\left(p_{T i}, \eta_{i}\right)-T R F_{d}^{a}\left(p_{T i}, \eta_{i}\right)}{T R F_{m}^{b}\left(p_{T i}, \eta_{i}\right)-T R F_{m}^{a}\left(p_{T i}, \eta_{i}\right)}
$$

where $T R F^{b}\left(T R F^{a}\right)$ is the TRF for the operating point below (above) the jet in question. This is referred to as a pseudo-continuous reweighting. In the limit of infinite operating points, this produces a smooth and continuous function to correct the simulation. Since an approximation of this many operating points is not feasible, this procedure is a suitable alternative. 

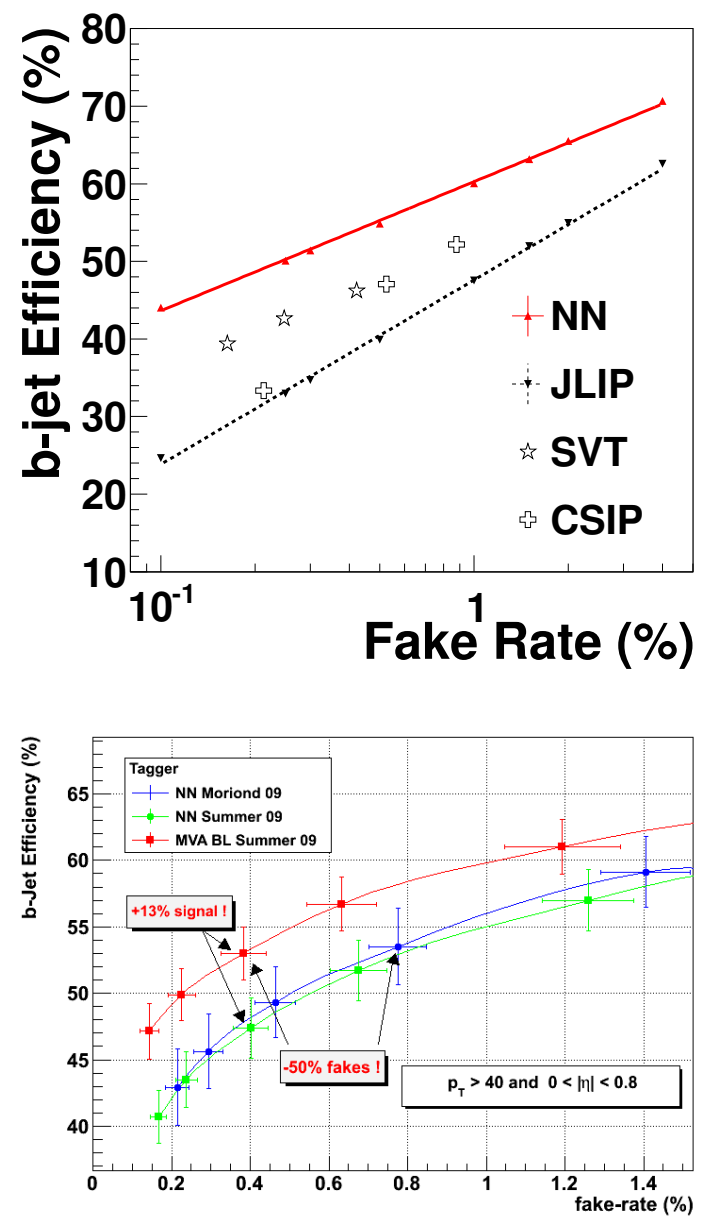

Figure 7.2: (left) The NN tagger compared to the JLIP, SVT, and CSIP taggers shows an efficiency increase of $\approx 20-50 \%$ for a fake rate of $0.2 \%$ and $\approx 15 \%$ for a fake rate of $4 \%$. (right) For jets of $p_{T}>40 \mathrm{GeV}$ and $0<\eta<0.8$ the MVA tagger identifies jets from b-quark hadronization $\approx 13 \%$ more efficiently while rejecting $\approx 50 \%$ more of the light jet background. 


\section{Chapter 8}

\section{Statistics}

In a typical HEP analysis, the simulation of the signal and background process and data selection results in a final set of events. For a discernible signal, this set is used to measure the cross section and/or some kinematic distributions while for sets where no signal-like events are identified, the associated theory predictions are limited or completely excluded. To this end, a statistical analysis is carried out on a final variable usually constructed to have maximal separation between background and signal process; a perfect time to use multivariate techniques such as those described in Chapter 6.

The two main approaches used to calculate and interpreted statistical results in HEP are the Frequentist and Bayesian methods [84,85,86]. The Frequentist approach interprets results as the frequency at which the observed outcome is likely to occur in a series of repeatable experiments. The Bayesian interpretation is a degree of belief that the result is the true value.

In the analysis described a modified Frequentist approach is used as implemented in the COLLIE [87] statistical package. The Bayesian method predominantly used by CDF for the Higgs search will not be discussed here. 


\subsection{Overview}

The goal of this type of statistical analysis is to evaluated the conditional probability of a model given the data. Even though this part of any analysis is statistical in nature, the methods themselves can be discussed in a way which explicitly relates to this field. To aid in the following discussion, series of terms are defined below:

- Null Hypothesis $\left(H_{0}\right)$ : This corresponds to the default or accepted model. In the HEP community, this also referred to as the background-only (B) model as only the background process are considered.

- Test Hypothesis $\left(H_{1}\right)$ : The alternative model chosen to be tested against the data and possibly replace the Null Hypothesis as the accepted model. This is referred to as the signal+background $(\mathrm{S}+\mathrm{B})$ model as it contains both the background and signal contributions. The difference between the Test and Null hypothesis is called the parameter of interest.

- Simple Hypothesis: Is a model in which all the parameters of the model are specified.

- Compound Hypothesis: A model in which all the parameters are not specified. This includes those in which a parameter is said to fall within a range referred to as a prior. A compound hypothesis is a set of simple hypothesis each corresponding to a possible value(s) of the undetermined parameter(s).

- Parameter of Interest: As stated, a parameter which specifies the difference between the Test and Null hypothesis. Potentially this can be several parameters for a pair of test and null hypothesis however only one parameter can be the tested parameter of interest. 
- Nuisance Parameters: Are the potential parameters of interest, but not the one of interest itself, and can be shared by both $H_{0}$ and $H_{1}$. If each of these is known exactly, the models are simple, else if at least has a range of possible values, or some uncertainty, then the models are compound.

The final set of events of a counting experiment is governed by Poisson statistics. The sample consists of events from the $\mathrm{S}, \mathrm{S}+\mathrm{B}$ model, and the data sample, given by $H_{0}, H_{1}$, and nature respectively, with each being a random sampling of the corresponding parent distribution. If a histogram is used to present the final sample, then each bin is thought of as a Poisson distribution with a mean equal to the bin content. Because of the inherent uncertainty, it only makes sense to construct probability density functions (PDFs) of a test statistic for each hypothesis in order to determine confidence levels. The D $\varnothing$ Higgs group uses the statistic, $\Gamma(D)$, described in Section 8.2, but for now it is sufficient to state it is a function of the $\mathrm{B}$ and $\mathrm{S}+\mathrm{B}$ yields as well as a sample, $D$, used to test the conditional probability. By evaluating the test statistic against $H_{0}$ and $H_{1}$ taking into account the Poisson nature of the expected values by independently altering the test and null hypothesis according to the Poisson uncertainty of each bin, an independent PDF of the test statistic is created for both models. This is a Frequentist approach. For simple models, these PDFs can be compared to $\Gamma($ Data $)$ to produce a p-value and confidence level as described in Section 8.3. Since HEP models are usually compound hypotheses, a procedure, Bayesian in nature, has been formulated to handle these additional degrees of freedom (nuisance parameters) estimated to be in some range (a prior) described by a Gaussian distribution. Every simple hypotheses inherently contained within a compound hypotheses would produce a different PDF with the above mentioned procedure. This information is included through a techniques known as marginalization which superimposes this set of simple models by integrating the nuisance parameters over the 
respective priors. Technically this is achieved at D $\varnothing$ by sampling the prior density for each nuisance parameter independently and adjusting the predicted yield accordingly. From there, the procedure is as stated above. The resultant yields are varied according to the Poisson uncertainty and evaluated in the test statistic against the appropriate model creating two PDFs. The distributions given to the test statistic

is known to statisticians as the "prior predictive ensemble" and as "pseudo-data" to the HEP community. It should be noted that nuisance parameters common to both $H_{0}$ and $H_{1}$ are not sampled independently therefore maintaining a correlation. With the nuisance parameters integrated into the PDFs, the p-value and confidence level can now be evaluated.

\subsection{The Test Statistic}

HEP experiments are counting experiments and therefore are governed by Poisson statistics motivating the choice of a Poisson Likelihood Ratio as the test statistic.

\subsubsection{The Poisson Likelihood Ratio}

The Poisson distribution is a discrete probability distribution conveying the probability of a number of occurrences (d) over a fixed interval (p) given an average rate as long as the occurrences are independent of each other:

$$
f(d, p)=\frac{p^{d}}{d !} e^{-p}
$$


Defined for $H_{0}$ and $H_{1}$ as

$$
\begin{aligned}
& f\left(d \mid H_{0}\right)=\frac{b^{d}}{d !} e^{-b} \\
& f\left(d \mid H_{1}\right)=\frac{(s+b)^{d}}{d !} e^{-(s+b)} .
\end{aligned}
$$

The likelihood ratio is then

$$
Q(s, b, d)=\frac{f\left(d \mid H_{1}\right)}{f\left(d \mid H_{0}\right)}=\left(\frac{s+b}{b}\right)^{d} e^{-s}
$$

where $\mathrm{s}$ and $\mathrm{b}$ are the signal and background expectations respectively and $\mathrm{d}$ is either the observed data or the pseudo-data created from $H_{0}$ or $H_{1}$ as described above. Considering the joint likelihood of multiple distributions, such as the bins of a histogram, is the product of the individual likelihoods, this ratio can be written as

$$
Q=\prod_{i}^{N_{b}}\left(1+\frac{s_{i}}{b_{i}}\right)^{d_{i}} e^{-s_{i}}
$$

where $N_{b}$ is the number of bins. The test statistic which has a non-negative change in sensitivity for each additional distribution (or bin) and is monotonically increasing in the number of data events is the the negative log-likelihood ratio (LLR), $\Gamma$, given by

$$
\mathrm{LLR}=\Gamma(D)=-2 \ln (Q)=2 \sum_{i}^{N_{b}}\left[s_{i}-d_{i} \ln \left(1+s_{i} / b_{i}\right)\right]
$$

Figure 8.1 shows an LLR distribution for the test and null hypotheses. 

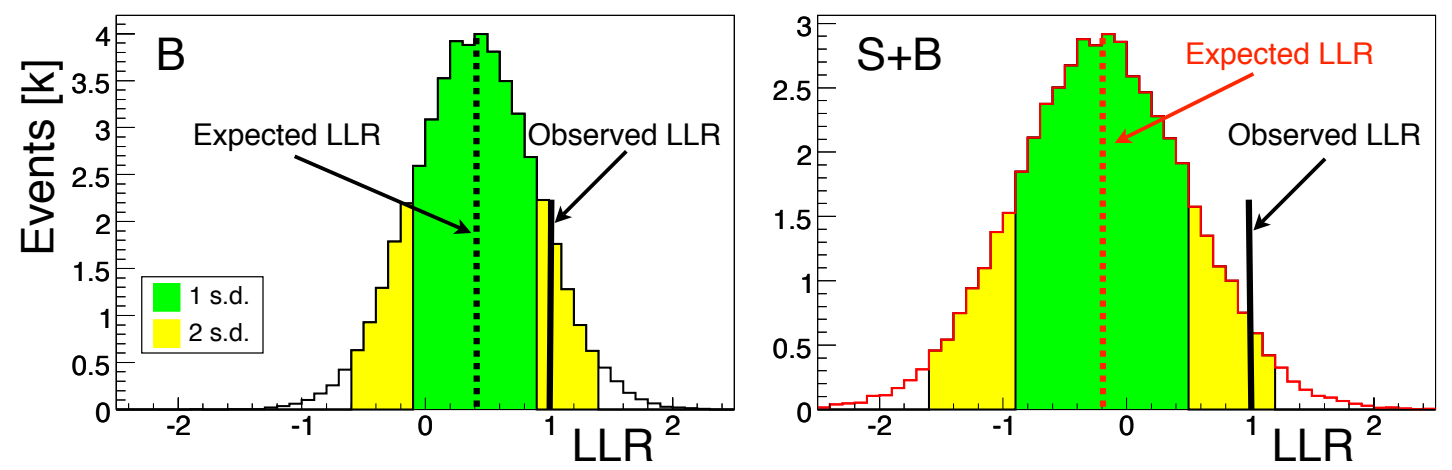

Figure 8.1: Example LLR distributions for the null (left) and test (right) hypotheses showing the expected value for each, the median, and an observed value.

\subsubsection{The Profile Likelihood Ratio}

An alternative statistic relying on the minimization of a Poisson $\chi^{2}$ as a function of the set of nuisance parameters, $\theta$, is also used [88]. Known as profiling, this minimization reveals the set of nuisance parameter values, or the simple hypothesis, which maximizes the likelihood function. The modified $\chi^{2}$ used to describe a specified hypotheses, $H$, is

$$
\chi^{2}(H)=-2 \ln P(\text { Data } \mid H, \theta)=2 \sum_{i}^{N_{b}}\left[\left(p(H)_{i}^{\prime}-d_{i}\right)-d_{i} \ln \left(\frac{p(H)_{i}^{\prime}}{d_{i}}\right)\right]+\sum_{k}^{N_{n}} R(H)_{k}^{2}
$$

where $p(H)_{i}^{\prime}$ is the predicted yields for a set of $N_{n}$ nuisance parameters $\eta$, and $R(H)_{k}$ is the deviation from the central value of the $k^{\text {th }}$ nuisance parameter, $\eta_{k}^{0}$, in units of the prior density Gaussian width, $\sigma_{k}$, given by

$$
R_{k}=\frac{\eta_{k}-\eta_{k}^{0}}{\sigma_{k}}
$$


The most reliable form of the test statistic if the negative log-likelihood of the likelihoods maximized independently for $H_{1}$ and $H_{0}$ :

$$
\begin{aligned}
\Gamma(D)=-2 \ln \left(Q\left(D \mid \theta_{0}, \theta_{1}\right)\right) & =-2 \ln \left(\frac{P\left(D \mid H_{1}, \hat{\theta}_{1}\right.}{P\left(D \mid H_{2}, \hat{\theta}_{2}\right.}\right) \\
& ==\chi^{2}\left(H_{0}\right)_{\text {min }}-\chi^{2}\left(H_{1}\right)_{\text {min }}
\end{aligned}
$$

where $\theta_{(0,1)}$ represents the set of nuisance parameters for $H_{(0,1)}$ and $\hat{\theta}_{(0,1)}$ represents the set of nuisance parameters values that maximize the likelihood for $H_{(0,1)}$.

\section{3 p-Values and Confidence Levels}

The probability that a hypothesis fluctuation gave rise to the observed data is known as the $p$-value and is determined as the fraction of the LLR distribution which is larger than the value from data.

The confidence level (CL), associated with a confidence interval or significance level, is a statement of how often the true value lies within the bounds of the interval. In hypothesis testing, a confidence interval with confidence level of $1-\alpha$ contains any value for the parameter of interest for which the null hypothesis is not rejected at significance level $\alpha$. In the case where the parameter of interest is bounded by 0 , such as a cross section, the CL and p-value can be interpreted in the same way. In this case the CL and p-value relationship is given by

$$
\begin{aligned}
C L_{B} & =1-P V_{B}=\int_{\Gamma_{r e f}}^{\mathrm{inf}} H_{S+B}(\Gamma \mid \sigma) d \Gamma \\
C L_{S+B} & =P V_{S+B}=\int_{\Gamma_{r e f}}^{\mathrm{inf}} H_{B}(\Gamma \mid \sigma) d \Gamma .
\end{aligned}
$$

where $\Gamma_{\text {ref }}$ is some reference value such as $\Gamma($ Data) (Figure 8.2). Since the test 
statistic is a function of the signal rate, the confidence level is therefore also a function of the signal rate $\sigma_{s}$.

In the Frequentist approach, $\mathrm{CL}_{\mathrm{S}+\mathrm{B}}$, is the confidence level of interest. This however does not protect making a strong statement constraining the test hypothesis due to a downward fluctuation in the data or a poor background model. This protection is found in the modified-Frequentist statistic $\mathrm{CL}_{\mathrm{S}}$ given by

$$
C L_{\mathrm{S}}\left(\sigma_{s}\right)=\frac{C L_{\mathrm{S}+\mathrm{B}}\left(\sigma_{s}\right)}{C L_{\mathrm{B}}\left(\sigma_{s}\right)}=\frac{P V_{\mathrm{S}}\left(\sigma_{s}\right)}{1-P V_{\mathrm{B}}\left(\sigma_{s}\right)}
$$

A $\operatorname{CL}_{S}\left(\sigma_{s}\right)<\alpha$ excludes the test hypothesis at a confidence level of $1-\alpha$.

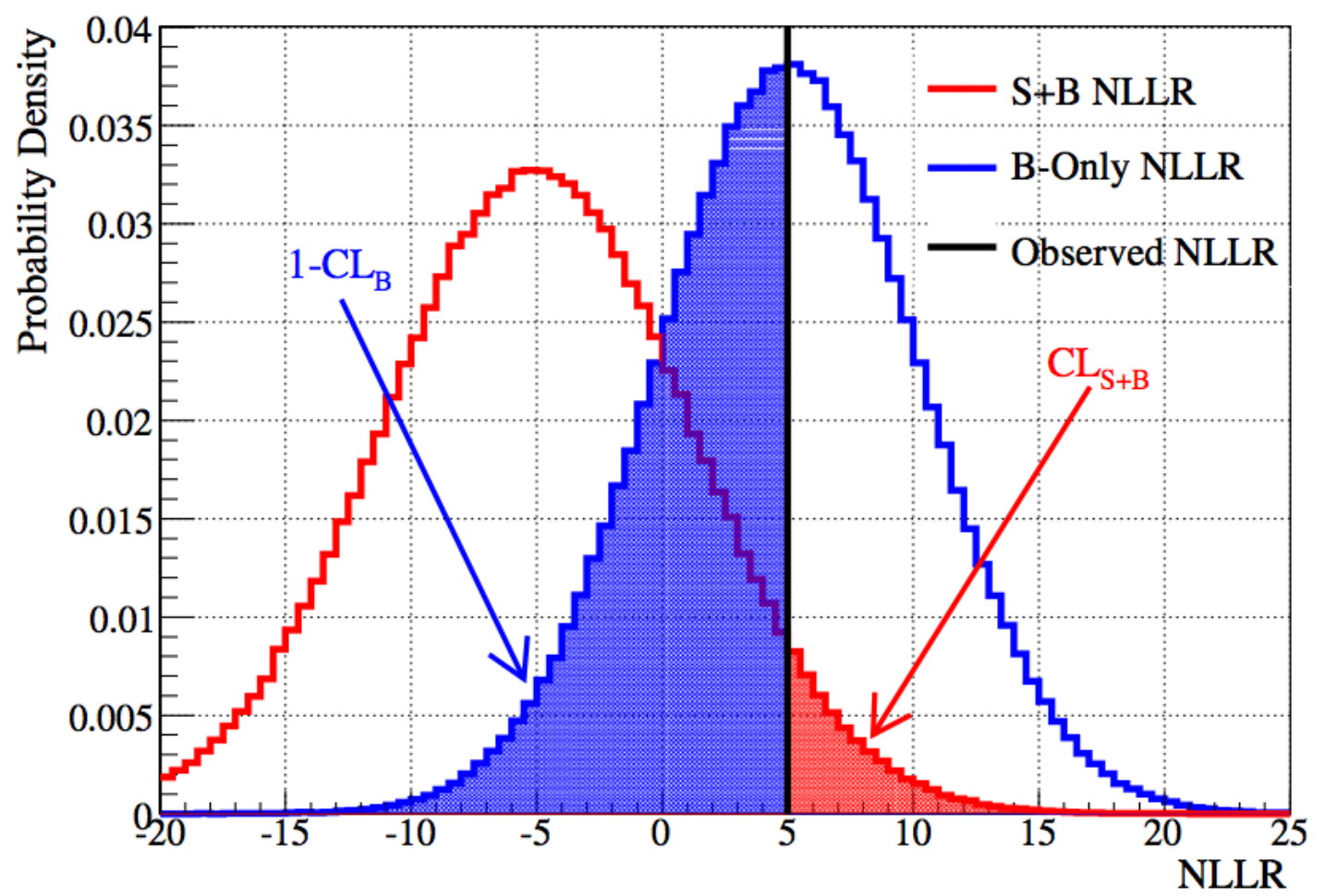

Figure 8.2: Graphical representation of $\mathrm{CL}_{\mathrm{B}}$ and $\mathrm{CL}_{\mathrm{S}+\mathrm{B}}$ shown for an example LLR distribution. 


\subsection{Cross Section Limits}

If no signal is observed, or the observed data is consistent with the null hypothesis, one would like to know how much the signal rate can be increased by before the given sample would be sensitive to it. Using the above formulation, a signal cross section, $\sigma_{s}$, or more generally the parameter of interest is found which satisfies the exclusion condition. If the rate need not be scaled to obtain sensitivity, the test hypothesis can be excluded at the given confidence level $1-\alpha$.

\subsection{Cross Section Measurements}

In the case where the signal is discernible in the sample, the value of the cross section of this signal in the data is of interest. This can be measured from the data by either leaving this free parameter unconstrained in the Poisson $\chi^{2}$ minimization or differentiating the Gaussian distribution created from the $1-C L_{S+B}$ values generated from a set of $H_{1}$ pseudo-experiments created from a range of parameters i.e. signal cross sections. The integral of this distribution for values lower than the observed $1-C L_{S+B}$ is used to build a curve the differential of which is a Gaussian and can be used to extract a confidence interval and the mean value for the parameter. Although the two methods are nominally equivalent the second one is affected by the choice of the granularity in the scan.

\subsection{Evaluation of Systematic Uncertainties}

Nuisance parameter priors interpreted as fraction uncertainties presented as Gaussian distributions with a mean of zero and some width are interpreted as systematics uncertainties. There are several systematics associated with the reconstruction and 
calibration of objects and Monte Carlo generation which are common across all analysis and are discussed below. These errors enter the calculation of confidence levels and cross section limits or measurements as either normalization uncertainties that only affect the expected signal and/or background yields, and as shape uncertainties that affect the distributions of the discriminant variables used to derive the results. In the case of the latter, the correction factors applied to the MC are shifted by $\pm 1 \sigma$ to determine the shape of the uncertainty.

Some care must be taken to ensure the systematic effect is not biased. MC events are weighted to correct for various reasons such as simulation deficiencies. In some cases limited statistics are available for a given sample leading to individual events carrying large weights in order have the desired yield. To evaluate some systematics the entire analysis chain is rerun selecting a slightly different subset of events. If an event which required a large weight no longer passes the analysis cuts, the systematic in question might be inflated due to an effect statistical in nature. This is a problem that must be dealt with creatively if more statistics is not available.

\subsubsection{Experimental Systematic Uncertainties}

The following discusses uncertainties having to do with the corrections applied to the creation, reconstruction, identification, or calibration of simulated objects in order to better model the behaviors seen in data. Some analysis consider data from multiple run epochs of the $\mathrm{D} \varnothing$ detector and some corrections are determined for each epoch individually. Those corrections which are determined for each epoch and have an error that is statistical in nature, the sampling of the prior density should be done independently. For those in which the error is dominated by the procedure or sample used to determine the correction, the prior density should be sampled in a correlated manner. All corrections below unless otherwise stated have statistically dominated 
errors.

\section{- Jet Energy Scale:}

Initial MC jet energies are shifted after modifying the JSSR shifting correction factors by $\pm 1 \sigma$ of their uncertainties.

\section{- Jet Energy Resolution:}

Initial MC jet energies were smeared after modifying the JSSR smearing correction factors by $\pm 1 \sigma$ of their uncertainties.

\section{- Jet Reconstruction and Identification:}

Scale factors calculated by the ratio of data and simulation efficiencies used to remove $\mathrm{MC}$ jets to account for data/MC differences in jet reconstruction and identification efficiencies were varied by $-1 \sigma$ of their uncertainties, and the result was symmetrized to obtain the $+1 \sigma$ error estimate.

\section{- Vertex confirmation and Taggability:}

The combined scale factors for vertex confirmation and taggability, or the individual scale factors, whichever where used in the analysis to account for data/MC differences are varied by $\pm 1 \sigma$ of their uncertainties.

\section{- b-tagging:}

Flavor-dependent scale-factors centrally provided by the b-ID group are used to weight $\mathrm{MC}$ events according to the flavor of the jets in the event to account

for data/MC differences in efficiencies for direct tagging. These weights were varied by $\pm 1 \sigma$ of their uncertainties.

\section{- Lepton identification:}

For each type of lepton multiple corrections are applied. The uncertainties associated with these various sources of inefficiency are combined in quadrature. 
Each of these lepton-ID efficiencies is then varied by $\pm 1 \sigma$ of its uncertainty.

The uncertainty on this corrections is systematically dominated.

- Lepton Resolution: As stated in Chapter 4 the simulated response of the detector is sharper then that of the real detector so the energy measurements of objects are smeared to match the data on average. Similar to jet resolutions these are varied within one sigma of the mean value.

\section{- Luminosity:}

A $6.1 \%$ uncertainty is applied to total integrated luminosity [89].

\section{- Trigger Efficiency:}

The modeling of trigger efficiencies is a common experimental uncertainty but will be discussed for each specific analysis since it is dependent on the trigger suite required in the data.

\subsubsection{Theoretical Systematic Uncertainties}

The following systematic uncertainties are of theoretical nature.

\section{- Cross sections:}

The systematic uncertainties on the cross sections of the various processes involved are considered. They range from $6 \%$ for associated Higgs production and for the inclusive production of $W / Z$, to $10 \%$ for single and double top production.

\section{- $V p_{T}$ reweighting:}

The impact of this reweighting in the two-jet sample was estimated as a flat $2 \%$ normalization uncertainty based on the study in Ref. [90]. 


\section{- Heavy flavor ratio:}

The uncertainty on the $(W / Z) b \bar{b}$ and $(W / Z) c \bar{c}$ cross sections is calculated within MCFM to be $+19 /-18 \%$ [91]. A conservative $20 \%$ uncertainty is assigned on the ratio of heavy to light flavor production.

\section{- ALPGEN parameters uncertainties:}

Uncertainties arise in ALPGEN from the choice of MLM matching $p_{T}$ threshold, the choice of MLM clustering radius, and from two scaling parameters, the $k_{\perp^{-}}$ and $Q$-factor. These are combined in Refs. [92] and [93] into two independent shape-only uncertainties: related to the MLM algorithm and related to the light and heavy flavor scaling parameters. The MLM algorithm uncertainty is only applied to $\mathrm{V}+$ jets samples with light flavor jets.

\section{- Underlying event and fragmentation modeling:}

From studies of the impact on the dijet mass of various PYTHIA tunes and of the comparison of ALPGEN interfaced with PYTHIA and with HERWIG, a shapeonly systematic uncertainty is applied to all $\mathrm{V}+$ jets samples as recommended in Reference [93].

\section{- Parton Distribution Function Uncertainty:}

The signal acceptance and modeling is sensitive to the parton distribution functions (pdfs) used in MC generation. To assess the impact of the uncertainties on these pdfs, a re-weighting is used. The signal has been generated using CTEQ6L1 pdf, but to assess the pdf uncertainty a per-event re-weighting is performed based on the properties of the incoming partons to CTEQ6.1M and the 20 pairs of associated error sets. The use of this error set is discussed for each analysis. 


\section{Chapter 9}

\section{$Z Z \rightarrow \ell^{-} \ell^{+} \nu \bar{\nu}$ Analysis}

$S U(2)$ kinetic energy terms lead to gauge coupling self interactions described by Equations 1.33 and 1.34. Testing these interactions is essentially a test of the symmetrybroken non-abelian gauge theory and any deviations observed would force a reconsideration of the Electroweak Theory or indicate physics beyond the SM. The test is achievable through a measurement of any of the di-boson processes. The $Z Z$ production can also be used to test for new physics in the form of anomalous couplings involving $Z Z Z, Z Z \gamma$, or $Z \gamma \gamma$ since these couplings are not included in the SM. Furthermore, this process is connected with the Higgs boson search as a background in both the low mass $Z H$ search and in the high mass $H \rightarrow W^{+} W^{-}$and $H \rightarrow Z Z$ channels. There being no tree level process with a cross section between $Z Z$ and $V H$ production, observing this process is necessary proof that the Higgs is within our reach. The methods of the Higgs search have fallen under some scrutiny lately since the combination of multiple channels from different collaborations is a nontrivial process with many pitfalls. Using this exact same procedure with identical analysis techniques is the benchmark to the Higgs search and a way to quench these concerns. Work on this benchmark measurement and the Higgs search itself is presented in 
Chapter 10. In this chapter we focus our attention on the measurement of the last diboson process on the road to the Higgs and the lessons learned from the experimental challenges that lie within.

This analysis centers on the search for a pair of $Z$ bosons where one decays to a pair of electrons or muons while the other decays into a pair of neutrinos evident from a large imbalance in the transverse energy measurement (see Fig. 9.1) As discussed in Chapter 5 , events represented by the tails of the resolution distributions can fake the $E_{T}$ signature and therefore mimic this signal. With the requirement of two leptons, Drell Yann $Z$ production $(q \bar{q} \rightarrow Z)$ with a cross section four orders of magnitude larger then $\sigma_{Z Z}$, easily dominates a selection based on the above requirements. Using the $\mathscr{H}_{T}{ }^{\prime}$ variable described in Section 5.1 this background is removed leaving mostly events with true $E_{T}$. A likelihood based discriminant is then built and used to measure the significance and cross section.

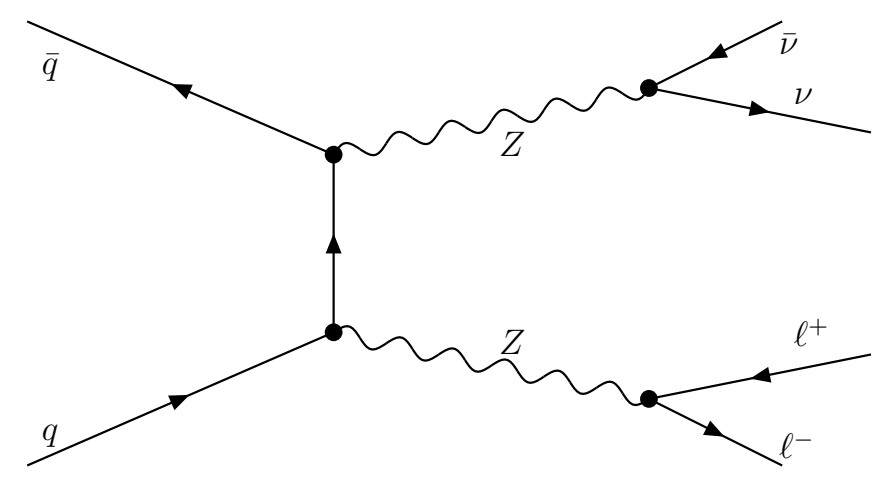

Figure 9.1: Feynman diagram of the leading order $Z Z \rightarrow \ell^{-} \ell^{+} \nu \bar{\nu}$ process 


\subsection{Dataset and Monte Carlo Samples}

\subsubsection{Data}

The data for this analysis were collected by the $\mathrm{D} \varnothing$ collaboration between October 2002 and February 2008 at the center-of-mass energy $\sqrt{s}=1.96 \mathrm{TeV}$. The integrated luminosity after data quality is $2.67 \pm 0.11 \mathrm{fb}^{-1}$ for the di-electron and di-muon channels.

\subsubsection{Monte Carlo}

The $W+l p$ sample was generated with ALPGEN+PYTHIAwhile the remaining were produced by PYTHIA and normalized to the NLO cross section. After the selection described in Section 9.2, these samples were scaled such that the absolute yield agrees with data. The $W+l p$ sample was normalized to the predicted multijet contribution as described in Section 9.3. The signal and background samples are listed in Tables 9.1.2 and 9.1.2.

\begin{tabular}{|c|c|c|c|}
\hline Process & Mass Range $[\mathrm{GeV}]$ & $\sigma \times \mathrm{BR}[\mathrm{pb}]$ & Events \\
\hline$\overline{\mathrm{Z} / \gamma^{*} \rightarrow \mu \mu}$ & $15<M<60$ & 465 & $2.1 \mathrm{M}$ \\
\hline $\mathrm{Z} / \gamma^{*} \rightarrow \mu \mu$ & $60<M<130$ & 241.6 & $3.4 \mathrm{M}$ \\
\hline $\mathrm{Z} / \gamma^{*} \rightarrow \mu \mu$ & $130<M<250$ & 1.96 & $414 \mathrm{k}$ \\
\hline $\mathrm{Z} / \gamma^{*} \rightarrow e e$ & $15<M<60$ & 465 & $3.0 \mathrm{M}$ \\
\hline $\mathrm{Z} / \gamma^{*} \rightarrow e e$ & $60<M<130$ & 241.6 & $3.7 \mathrm{M}$ \\
\hline $\mathrm{Z} / \gamma^{*} \rightarrow e e$ & $130<M<250$ & 1.96 & $407 \mathrm{k}$ \\
\hline $\mathrm{Z} / \gamma^{*} \rightarrow \tau \tau$ & $15<M<60$ & 465 & $1.8 \mathrm{M}$ \\
\hline $\mathrm{Z} / \gamma^{*} \rightarrow \tau \tau$ & $60<M<130$ & 241 & $6.9 \mathrm{M}$ \\
\hline $\mathrm{Z} / \gamma^{*} \rightarrow \tau \tau$ & $130<M<250$ & 1.96 & $409 \mathrm{k}$ \\
\hline$t \overline{\bar{t}} \rightarrow b b l l \nu \nu$ & & 0.67 & $325 \mathrm{k}$ \\
\hline$W W \rightarrow l \nu l \nu$ & & 1.29 & $1.2 \mathrm{M}$ \\
\hline$W Z \rightarrow l \nu l l$ & & 0.12 & $375 \mathrm{k}$ \\
\hline $\mathrm{ZZ} \rightarrow 1111$ & & 0.014 & $87 \mathrm{k}$ \\
\hline $\mathrm{ZZ} \rightarrow 11 \nu \nu$ & & 0.057 & $253 \mathrm{k}$ \\
\hline$W \gamma \rightarrow e \nu+\gamma($ road $)$ & & 8.76 & $57 \mathrm{k}$ \\
\hline$W \gamma \rightarrow l \nu+\gamma($ prod $)$ & & 5.58 & $35 \mathrm{k}$ \\
\hline$W+0 l p \rightarrow l \nu+X$ & & 674 & $14 \mathrm{M}$ \\
\hline$W+1 l p \rightarrow l \nu+X$ & & 190 & $7 \mathrm{M}$ \\
\hline$W+2 l p \rightarrow l \nu+X$ & & 44 & $4 \mathrm{M}$ \\
\hline$W+3 l p \rightarrow l \nu+X$ & & 11 & $2 \mathrm{M}$ \\
\hline$W+4 l p \rightarrow l \nu+X$ & & 2.52205 & $2 \mathrm{M}$ \\
\hline$W+5 l p \rightarrow l \nu+X$ & & 0.85 & $1.2 \mathrm{M}$ \\
\hline
\end{tabular}

Table 9.1: Run IIa signal and background Monte Carlo samples 


\begin{tabular}{|c|c|c|c|}
\hline Process & Mass Range [GeV] & $\sigma \times \mathrm{BR}[\mathrm{pb}]$ & Events \\
\hline$\overline{\mathrm{Z} / \gamma^{*} \rightarrow \mu \mu}$ & $15<M<60$ & $\begin{array}{l}465 \\
\end{array}$ & $2.0 \mathrm{M}$ \\
\hline $\mathrm{Z} / \gamma^{*} \rightarrow \mu \mu$ & $60<M<130$ & 241.6 & $2.0 \mathrm{M}$ \\
\hline $\mathrm{Z} / \gamma^{*} \rightarrow \mu \mu$ & $130<M<250$ & 1.96 & $519 \mathrm{k}$ \\
\hline $\mathrm{Z} / \gamma^{*} \rightarrow e e$ & $15<M<60$ & 465 & $1.9 \mathrm{M}$ \\
\hline $\mathrm{Z} / \gamma^{*} \rightarrow e e$ & $60<M<130$ & 241.6 & $3.8 \mathrm{M}$ \\
\hline $\mathrm{Z} / \gamma^{*} \rightarrow e e$ & $130<M<250$ & 1.96 & $404 \mathrm{k}$ \\
\hline $\mathrm{Z} / \gamma^{*} \rightarrow \tau \tau$ & $15<M<60$ & 465 & $2.0 \mathrm{M}$ \\
\hline $\mathrm{Z} / \gamma^{*} \rightarrow \tau \tau$ & $60<M<130$ & 241.6 & $2.2 \mathrm{M}$ \\
\hline $\mathrm{Z} / \gamma^{*} \rightarrow \tau \tau$ & $130<M<250$ & 1.96 & $409 \mathrm{k}$ \\
\hline$t \bar{t}$ inclusive & & 6.4 & $1.5 \mathrm{M}$ \\
\hline$W W \rightarrow l \nu l \nu$ & & 1.29 & $1.9 \mathrm{M}$ \\
\hline$W Z \rightarrow l \nu l l$ & & 0.12 & $1.0 \mathrm{M}$ \\
\hline $\mathrm{ZZ} \rightarrow 1111$ & & 0.014 & $607 \mathrm{k}$ \\
\hline$\overline{\mathrm{ZZ}} \rightarrow 11 \nu \nu$ & & 0.057 & $607 \mathrm{k}$ \\
\hline$W+0 l p \rightarrow l \nu+X$ & & 674424 & $2.3 \mathrm{M}$ \\
\hline$W+1 l p \rightarrow l \nu+X$ & & 190 & $2.2 \mathrm{M}$ \\
\hline$W+2 l p \rightarrow l \nu+X$ & & 44 & $919 \mathrm{k}$ \\
\hline$W+3 l p \rightarrow l \nu+X$ & & 11 & $403 \mathrm{k}$ \\
\hline$W+4 l p \rightarrow l \nu+X$ & & 2.5 & $406 \mathrm{k}$ \\
\hline$W+5 l p \rightarrow l \nu+X$ & & 0.85 & $238 \mathrm{k}$ \\
\hline
\end{tabular}

Table 9.2: Run IIb signal and background Monte Carlo samples

\section{Monte Carlo Corrections}

All the corrections discussed in Section 3.3 were applied except the unavailable $W p_{T}$ and MLM matching reweightings. In addition, to account for shortcomings in tracking modeling such as a simple geometry and improper modeling of dead material, tracks are randomly removed from the MC using the reconstruction inefficiency parameterized as a function of $\eta$ and $\phi$.

\subsection{Preselection Requirements}

Data events in the di-muon (di-electron) channel are required to have satisfied at least one single muon (electron) trigger and MC trigger efficiency corrections were used in the trigger decision simulation. Events are chosen with exactly two identified leptons required to have opposite charge and produce an invariant mass within the $Z$-mass window of $70<M_{\ell \ell}<110 \mathrm{GeV}$. To allow events with ISR or FSR jets, up to two jets can be in an event. No explicit cut on $\not_{T}$ is made at this point. 


\subsubsection{Electron Requirements}

The di-electron channel is defined by the presence of two electrons following the following criteria:

- $p_{T}>15.0 \mathrm{GeV}$

- $I D=10$ or $I D=11$

- isolation iso $<0.15$

- EM fraction $E M F>0.9$

- $H M x 7>50$

- matched to a central track with $p_{T}>5.0 \mathrm{GeV}$

- Likelihood $>0.85$

- $\left|\eta_{\text {det }}\right|<1.1$ or $1.5<\left|\eta_{\text {det }}\right|<2.5$

\subsubsection{Muon Requirements}

The di-muon channel is defined by the presence of energy deposits in the muon system matched to a track in the central tracking system as described in Section 4.3 which pass the following criteria:

- $p_{T}>15.0 \mathrm{GeV}$

- Loose muon ID

- A track with at least 1 SMT hit and $\chi^{2} / d o f<4.0$

- A distance of closest approach of $<0.02 \mathrm{~cm}$ on the track 
- $\left|\Sigma^{\text {cells }} E_{T} / p_{T}(\mu)\right|<0.1$ in the annulus $0.1<\Delta R<0.4$

- $\left|\Sigma^{\text {tracks }} p_{T} / p_{T}(\mu)\right|<0.1$ in the cone $\Delta R<0.5$

\subsubsection{Jet Requirements}

As previously stated, up to two jets which satisfy the following are allowed in an event:

- cone algorithm with $\Delta R<0.5$ (JCCB algorithm),

- JES corrected,

- $\Delta R($ jet - lepton $)>0.3$,

- $E_{T}>15 \mathrm{GeV}$.

\subsubsection{Additional Lepton Requirements}

Besides not being a signature of a $Z$ decay to leptons, additional charged leptons are a way to look for soft, non-isolated or poorly reconstructed objects.

\section{EM Cluster Requirements}

Events with one or more extra EM cluster which satisfies the following requirements are discarded:

- simple cone EM-cluster in $\mathrm{CC}$ or EC region,

- $I D=10$ or $I D=11$,

- isolation iso $<0.2$,

- EM fraction $E M F>0.9$, 
- $E_{T}>5 \mathrm{GeV}$,

- $\Delta R($ cluster, $\ell$ from Z) $>0.2$.

In addition the cluster must satisfy at least one of the following requirements:

- matched to a central track with $p_{T}>8 \mathrm{GeV}, \Delta z(P V)<1 \mathrm{~cm}$ and $d c a<$ $0.1 \mathrm{~cm}$,

- $H M x 7>12$ in $\mathrm{CC}$ or $H M x 8>20$ in EC.

\section{Muon Requirements}

Events with additional muons other than those produced by the $Z$ decay are rejected. For this purpose the muons are defined as:

- loose muons associated to a central track;

or

- medium muons;

In both cases the muon is required to have a $\Delta R>0.2$ from the primary leptons.

\section{Hadronic Tau Requirements}

A veto on the presence of reconstructed multi-prong hadronic tau leptons is applied. The tau objects are defined as:

- tau neural network $N N>0.3($ TauNN v1),

- tau type 3,

- $p_{T}>5 \mathrm{GeV}$,

- $\Delta R($ tau, $\ell$ from Z $)>0.2$,

- $\Delta z(P V)<1 \mathrm{~cm}$. 


\subsubsection{Track Requirements}

A veto on the presence of an isolated track rejects poorly isolated and/or reconstructed leptons and leptons produced in a region of poor or non-existing detector coverage. An isolated track must have $p_{T}$ greater than that of the sum of the surrounding tracks that satisfy the following criteria:

- $p_{T}>5 \mathrm{GeV}$

- $\Delta R($ track, iso.track $)<R$;

- $\Delta z($ primary - vertex, track $)<1 \mathrm{~cm}$;

- $\chi^{2}($ primary - vertex $)<25$;

- $E_{T}($ cone $) / p_{T}($ track $)<1$ for a cone of $R=0.7$.

- At least 1 SMT hit or a distance of closest approach of $\leq 0.1 \mathrm{~cm}$. 


\subsection{Instrumental Backgrounds}

Drell-Yan $Z$ production was already mentioned as an instrumental background with fake $\mathbb{E}_{T}$. Another type of instrumental background are those in which the $\mathbb{E}_{T}$ is from a neutrino, but the lepton criteria is faked by an object which is not a lepton. In events with a $W$ decaying to a lepton and a neutrino, if a jet or photon is reconstructed as the second lepton, the event can pass the preselection criteria. This class of events is rather difficult to simulate at the correct rate so the normalization is usually derived from a data sample while the Monte Carlo is used to describe the kinematic distributions. For this particular analysis the instrumental backgrounds described below have non-negligible contributions in only the di-electron channel.

\subsubsection{Wr Background}

This background arises from ISR or FSR of a photon $(\gamma)$ and from direct $W \gamma$ production through the trilinear gauge coupling where the photon is identified as an electron. The rate of photons faking electrons was measured in $Z \rightarrow \ell \ell \gamma$ events [95]. Photons were identified as EM clusters with

- electromagnetic fraction $E M F<0.9$,

- isolation variable $i s o<0.15$,

- transverse energy $E_{T}>15 \mathrm{GeV}$,

- $\left|\eta_{\text {det }}\right|<1.1$ or $1.5<\left|\eta_{\text {det }}\right|<2.5$

- No explicit $p_{T}$ requirement on the matched track

and the probability of a surviving EM cluster to pass an electron likelihood cut of 0.85 was measured to be $0.012 \pm 0.008$. 
For the $Z Z$ analysis, two PYTHIA generated samples were created: $W \rightarrow \ell \nu$ for the ISR and trilinear gauge coupling components and $W \rightarrow e \nu_{e}$ for the FSR contributions. The relative normalization was estimated with the Baur LO Monte Carlo generator [94] requiring electron $E_{T}>12 \mathrm{GeV}$ and $\Delta R(e, \gamma)>0.4$ to match PYTHIA generator cuts. The respective cross sections are shown in Tables 9.1.2 and 9.1.2. To obtain the $W \gamma$ yield the fake rate of 0.012 was applied to selected events in which one EM object passed the electron criteria from Section 9.2.1 and another satisfied the photon requirements listed above. The photon is matched to a generator level photon in order to avoid double counting with the $W+$ jets background.

\subsection{2 $W+$ jets Background}

This background originates from jets faking the electron signature. The resulting contribution is estimated using the "matrix-method" which involves solving a set of linear equations for the final yield of a given process.

Using matrix-method requires two samples to be defined in order to determine the rate at which the object selection criteria is faked by an object not of that type. The yield of the "tight" ("loose") sample in which the criteria in question is (not) imposed is $N_{t}\left(N_{l}\right)$. The efficiency for a real (fake) object to pass the tight criteria

having already passed the loose selection is $\varepsilon_{\text {real }}\left(\varepsilon_{\text {fake }}\right)$. With these four quantities the number of correctly $\left(N_{\text {sig }}\right)$ and incorrectly $\left(N_{b k g}\right)$ identified objets can be determined by solving the following equations:

$$
\begin{aligned}
& N_{l}=N_{b k g}+N_{s i g} \\
& N_{t}=\epsilon_{\text {fake }} N_{b k g}+\epsilon_{\text {real }} N_{\text {sig }}
\end{aligned}
$$

For the electron fake rate, a "tight" data sample enriched with multijet events is 
selected by requiring exactly one EM object and $\mathbb{E}_{T}<10 \mathrm{GeV}$ to reduce and $Z \rightarrow e e$ $W \rightarrow e \nu$ contamination respectively. By dropping the electron isolation cut, a "loose" sample is defined. In both cases the simulated events are subtracted from the data and the ratio of "tight" to "loose" events gives the efficiency for a fake electron to pass the isolation cut, $\varepsilon_{f a k e}$. To determine the efficiency for a real electron to pass the isolation cut, $\varepsilon_{\text {real }}$ the ratio of the two efficiencies measured by a tag-and-probe method by the electron-id group were used.

In the mass window of $70<M_{\ell \ell}<110 \mathrm{GeV}$, after all cuts listed above, the estimated number of $W+$ jets events in Run IIa was $1.64 \pm 0.5$ and $1.08 \pm 0.3$ for Run IIb. The $W+$ jets MC events generated with ALPGEN+PYTHIAwas scaled to this value.

\subsection{Monte Carlo Normalization}

After the selection described in Section 9.2, the PYTHIA generated samples were scaled such that the absolute yield agrees with data. Figure 9.2 shows the di-lepton invariant mass distribution for the four samples and Figure 9.3 shows the di-lepton $p_{T}$ spectrum. 

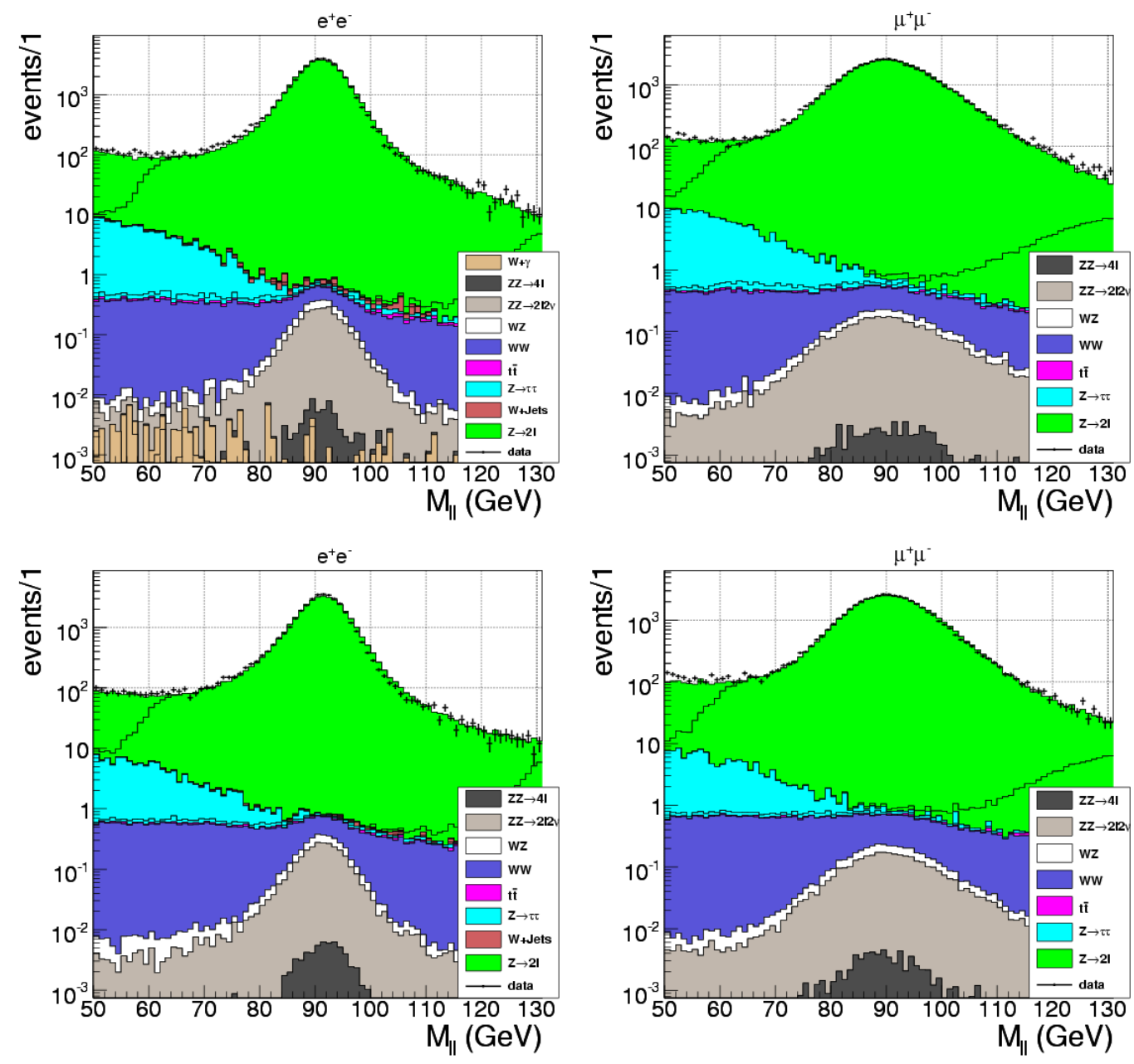

Figure 9.2: Di-lepton invariant mass evaluated after applying the mass cut and the extra activity vetoes for the di-electron (left) and di-muon (right) channels in Run IIa (top) and Run IIb (bottom). 

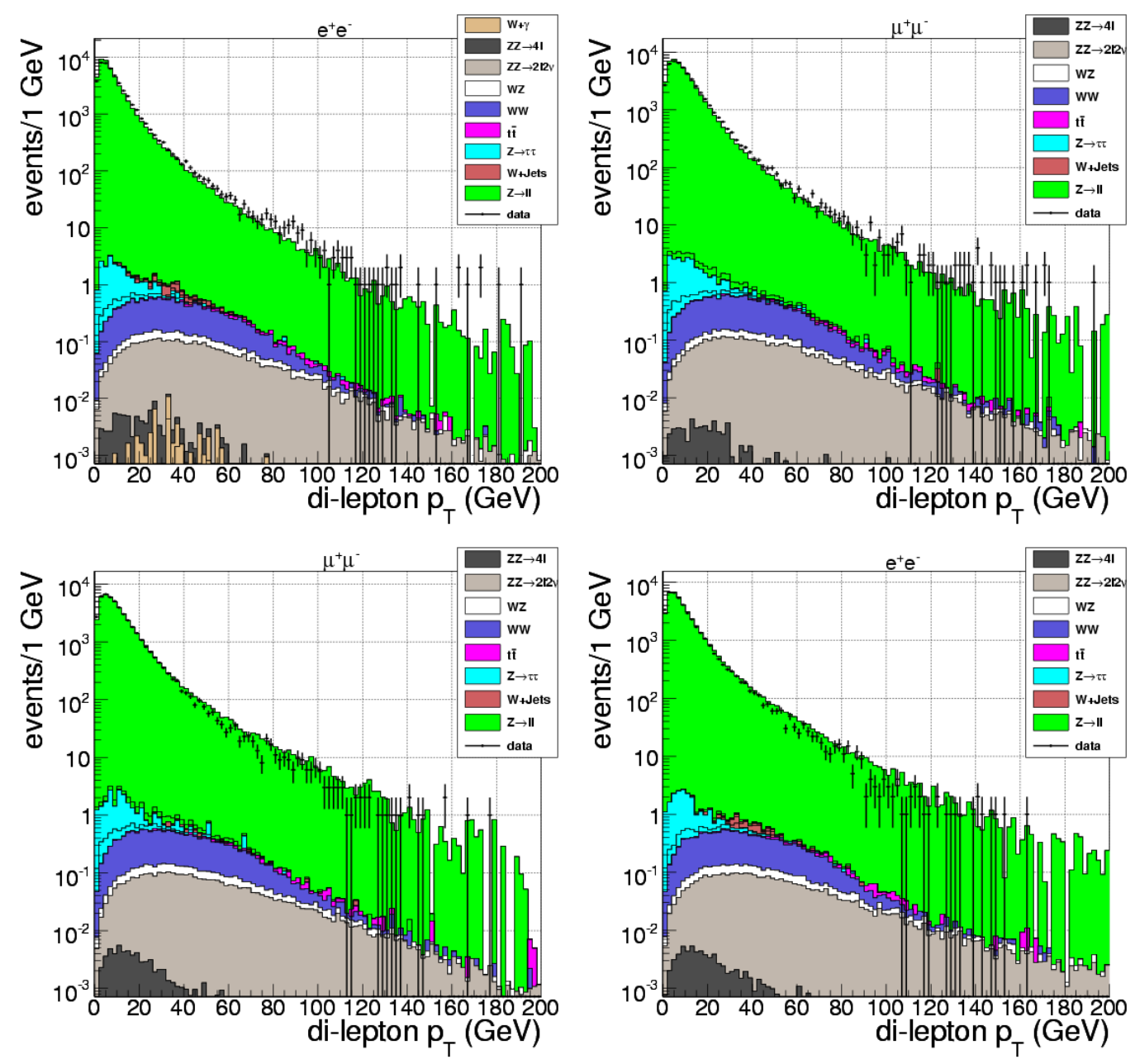

Figure 9.3: Di-lepton $p_{T}$ spectrum after applying the mass cut and the extra activity vetoes for the di-electron (left) and di-muon (right) channels in Run IIa (top) and Run IIb (bottom). 


\subsection{Alternative $E_{T}$ Selection}

Looking at the $\mathscr{E}_{T}$ distributions shown in Figure 9.4, it is clear a cut on this variable would not allow for a robust and sensitive result. The alternative $\mathscr{E}_{T}$ definition discussed in Section 5.1 was introduced to $\mathrm{D} \emptyset$ in this analysis to deal with this problem. Using the two leptons to define the thrust axis a cut on this variable increased the signal to background ratio by an order of magnitude.

When minimizing the two components of the di-lepton transverse momentum decomposed onto the thrust axis special consideration was given to electrons in the $\phi$-gap region of the central calorimeter. There is a non-zero probability for such electrons to have significantly underestimated energies. If the lower $p_{T}$ lepton is within the $\phi$-gap region of the central calorimeter its measurement uncertainty is additionally inflated by a factor of 15 but the fractional uncertainty is capped at unity. This has

the effect of reducing $a_{l}{ }^{\text {di-lept }}$ to zero in some cases. Figures 9.5, 9.6, and 9.7 show the lepton resolution, calorimeter measured, and tracking recoil corrections respectively for the full $2.2 \mathrm{fb}^{-1}$ data set. 

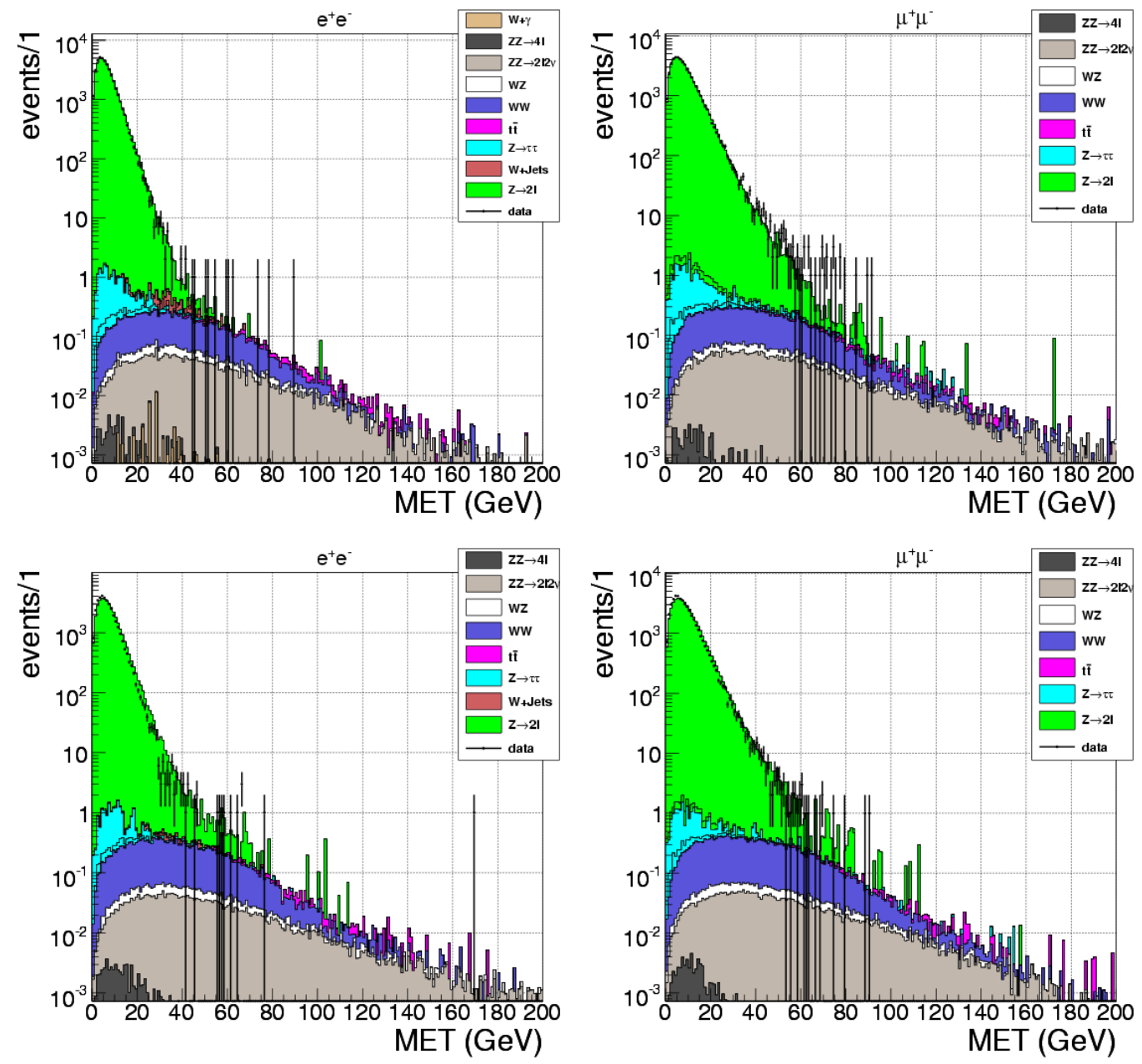

Figure 9.4: $\mathbb{E}_{T}$ of the event evaluated after applying the mass cut and the extra activity vetoes for the di-electron (left) and di-muon (right) channels in Run IIa (top) and Run IIb (bottom). 

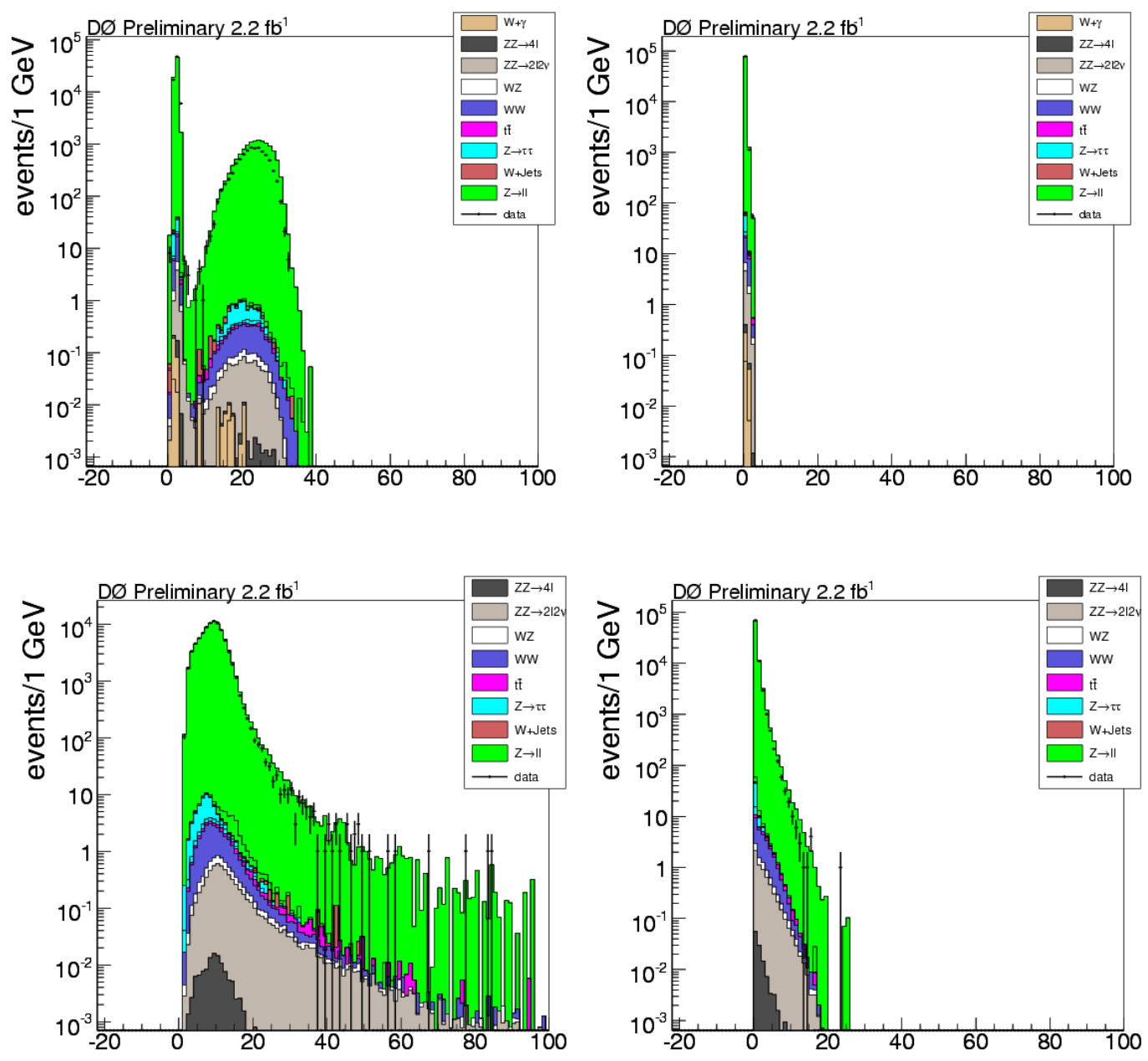

Figure 9.5: Correction due to lepton resolution for the longitudinal (left plots) and transverse (right plots) directions with respect to the thrust axis for the full $2.2 \mathrm{fb}^{-1}$ integrated luminosity. The top shows the di-electron channel while the bottom shows the di-muon channel. 

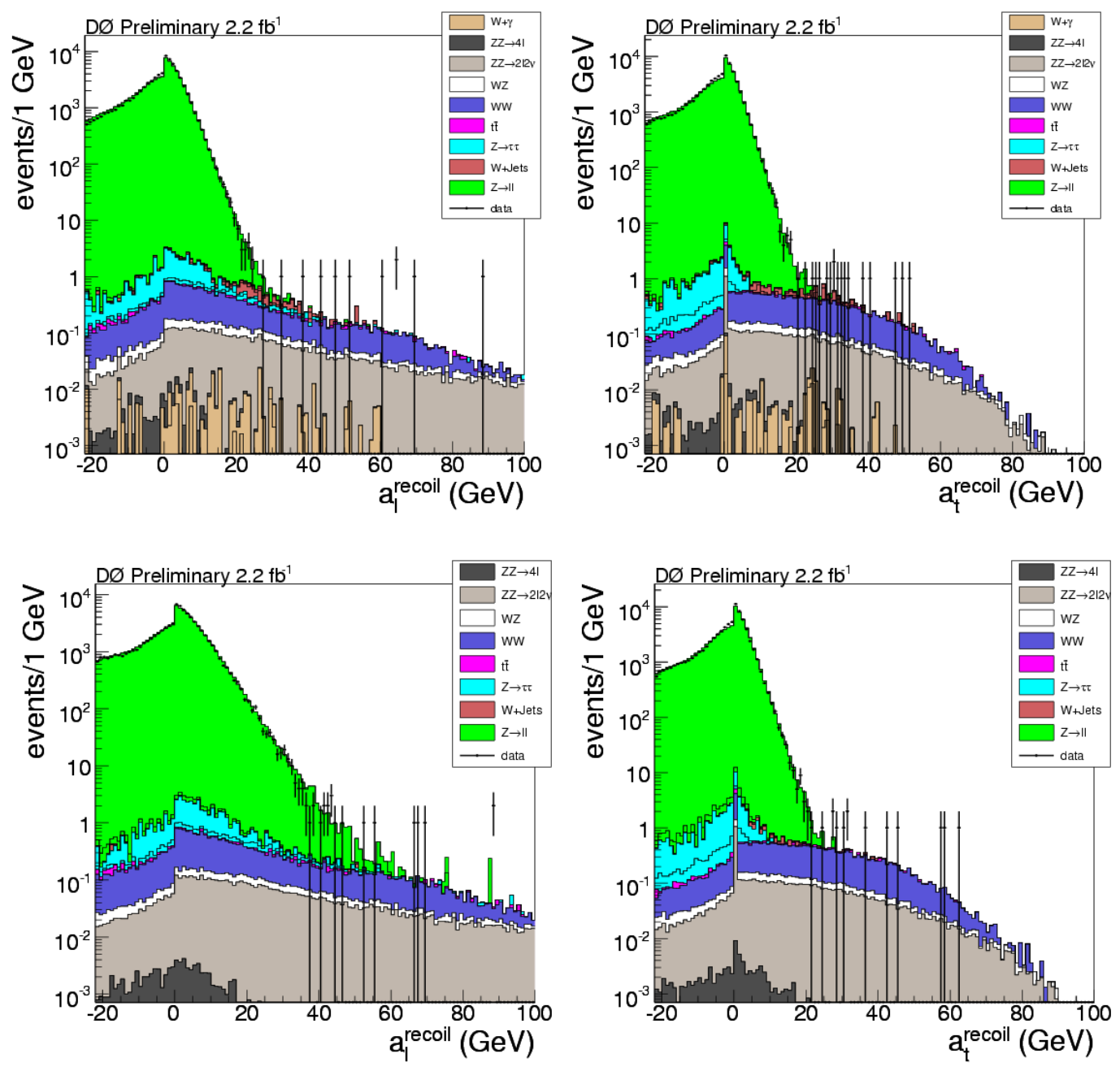

Figure 9.6: Calorimeter recoil activity in the longitudinal (left plots) and transverse (right plots) direction with respect to the thrust axis for the full $2.2 \mathrm{fb}^{-1}$ integrated luminosity. The top shows the di-electron channel while the bottom shows the di-muon channel. 

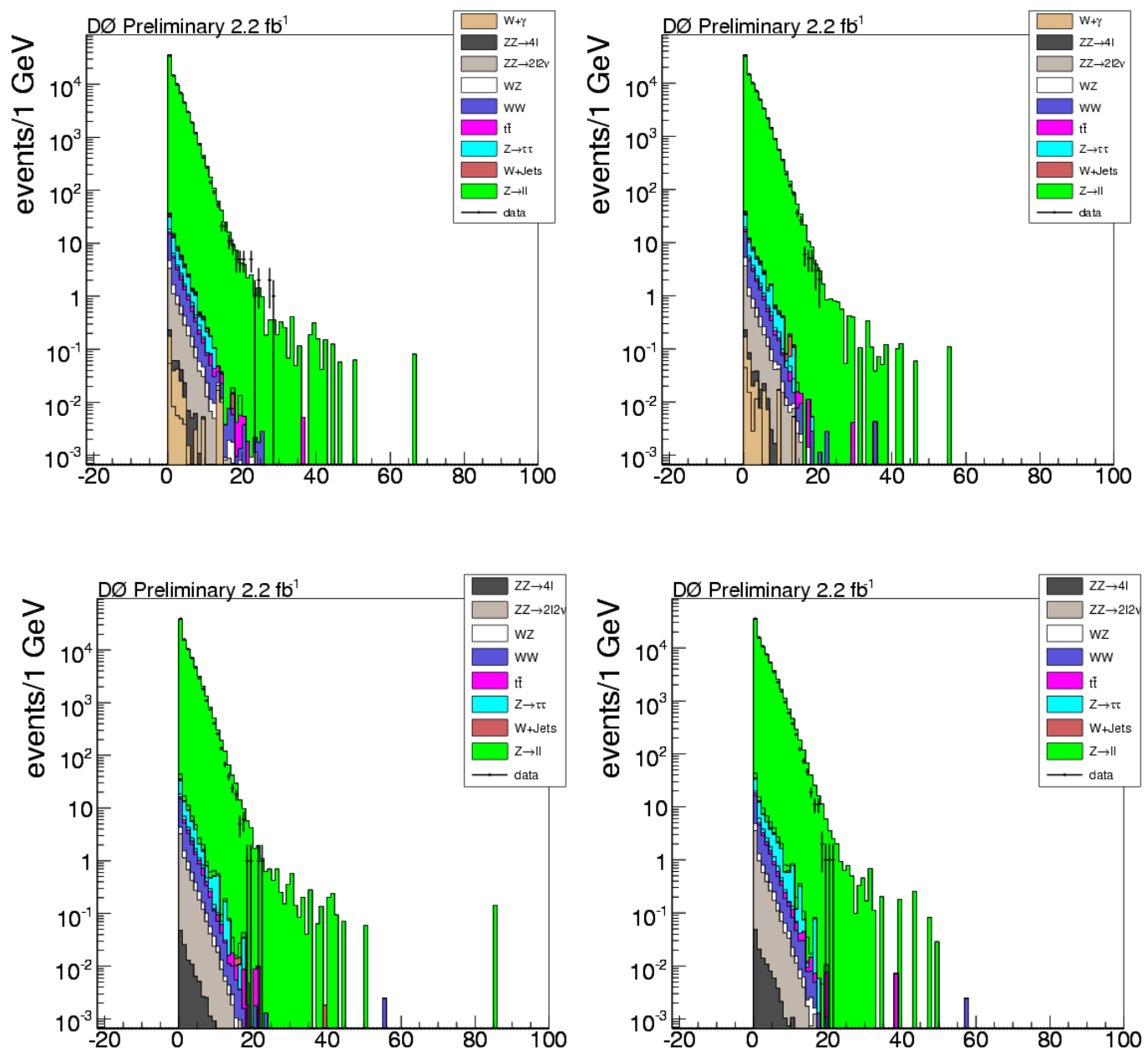

Figure 9.7: Tracking activity activity in the longitudinal (left plots) and transverse (right plots) direction with respect to the thrust axis for the full $2.2 \mathrm{fb}^{-1}$ integrated luminosity. The top shows the di-electron channel while the bottom shows the di-muon channel.

In Equation 5.11, the parameter $k$ and $k^{\prime}$ can be altered to optimize the selection cut. Maximization of the $S / \sqrt{B}$ at $25 \mathrm{GeV}$ is achieved with the values listed in Table 9.3. 


\begin{tabular}{|c|c|c|c|c|}
\hline & \multicolumn{2}{|c|}{ Run IIa } & \multicolumn{2}{c|}{ Run IIb } \\
\hline & di-electron & di-muon & di-electron & di-muon \\
\hline$k$ & 2.5 & 1.5 & 2.5 & 1.5 \\
$k^{\prime}$ & 2.2 & 1.5 & 2.2 & 1.5 \\
\hline
\end{tabular}

Table 9.3: The choice of $k$-factors for $\not_{T}{ }^{\prime}$ maximizes $S / \sqrt{B}$ at $25 \mathrm{GeV}$.

In order to reject the background from inclusive $Z$ production, the cuts listed in Table 9.4 are applied to the $\mathbb{E}_{T}{ }^{\prime}$ variable shown in Figure 9.8. These cuts were determined by examining the falling edge of the $\mathbb{F}_{T}{ }^{\prime}$ distributions in Monte Carlo and selecting a point which removed the bulk of the $Z$ production is rejected while allowing enough statistics to keep the associated systematics under control.

\begin{tabular}{|l|c|c|}
\hline & RunIIa & RunIIb \\
\hline di-electron & $27 \mathrm{GeV}$ & $27 \mathrm{GeV}$ \\
di-muon & $30 \mathrm{GeV}$ & $35 \mathrm{GeV}$ \\
\hline
\end{tabular}

Table 9.4: $\not_{T}{ }^{\prime}$ cut values for the di-electron and di-muon channels in RunIIa and RunIIb 

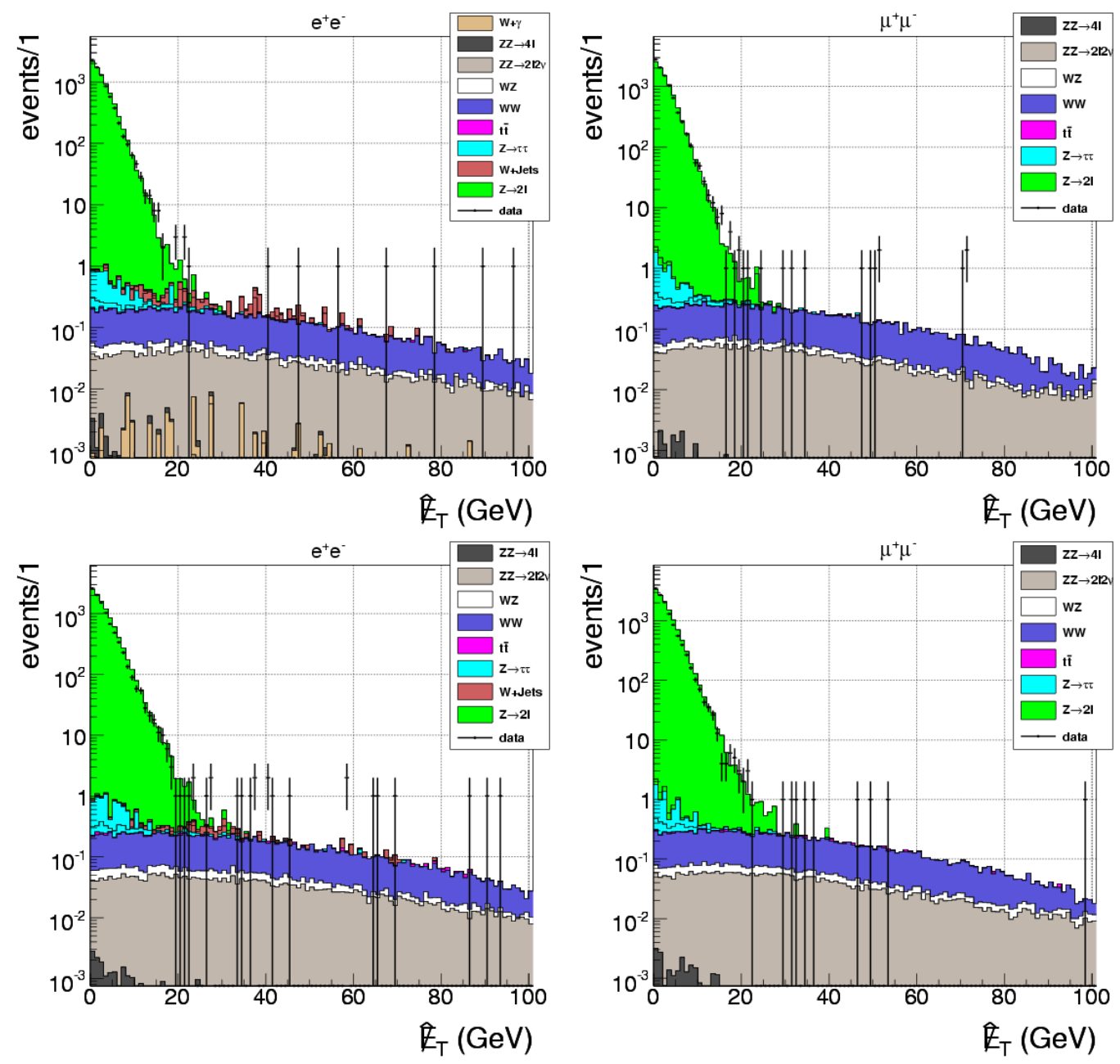

Figure 9.8: $\not_{T}{ }^{\prime}$ for the di-electron (left) and di-muon (right) final states in Run IIa (top) and Run IIb (bottom). 


\subsection{Yields}

\begin{tabular}{|lcccccc|}
\hline Samples & Pre-cuts & di-lepton sel & Jet Veto & Extra Activity & Charge & $\not_{T}{ }^{\prime}$ \\
\hline$Z \rightarrow \ell \ell$ & $58702.45 \pm 76.4$ & $53774.14 \pm 71.5$ & $53111.18 \pm 70.9$ & $43459.87 \pm 63.2$ & $42940.04 \pm 62.8$ & $0.04 \pm 0.0$ \\
$Z \rightarrow \tau \tau$ & $233.80 \pm 3.4$ & $21.21 \pm 1.0$ & $21.06 \pm 1.0$ & $17.52 \pm 0.9$ & $17.24 \pm 0.9$ & $0.13 \pm 0.0$ \\
$W+$ Jets & $33.97 \pm 1.4$ & $11.39 \pm 0.8$ & $11.18 \pm 0.8$ & $8.50 \pm 0.7$ & $5.23 \pm 0.6$ & $1.64 \pm 0.3$ \\
$W+\gamma$ & $5.94 \pm 0.1$ & $2.00 \pm 0.1$ & $1.98 \pm 0.1$ & $1.66 \pm 0.1$ & $0.05 \pm 0.0$ & $0.01 \pm 0.0$ \\
$t \bar{t}$ & $17.04 \pm 0.2$ & $6.83 \pm 0.1$ & $4.98 \pm 0.1$ & $0.96 \pm 0.0$ & $0.95 \pm 0.0$ & $0.15 \pm 0.0$ \\
WW & $32.82 \pm 0.2$ & $12.57 \pm 0.1$ & $12.40 \pm 0.1$ & $10.42 \pm 0.1$ & $10.35 \pm 0.1$ & $4.76 \pm 0.1$ \\
WZ & $10.13 \pm 0.1$ & $8.24 \pm 0.1$ & $7.97 \pm 0.1$ & $1.30 \pm 0.0$ & $1.19 \pm 0.0$ & $0.45 \pm 0.0$ \\
$Z Z \rightarrow \ell \ell \ell \ell$ & $1.44 \pm 0.0$ & $1.22 \pm 0.0$ & $1.13 \pm 0.0$ & $0.07 \pm 0.0$ & $0.06 \pm 0.0$ & $0.01 \pm 0.0$ \\
$Z Z \rightarrow \ell \ell \nu \nu$ & $4.07 \pm 0.0$ & $3.85 \pm 0.0$ & $3.80 \pm 0.0$ & $3.20 \pm 0.0$ & $3.17 \pm 0.0$ & $1.75 \pm 0.0$ \\
Tot bckg & $59031.65 \pm 76.5$ & $53835.59 \pm 71.5$ & $53169.90 \pm 70.9$ & $43498.64 \pm 63.2$ & $42975.07 \pm 62.9$ & $7.17 \pm 0.3$ \\
Tot MC & $59035.72 \pm 76.5$ & $53839.43 \pm 71.5$ & $53173.70 \pm 70.9$ & $43501.84 \pm 63.2$ & $42978.24 \pm 62.9$ & $8.92 \pm 0.3$ \\
\hline Data & $59099.00 \pm 243.1$ & $53255.00 \pm 230.8$ & $52562.00 \pm 229.3$ & $44163.00 \pm 210.1$ & $42973.00 \pm 207.3$ & $8.00 \pm 2.8$ \\
\hline
\end{tabular}

Table 9.5: Number of MC and data events after the major steps of the selection chain for the di-electron final state (Run IIa). The uncertainties are statistical only.

\begin{tabular}{|lcccccc|}
\hline Samples & Pre-cuts & di-lepton sel & Jet Veto & Extra Activity & Charge & $\mathscr{H}_{T}{ }^{\prime}$ \\
\hline$Z \rightarrow \ell \ell$ & $64562.41 \pm 84.8$ & $56675.57 \pm 76.6$ & $55762.64 \pm 75.7$ & $45368.61 \pm 67.2$ & $45368.61 \pm 67.2$ & $0.00 \pm 0.0$ \\
$Z \rightarrow \tau \tau$ & $271.66 \pm 3.9$ & $25.59 \pm 1.0$ & $24.97 \pm 1.0$ & $20.67 \pm 0.9$ & $20.67 \pm 0.9$ & $0.07 \pm 0.0$ \\
$t \bar{t}$ & $20.62 \pm 0.2$ & $7.12 \pm 0.1$ & $4.78 \pm 0.1$ & $0.90 \pm 0.0$ & $0.90 \pm 0.0$ & $0.08 \pm 0.0$ \\
$\mathrm{WW}$ & $41.96 \pm 0.2$ & $14.60 \pm 0.1$ & $14.31 \pm 0.1$ & $12.03 \pm 0.1$ & $12.03 \pm 0.1$ & $4.82 \pm 0.1$ \\
$\mathrm{WZ}$ & $10.37 \pm 0.1$ & $8.40 \pm 0.1$ & $7.91 \pm 0.1$ & $1.13 \pm 0.0$ & $1.13 \pm 0.0$ & $0.38 \pm 0.0$ \\
$Z Z \rightarrow \ell \ell \ell \ell$ & $1.59 \pm 0.0$ & $1.31 \pm 0.0$ & $1.06 \pm 0.0$ & $0.06 \pm 0.0$ & $0.06 \pm 0.0$ & $0.00 \pm 0.0$ \\
$Z Z \rightarrow \ell \ell \nu \nu$ & $5.02 \pm 0.0$ & $4.34 \pm 0.0$ & $4.26 \pm 0.0$ & $3.60 \pm 0.0$ & $3.60 \pm 0.0$ & $1.66 \pm 0.0$ \\
Tot bckg & $64908.62 \pm 84.8$ & $56732.59 \pm 76.6$ & $55815.68 \pm 75.7$ & $45403.40 \pm 67.2$ & $45403.40 \pm 67.2$ & $5.35 \pm 0.1$ \\
Tot MC & $64913.64 \pm 84.8$ & $56736.93 \pm 76.6$ & $55819.95 \pm 75.7$ & $45407.00 \pm 67.2$ & $45407.00 \pm 67.2$ & $7.01 \pm 0.1$ \\
\hline Data & $63631.00 \pm 252.3$ & $55049.00 \pm 234.6$ & $54081.00 \pm 232.6$ & $45407.00 \pm 213.1$ & $45407.00 \pm 213.1$ & $10.00 \pm 3.2$ \\
\hline
\end{tabular}

Table 9.6: Number of MC and data events after each step of the selection chain for the di-muon final state (Run IIa). The uncertainties are statistical only. 


\begin{tabular}{|lcccccc|}
\hline Samples & Pre-cuts & di-lepton sel & Jet Veto & Extra Activity & Charge \\
\hline$Z \rightarrow \ell \ell$ & $70663.84 \pm 83.3$ & $64693.54 \pm 78.2$ & $64135.85 \pm 77.8$ & $51010.41 \pm 68.2$ & $50518.75 \pm 67.9$ & $0.43 \pm 0.2$ \\
$W+$ Jets & $20.37 \pm 0.7$ & $6.85 \pm 0.4$ & $6.75 \pm 0.4$ & $4.97 \pm 0.3$ & $2.93 \pm 0.3$ & $1.08 \pm 0.2$ \\
$Z \rightarrow \tau \tau$ & $273.98 \pm 6.6$ & $27.04 \pm 2.0$ & $26.28 \pm 1.9$ & $21.16 \pm 1.7$ & $20.99 \pm 1.7$ & $0.22 \pm 0.0$ \\
$t \bar{t}$ & $25.90 \pm 0.4$ & $9.53 \pm 0.2$ & $7.36 \pm 0.2$ & $1.12 \pm 0.1$ & $1.11 \pm 0.1$ & $0.19 \pm 0.0$ \\
WW & $43.76 \pm 0.2$ & $15.47 \pm 0.1$ & $15.31 \pm 0.1$ & $12.58 \pm 0.1$ & $12.52 \pm 0.1$ & $5.84 \pm 0.1$ \\
WZ & $13.85 \pm 0.1$ & $11.00 \pm 0.1$ & $10.72 \pm 0.1$ & $1.64 \pm 0.0$ & $1.52 \pm 0.0$ & $0.63 \pm 0.0$ \\
$Z Z \rightarrow \ell \ell \ell \ell$ & $2.09 \pm 0.0$ & $1.72 \pm 0.0$ & $1.60 \pm 0.0$ & $0.10 \pm 0.0$ & $0.09 \pm 0.0$ & $0.01 \pm 0.0$ \\
$Z Z \rightarrow \ell \ell \nu \nu$ & $5.37 \pm 0.0$ & $5.00 \pm 0.0$ & $4.95 \pm 0.0$ & $4.03 \pm 0.0$ & $4.01 \pm 0.0$ & $2.28 \pm 0.0$ \\
Tot bckg & $71043.79 \pm 83.6$ & $64765.15 \pm 78.3$ & $64203.86 \pm 77.8$ & $51051.98 \pm 68.2$ & $50557.92 \pm 67.9$ & $8.40 \pm 0.3$ \\
Tot MC & $71049.16 \pm 83.6$ & $64770.14 \pm 78.3$ & $64208.80 \pm 77.8$ & $51056.02 \pm 68.2$ & $50561.93 \pm 67.9$ & $10.68 \pm 0.3$ \\
\hline Data & $72435.00 \pm 269.1$ & $65595.00 \pm 256.1$ & $64668.00 \pm 254.3$ & $51844.00 \pm 227.7$ & $50559.00 \pm 224.9$ & $20.00 \pm 4.5$ \\
\hline
\end{tabular}

Table 9.7: Number of MC and data events after the major steps of the selection chain for the di-electron final state (Run IIb). The uncertainties are statistical only.

\begin{tabular}{|lcccccc|}
\hline Samples & Pre-cuts & di-lepton sel & Jet Veto & Extra Activity & Charge & $E_{T}{ }^{\prime}$ \\
\hline$Z \rightarrow \ell \ell$ & $82138.36 \pm 127.6$ & $73615.24 \pm 120.2$ & $72855.28 \pm 119.3$ & $57669.79 \pm 104.4$ & $57669.79 \pm 104.4$ & $0.11 \pm 0.1$ \\
$Z \rightarrow \tau \tau$ & $274.56 \pm 6.3$ & $27.72 \pm 1.9$ & $27.11 \pm 1.8$ & $22.21 \pm 1.7$ & $22.21 \pm 1.7$ & $0.02 \pm 0.0$ \\
$t \bar{t}$ & $24.12 \pm 0.4$ & $8.84 \pm 0.2$ & $6.53 \pm 0.2$ & $1.14 \pm 0.1$ & $1.14 \pm 0.1$ & $0.13 \pm 0.0$ \\
$\mathrm{WW}$ & $49.19 \pm 0.2$ & $17.43 \pm 0.1$ & $17.22 \pm 0.1$ & $14.08 \pm 0.1$ & $14.08 \pm 0.1$ & $4.83 \pm 0.1$ \\
$\mathrm{WZ}$ & $11.93 \pm 0.1$ & $9.94 \pm 0.1$ & $9.52 \pm 0.1$ & $1.55 \pm 0.0$ & $1.55 \pm 0.0$ & $0.44 \pm 0.0$ \\
$Z Z \rightarrow \ell \ell \ell \ell$ & $1.87 \pm 0.0$ & $1.58 \pm 0.0$ & $1.34 \pm 0.0$ & $0.09 \pm 0.0$ & $0.09 \pm 0.0$ & $0.00 \pm 0.0$ \\
$Z Z \rightarrow \ell \ell \nu \nu$ & $5.76 \pm 0.0$ & $5.14 \pm 0.0$ & $5.08 \pm 0.0$ & $4.16 \pm 0.0$ & $4.16 \pm 0.0$ & $1.73 \pm 0.0$ \\
Tot bckg & $82500.05 \pm 127.7$ & $73680.76 \pm 120.2$ & $72917.01 \pm 119.4$ & $57708.84 \pm 104.4$ & $57708.84 \pm 104.4$ & $5.54 \pm 0.1$ \\
Tot MC & $82505.81 \pm 127.7$ & $73685.90 \pm 120.2$ & $72922.09 \pm 119.4$ & $57713.00 \pm 104.4$ & $57713.00 \pm 104.4$ & $7.27 \pm 0.1$ \\
\hline Data & $82989.00 \pm 288.1$ & $72911.00 \pm 270.0$ & $71707.00 \pm 267.8$ & $57713.00 \pm 240.2$ & $57713.00 \pm 240.2$ & $5.00 \pm 2.2$ \\
\hline
\end{tabular}

Table 9.8: Number of MC and data events after each step of the selection chain for the di-muon final state (Run IIb). The uncertainties are statistical only. 


\subsection{Likelihood Discriminant}

After removing events with artificial $\not_{T}$ events with true $\not_{T}$ such as $W+$ jets and $W W$ production are the largest remaining backgrounds. To distinguish between these process and the signal a likelihood discriminant is deployed with the following three input variables are used in both channels

- Transverse momentum of the leading lepton, $p_{T}^{\text {lead }}$, since the leading lepton in the $Z$ decay is always a charged lepton.

- Cosine of the scattering angle of the negatively charged lepton measured in the di-lepton Collins-Soper rest frame, $\cos \left(\theta^{*}\right)$, to exploit polarization differences between signal and background. The Collins-Soper frame is defined as where the z-axis bisects the angle formed by the momentum of the proton and the negative-momentum of the antiproton.

- Opening angle between the di-lepton system and the leading lepton, $\Delta \phi\left(\ell_{\text {lead }}, Z\right)$, because the angular correlations in $Z \rightarrow \ell^{-} \ell^{+}$differ from the independent leptons of the backgrounds.

Additionally in the di-electron channel the di-lepton invariant mass is used $\left(M_{\ell \ell}\right)$ to exploit the $Z$ peak, while in the di-muon channel a $\chi^{2}$ probability is used again exploiting the $Z$ peak information. This $\chi^{2}$ is obtained from minimizing

$$
\chi^{2}\left(k_{1}\right)=\left(\frac{k_{1}^{o b s}-k_{1}^{f i t}}{\sigma\left(1 / p_{T}^{1}\right)}\right)^{2}+\left(\frac{k_{2}^{o b s}-k_{2}^{f i t}}{\sigma\left(1 / p_{T}^{2}\right)}\right)^{2}
$$

where

$$
k_{i}=1 / p_{T}^{i} .
$$


The function is minimized using MINUIT imposing the mass constraint:

$$
p_{2}^{f i t}=\frac{M_{Z}^{2}}{2[1-\cos \alpha] p_{1}^{f i t}},
$$

where $\alpha$ is the opening angle between the two momenta. The uncertainties on the measured transverse momenta are obtained from the transfer functions. As expected, the signal distribution behaves like a $\chi^{2}$ for 1 dof. The corresponding probability is therefore used as input to the likelihood discriminant. Figures 9.9 through 9.12 show the input variable distributions for each of the four sub-channels. 

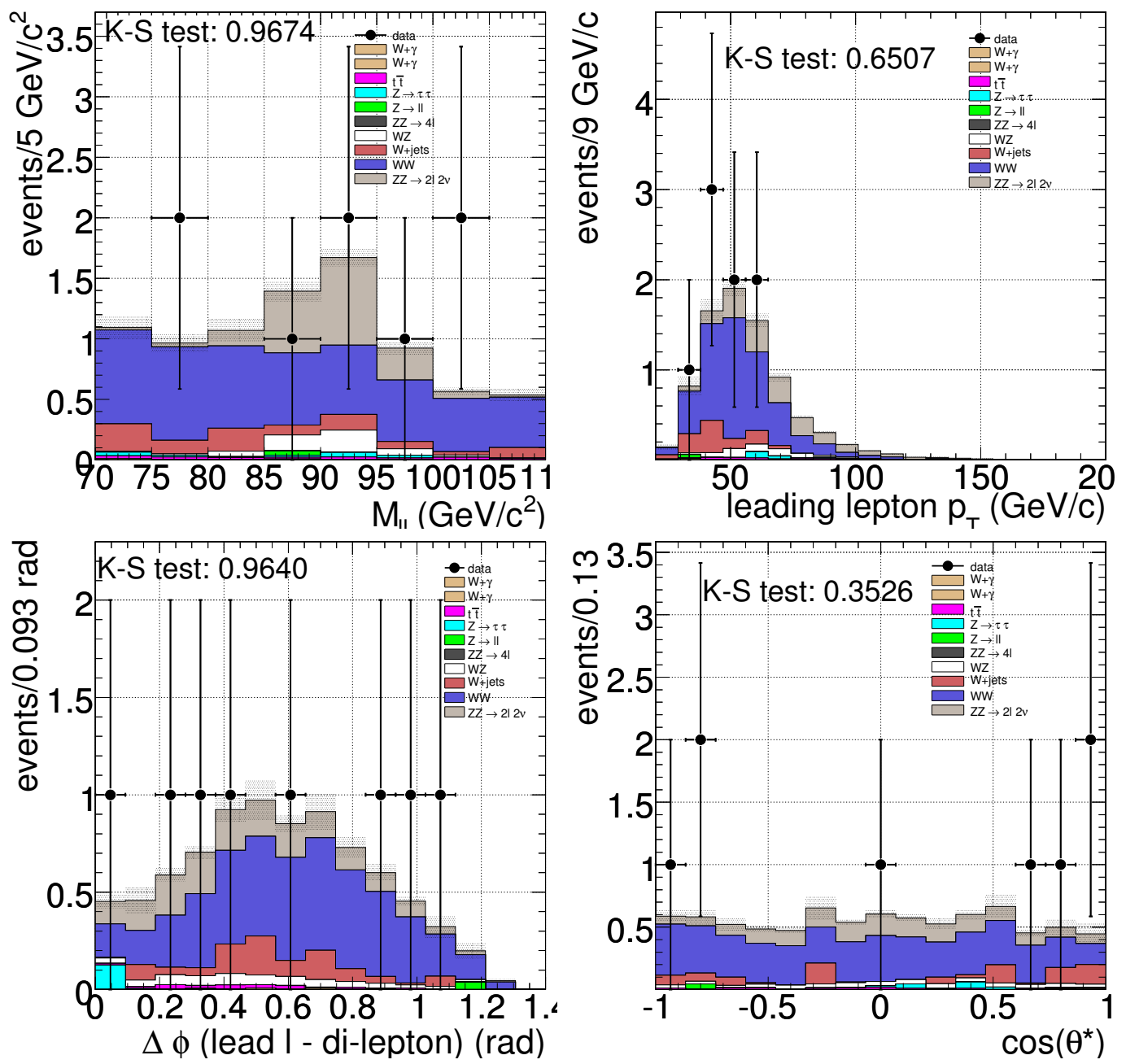

Figure 9.9: Distribution of the input variables of the likelihood discriminant for data and MC in the di-electron channel (Run IIa dataset). 

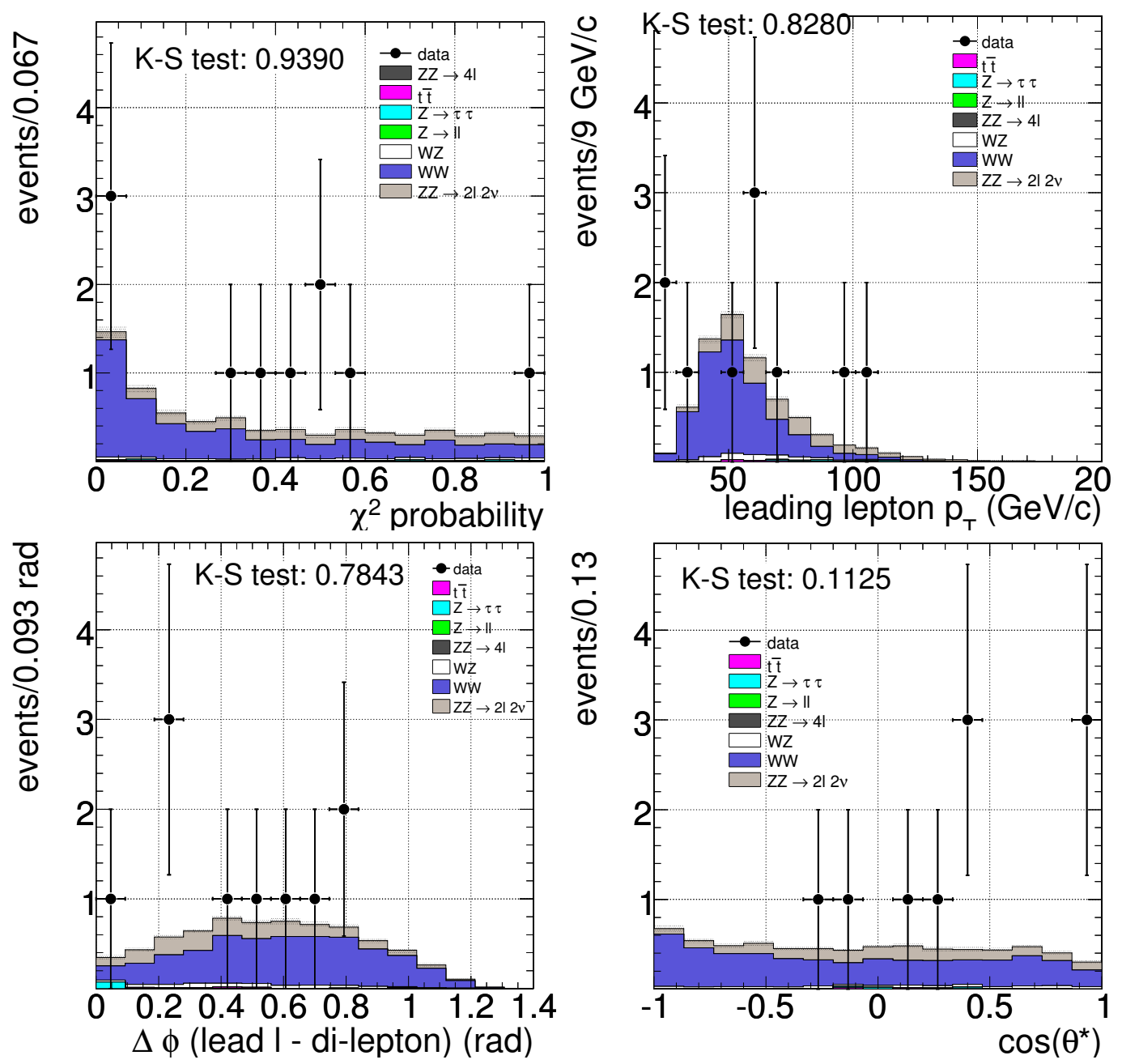

Figure 9.10: Distribution of the input variables of the likelihood discriminant for data and $\mathrm{MC}$ in the di-muon channel (Run IIa dataset). 

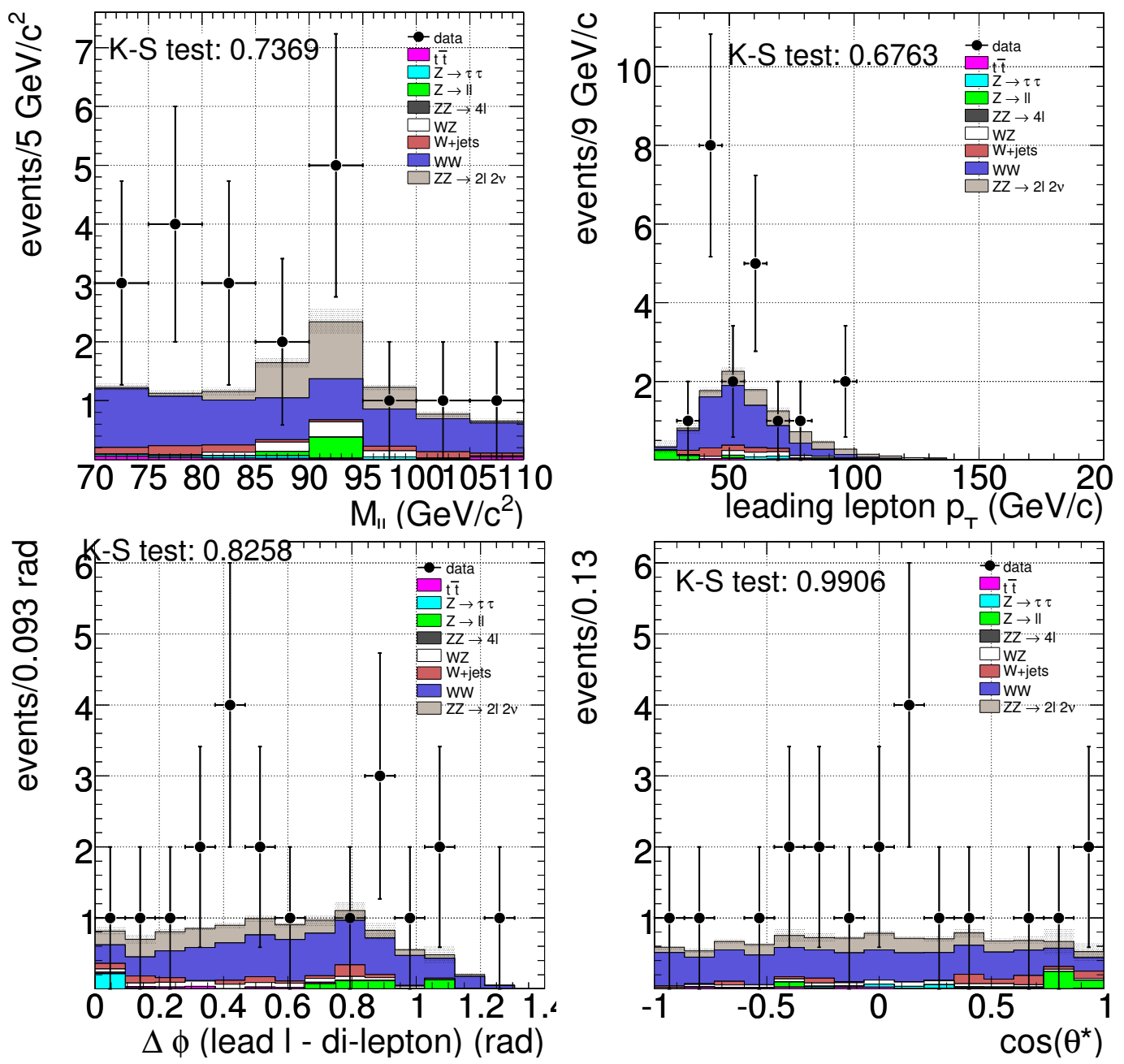

Figure 9.11: Distribution of the input variables of the likelihood discriminant for data and MC in the di-electron channel (Run IIb dataset). 

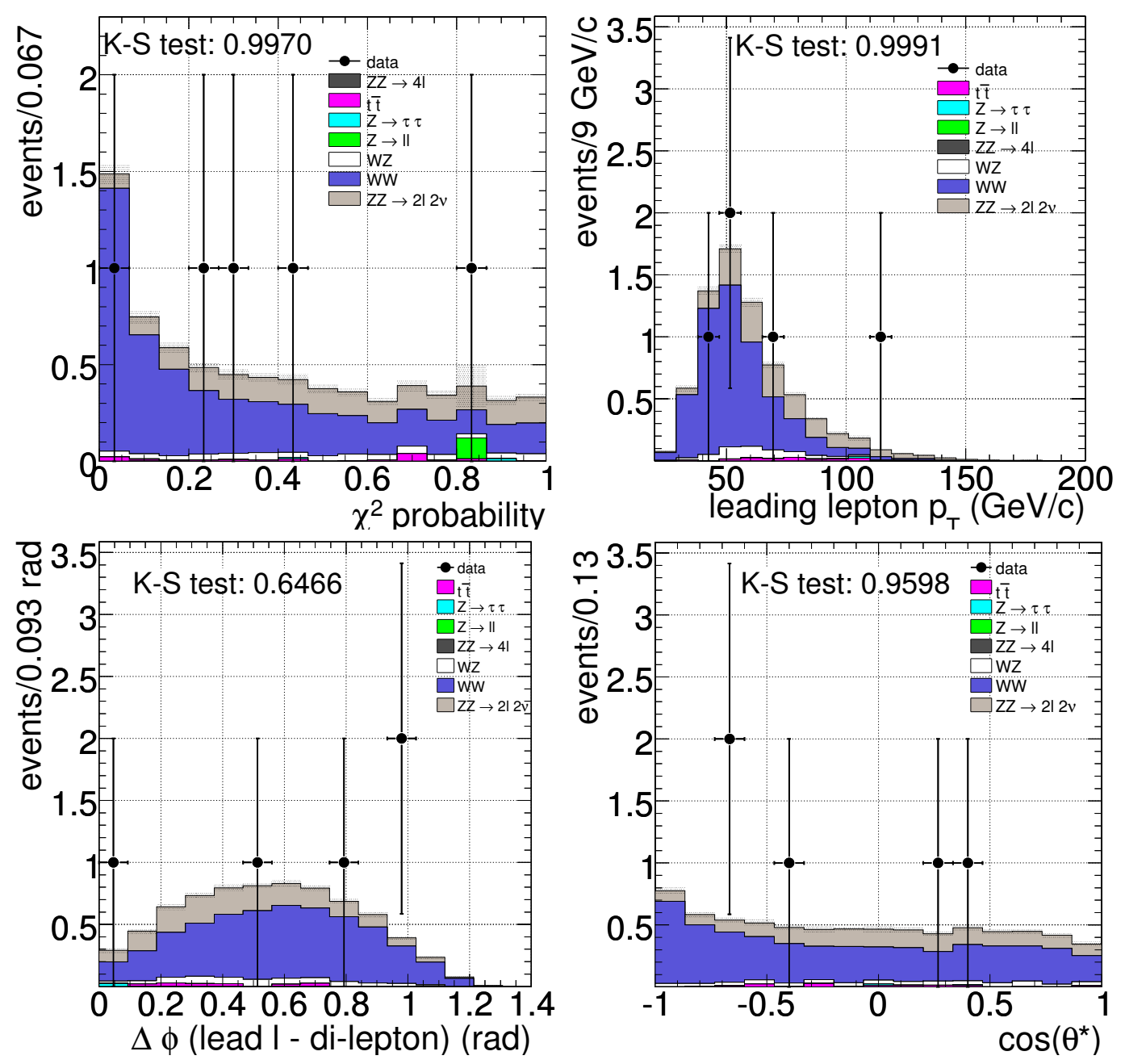

Figure 9.12: Distribution of the input variables of the likelihood discriminant for data and $\mathrm{MC}$ in the di-muon channel (Run IIb dataset).

The available MC statistics was divided into two even subsamples. In one a fit was performed of the signal over background distribution used to build the likelihood and in the other the likelihood was evaluated. Limited statistics of the $W+$ jets and low $Z-p_{T}$ samples prevented their inclusion in the training. The normalized likelihoods for signal and background are shown separately in Figure 9.13 and the evaluated likelihood output is shown in Figure 9.14. These likelihood distributions are the inputs to the significance and cross-section calculations. 

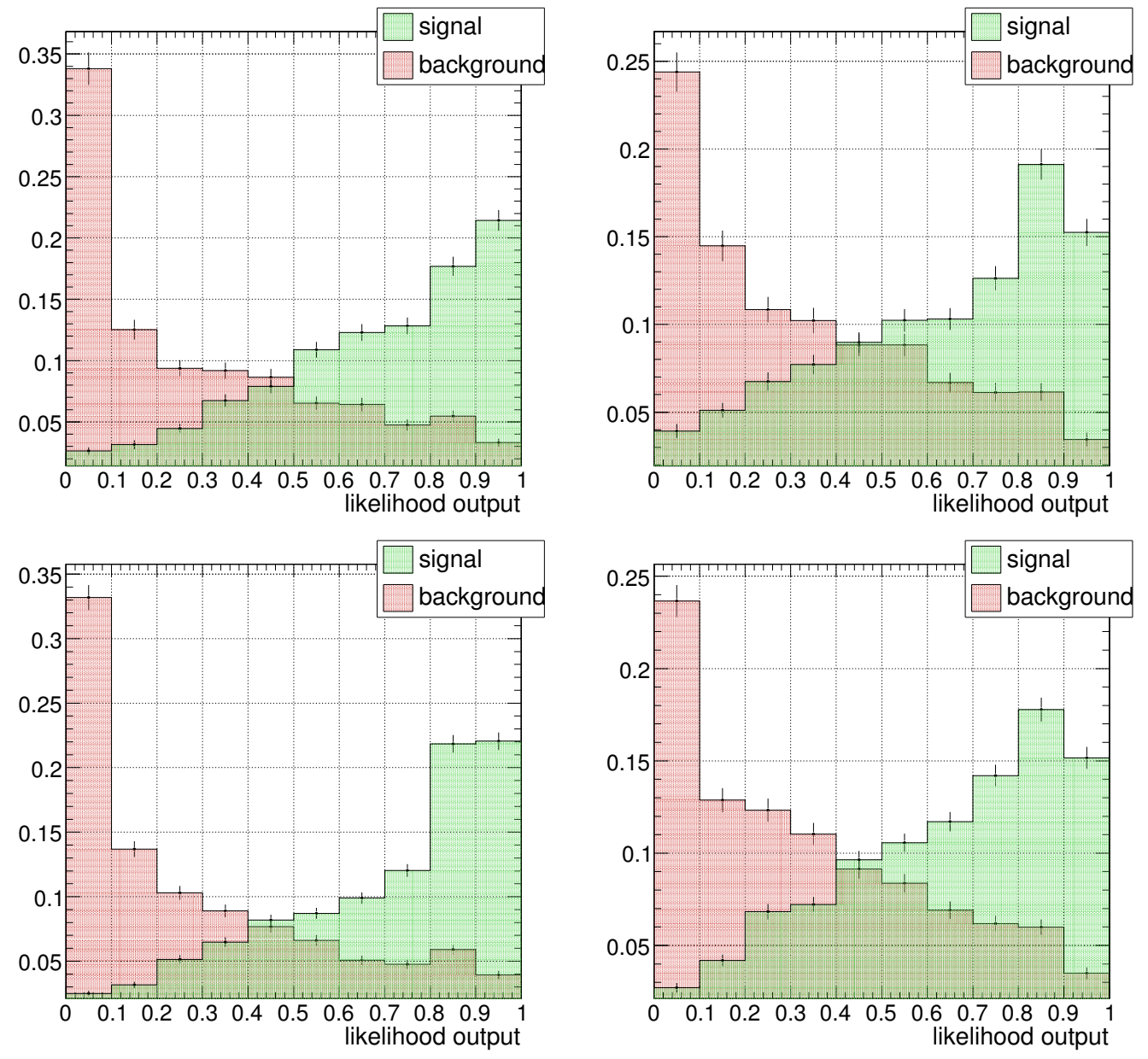

Figure 9.13: Output of the likelihood discriminant for the di-electron (left) and di-muon (right) final states in Run IIa (top) and Run IIb (bottom). The signal and background distribution are normalized to 1 . 

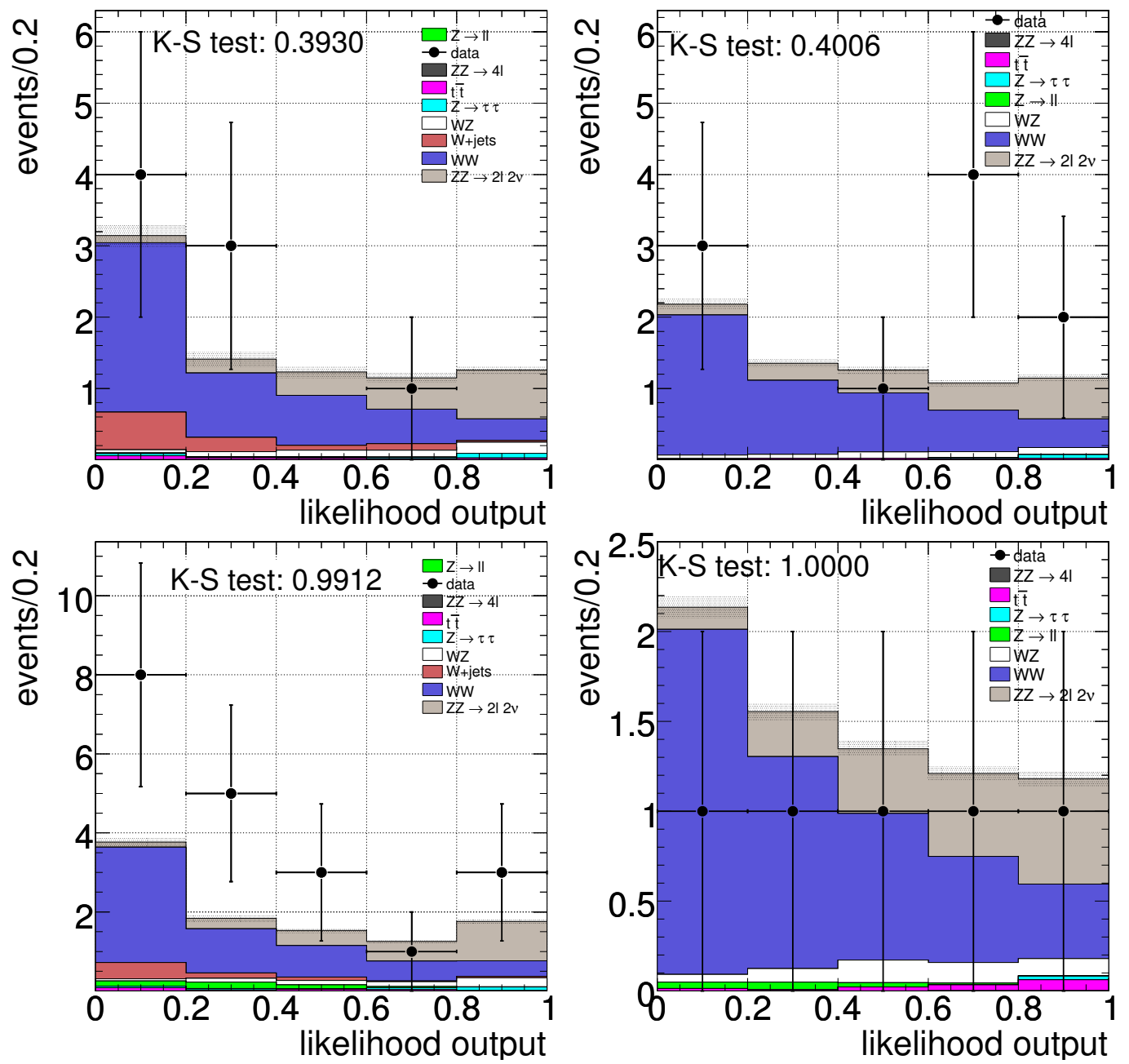

Figure 9.14: Output of the likelihood discriminant for the di-electron (left) and di-muon (right) final states in Run IIa (top) and Run IIb (bottom). 


\subsection{Systematic Uncertainties}

The following is a list of the systematics which must be considered for this analysis with respect to the likelihood discriminant output. Unless noted here, the nature and evaluation of these systematics is described in Section 8.6.

\subsubsection{Significance Systematics}

The following errors must be considered in the significance calculation.

\section{Jet Related Errors}

Since none of the input variables to the likelihood are directly related to jet, no variation in the shape is expected or observed. Jet Energy Scale (JES), Jet Energy Resolution (JER), and Jet Reconstruction Efficiency (Jet Eff) are all then taken as normalization uncertainties.

The relative uncertainties estimated with these procedures are listed in Tables 9.8.1 and 9.8.1.

As discussed in Section 8.6, statistical errors can inflate the estimation of systematic effects. To combat the low statistics problems in this analysis, a flat $3 \mathrm{GeV}$ width was given to each event. Therefore when a large weighted event shifts from

above the $\mathscr{H}_{T}{ }^{\prime}$ cut to below the cut, not all of its weight is lost from the final yield. This is a rough approximation of increasing the statistics of the sample and avoids non-physical effects such as negative events in pseudo-data events arising from over estimated systematics. All the jet uncertainties were added in quadrature and treated as one, and this yielded a similar result to treating them separately with and without the use of the profiling technique. These cross checks give some validity to the procedure. 


\begin{tabular}{|c|c|c|c|c|c|c|}
\hline \multirow[t]{2}{*}{ Sample } & \multicolumn{2}{|c|}{ Absolute JES } & \multicolumn{2}{|c|}{ Jet res. } & \multicolumn{2}{|c|}{ Jet eff. } \\
\hline & positive(\%) & negative $(\%)$ & positive(\%) & negative $(\%)$ & positive(\%) & negative $(\%)$ \\
\hline \multicolumn{7}{|c|}{ Run IIa } \\
\hline$Z \rightarrow l l$ & 50.66 & $2.00 \mathrm{e}-04$ & 0.0 & $2.00 \mathrm{e}-04$ & 45.81 & 0.0 \\
\hline$Z \rightarrow \tau \tau$ & 0.0 & $8.00 \mathrm{e}-04$ & 0.0 & $8.00 \mathrm{e}-04$ & 0.0 & 0.12 \\
\hline$t \bar{t}$ & 0.41 & 0.0 & $6.20 \mathrm{e}-03$ & 0.91 & 0.0 & 5.56 \\
\hline$W W$ & 0.17 & 0.0 & $2.50 \mathrm{e}-03$ & $3.18 \mathrm{e}-02$ & 0.0 & $5.33 \mathrm{e}-02$ \\
\hline$W Z$ & $6.22 \mathrm{e}-02$ & 0.0 & $9.00 \mathrm{e}-02$ & 0.0 & 0.0 & 0.34 \\
\hline$Z Z \rightarrow l l v v$ & 0.11 & $9.33 \mathrm{e}-02$ & $7.60 \mathrm{e}-03$ & $3.50 \mathrm{e}-03$ & $5.57 \mathrm{e}-02$ & 0.0 \\
\hline$Z Z \rightarrow l l l l$ & $2.00 \mathrm{e}-04$ & $2.00 \mathrm{e}-04$ & 0.0 & $2.00 \mathrm{e}-04$ & 1.65 & 0.0 \\
\hline$W+$ jets & $4.24 \mathrm{e}-02$ & $2.28 \mathrm{e}-02$ & 0.0 & 0.0 & 3.45 & 0.0 \\
\hline$W \gamma$ & 0.0 & 2.09 & 0.0 & 3.35 & 0.0 & 1.60 \\
\hline \multicolumn{7}{|c|}{ Run IIb } \\
\hline$Z \rightarrow l l$ & 8.91 & 95.97 & 33.54 & 7.70 & 21.09 & 0.0 \\
\hline$Z \rightarrow \tau \tau$ & 0.76 & 0.96 & 3.65 & 1.97 & 0.0 & 2.18 \\
\hline$t \bar{t}$ & 10.76 & 0.0 & 18.17 & 0.0 & 3.78 & 0.0 \\
\hline$W W$ & 0.84 & 0.41 & 0.50 & 0.51 & $9.72 \mathrm{e}-02$ & 0.0 \\
\hline$W Z$ & 2.98 & 0.82 & 3.27 & 0.0 & 0.81 & 0.0 \\
\hline$Z Z \rightarrow l l v v$ & 0.24 & 0.58 & 0.63 & 0.71 & 0.0 & 0.52 \\
\hline$Z Z \rightarrow l l l l$ & 5.97 & 0.28 & 4.44 & 2.98 & 7.15 & 0.0 \\
\hline$W+$ jets & 0.41 & 0.58 & 2.01 & 1.13 & 0.0 & $7.50 \mathrm{e}-03$ \\
\hline
\end{tabular}

Table 9.9: Jet related systematic uncertainties for the di-electron final state.

\begin{tabular}{|c|c|c|c|c|c|c|}
\hline \multirow[t]{2}{*}{ Sample } & \multicolumn{2}{|c|}{ Absolute JES } & \multicolumn{2}{|c|}{ Jet res. } & \multicolumn{2}{|c|}{ Jet eff. } \\
\hline & positive(\%) & negative(\%) & positive(\%) & negative(\%) & positive(\%) & negative $(\%)$ \\
\hline \multicolumn{7}{|c|}{ Run IIa } \\
\hline$Z \rightarrow l l$ & 67.24 & $2.00 \mathrm{e}-02$ & $2.00 \mathrm{e}-02$ & 170.41 & 0.0 & 116.46 \\
\hline$Z \rightarrow \tau \tau$ & 0.0 & 0.40 & $2.00 \mathrm{e}-02$ & 0.0 & 1.15 & 0.0 \\
\hline$t \bar{t}$ & 0.0 & 2.15 & 0.0 & 0.12 & 0.0 & 0.74 \\
\hline$W W$ & 0.21 & 0.0 & 0.0 & 0.10 & 0.0 & $6.00 \mathrm{e}-02$ \\
\hline$W Z$ & 0.29 & 0.0 & 0.0 & 0.21 & 0.0 & 0.57 \\
\hline$Z Z \rightarrow l l v v$ & 0.26 & 0.0 & 0.0 & $4.00 \mathrm{e}-02$ & 0.0 & 0.28 \\
\hline$Z Z \rightarrow l l l l$ & 2.51 & 0.26 & 1.42 & 0.0 & 0.0 & 3.71 \\
\hline \multicolumn{7}{|c|}{ Run IIb } \\
\hline$Z \rightarrow l l$ & 5.92 & 0.0 & 6.48 & 0.85 & $2.00 \mathrm{e}-02$ & 0.0 \\
\hline$Z \rightarrow \tau \tau$ & 29.64 & 0.0 & 32.82 & 0.0 & 9.83 & 0.0 \\
\hline$t \bar{t}$ & 0.62 & 4.19 & 2.29 & 2.65 & 0.0 & 0.47 \\
\hline$W W$ & 0.98 & 2.13 & 0.60 & 0.72 & 0.0 & 0.12 \\
\hline$W Z$ & 1.84 & 2.50 & 0.68 & 0.27 & 0.0 & $7.00 \mathrm{e}-02$ \\
\hline$Z Z \rightarrow l l v v$ & 0.51 & 1.80 & 0.19 & 0.56 & 0.0 & 0.14 \\
\hline$Z Z \rightarrow l l l l$ & 14.83 & 0.0 & 12.06 & 0.0 & 7.96 & 0.0 \\
\hline
\end{tabular}

Table 9.10: Jet related systematic uncertainties for the di-muon final state. 


\section{Lepton Resolution}

Electron smearing for Run IIa was not available so the fractional error from the Run

IIb sample was used for both. A conservative approach was maintained and a symmetric error set to the largest variation was assumed for each bin. The effect of these uncertainties on the output of the likelihood discriminant are shown in Figures 9.15 and 9.16 for the di-electron and di-muon final states. This error ranges from $1-6 \%$. 

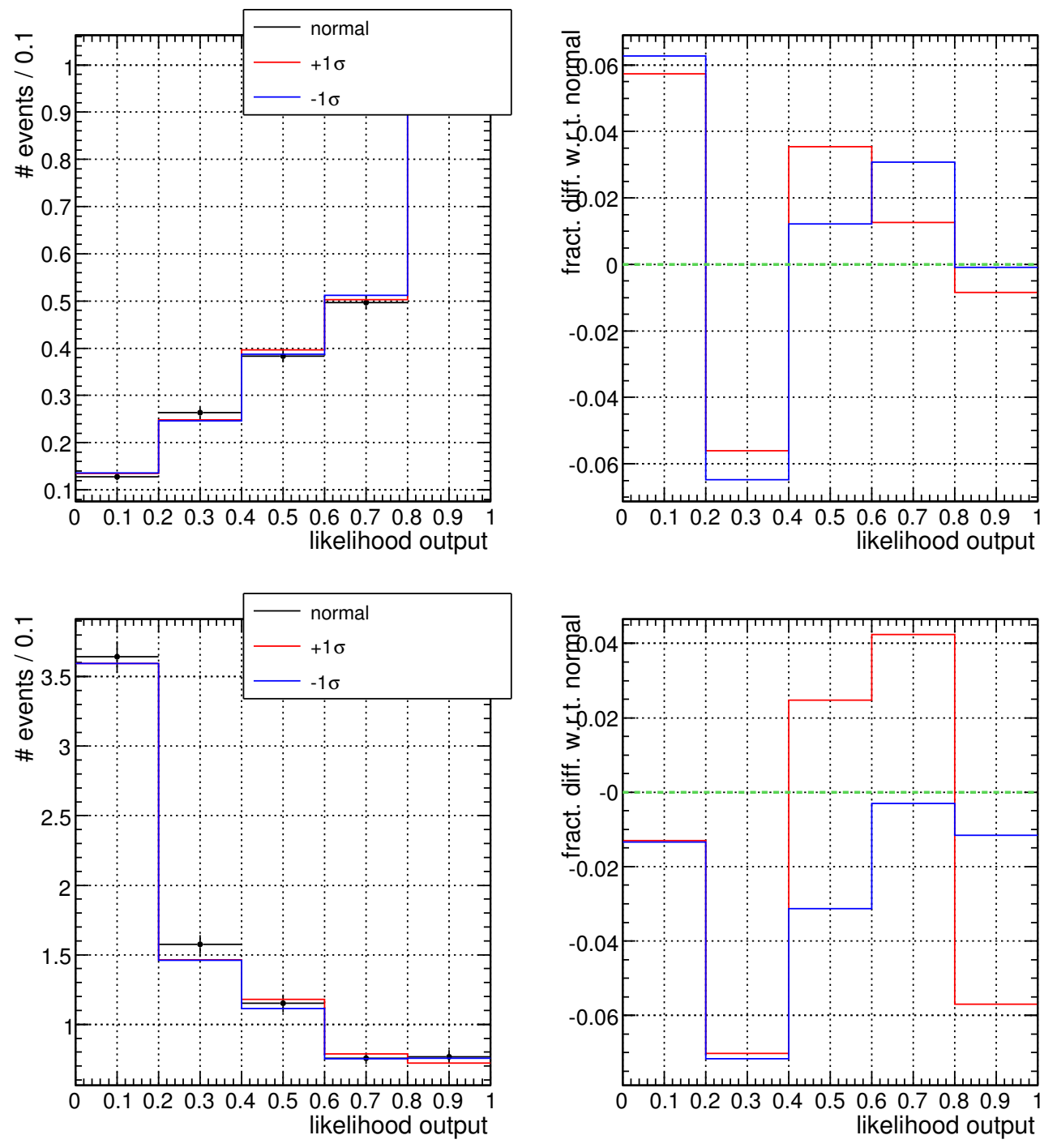

Figure 9.15: Absolute variation (left) and fractional difference (right) of the output of the likelihood discriminant for signal (top) and background (bottom) due to a $\pm 1 \sigma$ variation in the parameters of the electron smearing in $\mathrm{MC}$. 

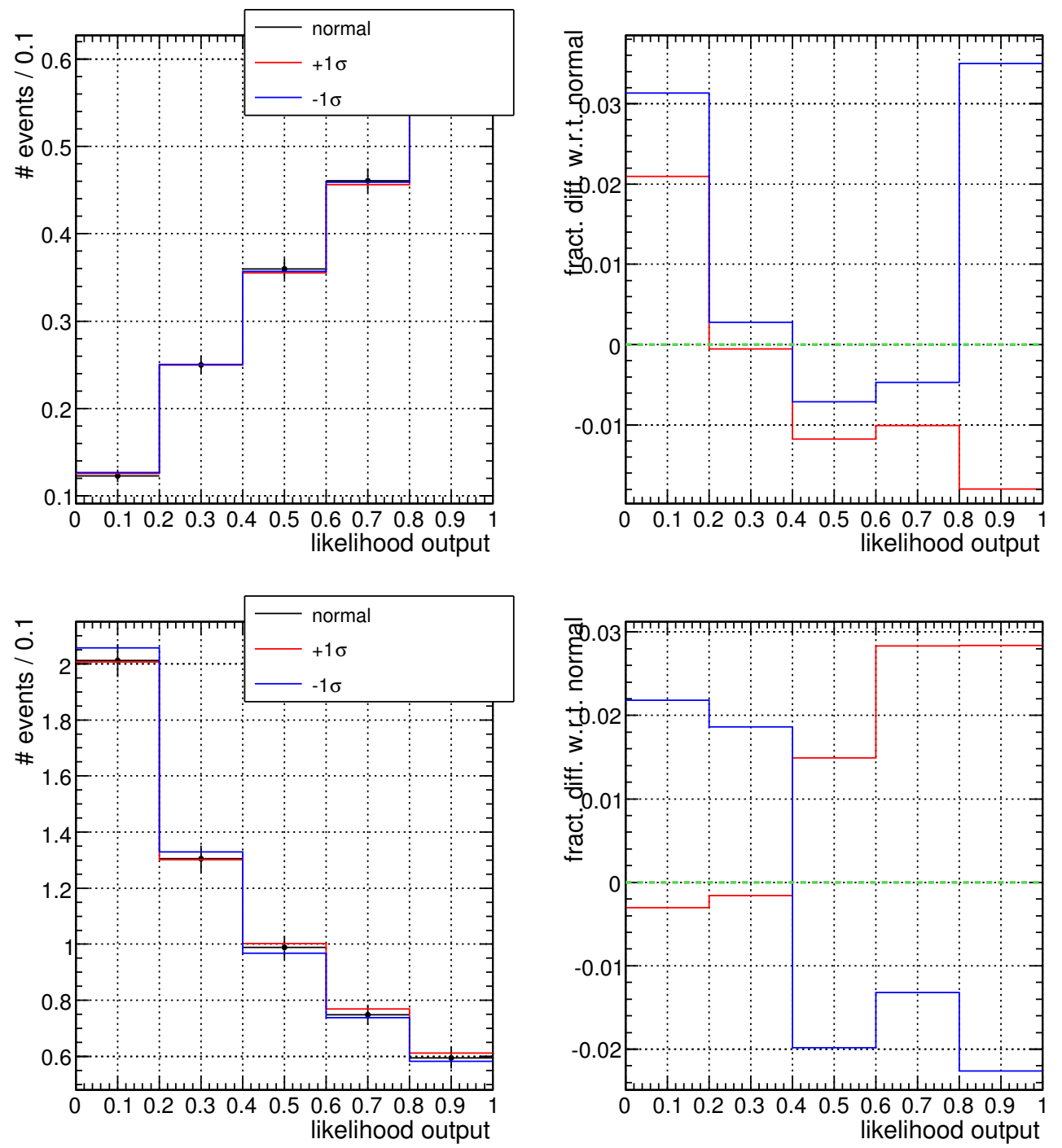

Figure 9.16: Absolute variation (left) and fractional difference (right) of the output of the likelihood discriminant for signal (top) and background (bottom) due to a $\pm 1 \sigma$ variation in the parameters of the muon smearing in MC.

\section{$W+$ jets Normalization}

The matrix-method itself provides the uncertainty on this background through simple error propagation. Limited statistics available for the derivation of the matrix-method inputs leads to a relatively large uncertainty. The values of these uncertainties for 
Run IIa and Run IIb datasets are listed in Table 9.8.1.

\begin{tabular}{|c|c|c|}
\hline Channel & Normalization & Relative uncertainty \\
\hline \multicolumn{3}{|c|}{ Run IIa } \\
\hline di-electron & $1.64 \pm 0.3$ & $30 \%$ \\
\hline \multicolumn{3}{|c|}{ Run IIb } \\
\hline di-electron & $1.08 \pm 0.3$ & $28 \%$ \\
\hline
\end{tabular}

Table 9.11: Uncertainties on the $W+$ jets normalization.

\section{$E_{T}^{\prime}$ Description}

With so few inclusive $Z$ events passing the cut on $E_{T}{ }^{\prime}$, three estimates of the expected number of surviving events were obtained. The first two are determined by integrating an exponential function which is fit to the falling edge of the variable for the Data and the MC. The third is calculated by summing the predicted MC events above the cut. The relevant curves are shown in Figures 9.17 and 9.20. The central value of the $\mathrm{Z}$ contribution is computed from the number of surviving $\mathrm{MC}$ events while the uncertainty on this number is estimated comparing the extrapolation of the $\mathrm{MC}$ and data fits.

\begin{tabular}{|lcccc|}
\hline \multicolumn{5}{c|}{ Run IIa } \\
\hline Channel & MC & Fit to MC & Fit to Data & Uncertainty \\
\hline di-electron & 0.04 & 0.07 & 0.07 & $6.7 \%$ \\
di-muon & 0.009 & 0.009 & 0.01 & $32 \%$ \\
\hline \multicolumn{5}{c}{ Run IIb } \\
\hline di-electron & 0.43 & 0.36 & 0.27 & $20 \%$ \\
di-muon & 0.11 & 0.009 & 0.007 & $2.0 \%$ \\
\hline
\end{tabular}

Table 9.12: Number of $\mathrm{Z}$ events surviving the $\mathscr{E}_{T}{ }^{\prime}$ cut as estimated from the MC and from the exponential fits to the falling edge of the $\mathscr{H}_{T}{ }^{\prime}$ distribution in data and MC. 


\section{$W W, W Z$ Production Cross Sections Uncertainty}

A 7\% uncertainty on the theoretical cross section for the WW and WZ [97] production processes has been applied to the normalization of these backgrounds.

\subsubsection{Cross Section Systematics}

In addition to all the systematic uncertainties listed above the cross section computation is also affected by the following uncertainties:

- Uncertainties on the $Z / \gamma^{*} \rightarrow \ell^{-} \ell^{+}$theoretical cross-section

- Uncertainty on the ratio of $Z$ to $Z Z$ acceptance $\left(\frac{A_{Z}}{A_{Z Z}}\right)$ due to pdf uncertainties. As described in Section 8.620 sets of variations are obtained for the pdf error estimate. The difference of the ratio $\frac{A_{Z}}{A_{Z Z}}$ with respect to the central value are added in quadrature for each of the 20 sets. The resulting uncertainties are listed in Table 9.8.2 for the di-electron and di-muon final states. The conservative value of $1.8 \%$ is used in the estimate of the cross-section uncertainties.

\begin{tabular}{|c|c|}
\hline di-electron & di-muon \\
\hline$+1.5 \%+1.8 \%$ & $-1.8 \%+1.7 \%$ \\
\hline
\end{tabular}

Table 9.13: Pdf uncertainties on the $\frac{A_{Z}}{A_{Z Z}}$ ratio as estimated with the CTEQ prescription.

- Uncertainty on the $\frac{A_{Z}}{A_{Z Z}}$ ratio due to modeling of the veto efficiency. A systematic uncertainty is assigned to the ratio $\frac{A_{Z}}{A_{Z Z}}$ to account for the modeling of the veto efficiency in the MC. The size of the uncertainty is estimated to be $0.8 \%$ based on the measured difference of efficiency in data and MC.

- Uncertainty on the $\frac{A_{Z}}{A_{Z Z}}$ ratio due to modeling of the $Z Z p_{T}$ spectrum. We apply a re-weighting function on the $Z Z p_{T}$, derived by fitting the $p_{T}$ spectra of 
$W W$ in Sherpa and Pythia. A 3.0\% uncertainty is estimated from the effect on the $Z Z$ acceptance. 


\section{\# surviving events}

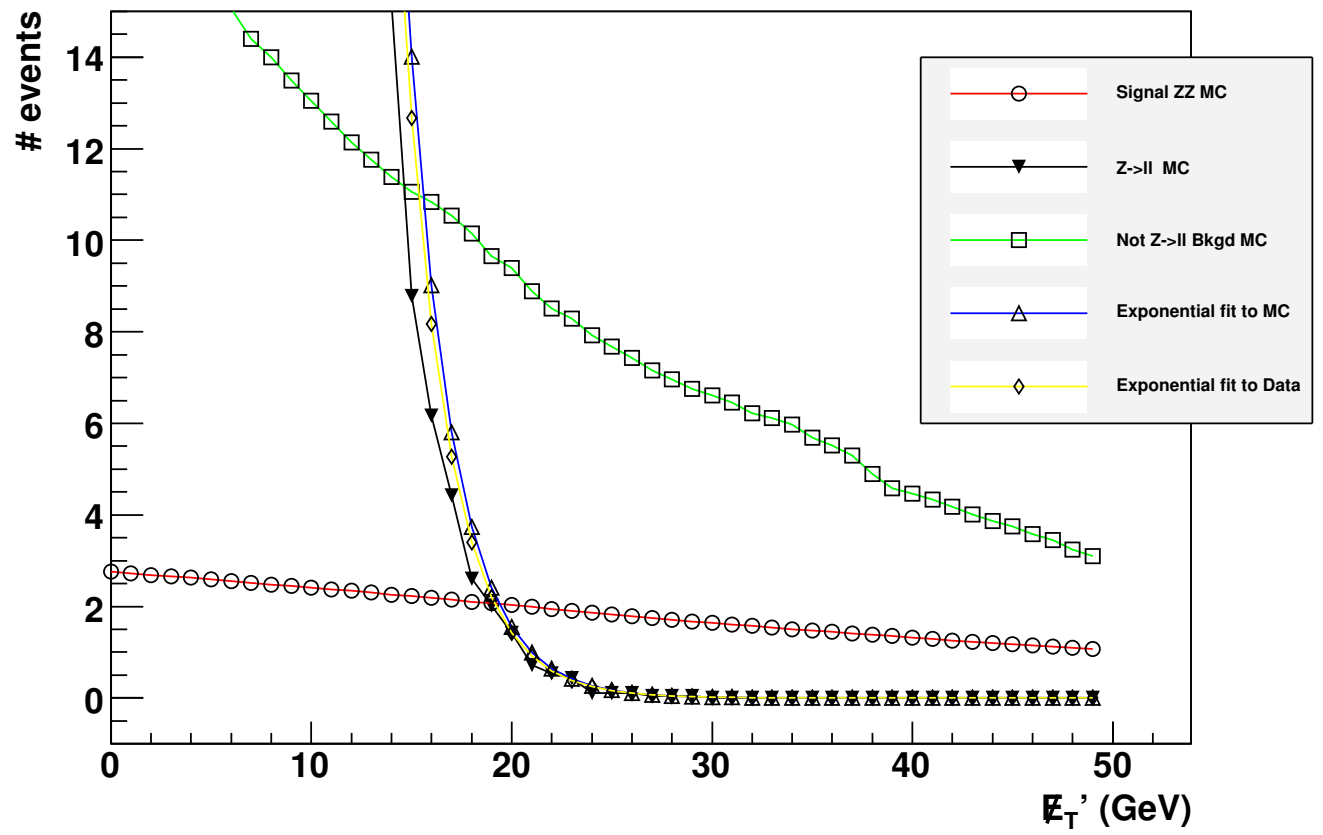

\section{cut efficiency}

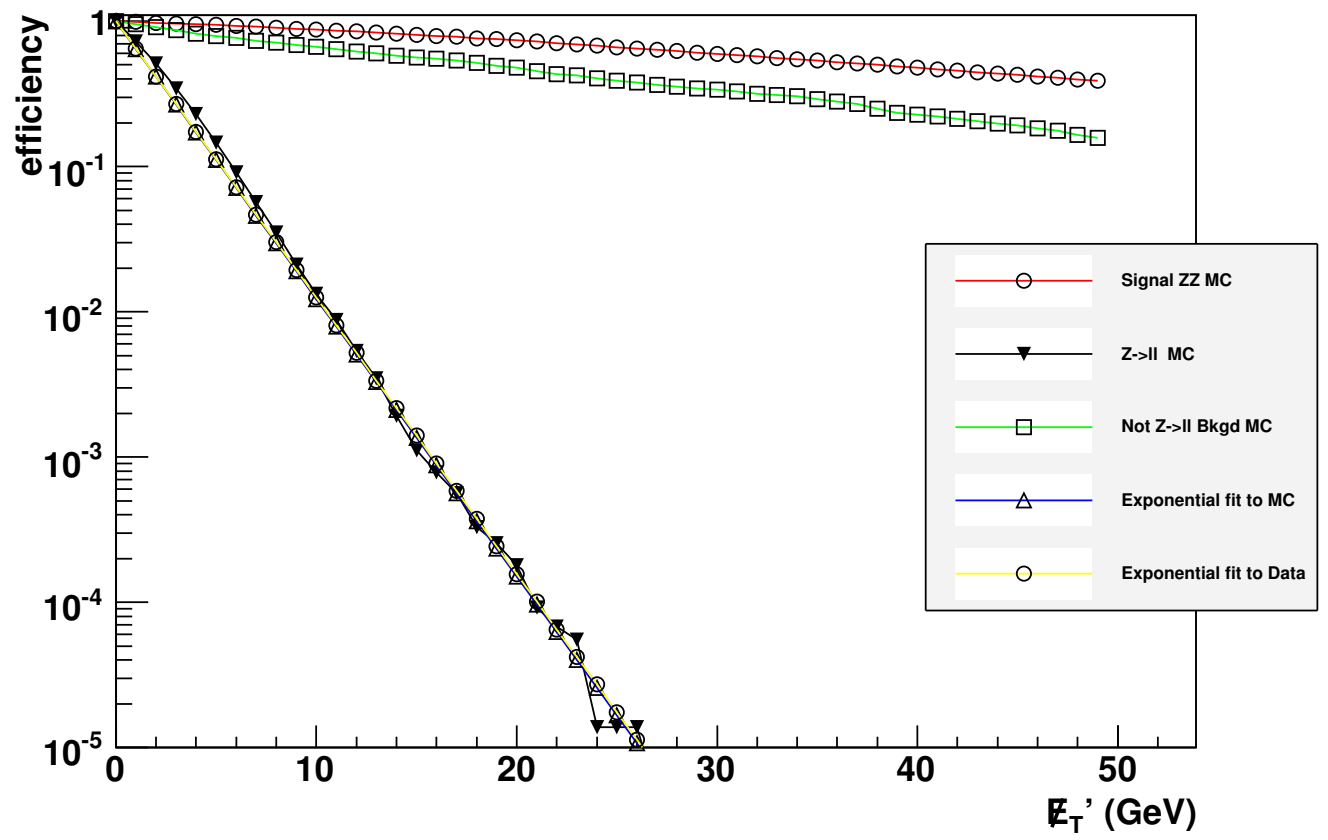

Figure 9.17: Number of events (top) and fraction remaining (bottom) varying the cut (along the $\mathrm{x}$ axis) on the corrected missing energy of the event in the Run IIa dielectron channel. The red line results in summing events in the signal MC, the black line in summing the $\mathrm{Z} \rightarrow$ ee $\mathrm{MC}$, and the green line in summing all the other background MC. The blue and yellow lines are obtained by integrating an exponential which has been fit to the falling edge in the $\mathrm{MC}$ and Data respectively. 


\section{\# surviving events}

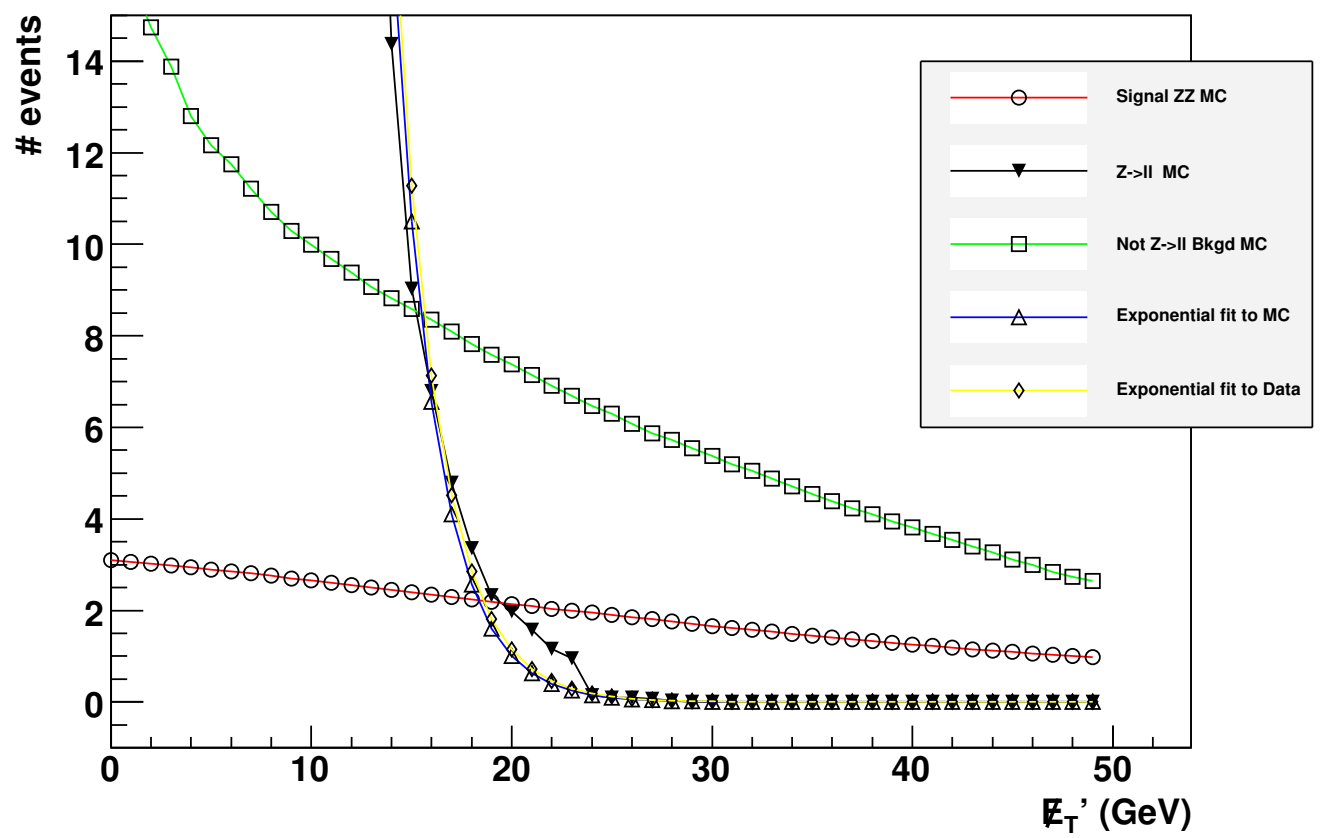

\section{cut efficiency}

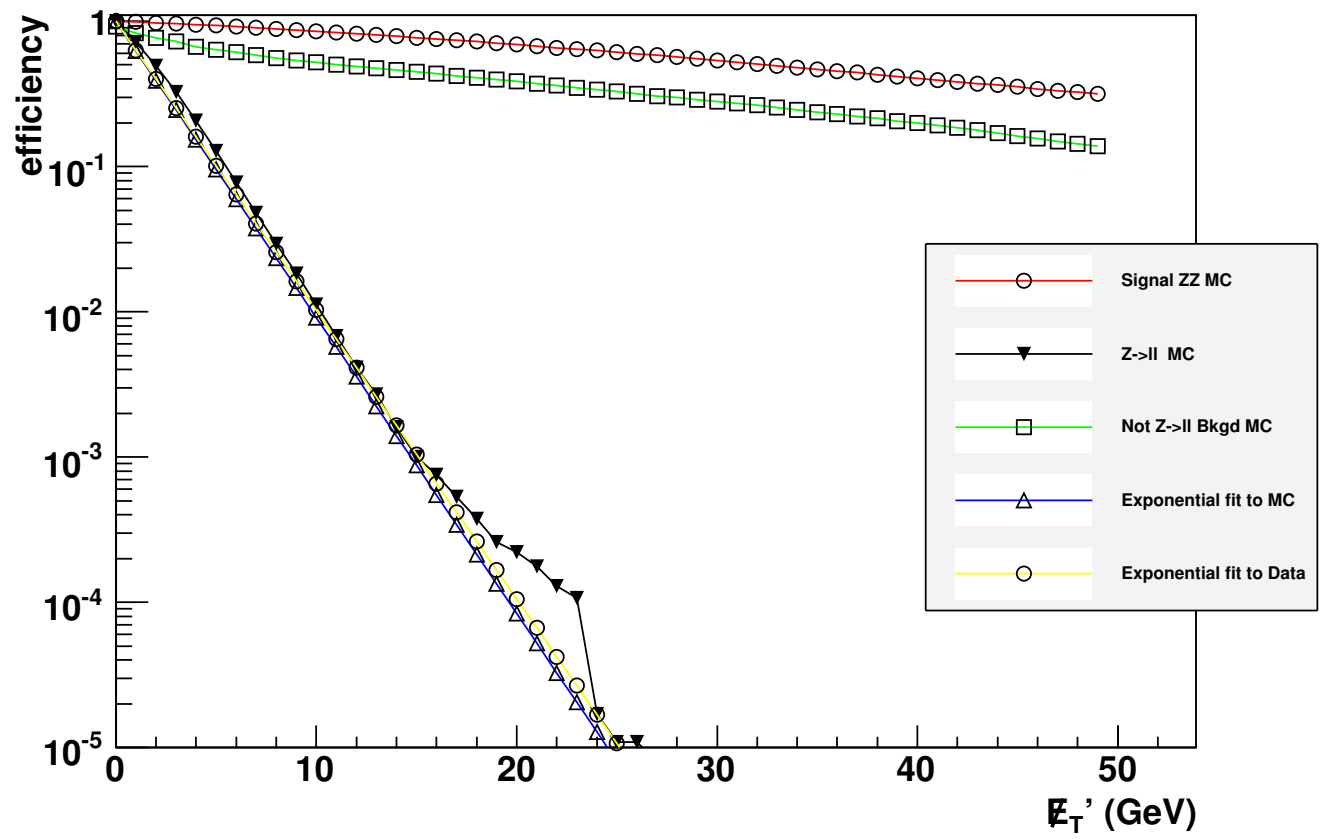

Figure 9.18: Number of events (top) and fraction remaining (bottom) varying the cut (along the $\mathrm{x}$ axis) on the corrected missing energy of the event in the Run IIa dimuon channel. The red line results in summing events in the signal MC, the black line in summing the $\mathrm{Z} \rightarrow \mu \mu \mathrm{MC}$, and the green line in summing all the other background MC. The blue and yellow lines are obtained by integrating an exponential which has been fit to the falling edge in the MC and Data respectively. 


\section{\# surviving events}

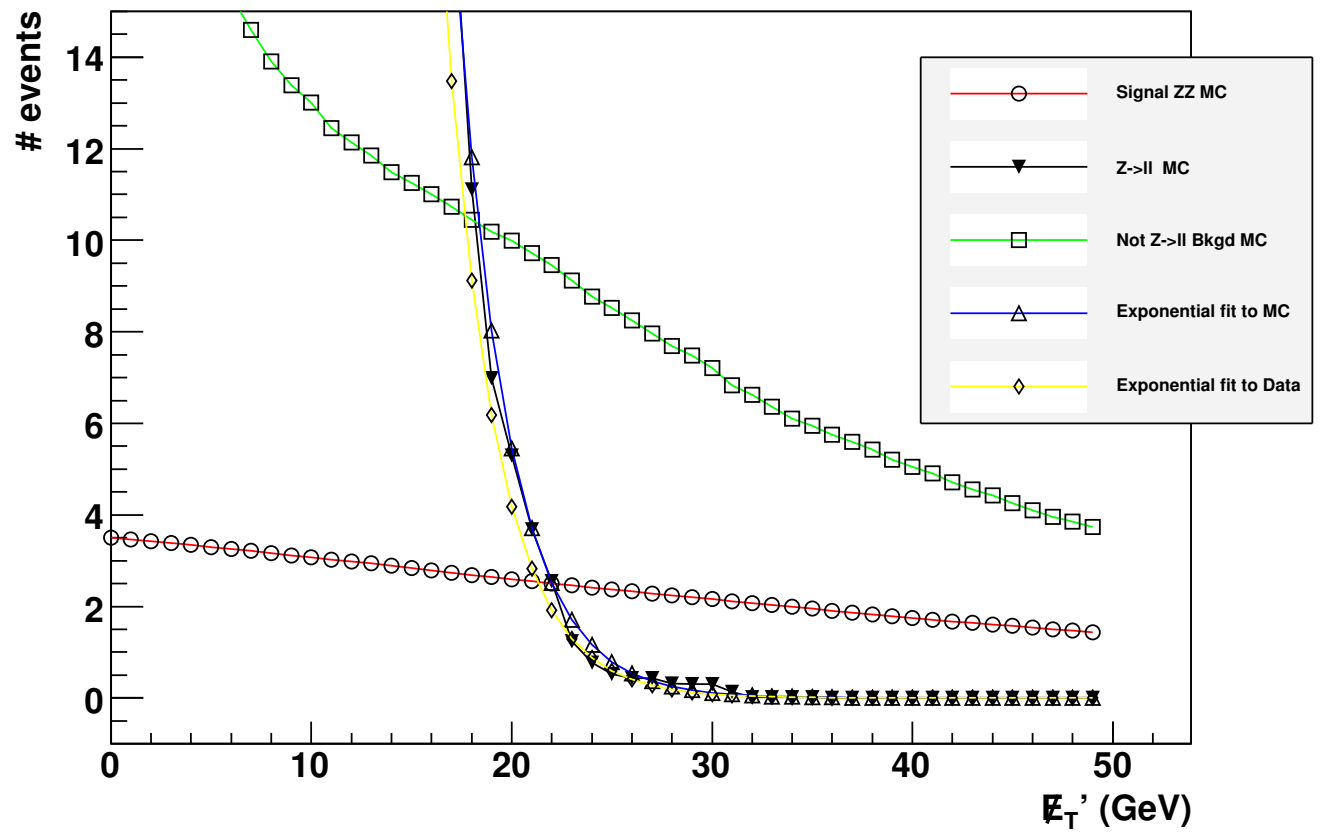

\section{cut efficiency}

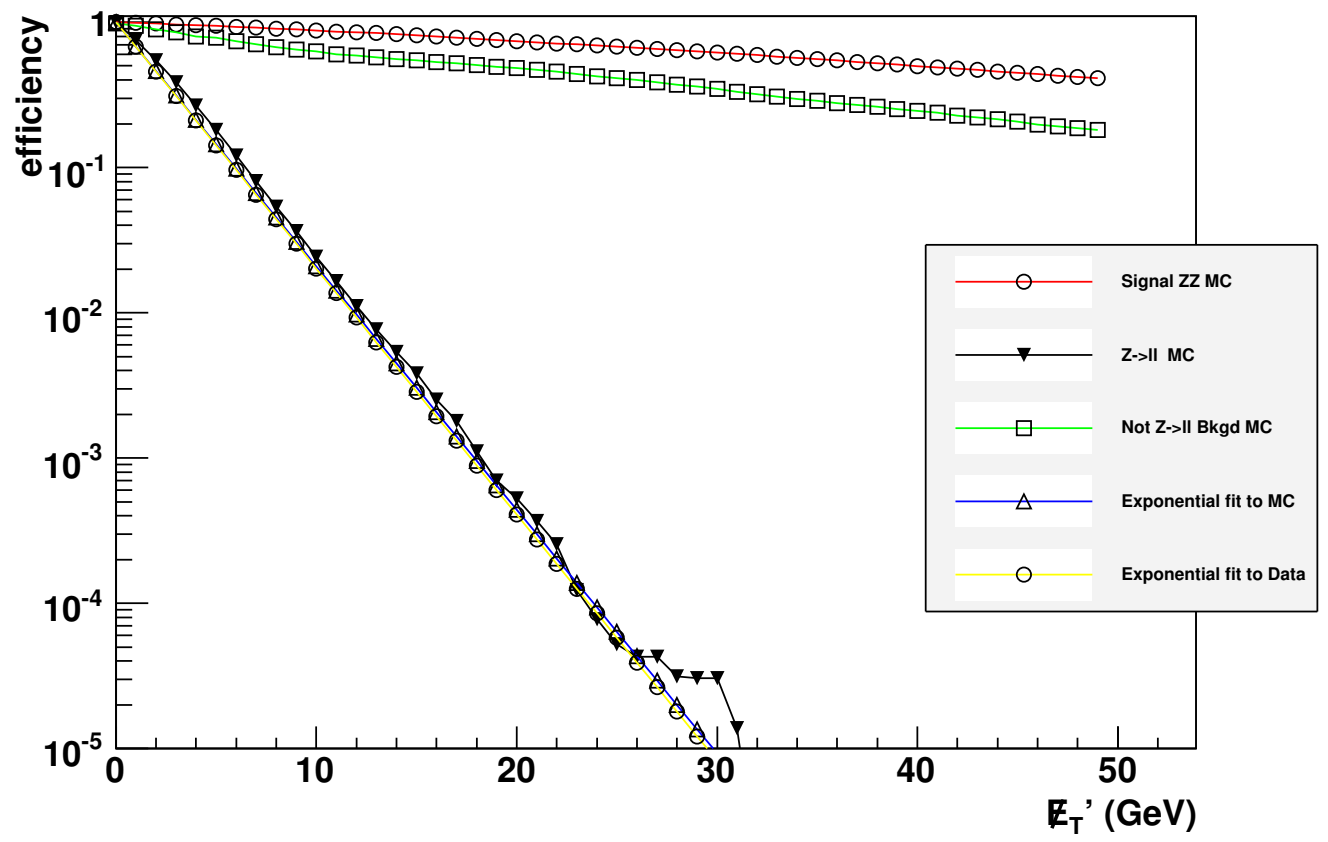

Figure 9.19: Number of events (top) and fraction remaining (bottom) varying the cut (along the $\mathrm{x}$ axis) on the corrected missing energy of the event in the Run IIb dielectron channel. The red line results in summing events in the signal MC, the black line in summing the $\mathrm{Z} \rightarrow$ ee $\mathrm{MC}$, and the green line in summing all the other background MC. The blue and yellow lines are obtained by integrating an exponential which as been fit to the falling edge in the MC and Data respectively. 


\section{\# surviving events}

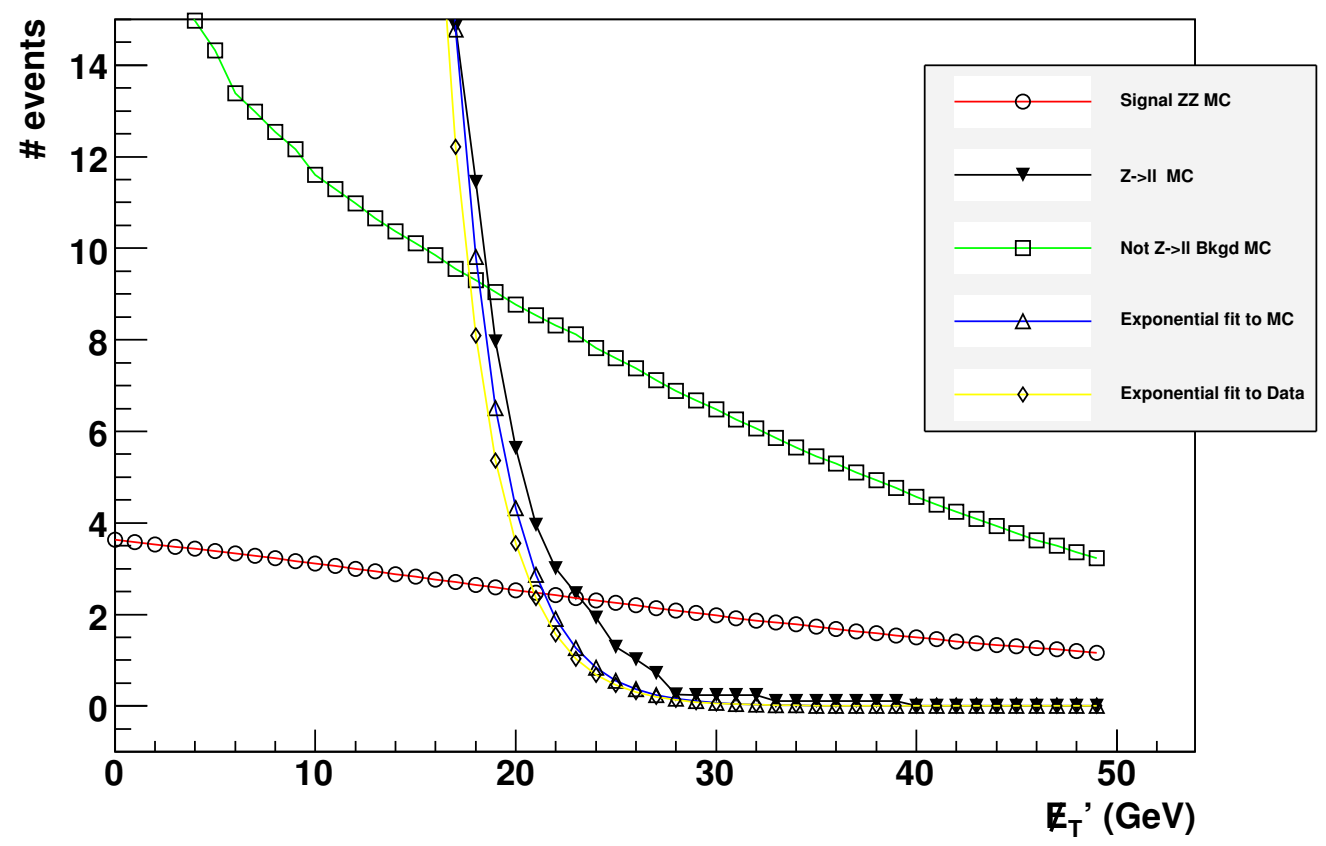

\section{cut efficiency}

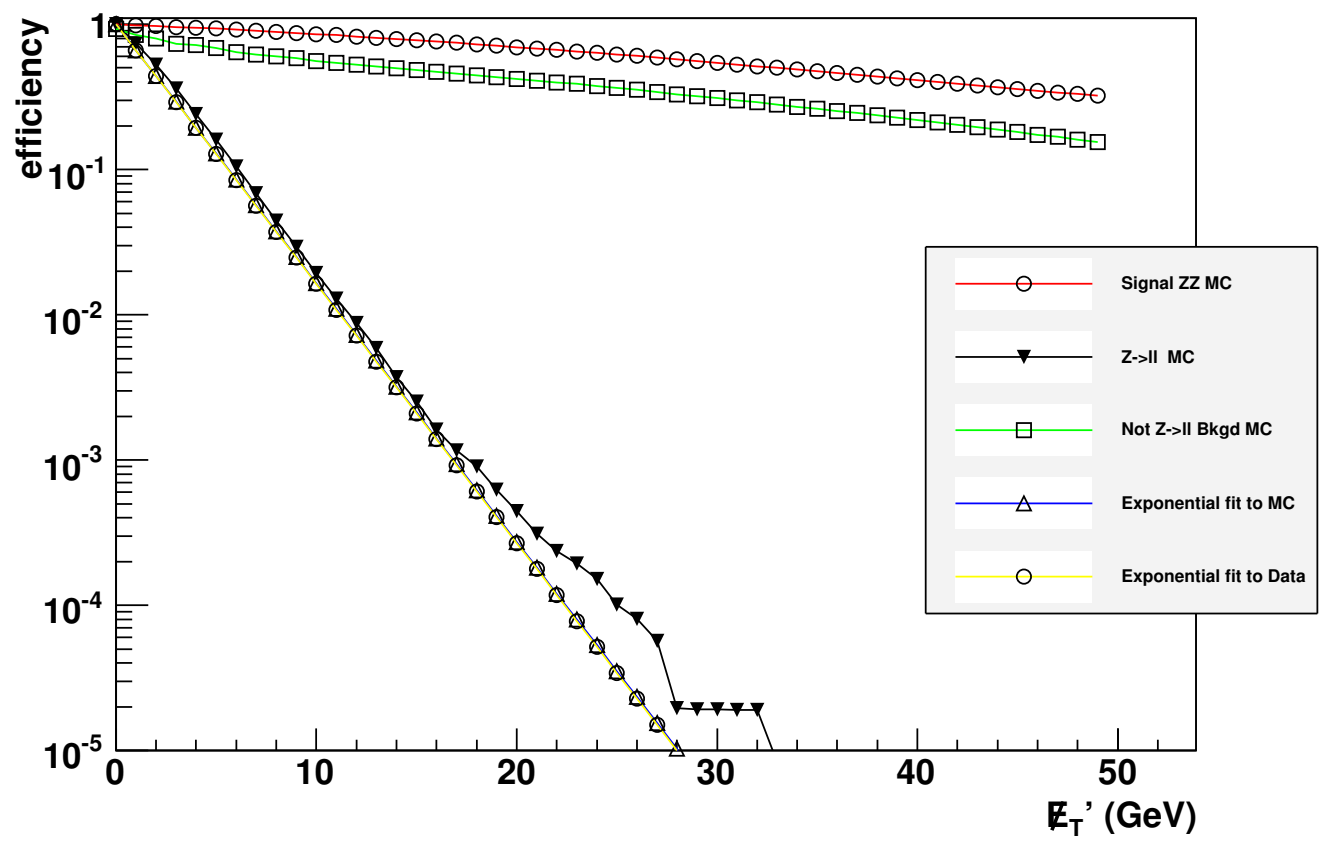

Figure 9.20: Number of events (top) and fraction remaining (bottom) varying the cut (along the $\mathrm{x}$ axis) on the corrected missing energy of the event in the Run IIb dimuon channel. The red line results in summing events in the signal MC, the black line in summing the $\mathrm{Z} \rightarrow \mu \mu \mathrm{MC}$, and the green line in summing all the other background MC. The blue and yellow lines are obtained by integrating an exponential which as been fit to the falling edge in the MC and Data respectively. 


\subsection{Significance and Cross Section Measurement}

The binned output of the likelihood distribution described in Section 9.7 is give to COLLIE, described in Chapter 8. The plots in Figure 9.21 show the distribution of the LLR test statistics computed by COLLIE as a result of 200000 pseudo-experiments for the signal plus background hypothesis and for the background only hypothesis.
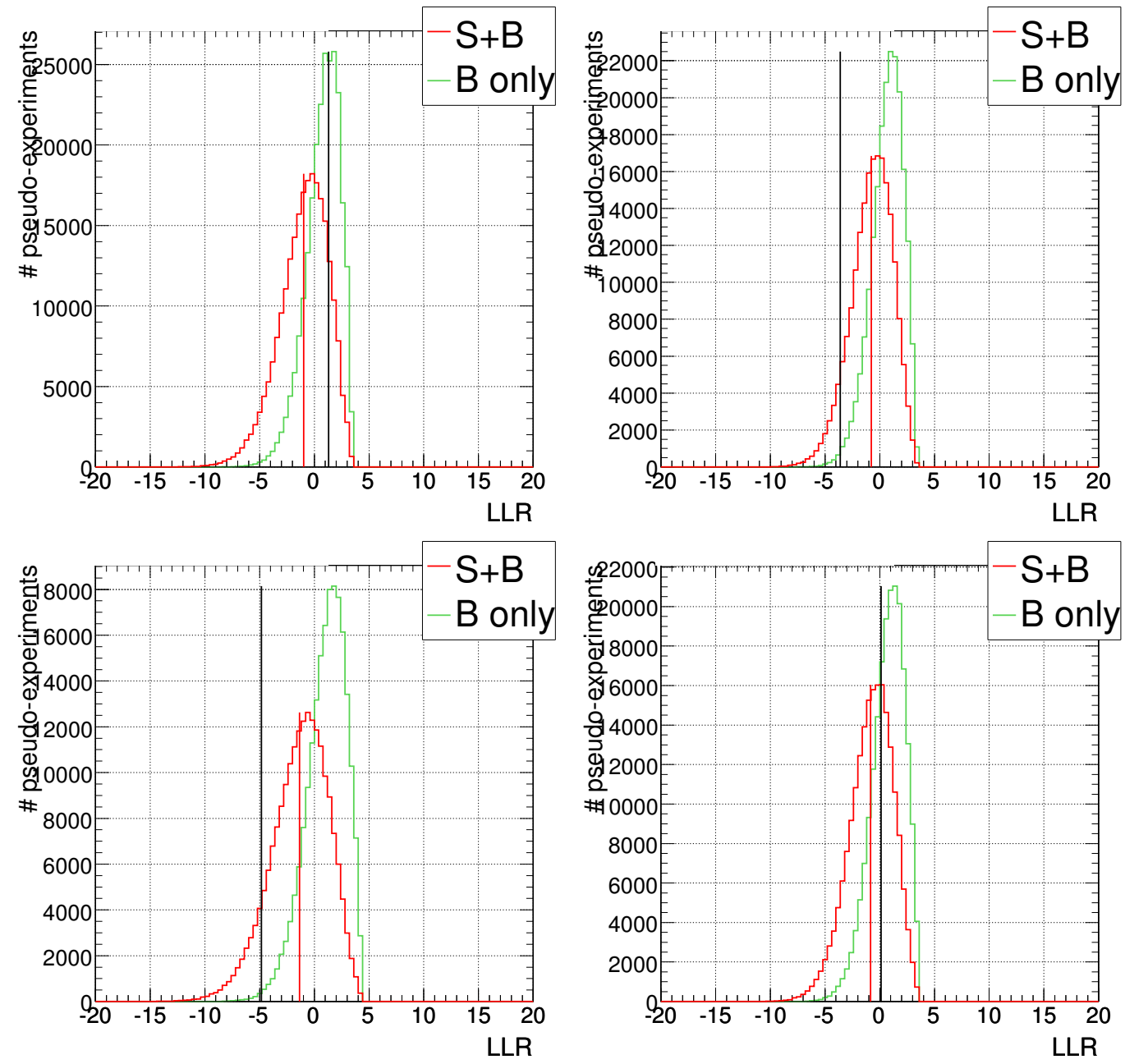

Figure 9.21: LLR distribution computed by COLLIE in 200000 pseudo-experiment for the signal plus backgrounds $(\mathrm{S}+\mathrm{B})$ and background only (B only) hypothesis in the di-electron (left) and di-muon (right) channels using Run IIa and Run IIb datasets. The vertical red line indicates the median of the $\mathrm{S}+\mathrm{B}$ distribution while the vertical black line indicates the actual value measured in the data.

The values of $C L_{b}, 1-C L_{b}$ and the significance measured and expected in the 
di-electron and di-muon channels is reported in Table 9.9 for Run IIa and Run IIb data.

The two final states and the two sets of data are finally combined and a global significance is then estimated. The resulting LLR distributions are shown in Figure 9.22. We observe a signal with $+2.63 \sigma$ significance $(+1.97 \sigma$ expected $)$ and measure a cross-section $\sigma(p \bar{p} \rightarrow Z Z)=2.0 \pm 0.93($ stat. $) \pm 0.29($ sys. $)$ pb, as reported in the last column of Table 9.9.

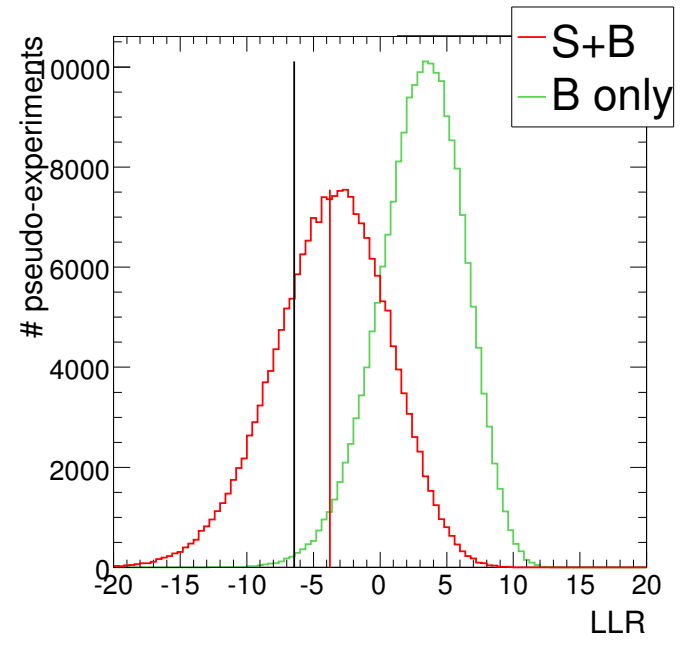

Figure 9.22: LLR distribution computed by COLLIEin several pseudo-experiment for the signal plus backgrounds ( $\mathrm{S}+\mathrm{B})$ and background only (B only) hypothesis combining the di-electron and di-muon final states for the Run IIa and Run IIb datasets.

The same test statistics used for the significance computation is used to measure cross-section measurement by keeping the the signal cross section as a free parameter in the Poisson likelihood maximization, as discussed in Section 8.5. This procedure allows to determine a scale factor, together with its error, with respect to the nominal cross section:

$$
f=1.42 \pm 0.69
$$

A cross check was performed by scanning the cross sections in a procedure also de- 


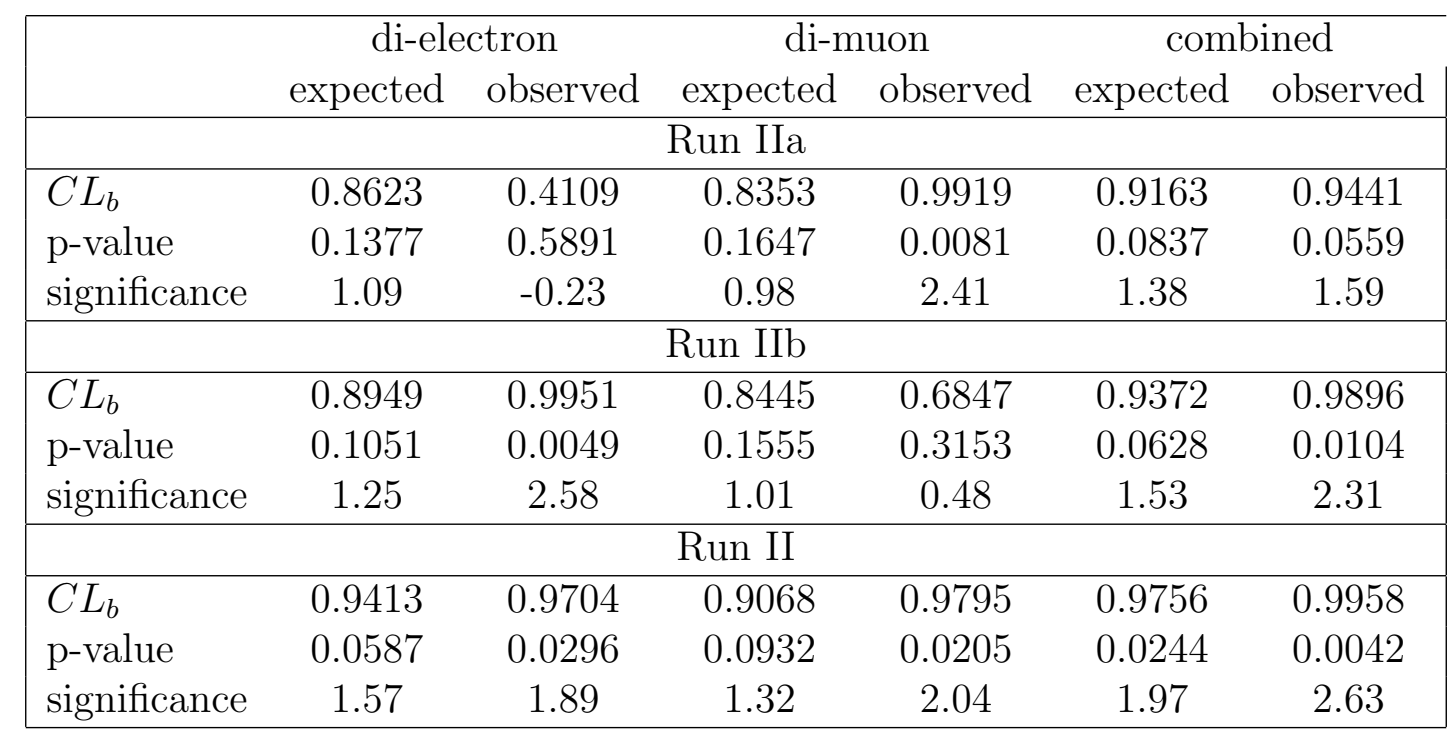

Table 9.14: $Z Z \rightarrow \ell^{-} \ell^{+} \nu \bar{\nu}$ significance values estimated using COLLIE for the di-electron and di-muon channels and for the combination of the two. Systematics have been included in the computation.

scribed in Section 8.5. The resulting scale factor is $f=1.53 \pm 0.67$. The corresponding cross section can be computed scaling the number of events foreseen by the MC by this scale factor $f=1.42$.

$$
\sigma^{Z Z}=\sigma^{Z} \frac{A_{Z}}{A_{Z Z}} \frac{f N_{Z Z}^{M C}}{N_{Z}}
$$

where $A_{Z}$ and $A_{Z Z}$ are the acceptances $\times$ efficiencies for the $Z / \gamma^{*} \rightarrow \ell \ell\left(60<M_{\ell \ell}<\right.$ $130 \mathrm{GeV})$ and $Z Z \rightarrow \ell \ell \nu \nu$ respectively computed after the extra activity cut. $N_{Z}$ and $N_{Z Z}^{M C}$ are the number of events of the two samples after the normalization to data explained in Section 9.4. The values for these parameter are listed in Tabel 9.9.

The resulting cross section for the production of $Z Z \rightarrow \ell \ell \nu \nu$ is:

$$
\sigma^{Z Z} \times B R(\ell \ell \nu \nu)=0.081 \pm 0.037 \pm 0.012 \mathrm{pb}
$$

In this expression, $\ell \ell$ represents either $e e, \mu \mu$, or $\tau \tau$. To convert $\sigma \times B R$ into a total cross section, we use the Particle Data Group [96] (PDG) values for the $Z$ branching 


\begin{tabular}{|c|c|c|}
\hline \multicolumn{3}{|c|}{ Run IIa } \\
\hline & di-electron & di-muon \\
\hline$A_{Z}$ & $16.69 \pm 0.02 \%$ & $19.12 \pm 0.03 \%$ \\
\hline$A_{Z Z}$ & $5.83 \pm 0.05 \%$ & $6.77 \pm 0.06 \%$ \\
\hline$N_{Z}$ & $42145.21 \pm 60.24$ & $46877.33 \pm 67.50$ \\
\hline$N_{Z Z}^{M C}$ & $3.48 \pm 0.03$ & $4.14 \pm 0.04$ \\
\hline \multicolumn{3}{|c|}{ Run IIb } \\
\hline$A_{Z}$ & $11.33 \pm 0.02 \%$ & $12.19 \pm 0.02 \%$ \\
\hline$A_{Z Z}$ & $3.87 \pm 0.03 \%$ & $3.95 \pm 0.03 \%$ \\
\hline$N_{Z}$ & $36213.56 \pm 51.89$ & $37664.15 \pm 72.56$ \\
\hline$N_{Z Z}^{M C}$ & $2.92 \pm 0.02$ & $2.88 \pm 0.02$ \\
\hline
\end{tabular}

Table 9.15: Acceptances $\times$ efficiency and number of events after the extra activity cut for the $Z / \gamma^{*} \rightarrow \ell \ell\left(60<M_{\ell \ell}<130 \mathrm{GeV}\right)$ and the $Z Z \rightarrow \ell \ell \nu \nu$ samples.

fractions: $B R(Z \rightarrow e e)=0.03363 \pm 0.00004, B R(Z \rightarrow \mu \mu)=0.03366 \pm 0.00007$, $B R(Z \rightarrow \tau \tau=0.03370 \pm 0.00008), B R(Z \rightarrow \nu \nu)=0.2000 \pm 0.0006$. This gives $B R(Z Z \rightarrow \ell \ell \nu \nu)=0.0403 \pm 0.0001)$. The measured value for the inclusive $Z$ pair cross section is then:

$$
\sigma(p \bar{p} \rightarrow Z Z)=2.0 \pm 0.93 \pm 0.29 \mathrm{pb}
$$

This can be compared with the predicted standard model cross section of $1.4 \pm$ $0.1 \mathrm{pb}[97]$.

The statistical error on this number was estimated using COLLIE without including the systematics.

\subsection{First Observation of the $Z Z$ Production Pro- cess}

[2] A separate search for $Z Z$ production was carried out in the four charged lepton channel where four muon $(4 \mu)$, four electron $(4 e)$, and two electron and two muon 

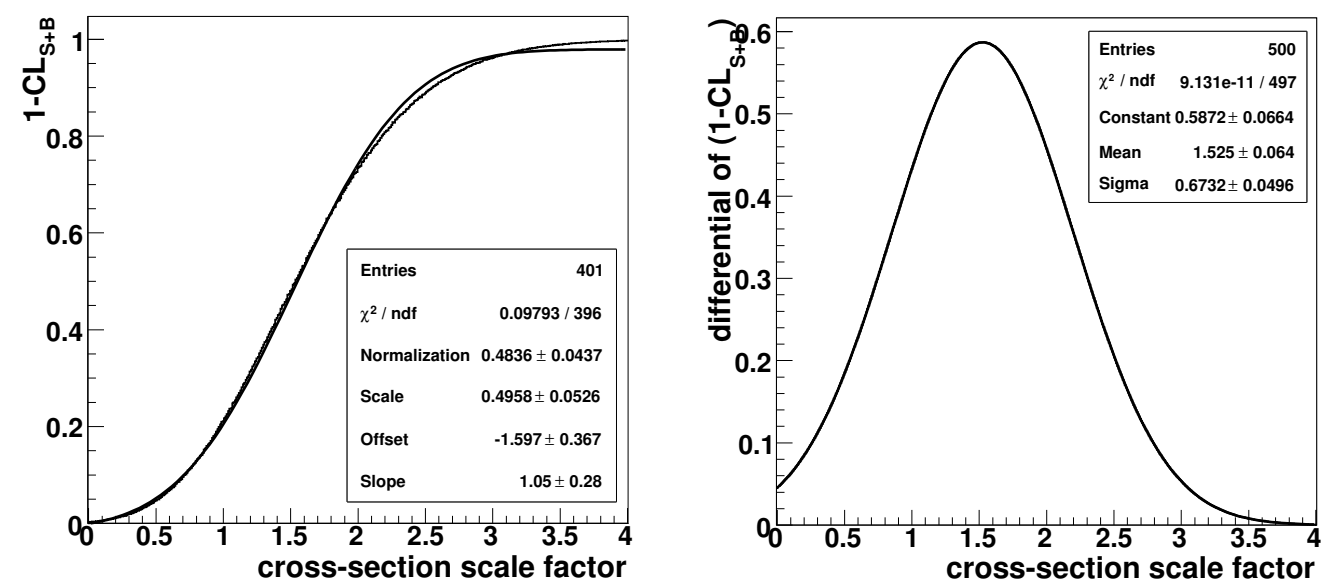

Figure 9.23: $1-C L_{S+B}$ distribution (left) and differential curve of $1-C L_{S+B}$ (right) as a function of the cross-section scaling factor as estimated by COLLIEon the d-electron and di-muon combined using RunIIa and RunIIb datasets. 50000 pseudo-experiment are generated for each different hypothesis on the signal cross-section, scanning the range from 0 to 4 with a granularity of 0.01 .

$(2 e 2 \mu)$ events were considered. This analysis was combined with the above described $Z Z \rightarrow \ell^{-} \ell^{+} \nu \bar{\nu}$ search and a previous version of the four lepton search using Run IIa data. This combination produced the first observation of $Z Z$ production at a hadron collider.

\subsection{1 $Z Z \rightarrow \ell^{-} \ell^{+} \ell^{\prime-} \ell^{\prime+}$ Analysis}

\section{Event Selection}

Electrons satisfying the following criteria are considered

- $I D=10$ or $I D=11$

- EM fraction $E M F>0.90$

- isolation iso $<0.20$

- Likelihood $>0.2$ (CC only) 
- $\mathrm{HMx} 8<20.0$ (EC only)

- $\left|\eta_{\text {det }}\right|<1.1(\mathrm{CC})$ or $1.5<\left|\eta_{\text {det }}\right|<3.2(\mathrm{EC})$

Note that the likelihood requirement implies a track match and is not used in the forward region due to a low efficiency. Instead a stricter requirement is put on the shower shape. In the $4 e$ channel, four electrons with transverse energy $E_{T}>30,20,15$, and $15 \mathrm{GeV}$ with at least two being located in the CC are selected. This channel if further subdivided by the number of electrons in the $\mathrm{CC}(2 \mathrm{CC}, 3 \mathrm{CC}$, or $4 \mathrm{CC})$ since the background contributions will differ for each.

For muons, the following requirements must be met:

- Loose muon ID

- matched to a central track

- cosmic veto ( scintillator timing )

- dca $<0.02(0.2)$ cm for tracks with (without) SMT hits

- Calorimeter Isolation $E_{T}($ halo $)<2.5 \mathrm{GeV}$ for $n s e g=1$ muons

In the $4 \mu$ channel only three have to pass the calorimeter isolation cut and the transverse momenta must satisfy $p_{T}>30,25,15$, and $15 \mathrm{GeV}$.

In the $2 e 2 \mu$ channel, only one muon has to pass the calorimeter isolation cut and no requirement is imposed on the number of electrons in the CC. A spatial separation of $\Delta R>0.2$ is required between the muons and electrons to reject $Z \rightarrow \mu \mu$ events where the muons radiate photons.

The four leptons are combined into all possible opposite signed (not in the $4 e$ channel) same flavor pairs and if a configuration exists such that the di-lepton mass of one pair is $>70 \mathrm{GeV}$ and the other is $>50 \mathrm{GeV}$ then the event is kept for further consideration. 


\section{Background Modeling}

Top pair production in which both $W$ s decay leptonicly was modeled using MC. $Z+$ jets and $Z+\gamma+$ jets productions can contaminate the event selection if jets or photons fake electron signatures or jets containing a real muon from in-flight decays of pions, kaons, or a heavy quarks are not reconstructed properly. This background contribution was estimated applying fake rates derived from a data sample collected with jet triggers to events containing jets and passing all other selection criteria. The final background process to consider is events from the beam halo and cosmic muons. A data sample is used where four muons are selected with loose criteria. Each criterium is tightened individually to obtain the rejection rate and these rates are combined for all criteria and applied to loosely selected data.

Three data events were observed with $1.89 \pm 0.08$ signal and $0.14_{-0.02}^{+0.03}$ background events were expected. The final sample is shown in Figure 9.24.

\section{Systematic Uncertainties}

Here only a list of systematic uncertainties are given

- Integrated Luminosity

- Data Quality

- Luminosity Profile Reweighting

- Beam Position Reweighting

- $Z Z p_{T}$ Spectrum Reweighting

- Electron Identification, Energy Resolution, Fake-rate

- Muon Identification, Track, Isolation, Momentum Resolution, Fake-rate 


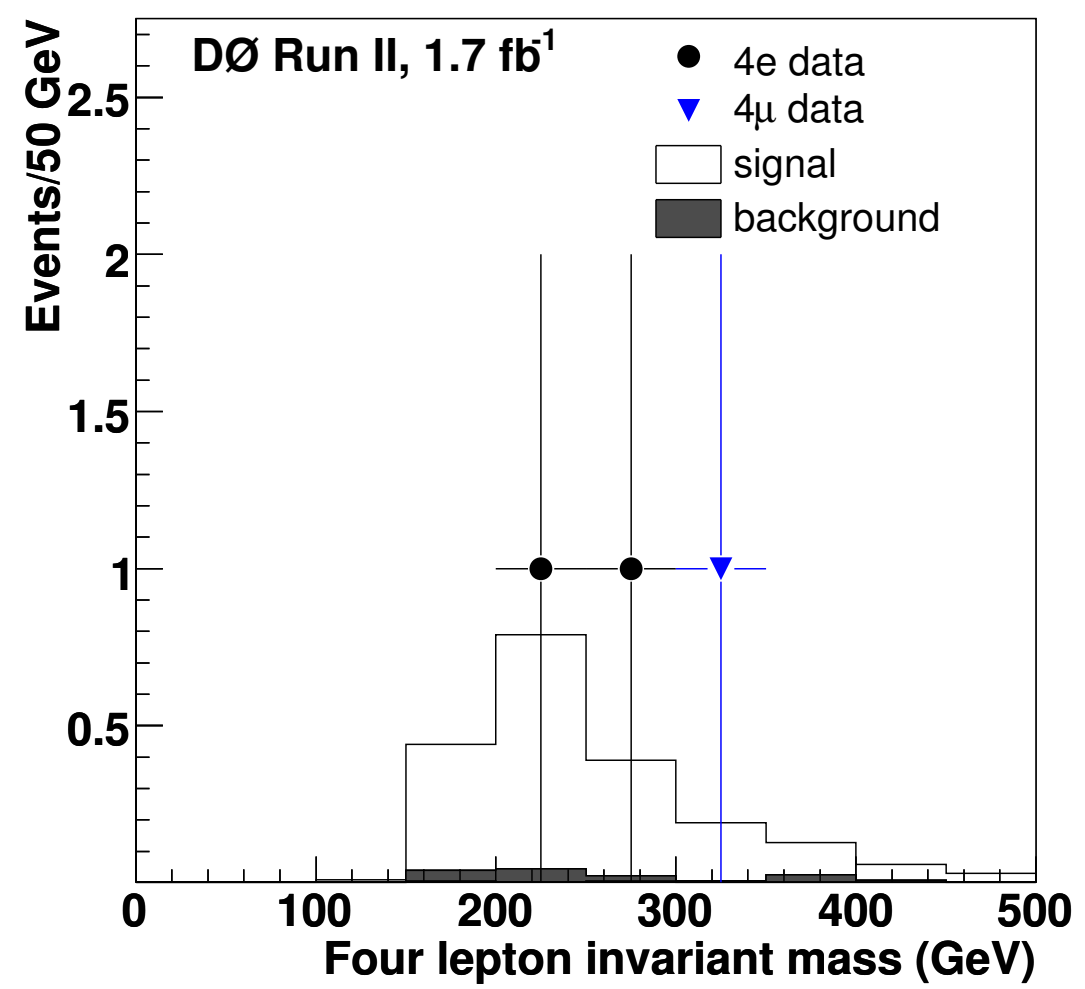

Figure 9.24: The final sample of the Run IIb $Z Z \rightarrow \ell^{-} \ell^{+} \ell^{\prime-} \ell^{\prime+}$ Analysis. Three data events were observed with $1.89 \pm 0.08$ signal and $0.14_{-0.02}^{+0.03}$ background events were expected.

- $t \bar{t}$ Cross Section

- Parton Distribution Functions

- $Z Z$ cross section uncertainty

\section{Significance and Cross Section Measurement}

The significance of the observed event distribution was calculated using COLLIE and was observed to be $5.7 \sigma$ while $3.7 \sigma$ was expected. A cross section measurement was done scanning a scaling factor from 0 to 8 in a Poisson likelihood function with $300 \mathrm{M}$ 
pseudo experiments. A measured value of

$$
\sigma(p \bar{p} \rightarrow Z Z)=1.75_{-0.86}^{+1.27}(\text { stat. }) \pm 0.08(\text { syst. }) \pm 0.10(\text { lumi }) p b
$$

was found.

A similar analysis was conducted on Run IIa data with a looser di-lepton mass cut and object $p_{T}$ cut as well as not having the muon calorimeter isolation cut and the channels were not further divided by the number of $\mathrm{CC}$ electrons. To have a fair comparison with Run IIb, the Run IIb criteria were relaxed to those of Run IIa. Then the mass cut was increased to $50 \mathrm{GeV}$ and $70 \mathrm{GeV}$. Taking the ratio of these two relaxed Run IIb samples, a scale factor was found for each channel to estimate the Run IIa yield using the Run IIb cuts. The one data event observed in the Run IIa analysis was rejected due to its failure to meet the $M_{\ell \ell}$ requirements of the Run IIb analysis.

\subsubsection{Combination}

Systematics in each of the $Z Z \rightarrow \ell^{+} \ell^{-} \nu \bar{\nu}$ and $Z Z \rightarrow \ell^{+} \ell^{-} \ell^{\prime+} \ell^{\prime-}$ analysis are shown in Tables 9.10.2 and 9.10.2 and those which are common to both are shown in Table 9.10.2. Below is a list of common uncertainties with a note on why each is correlated or not across the two analysis.

\section{Correlated Shared Systematic Uncertainties}

This section lists only those systematic uncertainties which affect both analyses and

are treated as correlated between them. 100\% correlation is assumed, since COLLIE is able to treat uncertainties only as either $100 \%$ correlated or uncorrelated. Note that there is no cross-correlation among the individual systematic uncertainties in this list. 
- Electron and Muon Resolution: Uncertainties arise from the same set of over-smearing parameters used the the tools package common to all $\mathrm{D} \emptyset$ analyses.

- $Z Z p_{T}$ spectrum: Uncertainties are measured by re-weighting events with respect to the di-boson $p_{T}$ spectrum. This reweighting is done so that the distributions from Pythia [31] match ShERPa.

\section{Uncorrelated Shared Systematic Uncertainties}

This section lists only those systematic uncertainties which affect both analyses and are uncorrelated between them. Note that there is no cross-correlation among the individual systematic uncertainties in this list.

- Electron Misidentification: Uncertainties are taken to be uncorrelated because they are determined using two different methods. The $\ell^{+} \ell^{-} \ell^{\prime+} \ell^{\prime-}$ uses the so-called "Tag and Probe" method using a subset of two jet events while the $\ell^{+} \ell^{-} \nu \bar{\nu}$ analysis uses the so-called "Matrix Method" with no requirement on jets.

- Multihadron Sample Statistics: Uncertainties are taken to be uncorrelated. Although both analyses estimate their QCD background from statistically limited data samples, the regions of phase-space used are not the same.

\section{Significance Calculation}

The two final states and the two sets of data from the $Z Z \rightarrow \ell^{+} \ell^{-} \nu \bar{\nu}$ analysis are combined with the two $Z Z \rightarrow \ell^{+} \ell^{-} \ell^{\prime+} \ell^{\prime-}$ data sets to obtain a global significance. The resulting LLR distributions are shown in Figure 9.25. A significance of $5.7 \sigma$ was observed with $5.2 \sigma$ expected as shown in Table 9.19. 


\begin{tabular}{|c|c|c|c|}
\hline Systematic Uncertainty & Type & Sample & Correlated? \\
\hline Electron Misidentification & Flat & $\mathrm{W} \gamma$ & No \\
\hline Multihadron Sample Statistics & Flat & $\mathrm{W}+$ Jets & Epoch \\
\hline Extrapolation of the Recoil Activity & Flat & Inclusive Z & No \\
\hline Jet Energy Scale & Flat & \multirow{3}{*}{ B \& S } & \multirow{3}{*}{ Yes } \\
\hline Jet Energy Resolution & Flat & & \\
\hline Jet Reconstruction Efficiency & Flat & & \\
\hline Electron Resolution & Shape & \multirow{2}{*}{$\mathrm{B} \& \mathrm{~S}$} & \multirow{2}{*}{ Epoch } \\
\hline Muon Resolution & Shape & & \\
\hline$W W$ Production Cross Section & Flat & WW & \multirow{2}{*}{ All WW \& WZ } \\
\hline$W Z$ Production Cross Section & Flat & WZ & \\
\hline$Z / \gamma^{*}$ theoretical cross section & Flat & $\mathrm{S}$ & $\mathrm{No}^{\dagger}$ \\
\hline Veto Efficiency & Flat & Inclusive Z & No \\
\hline PDF Uncertainties & Flat & S \& Inclusive Z & No \\
\hline$Z Z p_{T}$ Spectrum & Flat & $\mathrm{S}$ & No \\
\hline$W W p_{T}$ Spectrum & Flat & WW & Yes \\
\hline
\end{tabular}

$\mathrm{B}=$ All Backgrounds, $\mathrm{S}=$ Signal, Epoch $=$ Correlation among Run IIa and Run IIb $\dagger$ denotes systematic uncertainties not handled by COLLIE.

Table 9.16: Sources of Systematic Uncertainties in the $Z Z \rightarrow \ell^{+} \ell^{-} \nu \bar{\nu}$ channel. All correlations noted refer to uncertainties within this channel alone.

\begin{tabular}{|lcc|}
\hline Systematic Uncertainty & Sample & Correlated? \\
\hline Luminosity & $\mathrm{S}, \mathrm{C}, \mathrm{t} \bar{t}$ & Yes \\
Luminosity Profile Reweighting & $\mathrm{S}, \mathrm{C}, t \bar{t}$ & Yes \\
Data Quality & All & Yes \\
\hline Electron Identification & $\mathrm{S}, \mathrm{C}, t \bar{t}($ e only $)$ & Yes \\
Electron Resolution & $\mathrm{S}, \mathrm{C}, t \bar{t}(\mu$ only $)$ & Yes \\
\hline Muon Identification & $\mathrm{S}, \mathrm{C}$ & Yes \\
Muon Resolution & $\mathrm{S}, \mathrm{C}, t \bar{t}$ & Yes \\
\hline$Z Z p_{T}$ Spectrum & $\mathrm{QCD}(\mathrm{e}$ only $)$ & Yes \\
$Z$ Vertex Reweighting & $\mathrm{QCD}(\mu$ only $)$ & Yes \\
QCD Electron Misidentification & $\mathrm{S}, \mathrm{C}, t \bar{t}$ & Yes \\
QCD Muon Misidentification & $t \bar{t}$ & No \\
PDF Uncertainties & All & No \\
$t \bar{t}$ Production Cross Section & Monte Carlo Statistics & \\
\hline
\end{tabular}

$$
\mathrm{S}=\text { Signal, } \mathrm{C}=\text { Contribution due to combinatorics }
$$

Table 9.17: Sources of Systematic Uncertainties in the $Z Z \rightarrow \ell^{+} \ell^{-} \ell^{\prime+} \ell^{\prime-}$ channel. All correlations noted refer to uncertainties within this channel alone. 


\begin{tabular}{|lc|}
\hline Systematic Uncertainty & Correlated? \\
\hline Electron Misidentification & No \\
Multihadron Sample Statistics & No \\
Electron Resolution & Yes \\
Muon Resolution & Yes \\
pdf uncertainties & Yes \\
$Z Z p_{T}$ spectrum & Yes \\
\hline
\end{tabular}

Table 9.18: Sources of systematic uncertainties present in both $Z Z$ analyses.

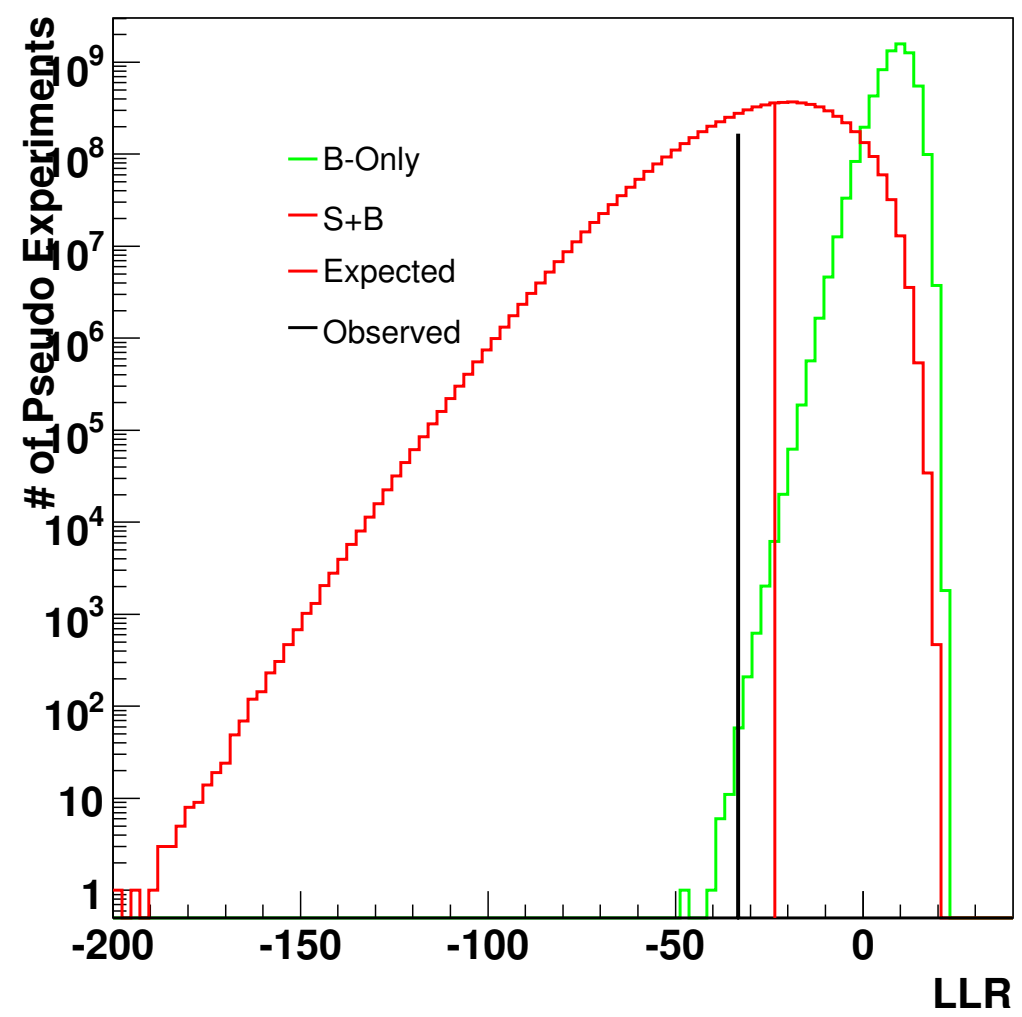

Figure 9.25: LLR distribution computed by COLLIE in $3 * 10^{9}$ pseudo-experiments for the signal-plus-background ( $\mathrm{S}+\mathrm{B}$ ) and background only (B only) hypothesis. The value measured in the combination of the two analyses is indicated by the vertical black line.

\section{Cross Section Calculation}

The same test statistic used for the significance computation is used for the crosssection measurement with COLLIE. Leaving the signal cross section unconstrained in 


\begin{tabular}{|c|c|c|c|c|c|c|c|c|}
\hline & \multicolumn{2}{|c|}{$Z Z \rightarrow e^{+} e^{-} \nu \bar{\nu}$} & \multicolumn{2}{|c|}{$Z Z \rightarrow \mu^{+} \mu^{-} \nu \bar{\nu}$} & \multicolumn{2}{|c|}{$Z Z \rightarrow \ell^{+} \ell^{-} \ell^{\prime+} \ell^{\prime-}$} & \multicolumn{2}{|c|}{ combined } \\
\hline & expected & observed & expected & observed & expected & observed & expected & observed \\
\hline \multicolumn{9}{|c|}{ Run IIa } \\
\hline$C L_{b}$ & 0.8599 & 0.4211 & 0.8342 & 0.9917 & 0.988129 & 0 & 0.998379 & 0.891469 \\
\hline p-value & 0.1401 & 0.5789 & 0.1658 & 0.0083 & 0.0118711 & 1 & 0.0016213 & 0.108531 \\
\hline significance & 1.08 & -0.20 & 0.97 & 2.40 & 2.26 & 0 & 2.94 & 1.23 \\
\hline \multicolumn{9}{|c|}{ Run IIb } \\
\hline$C L_{b}$ & 0.8924 & 0.9965 & 0.8448 & 0.6783 & 0.999582 & 1 & 0.999905 & 1 \\
\hline p-value & 0.1076 & 0.0035 & 0.1552 & 0.3217 & $4.17631 \times 10^{-4}$ & $4.26009 \times 10^{-8}$ & $9.48069 \times 10^{-5}$ & $2.18391 \times 10^{-9}$ \\
\hline significance & 1.24 & 2.70 & 1.01 & 0.46 & 3.34 & 5.36 & 3.73 & 5.87 \\
\hline \multicolumn{9}{|c|}{ combined } \\
\hline$C L_{b}$ & 0.9384 & 0.9805 & 0.9067 & 0.9778 & 0.999993 & 1 & 0.999999 & 1 \\
\hline p-value & 0.0616 & 0.0195 & 0.0933 & 0.0222 & $7.2642 \times 10^{-6}$ & $2.878 \times 10^{-7}$ & $9.07986 \times 10^{-7}$ & $6.22441 \times 10^{-9}$ \\
\hline significance & 1.54 & 2.06 & 1.32 & 2.01 & 4.34 & 5.00 & 4.77 & 5.69 \\
\hline
\end{tabular}

Table 9.19: Significance values estimated for $Z Z \rightarrow \ell^{+} \ell^{-} \nu \bar{\nu}$ and $Z Z \rightarrow \ell^{+} \ell^{-} \ell^{+} \ell^{-}$analyses using COLLIE. Systematics have been included in the computation. No profiling has been applied

the Poisson $\chi^{2}$ minimization yields a scale factor for the nominal cross section of:

$$
f=1.13 \pm 0.438 \text { (stat.) } \pm 0.111 \text { (syst.). }
$$

We use the PDG values for the $Z$ branching fractions: $B R(Z \rightarrow e e)=0.03363 \pm$ $0.00004, B R(Z \rightarrow \mu \mu)=0.03366 \pm 0.00007, B R(Z \rightarrow \tau \tau=0.03370 \pm 0.00008)$, $B R(Z \rightarrow \nu \nu)=0.2000 \pm 0.0006$ to obtain a total cross section. This gives $B R(Z Z \rightarrow$ $\ell \ell \nu \nu)=0.0403 \pm 0.0001)$. The measured value for the inclusive $Z$ pair cross section is then:

$$
\sigma(p \bar{p} \rightarrow Z Z)=1.60 \pm 0.63(\text { stat. })_{-0.17}^{+0.16} \text { (syst.) } p b
$$

The statistical uncertainty on this number was estimated running COLLIE without including the systematics. This can be compared with the predicted standard model cross section of $1.4 \pm 0.1 \mathrm{pb}[97]$. 


\section{Chapter 10}

\section{$Z H \rightarrow \nu \bar{\nu} b \bar{b}$ Analysis}

The existence of the Higgs boson is the only fundamental element of the SM that has yet to be confirmed. Its observation would be a key step in establishing the mechanism of electroweak symmetry breaking and mass generation. Associated $Z H$ production in $p \bar{p}$ collisions, with $Z \rightarrow \nu \bar{\nu}$ and $H \rightarrow b \bar{b}$, is among the most sensitive processes for seeking a Higgs boson with a mass $m_{H} \lesssim 135 \mathrm{GeV}$ at the Fermilab Tevatron Collider [98].

The final-state topology considered in this analysis comprises a pair of $b$ jets from the decay of the Higgs boson, and $\not_{T}$ due to the two escaping neutrinos from $Z$ decay (Figure 10.1). The search is therefore also sensitive to those events in $W H$ channel where the charged lepton from $W \rightarrow \ell \nu$ decay is not detected. The main backgrounds arise from $(W / Z) b \bar{b},(W / Z)+$ (non- $b$ jets) due to flavor misidentification (mistagging), top-quark production, e.g., $t \bar{t} \rightarrow \ell \nu b q \bar{q}^{\prime} \bar{b}$ or $t(q) \bar{b} \rightarrow \ell \nu b(q) \bar{b}$, diboson production such as $W Z \rightarrow q \bar{q}^{\prime} \nu \bar{\nu}$ or $Z Z \rightarrow b \bar{b} \nu \bar{\nu}$, and from multijet events produced via the strong interaction, containing true $b$ jets or mistagged light-parton jets, and $\not_{T}$ arising from fluctuations in measurement of jet energies.

A kinematic based boosted-decision-tree discriminant is first used to reject most 
of the multijet events. Then jets expected to arise from Higgs-boson decays are required to be identified as $b$ jets. Finally, discrimination between signal and remaining backgrounds is achieved by means of a boosted-decision-tree technique.

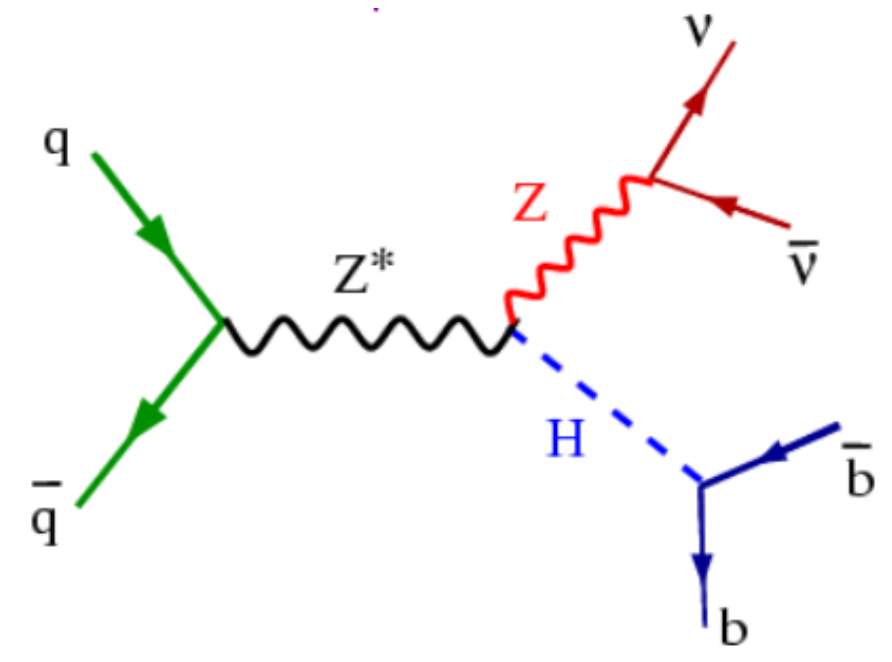

Figure 10.1: Feynman diagram of the leading order $Z H \rightarrow \nu \nu b \bar{b}$ process

\subsection{Dataset and Monte Carlo Samples}

\subsubsection{Data}

The data for this analysis were collected by the $\mathrm{D} \varnothing$ collaboration between June 9th, 2006 and March 112010 at the center-of-mass energy $\sqrt{s}=1.96 \mathrm{TeV}$, during Run IIb. The data is further segmented due to an increase in the maximum instantaneous luminosity, separating Run IIb1 from IIb2, and recovery of dead channels in the SMT, separating Run IIb2 and IIb3 (Table 10.1). The integrated luminosity after data quality is $6.2 \pm 0.11 \mathrm{fb}^{-1}$. 


\begin{tabular}{|l|lr|}
\hline Mode & \multicolumn{2}{|c|}{ Run IIb } \\
& $\sigma(\mathrm{pb}) \times \mathrm{BR}$ & \# of events \\
\hline$Z H \rightarrow \nu \nu b b\left(m_{h}=115\right)$ & 0.015671 & 422956 \\
$Z H \rightarrow l l b b\left(m_{h}=115\right)$ & 0.007912 & 279468 \\
$Z H \rightarrow \nu \nu c c\left(m_{h}=115\right)$ & 0.000690 & 180707 \\
$Z H \rightarrow \nu \nu \tau \tau\left(m_{h}=115\right)$ & 0.001587 & 179077 \\
\hline
\end{tabular}

Table 10.2: Signal $Z H$ MC samples with cross sections for $115 \mathrm{GeV}$ Higgs. The number of events is calculated after applying the DQ cuts and duplicate events removal.

\begin{tabular}{|l|c|c|c|}
\hline Run & Start Date & End Date & Luminosity $\left(\mathrm{pb}^{-1}\right)$ \\
\hline IIb1 & June 9, 2006 & August 4, 2007 & $1,221.2$ \\
IIb2 & October 28, 2007 & June 13, 2009 & $2,997.9$ \\
IIb3 & September 15, 2009 & March 11, 2010 & $1,945.7$ \\
\hline IIb & June 9, 2006 & March 11, 2010 & $6,164.8$ \\
\hline
\end{tabular}

Table 10.1: Data taking epochs.

\subsubsection{Monte Carlo}

For all PYTHIA samples version v6.409 with "D0 tune A" and the CTEQ6L1 LO pdf set is used. All $W / Z+$ jets and $t \bar{t}$ processes were generated with ALPGEN v2.11 interfaced with PYTHIA. The inclusive di-boson MC samples were produced with PYTHIA. The single top samples were produced with COMPHEP, interfaced with PYthiA. The $W H$ and $Z H$ signal samples were simulated with PYthiA. A list of cross-sections and branching ratios for $115 \mathrm{GeV}$ Higgs MC can be found in Table 10.2. Two sets of MC were used to properly model the different features of the different data epochs. One was used for Run IIb1 data and another for Run IIb2-3. 


\section{Custom Monte Carlo Corrections}

In addition to the luminosity and beam profile, $\mathrm{W} / \mathrm{Z} p_{T}$, and MLM matching reweightings, described in Section 3.3, a custom reweighting was also applied. It was found that in many selections ALPGEN samples do not perfectly model angular distributions. For this reason reweighting functions were derived which should correct for this generator level imperfections. This reweighting function was derived on our independent electroweak control sample and is described in Appendix C. After deriving and applying a reweighting function to the $\Delta \eta$ distribution between the two leading jets, the modeling of the $\Delta \phi$ between the two leading jets and the dijet mass distribution were also improved. For this reason we keep this simple form of corrections.

In terms of the JSSR procedure, as suggested in Ref. [99], shifting is not done for quark dominated final states (double and single top, dibosons, $V+b b / c c$, and signal). The energy changes resulting from this processor are then propagated to the $\mathbb{E}_{T}$.

\subsection{Trigger Description}

Three jets $+E_{T}$ triggers were designed corresponding to different topologies: mono-, di- and multi-jet $+\mathbb{E}_{T}$ topologies (cf [100], [101]). An ORing of mono, di and multi-jet requirements was designed at $\mathrm{L} 1$ for the di-jet $+E_{T}$ trigger, in order to increase the $Z H$ signal efficiency. At L1, considerable $\mathbb{E}_{T}$ and energetic jets are required where the $\Delta \phi$ between the two leading jets is less than $170^{\circ}$. Events with many energetic jets without considerable $E_{T}$ are also selected. At L2, this selection is refined with a tighter cut on the leading jet $\Delta \phi$ and requirements on the $H_{T}$ and $H_{T}$. $H_{T}$ is defined the same way as $E_{T}$ but only using identified jets and $H_{T}$ is the scalar sum of the jet transverse energies giving a measure of how energetic the event is. In the data taken before early March 2008, a requirement was put on the $H_{T}$ while after this date, the 
$\#_{T}$ itself was used instead.

This complicated topological trigger was parameterized using $W \rightarrow \mu \nu$ events. The Level 1 decision was broken down into the probability of the event passing the jet requirements then the $\mathscr{E}_{T}$ requirements and computed as a probability as a function of jet $p_{T}$ and $\left|E_{T}\right|$ respectively. The jet turn-on curves are found for three different regions of the detector $\left(\left|\eta_{d}\right|<0.8,0.8<\left|\eta_{d}\right|<1.6\right.$, and $\left.1.6<\left|\eta_{d}\right|<3.2\right)$ and before and after March 2008. In early March 2008, a new calibration for the L1 calorimeter trigger was introduced slightly changing the jet turn-ons. Level 2 is almost $100 \%$ efficient. The slight inefficiency is parameterized in terms of the leading jet $p_{T}$. L3 was parameterized in the same subdivisions as L1 using the data and MC JES and MC JSSR corrections to map uncorrected MC jet energies to uncorrected data jet energies in order to obtain the energy available to L3.

\subsection{Event Selection}

The initial preselection criteria are meant to minimize the overwhelming background from multijet events, but retain high efficiency for signal. At the second stage, the selection enhances the sensitivity by first making use of kinematic criteria, and secondly of $b$ tagging. These selection cuts are optimized for a Higgs-boson mass of $115 \mathrm{GeV}$. A multivariate discriminant is also employed before and after $b$ tagging to separate the signal from the multijet background and remaining SM backgrounds respectively.

\subsubsection{Preselection}

In addition to our trigger requirements, and basic data quality requirements, the following criteria is also required during the preselection.

- The primary vertex (PV) must be reconstructed within the acceptance of the 
silicon vertex detector $\left(\left|z_{\mathrm{PV}}\right|<40 \mathrm{~cm}\right.$, where $z$ is measured from the center of the detector along the beam direction), and at least three charged particle tracks have to originate from that vertex.

- Only jets with $p_{T}>15 \mathrm{GeV}$ within $\left|\eta_{\text {det }}\right|<3.2$ are considered in the analysis, and are ordered in decreasing $p_{T}$. One of these jets must be taggable and there must be two or more jets in the event with $p_{T}>20 \mathrm{GeV}$ within $|\eta|<2.5$ and the missing transverse energy is required to be larger than $30 \mathrm{GeV}$.

The numbers of events after each cut for the MC signal samples and the observed data events can be seen in Tables 10.3 and 10.4.

After preselection, additional criteria are used to define four distinct samples:

- The signal sample (Section 10.7) - used to search for the Higgs-boson signal. Here, further topological criteria are applied to reduce the multijet background, among which is a tighter cut on the $\mathscr{H}_{T}$ and a requirement that there must be no bad jets in the event with $p_{T}>15 \mathrm{GeV}$, not considering those which only fail the EM fraction cut of the good-jet criterion. This is discussed further in Section 10.3.2. At least two and not more than three taggable jets are required. In addition, a veto on isolated leptons is applied to reduce the background from $W \rightarrow \ell \nu+$ jets and to make the sample exactly orthogonal to the $W H \rightarrow l \nu b b$ channel;

- The electroweak control sample (Section 10.5) - enriched in $(W \rightarrow \mu \nu)+$ jets events has a jet system with similar topology to the signal sample and is used to validate the SM background simulation. The selection is the same as the one used for the signal sample, except that the veto on isolated muons is reversed; 
- The "MJ-model" sample - dominated by multijet events, and used to model the multijet background. This sample is selected in the same way as the signal sample, except the last topological selection criterion, a cut on $\mathcal{D}=$ $\Delta \phi\left(\not_{T}, \not p_{T}^{t r k}\right)$, is reversed;

- The MJ control sample (Section 10.6) - used to validate the MJ modeling procedure. Here, the topological selection criteria are sufficiently relaxed to lead to a sample largely dominated by multijet events.

Once these selection criteria have been applied, we use "multijet decision tree" (MJ-DT) to discriminate the signal from the multijet background (Section 10.8). Discrimination against SM backgrounds is achieved by defining $b$-tagged samples (Section 10.3.7) and using "physics decision trees" within those b-tagged samples (Section 10.8). 


\begin{tabular}{|c|c|}
\hline 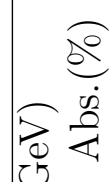 & 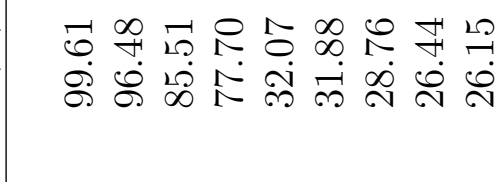 \\
\hline 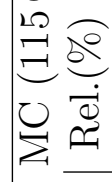 & 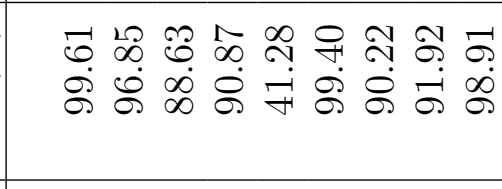 \\
\hline 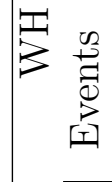 & 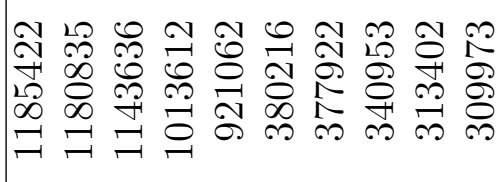 \\
\hline 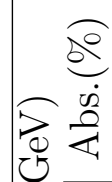 & 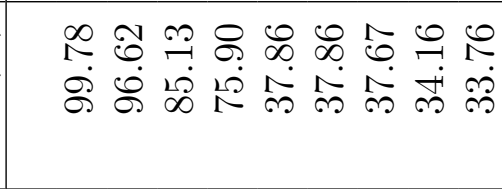 \\
\hline 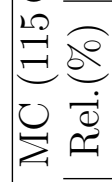 & 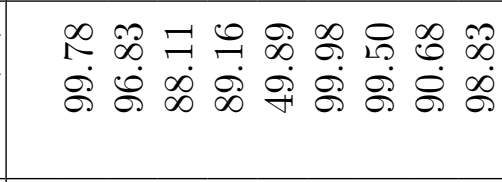 \\
\hline 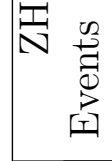 & 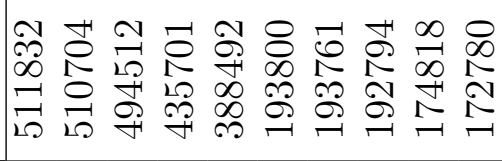 \\
\hline$\underbrace{2}_{\substack{20 \\
\frac{0}{4}}}$ & 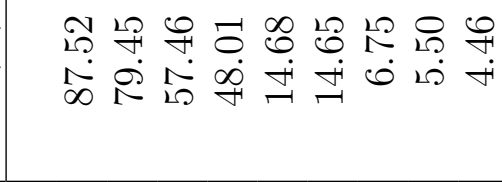 \\
\hline 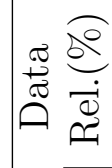 & 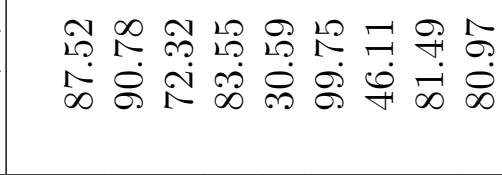 \\
\hline 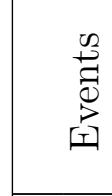 & 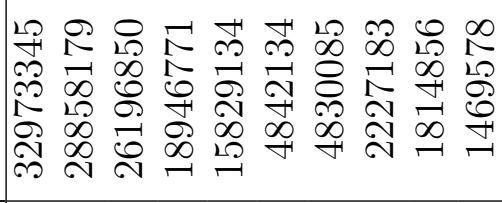 \\
\hline $\begin{array}{l}7 \\
.0 \\
\stackrel{0}{0} \\
0 \\
0 \\
0 \\
0\end{array}$ & 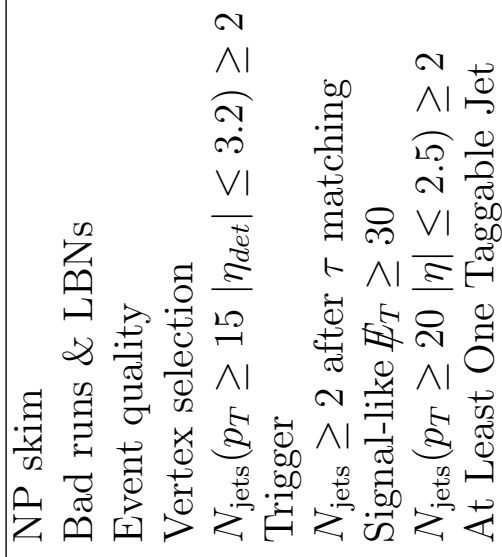 \\
\hline
\end{tabular}




\begin{tabular}{|c|c|}
\hline 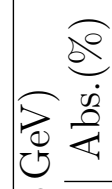 & 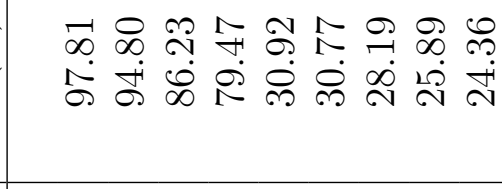 \\
\hline 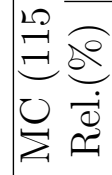 & 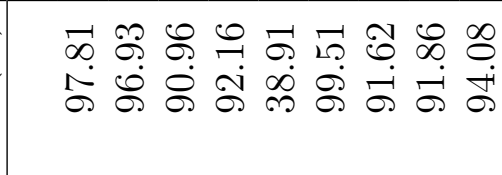 \\
\hline 焉 & 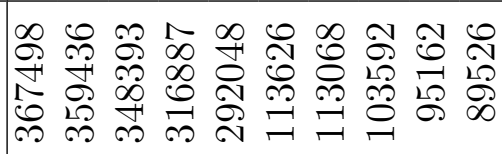 \\
\hline 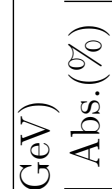 & 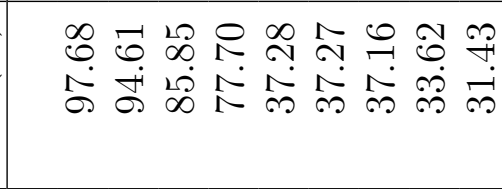 \\
\hline 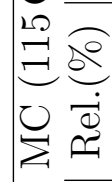 & 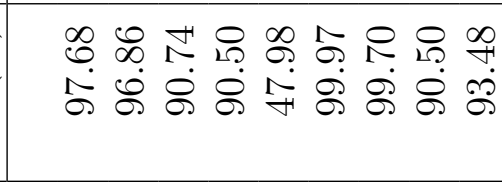 \\
\hline 志 & 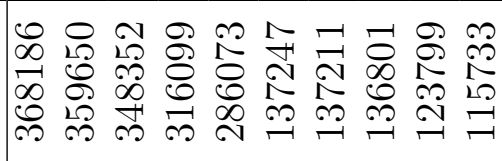 \\
\hline$\underbrace{\overparen{1}}_{\frac{\dot{d}}{2}}$ & 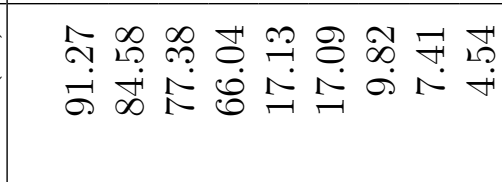 \\
\hline 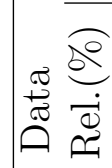 & 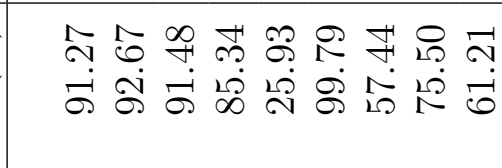 \\
\hline 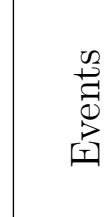 & 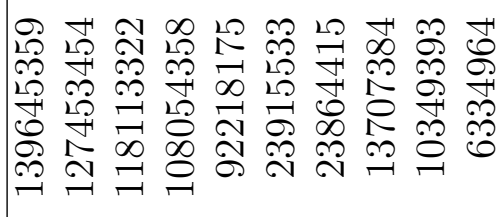 \\
\hline 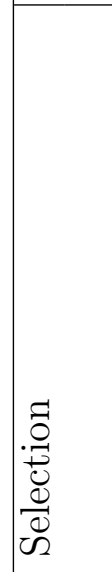 & 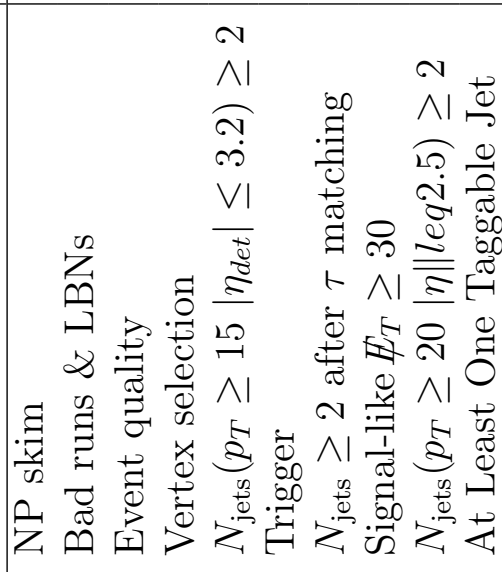 \\
\hline
\end{tabular}


Before describing the samples themselves, the object identification should be discussed.

\subsubsection{Lepton Identification}

To ensure orthogonality to the WH analysis [102] in the signal sample we veto events that contain isolated leptons satisfying the following definitions:

A MC event with a lepton is removed only if a random number uniformly generated between 0 and 1 , is lower than the product of the ratios of data/MC identification efficiencies.

Electrons:

- Point1 selection criteria within $\left|\eta_{\text {det }}\right|<1.1$

- Point2 selection criteria within $1.5<\left|\eta_{\text {det }}\right|<2.5$

- $p_{T}>15 \mathrm{GeV}$

Muons:

- Medium quality selection criteria

- $\left|\eta_{\text {det }}\right|<2.0$

- $p_{T}>15 \mathrm{GeV}$

- Track new medium track quality

- Tight track scaled isolation

The veto on the above defined leptons does not remove all leptons from the events. There remains both muon and electrons which fail the above criteria and hadronicly 
decaying tau leptons. The criteria for these objects is as follows in order from highest to lowest quality. We classify these leptons in an exclusive manner using the highest quality definition satisfied. The modeling of these objects was found to be in good agreement for all kinematic variables.

Point1 Electrons:

- Point1 selection criteria within $1.5<\left|\eta_{\text {det }}\right|<2.5$

- $p_{T}>15 \mathrm{GeV}$

Point0 Electrons:

- Point0 selection criteria within $\left|\eta_{\text {det }}\right|<1.1$ or $1.5<\left|\eta_{\text {det }}\right|<2.5$

- $p_{T}>15 \mathrm{GeV}$

The minimal electron quality:

- CAL isolation $<0.15$

- EM fraction $>0.9$

- HMatrix $8<10000$

- $\left|\eta_{\text {det }}\right|<2.5$

- $p_{T}>10 \mathrm{GeV}$

Muons selected in high mass Higgs searches.

- Loose quality selection criteria

- $\left|\eta_{\text {det }}\right|<2.0$ 
- $p_{T}>15 \mathrm{GeV}$

- Track new medium track quality

- Loose track scaled isolation

The minimal muon quality:

- 'MediumNSeg3', cosmic veto, and $\chi^{2}$ from global matching $<100$

- $\left|\eta_{\text {det }}\right|<2.0$

- $p_{T}>10 \mathrm{GeV}$

- no track quality

- $\Delta \mathrm{R}($ all jets $) \geq 0.5$ isolation

Tau Type1:

- Not overlapping with a muon

- $E_{T}>12.5 \mathrm{GeV}$

- $p_{\text {Ttrk }}>7.0 \mathrm{GeV}$

- $E / p>0.65$

- $\tau_{N N}>0.92$

- $\left|\eta_{\text {det }}\right|<2.0$

Tau Type2:

- Not overlapping with a muon

- $E_{T}>12.5 \mathrm{GeV}$ 
- $p_{\text {Ttrk }}>5.0 \mathrm{GeV}$

- $E / p>0.50$

- $\tau_{N N}>0.9$

- $\left|\eta_{\text {det }}\right|<2.0$

By identifying every object in the event, understanding of the data set has improved. Also these leptons provide a handle by which ' $Z H$ '-like and ' $W H$ '-like events can be separated. By rejecting events which contain these leptons, a $Z+$ jets dominated sample is selected, providing further cross checks of the background modeling.

\section{Jets}

In this analysis all good jets are corrected with the final Jet Energy Scale and those where a muon is found within the cone the JESMU correction is applied. As described in Section 4.6.4, JSSR is applied to MC jets.

The uncorrected $\not_{T}$ is computed from all calorimeter cells, except the cells in the coarse hadronic calorimeter which were not included in a good reconstructed jet. All corrections on the jets are propagated to $\mathbb{E}_{T}$. This includes JES for data and MC, and JSSR jet corrections for the MC. We treat the overlap between jets and EM clusters by removing jets which overlap with an electron which satisfies the Point0 criteria with $p_{T}>15 \mathrm{GeV}$. However, when correcting the $\not_{T}$, the energy in the calorimeter cells associated with jets overlapping electrons passing the minimal electron requirement (see section 10.3.2) and have transverse momentum greater then $5 \mathrm{GeV}$ are corrected to the electron's energy not the JES corrected jet energy. Jets overlapping type 1 and 2 taus which satisfy the criteria outlined in Section 10.3.2 are also treated as taus and the correction for these objects is propagated to the $\not_{T}$. $\mathbb{E}_{T}$ is also corrected for the presence of the muons passing the minimum quality requirements in Section 10.3.2. 


\section{Bad Jets}

Events that contain bad jets with $p_{T}>15 \mathrm{GeV}$ are rejected. In this context we denote a bad jets as those which do not pass the Good Jet criteria outlined in Section 4.6.2. Events with bad jets contribute largely to the instrumental background for two reasons: a) the JES correction is not applied to these jets, thus their $p_{T}$ is mis-measured which is subsequently propagated to the $\not_{T}$; b) coarse hadronic cells for the $\mathbb{E}_{T}$ computation are only taken into account if they belong to jets passing the Good Jet criteria. One of the reasons why these bad jets fail the criteria is the CHF being larger than 0.4. Thus, we expect in these events a large energy deposited in the coarse hadronic part of the calorimeter which is not taken into account for the $E_{T}$ computation.

To check the effect of the bad jet veto on the multijet background, and overall modeling of the data, the veto was inverted in the "signal sample" (described in Section 10.3.3). It can be seen from Figure 10.2 that the modeling (purple histogram) of the bad jets matches very well the data (data points), once the small standard model contributions are included. This gives confidence that the bad-jet veto does not bias the multijet modeling.

\section{EM Jets}

Events with two good jets are not rejected if they have only one bad jet with the only failed criterion being the EM fraction $>0.95$, to retain acceptance for the $W H$ signal where the electron from the $W$ decay fakes a jet. The JES corrections are not applied to these jets as they are misidentified EM objects. All good jets and retained bad jets are propagated to the $H_{T}$ and $H_{T}$ calculation. 

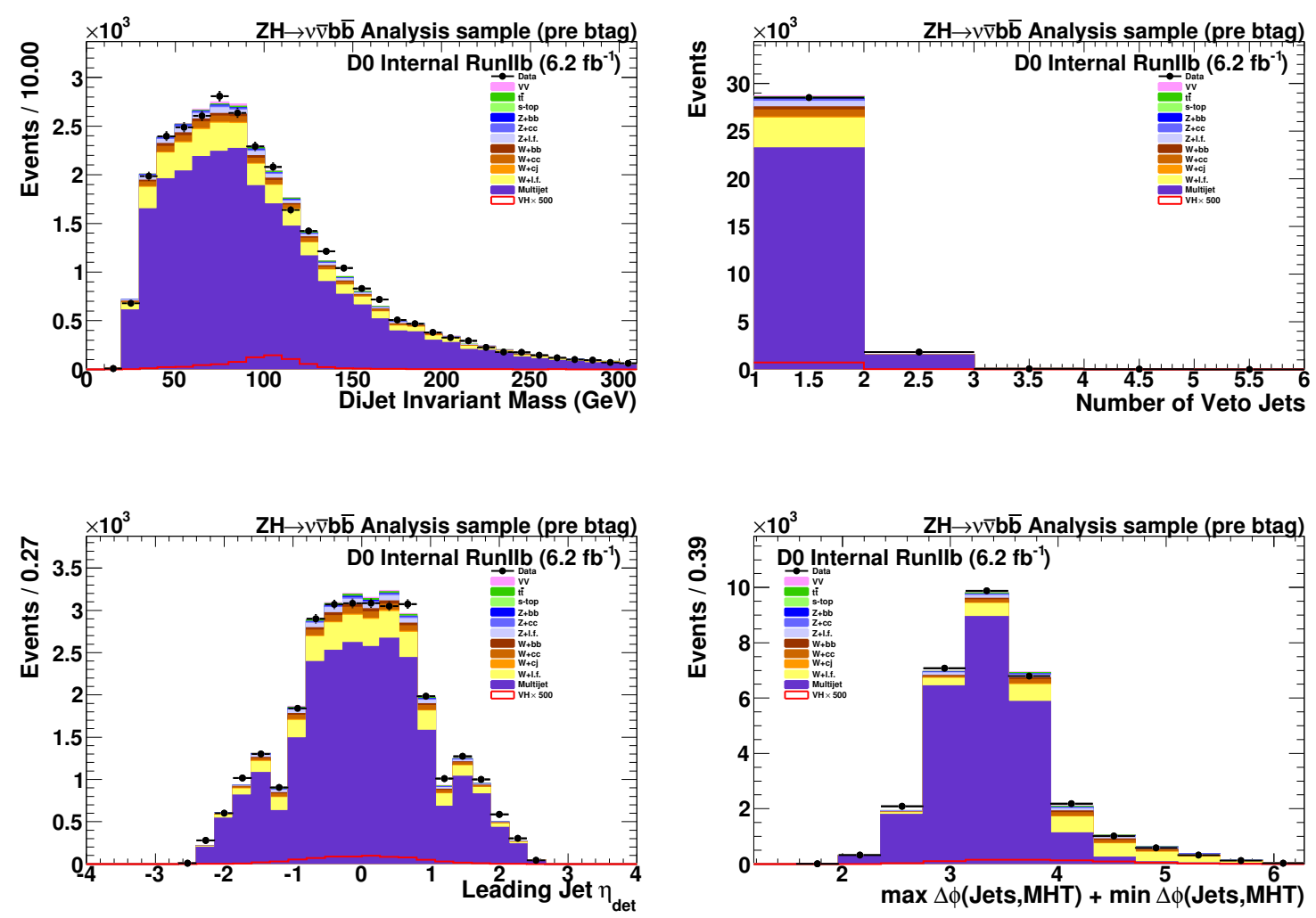

Figure 10.2: Distributions in events where a jet fails the Good Jet criteria not considering those for which the only bad-jet criterion is EM fraction $>0.95$ and which otherwise pass the cuts applied in the signal sample. 


\subsubsection{Signal Selection}

The signal sample is selected as follows:

- The highest $p_{T}$ good jet in the event has to be taggable.

- At least two but no more than three taggable jets with $p_{T}>20 \mathrm{GeV}$ and $|\eta|<2.5$

- Acoplanarity $\Delta \phi\left(\right.$ jet $_{1}$, jet $\left._{2}\right)<165^{\circ}$. The two leading jets must not be back-toback in the plane transverse to the beam direction.

- $E_{T}>40 \mathrm{GeV}$

- $E_{T}$ Significance $>5$.

- Veto on isolated electrons and muons as described in section 10.3.2.

- $\mathcal{D}<\pi / 2$, where $\mathcal{D}=\Delta \phi\left(\not_{T}, \not p_{T}^{\text {trk }}\right)$. For signal, the missing track- $p_{T}, \not p_{T}^{\text {trk }}$, defined as the opposite of the vectorial sum of the charged particle transverse momenta, is expected to point in a direction similar to that of $\mathbb{E}_{T}$. This is not expected in multijet events, in which the $\mathbb{E}_{T}$ originates mainly from mismeasurements of jet energies. This defines the "Signal Band".

\subsubsection{Sideband and MJ-model Sample}

The variable $\mathcal{D}$ is used to define the "signal sideband". It is selected in the same way as the signal sample, except that the previous requirement $\mathcal{D}<\pi / 2$ is now inverted. To define the multijet model (MJ-model), the small contribution from SM processes in that $\mathcal{D}>\pi / 2$ sideband is subtracted, and the resulting sample is used to model the multijet background shape in the signal sample, i.e., in the $\mathcal{D}<\pi / 2$ region. The MJ-model sample normalization is explained in Section 10.4. 
An MJ-model sample is constructed for the multijet control sample (Section 10.6) using the same procedure with a normalization explained in Section 10.4.

\subsubsection{Cut Flow}

The number of events after each selection cut for the MC signal samples and the observed data events in the signal sample for Run IIb1 and Run IIb2-3 can be seen in Table 10.5 and 10.6 respectively. 


\begin{tabular}{|c|c|}
\hline 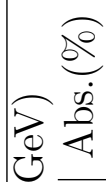 & 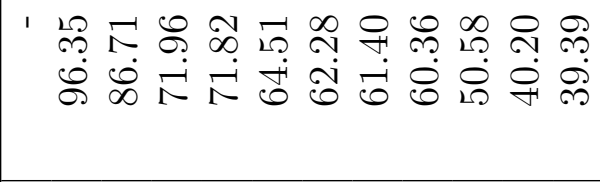 \\
\hline 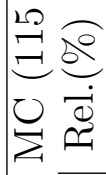 & 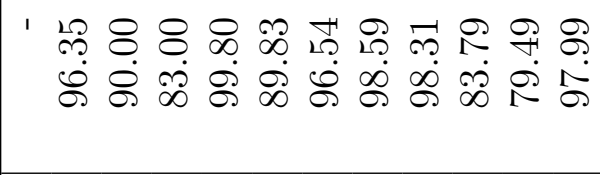 \\
\hline 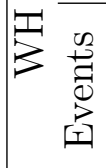 & 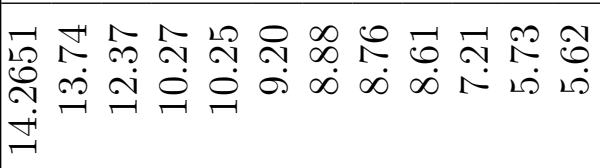 \\
\hline 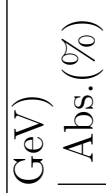 & 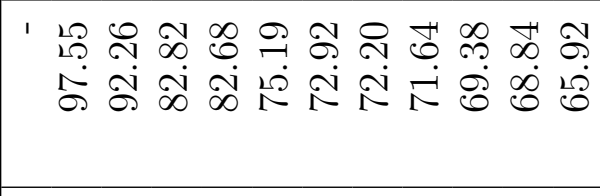 \\
\hline 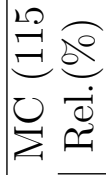 & 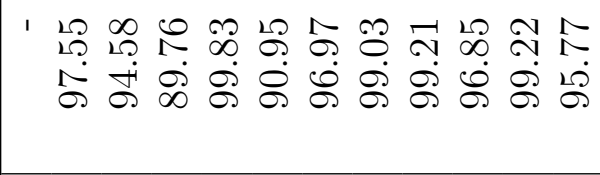 \\
\hline 焉 & 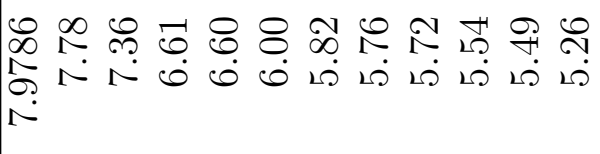 \\
\hline$\underbrace{\frac{0}{4}}_{\frac{\dot{0}}{2}}$ & 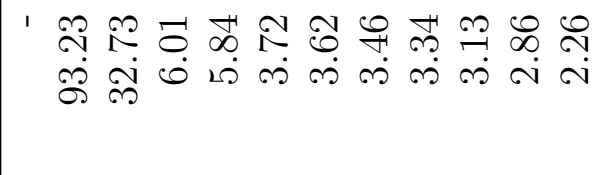 \\
\hline 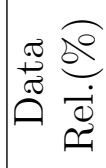 & 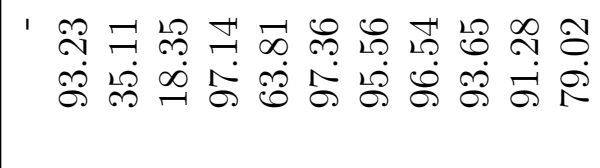 \\
\hline 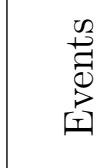 & 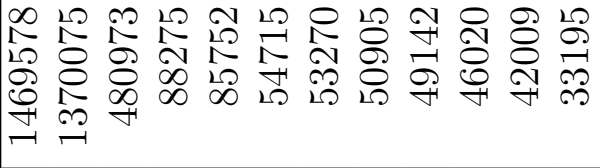 \\
\hline 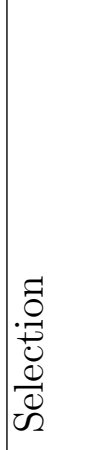 & 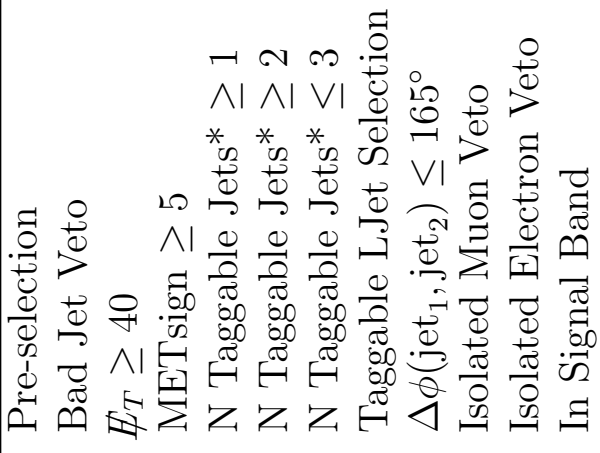 \\
\hline
\end{tabular}




\begin{tabular}{|c|c|}
\hline 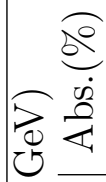 & 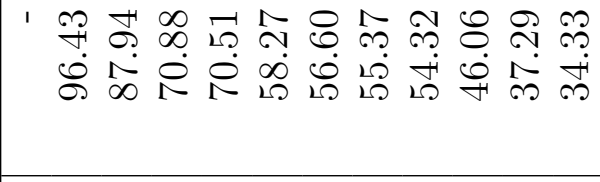 \\
\hline 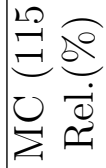 & 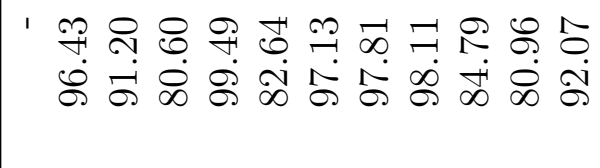 \\
\hline 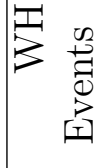 & 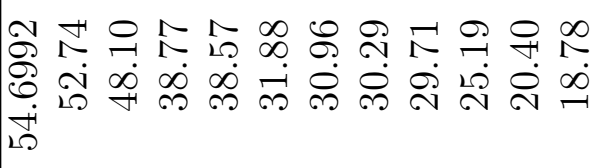 \\
\hline 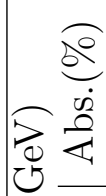 & 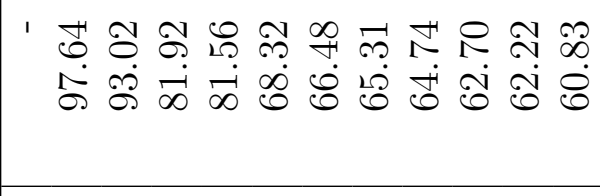 \\
\hline 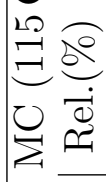 & 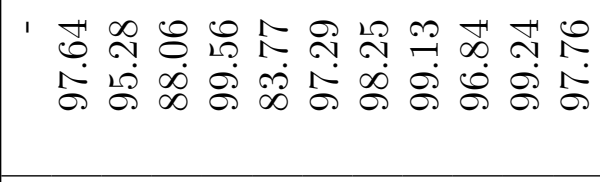 \\
\hline 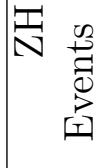 & 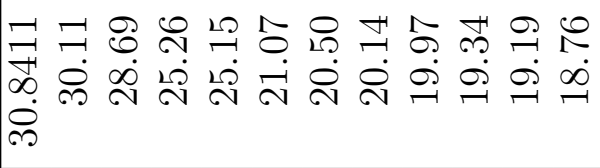 \\
\hline$\underbrace{\underbrace{2}_{1}}_{\frac{\dot{\theta}}{2}}$ & 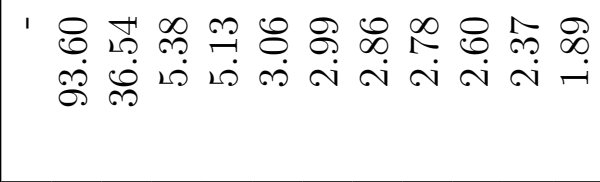 \\
\hline 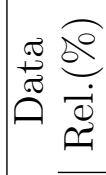 & 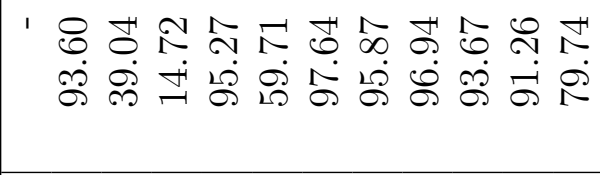 \\
\hline 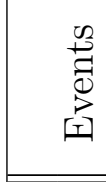 & 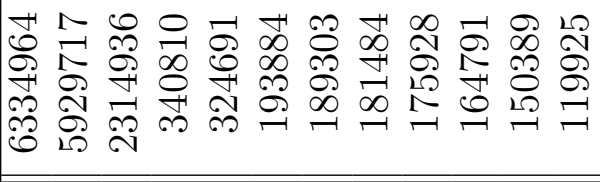 \\
\hline 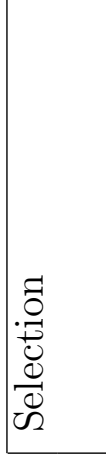 & 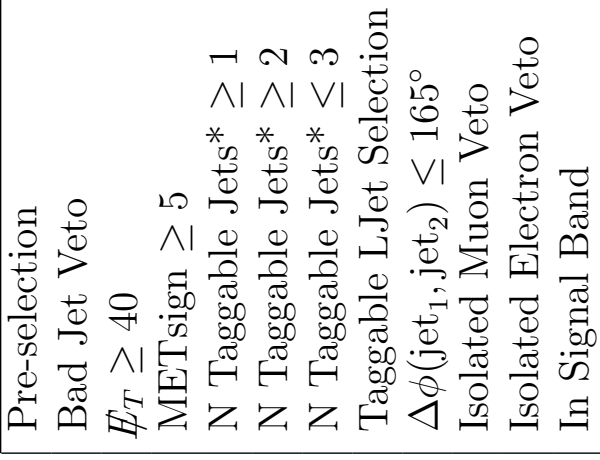 \\
\hline
\end{tabular}




\subsection{6 $\quad E_{T}$ Quality}

As discussed in Chapter 5 , the $\mathscr{E}_{T}{ }^{\prime}$ and $\mathscr{E}_{T}$ Significance distinguish between events with real and fake $\not_{T}$. The multijet background, discussed in Section 10.3.4, predominantly arises from fluctuations in the measurement of jet energies. Either one or both of these $\mathbb{E}_{T}$-based variables should be able to assist us in controlling this multijet background. We found that the most sensitive part of the $\mathscr{E}_{T}{ }^{\prime}$ variable was the transverse component of the di-jet $p_{T}$ reduced after considering the jet resolutions and the calorimeter recoil correction (Figure 10.3). Dropping the missing $E_{T}$ significance cut and comparing the two we see that the $\mathbb{E}_{T}$ significance outperforms this component of $E_{T}{ }^{\prime}$. Cutting above $50 \mathrm{GeV}$ on the recoil-subtracted di-jet $p_{T}$ transverse to the thrust axis would remove a lot of the multijet background, but the separation in the $E_{T}$ significance variable is much better (Figure 10.4). This component of $\not_{T}{ }^{\prime}$ provides valuable information to the multijet DT through the use of the recoil subtracted dijet $p_{T}$ transverse component. The track activity correction is not applied since the MJ-model definition somewhat biases the description of the tracking variables. 

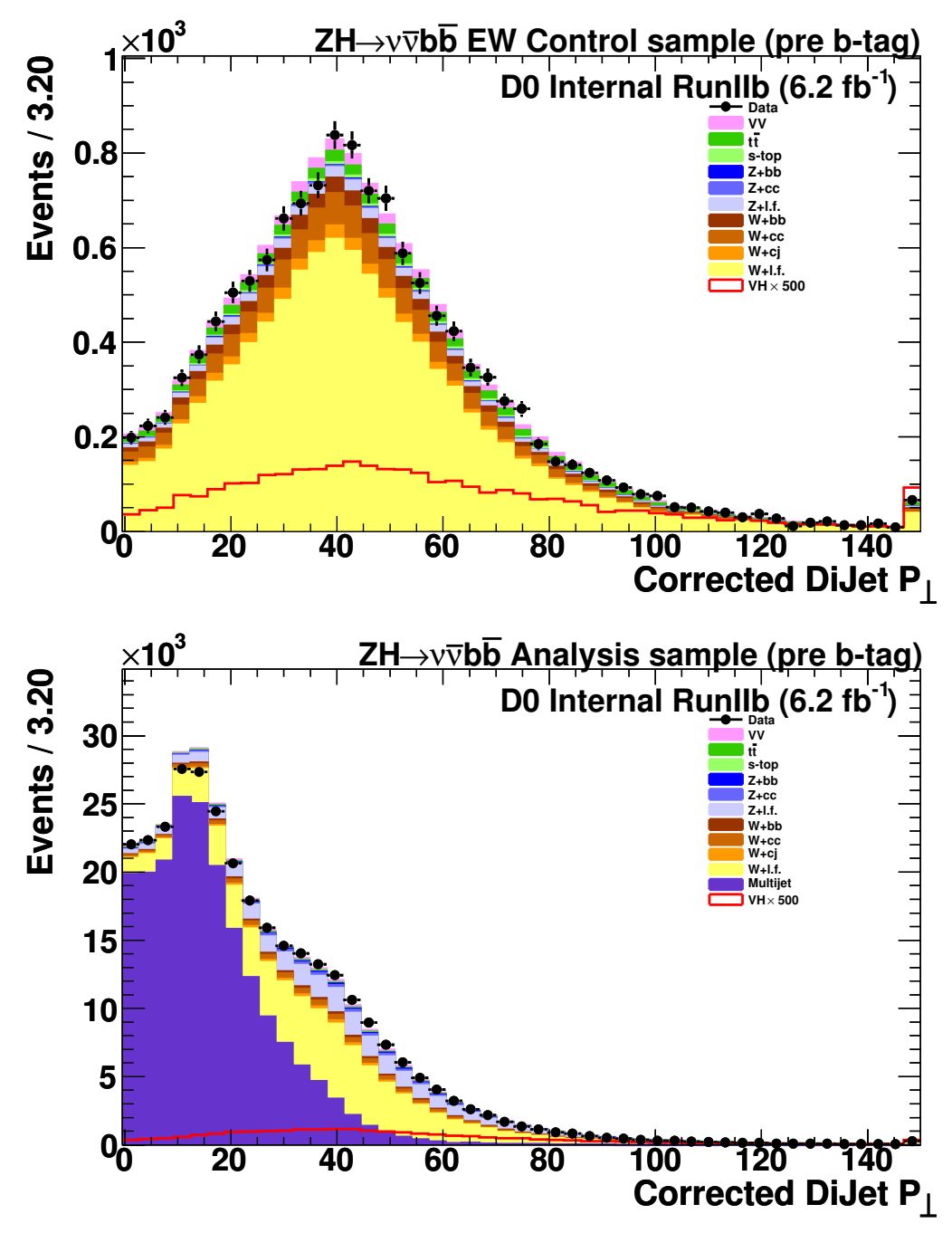

Figure 10.3: The transverse component of the di-jet $p_{T}$ with respect to the thrust axis after the jet resolution and recoil activity corrections in the electroweak control sample (top) and the analysis sample (bottom) without any cut on $\not_{T}$ significance. It is clear that the shape of the instrumental and physics backgrounds differ, however the separation is not as clear as with $\not_{T}$ significance. 

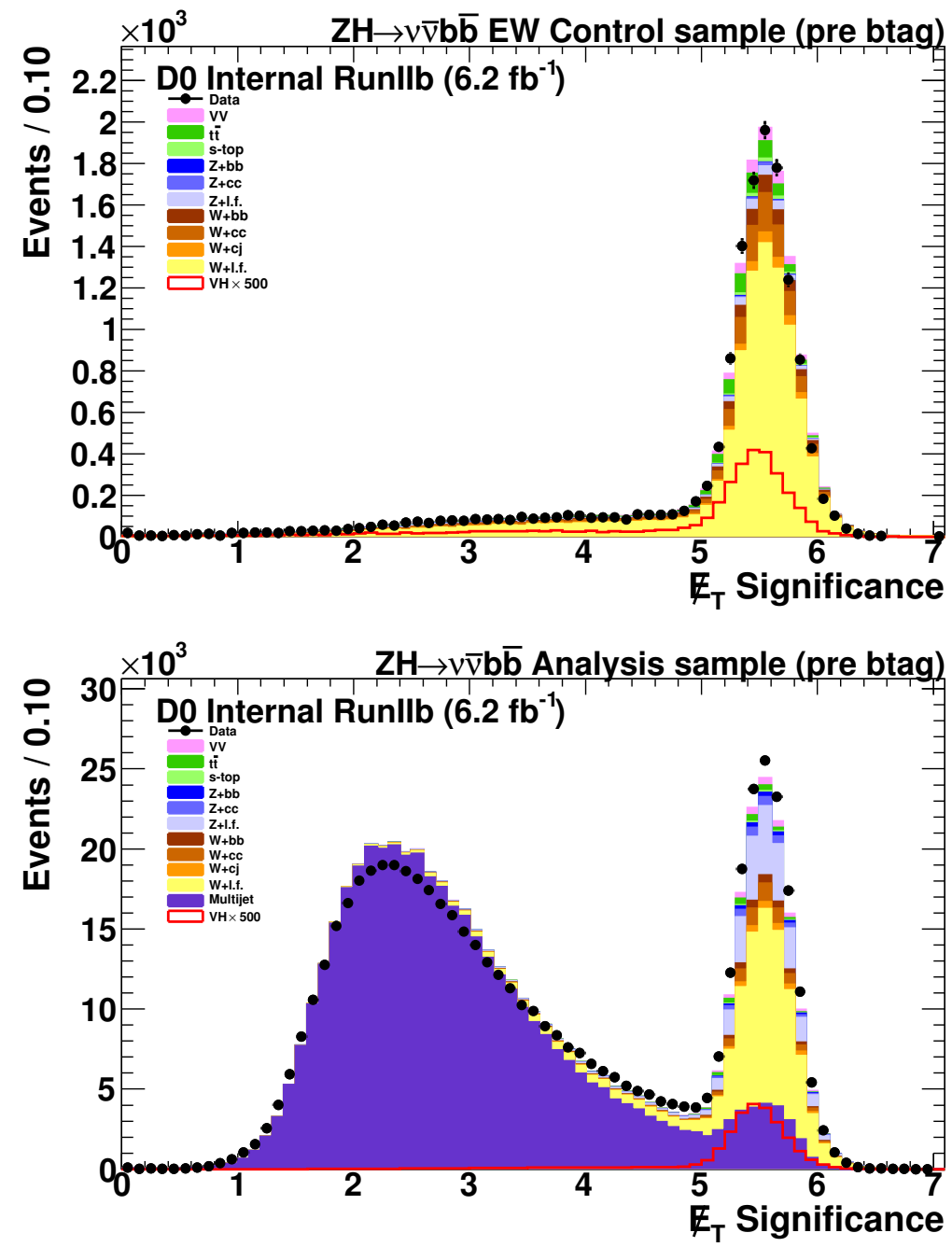

Figure 10.4: Missing $E_{T}$ significance in the electroweak control sample (top) and in the analysis sample (bottom) before any cut is applied.

\subsection{7 b-tagging}

We use the standard DØ MVA b-tagging algorithm (Chapter 7) on the two leading taggable jets selected by the analysis cuts. In previous results from this analysis we chose to have one tight (VT) and loose (L3) tagged jet (VT-L3) in the asymmetric double tag sample. Similarly, we defined an orthogonal single tag sample, VT-!L3. 
Events which fail the criteria for the double and single tag channel are gathered in the zero tag channel. Since then we have moved to a symmetric tags at the loosest operating point (L6) producing a L6-L6 double tag and consequently a L6-!L6 single tag sample. This L6 operating point is defined as 0.02 on the $b$-tagging MVA output. Looking at the distributions shown in Appendix D, cutting at this point leaves most of the information contained in this distribution unused. This allows for the unused $b$-tagging information to be included in the multivariate analysis used to discriminate signal from SM backgrounds after $b$-tagging. Using this procedure, we have found a $14 \%$ improvement in the limit when only considering statistical uncertainties. Also allowing more events into the final sample allows the profiling technique to further constrain the systematics in the final limit setting.

\subsection{Normalization}

All of the SM process have been normalized with the theoretical cross section and the total integrated luminosity. The multijet background yield is unknown and therefore estimated from the selected sample itself. In the following section, we adopt a data-driven normalization procedure for the main SM backgrounds and the multijet background. The purpose is to disentangle the shape issues from the overall normalizations when comparing data and MC predictions, and to have the best background description in the training of the decision trees used to discriminate signal from backgrounds. It must however be kept in mind that the final results are obtained by providing input distributions to COLLIE in which the nominal SM background normalizations and uncertainties are maintained.

The processes for which we calculate normalization (or scale) factors are: $(W / Z)+$ jets, the heavy flavor fraction within $(W / Z)+$ jets, top production and the MJ components 
in the 0,1 , and 2 tag samples separately.

In the signal sample before MJ-DT cut, the normalization of all the above samples is calculated by performing a $\chi^{2}$ fit to the jet multiplicity and MJ-DT distributions in the 0,1 and 2 tag samples. The heavy flavor and top scale factors are constrained within their a priori uncertainties, taken to be Gaussian, of $20 \%$ and 10\%, respectively, while the overall $(W / Z)+$ jets scale factor is left unconstrained in the fits. The multijet normalization calculated in the fit is subsequently used in signal sample after MJ-DT cut.

For the electroweak and signal sample after MJ-DT cut, the MJ normalization is removed from the fit with only the jet multiplicity distributions used in the 0,1 and 2-tag samples.

Additionally, in the multijet control sample (Sec. 10.6) the multijet background is normalized in pre, 0,1 , and 2 b-tag sample individually such that the simulation yield is equal to that of the observed events. This normalization is used only for the comparison plots in the MJ control sample. The normalization factor for the multijet model is 1.38 for RunIIb1 and 1.23 for RunIIb2-3 for the pre-tag sample and 1.40 and 1.25 for RunIIb1 and 1.41 and 1.29 for RunIIb2-3 single and double tag, respectively.

The results of these various fits are given in Table 10.7. Since a set of DTs is trained at each mass point, we checked that the training itself does not bias the scale factor determination. We found the variation of these scale factors as a function of Higgs mass is within the uncertainty of the fit errors.

\subsection{Electroweak Control Sample}

We use a $W+$ jets dominated sample to test MC modeling of the electroweak (EW) backgrounds, the trigger simulation and the $b$-tagging performance. This sample is 


\begin{tabular}{|lccc|}
\hline Sample & Electroweak & signal pre-MJDT cut & signal after MJDT cut \\
\hline \multicolumn{3}{c|}{ RunIIb1 } \\
\hline$(W / Z)+$ jets & $1.00 \pm 0.03$ & $1.03 \pm 0.02$ & $1.02 \pm 0.02$ \\
Heavy flavors & $0.88 \pm 0.16$ & $0.76 \pm 0.10$ & $0.76 \pm 0.12$ \\
Top & $0.96 \pm 0.09$ & $0.93 \pm 0.09$ & $0.96 \pm 0.09$ \\
\hline \multicolumn{3}{c}{ RunIIb2-3 } \\
\hline$(W / Z)+$ jets & $1.01 \pm 0.02$ & $1.07 \pm 0.01$ & $1.02 \pm 0.01$ \\
Heavy flavors & $1.02 \pm 0.12$ & $0.79 \pm 0.06$ & $0.96 \pm 0.07$ \\
Top & $1.00 \pm 0.08$ & $1.04 \pm 0.07$ & $0.99 \pm 0.08$ \\
\hline
\end{tabular}

Table 10.7: Scale factors obtained from fits in the various samples.

orthogonal to the signal sample, but has similar event topology, and is virtually void of instrumental background.

A tight isolated muon is acquired by reversing the muon veto in the basic selection $\left(p_{T}>15 \mathrm{GeV},|\eta|<2\right)$. The muon track information is removed from all variables to simulate the event topology of our signal sample. On top of the cuts used in the analysis sample the tight muon-corrected $\mathscr{H}_{T}>20 \mathrm{GeV}$ and the transverse mass of the $W$ candidate is greater than $30 \mathrm{GeV}$ are required. These additional cuts are used to reject the remaining multijet contribution, which has been verified to be less than $0.1 \%$ [103]. Therefore the multijet background is neglected in this sample.

With the scale factors from Table 10.7, the numbers of events observed and expected from the various background sources are given in Table 10.8 before $b$ tagging, for an exclusive L6 tag, and for a symmetric L6-L6 double tag.

Plots of various variable distributions before $b$-tagging can be seen in Figures 10.510.6, for a sample with one L6 b-tag that is orthogonal to the double tag sample in Figures 10.7-10.8, and after double symmetric L6-L6 tagging in Figures 10.9-10.10. Overall, there is good agreement between the observed data and the expected background, both before and after $b$-tagging. 


\begin{tabular}{|l|rl|rl|rl|}
\hline Sample & \multicolumn{2}{|c|}{$\begin{array}{c}\text { Before } \\
\text { b-tagging }\end{array}$} & \multicolumn{2}{|c|}{$\begin{array}{c}\text { L6 and } 1 ! \mathrm{L} 6 \\
\text { tag }\end{array}$} & \multicolumn{2}{c|}{$\begin{array}{c}\text { L6 } \\
\text { tags }\end{array}$} \\
\hline$Z H(115 \mathrm{GeV})$ & 0.27 & \pm 0.01 & 0.12 & \pm 0.00 & 0.12 & \pm 0.00 \\
$W H(115 \mathrm{GeV})$ & 4.95 & \pm 0.06 & 2.05 & \pm 0.04 & 2.36 & \pm 0.04 \\
\hline$W+$ jets & 8109.87 & \pm 34.20 & 1673.00 & \pm 12.52 & 114.57 & \pm 2.85 \\
$W+\mathrm{c}+$ jet & 318.45 & \pm 6.72 & 133.46 & \pm 4.20 & 14.02 & \pm 1.29 \\
$W+\mathrm{cc}$ & 1020.73 & \pm 10.94 & 375.15 & \pm 6.38 & 69.34 & \pm 2.60 \\
$W+$ bb & 465.10 & \pm 5.87 & 237.54 & \pm 4.30 & 101.89 & \pm 2.60 \\
$Z+$ jets & 263.51 & \pm 5.49 & 57.04 & \pm 2.10 & 4.46 & \pm 0.47 \\
$Z+\mathrm{cc}$ & 49.12 & \pm 8.47 & 17.21 & \pm 2.17 & 9.45 & \pm 6.84 \\
$Z+$ bb & 19.11 & \pm 0.51 & 10.25 & \pm 0.38 & 4.12 & \pm 0.21 \\
single top & 104.16 & \pm 0.88 & 58.32 & \pm 0.63 & 29.46 & \pm 0.47 \\
$t \bar{t}$ & 525.95 & \pm 5.36 & 258.13 & \pm 3.95 & 188.78 & \pm 2.73 \\
di-boson & 386.38 & \pm 3.22 & 119.24 & \pm 1.74 & 17.52 & \pm 0.65 \\
\hline Total Bgrd & 11262.40 & \pm 38.87 & 2939.36 & \pm 16.18 & 553.62 & \pm 8.86 \\
Observed & 11270.00 & 2910.00 & 540.00 \\
\hline
\end{tabular}

Table 10.8: Run IIb number of events after applying all analysis cuts including requiring the transverse mass of the $W$ candidate to be greater than $30 \mathrm{GeV}$ and after b-tagging using different combinations of the L6 MVA operation points in the Electro-Weak control sample Errors are statistical errors only. 

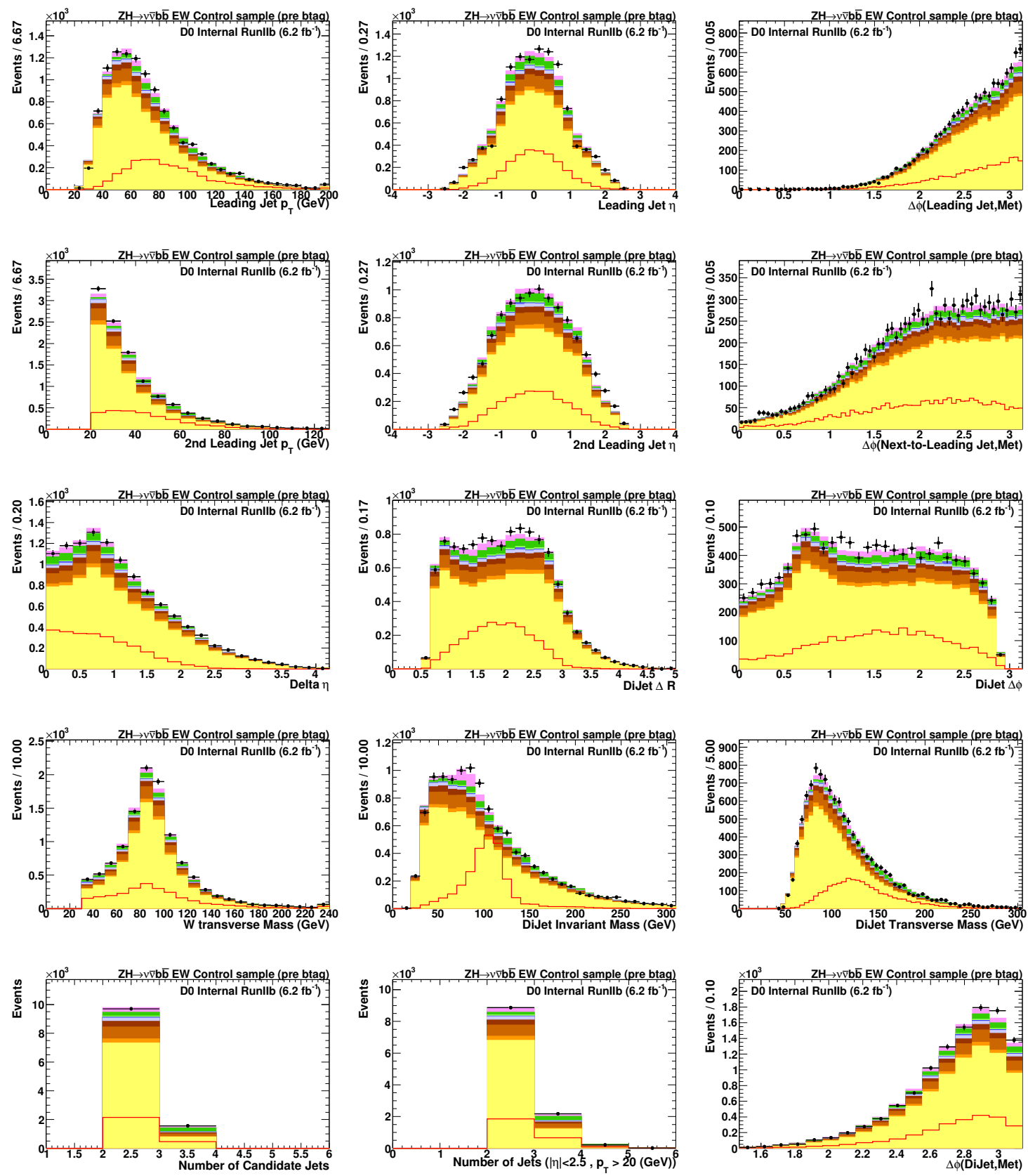

Figure 10.5: Electroweak control sample with before $b$-tagging. 

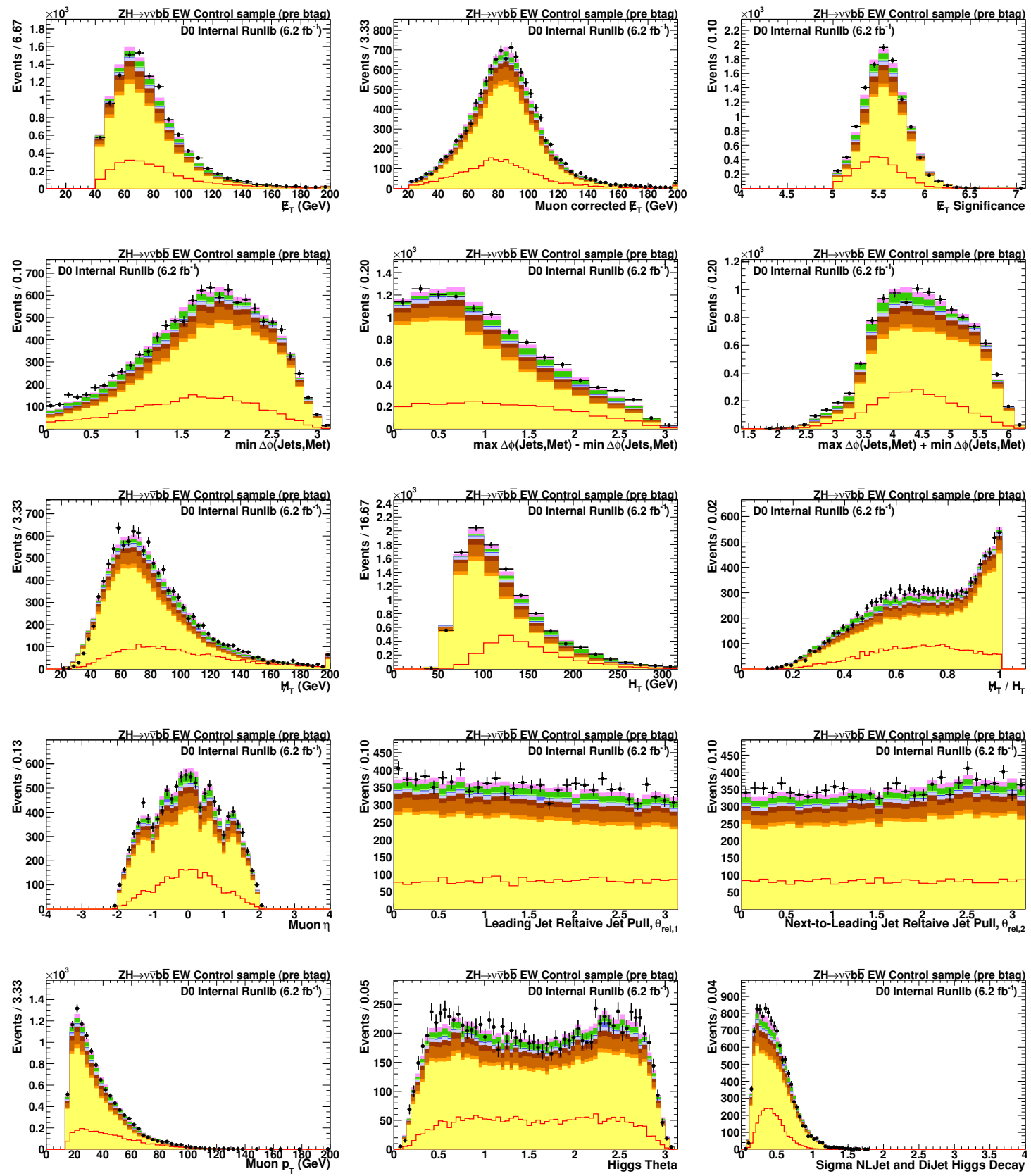

Figure 10.6: Electroweak control sample with before $b$-tagging. 

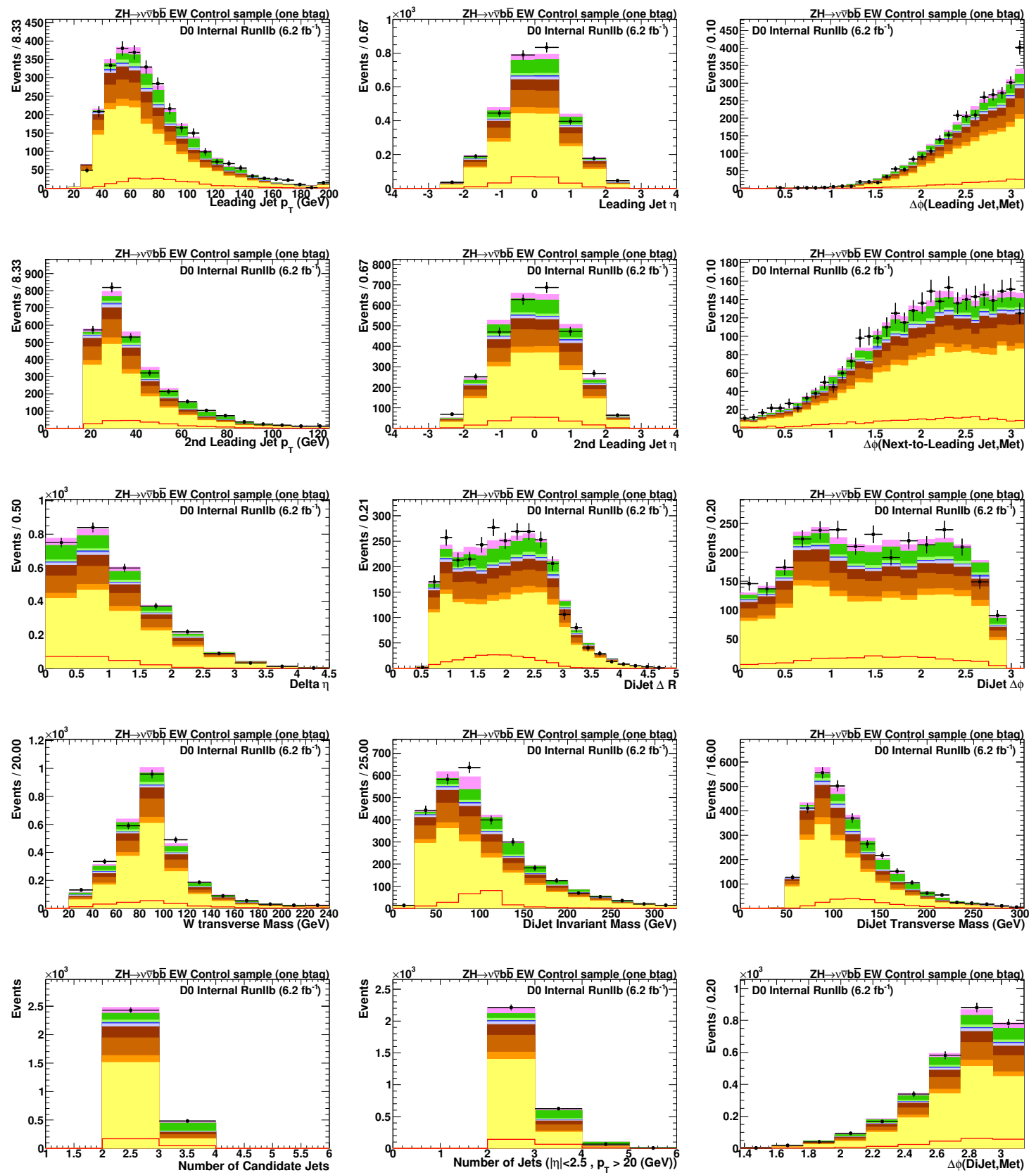

Figure 10.7: Electroweak control sample with one $b$-tags. 

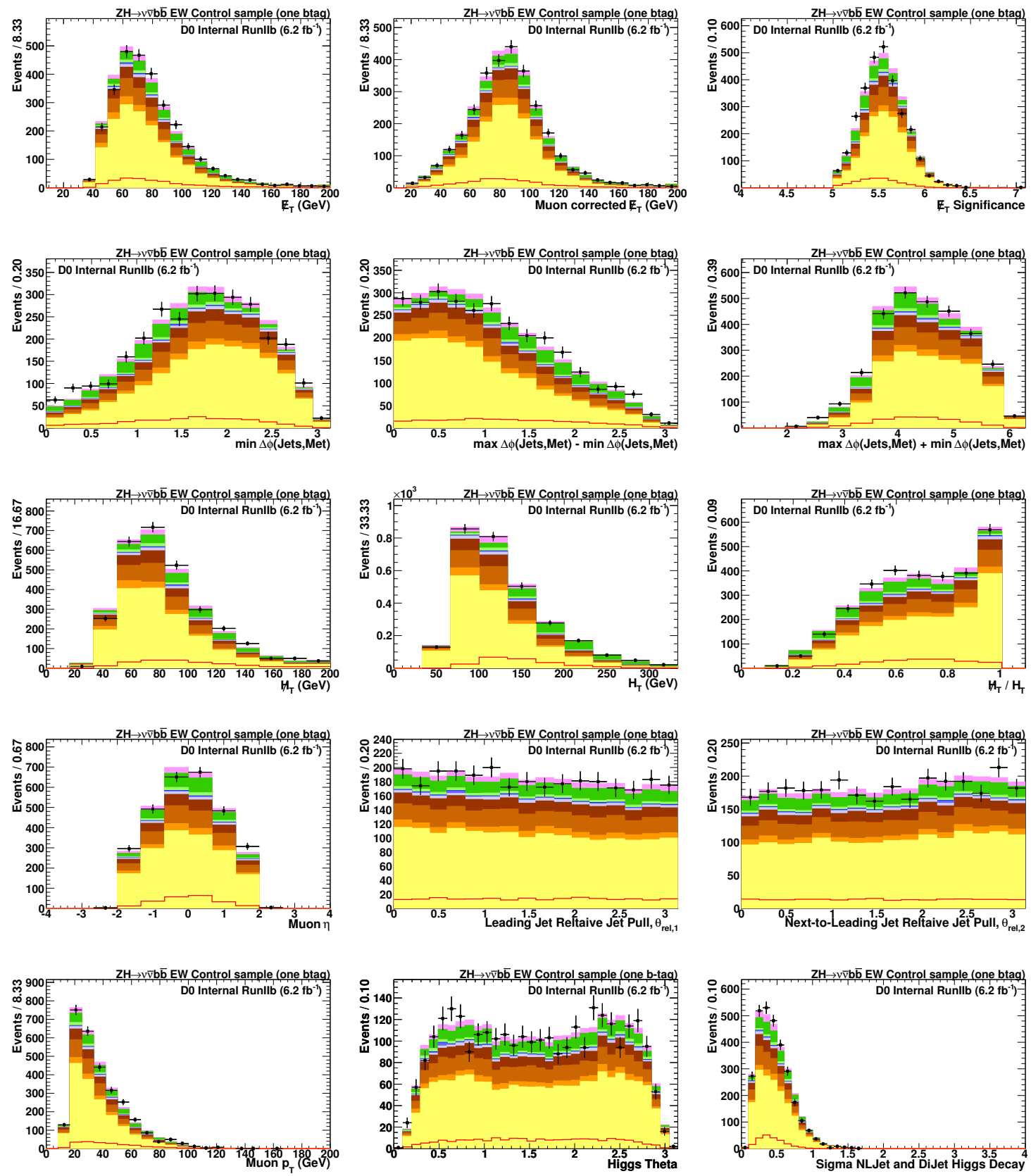

Figure 10.8: Electroweak control sample with one $b$-tags. 

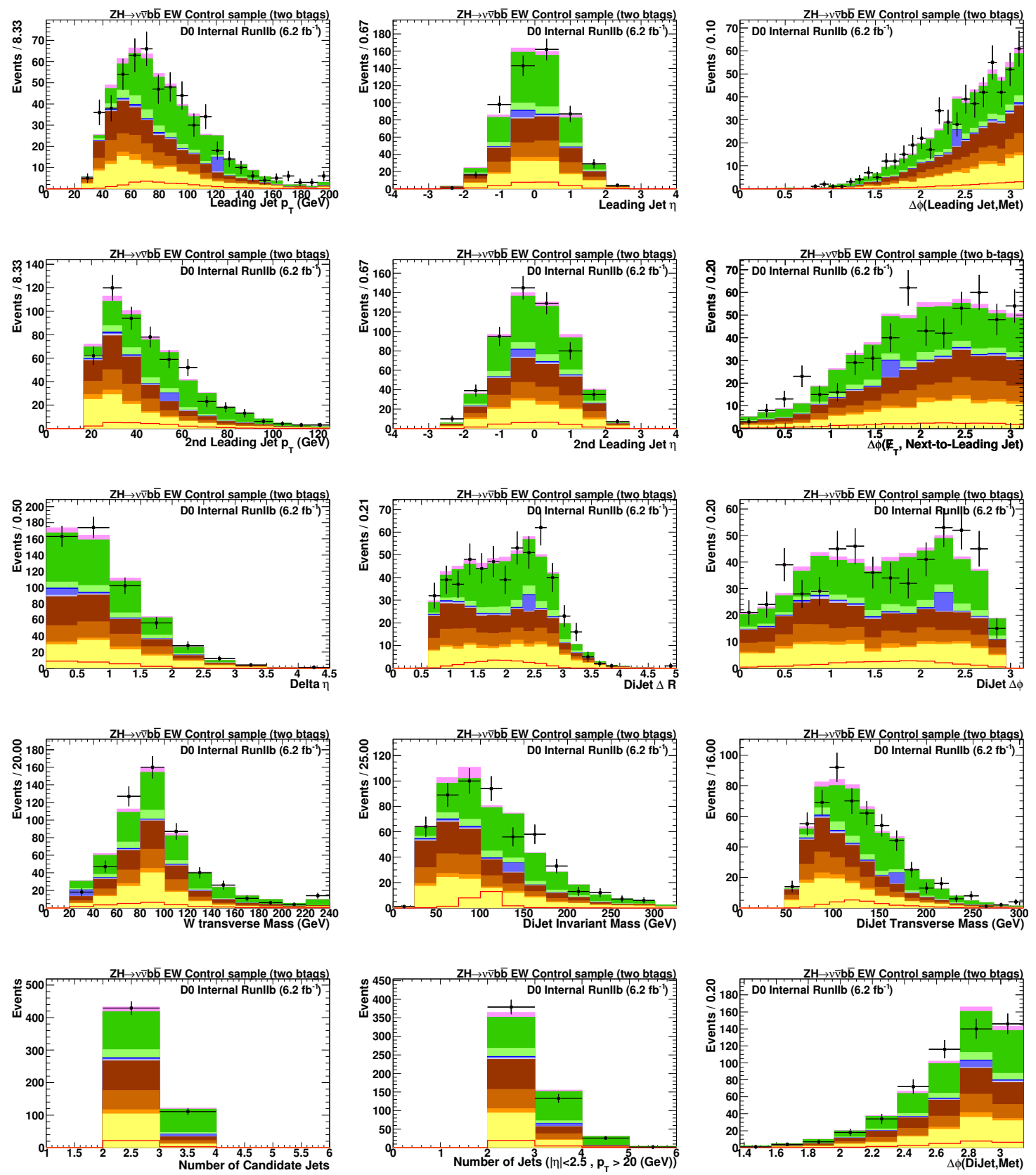

Figure 10.9: Electroweak control sample with two $b$-tags. 

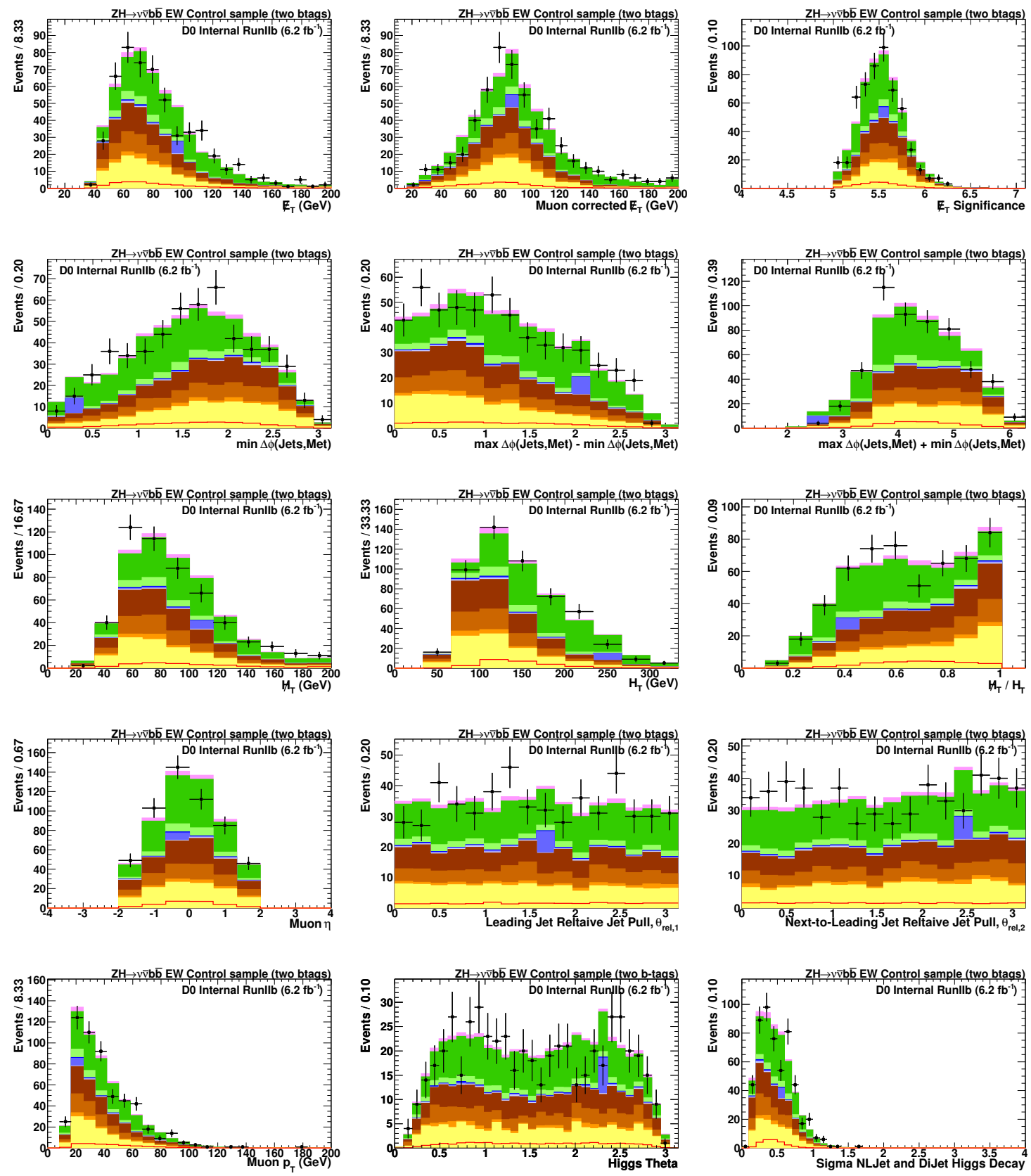

Figure 10.10: Electroweak control sample with two $b$-tags. 


\subsection{Multijet Control Sample}

As described in Section 10.3.4, the variable $\mathcal{D}=\Delta \phi\left(\not_{T}, \not p_{T}^{\mathrm{trk}}\right)$ is used to define a sample dominated by the multijet background. It is selected in the same way as the signal sample, except that the cut $\mathcal{D}<\pi / 2$ is now inverted, we call this our signal sideband. The $\not p_{T}^{\text {trk }}$ is computed only with tracks that originate from the primary vertex within $d c a<2 \mathrm{~mm}$ and $d c a_{z}<5 \mathrm{~mm}$ and have a $p_{T} \leq 400 \mathrm{GeV}$. The latter cut is used to reject fake tracks, as most tracks with a very high $p_{T}$ are fake. After SM background subtraction, the signal sideband is used as our multijet-model in the signal sample, i.e., in the $\mathcal{D}<\pi / 2$ region. The distribution of $\mathcal{D}$ after applying all selection cuts, before b-tagging, is plotted in Figure 10.11 for the analysis sample and in Figure 10.12 for the electroweak control sample. Note that a discontinuity in the $\mathcal{D}$ distribution is expected because of the triangle cut in the sideband region as explained below. The normalization of this sample is explained in Section 10.4.
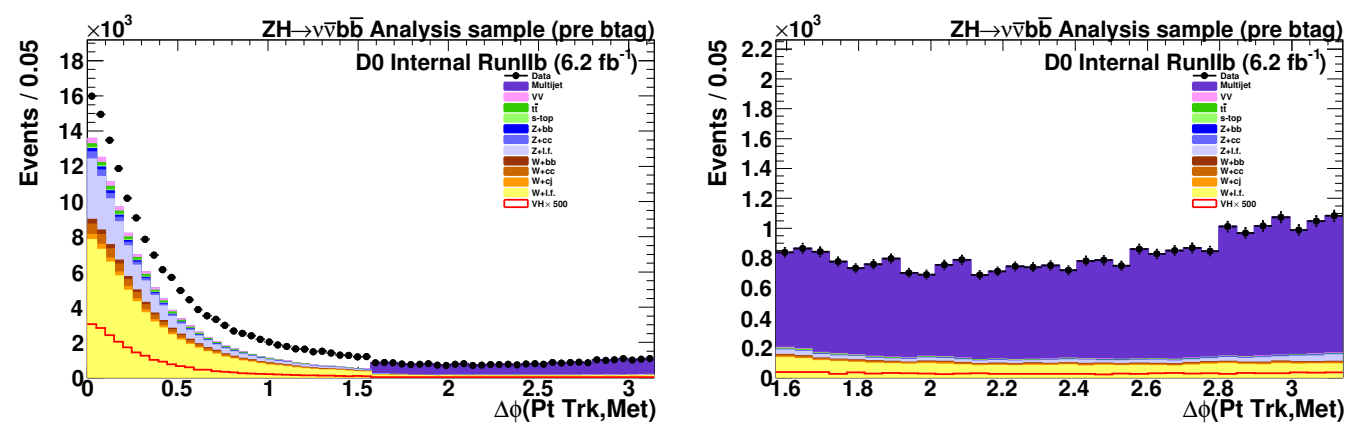

Figure 10.11: The left plot shows the $\mathcal{D}$ variable. The purple distribution is used to model the multijet background (MJ-model sample). The right plot shows in more detail the $\mathcal{D}>\pi / 2$ region and the SM contribution which is subtracted from the data to obtain the multi-jet model.

To test our multijet-background modeling procedure, a multijet control sample is defined which is greatly enhanced in multijet events. This sample is selected in the same way as the signal sample, but the $\not_{T}$ cut is relaxed from 40 to $30 \mathrm{GeV}$ and the 

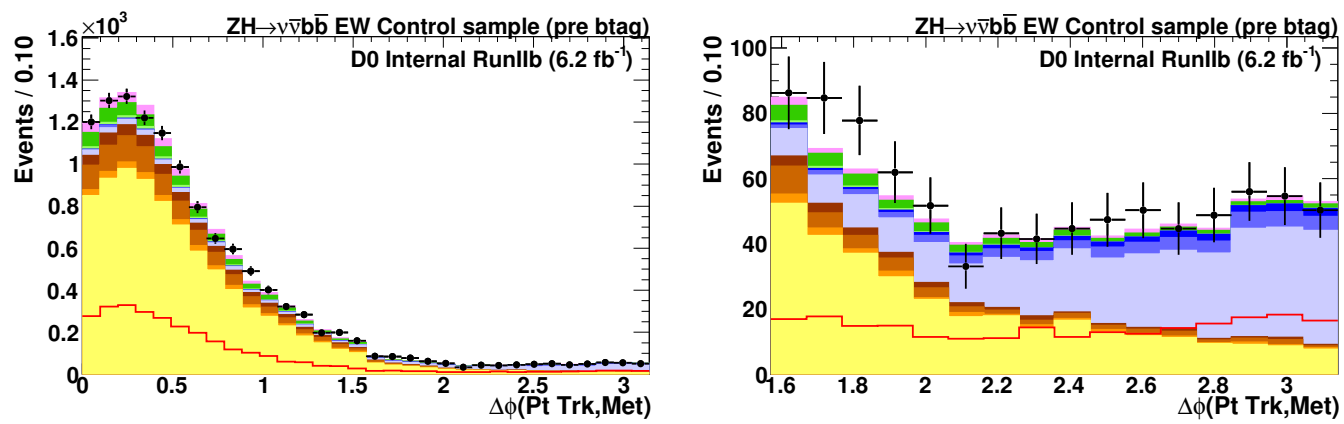

Figure 10.12: The left plot shows the $\mathcal{D}$ variable in the electroweak control sample which has negligible multijet contributions. The right plot shows in more detail the $\mathcal{D}>\pi / 2$ region.

cut $\mathcal{S}>5$ on the $\mathscr{E}_{T}$ significance is dropped. A multijet-model sample is then defined in the same way as for the signal sample, and compared with the multijet control sample in the $\mathcal{D}<\pi / 2$ region and the normalization is explained in Section 10.4.

The $\mathcal{D}>\pi / 2$ cut is designed to model events where a calorimeter jet energy is mis-measured, thus leading to significant $\mathbb{E}_{T}$ close to one of the jets. However, the $\mathbb{E}_{T}$ can be also opposite to $\not p_{T}^{\text {trk }}$ in a perfect signal-like event if the tracks of the highest energy jet are missing. This is more likely to happen for jets in the forward region if an explicit requirement is placed on the number of CFT hits causing an unwanted bias.

To further suppress events in the multijet-model samples with such nature we compare the leading jet $p_{T}$ measured with the calorimeter and the tracking in the multijet control sample. These two dimensional distributions are plotted for the $\mathcal{D}<\pi / 2$ and $\mathcal{D}>\pi / 2$ regions in Figure 10.13. As can be seen in these plots, the events from the multijet-model have more events with small Jet $p_{T}^{\text {trk }}$. To reject events from the multijet-model sample with missing tracks we further define the multi-jet model to only include events with $3.0 \times \sum^{l-j e t t r k s} \overrightarrow{p_{T}}+20>p_{T}^{l-j e t}$ where $\sum^{l-j e t t r k s} \overrightarrow{p_{T}}$ is the vectorial sum of the $p_{T}$ of the tracks in the leading jet and $p_{T}{ }^{l-j e t}$ is the jet $p_{T}$ 
measured with the calorimeter. The improvements due to these changes are discussed in detail in [104].
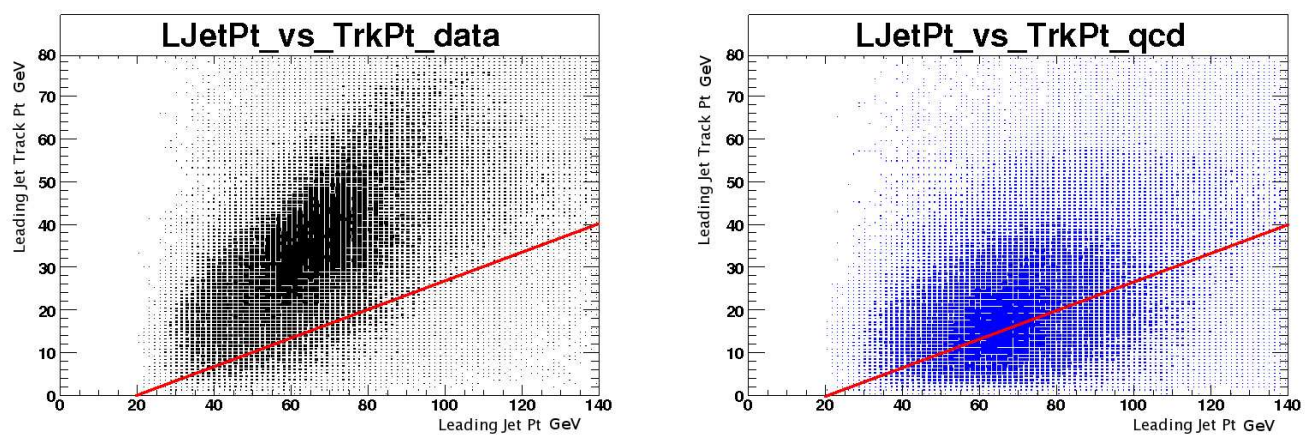

Figure 10.13: Data events from the signal like selection (black) and from the multijet sideband region (blue) in the Leading Jet Pt vs. Leading Jet Track Pt plane. The red line shows the triangle cut on the 2-D plane.

All the calorimeter related variables in the multijet control sample are very well described by our multijet model, as shown before $b$ tagging in Figure 10.14 and Figure 10.15. Plots for the single tag and double tag multijet control sample are shown in Figs.10.16-10.19. 

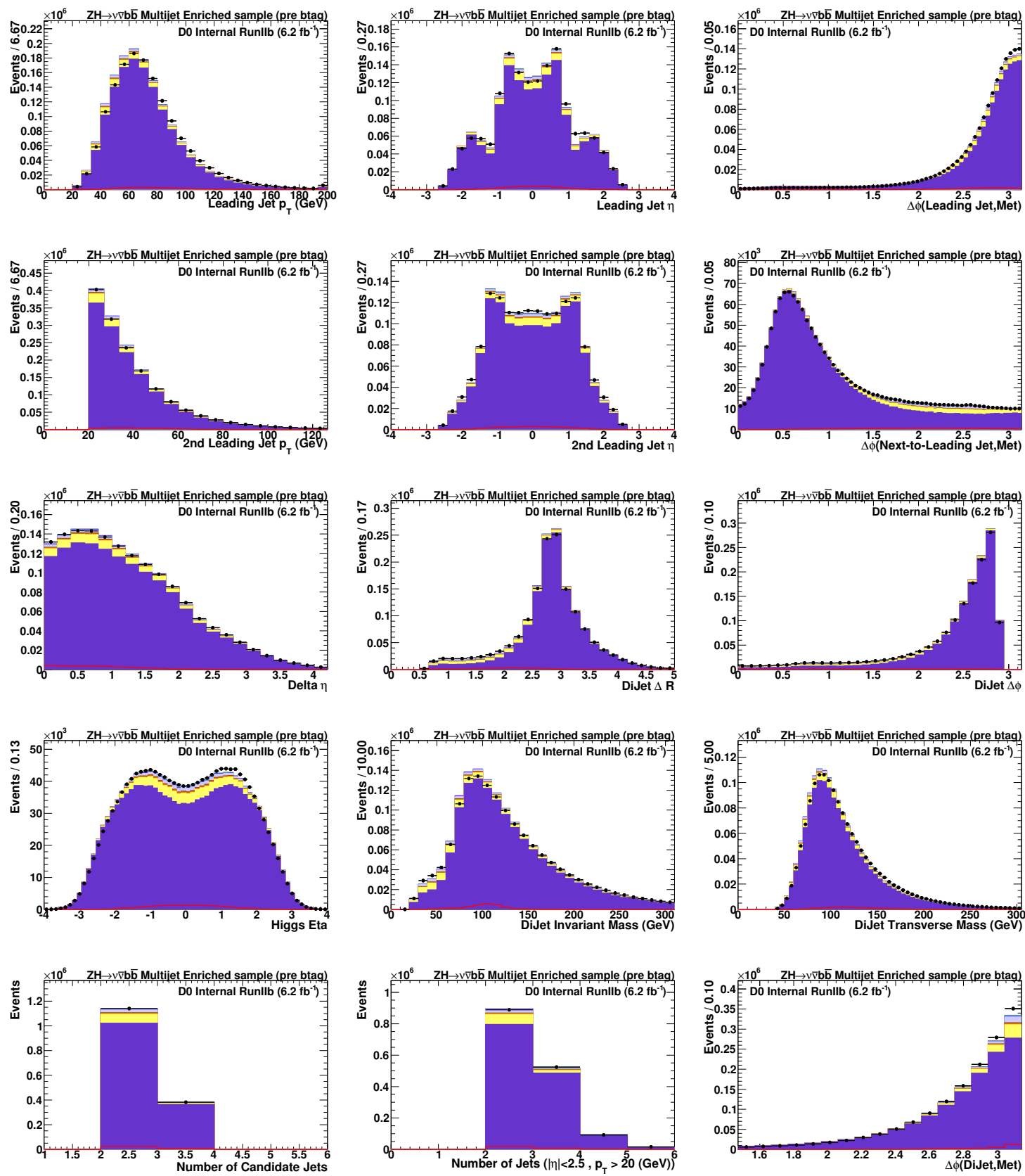

Figure 10.14: Multijet control sample with before $b$-tagging. 

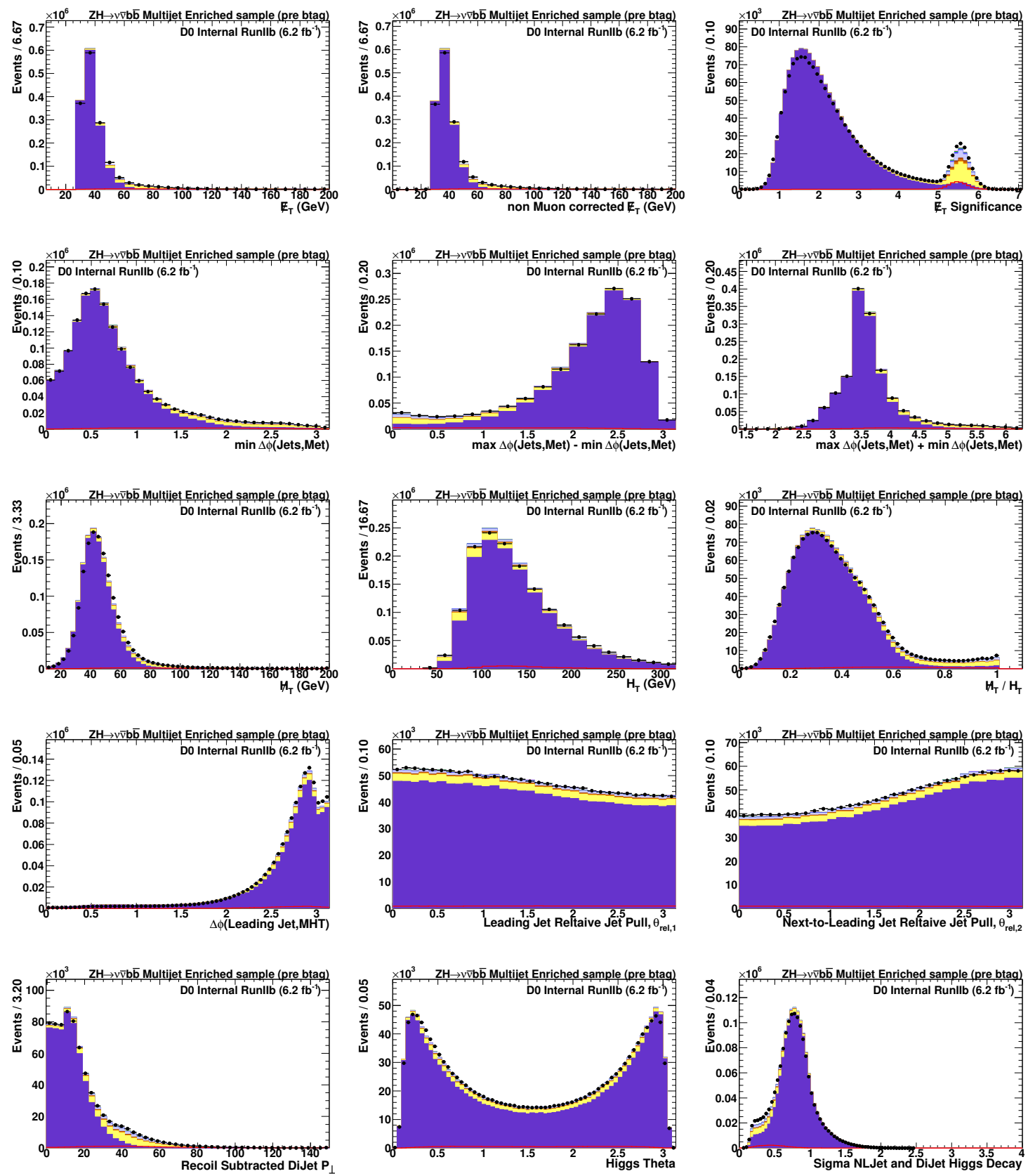

Figure 10.15: Multijet control sample with before $b$-tagging. 

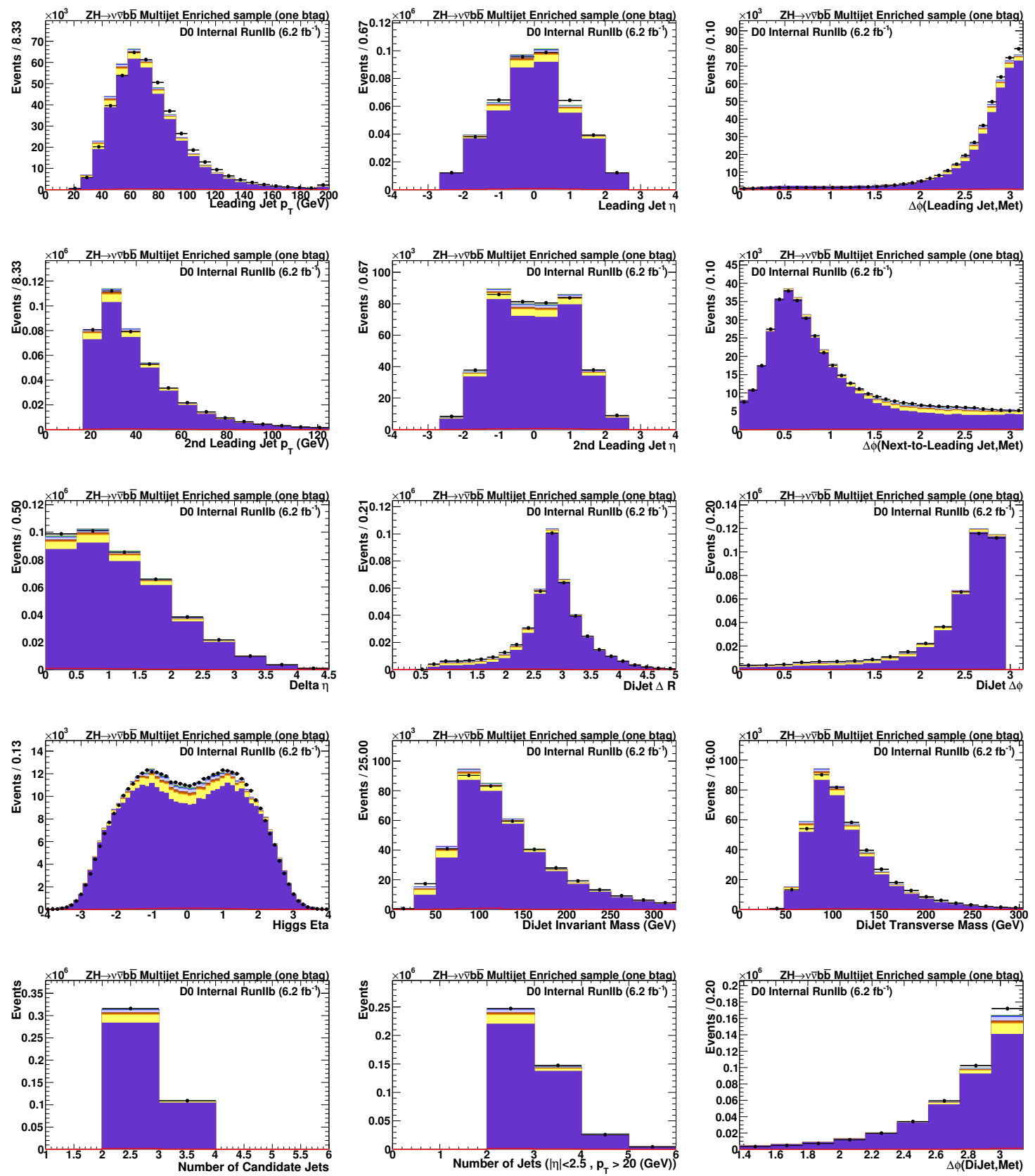

Figure 10.16: Multijet control sample with one $b$-tag. 

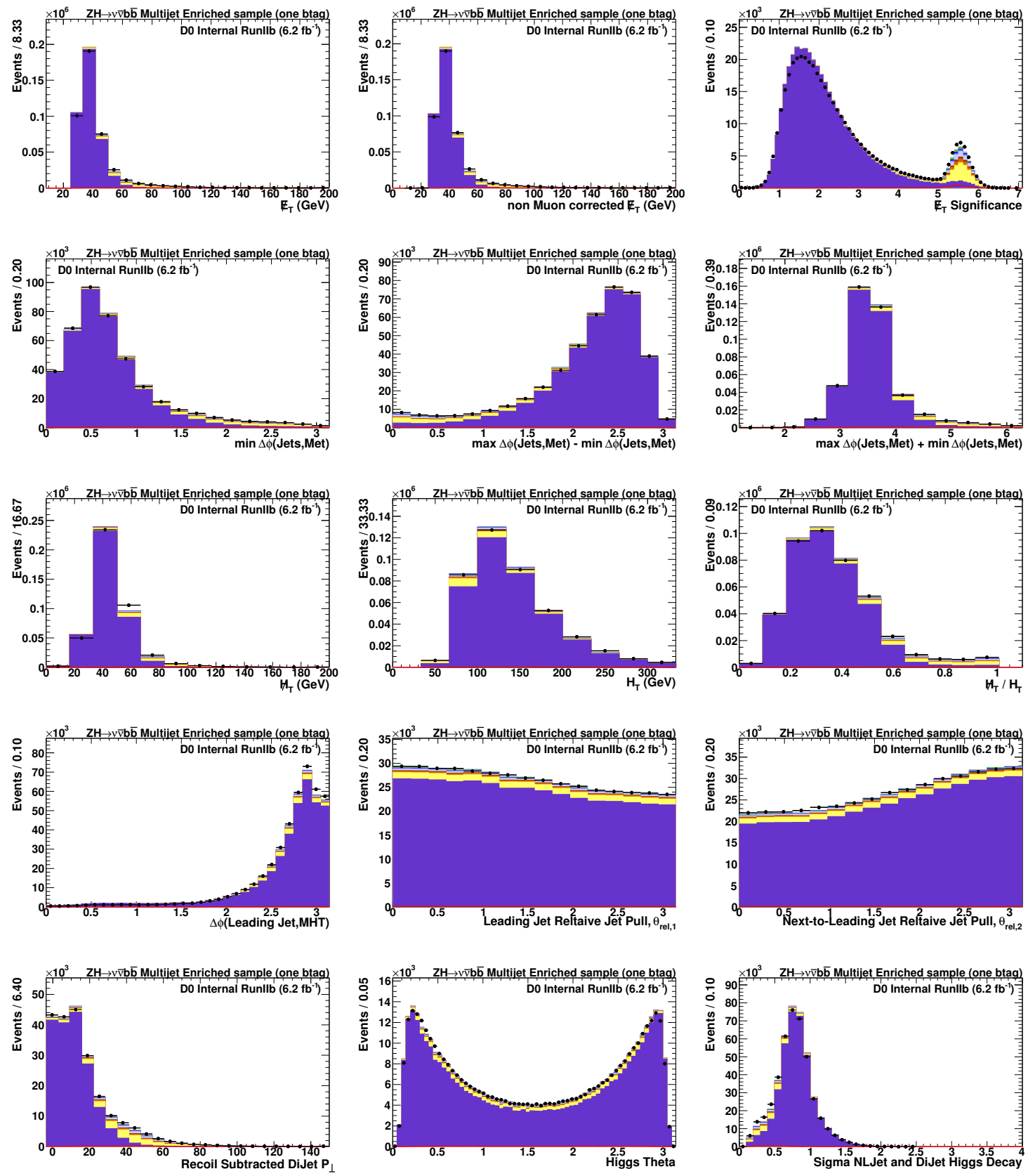

Figure 10.17: Multijet control sample with one $b$-tag. 

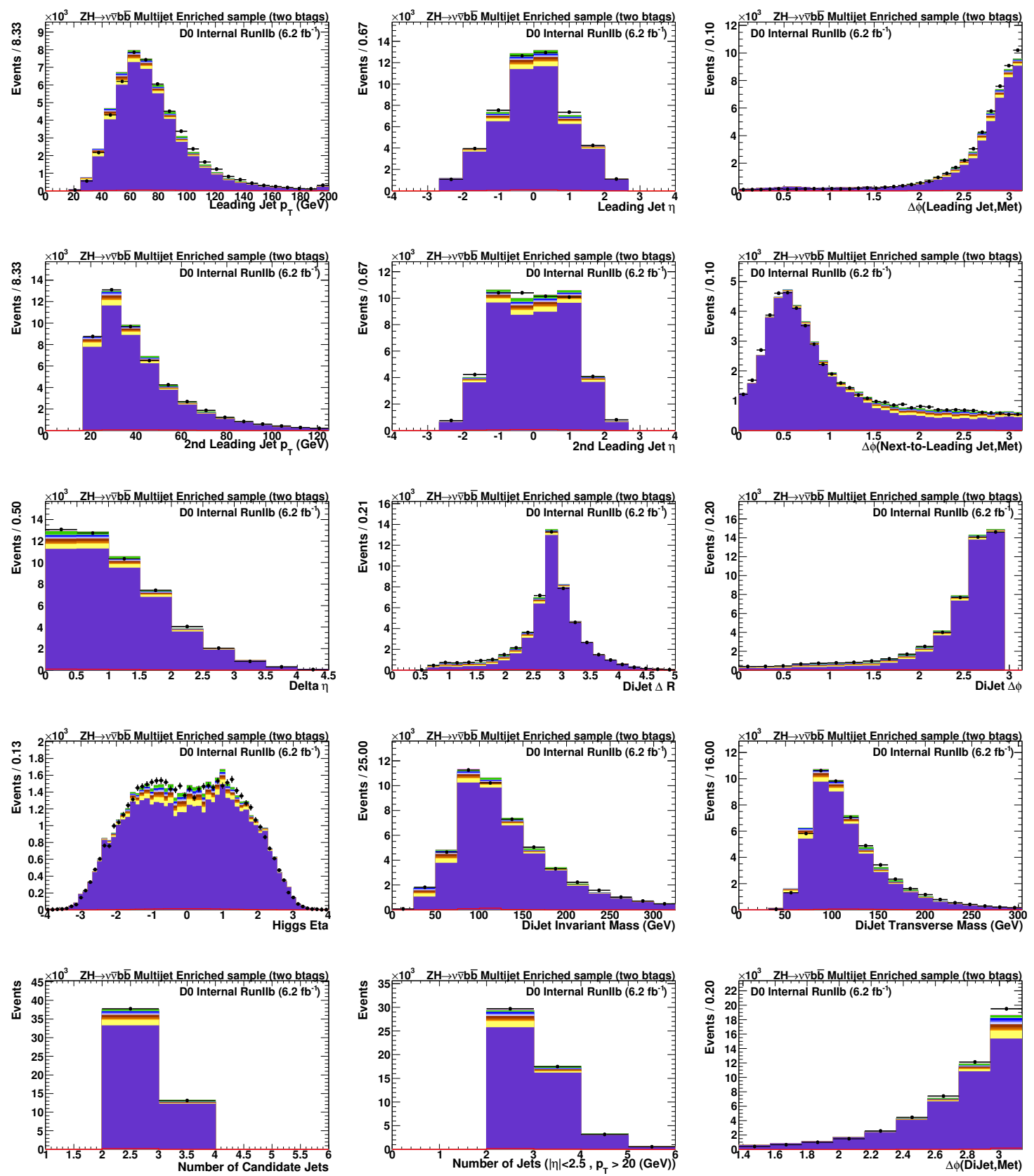

Figure 10.18: Multijet control sample with two $b$-tags. 

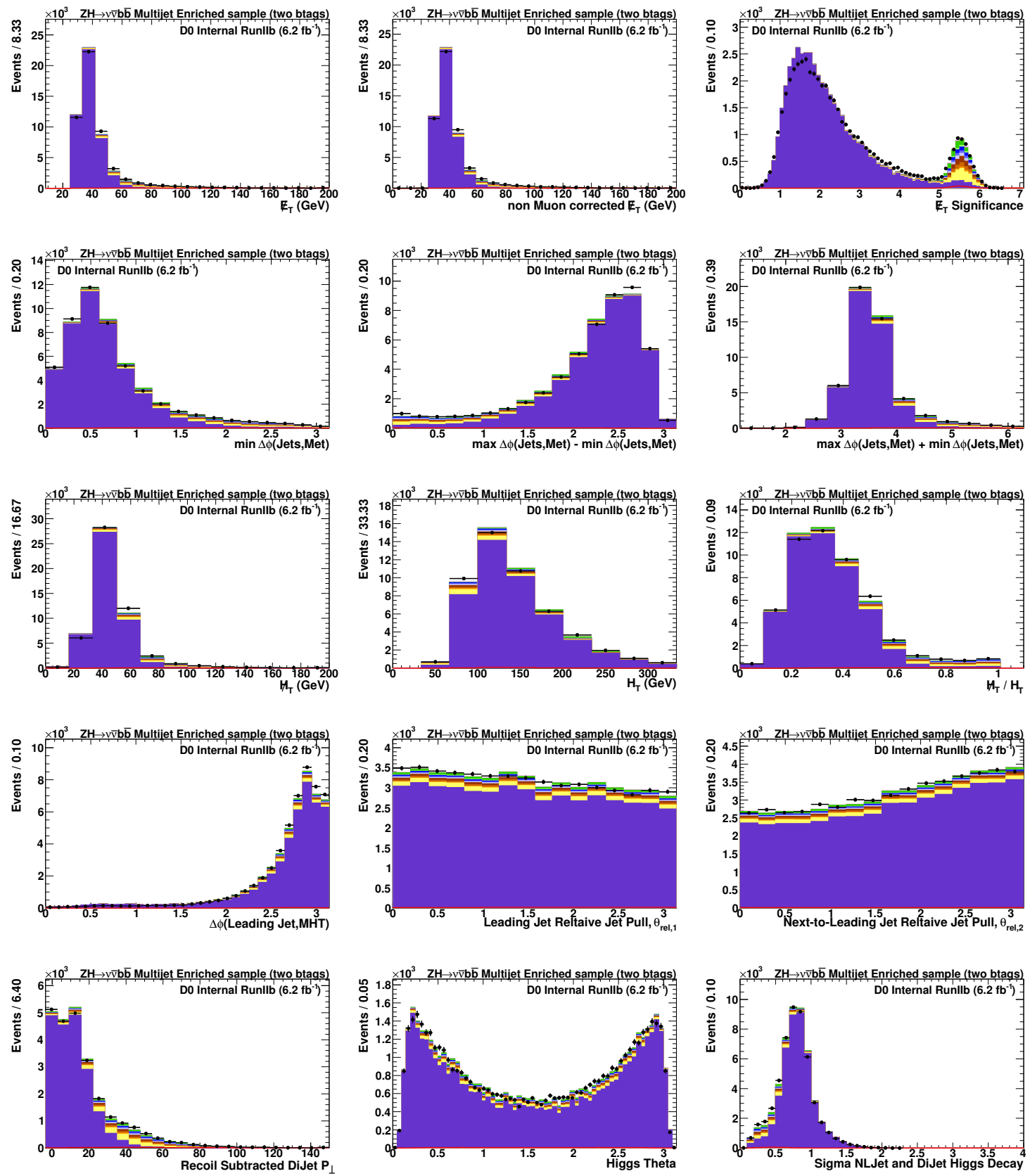

Figure 10.19: Multijet control sample with two $b$-tags. 


\subsection{Signal Sample Sample}

The signal sample, also called analysis sample, is selected as described in Section 10.3.3. The background normalizations are obtained as explained in Section 10.4.

The numbers of events observed and expected from the various background sources are given in Table 10.9 before $b$ tagging, for an exclusive L6 tag, and for an symmetric L6-L6 double tag. There is agreement between numbers of events expected and observed in the $b$-tagged samples.

Plots of various variable distributions before $b$-tagging can be seen in Figures 10.2010.21, for a sample with one L6 b-tag that is orthogonal to the double tag sample in Figures 10.22-10.23, and after double asymmetric L6-L6 tagging in Figures 10.2410.25. Overall, there is good agreement between the observed data and the expected background, both before and after $b$-tagging. There are some exceptions. Recently the modeling has been improved through the use of two separate $\mathrm{MC}$ samples for the different data taking epochs with the object reconstruction efficiencies computed separately for each. This as uncovered a bias in the multijet model in the signal sample which is covered by the systematics. A solution is in development. The same disagreement is not seen in the electroweak control sample and after cutting on the multijet DT these discrepancies are negligible in the signal sample which supports the claim that signal sample multijet model is biased. Since there is very little of the multijet background in the final sample, there is no impact on the limit setting. 


\begin{tabular}{|c|c|c|c|c|c|c|}
\hline Sample & \multicolumn{2}{|c|}{$\begin{array}{c}\text { Before } \\
\text { b-tagging }\end{array}$} & \multicolumn{2}{|c|}{$\begin{array}{c}1 \text { L6 } 6 \text { and } 1 \text { !L6 } \\
\text { tag }\end{array}$} & \multicolumn{2}{|c|}{$\begin{array}{l}2 \mathrm{~L} 6 \\
\operatorname{tags}\end{array}$} \\
\hline$Z H(115 \mathrm{GeV})$ & 24.02 & \pm 0.09 & 10.34 & \pm 0.06 & 11.12 & \pm 0.06 \\
\hline$W H(115 \mathrm{Ge}$ & 24.41 & \pm 0.15 & 10.61 & \pm 0.10 & 11.09 & \pm 0.10 \\
\hline$W+$ jets & 68834.98 & \pm 110.06 & 16646.31 & \pm 48.24 & 1372.31 & \pm 12.35 \\
\hline$W+\mathrm{c}+$ jet & 66.96 & \pm 21.24 & 1178.64 & \pm 13.69 & 173.23 & \pm 4.73 \\
\hline$W+\mathrm{cc}$ & 59.08 & \pm 23 . & 2057.21 & \pm 13.96 & 360.85 & \pm 5.49 \\
\hline$W-$ & 05.07 & \pm 1 & 270.74 & \pm 8.97 & 507.80 & \pm 5.59 \\
\hline$Z+\mathrm{j}$ & 23203.98 & & 4935.23 & \pm 44.03 & 355.15 & \pm 9.38 \\
\hline$Z+c$ & 16.13 & \pm 2 & 1090.85 & \pm 12.68 & 205.60 & \pm 4.92 \\
\hline$Z+\mathrm{bb}$ & 1341.78 & \pm 8.83 & 688.09 & \pm 6.23 & 309.01 & \pm 3.92 \\
\hline single top & 607.29 & \pm 2.33 & 337.00 & \pm 1.61 & 166.75 & \pm 0.89 \\
\hline$t \bar{t}$ & 2273.23 & \pm 16.70 & 1187.51 & \pm 14.16 & 694.24 & \pm 6.52 \\
\hline di-bo & 2594.53 & \pm 8.38 & 813.38 & \pm 4.51 & 119.88 & \pm 1.46 \\
\hline Total Pl & 112503.00 & \pm 166.77 & 30204.97 & \pm 71.77 & 4264.82 & \pm 20.23 \\
\hline Instr. Bgrd & 39290.00 & \pm 403.30 & 12541.17 & \pm 234.45 & 1479.35 & \pm 80.59 \\
\hline Total Bgrd & 151793.00 & \pm 436.42 & 42746.14 & \pm 245.19 & 5744.16 & \pm 83.09 \\
\hline Observed & 1531 & 0.00 & 426 & & & \\
\hline
\end{tabular}

Table 10.9: Run IIb number of events after applying all analysis cuts and after b-tagging using different combinations of the L6 MVA operating point. Errors are statistical errors only. 

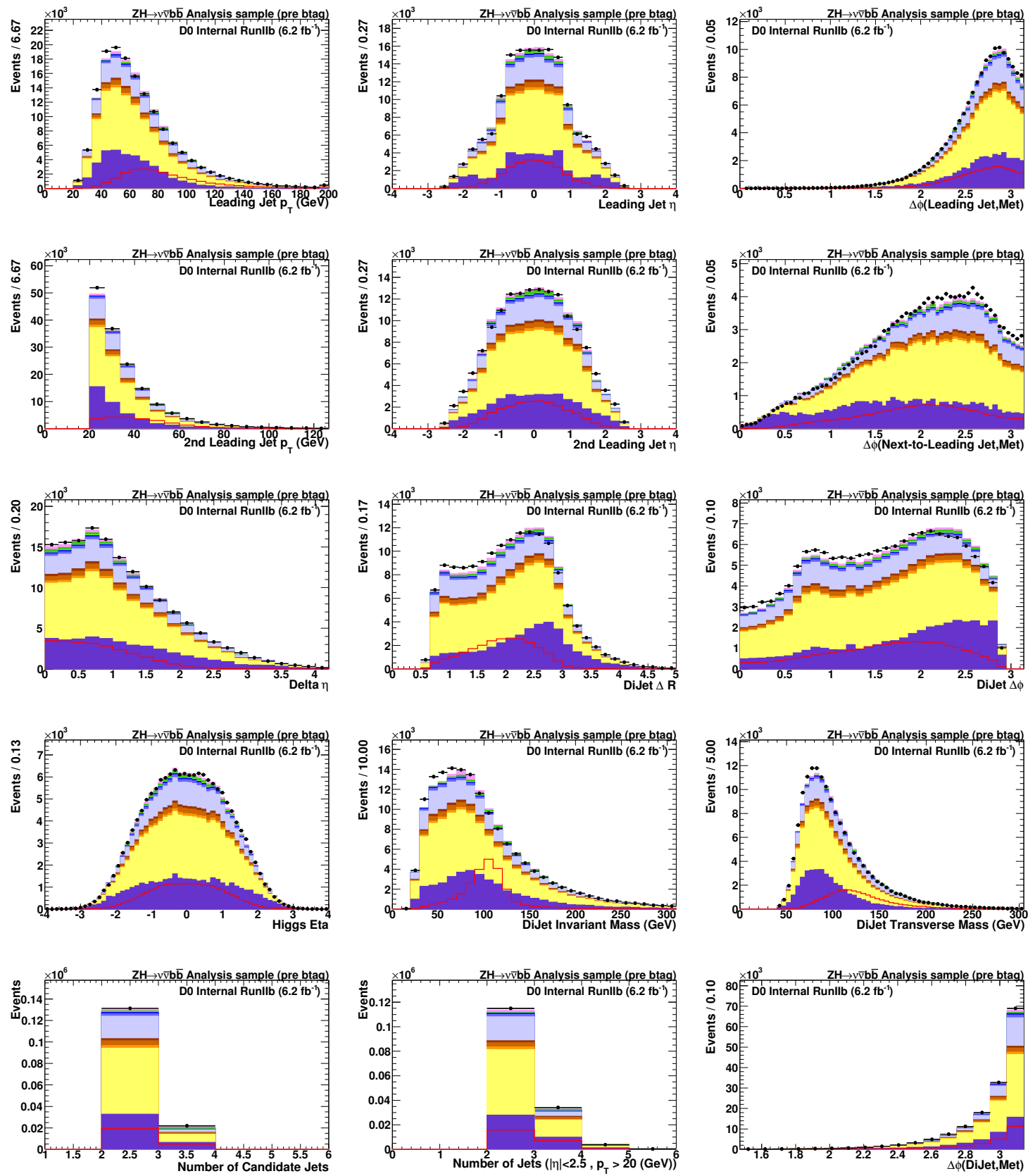

Figure 10.20: Signal sample before $b$-tagging 

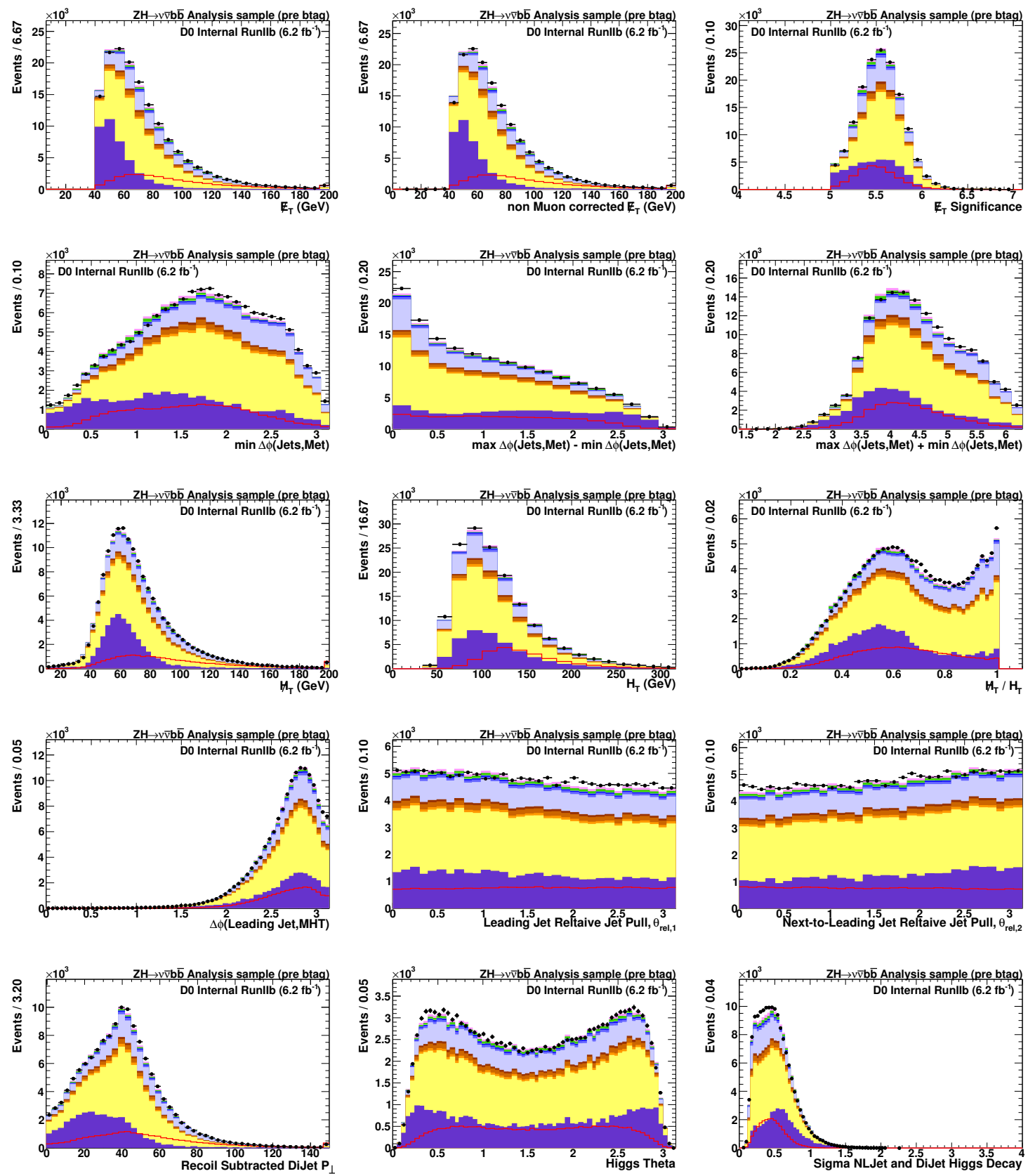

Figure 10.21: Signal sample before $b$-tagging 

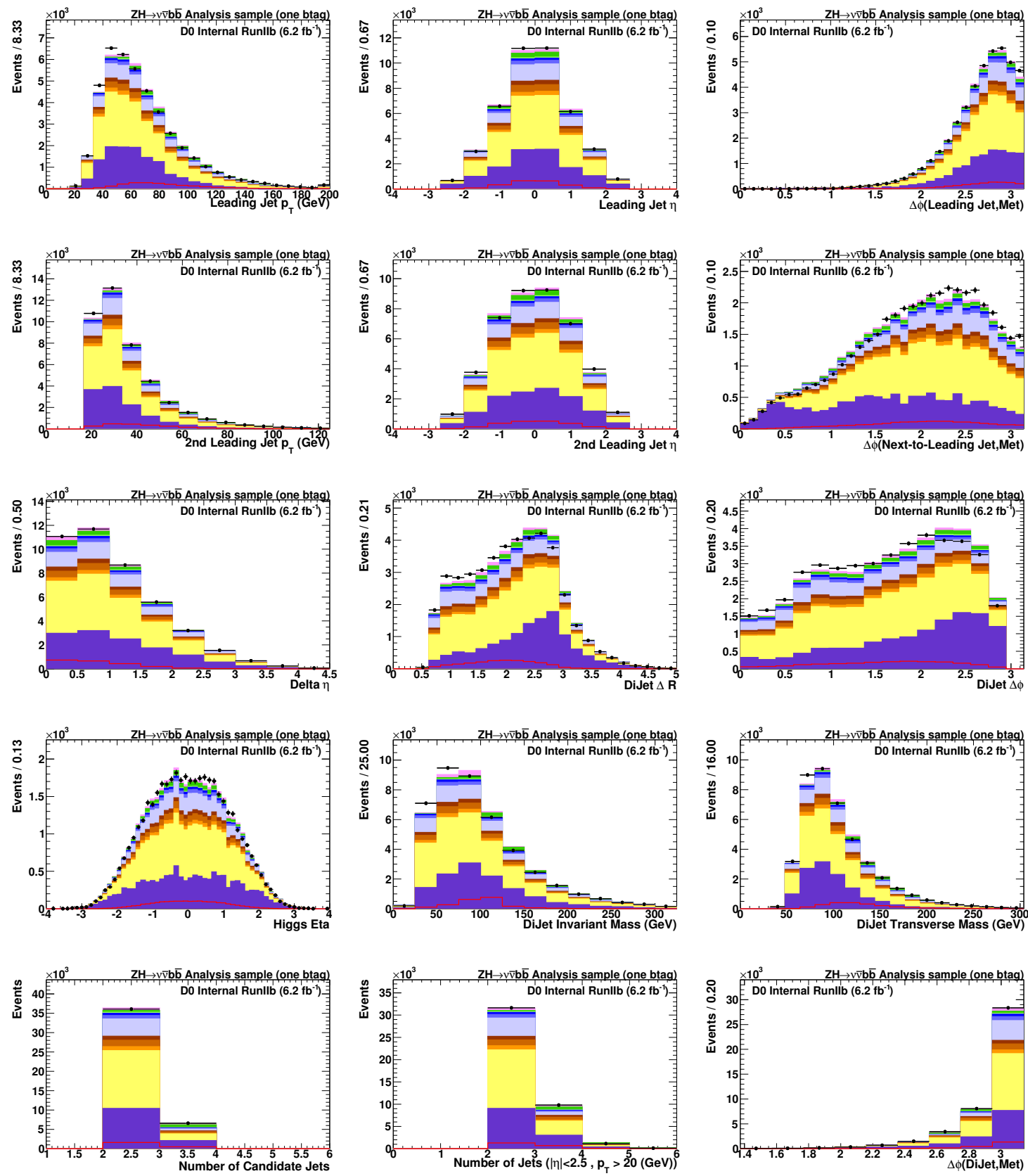

Figure 10.22: Signal sample with one L6 b-tag 

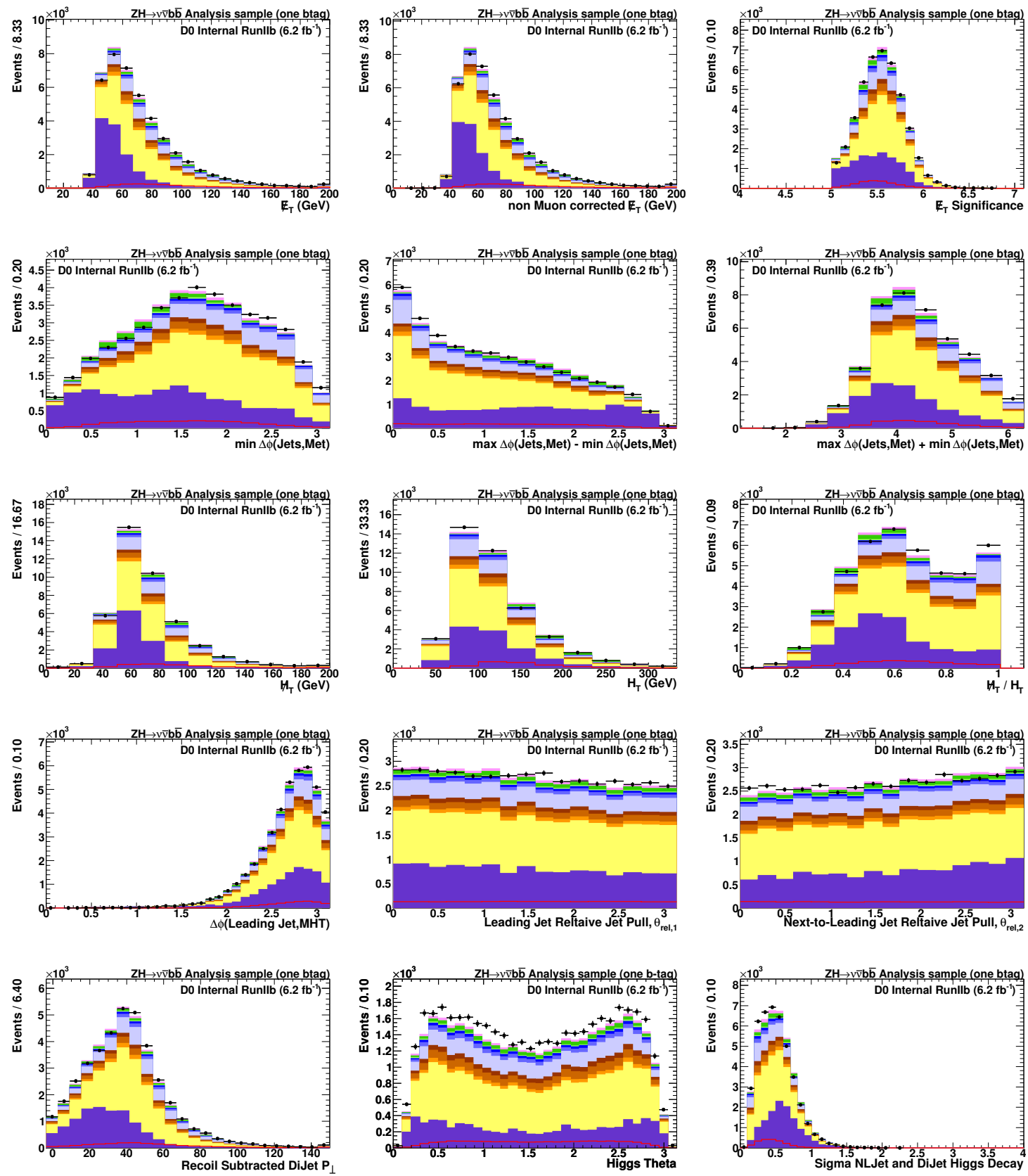

Figure 10.23: Signal sample with one L6 b-tag 

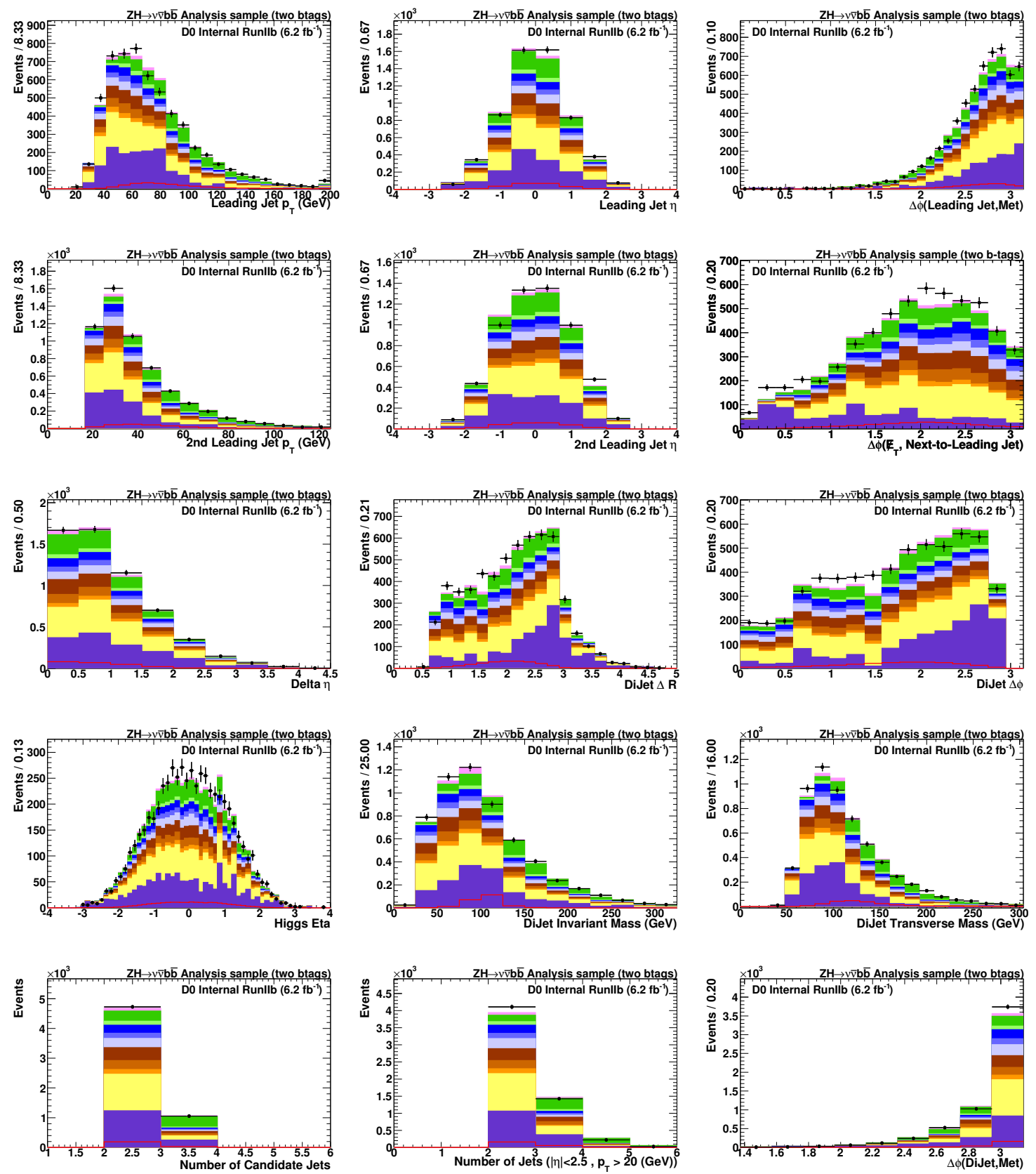

Figure 10.24: Signal sample with two b-tags 

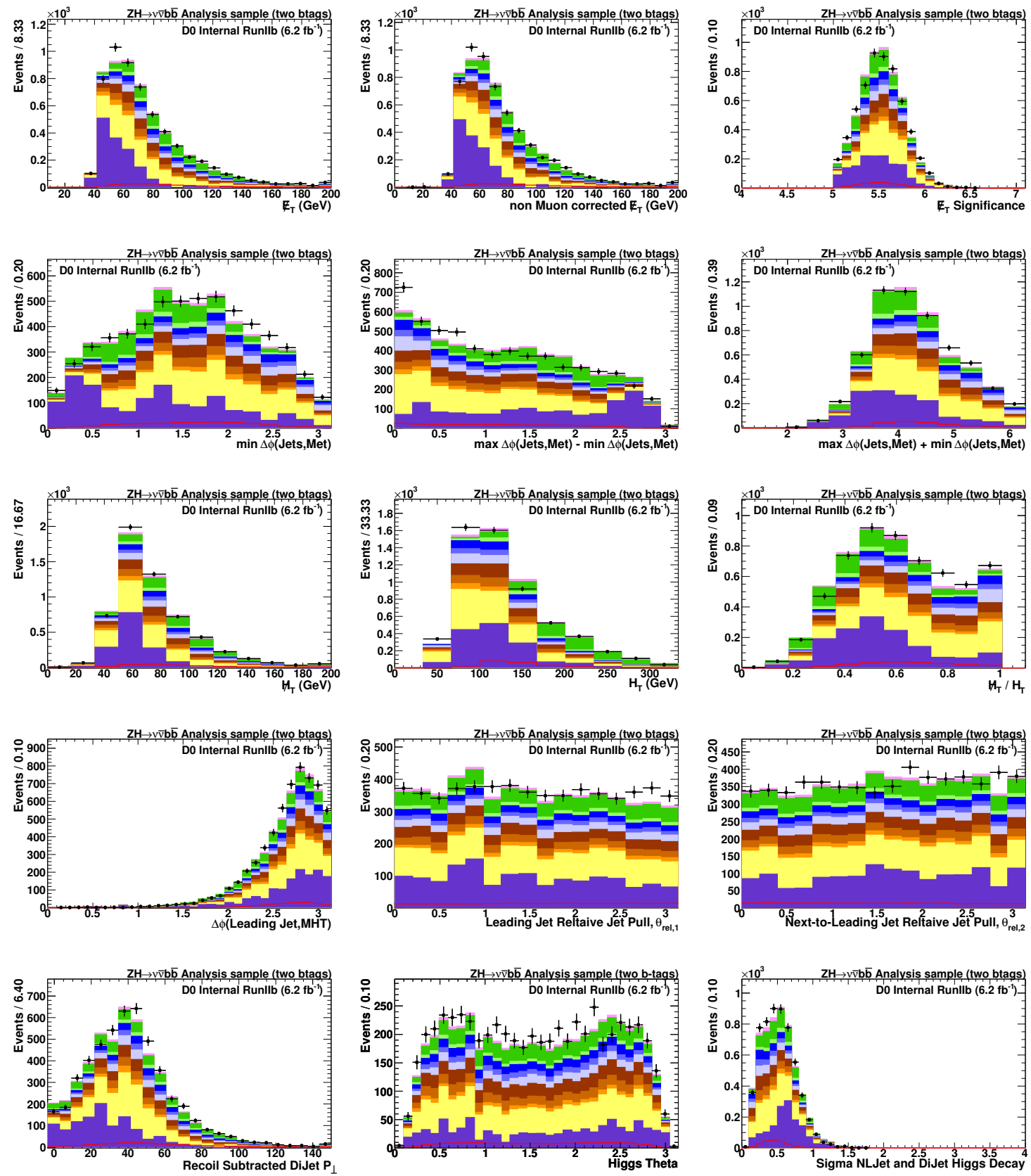

Figure 10.25: Signal sample with two b-tags 


\subsection{Multijet and Physics Decision Tree}

One set of three decision trees were used for each Higgs boson mass point. In each set, a first tree ("multijet DT") is trained to separate $V H$ signal from multijet background (see Appendices $\mathrm{G}$ and $\mathrm{H}$ ) using 20 kinematic variables. The variables are described in Tables E.1 and E.2. This tree is trained at pre-tag level on the multijet-model for the signal sample, and applied to the pre-tagged and tagged signal samples. In pervious iterations of this analysis, several kinematic cuts were applied to reduce the multijet background. When first introduced the multijet DT increased the $S / \sqrt{B}$ by $36 \%$. The multijet DT was improved upon by altering the boosting procedure and now accepts $13 \%$ more signal for the same background rejection. Currently this DT rejects $90 \%$ of the multijet background while keeping $86 \%$ of the signal. Two other trees ("Physics DT") are trained to separate $V H$ from top, $V+$ jets, and diboson backgrounds, one at the single-tag level, and the other at the double-tag level (see Appendices G.1 and H.1). These trees use the same input variables as the multijet DT as well as the the MVA b-tagger output of the leading two jets. The various backgrounds are weighted according to their expected contributions. All trees were trained as stochastic gradient boosted decision trees (see Section 6.2) using the parameters in Table 10.10.

\begin{tabular}{|ll|}
\hline \multicolumn{2}{|c|}{ Decision Tree Settings } \\
\hline$N_{\text {trees }}=200$ & shrinkage $=0.90$ \\
Bagging fraction $=0.6$ & Gini index for separation \\
$N_{\text {cuts,gini }}=20$ & $N_{\text {nodes } \text { max }}=15$ \\
\hline
\end{tabular}

Table 10.10: The settings for the training of the Stochastic Gradient Boosted Decision Trees

For the final results we use the Physics Decision Tree outputs, after a cut on the multijet Decision Tree and requiring b-tagged jets. The distributions of the multijet decisions tree output are shown for a Higgs mass of $115 \mathrm{GeV}$ in Figure 10.26 for Run 


\begin{tabular}{|c|c|}
\hline Variable & Ranking \\
\hline$E_{T}$ & 0.263 \\
\hline dijet mass & 0.144 \\
\hline second jet $p_{T}$ & 0.08486 \\
\hline $\max \Delta \phi\left(\mathbb{E}_{T}\right.$, jet $\left._{\mathrm{i}}\right)-\min \Delta \phi\left(\mathbb{E}_{T}\right.$, jet $\left._{\mathrm{i}}\right)$ & 0.08206 \\
\hline Transverse mass & 0.07802 \\
\hline$\Delta R\left(\right.$ jet $_{1}$, jet $\left._{2}\right)$ & 0.05408 \\
\hline recoil subtracted dijet $P_{\perp}$ & 0.04867 \\
\hline$\Delta \eta\left(\right.$ jet $_{1}$, jet $\left._{2}\right)$ & 0.03849 \\
\hline $\max \Delta \phi\left(\mathbb{E}_{T}\right.$, jet $\left._{\mathrm{i}}\right)+\min \Delta \phi\left(\mathbb{E}_{T}\right.$, jet $\left._{\mathrm{i}}\right)$ & 0.03784 \\
\hline & 0.02808 \\
\hline second jet $\phi_{\text {color }}$ & 0.026 \\
\hline$H_{T} / H_{T}$ & 0.02231 \\
\hline$\Delta \phi\left(\right.$ jet $_{1}$, jet $\left._{2}\right)$ & 0.01692 \\
\hline$\Sigma\left(\right.$ jet $_{2}$, dijet $\left._{\text {higgs }}\right)$ & 0.0169 \\
\hline leading jet $p_{T}$ & 0.01511 \\
\hline$\theta_{\text {Higgs }}$ & 0.01332 \\
\hline leading jet $\phi_{\text {color }}$ & 0.01121 \\
\hline$\eta_{\text {Higgs }}$ & 0.01071 \\
\hline$\Delta \phi\left(\mathbb{E}_{T}, \mathrm{jet}_{2}\right)$ & 0.005829 \\
\hline Number of taggable jets & 0.002552 \\
\hline
\end{tabular}

Table 10.11: Variables and rankings of inputs to the Run IIb1 multi-jet decision tree.

IIb1 and IIb2-3. A cut of 0.0 was chosen based on optimization studies of the limit of the standard model cross section of a $115 \mathrm{GeV}$ Higgs only considering statistical uncertainties. The default approach is to train the Physics DT before cutting on the multijet DT to maximize the statistics available. It was verified that no improvement is obtained by training the Physics DT on samples with the multijet DT cut is applied.

A ranking of the input variables is found by counting how often the variables are used and weighting each split occurrence by the separation gain-squared achieved and the number of events in the node. The variables and their rankings used in the decision trees are given in Tables 10.11- 10.16.

A comparison of the signal and background for the input variables of the three decision trees for a Higgs mass of $115 \mathrm{GeV}$ can be seen in Section E. 


\begin{tabular}{|c|c|}
\hline Variable & Ranking \\
\hline second jet $\phi_{\text {color }}$ & 0.07883 \\
\hline leading jet MVA bl output & 0.06655 \\
\hline$\Delta R\left(\right.$ jet $_{1}$, jet $\left._{2}\right)$ & 0.06486 \\
\hline$H_{T} / H_{T}$ & 0.06476 \\
\hline$\Delta \eta\left(\right.$ jet $_{1}$, jet $\left._{2}\right)$ & 0.06237 \\
\hline dijet mass & 0.062 \\
\hline Transverse mass & 0.05537 \\
\hline recoil subtracted dijet $P_{\perp}$ & 0.05331 \\
\hline leading jet $\phi_{\text {color }}$ & 0.04958 \\
\hline$E_{T}$ & 0.04626 \\
\hline$\Delta \phi\left(H_{T}\right.$, jet $\left._{2}\right)$ & 0.04432 \\
\hline leading jet $p_{T}$ & 0.04218 \\
\hline second jet $p_{T}$ & 0.04103 \\
\hline$\Delta \phi\left(\right.$ jet $_{1}$, jet $\left._{2}\right)$ & 0.04037 \\
\hline $\max \Delta \phi\left(H_{T}\right.$, jet $\left._{\mathrm{i}}\right)-\min \Delta \phi\left(\mathscr{H}_{T}\right.$, jet $\left._{\mathrm{i}}\right)$ & 0.04002 \\
\hline$\theta_{\text {Higgs }}$ & 0.03926 \\
\hline $\max \Delta \phi\left(\mathscr{H}_{T}\right.$, jet $\left._{\mathrm{i}}\right)+\min \Delta \phi\left(\mathscr{H}_{T}\right.$, jet $\left._{\mathrm{i}}\right)$ & 0.03549 \\
\hline$\eta_{\text {Higgs }}$ & 0.0334 \\
\hline$\Sigma\left(\right.$ jet $_{2}$, dijet $\left._{\text {higgs }}\right)$ & 0.03217 \\
\hline$H_{T}$ & 0.03214 \\
\hline Number of taggable jets & 0.01573 \\
\hline
\end{tabular}

Table 10.12: Variables and rankings of inputs to the Run IIb1 single tag physics decision tree. 


\begin{tabular}{|c|c|}
\hline Variable & Ranking \\
\hline leading jet MVA bl output & 0.1471 \\
\hline second jet MVA bl output & 0.1455 \\
\hline dijet mass & 0.1204 \\
\hline $\max \Delta \phi\left(\mathscr{E}_{T}\right.$, jet $\left._{\mathrm{i}}\right)-\min \Delta \phi\left(\mathbb{E}_{T}\right.$, jet $\left._{\mathrm{i}}\right)$ & 0.06299 \\
\hline$\Delta R\left(\right.$ jet $_{1}$, jet $\left._{2}\right)$ & 0.05799 \\
\hline Transverse mass & 0.05271 \\
\hline$\Delta \eta\left(\right.$ jet $_{1}$, jet $\left._{2}\right)$ & 0.04674 \\
\hline recoil subtracted dijet $P_{\perp}$ & 0.03737 \\
\hline$E_{T}$ & 0.03583 \\
\hline$\Delta \phi\left(E_{T}\right.$, jet $\left._{2}\right)$ & 0.03536 \\
\hline$H_{T} / H_{T}$ & 0.03335 \\
\hline leading jet $\phi_{\text {color }}$ & 0.0283 \\
\hline$H_{T}$ & 0.02729 \\
\hline second jet $p_{T}$ & 0.02584 \\
\hline second jet $\phi_{\text {color }}$ & 0.02464 \\
\hline$\Delta \phi\left(\right.$ jet $_{1}$, jet $\left._{2}\right)$ & 0.02195 \\
\hline$\eta_{\text {Higgs }}$ & 0.02004 \\
\hline$\theta_{\text {Higgs }}$ & 0.01845 \\
\hline $\max \Delta \phi\left(E_{T}\right.$, jet $\left._{\mathrm{i}}\right)+\min \Delta \phi\left(E_{T}\right.$, jet $\left._{\mathrm{i}}\right)$ & 0.01839 \\
\hline$\Sigma\left(\right.$ jet $_{2}$, dijet $\left._{\text {higgs }}\right)$ & 0.01831 \\
\hline leading jet $p_{T}$ & 0.01315 \\
\hline Number of taggable jets & 0.008316 \\
\hline
\end{tabular}

Table 10.13: Variables and rankings of inputs to the Run IIb1 double tag physics decision tree. 


\begin{tabular}{|c|c|}
\hline Variable & Ranking \\
\hline $\bar{H}_{T}$ & 0.1389 \\
\hline dijet mass & 0.08746 \\
\hline$H_{T}$ & 0.07494 \\
\hline $\max \Delta \phi\left(\mathscr{F}_{T}\right.$, jet $\left._{\mathrm{i}}\right)-\min \Delta \phi\left(\mathscr{E}_{T}\right.$, jet $\left._{\mathrm{i}}\right)$ & 0.06798 \\
\hline recoil subtracted dijet $P_{\perp}$ & 0.06639 \\
\hline$H_{T} / H_{T}$ & 0.05947 \\
\hline $\max \Delta \phi\left(H_{T}\right.$, jet $\left._{\mathrm{i}}\right)+\min \Delta \phi\left(H_{T}\right.$, jet $\left._{\mathrm{i}}\right)$ & 0.05646 \\
\hline second jet $p_{T}$ & 0.0492 \\
\hline Transverse mass & 0.04649 \\
\hline$\Delta \eta\left(\right.$ jet $_{1}$, jet $\left._{2}\right)$ & 0.04494 \\
\hline$\Delta \phi\left(B_{T}\right.$, jet $\left._{2}\right)$ & 0.0418 \\
\hline$\eta_{\text {Higgs }}$ & 0.03979 \\
\hline second jet $\phi_{\text {color }}$ & 0.03815 \\
\hline leading jet $p_{T}$ & 0.0367 \\
\hline$\theta_{\text {Higgs }}$ & 0.03164 \\
\hline leading jet $\phi_{\text {color }}$ & 0.03135 \\
\hline$\Delta \phi\left(\right.$ jet $_{1}$, jet $\left._{2}\right)$ & 0.02633 \\
\hline$\Sigma\left(\right.$ jet $_{2}$, dijet $\left._{\text {higgs }}\right)$ & 0.0261 \\
\hline$\Delta R\left(\right.$ jet $_{1}$, jet $\left._{2}\right)$ & 0.02333 \\
\hline Number of taggable jets & 0.01254 \\
\hline
\end{tabular}

Table 10.14: Variables and rankings of inputs to the Run IIb2-3 multi-jet decision tree. 


\begin{tabular}{|c|c|}
\hline Variable & Ranking \\
\hline leading jet MVA bl output & 0.07446 \\
\hline$H_{T} / H_{T}$ & 0.06851 \\
\hline $\max \Delta \phi\left(\mathscr{H}_{T}, \mathrm{jet}_{\mathrm{i}}\right)-\min \Delta \phi\left(\mathscr{H}_{T}, \mathrm{jet}_{\mathrm{i}}\right)$ & 0.06743 \\
\hline$\Delta \phi\left(E_{T}\right.$, jet $\left._{2}\right)$ & 0.06371 \\
\hline$\Delta \eta\left(\right.$ jet $_{1}$, jet $\left._{2}\right)$ & 0.05292 \\
\hline recoil subtracted dijet $P_{\perp}$ & 0.0525 \\
\hline leading jet $\phi_{\text {color }}$ & 0.05232 \\
\hline dijet mass & 0.05216 \\
\hline second jet $\phi_{\text {color }}$ & 0.0479 \\
\hline$H_{T}$ & 0.04721 \\
\hline$\eta_{\text {Higgs }}$ & 0.04554 \\
\hline second jet $p_{T}$ & 0.04235 \\
\hline$\Delta R\left(\right.$ jet $_{1}$, jet $\left._{2}\right)$ & 0.04129 \\
\hline $\max \Delta \phi\left(\mathbb{E}_{T}\right.$, jet $\left._{\mathrm{i}}\right)+\min \Delta \phi\left(\mathbb{E}_{T}\right.$, jet $\left._{\mathrm{i}}\right)$ & 0.04001 \\
\hline$\Delta \phi\left(\right.$ jet $_{1}$, jet $\left._{2}\right)$ & 0.03988 \\
\hline$\theta_{\text {Higgs }}$ & 0.03896 \\
\hline leading jet $p_{T}$ & 0.03879 \\
\hline$E_{T}$ & 0.03774 \\
\hline$\Sigma\left(\right.$ jet $_{2}$, dijet $\left._{\text {higgs }}\right)$ & 0.03735 \\
\hline Transverse mass & 0.03533 \\
\hline Number of taggable jets & 0.02363 \\
\hline
\end{tabular}

Table 10.15: Variables and rankings of inputs to the Run IIb2-3 single tag physics decision tree. 


\begin{tabular}{|c|c|}
\hline Variable & Ranking \\
\hline dijet mass & 0.06737 \\
\hline $\max \Delta \phi\left(\mathscr{H}_{T}\right.$, jet $\left._{\mathrm{i}}\right)-\min \Delta \phi\left(\mathscr{E}_{T}\right.$, jet $\left._{\mathrm{i}}\right)$ & 0.06409 \\
\hline$H_{T} / H_{T}$ & 0.05841 \\
\hline second jet MVA bl output & 0.05168 \\
\hline $\max \Delta \phi\left(E_{T}\right.$, jet $\left._{\mathrm{i}}\right)+\min \Delta \phi\left(E_{T}\right.$, jet $\left._{\mathrm{i}}\right)$ & 0.05101 \\
\hline$\eta_{\text {Higgs }}$ & 0.05039 \\
\hline$\Delta \phi\left(\mathbb{H}_{T}, \mathrm{jet}_{2}\right)$ & 0.04954 \\
\hline leading jet MVA bl output & 0.04895 \\
\hline second jet $p_{T}$ & 0.04796 \\
\hline$\Delta \eta\left(\right.$ jet $_{1}$, jet $\left._{2}\right)$ & 0.04623 \\
\hline leading jet $\phi_{\text {color }}$ & 0.04595 \\
\hline$\theta_{\text {Higgs }}$ & 0.04562 \\
\hline$\Delta R\left(\right.$ jet $_{1}$, jet $\left._{2}\right)$ & 0.04389 \\
\hline second jet $\phi_{\text {color }}$ & 0.04315 \\
\hline$E_{T}$ & 0.04246 \\
\hline$\Sigma\left(\right.$ jet $_{2}$, dijet $\left._{\text {higgs }}\right)$ & 0.04093 \\
\hline$H_{T}$ & 0.03939 \\
\hline$\Delta \phi\left(\right.$ jet $_{1}$, jet $\left._{2}\right)$ & 0.03839 \\
\hline recoil subtracted $\operatorname{dijet} P_{\perp}$ & 0.03716 \\
\hline leading jet $p_{T}$ & 0.03484 \\
\hline Transverse mass & 0.03059 \\
\hline Number of taggable jets & 0.02199 \\
\hline
\end{tabular}

Table 10.16: Variables and rankings of inputs to the Run IIb2-3 double tag physics decision tree. 


\begin{tabular}{|l|rl|rl|rl|}
\hline Sample & \multicolumn{2}{|c|}{$\begin{array}{c}\text { Before } \\
\text { b-tagging }\end{array}$} & \multicolumn{2}{c|}{$\begin{array}{c}\text { L6 and 1 !L6 } \\
\text { tag }\end{array}$} & \multicolumn{2}{c|}{$\begin{array}{c}\text { L6 } \\
\text { tags }\end{array}$} \\
\hline$Z H(115 \mathrm{GeV})$ & 20.69 & \pm 0.08 & 8.66 & \pm 0.05 & 9.95 & \pm 0.06 \\
$W H(115 \mathrm{GeV})$ & 19.79 & \pm 0.13 & 8.36 & \pm 0.09 & 9.41 & \pm 0.09 \\
\hline$W+$ jets & 28545.21 & \pm 67.19 & 6857.72 & \pm 28.78 & 575.12 & \pm 7.39 \\
$W+\mathrm{c}+$ jet & 1020.29 & \pm 12.19 & 444.69 & \pm 7.95 & 60.64 & \pm 2.54 \\
$W+\mathrm{cc}$ & 3233.83 & \pm 19.18 & 1204.59 & \pm 11.23 & 221.89 & \pm 4.49 \\
$W+$ bb & 1507.22 & \pm 10.25 & 764.96 & \pm 7.33 & 325.78 & \pm 4.72 \\
$Z+$ jets & 10504.83 & \pm 72.90 & 2332.34 & \pm 28.10 & 171.20 & \pm 6.49 \\
$Z+\mathrm{cc}$ & 1781.12 & \pm 17.85 & 700.79 & \pm 10.69 & 136.51 & \pm 4.24 \\
$Z+$ bb & 869.15 & \pm 7.52 & 446.59 & \pm 5.32 & 206.37 & \pm 3.41 \\
single top & 354.60 & \pm 1.84 & 192.24 & \pm 1.17 & 103.91 & \pm 0.68 \\
$t \bar{t}$ & 1784.41 & \pm 15.08 & 934.46 & \pm 12.97 & 532.23 & \pm 5.49 \\
di-boson & 1753.03 & \pm 6.86 & 551.73 & \pm 3.68 & 82.78 & \pm 1.17 \\
\hline Total Physics & 51353.70 & \pm 105.37 & 14430.13 & \pm 46.76 & 2416.43 & \pm 14.40 \\
Instr. Bgrd & 4413.36 & \pm 178.13 & 1588.17 & \pm 106.02 & 186.60 & \pm 39.10 \\
\hline Total Bgrd & 55767.05 & \pm 206.96 & 16018.31 & \pm 115.88 & 2603.02 & \pm 41.67 \\
Observed & 56017.00 & 15863.00 & 2501.00 \\
\hline
\end{tabular}

Table 10.17: Run IIb number of events after applying all analysis cuts and after cutting on the multijet-DT output and after b-tagging using different combinations of the L6 MVA operating point. Errors are statistical errors only.

The numbers of events observed and expected from the various background sources are given in Table 10.17 after the cut at 0.0 on the multijet DT output before $b$ tagging, for an exclusive L6 tag, and for a L6 double tag. The effect of the cut on the multijet-DT output can be seen by comparing with Table 10.9.

Kinematic variable distributions after the cut at 0.0 on the multijet DT output are shown here for a Higgs boson mass of $115 \mathrm{GeV}$. Distributions before $b$-tagging can be seen in Figs. 10.29-10.30, for a sample with one L6 b-tag that is orthogonal to the double tag sample in Figs. 10.31-10.32, and after double (L6-L6) tagging in Figs. 10.33-10.34. Overall, there is good agreement between the observed data and the expected background, both before and after $b$-tagging.

The physics DT output distributions are shown in Appendices F.1 through F.4 for the single and double tag signal samples. They are also show in Figure 10.27 and 
10.28 for a Higgs mass of $115 \mathrm{GeV}$ in Run IIb1 and IIb2-3. 

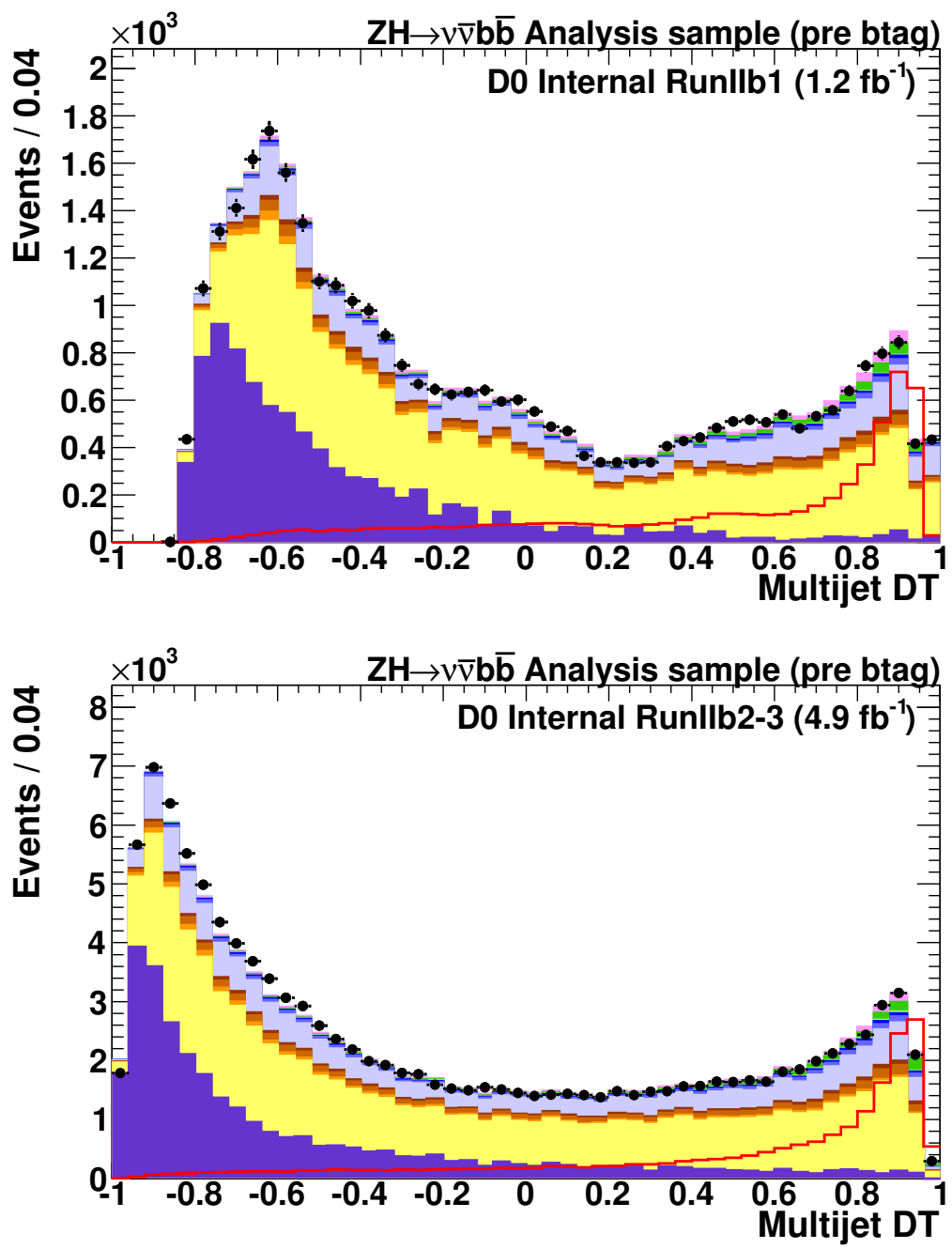

Figure 10.26: Multijet decision trees for a Higgs mass of $115 \mathrm{GeV}$ in Run IIb1 (top) and Run IIb2-3 (bottom). 

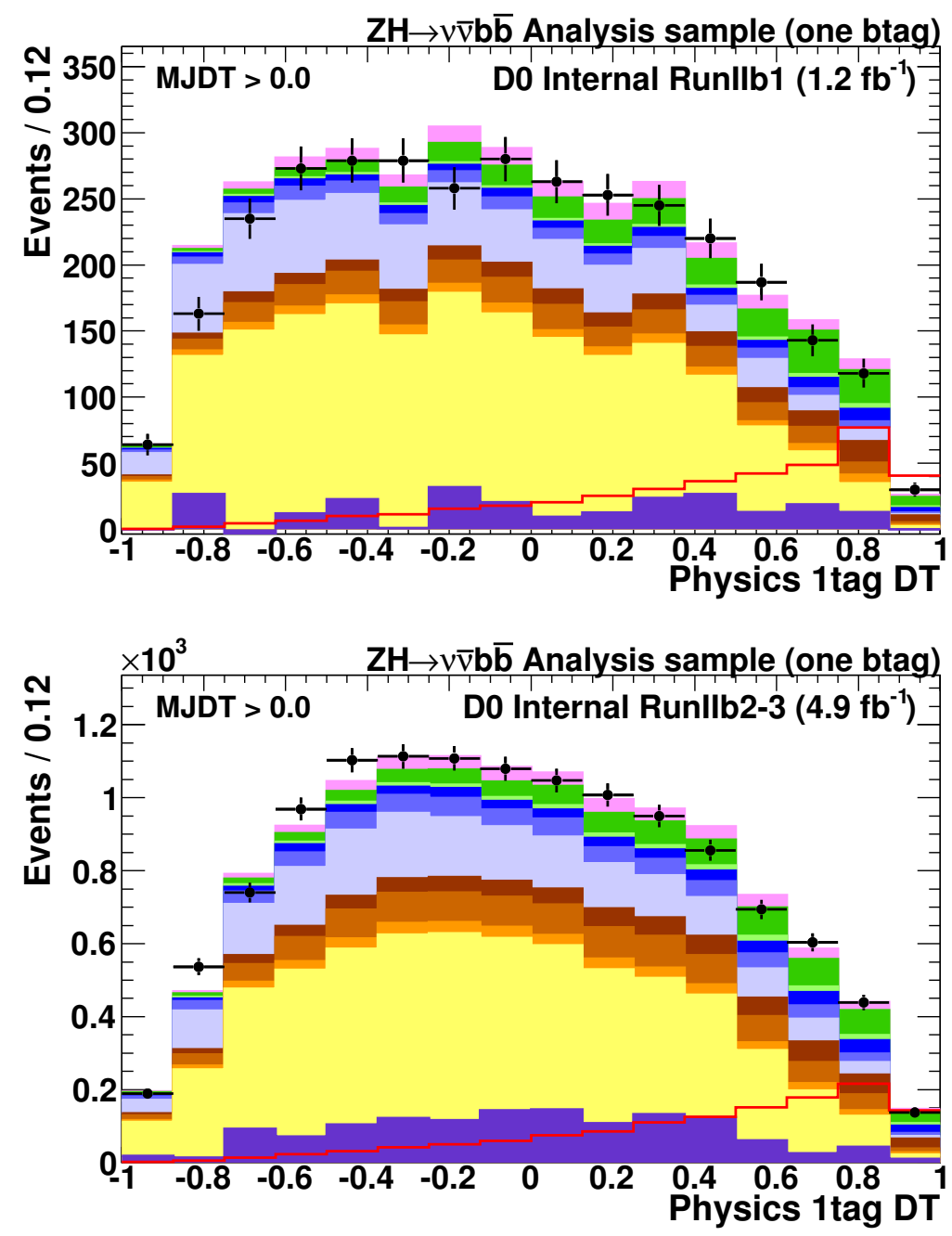

Figure 10.27: Single tag physics decision trees for a Higgs mass of $115 \mathrm{GeV}$ in Run IIb1 (top) and Run IIb2-3 (bottom). 

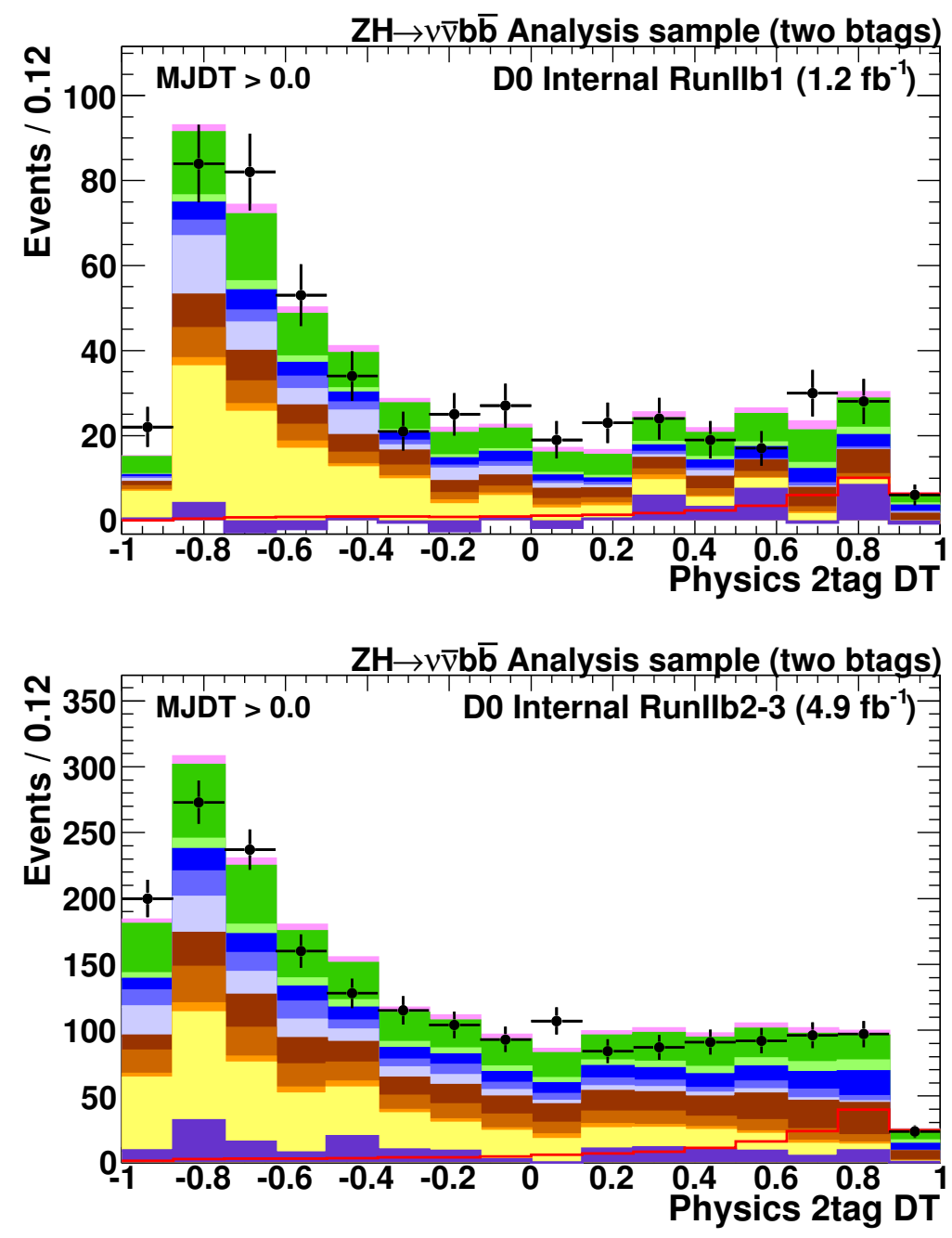

Figure 10.28: Double tag physics decision trees for a Higgs mass of $115 \mathrm{GeV}$ in Run IIb1 (top) and Run IIb2-3 (bottom). 

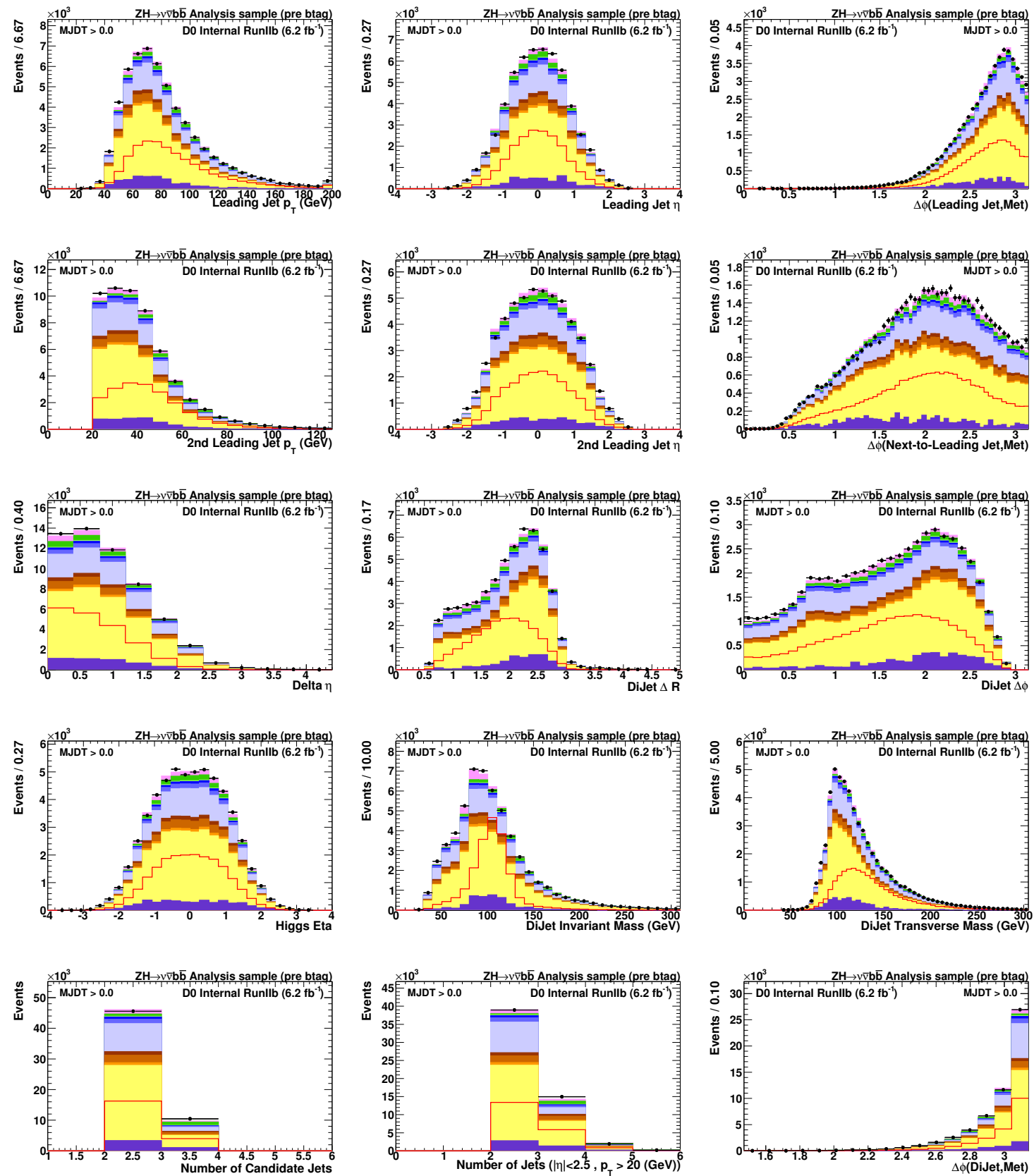

Figure 10.29: Pre b-tag signal sample after requiring a multijet decision tree cut of $D T>$ 0.0 . 

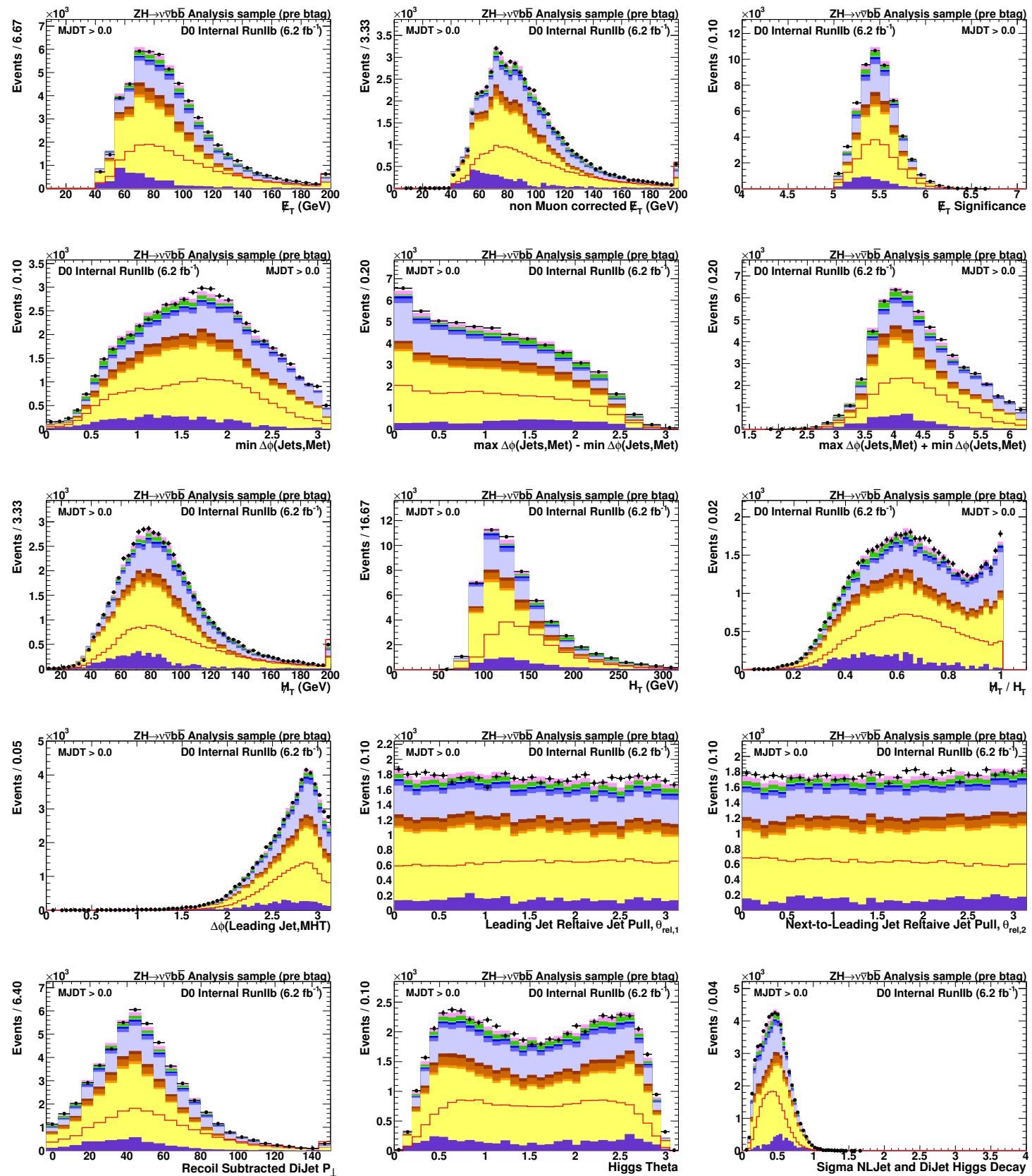

Figure 10.30: Pre b-tag signal sample after requiring a multijet decision tree cut of $D T>$ 0.0 . 

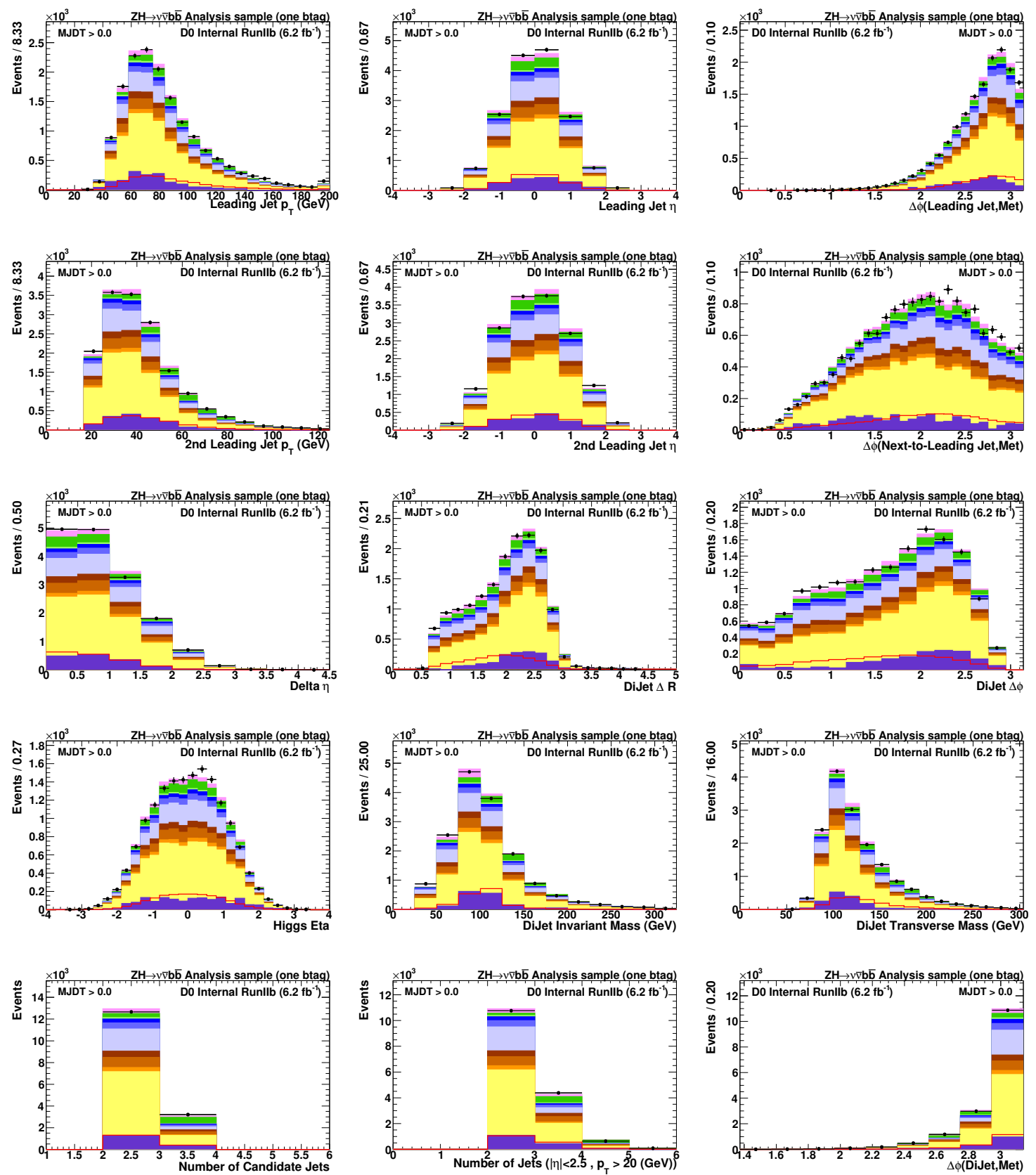

Figure 10.31: Signal sample with one b-tag after requiring a multijet decision tree cut of $D T>0.0$. 

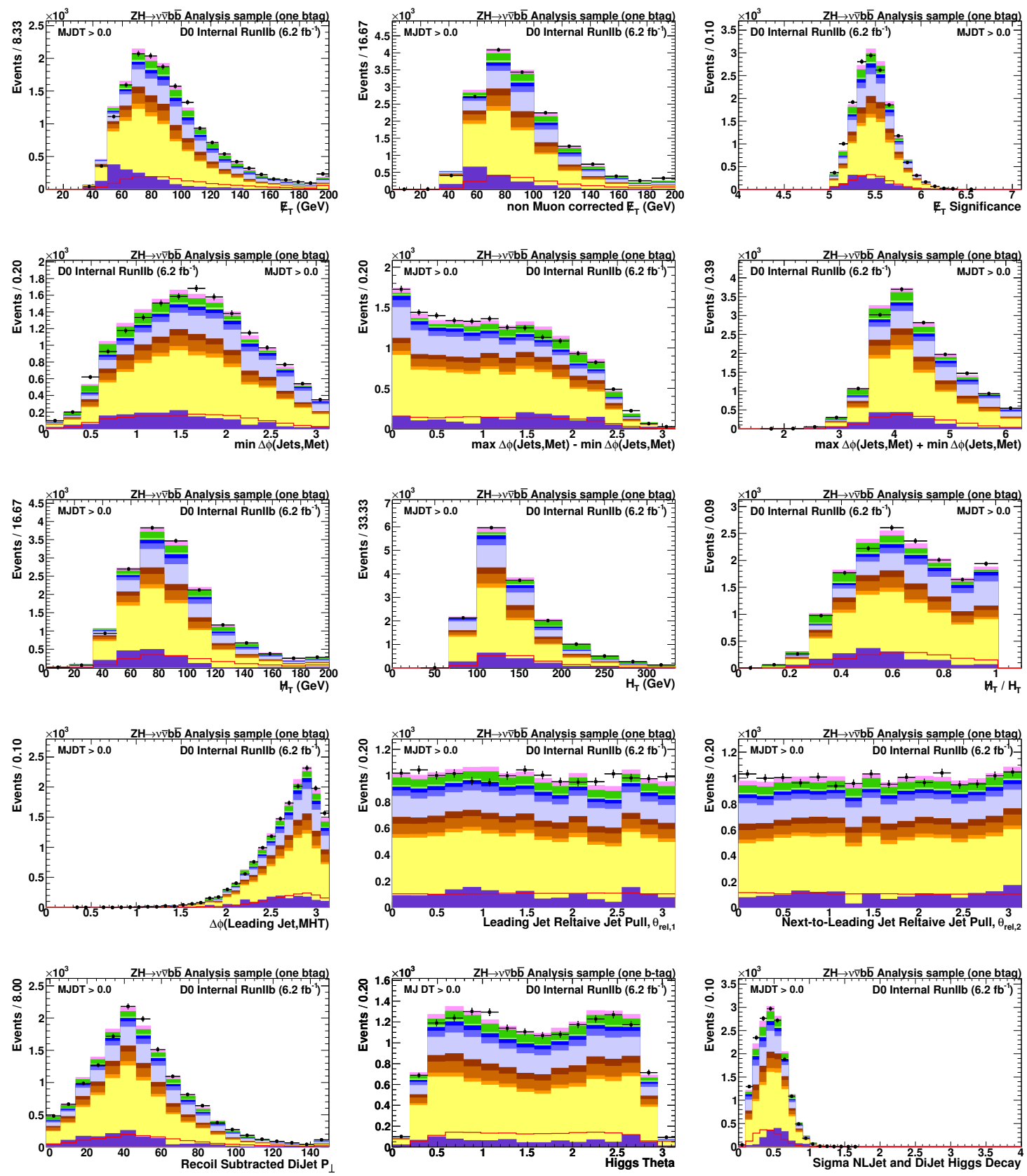

Figure 10.32: Signal sample with one b-tag after requiring a multijet decision tree cut of $D T>0.0$. 

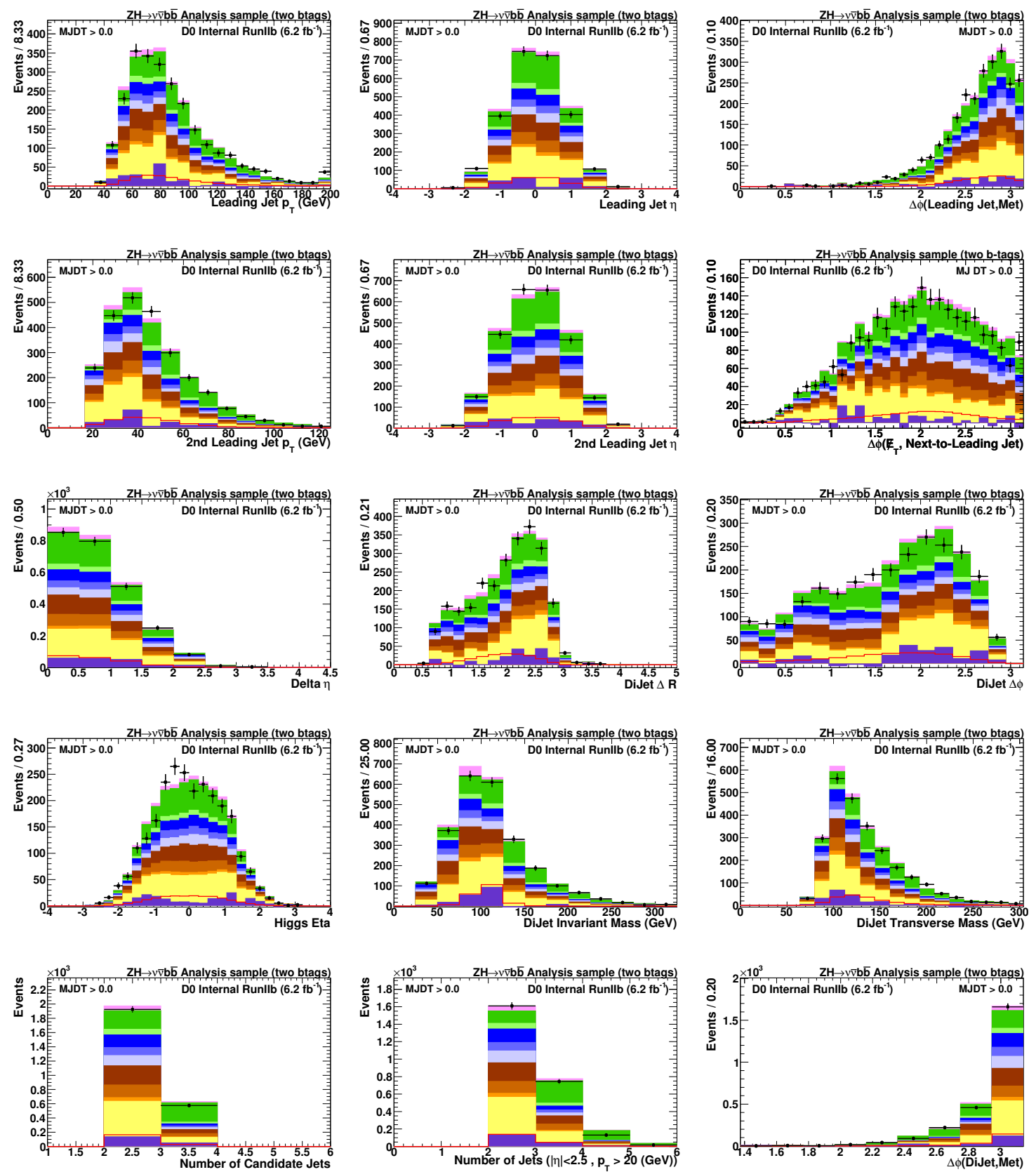

Figure 10.33: Signal sample with two L6 b-tags after requiring a multijet decision tree cut of $D T>0.0$. 

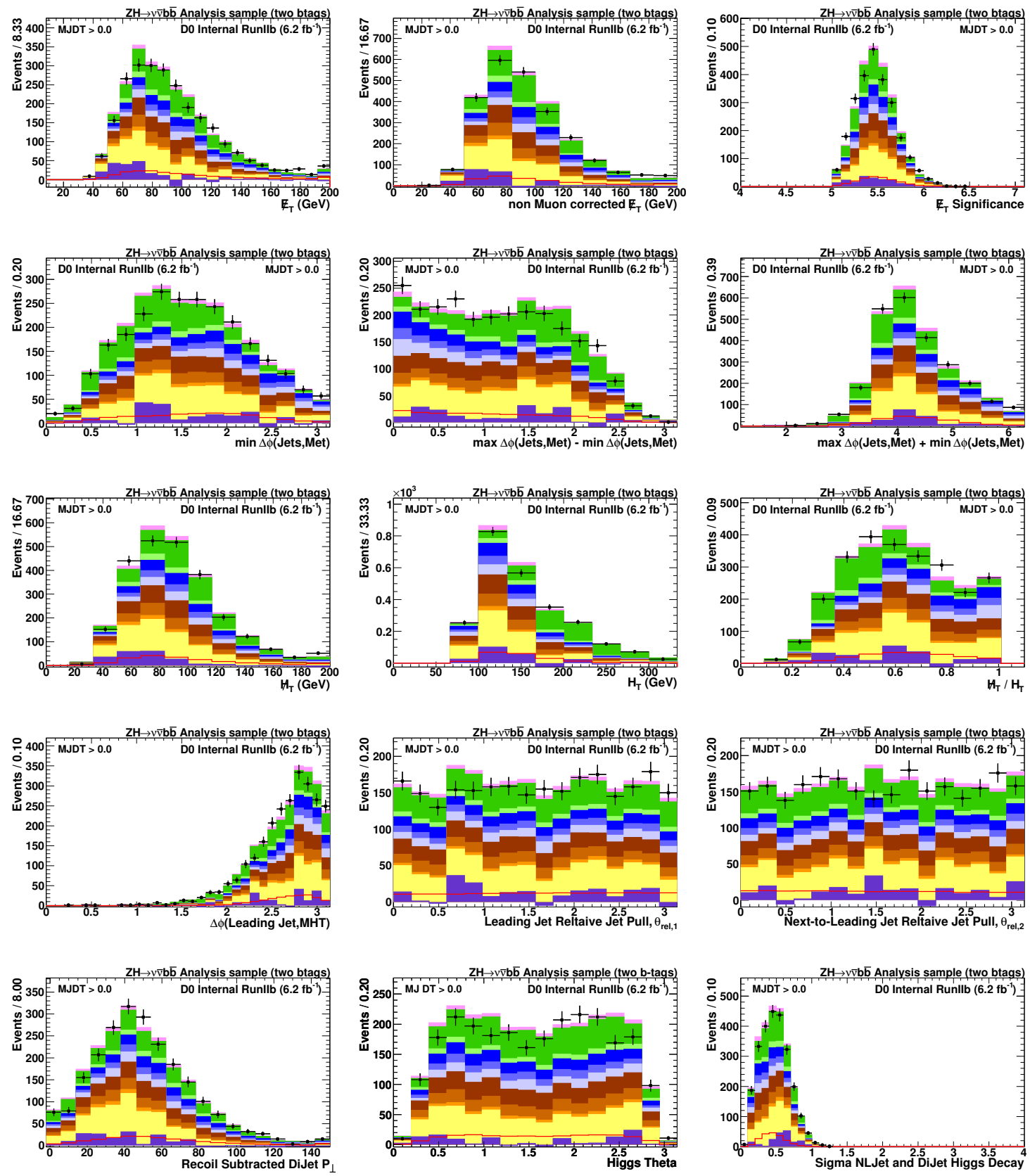

Figure 10.34: Signal sample with two L6 b-tags after requiring a multijet decision tree cut of $D T>0.0$. 


\subsection{Systematic Uncertainties}

Systematic uncertainties enter the calculation of the Higgs-boson production crosssection upper limit as normalization uncertainties that only affect the expected signal and/or background yields, and as shape uncertainties that affect the distributions of the discriminant variables used to derive the results.

The following experimental systematic uncertainties have been considered. Those not discussed in Section 8.6.1 are explained further. The correlations of these systematics are shown in Table 10.18.

- $\not_{T}+$ Jets Trigger:

We currently assign a flat error of $2 \%$ to account for the difference between the simulated and true decision compared in $W \rightarrow \mu \nu$ data.

- Jet energy scale

- Jet energy resolution

- Jet reconstruction and identification

- Vertex confirmation and Taggability

- $b$ tagging

- Lepton identification

- Multijet modeling

- Luminosity

The shape of these uncertainties is shown Appendices I to L.

The following systematic uncertainties are of theoretical nature. Those not discussed in Section 8.6.2 are explained further. 
- Cross sections

- Heavy flavor ratio

- ALPGEN parameters uncertainties

- Underlying event and fragmentation modeling

- Parton Distribution Function Uncertainty:

For the final shape uncertainty the largest pair of uncertainties for signal and background separately are used.

Tables 10.19-10.22 summarize all normalization systematic uncertainties in the RunIIb analysis evaluated for SM background expectations and for signal efficiencies. The signal is evaluated for a Higgs-boson mass of $m_{H}=115 \mathrm{GeV}$, summing the $Z H \rightarrow \nu \bar{\nu} b \bar{b}$ and $W H \rightarrow \ell^{ \pm} \nu b \bar{b}$ contributions. The $t \bar{t}$ and single top contributions are added together as well.

\subsection{Upper Limits on $Z H \rightarrow \nu \bar{\nu} b \bar{b}$ and $W H \rightarrow \ell^{ \pm} \nu b \bar{b}$ Production}

The package COLLIE discussed in Chapter 8 is used to derive expected and observed upper limits on combined $Z H \rightarrow \nu \bar{\nu} b \bar{b}$ and $W H \rightarrow \ell^{ \pm} \nu b \bar{b}$ production, for Higgsboson masses ranging from 100 to $150 \mathrm{GeV}$, in $5 \mathrm{GeV}$ increments. The inputs to the limit calculation are the physics-DT outputs, after requiring a multijet-DT output greater than 0.0. Limits are calculated for the Run IIb1 and Run IIb2-3 epochs separately, and for the full dataset, as well as for the single and double-tag samples separately, and for both samples combined. Two approaches to limit setting are used: CLfast, with systematic uncertainties ignored; (Table 10.23); CLfit2, with systematic 


\begin{tabular}{|l|cccc|}
\hline Systematic Uncertainty & COLLIE Tag & Type & Sample & Correlated \\
\hline \hline$\sigma_{Z H}$ & XS_ZH & Flat Norm & S & Full \\
\hline Jet Energy Scale & JES & Shape \& Norm & B \& S & Within Run \\
Jet Reco*ID & EFF & Shape \& Norm & B \& S & Within Epoch \\
Jet Resolution & RES & Shape \& Norm & B \& S & Within Run \\
\hline$\sigma_{\text {Diboson }}$ & Bkgd_XS_EW & Flat Norm & Diboson & Full \\
$\sigma_{T o p}$ & Bkgd_XS_Top & Flat Norm & Top & Full \\
$\sigma_{V+J e t s}$ & V+jets & Flat Norm & $(W / Z)+$ Jets & Full \\
$\sigma_{V+h f}$ & V+hf & Flat Norm & $(W / Z)+h f$ & Full \\
$V p_{T}$ reweighting & V+jets & Flat Norm & $(W / Z)+$ Jets & Full \\
Mulit-jet Normalization & MJ & Flat Norm & Mulit-jet & None \\
Parton Distribution Functions & PDF & Shape & B \& S & Full \\
\hline Vertex Conf./ Taggability & TAG & Shape \& Norm & B \& S & Within Run \\
B-Tagging HF Rate & btagHF & Shape \& Norm & B \& S & Within Run \\
B-Tagging LF Fake Rate & btagLF & Shape \& Norm & B \& S & Within Run \\
\hline Trigger Simulation & TRIG_D_MC & Shape \& Norm & B \& S & Within Run \\
\hline$\mu$ ID & MUID & Flat Norm & B \& S & Within Run \\
EM ID & EMID & Flat Norm & B \& S & Within Run \\
\hline Alpgen MLM & ALPGEN_MLM & Shape & V +lf & Full \\
Alpgen Event Scale & ALPGEN_S & Shape & $V+$ Jets & Full \\
Alpgen Underlying Event & ALPGEN_UE & Shape & $V+$ Jets & Full \\
Alpgen Angular RW & ALPGEN_RW & Shape & V + Jets & Full \\
\hline Luminosity & Luminosity & Flat Norm & B \& S & Full \\
\hline
\end{tabular}

Table 10.18: Table of correlations among systematics; $B$ denotes all Monte Carlo backgrounds, $S$ denotes signal, Full denotes correlations across data taking epochs (IIa, IIb1, and IIb2-3 all correlated). WithinRun denotes correlations within individual data taking runs (IIa and IIb uncorrelated). WithinEpoch denotes correlations only within individual data taking epochs (IIa, IIb1, and IIb2-3 all uncorrelated). The uncertainties affecting both $W+$ jets and $Z+$ jets are correlated between the two processes. 


\begin{tabular}{|l|c|c|c|c|}
\hline & B Bkgd & signal & Top & Diboson \\
\hline \hline Jet energy scale & \pm 3.9 & \pm 0.8 & $\mp 1.7$ & \pm 4.2 \\
Jet resolution & \pm 1.6 & $\mp 0.7$ & $\mp 2.4$ & \pm 1.4 \\
Jet reco ${ }^{*}$ ID & \pm 1.9 & \pm 2.0 & \pm 2.0 & \pm 2.0 \\
VC+Taggability & \pm 5.8 & \pm 6.8 & \pm 10.9 & \pm 7.0 \\
MC b-tag Heavy Flavor & \pm 3.6 & \pm 13.9 & \pm 13.0 & \pm 5.6 \\
MC b-tag Light Flavor & \pm 8.1 & \pm 1.0 & \pm 1.9 & \pm 6.9 \\
Trigger & \pm 1.9 & \pm 2.0 & \pm 2.0 & \pm 2.0 \\
Electron identification & \pm 0.3 & \pm 0.3 & \pm 0.7 & \pm 0.4 \\
Muon identification & \pm 0.4 & \pm 0.4 & \pm 0.8 & \pm 0.6 \\
Heavy-flavor fractions & \pm 4.1 & - & - & - \\
Cross section & \pm 5.5 & \pm 6.0 & \pm 10.0 & \pm 7.0 \\
Luminosity & \pm 6.1 & \pm 6.1 & \pm 6.1 & \pm 6.1 \\
\hline \hline & $\mathrm{Z}+\mathrm{lf}$ & $\mathrm{Z}+\mathrm{hf}$ & $\mathrm{W}+\mathrm{lf}$ & $\mathrm{W}+\mathrm{hf}$ \\
\hline Jet energy scale & \pm 3.6 & \pm 3.3 & \pm 4.3 & \pm 8.4 \\
Jet resolution & \pm 3.1 & \pm 0.6 & \pm 2.2 & \pm 1.2 \\
Jet reco ${ }^{\text {ID }}$ & \pm 2.0 & \pm 2.0 & \pm 2.0 & \pm 2.0 \\
VC+Taggability & \pm 4.3 & \pm 6.3 & \pm 5.4 & \pm 8.2 \\
MC b-tag Heavy Flavor & \pm 0.1 & \pm 8.9 & \pm 1.3 & \pm 8.0 \\
MC b-tag Light Flavor & \pm 12.6 & \pm 3.7 & \pm 10.7 & \pm 4.0 \\
Trigger & \pm 2.0 & \pm 2.0 & \pm 2.0 & \pm 2.0 \\
Electron identification & - & - & \pm 0.4 & \pm 0.4 \\
Muon identification & \pm 0.1 & - & \pm 0.5 & \pm 0.6 \\
Heavy-flavor fractions & - & \pm 20.0 & - & \pm 20.0 \\
Cross section & \pm 6.3 & \pm 6.3 & \pm 6.3 & \pm 6.3 \\
Luminosity & \pm 6.1 & \pm 6.1 & \pm 6.1 & \pm 6.1 \\
\hline \hline
\end{tabular}

Table 10.19: Relative systematic uncertainties (in \%) on SM background expectations and on the $Z H / W H$ signal $\left(m_{H}=115 \mathrm{GeV}\right)$ for the RunIIb1 single tag analysis. Shape-only systematic uncertainties are omitted and can be seen in Appendix I. Top includes $t \bar{t}$ and single top. Shape dependent errors are in italic and the quoted numbers are just the integral values to give an order estimate. 


\begin{tabular}{|l|c|c|c|c|}
\hline & $\sum$ Bkgd & signal & Top & Diboson \\
\hline \hline Jet energy scale & \pm 2.7 & \pm 0.8 & $\mp 2.3$ & \pm 3.3 \\
Jet resolution & - & $\mp 1.5$ & $\mp 2.0$ & \pm 1.0 \\
Jet reco ${ }^{*}$ ID & \pm 1.9 & \pm 2.0 & \pm 2.0 & \pm 2.0 \\
VC+Taggability & \pm 6.8 & \pm 5.7 & \pm 10.6 & \pm 7.0 \\
MC b-tag Heavy Flavor & \pm 10.4 & \pm 17.9 & \pm 19.1 & \pm 10.5 \\
MC b-tag Light Flavor & \pm 7.9 & \pm 0.6 & \pm 1.8 & \pm 8.4 \\
Trigger & \pm 1.9 & \pm 2.0 & \pm 2.0 & \pm 2.0 \\
Electron identification & \pm 0.4 & \pm 0.4 & \pm 0.9 & \pm 0.8 \\
Muon identification & \pm 0.5 & \pm 0.5 & \pm 1.1 & \pm 0.2 \\
Heavy-flavor fractions & \pm 6.9 & - & - & - \\
Cross section & \pm 5.1 & \pm 6.0 & \pm 10.0 & \pm 7.0 \\
Luminosity & \pm 6.1 & \pm 6.1 & \pm 6.1 & \pm 6.1 \\
\hline \hline & $\mathrm{Z}+\mathrm{lf}$ & $\mathrm{Z}+\mathrm{hf}$ & $\mathrm{W}+\mathrm{lf}$ & $\mathrm{W}+\mathrm{hf}$ \\
\hline Jet energy scale & \pm 6.7 & \pm 1.8 & \pm 4.7 & \pm 5.5 \\
Jet resolution & \pm 2.6 & \pm 0.3 & \pm 0.8 & \pm 0.2 \\
Jet reco*ID & \pm 2.0 & \pm 2.0 & \pm 2.0 & \pm 2.0 \\
VC+Taggability & \pm 2.9 & \pm 5.3 & \pm 5.2 & \pm 7.7 \\
MC b-tag Heavy Flavor & \pm 0.3 & \pm 14.3 & \pm 2.1 & \pm 13.1 \\
MC b-tag Light Flavor & \pm 19.1 & \pm 3.8 & \pm 16.3 & \pm 4.4 \\
Trigger & \pm 2.0 & \pm 2.0 & \pm 2.0 & \pm 2.0 \\
Electron identification & - & - & \pm 0.2 & \pm 0.4 \\
Muon identification & \pm 0.2 & \pm 0.3 & \pm 0.4 & \pm 0.4 \\
Heavy-flavor fractions & - & \pm 20.0 & - & \pm 20.0 \\
Cross section & \pm 6.3 & \pm 6.3 & \pm 6.3 & \pm 6.3 \\
Luminosity & \pm 6.1 & \pm 6.1 & \pm 6.1 & \pm 6.1 \\
\hline
\end{tabular}

Table 10.20: Relative systematic uncertainties (in \%) on SM background expectations and on the $Z H / W H$ signal $\left(m_{H}=115 \mathrm{GeV}\right)$ for the RunIIb1 two tags analysis. Shape-only systematic uncertainties are omitted and can be seen in Appendix J. Top includes $t \bar{t}$ and single top. Shape dependent errors are in italic and the quoted numbers are just the integral values to give an order estimate. 


\begin{tabular}{|l|c|c|c|c|}
\hline & B Bkgd & signal & Top & Diboson \\
\hline \hline Jet energy scale & \pm 2.3 & $\mp 0.4$ & $\mp 3.0$ & \pm 1.3 \\
Jet resolution & \pm 0.9 & $\mp 1.2$ & $\mp 2.4$ & $\mp 0.6$ \\
Jet reco ${ }^{*}$ ID & \pm 1.8 & \pm 2.0 & \pm 2.0 & \pm 2.0 \\
VC+Taggability & \pm 2.8 & \pm 3.3 & \pm 5.0 & \pm 3.6 \\
MC b-tag Heavy Flavor & \pm 1.7 & \pm 5.7 & \pm 4.8 & \pm 2.9 \\
MC b-tag Light Flavor & \pm 2.0 & \pm 0.3 & \pm 0.5 & \pm 1.7 \\
Trigger & \pm 1.8 & \pm 2.0 & \pm 2.0 & \pm 2.0 \\
Electron identification & \pm 0.2 & \pm 0.3 & \pm 0.6 & \pm 0.4 \\
Muon identification & \pm 0.3 & \pm 0.4 & \pm 0.9 & \pm 0.4 \\
Heavy-flavor fractions & \pm 4.2 & - & - & - \\
Cross section & \pm 5.3 & \pm 6.0 & \pm 10.0 & \pm 7.0 \\
Luminosity & \pm 6.1 & \pm 6.1 & \pm 6.1 & \pm 6.1 \\
\hline \hline & $\mathrm{Z}+\mathrm{lf}$ & $\mathrm{Z}+\mathrm{hf}$ & $\mathrm{W}+\mathrm{lf}$ & $\mathrm{W}+\mathrm{hf}$ \\
\hline Jet energy scale & \pm 3.2 & \pm 2.2 & \pm 3.6 & \pm 1.7 \\
Jet resolution & \pm 2.1 & \pm 0.6 & \pm 1.7 & \pm 0.2 \\
Jet reco ${ }^{\text {ID }}$ & \pm 2.0 & \pm 2.0 & \pm 2.0 & \pm 2.0 \\
VC+Taggability & \pm 2.2 & \pm 3.4 & \pm 2.7 & \pm 4.1 \\
MC b-tag Heavy Flavor & \pm 0.1 & \pm 4.0 & \pm 0.9 & \pm 4.0 \\
MC b-tag Light Flavor & \pm 3.3 & \pm 1.0 & \pm 2.8 & \pm 1.0 \\
Trigger & \pm 2.0 & \pm 2.0 & \pm 2.0 & \pm 2.0 \\
Electron identification & - & - & \pm 0.3 & \pm 0.4 \\
Muon identification & \pm 0.1 & - & \pm 0.4 & \pm 0.5 \\
Heavy-flavor fractions & - & \pm 20.0 & - & \pm 20.0 \\
Cross section & \pm 6.3 & \pm 6.3 & \pm 6.3 & \pm 6.3 \\
Luminosity & \pm 6.1 & \pm 6.1 & \pm 6.1 & \pm 6.1 \\
\hline \hline
\end{tabular}

Table 10.21: Relative systematic uncertainties (in \%) on SM background expectations and on the $Z H / W H$ signal $\left(m_{H}=115 \mathrm{GeV}\right)$ for the RunIIb23 single tag analysis. Shape-only systematic uncertainties are omitted and can be seen in Appendix K. Top includes $t \bar{t}$ and single top. Shape dependent errors are in italic and the quoted numbers are just the integral values to give an order estimate. 


\begin{tabular}{|l|c|c|c|c|}
\hline & $\sum$ Bkgd & signal & Top & Diboson \\
\hline \hline Jet energy scale & \pm 0.9 & $\mp 0.7$ & $\mp 3.1$ & \pm 1.4 \\
Jet resolution & $\mp 0.3$ & $\mp 2.3$ & $\mp 2.5$ & $\mp 1.0$ \\
Jet reco ${ }^{*}$ ID & \pm 1.9 & \pm 2.0 & \pm 2.0 & \pm 2.0 \\
VC+Taggability & \pm 3.1 & \pm 2.9 & \pm 4.5 & \pm 3.4 \\
MC b-tag Heavy Flavor & \pm 5.0 & \pm 8.4 & \pm 7.7 & \pm 5.1 \\
MC b-tag Light Flavor & \pm 1.8 & \pm 0.1 & \pm 0.4 & \pm 2.1 \\
Trigger & \pm 1.9 & \pm 2.0 & \pm 2.0 & \pm 2.0 \\
Electron identification & \pm 0.4 & \pm 0.3 & \pm 0.8 & \pm 0.4 \\
Muon identification & \pm 0.4 & \pm 0.4 & \pm 0.7 & \pm 0.6 \\
Heavy-flavor fractions & \pm 7.3 & - & - & - \\
Cross section & \pm 5.0 & \pm 6.0 & \pm 10.0 & \pm 7.0 \\
Luminosity & \pm 6.1 & \pm 6.1 & \pm 6.1 & \pm 6.1 \\
\hline \hline & $\mathrm{Z}+\mathrm{lf}$ & $\mathrm{Z}+\mathrm{hf}$ & $\mathrm{W}+\mathrm{lf}$ & $\mathrm{W}+\mathrm{hf}$ \\
\hline Jet energy scale & \pm 3.2 & \pm 1.5 & \pm 3.7 & \pm 1.7 \\
Jet resolution & \pm 2.6 & $\mp 0.6$ & \pm 1.3 & - \\
Jet reco*ID & \pm 2.0 & \pm 2.0 & \pm 2.0 & \pm 2.0 \\
VC+Taggability & \pm 1.9 & \pm 2.9 & \pm 2.5 & \pm 3.6 \\
MC b-tag Heavy Flavor & \pm 0.2 & \pm 6.9 & \pm 1.6 & \pm 6.8 \\
MC b-tag Light Flavor & \pm 5.3 & \pm 0.9 & \pm 4.3 & \pm 0.9 \\
Trigger & \pm 2.0 & \pm 2.0 & \pm 2.0 & \pm 2.0 \\
Electron identification & \pm 0.1 & - & \pm 0.3 & \pm 0.4 \\
Muon identification & - & - & \pm 0.3 & \pm 0.6 \\
Heavy-flavor fractions & - & \pm 20.0 & - & \pm 20.0 \\
Cross section & \pm 6.3 & \pm 6.3 & \pm 6.3 & \pm 6.3 \\
Luminosity & \pm 6.1 & \pm 6.1 & \pm 6.1 & \pm 6.1 \\
\hline
\end{tabular}

Table 10.22: Relative systematic uncertainties (in \%) on SM background expectations and on the $Z H / W H$ signal $\left(m_{H}=115 \mathrm{GeV}\right)$ for the RunIIb23 two tags analysis. Shape-only systematic uncertainties are omitted and can be seen in Appendix L. Top includes $t \bar{t}$ and single top. Shape dependent errors are in italic and the quoted numbers are just the integral values to give an order estimate. 
uncertainties constrained by our data through a profiling technique. (Table 10.24). Systematic uncertainties are correlated across data taking epochs and across single and double-tag samples as detailed in Section 10.9.

Figures 10.35 and 10.36 show the results from the individual data taking epochs

- in terms of expected and observed excluded cross sections, relative to the standard model expectation, and

- in terms of log-likelihood ratios for the signal+background and background-only hypotheses, and as observed in the data.

\subsubsection{Run IIb Results}

Results based on the physics-DT outputs, as obtained with CLfit2, are presented in Figures 10.37 through 10.39 for the full RunIIb data set for the combined single and double tag, double tag only, and single tag only respectively. Details on the limits obtained with CLfast and CLFit2 using the physics-DT outputs, are given in Tables 10.23-10.24.

\subsubsection{Comments on the limit setting procedure}

Several technical statements must be made concerning the configuration of COLLIE for the limit calculation. The utility known as Equal Probable Difference was used only on jet related systematics. This is meant to reduce the effect of statistical fluctuations in the the tails when evaluating systematic errors. Bins in the nominal distribution which contain less than $40 \%$ of the maximum bin content are combined with neighboring bins. This continues until the integral of these combined bins is no longer less than $40 \%$ of the maximum bin content. The fractional difference is calculated 

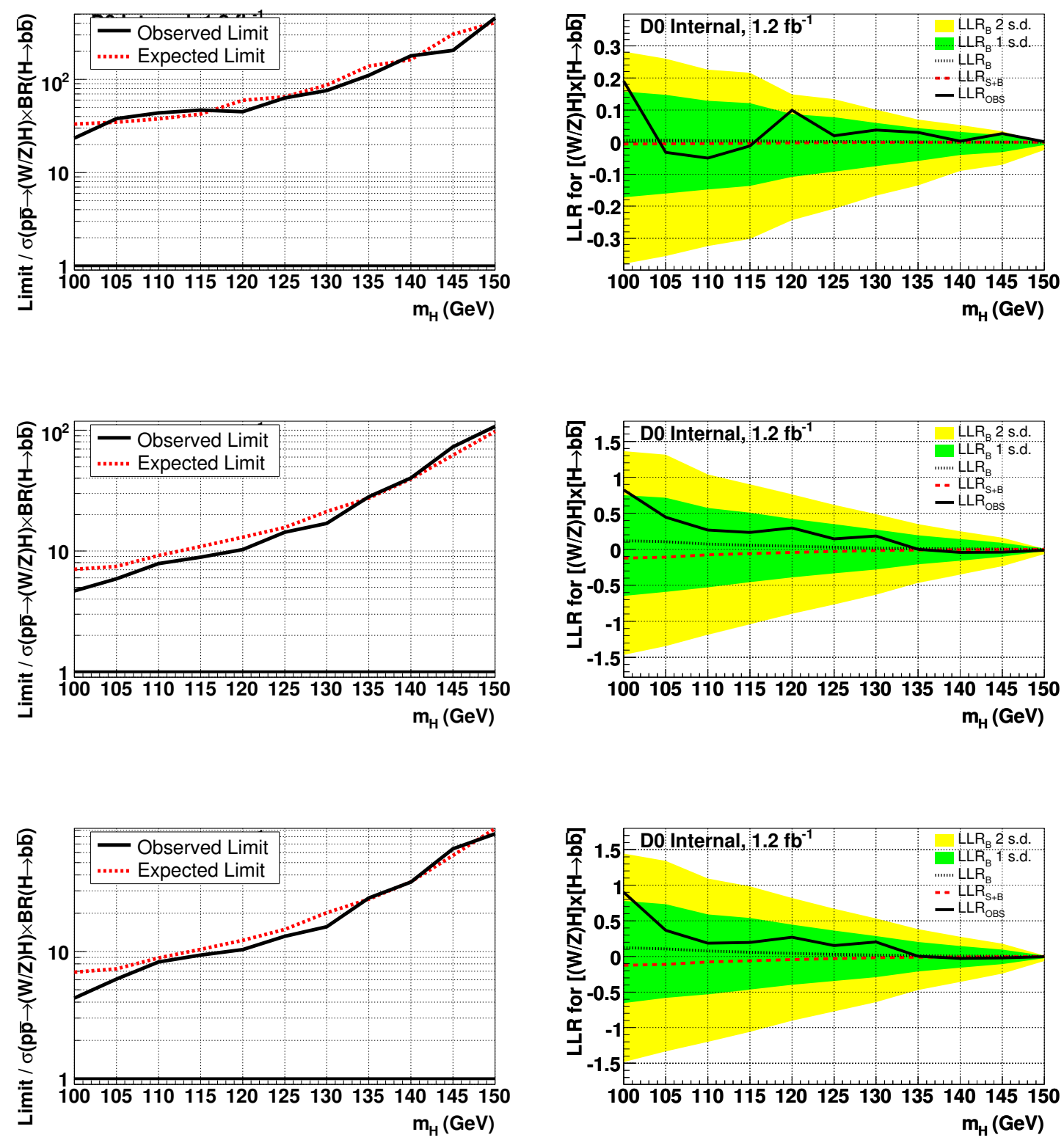

Figure 10.35: CLFit2 expected and observed 95\% CL upper limits on the cross section ratios and log-likelihood ratios for the Run IIb1 single \& double tag samples separately and combined using boosted decision trees. 

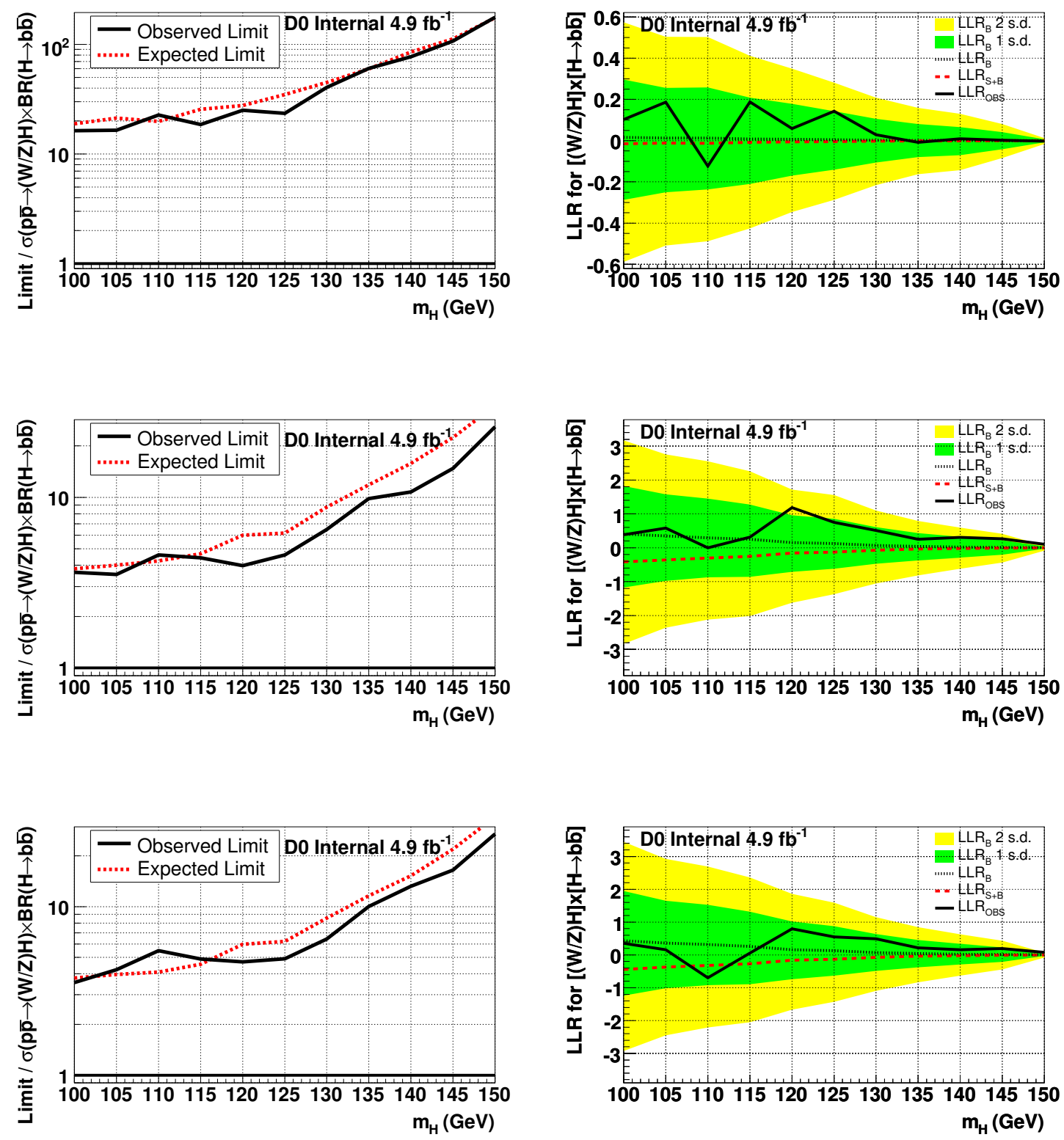

Figure 10.36: CLFit2 expected and observed 95\% CL upper limits on the cross section ratios and log-likelihood ratios for the Run IIb2-3 single \& double tag samples separately and combined using boosted decision trees. 
using the resultant binning then mapped back to the original binning. COLLIE has an automatic flattening procedure where shape systematics can be flattened if the fractional difference varies wildly from bin to bin. This utility, which is used in the default settings, was not used.
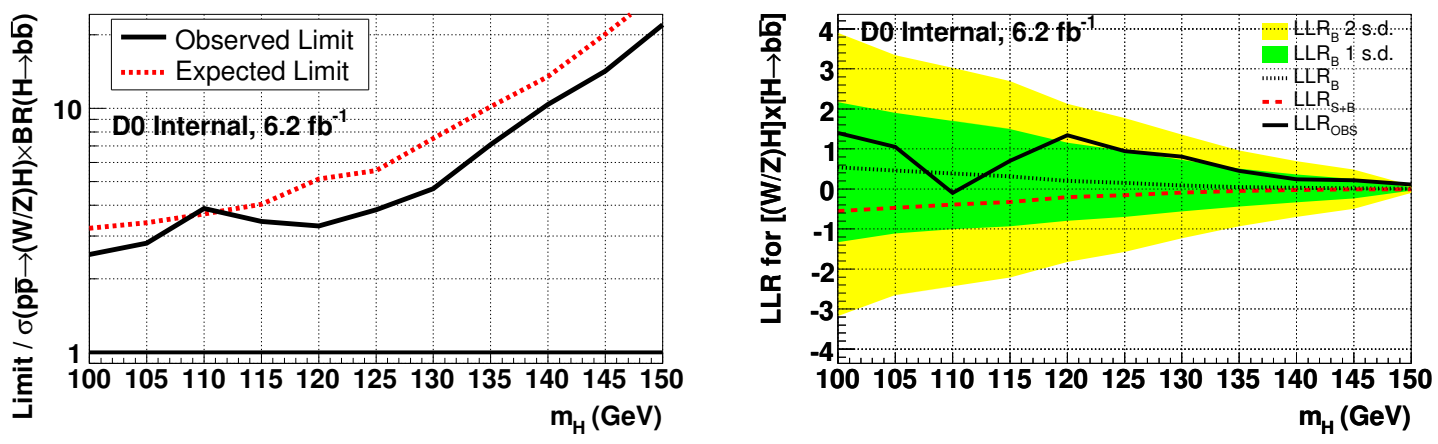

Figure 10.37: CLFit2 expected and observed 95\% CL upper limits on the cross section ratios and log-likelihood ratios for the full Run IIb single \& double tag combination using boosted decision trees.
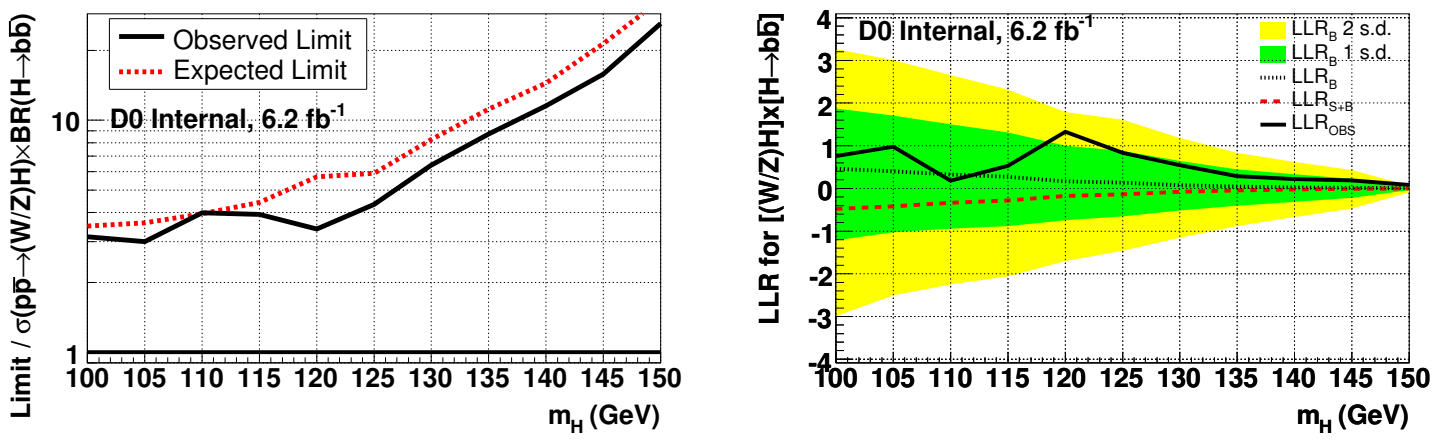

Figure 10.38: CLFit2 expected and observed 95\% CL upper limits on the cross section ratios and log-likelihood ratios for the full Run IIb double tag sample using boosted decision trees. 

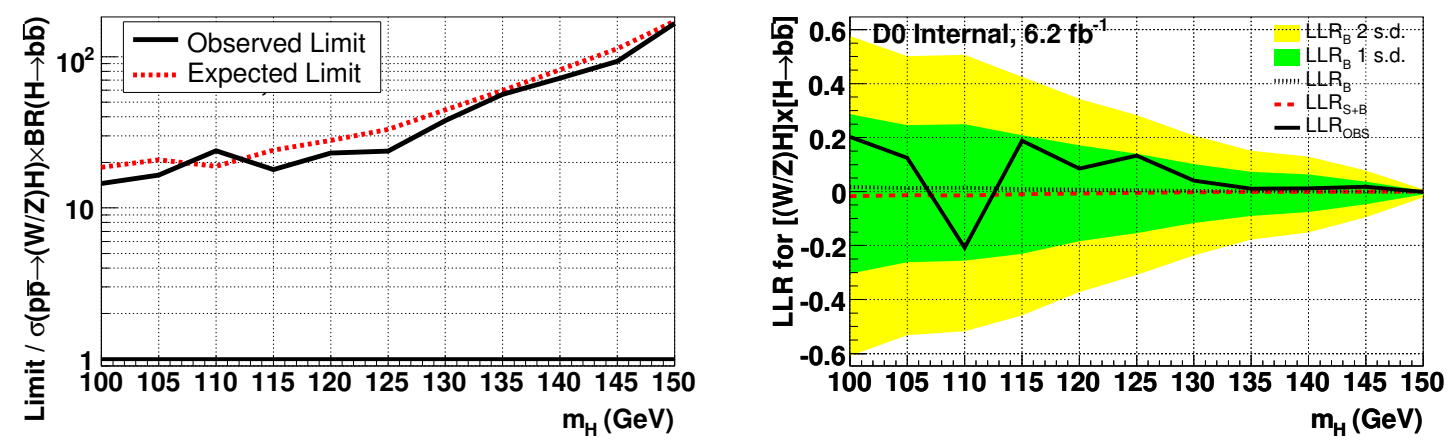

Figure 10.39: CLFit2 expected and observed 95\% CL upper limits on the cross section ratios and log-likelihood ratios for the full Run IIb single tag sample using boosted decision trees. 


\begin{tabular}{|c|c|c|c|c|c|c|}
\hline \multicolumn{7}{|c|}{ BDT CLfast Limits } \\
\hline \multirow{2}{*}{$\begin{array}{l}\text { Tagging } \\
\text { Point }\end{array}$} & \multicolumn{2}{|c|}{ Run IIb1 } & \multicolumn{2}{|c|}{ Run IIb2-3 } & \multicolumn{2}{|c|}{ Run IIb } \\
\hline & Exp. & Obs. & Exp. & Obs. & Exp. & Obs. \\
\hline & \multicolumn{6}{|c|}{100} \\
\hline $1 \mathrm{~L} 6$ & 12.791 & 1.308 & 7.271 & 10.696 & 6.333 & 4.793 \\
\hline 2 L6 & 4.579 & 2.763 & 2.204 & 1.185 & 1.955 & 0.968 \\
\hline \multirow[t]{2}{*}{ Combined } & 4.332 & 1.637 & 2.108 & 1.264 & 1.881 & 0.955 \\
\hline & \multicolumn{6}{|c|}{105} \\
\hline $1 \mathrm{~L} 6$ & 13.693 & 4.266 & 7.963 & 8.069 & 6.881 & 4.288 \\
\hline 2 L6 & 4.996 & 3.657 & 2.432 & 1.315 & 2.154 & 1.051 \\
\hline \multirow[t]{2}{*}{ Combined } & 4.646 & 2.605 & 2.310 & 1.283 & 2.042 & 0.949 \\
\hline & \multicolumn{6}{|c|}{110} \\
\hline $1 \mathrm{~L} 6$ & 14.975 & 6.869 & 8.682 & 11.937 & 7.394 & 6.353 \\
\hline 2 L6 & 5.759 & 3.967 & 2.661 & 1.666 & 2.380 & 1.340 \\
\hline \multirow[t]{2}{*}{ Combined } & 5.371 & 3.075 & 2.531 & 1.724 & 2.279 & 1.225 \\
\hline & \multicolumn{6}{|c|}{115} \\
\hline $1 \mathrm{~L} 6$ & 17.436 & 7.830 & 10.136 & 7.726 & 8.686 & 4.551 \\
\hline 2 L6 & 6.534 & 4.715 & 2.939 & 1.808 & 2.657 & 1.527 \\
\hline \multirow[t]{2}{*}{ Combined } & 6.066 & 3.284 & 2.831 & 1.691 & 2.547 & 1.276 \\
\hline & \multicolumn{6}{|c|}{120} \\
\hline $1 \mathrm{~L} 6$ & 21.333 & 0.553 & 12.132 & 10.829 & 10.469 & 5.161 \\
\hline 2 L6 & 7.780 & 4.834 & 3.668 & 1.622 & 3.285 & 1.479 \\
\hline \multirow[t]{2}{*}{ Combined } & 7.249 & 2.573 & 3.528 & 1.463 & 3.108 & 0.753 \\
\hline & \multicolumn{6}{|c|}{125} \\
\hline $1 \mathrm{~L} 6$ & 24.907 & 8.962 & 14.794 & 9.194 & 12.626 & 4.860 \\
\hline 2 L6 & 9.317 & 5.957 & 4.268 & 2.605 & 3.832 & 2.015 \\
\hline \multirow[t]{2}{*}{ Combined } & 8.623 & 4.532 & 4.094 & 2.193 & 3.656 & 1.736 \\
\hline & \multicolumn{6}{|c|}{130} \\
\hline $1 \mathrm{~L} 6$ & 31.473 & 11.501 & 19.038 & 20.288 & 16.153 & 10.195 \\
\hline 2 L6 & 11.793 & 6.582 & 5.508 & 3.167 & 4.940 & 2.411 \\
\hline \multirow[t]{2}{*}{ Combined } & 11.023 & 6.006 & 5.284 & 3.029 & 4.690 & 2.295 \\
\hline & \multicolumn{6}{|c|}{135} \\
\hline $1 \mathrm{~L} 6$ & 43.015 & 4.632 & 25.067 & 25.150 & 21.525 & 12.017 \\
\hline 2 L6 & 15.414 & 12.247 & 7.341 & 4.580 & 6.526 & 3.831 \\
\hline \multirow[t]{2}{*}{ Combined } & 14.493 & 7.395 & 6.947 & 4.383 & 6.244 & 3.355 \\
\hline & \multicolumn{6}{|c|}{140} \\
\hline $1 \mathrm{~L} 6$ & 57.777 & 18.255 & 35.009 & 25.197 & 29.659 & 14.297 \\
\hline 2 L6 & 21.180 & 14.848 & 10.120 & 5.963 & 8.956 & 4.771 \\
\hline Combined & 19.866 & 10.921 & 9.616 & 5.296 & 8.521 & 4.445 \\
\hline & & & & & & \\
\hline $1 \mathrm{~L} 6$ & 90.985 & 1.440 & 50.529 & 47.373 & 43.696 & 21.846 \\
\hline 2 L6 & 32.802 & 22.947 & 14.904 & 9.104 & 13.324 & 7.708 \\
\hline Combined & 30.566 & 13.087 & 14.284 & 7.972 & 12.672 & 6.065 \\
\hline & & & & & & \\
\hline $1 \mathrm{~L} 6$ & 138.106 & 58.703 & 78.694 & 77.306 & 68.118 & 45.016 \\
\hline 2 L6 & 51.567 & 39.382 & 23.550 & 14.284 & 20.990 & 11.951 \\
\hline Combined & 47.844 & 28.149 & 22.625 & 14.127 & 20.055 & 10.442 \\
\hline
\end{tabular}

Table 10.23: Ratio of the expected (observed) limit to the Standard Model prediction for different Higgs masses. Numb\&86 are given for Run IIb1 and IIb2-3, as well as for the combined result, using the BDT outputs as input and ignoring systematic uncertainities (CLfast). 


\begin{tabular}{|c|c|c|c|c|c|c|}
\hline \multicolumn{7}{|c|}{ BDT CLfit2 Limits } \\
\hline \multirow{2}{*}{$\begin{array}{l}\text { Tagging } \\
\text { Point }\end{array}$} & \multicolumn{2}{|c|}{ Run IIb1 } & \multicolumn{2}{|c|}{ Run IIb2-3 } & \multicolumn{2}{|c|}{ Run IIb } \\
\hline & Exp. & Obs. & Exp. & Obs. & Exp. & Obs. \\
\hline & \multicolumn{6}{|c|}{100} \\
\hline $1 \mathrm{~L} 6$ & 33.217 & 23.437 & 18.880 & 16.295 & 18.512 & 14.465 \\
\hline 2 L6 & 7.055 & 4.661 & 3.810 & 3.633 & 3.498 & 3.143 \\
\hline \multirow[t]{2}{*}{ Combined } & 6.873 & 4.310 & 3.770 & 3.544 & 3.227 & 2.516 \\
\hline & \multicolumn{6}{|c|}{105} \\
\hline $1 \mathrm{~L} 6$ & 34.809 & 38.053 & 21.307 & 16.482 & 20.755 & 16.505 \\
\hline 2 L6 & 7.441 & 5.884 & 4.002 & 3.534 & 3.616 & 3.002 \\
\hline \multirow[t]{2}{*}{ Combined } & 7.309 & 6.084 & 3.961 & 4.223 & 3.399 & 2.800 \\
\hline & \multicolumn{6}{|c|}{110} \\
\hline $1 \mathrm{~L} 6$ & 37.755 & 43.772 & 19.799 & 22.697 & 18.832 & 23.813 \\
\hline 2 L6 & 9.166 & 7.876 & 4.241 & 4.596 & 3.950 & 3.991 \\
\hline \multirow[t]{2}{*}{ Combined } & 8.899 & 8.274 & 4.091 & 5.479 & 3.686 & 3.880 \\
\hline & \multicolumn{6}{|c|}{115} \\
\hline $1 \mathrm{~L} 6$ & 42.433 & 46.809 & 25.555 & 18.581 & 24.128 & 17.881 \\
\hline 2 L6 & 10.915 & 8.888 & 4.668 & 4.424 & 4.418 & 3.928 \\
\hline \multirow[t]{2}{*}{ Combined } & 10.384 & 9.392 & 4.555 & 4.890 & 4.043 & 3.433 \\
\hline & \multicolumn{6}{|c|}{120} \\
\hline $1 \mathrm{~L} 6$ & 59.631 & 44.941 & 27.775 & 25.102 & 27.983 & 23.051 \\
\hline $2 \mathrm{~L} 6$ & 13.032 & 10.266 & 6.004 & 3.980 & 5.700 & 3.403 \\
\hline \multirow[t]{2}{*}{ Combined } & 12.265 & 10.363 & 5.990 & 4.699 & 5.136 & 3.294 \\
\hline & \multicolumn{6}{|c|}{125} \\
\hline $1 \mathrm{~L} 6$ & 65.213 & 63.411 & 34.950 & 23.555 & 32.999 & 23.703 \\
\hline 2 L6 & 15.704 & 14.294 & 6.173 & 4.598 & 5.902 & 4.334 \\
\hline \multirow[t]{2}{*}{ Combined } & 14.906 & 13.184 & 6.220 & 4.903 & 5.553 & 3.838 \\
\hline & \multicolumn{6}{|c|}{130} \\
\hline $1 \mathrm{~L} 6$ & 86.821 & 76.062 & 45.000 & 40.536 & 44.446 & 37.703 \\
\hline $2 \mathrm{~L} 6$ & 21.227 & 16.918 & 8.761 & 6.483 & 8.258 & 6.408 \\
\hline \multirow[t]{2}{*}{ Combined } & 20.194 & 15.673 & 8.562 & 6.434 & 7.519 & 4.686 \\
\hline & \multicolumn{6}{|c|}{135} \\
\hline $1 \mathrm{~L} 6$ & 138.754 & 110.766 & 59.868 & 60.530 & 59.720 & 56.321 \\
\hline 2 L6 & 27.407 & 28.110 & 11.850 & 9.814 & 11.165 & 8.729 \\
\hline \multirow[t]{2}{*}{ Combined } & 25.821 & 26.392 & 11.607 & 10.015 & 10.155 & 7.088 \\
\hline & \multicolumn{6}{|c|}{140} \\
\hline $1 \mathrm{~L} 6$ & 162.982 & 179.432 & 85.388 & 77.105 & 82.139 & 72.082 \\
\hline 2 L6 & 39.534 & 40.234 & 15.781 & 10.754 & 14.451 & 11.523 \\
\hline Combined & 35.340 & 35.250 & 15.253 & 13.212 & 13.503 & 10.354 \\
\hline & & & & & & \\
\hline $1 \mathrm{~L} 6$ & 306.201 & 205.242 & 112.477 & 107.253 & 112.704 & 93.038 \\
\hline $2 \mathrm{~L} 6$ & 62.122 & 72.974 & 22.427 & 14.755 & 21.397 & 15.812 \\
\hline Combined & 56.997 & 64.245 & 21.941 & 16.477 & 20.153 & 14.217 \\
\hline & & & & & & \\
\hline $1 \mathrm{~L} 6$ & 406.324 & 456.108 & 175.427 & 177.263 & 171.517 & 166.114 \\
\hline 2 L6 & 97.813 & 107.859 & 34.394 & 25.872 & 32.901 & 26.082 \\
\hline Combined & 92.638 & 84.513 & 33.922 & 27.012 & 30.565 & 22.025 \\
\hline
\end{tabular}

Table 10.24: Ratio of the expected (observerd) different Higgs masses. Numbers are given for Run IIb1 and IIb2-3, as well as for the combined result, using the BDT outputs as input, with all systematic uncertainities included and with profiling (CLfit2). 


\section{$10.11 Z Z \rightarrow \nu \bar{\nu} b \bar{b}$ Interpretation}

To validate the techniques used in the Higgs search outlined in this note and the combination with similar searches throughout $\mathrm{D} \emptyset$ the result can be reinterpreted as measurement of di-boson production with an analogous final state. This analysis is sensitive to $Z Z$ and $W Z$ production where a $Z$ decaying to a pair of b-quarks takes the place of the Higgs boson. The only deviation from the Higgs search is the re-training of the three decision trees with the above mentioned di-boson samples as the signal and the remaining di-boson production processes are maintained as a background. The multi-jet decision trees are shown in Figure 10.40 while the single and double tag decision trees are shown in Figures 10.41 and 10.42. Unfortunately this analysis alone is not yet sensitive to these di-boson production processes. The upper limit on di-boson producing with a $Z$ decaying to a pair of b-quarks can be found in Table 10.25. The LLR distribution for the full Run IIb single and double tag combined result can be found in Figure 10.43.

\begin{tabular}{|l|cc|cc|cc|}
\hline \multicolumn{7}{|c|}{ BDT CLfit2 Limits } \\
\hline Tagging & \multicolumn{2}{|c|}{ Run IIb1 } & \multicolumn{2}{c|}{ Run IIb2-3 } & \multicolumn{2}{c|}{ Run IIb } \\
Point & Exp. & Obs. & Exp. & Obs. & Exp. & Obs. \\
\hline & \multicolumn{7}{|c|}{ V(Z $\rightarrow$ bb $)$} \\
\hline 1 L6 & 17.502 & 17.008 & 14.126 & 10.304 & 13.167 & 8.869 \\
2 L6 & 3.909 & 3.697 & 2.404 & 2.378 & 2.159 & 2.052 \\
Combined & 3.805 & 3.561 & 2.372 & 2.577 & 2.039 & 1.747 \\
\hline
\end{tabular}

Table 10.25: Ratio of the expected (observed) limit to the Standard Model prediction for a di-boson search. Numbers are given for Run IIb1 and IIb2-3, as well as for the combined result. 

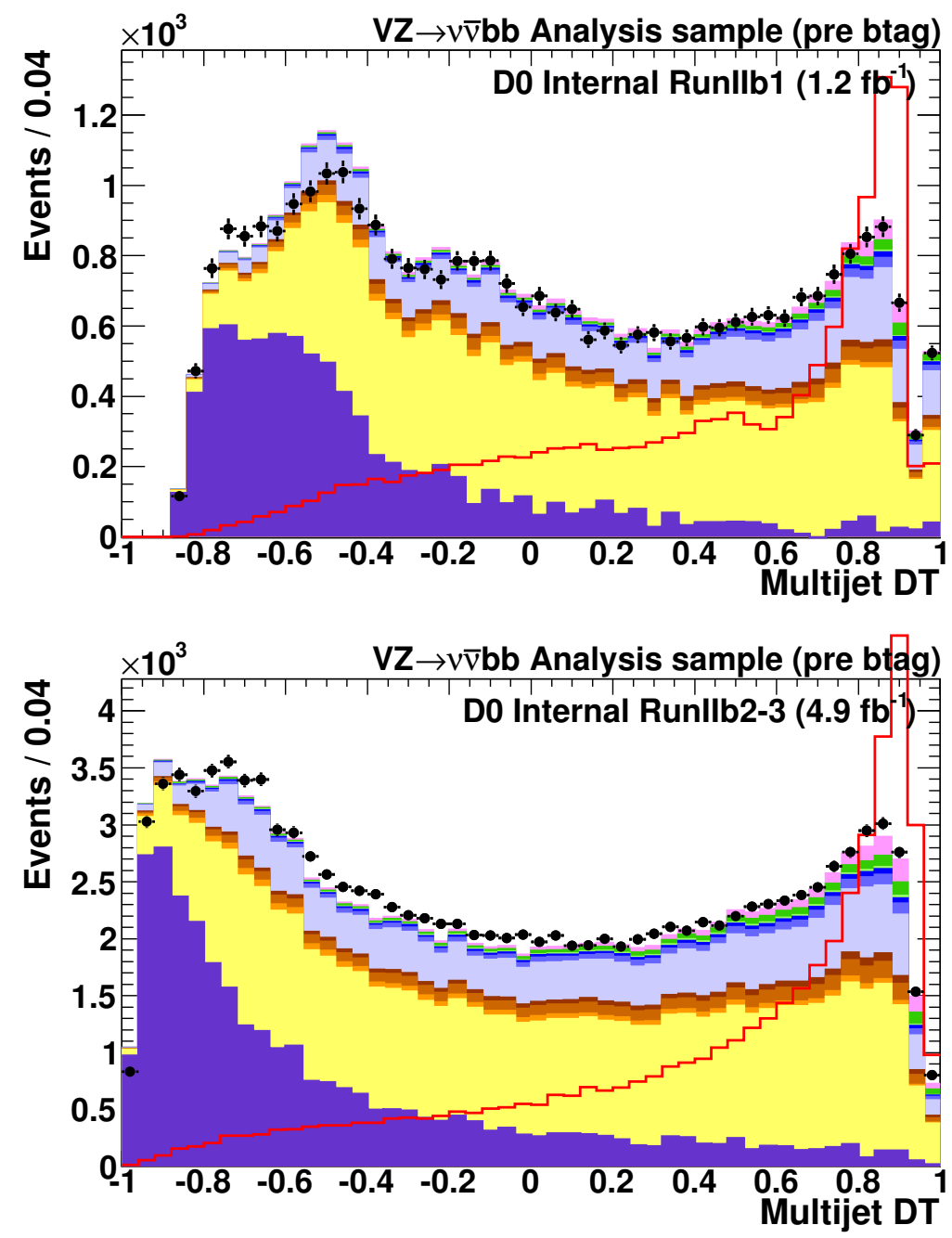

Figure 10.40: Multijet decision trees for a di-boson search in Run IIb1 (top) and Run IIb2-3 (bottom). 

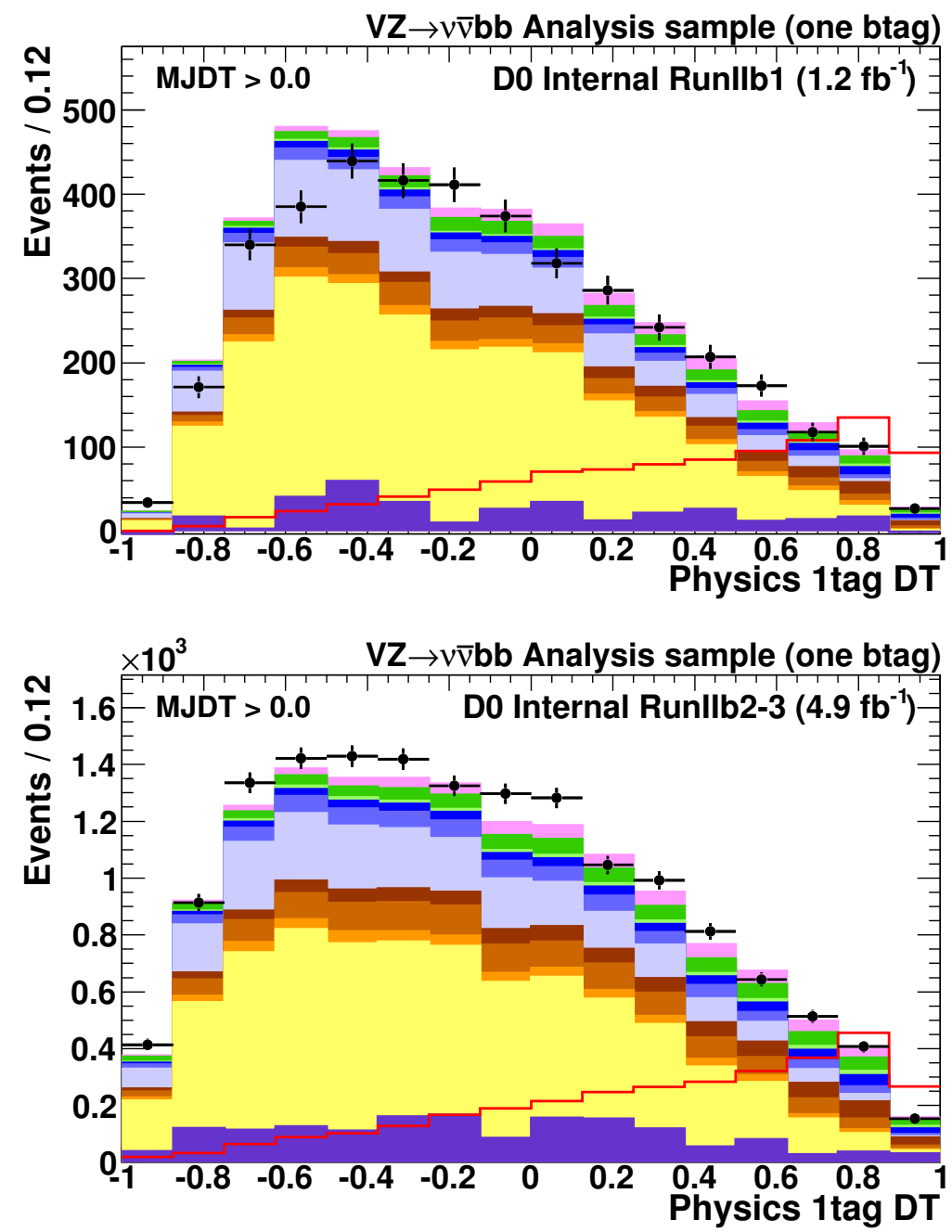

Figure 10.41: Single tag physics decision trees for a di-boson search in Run IIb1 (top) and Run IIb2-3 (bottom). 

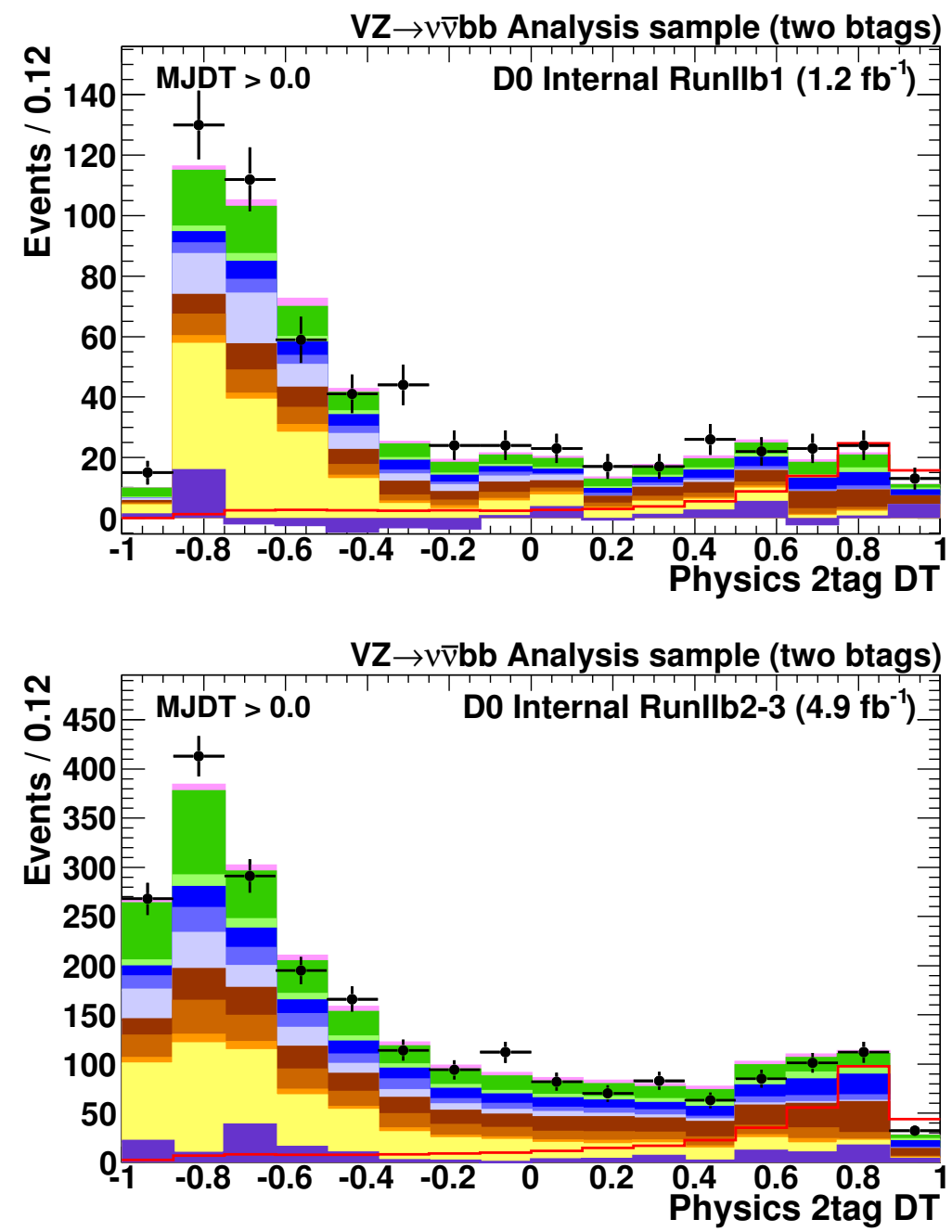

Figure 10.42: Double tag physics decision trees for a di-boson search in Run IIb1 (top) and Run IIb2-3 (bottom). 


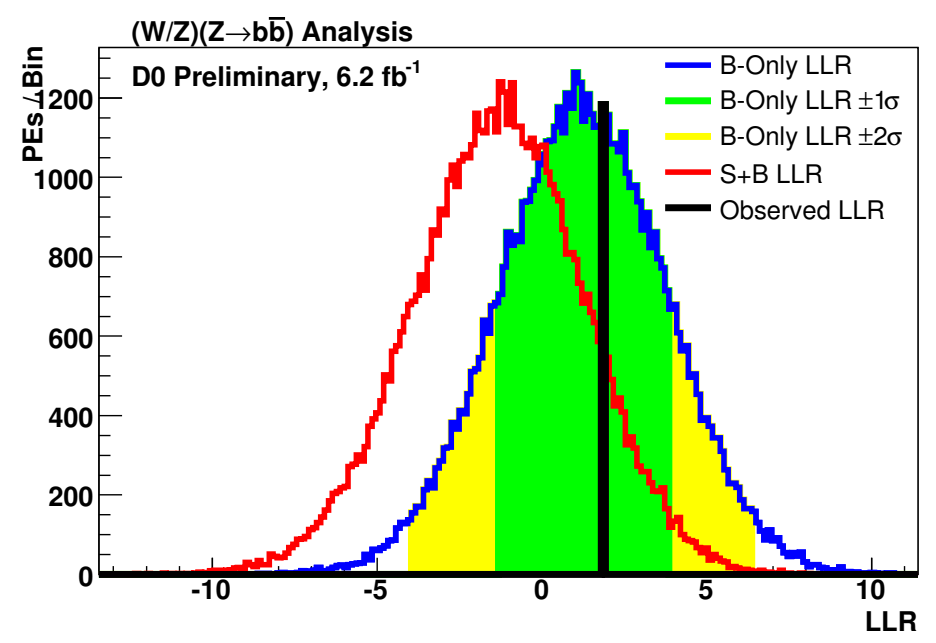

Figure 10.43: LLR distribution for the full Run IIb single and double tag combined result. 


\section{Chapter 11}

\section{Conclusions and Outlook}

Two results from the D $\emptyset$ experiment have been presented. The search and measurement of $Z Z$ di-boson production was proof that rare processes are within reach with clever techniques. This work resulted in a measurement of the $Z Z$ cross section which agrees with the $1.4 \mathrm{pb}$ theoretical value and therefore no hint of new physics in the form of triple gauge boson interactions involving $Z Z Z, Z Z \gamma$, or $Z \gamma \gamma$ was seen.

The search of the Higgs boson in the $Z H$ channel proves to be quite a challenge. This is the most difficult analysis in the low mass Higgs search and remains the most sensitive at DØ. With rapid development of new and innovative techniques the sensitivity of this analysis has improved $\sim 15 \%$ every six months. This is a testament to the hard work and dedication delivered by the groups which maintain common tools for the experiment and the analysis team itself.

Looking toward the future, the Tevatron's ability to observe the Higgs boson is an interesting question. The current run is planned to end in September, 2011, a few short months from now. By then $\mathrm{D} \varnothing$ will have collected $10 \mathrm{fb}^{-1}$ of data and the current projections estimate that by combining with $\mathrm{CDF}$, we might be able to have a $3 \sigma$ evidence in the most likely mass region (Figure 11.1). If the Higgs does not 
exist, this should be sufficient to exclude the entire mass range. The Tevatron is hard at work but the LHC experiments are rapidly approaching sensitivity.

At the LHC, the Higgs search has some different features. In the high mass the $g g \rightarrow H$ process has a cross section $\sim 15$ times larger at $7 \mathrm{TeV}$ compared to $2 \mathrm{TeV}$. With more signal, the high mass search will be more sensitive with less data. In the low mass, the associated production rates jump a factor of three when moving from $2 \mathrm{TeV}$ to $7 \mathrm{TeV}$ but the major background rates $(W / Z+b \bar{b}$ and $t \bar{t})$ also increase due to the rise in the $g g$ cross section. At high mass the brunt of the sensitivity will still be carried by the $H \rightarrow W W \rightarrow \ell \nu \ell^{\prime} \nu^{\prime}$ channel. The golden channel is $H \rightarrow Z Z \rightarrow$ $\ell^{+} \ell^{-} \ell^{+\prime} \ell^{-\prime}$ because even though the branching ratio is small, the backgrounds are even smaller as seen in the similar $Z Z$ analysis discussed in Section 9.10. In the low mass the $H \rightarrow \gamma \gamma$ channel is the preferred as the a clean di-photon mass peak can be picked out of the falling spectrum of the the QCD background.

If the Tevatron can produce an observation or exclusion it would be a milestone and a triumph for the experiment. Assuming the Higgs does exist, the work of measuring its precisely properties, must be left to the LHC collaborations. However the task of the Tevatron is not only to make a statement about the Higgs existence but also to validate the methods and combination procedure. As stated previous this is done by measuring the di-boson processes analogous to the low mass higgs search in the associated production channels with the $H \rightarrow b \bar{b}$ replaced by $Z \rightarrow b \bar{b}$. The road to the Higgs might not end at the Tevatron, but this machine's contribution to paving the path is far from over! 
Tevatron Higgs Search Projection

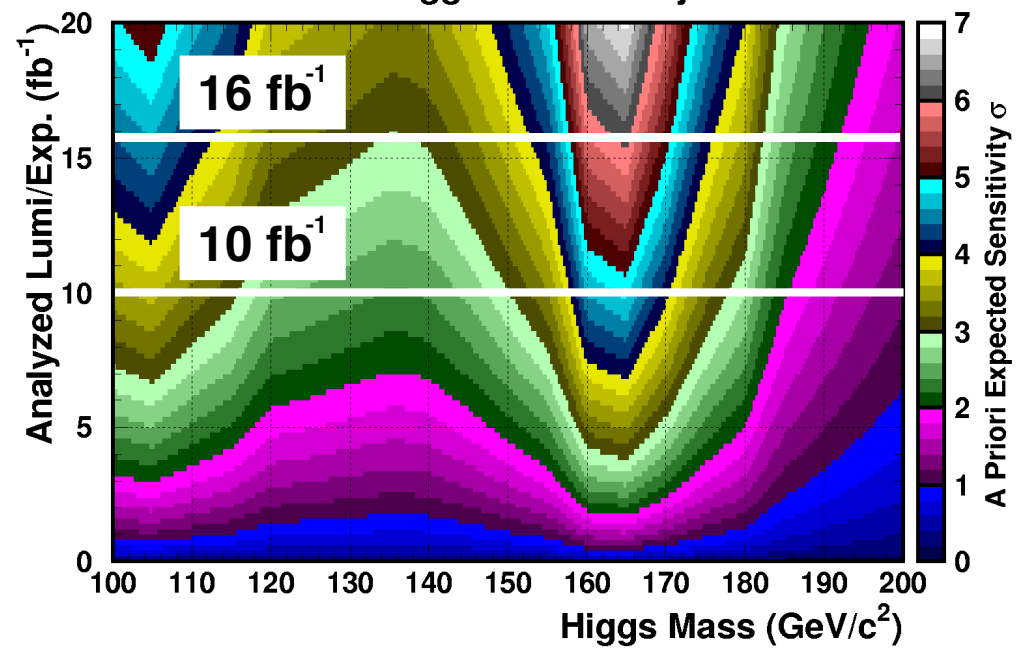

Figure 11.1: The projection indicate that with the rate of improvement seen in the individual channels, the Tevatron could have $3 \sigma$ evidence for the SM Higgs anywhere in the allowed mass range with $16 \mathrm{fb}^{-1}$ of data. At the upcoming stop date of September 2011, both DØ and CDF should have $\sim 10 \mathrm{fb}^{-1}$ of data which could yield a $3 \sigma$ evidence if the Higgs has a mass of $115 \mathrm{GeV}$. 


\section{Appendix A}

\section{Nomenclature}

\section{A.1 Units}

In this document "Planck units" or "God-given units" where constants such as the unit of quantum, $\hbar$, and the speed of light, $c$, are equal to unity.

$$
c=\hbar=1
$$

Therefore mass is expressed as $\mathrm{GeV}$ rather than $\mathrm{GeV} / \mathrm{c}^{2}$. Similarly momentum is $\mathrm{GeV}$ instead of $\mathrm{GeV} / \mathrm{c}$. Cross sections $(\sigma)$ are quoted in units of barns (b) where

$$
1 \mathrm{~b}=10^{-28} \mathrm{~m}^{2} .
$$

One barn is roughly the cross sectional area of a uranium nucleus. A convenient measure of instantaneous luminosity $(\mathcal{L})$ is $\mathrm{b}^{-1} \mathrm{~s}^{-1}$, inverse barns per second. Therefore luminosity (L) is expressed in $\mathrm{b}^{-1}$ and the predicted number of events of a given process created inside the detector can then be given by $L \cdot \sigma$. 


\section{A.2 List of Abbreviations}

\begin{tabular}{|c|c|}
\hline$p_{T}$ & Transverse momentum \\
\hline$E_{T}$ & "Transverse" Energy $\equiv E / \cosh \eta \equiv E \sin \theta$ \\
\hline$\phi_{T}$ & Imbalance in Transverse Momentum ("Missing $E_{T}$ ") \\
\hline $\mathrm{ADC}$ & Analog-to-Digital Converter \\
\hline ATC & Analog-to-Digital Filter Transition Card \\
\hline $\mathrm{BDT}$ & Boosted Decision Tree \\
\hline BLS & Baseline subtract \\
\hline $\mathrm{CC}$ & Central Calorimeter \\
\hline CERN & European Organization for Nuclear Research \\
\hline $\mathrm{CFT}$ & Central Fiber Tracker \\
\hline $\mathrm{CH}$ & Coarse Hadronic layers of the calorimeter \\
\hline $\mathrm{CL}$ & Confidence Level \\
\hline CPS & Central Preshower detector \\
\hline $\mathrm{DCA}$ & Distance of Closest Approach \\
\hline dof & Degrees of Freedom \\
\hline $\mathrm{DT}$ & Decision Tree \\
\hline $\mathrm{EC}$ & Endcap Calorimeter \\
\hline EM & Electromagnetic \\
\hline $\mathrm{FH}$ & Fine Hadronic layers of the calorimeter \\
\hline Fermilab & Fermi National Accelerator Laboratory \\
\hline FSR & Final Stat Radiation \\
\hline GAB & Global Algorithm Board \\
\hline $\mathrm{HD}$ & Hadronic \\
\hline
\end{tabular}




$\begin{array}{ll}\text { ICR } & \text { Inner-Cryostat Region } \\ \text { IP } & \text { Impact Parameter } \\ \text { ISR } & \text { Initial State Radiation } \\ \text { JES } & \text { Jet Energy Scale } \\ \text { LEP } & \text { Large ElectronPositron Collider } \\ \text { LHC } & \text { Large Hadron Collider } \\ \text { LO } & \text { Leading Order } \\ \text { LVDS } & \text { Low-Voltage Differential Signaling cable } \\ \text { JSSR } & \text { Jet Smearing, Shifting, and Removal } \\ \text { MC } & \text { Monte Carlo } \\ \text { MDT } & \text { Mini Drift Tube } \\ \text { MVA } & \text { Multivariate Discriminant } \\ \text { NLO } & \text { Next-to-Leading Order } \\ \text { NN } & \text { Neural Network } \\ \text { PDF } & \text { Probability Distribution Function } \\ \text { pdf } & \text { Parton Distribution Function } \\ \text { PDT } & \text { Proportional Drift Tube } \\ \text { PLC } & \text { Pleated Foil Cable } \\ \text { PPC } & \text { Patch Panel Card } \\ \text { PV } & \text { Primary Vertex } \\ \text { QED } & \text { Quantum Chromodynamics } \\ \text { Quantum Electrodynamics } \\ \text { Q }\end{array}$




$\begin{array}{ll}\text { ROI } & \text { Region of Interest } \\ \text { RF } & \text { Random Forest } \\ \text { SCL } & \text { Serial Command Link } \\ \text { SCLD } & \text { Serial Command Link Distributor card } \\ \text { SLC } & \text { Stanford Linear Collider } \\ \text { SM } & \text { Standard Model (of particle physics) } \\ \text { SMT } & \text { Silicon Microstrip Tracker } \\ \text { TAB } & \text { Trigger Algorithm Board } \\ \text { TCC } & \text { Trigger Control Computer } \\ \text { TFW } & \text { Trigger Frame Work } \\ \text { TTCL } & \text { Trigger Tower Clusters } \\ \text { VME } & \text { VERSA-module Eurocard }\end{array}$




\section{Appendix B}

\section{Level 1 Calorimeter Trigger}

The accelerator complex was upgraded between Run I and Run II to achieve a higher center of mass energy and maintain 36 (instead of 6 ) bunch crossings in a single turn. Further upgrades were planned in order to increase the total integrated luminosity. This included reducing the bunch spacing from 396 ns to 132 ns (159 potential bunch crossings) and increasing the density of these bunches. To prepare for this configuration the $\mathrm{D} \emptyset$ trigger system was upgraded [30]. One of the major cornerstones of the Run IIb upgrade was the introduction of the new Level 1 Calorimeter Trigger (L1Cal). Although the reduced bunch spacing was never realized, a modification was needed in order to maintain L1 and L2 rates below the values dictated by the fundamental features of the detector system described in Section 2.3. In Run I and Run IIa, the L1Cal trigger counted individual trigger towers above predefined thresholds in transverse energy. This lead to fairly sharp turn-on curves for electron and photon triggers but the opposite for jet triggers since jets shower into multiple towers while electromagnetic showers tend to be contained within one to two towers. Therefore a clustering algorithm was chosen to be implemented over simply increasing the

thresholds used for tower counting as this would result in a significant loss in signal 
efficiency. This system was designed with processes like $p \bar{p} \rightarrow Z H \rightarrow \nu \bar{\nu} b \bar{b}$ in mind. A sensitive search in that channel is impossible with the integrated luminosity collected without the implementation and efficient running of this trigger. In the following a more in depth look at this trigger is given while an exhaustive description can be found in References [106] and [107].

\section{B.1 Architecture}

Custom electronics boards are used to receive and process the calorimeter data as well as pass along the L1Cal information downstream (Figure B.1). The Analog and Digital Filter (ADF) System receives the analog trigger tower (TT) signals from the calorimeter readout path and performs some basic processing. The Trigger Algorithm Board/Global Algorithm Board (TAB/GAB) System runs the clustering algorithms and produces the trigger terms and when an L1 accept is issued, sends information out of the system. L1Cal is configured and monitored via the L1Cal Trigger Control Computer (L1Cal TCC) and timing information comes from the trigger framework (TFW) which also send control commands and receives the L1Cal and/or terms over a serial command link (SCL).

The analog signal from the calorimeter cells arrives at the baseline subtraction card (BLS) where it is shaped, baseline subtracted, and stored awaiting L1 and L2 decisions. This is the precision readout path which provides inputs to L3 and is used at the analysis level. Before the signal can be shaped, the analog signal is also sent down the trigger sum pickoff path where it is quickly shaped with a triangular pulse then sent to an analog summer. The summers adds the EM (FH) cells in $0.2 \times 0.2 \Delta \eta \times \Delta \phi$ region separately to create EM (HD) TTs. The CH layer, ICD, and towers with $|\eta|>3.2$ do not participate in the trigger. This system is configured 
and controlled by the TCC over a VME bus and timing information comes from an SCLDistributor card (SCLD) via a Low-voltage differential signaling (LVDS) cable.

\section{B.1.1 The ADF System}

The 1280 BLS trigger cables could not physically fit into the ADF system directly so a passive transition system lie between the two. Patch Panel Cards (PPC) condense 16 BLS cables worth of information into one Pleated Foil Cable (PLC) which connect to passive cards connected to the ADF crate backplane known as ADF Transition Cards (ATC). The Patch Panels housing the PPC is a convenient place to tap into the system with an oscilloscope and view the signal from the calorimeter before it processed by the L1Cal. When identifying and diagnosing noise in or sent to the system this is one of the first places to look.

On each ADF card the analog signals for a $4 \times 4$ array of TTs are passed through a frequency filter, converted to a digital format, and through the use of FPGAs the difference in arrival times is corrected for before being passed through a Digital Filter. The Digital Filter removes high frequency noise and low frequency shifts as well as converts energy to its transverse component through an $E$ to $E_{T}$ Lookup table such that one ADC count corresponds to $0.25 \mathrm{GeV}$ of $E_{T}$ on top of an eight count pedestal. The digitized signal from each ADF card is sent to three TABs for further processing.

\section{B.1.2 The TAB/GAB System}

The Trigger Algorithm Boards (TAB) each receive, through the backplane, inputs from 30 ADF cards, covering a $40 \times 12$ region in $\eta \times \phi$ which pass to ten Sliding Windows Algorithm (SWA) FPGAs. Each FPGA finds objets in a $4 \times 4, \eta \times \phi$ grid

which requires a $9 \times 9$ region of input. Neighboring FPGAs share information as they 
are adjacent in $\eta$. The algorithm output from each SWA is given to a single TAB global FPGA which calculates regional sums in a $31 \times 4$ gird to be sent through the front of the board to the GAB and the L1CalTrk system. The GAB receives the inputs from the eight TABs and computes the and/or terms using the objects found in the entire calorimeter. Upon receiving an L1 accept the global information is sent to L2 and L3 from both the individual TABs and the GAB. Because of the high density of inputs to the algorithm boards a backplane VME connection is impossible therefore a front-end VME/SCLcard is employed.

\section{B.2 Sliding Windows Algorithms}

The sliding windows algorithm (SWA) is the clustering algorithm of choice to locate local maxima in $E_{T}$ deposition by comparing groups of TTs to nearby groups. A similar algorithm is used at other HEP experiments such as the ATLAS detector at CERN [108]. Three steps are needed to identify EM, tau, and jet objects. In the first step, at each point in trigger tower space, several TTs are combined into Trigger Tower Clusters (TCCL) or Regions of Interest (ROI) as shown in the first panel of Figure B.2. For jets and hadronicly decaying taus, a $2 \times 2 \mathrm{TT}$ is used while a TCCL for an EM object is made of two EM towers. Each TCCL is indexed by the coordinates of the TT just to the left/bottom of the center point. After these objects have been identified, the next step is to locate the local maxima (LM) in transverse energy by comparing the $E_{T}$ sum of contiguous TCCLs. As shown in the third panel of Figure B.2, multiple counting of objects is avoided by requiring at least one TT to separate LMs. The final step to apply a customized criteria based on the object in question. Jets have an average lateral size of one unit in $\eta, \phi$ space and it was found that using the energy sum of a $4 \times 4$ in TT space gave the best energy and position 
resolution. Since electrons and photon have lateral sizes smaller than one TT and deposit energy in the electromagnetic layers of the calorimeter cuts are made on the isolation and the EM fraction. The EM energy deposits in the object are compared to that of the four adjacent TTs and to the HD energy of the TT directly behind the object itself to obtain the isolation and EM fraction respectively (Figure B.3). This choice was made to optimize the efficiency of selecting electrons from $W$ decays and $J / \psi \rightarrow e^{+} e^{-}$. Taus, when decaying hadronicly, look like narrow jets so for these objects a isolation is calculated from the ratio of the transverse energy in the $2 \times 2$ tau object with the $4 \times 4$ region which would be used for a jet object. Since some information is lost in the above mentioned steps, the energy of a jet closest in $\phi$ is used to calculate the isolation. With all these objects identified and a $\mathbb{F}_{T}$ created from the vector sum of all TTs, complex trigger terms can be created and tested in the GAB. For example in the $Z H \rightarrow \nu \bar{\nu} b \bar{b}$ channel, at L1, two jets acolinear in the transverse plane required as well as significant missing transverse energy.

\section{B.3 Operation}

The L1Cal system is extremely stable. As stated above, the pedestal of eight ADC counts is used for the differential signals. This must be considered when testing the energy of an object against a predefined threshold. To do so in a manner in which a time dependent systematic shift in the pedestal will not effect the rates, a dynamic pedestal is calculated by averaging the ADC counts of each TT over a two minute period and adding this value to the thresholds. There was some skepticism that this would add instability to the system but it has proven to be a stable and clever solution. Any TT can be masked from the SWA by sending the eight count pedestal from the ADF to the TAB. This is done if a significant amount of noise is seen in a 
given channel until the problem can be resolved. Of the 1280 separate EM and HD TTs, on average two to three are masked at any given time.

The hardware itself is monitored by an emulation which reproduces the trigger decision exactly in all but one case. When the beam halo is being removed before the beginning of high energy collisions, the calorimeter has almost $100 \%$ occupancy with energy levels much larger than those expected from HEP events. In this condition, when data is not being recorded anyway, the emulation does not properly model this level of overflow in hardware.

Recall one of the design goals of this system was to sharpen the jet turn on curve and reduce the background trigger rates. Trigger performance was measured using a minimally biased data set with instantaneous luminosities greater than $1 \times$ $10^{32} \mathrm{~cm}^{-2} \mathrm{~s}^{-1}$. Figure B.4 summarizes some of the results from these performance tests. Panel (a) shows the turn-on curve for requiring two TTs with $E_{T}>5 \mathrm{GeV}$ as in Run IIa and a jet-object with $15 \mathrm{GeV}$ as in Run IIb. Panel (b) shows the efficiency for finding an $E_{T}$ imbalance greater than $20 \mathrm{GeV}$ as a function of $E_{T}$ which is better than or comparable to Run IIa L1Cal performance. The performance of triggering on EMs and taus are shown in panels (c) and (d). In (c), the Run IIb performance was evaluated requiring an EM object with $19 \mathrm{GeV}$ of transverse energy or an isolated EM object with $E_{T}>16 \mathrm{GeV}$ while the equivalent Run IIa requirement is also shown which corresponds to a single TT with EM $E_{T}>16.5 \mathrm{GeV}$ or two TTs with $E_{T}>8.25 \mathrm{GeV}$. The trigger rates of both epoch requirements are about the same but the Run IIb is sharper allowing for a lower effective threshold and therefore a higher efficiency for selecting $Z \rightarrow e^{+} e^{-}$events. Finally in panel (d) using $Z \rightarrow \tau^{+} \tau^{-}$the Run IIb efficiency for finding a $15 \mathrm{GeV}$ tau compared to a $20 \mathrm{GeV}$ in jet in Run IIa.

The stable performance due to the well planned design of the L1Cal trigger has allowed D $\varnothing$ to conduct a broad and successful physics program with Run IIb data. 
Presented in Chapter 10 is an analysis which would be impossible without this trigger.

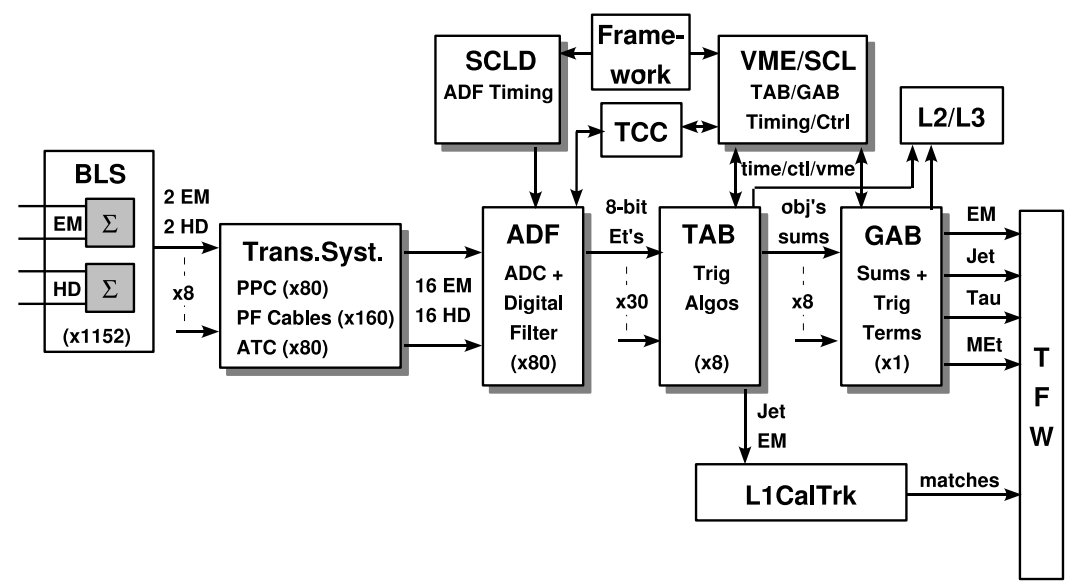

Figure B.1: The main hardware components of the Level 1 Calorimeter Trigger and their interconnections shown in a block diagram.

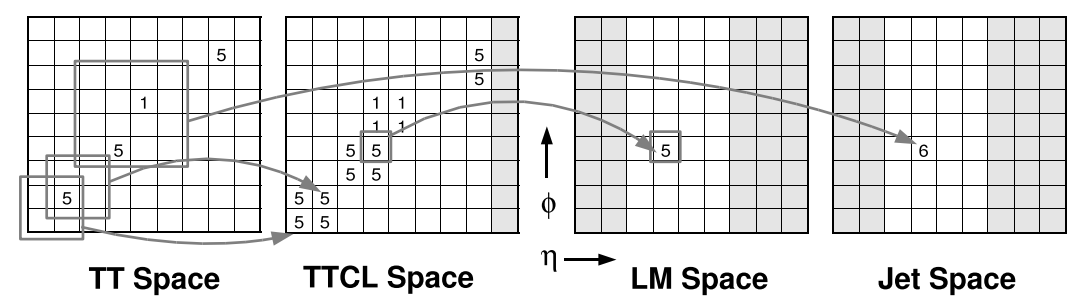

Figure B.2: The stages of the sliding windows algorithm starting with the formation of TTCLs, to the location of LMs to the creation of the final objects, in this case a jet. 


\section{Horizontal Vertical}

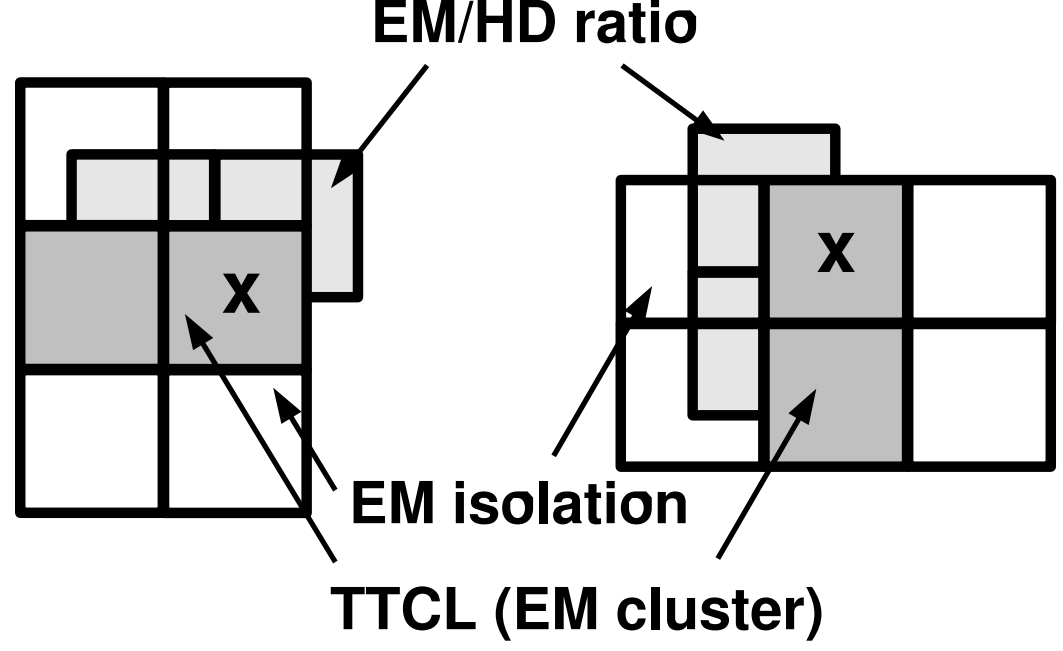

Figure B.3: Level 1 Calorimeter EM object definitions.
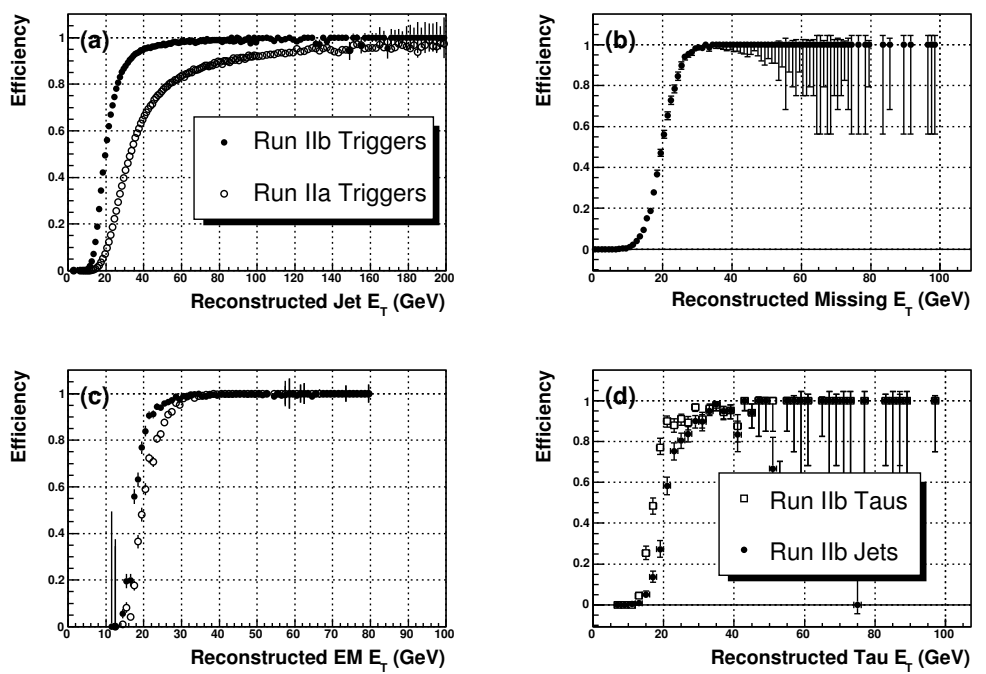

Figure B.4: Performance of the Run IIa and IIb L1Cal triggers. (a) Shows the comparison of jet trigger turn-ons, (b) $\mathbb{E}_{T}$ efficiency in Run IIb, (c) comparison of Run IIa and IIb electron trigger efficiencies, and (d) Run IIb tau triggering compared to the equivalent Run IIa jet requirements. 


\section{Appendix C}

\section{ALPGEN Angular Reweightings}

D0 currently has a set of data-driven corrections derived to reconcile the differences, observed in many analyses, between the kinematic distributions produced by ALPGEN and those in data [92]. Using the same technique we derive a similar correction in the $\Delta \eta$ distribution in the multijet free electroweak control sample. We calculate the $V+$ jets contribution in data by subtracting the top and diboson contributions from data. We then take the ratio of this data estimate to the $V+$ jets MC. The reweighting function is a third degree polynomial fit to this ratio and is applied to all signal and control sample $V+$ jets $\mathrm{MC}$. Below are some distributions before and after reweighting. 


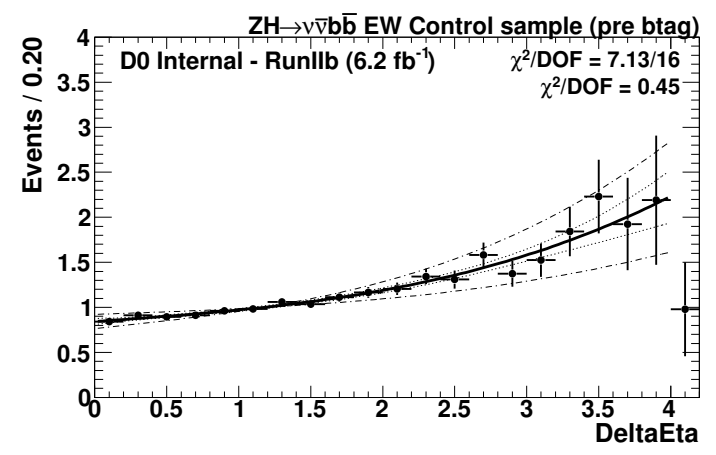

Figure C.1: Third degree polynomial fit to the ratio of data - top $_{M C}-$ diboson $_{M C}$ to $V+$ jets $_{M C}$. The solid black line represents the RunIIb fit used in this note, the dotted line is the error from covariance matrix and the dashed line is the systematic uncertainty. The systematic is estimated as half of the functions distance from unity.
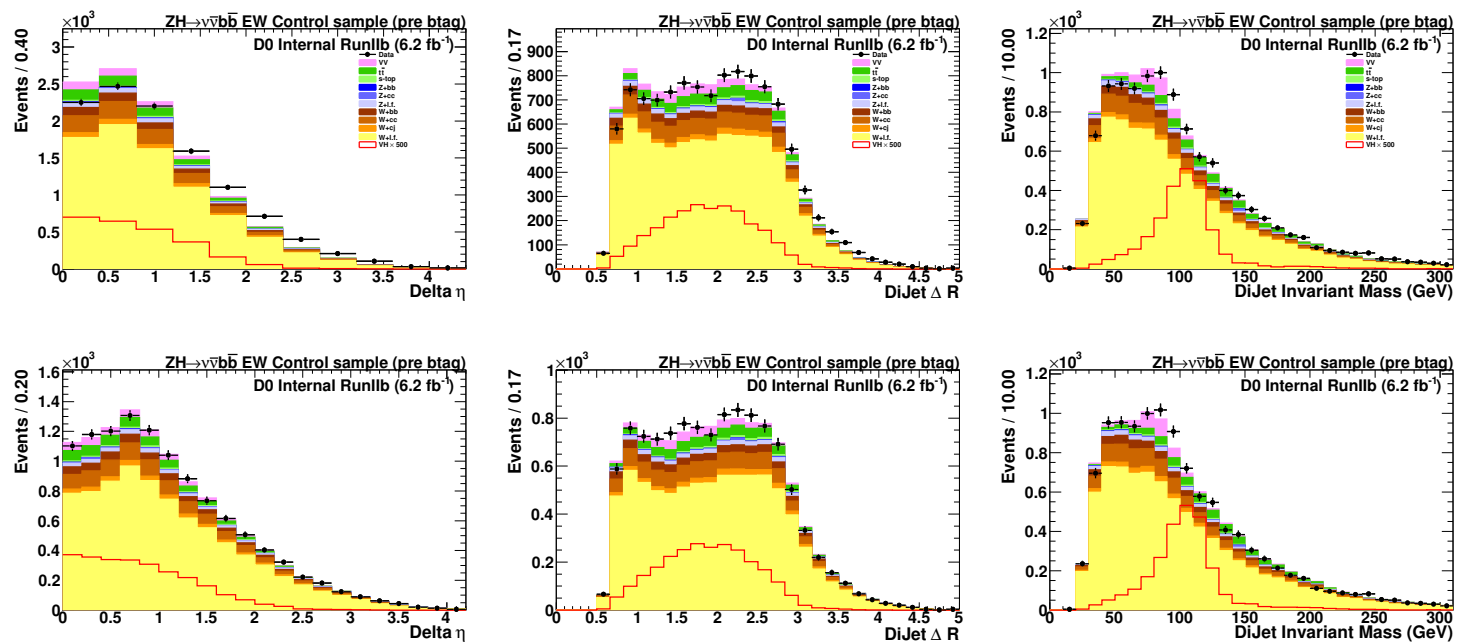

Figure C.2: $\Delta \eta$ (left column row), $\Delta R$ (middle column), and dijet invariant mass (right column) distributions in the pre-tag electroweak control sample before (top row) and after (bottom) the reweighting function is applied on the full RunII dataset 

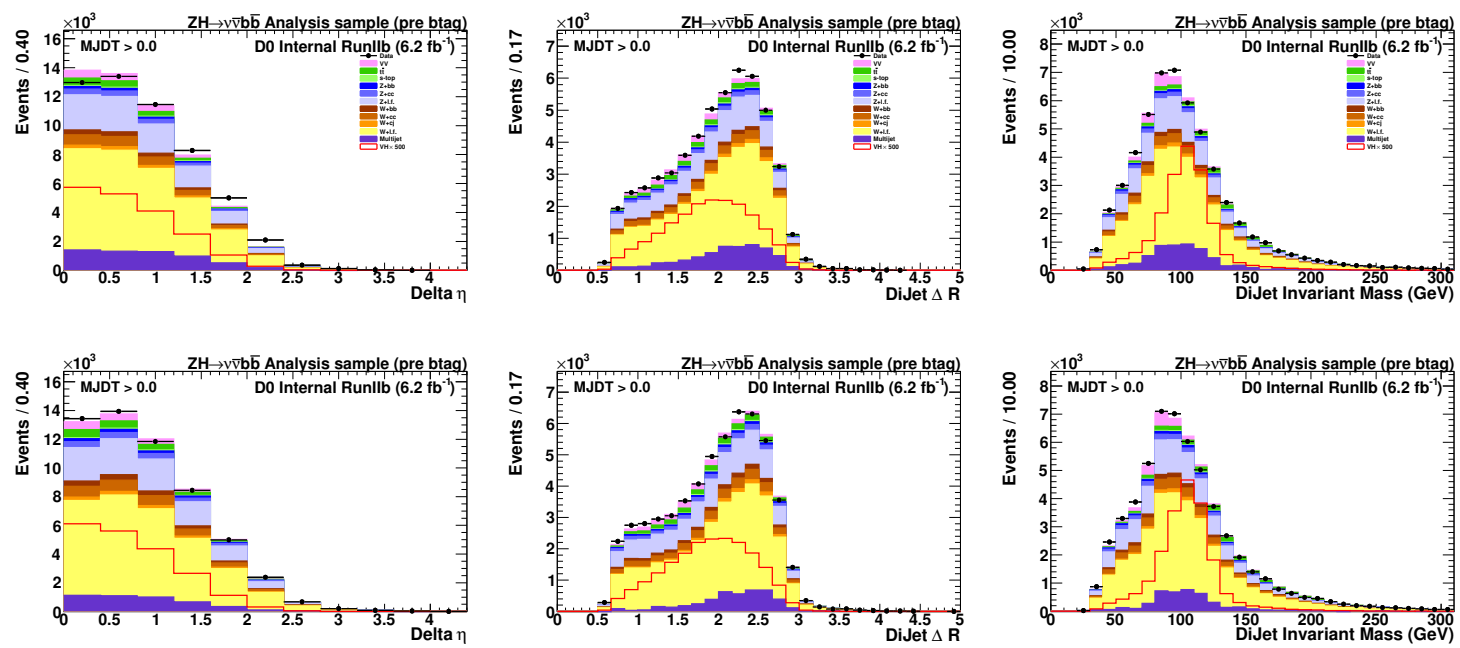

Figure C.3: $\Delta \eta$ (left column), $\Delta R$ (middle column), and dijet invariant mass (right column) distributions in the pre-tag signal sample after the multijet decision tree cut and before (top row) and after (bottom row) the reweighting function is applied on the full RunII dataset 


\section{Appendix D}

\section{b-tagging MVA Distributions}
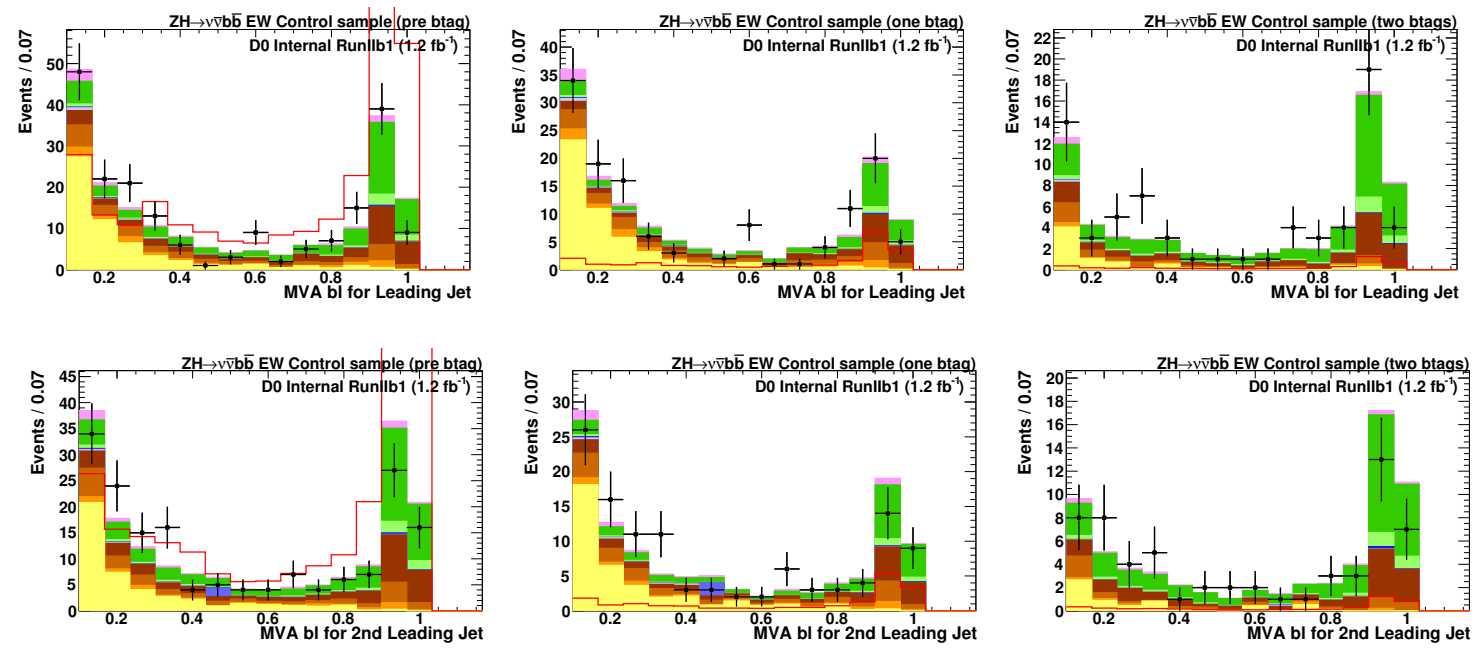

Figure D.1: Output of b-ID MVA bl distribution for the leading and next-to-leading jets in the Run IIb1 electroweak control sample 

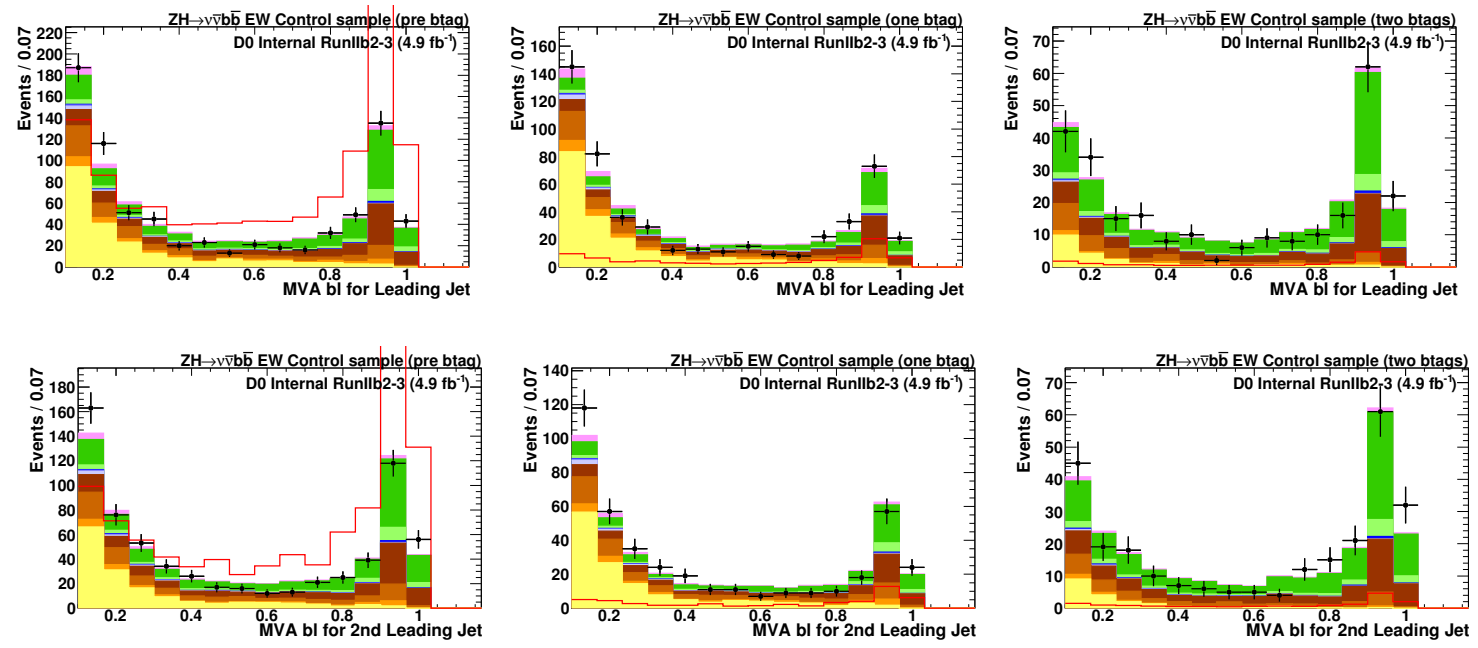

Figure D.2: Output of b-ID MVA bl distribution for the leading and next-to-leading jets in the Run IIb2-3 electroweak control sample
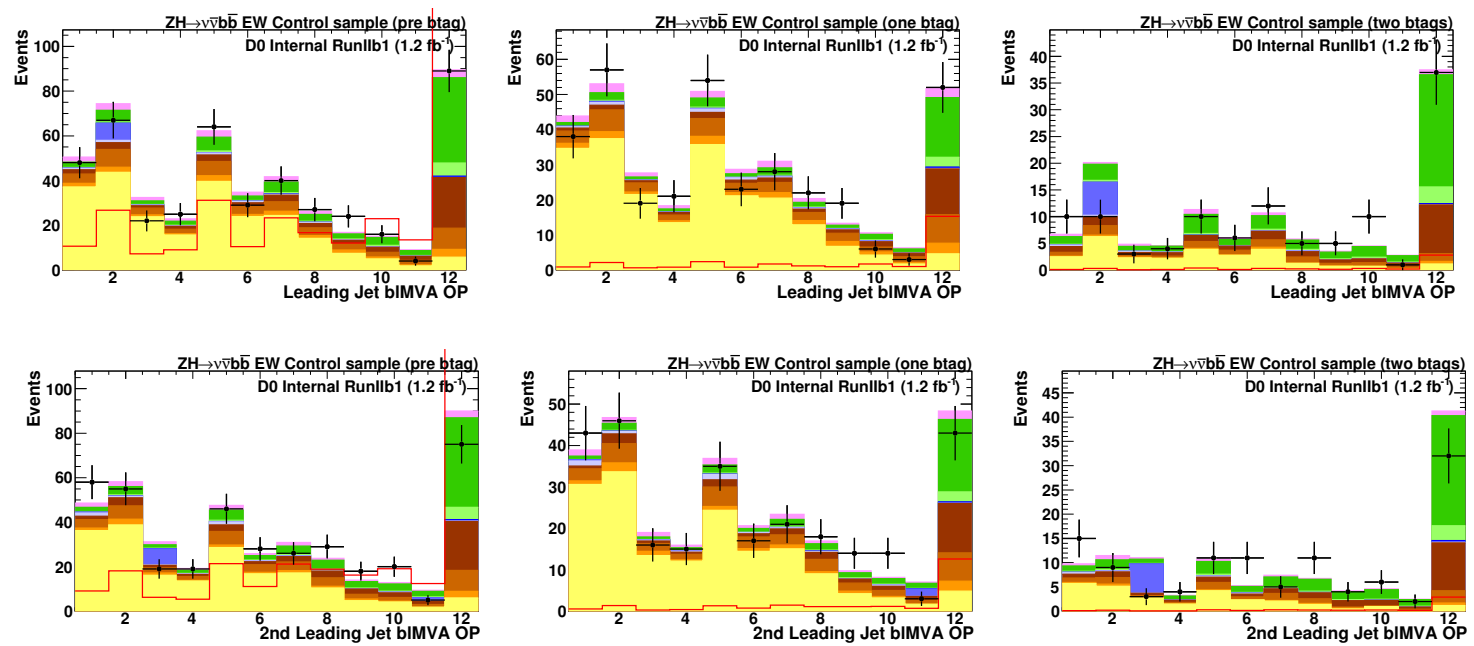

Figure D.3: Output of b-ID MVA bl distribution operating points for the leading and nextto-leading jets in the Run IIb1 electroweak control sample 

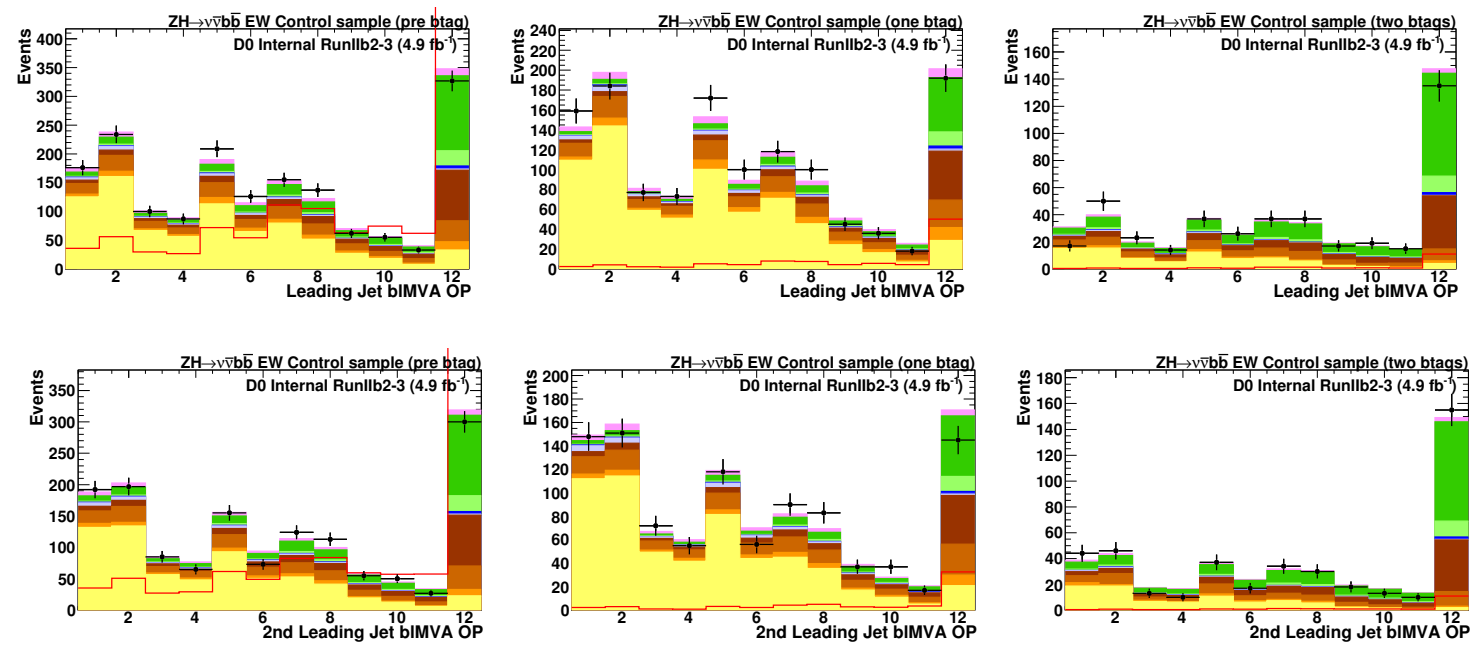

Figure D.4: Output of b-ID MVA bl distribution operating points for the leading and nextto-leading jets in the Run IIb2-3 electroweak control sample
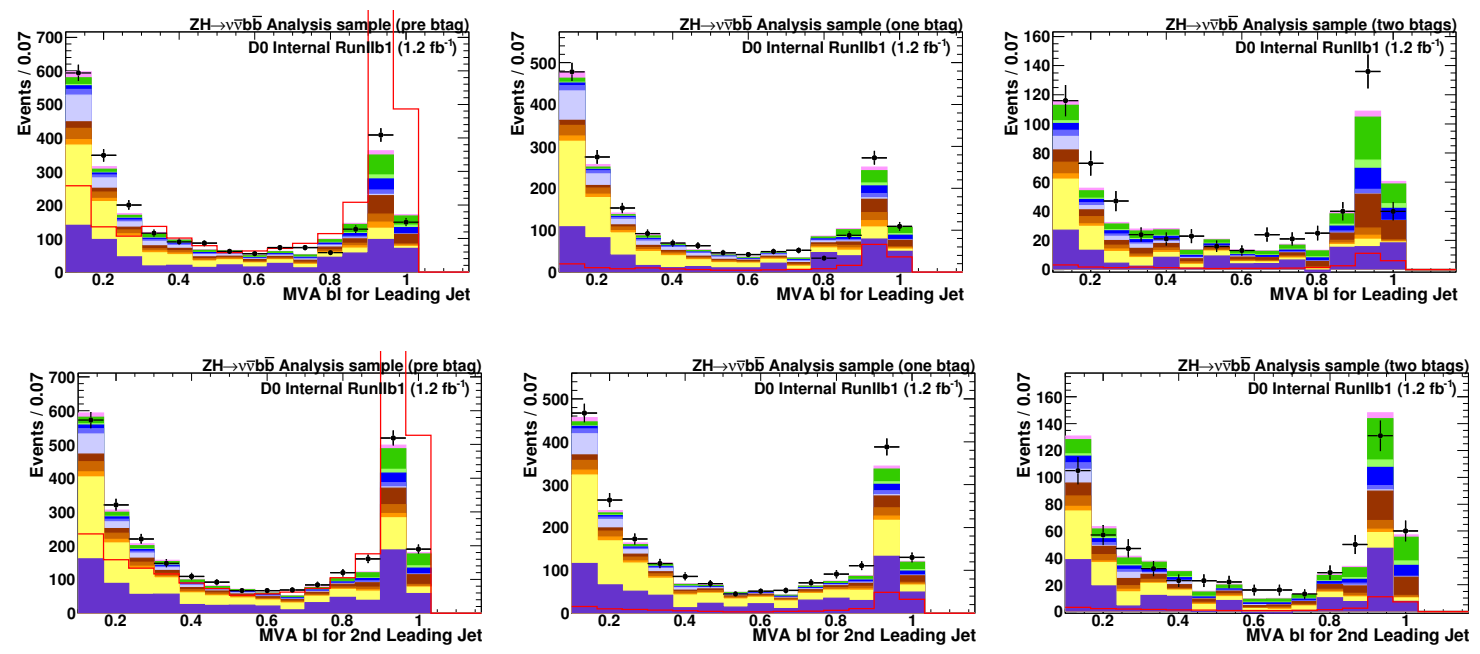

Figure D.5: Output of b-ID MVA bl distribution for the leading and next-to-leading jets in the Run IIb1 analysis sample. The pretag signal is scaled by 50 . 

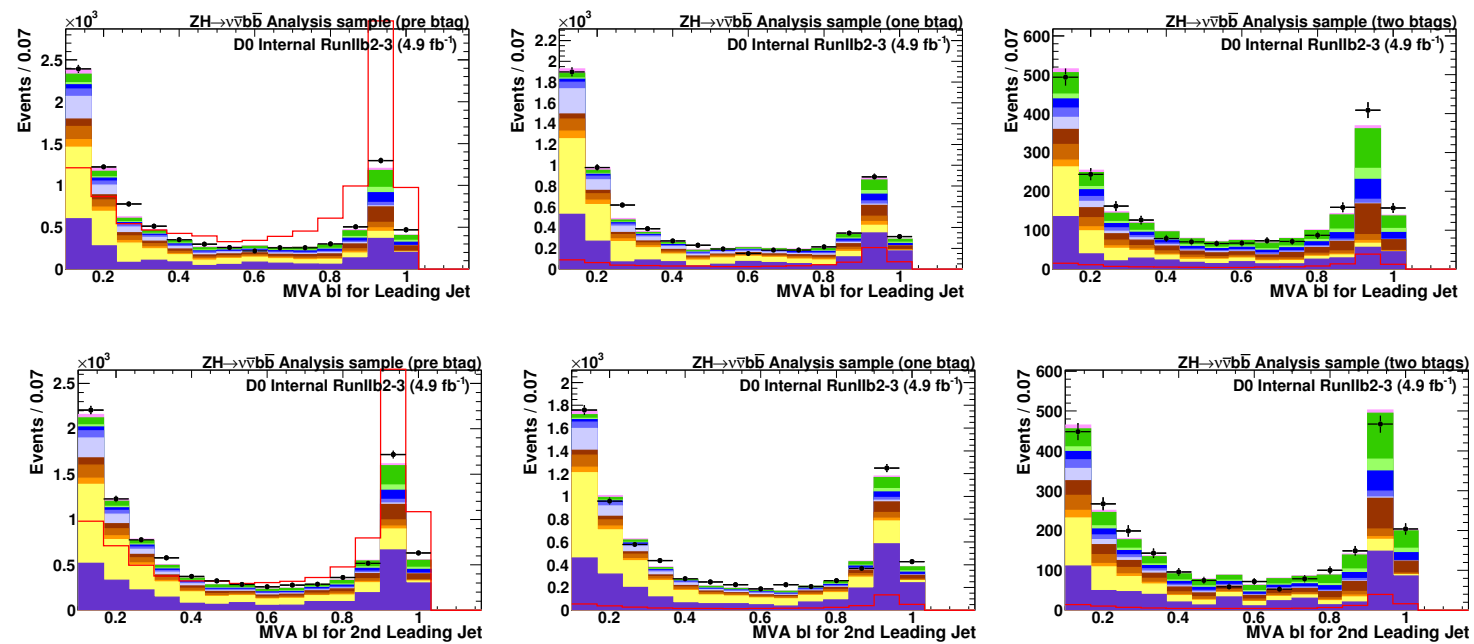

Figure D.6: Output of b-ID MVA bl distribution for the leading and next-to-leading jets in the Run IIb2-3 analysis sample. The pretag signal is scaled by 50 .
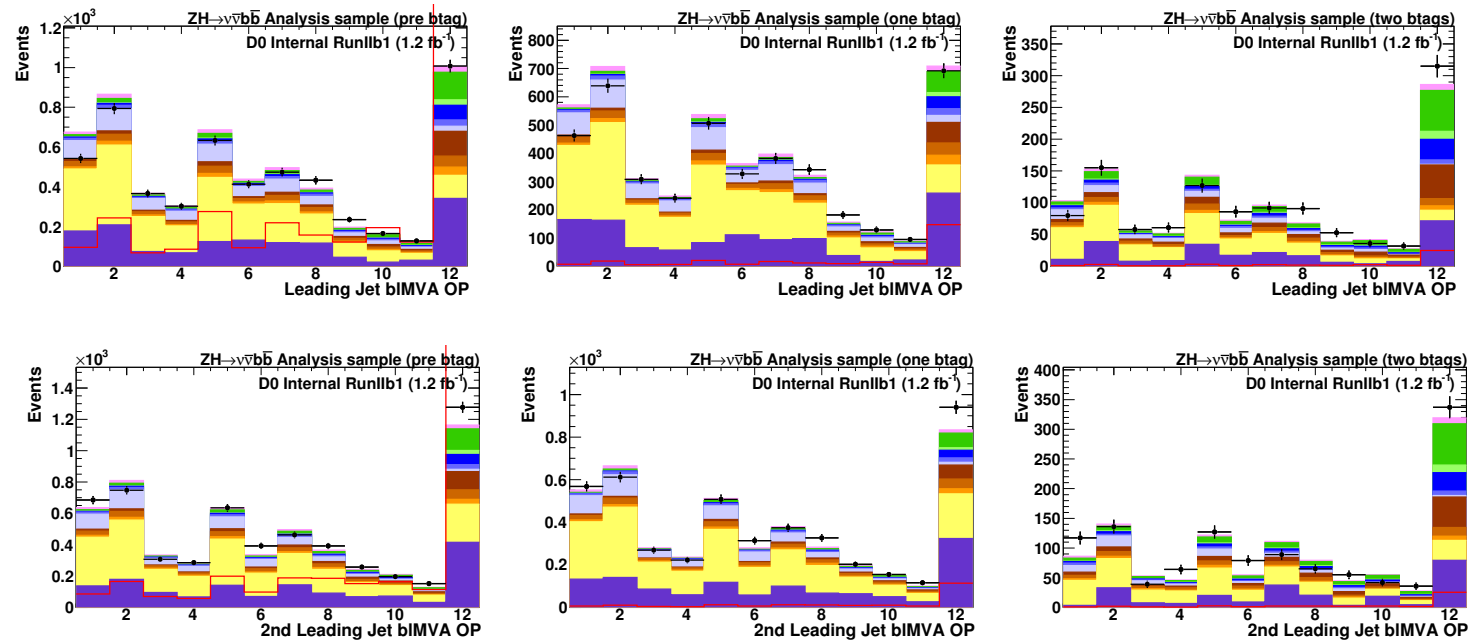

Figure D.7: Output of b-ID MVA bl operating points distribution for the leading and nextto-leading jets in the Run IIb1 analysis sample. The pretag signal is scaled by 50 . 

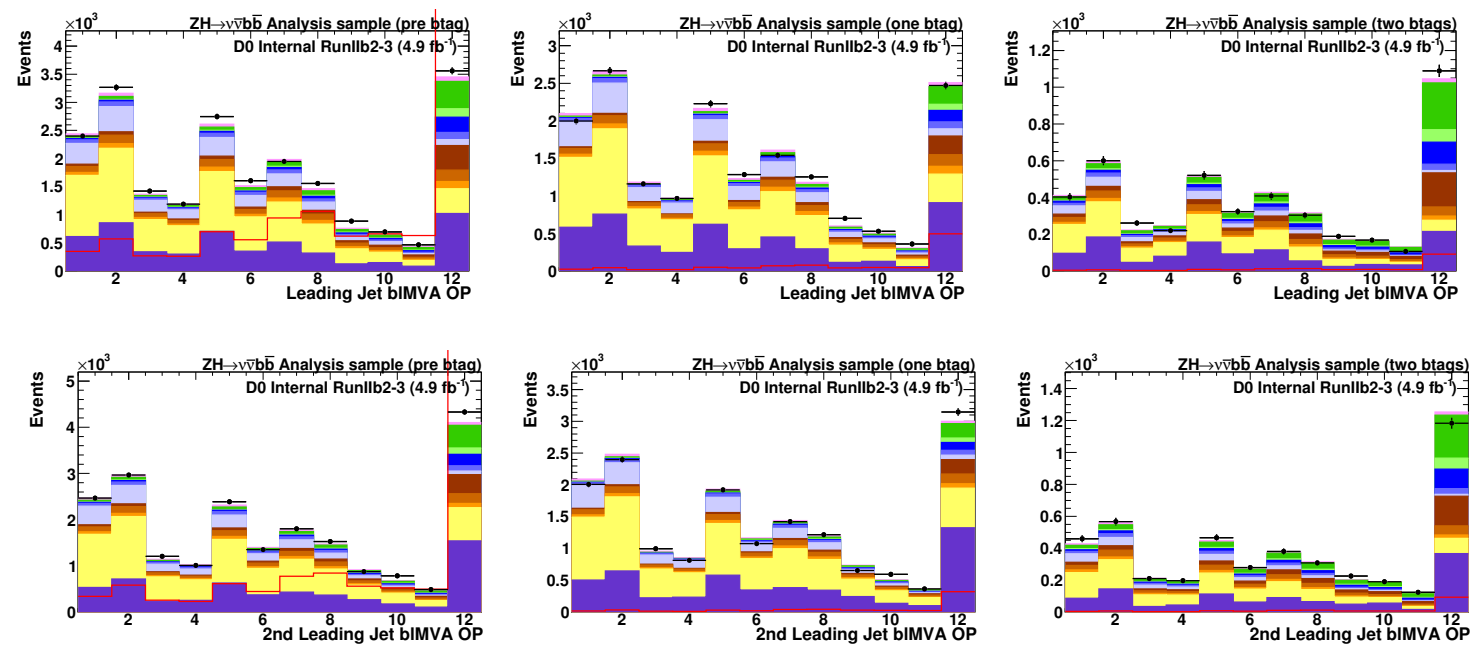

Figure D.8: Output of b-ID MVA bl operating points distribution for the leading and nextto-leading jets in the Run IIb2-3 analysis sample. The pretag signal is scaled by 50 . 
Appendix E

Decision Tree Training Input Variables 
1. Missing transverse energy $\left(\mathbb{E}_{T}\right)$

2. Dijet invariant mass constructed from the leading two jets

3. The difference in $\phi$ of the leading two jets $\left(\Delta \phi\left(\right.\right.$ jet $_{1}$, jet $\left.\left._{2}\right)\right)$

4. Difference in $\eta$ of the two leading jets $\Delta \eta\left(\right.$ jet $_{1}$, jet $\left._{2}\right)$

5. Distance in $\eta-\phi$ space between the two leading jets $\Delta R\left(\right.$ jet $_{1}$, jet $\left._{2}\right)$

6. The transverse momentum of the leading jet

7. The transverse momentum of the next-to-leading jet

8. The $p_{T}$ weighted distance in $(\eta-\phi)$ space between the second leading jet and other jets $\left(\Sigma\left(\right.\right.$ jet $\left.\left._{2}\right)=\frac{\Sigma_{i} \Delta R\left(\text { jet }_{2}, j e t_{i}\right) \times p_{T}^{\text {jet }}}{\Sigma_{\text {jets }} p_{T}^{\text {jet }}}\right)$

9. Transverse mass of the two leading jets

10. The color-flow variable [105] for the leading jet is used to help discriminate a pair of $b$ jets originating from a color singlet, the Higgs, and a color octet, a gluon. The angle in $\phi-\eta$ space between the jet pull, $\vec{\rho}=\Sigma_{i} \frac{E_{T}^{i}\left|r_{i}\right|}{E_{T}^{\text {jet }} \vec{r}_{i}}$ where the summation is over the cells that make up the jet and $\vec{r}_{i}$ is in the direction of the jet center to the cell, and the direction from the center of jet to jet $_{2}$ is defined as the leading jet color-flow.

Table E.1: Ten of the twenty kinematic variables used in the DTs 
1. The number of taggable jets

2. Difference in $\phi$ between the $\mathbb{F}_{T}$ and the next-to-leading jet $\left(\Delta \phi\left(\mathscr{H}_{T}\right.\right.$, jet $\left.\left.{ }_{2}\right)\right)$

3. Mismeasurements of jet energies, which plague the multijet background, can cause di-jet events to have a jet alligned with the $\mathbb{E}_{T}$ while jets back-to-back with the $E_{T}$ is expected from Higgs decays. To optimize this topological difference the sum of the maximum and minimum difference in $\phi$ of the $\mathscr{H}_{T}$ with any jet $\left(\max \Delta \phi\left(\mathbb{E}_{T}\right.\right.$, jet $\left._{\mathrm{i}}\right)+\min \Delta \phi\left(\mathbb{E}_{T}\right.$, jet $\left.\left._{\mathrm{i}}\right)\right)$.

4. With similar motivation to the previous variable we use the difference of the maximum and minimum difference in $\phi$ of the $\mathbb{F}_{T}$ with any jet $\left(\max \Delta \phi\left(\mathscr{E}_{T}\right.\right.$, jet $\left._{\mathrm{i}}\right)-\min \Delta \phi\left(\mathscr{E}_{T}\right.$, jet $\left.\left._{\mathrm{i}}\right)\right)$

5. The color-flow variable for the next-to-leading jet where the jet pull is defined

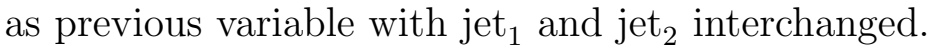

6. The angle with respect to the beamline $(\theta)$ of the Higgs (di-jet) system. This variable capitalizes on the angular difference between gluon splitting and a scalar particle decay.

7. $\eta$ of the Higgs (di-jet) system.

8. The modified "recoil-subtracted" dijet $p_{T}$ component along the unit vector $\hat{a}_{T}$ perpendicular to the di-jet thrust axis. This is a component of the $\mathbb{F}_{T}{ }^{\prime}$ variable discussed in Section 5.1. i

9. Vector sum of all jest $\left(H_{T}\right)$

10. Ratio of vector sum and scalar sum of all jets $\left(H_{T} / H_{T}\right)$

Table E.2: Ten of the twenty kinematic variables used in the DTs 

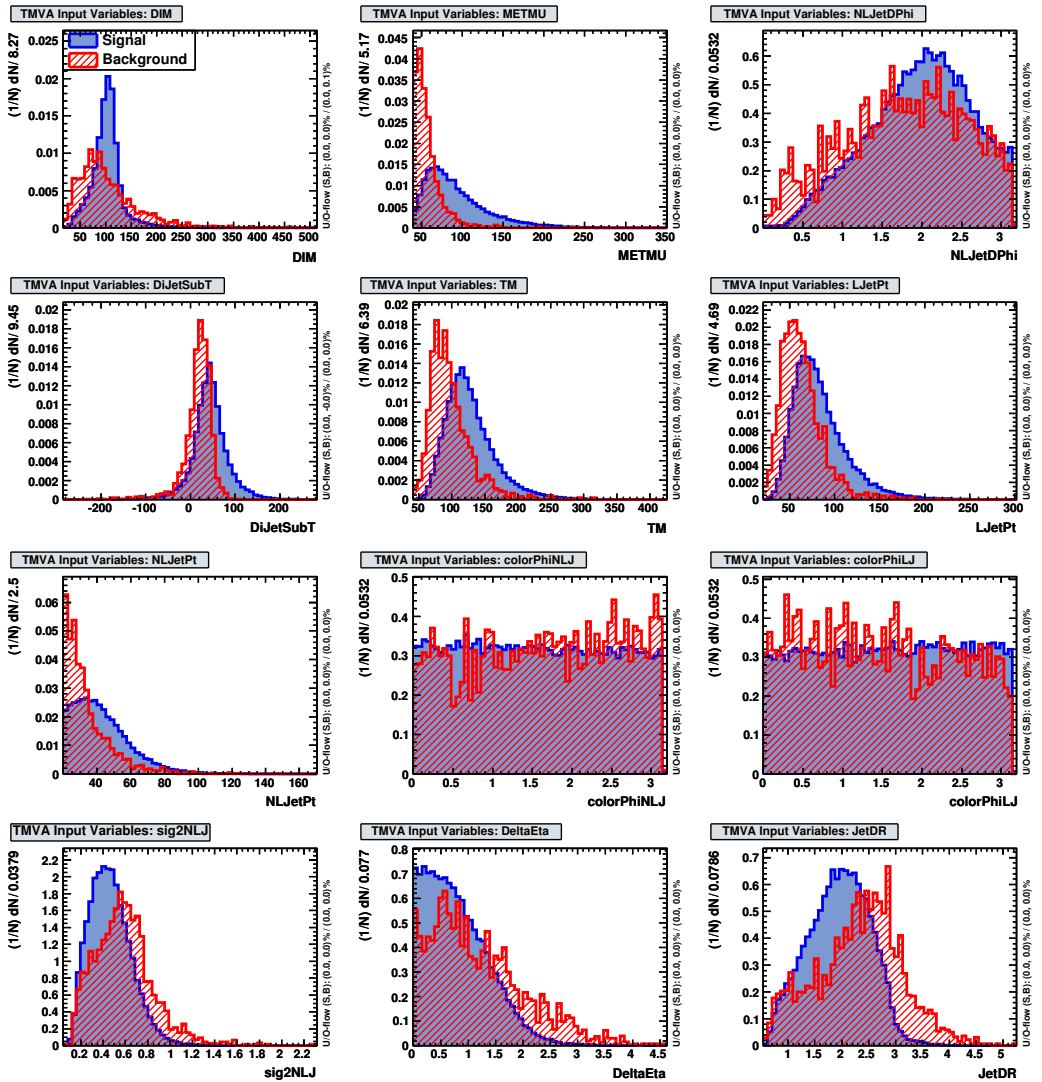

Figure E.1: Comparison of signal and background distributions for multijet decision tree input variables in Run IIb1 for a Higgs mass of $115 \mathrm{GeV}$. 

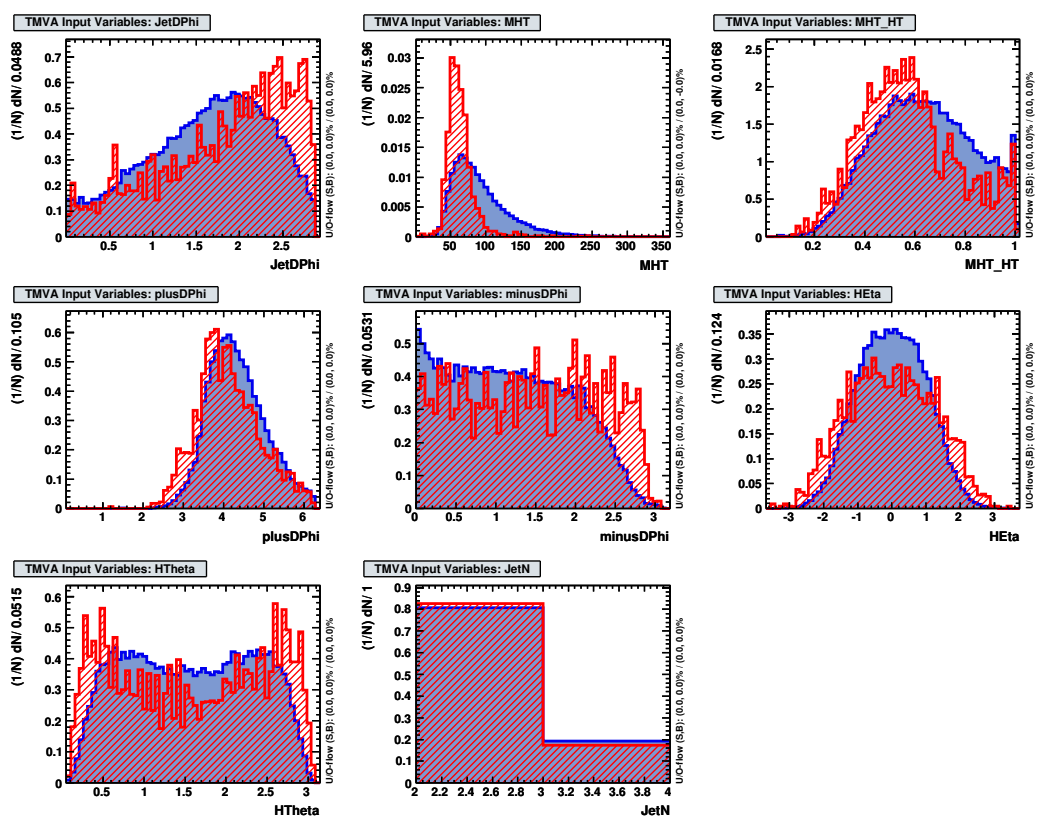

Figure E.2: Comparison of signal and background distributions for multijet decision tree input variables in Run IIb1 for a Higgs mass of $115 \mathrm{GeV}$. 

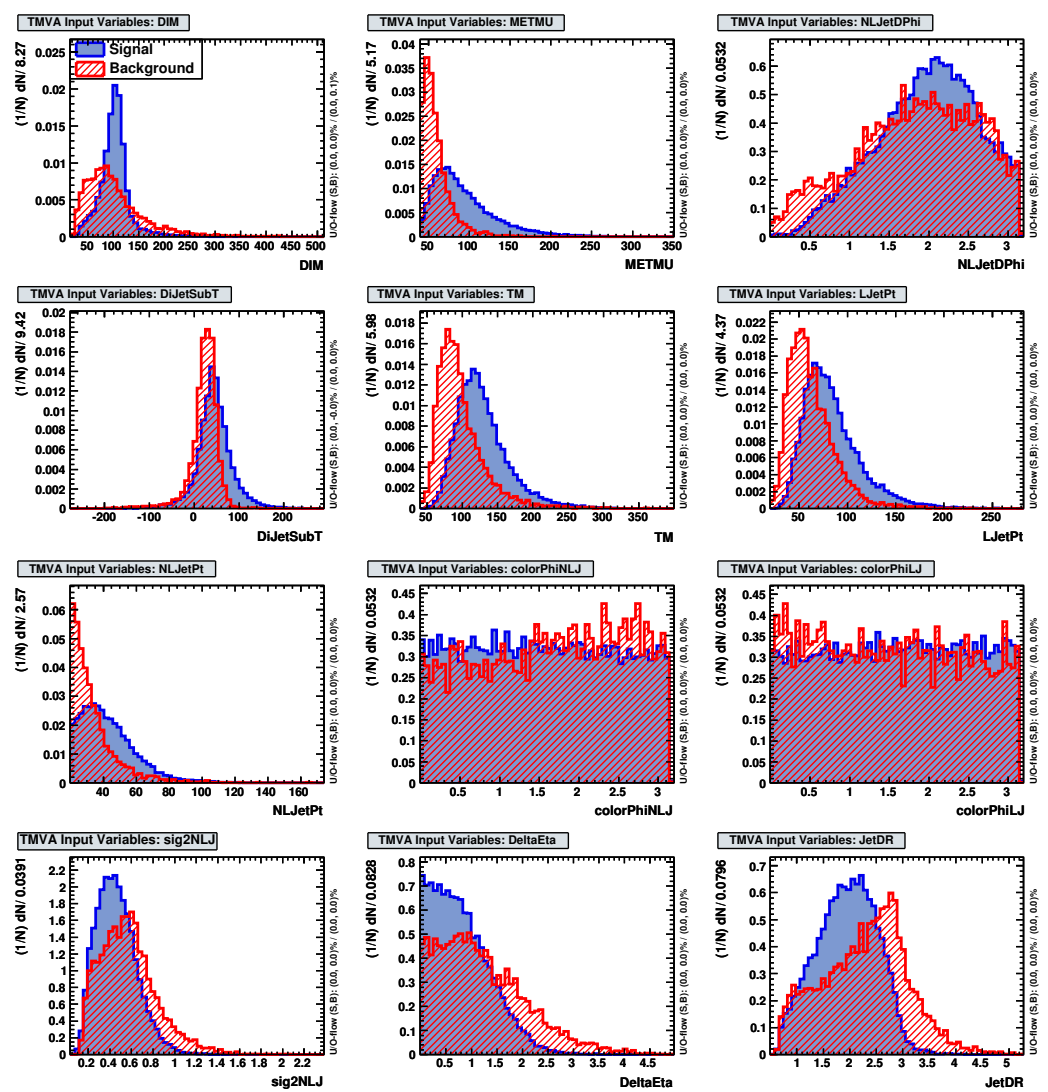

Figure E.3: Comparison of signal and background distributions for multijet decision tree input variables in Run IIb2-3 for a Higgs mass of $115 \mathrm{GeV}$. 

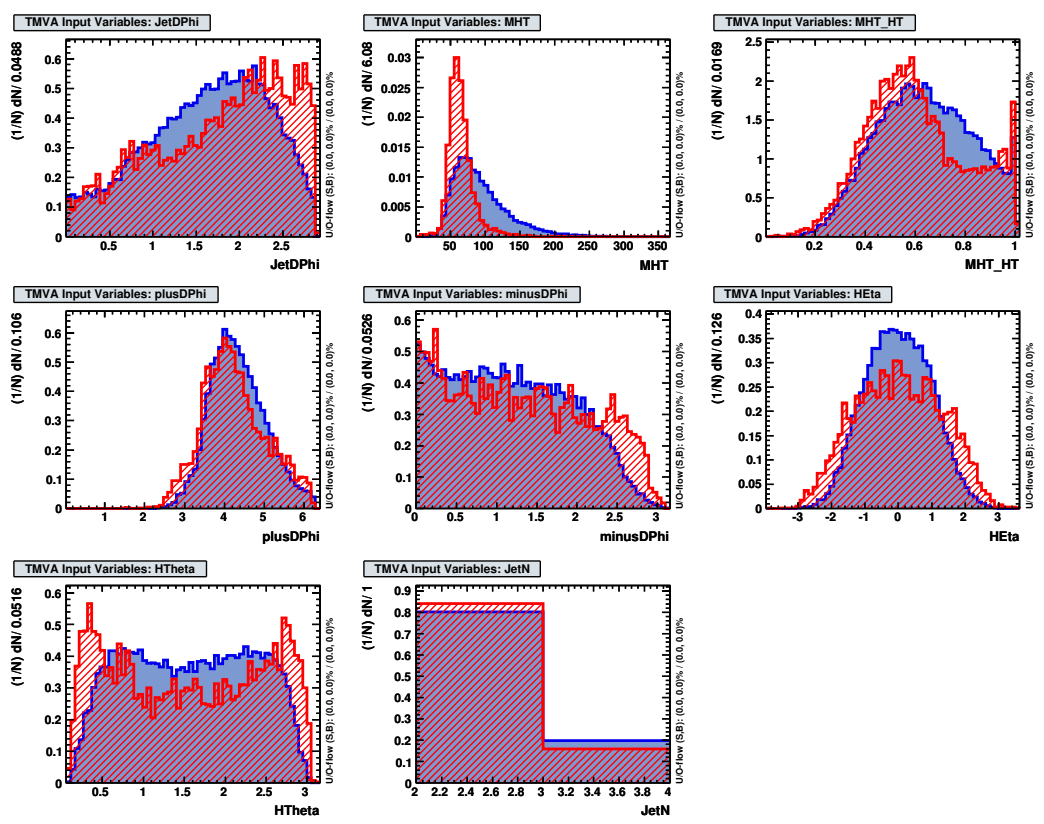

Figure E.4: Comparison of signal and background distributions for multijet decision tree input variables in Run IIb2-3 for a Higgs mass of $115 \mathrm{GeV}$. 

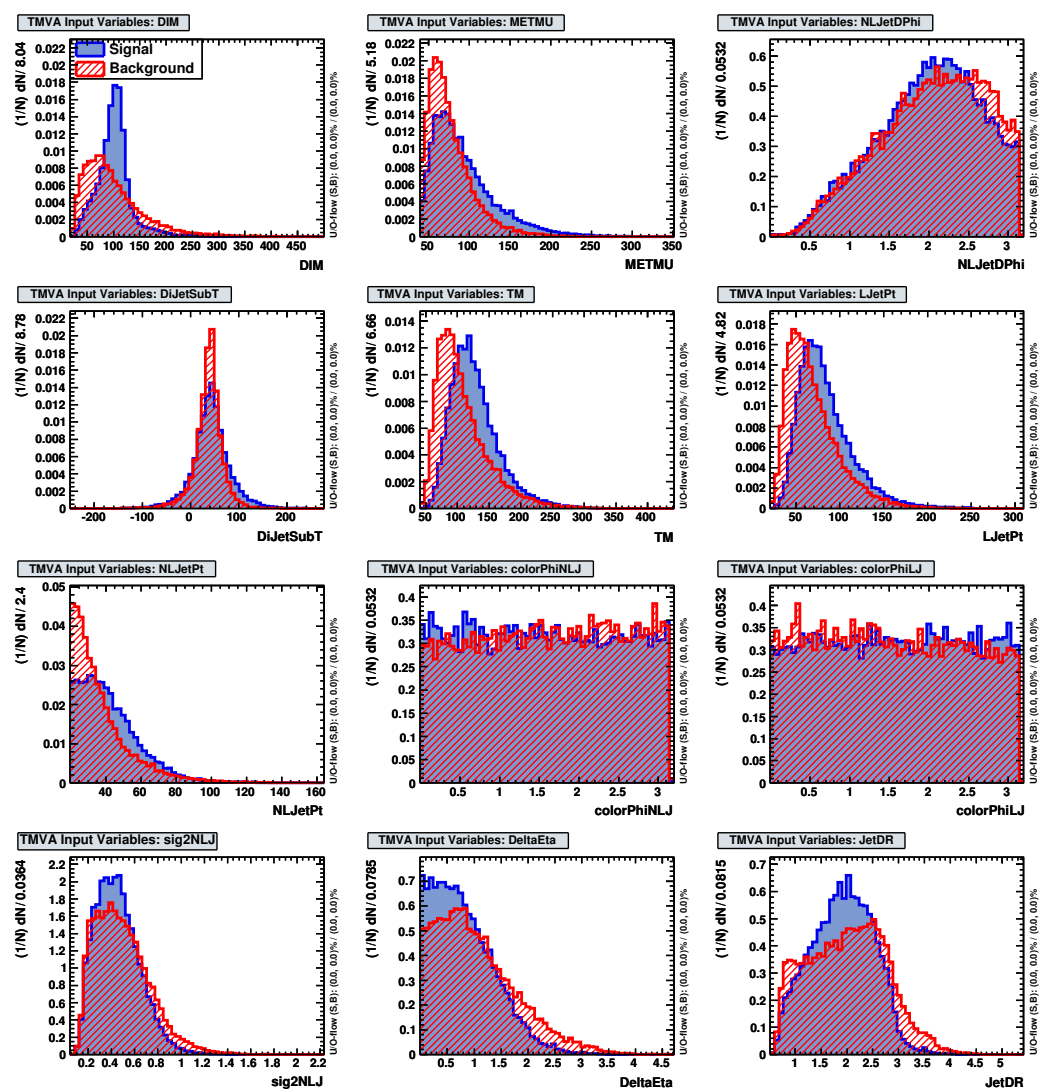

Figure E.5: Comparison of signal and background distributions for multijet decision tree input variables in Run IIb1 for a Higgs mass of $115 \mathrm{GeV}$. 

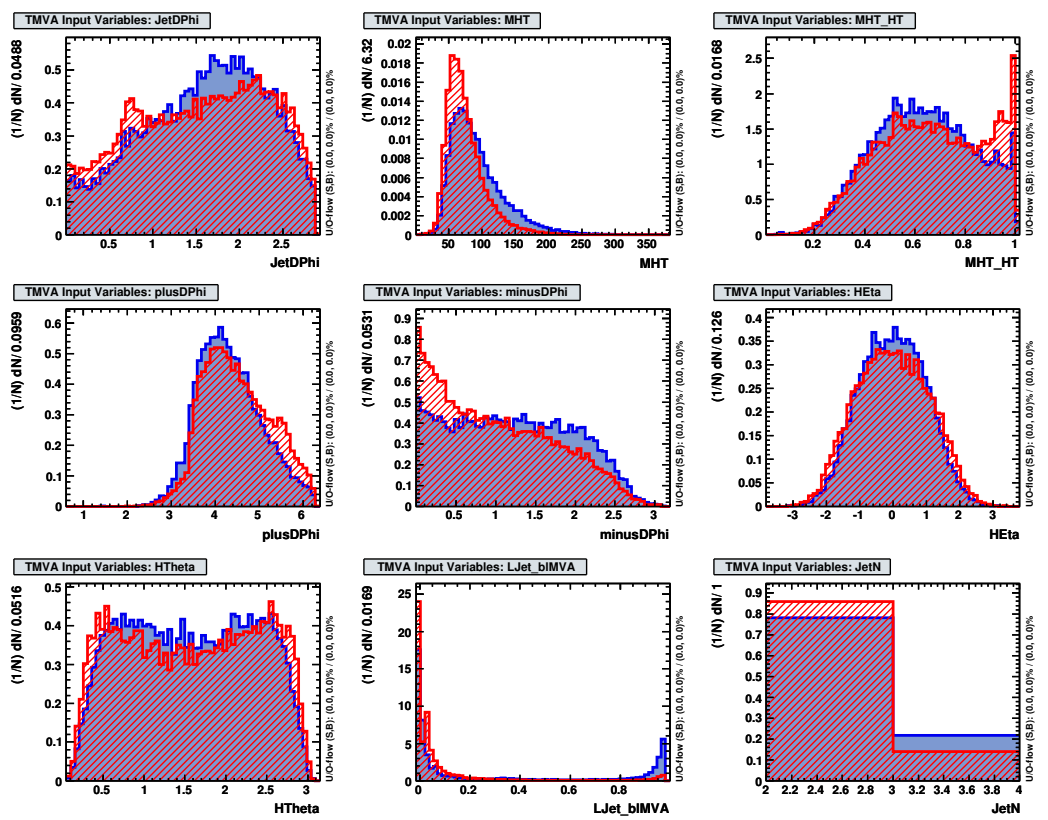

Figure E.6: Comparison of signal and background distributions for multijet decision tree input variables in Run IIb1 for a Higgs mass of $115 \mathrm{GeV}$. 

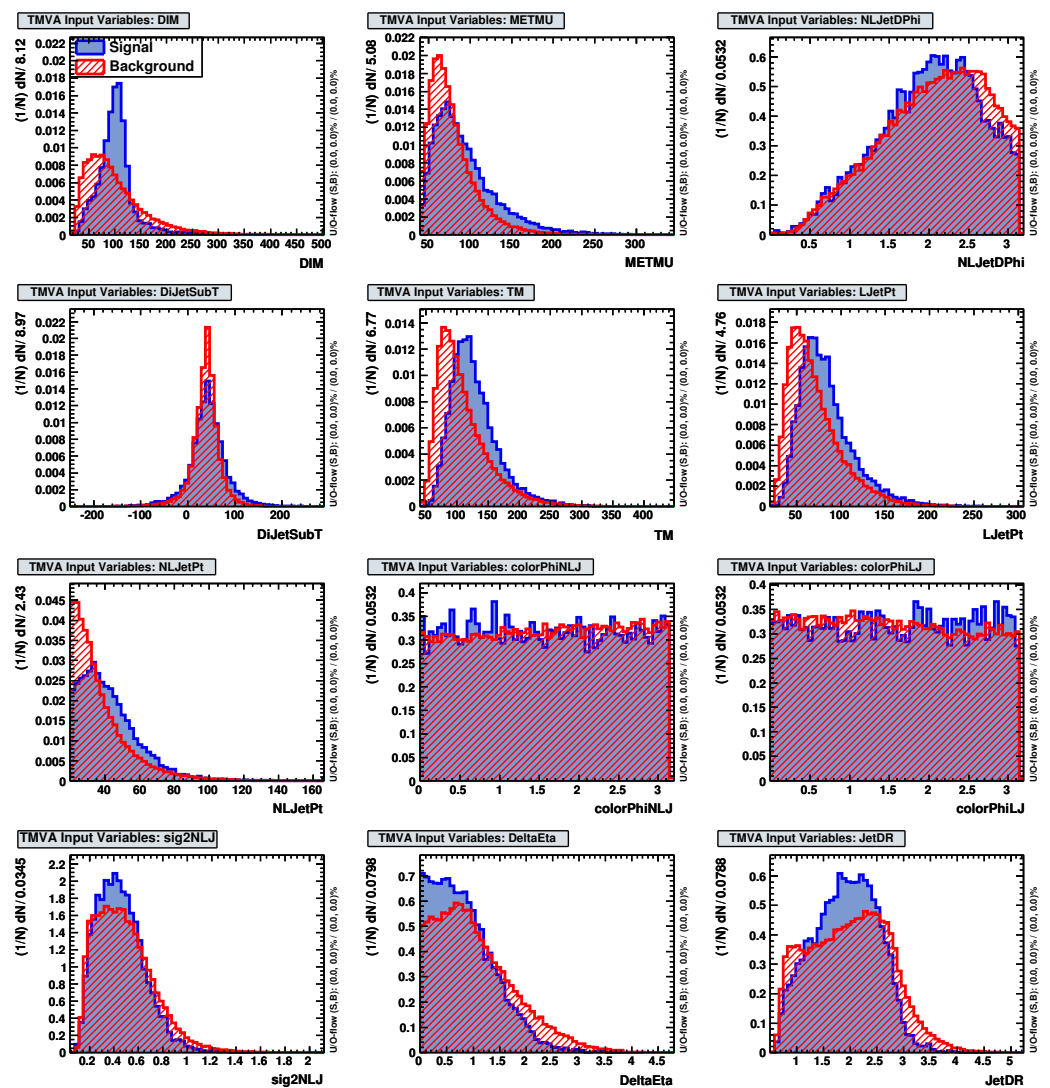

Figure E.7: Comparison of signal and background distributions for multijet decision tree input variables in Run IIb2-3 for a Higgs mass of $115 \mathrm{GeV}$. 

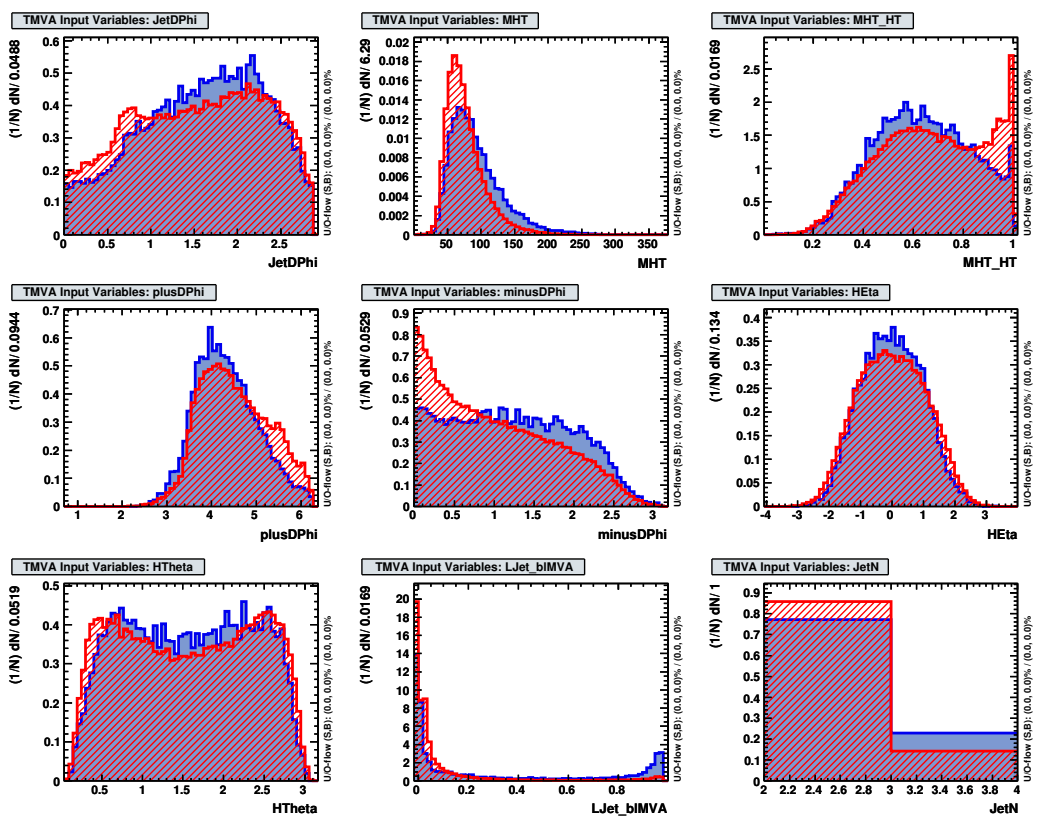

Figure E.8: Comparison of signal and background distributions for multijet decision tree input variables in Run IIb2-3 for a Higgs mass of $115 \mathrm{GeV}$. 

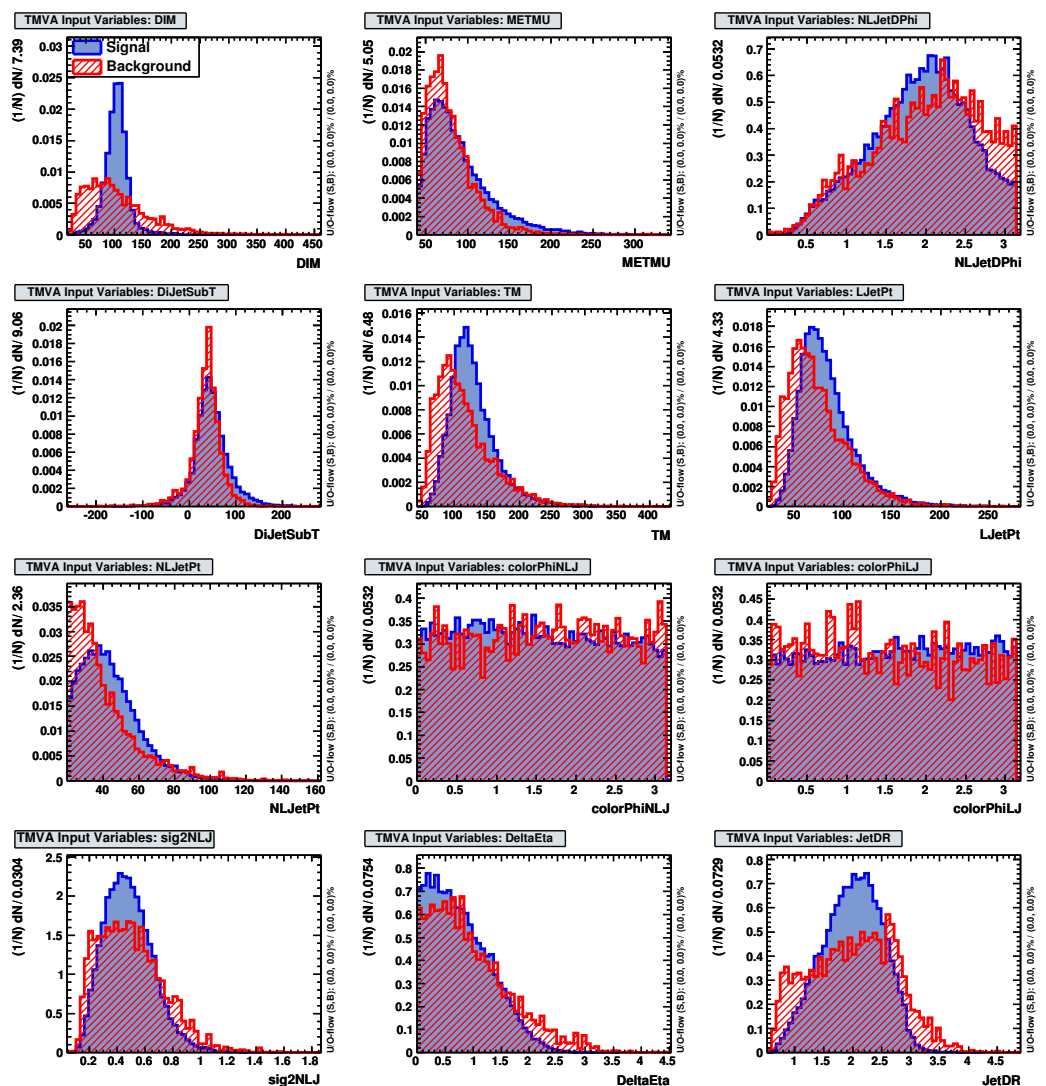

Figure E.9: Comparison of signal and background distributions for multijet decision tree input variables in Run IIb1 for a Higgs mass of $115 \mathrm{GeV}$. 

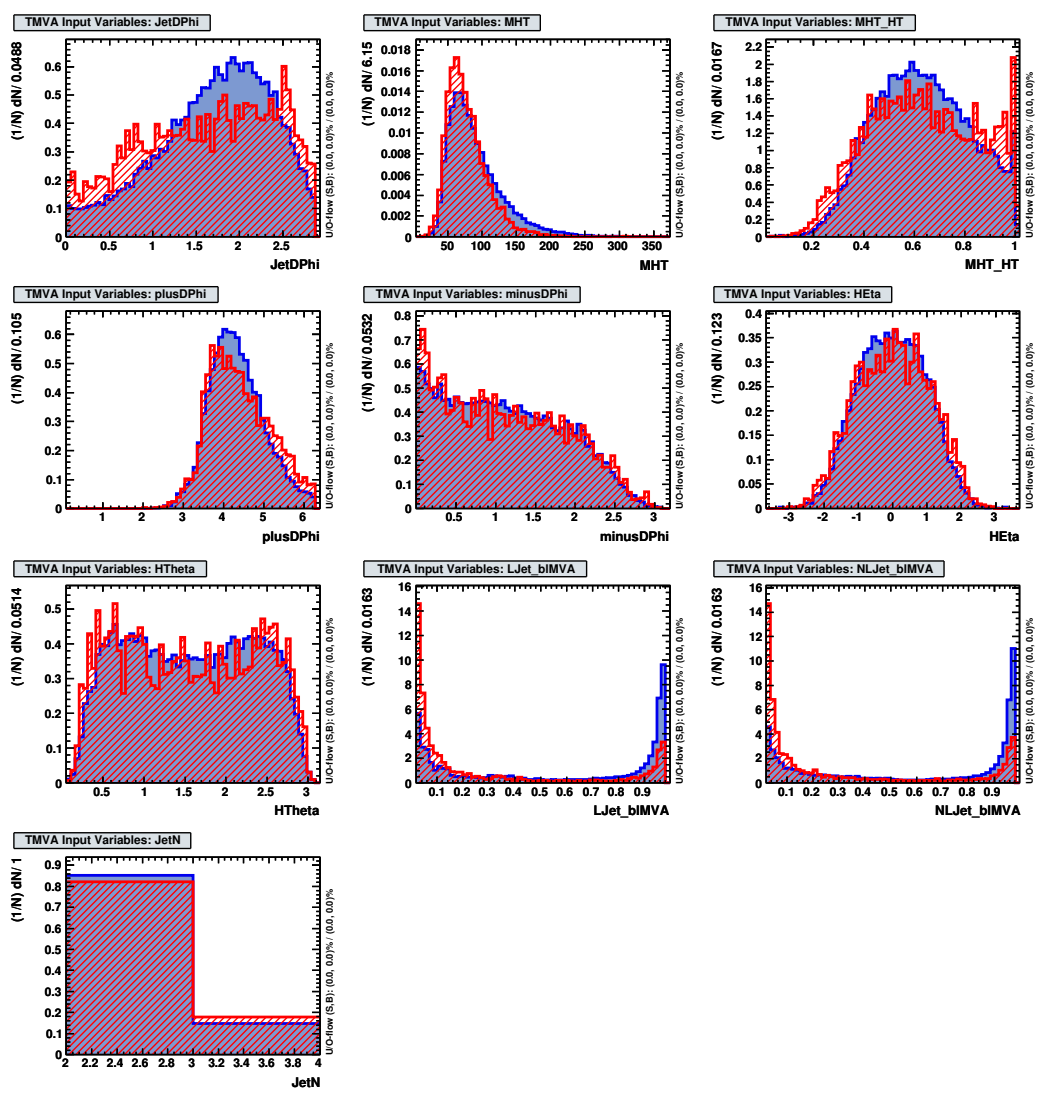

Figure E.10: Comparison of signal and background distributions for multijet decision tree input variables in Run IIb1 for a Higgs mass of $115 \mathrm{GeV}$. 

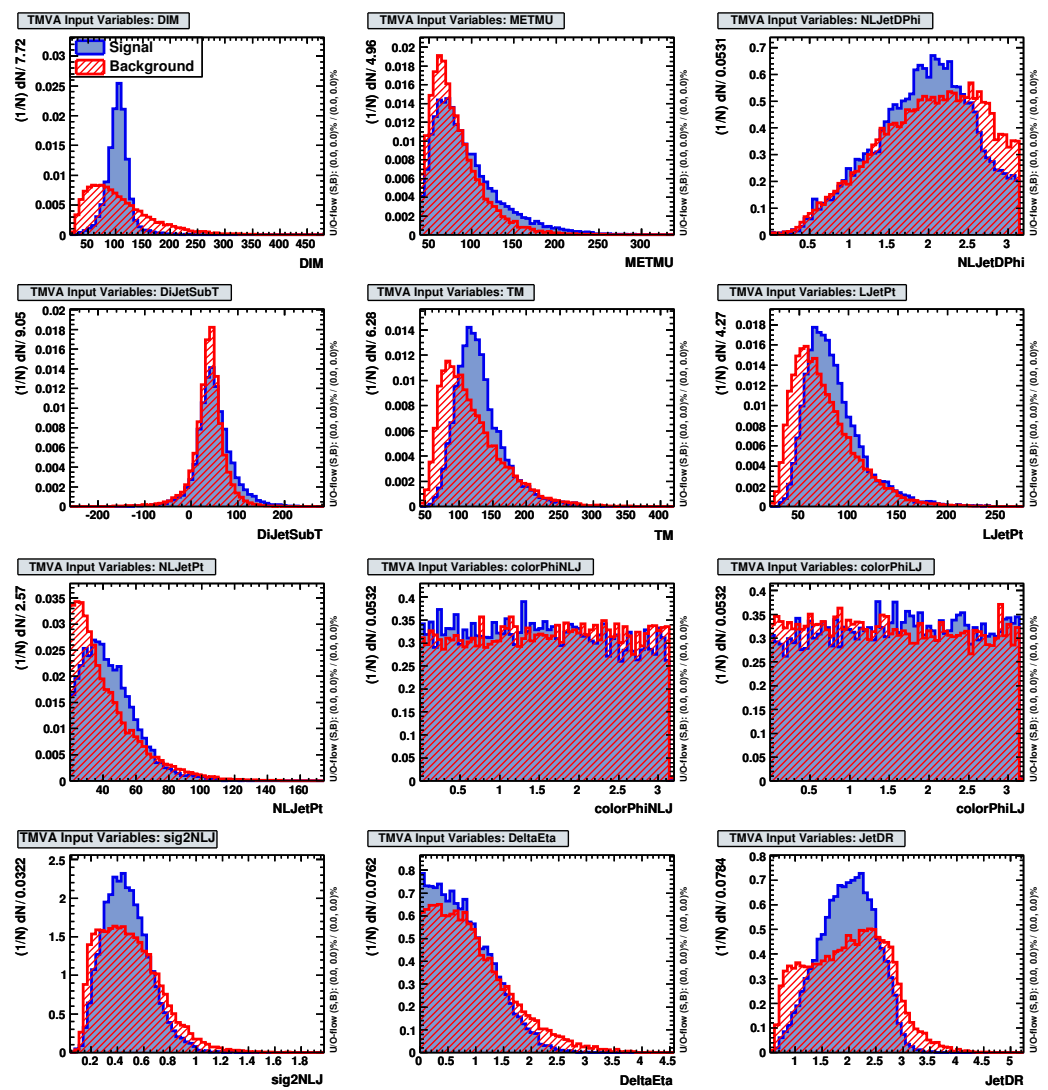

Figure E.11: Comparison of signal and background distributions for multijet decision tree input variables in Run IIb2-3 for a Higgs mass of $115 \mathrm{GeV}$. 

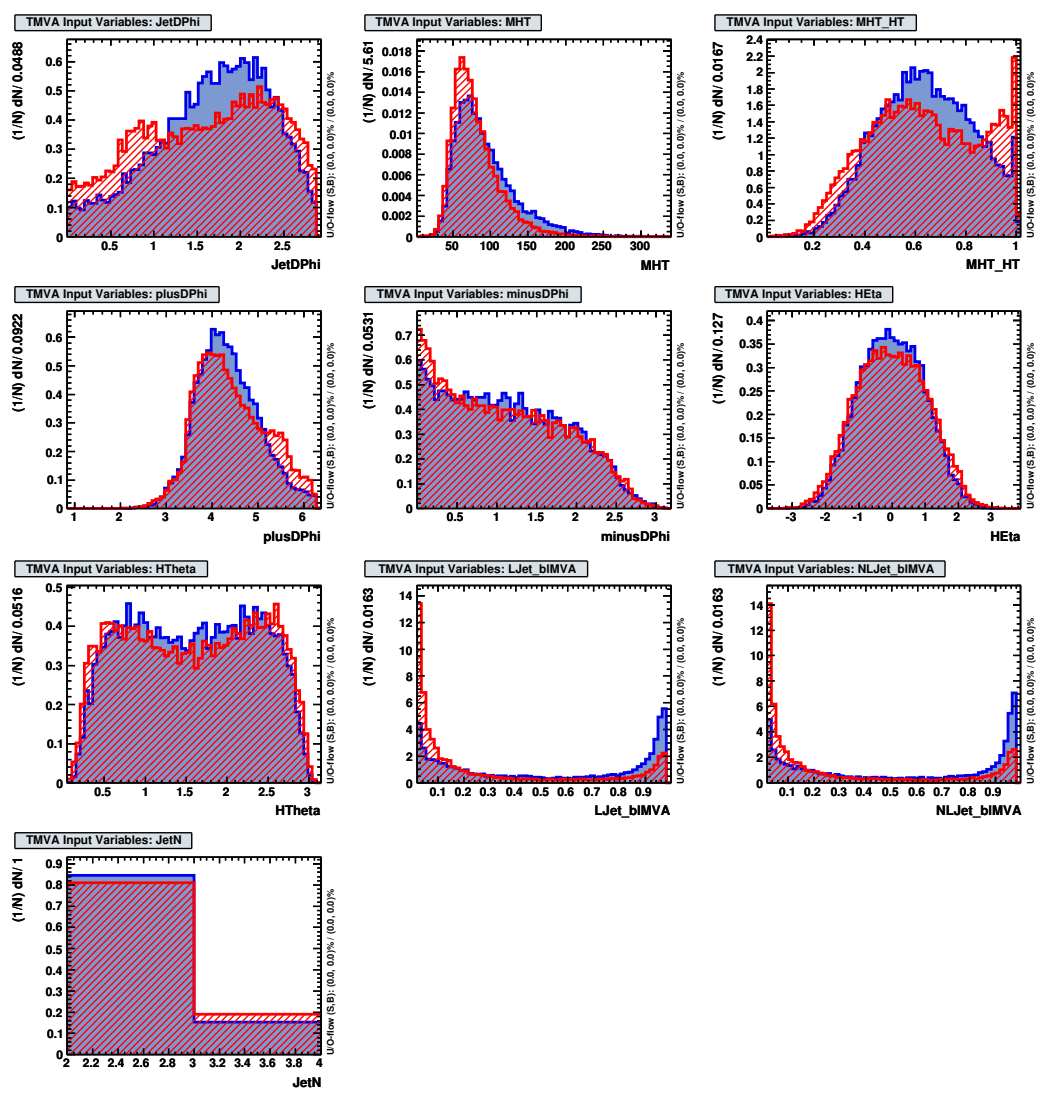

Figure E.12: Comparison of signal and background distributions for multijet decision tree input variables in Run IIb2-3 for a Higgs mass of $115 \mathrm{GeV}$. 


\section{E.1 Overtraining Check for Decision Tree Training}
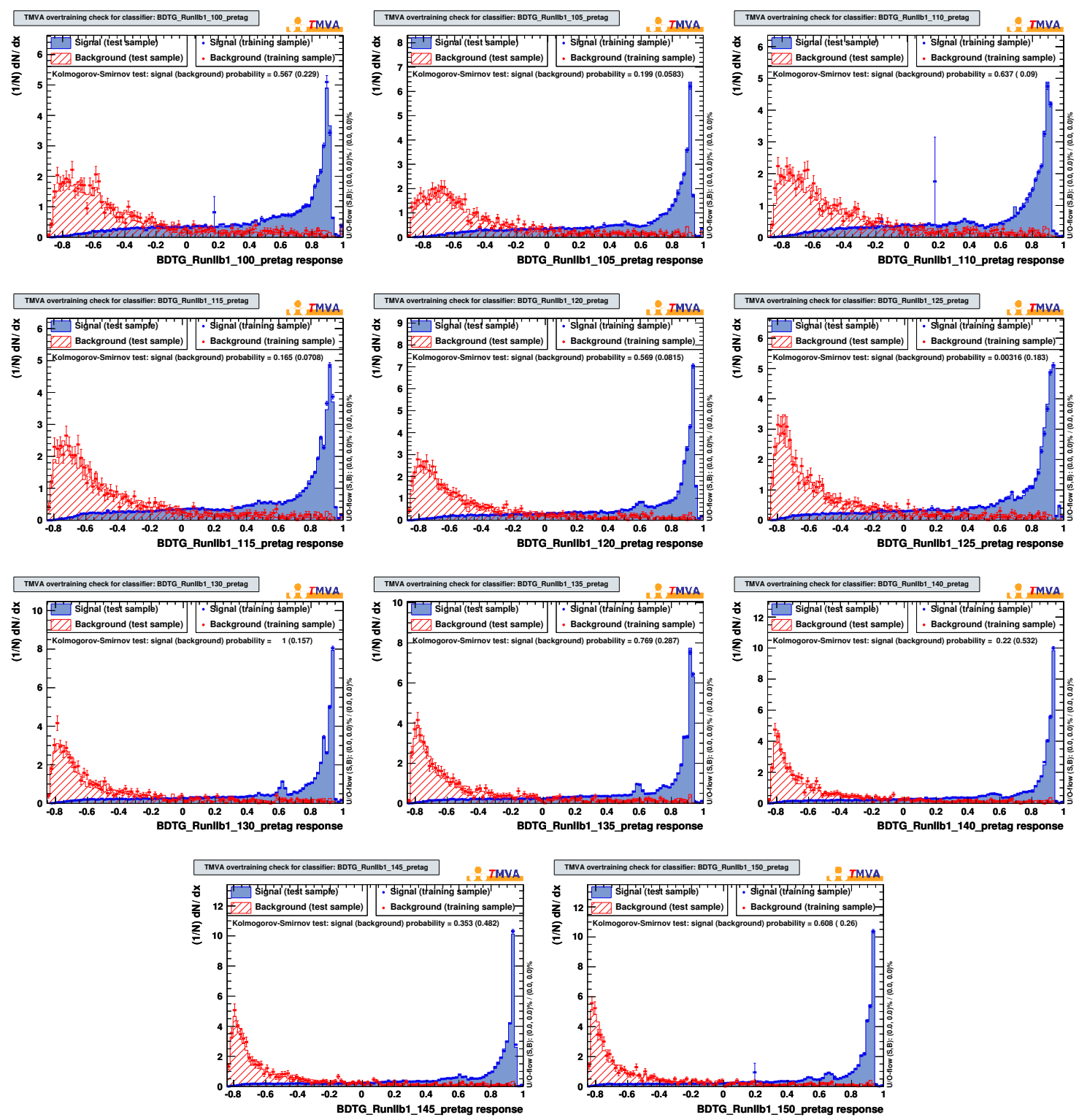

Figure E.13: Overtraining check for the multijet decision tree output. Decision trees are trained for different Higgs masses $(100 \mathrm{GeV}$ in first plot increasing in $5 \mathrm{GeV}$ increments) in the pre-tag sample Run IIb1 dataset. 

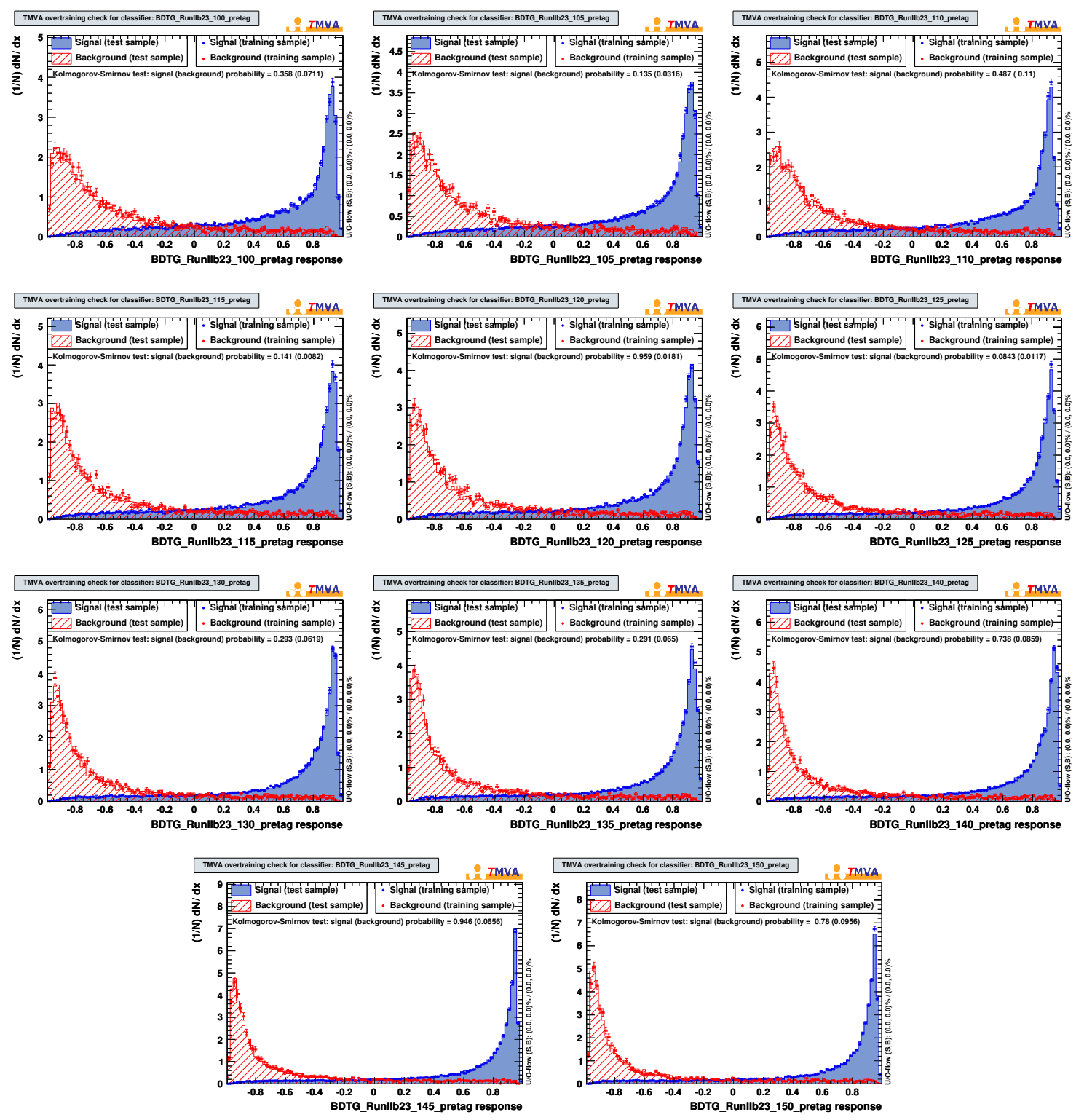

Figure E.14: Overtraining check for the multijet decision tree output. Decision trees are trained for different Higgs masses $(100 \mathrm{GeV}$ in first plot increasing in $5 \mathrm{GeV}$ increments) in the pre-tag sample Run IIb2-3 dataset. 

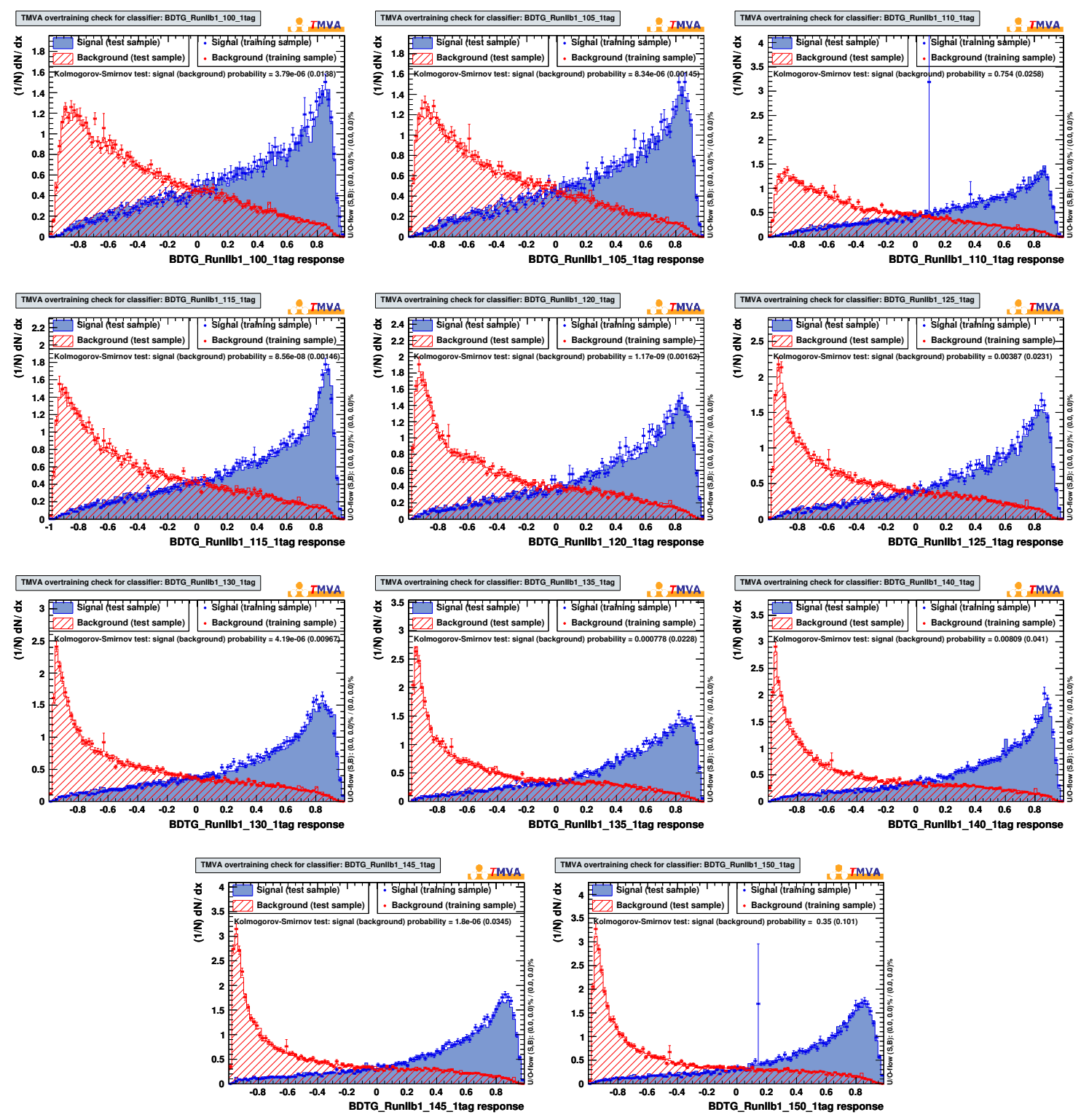

Figure E.15: Overtraining check for the multijet decision tree output. Decision trees are trained for different Higgs masses $(100 \mathrm{GeV}$ in first plot increasing in $5 \mathrm{GeV}$ increments) in the 1-tag sample Run IIb1 dataset. 

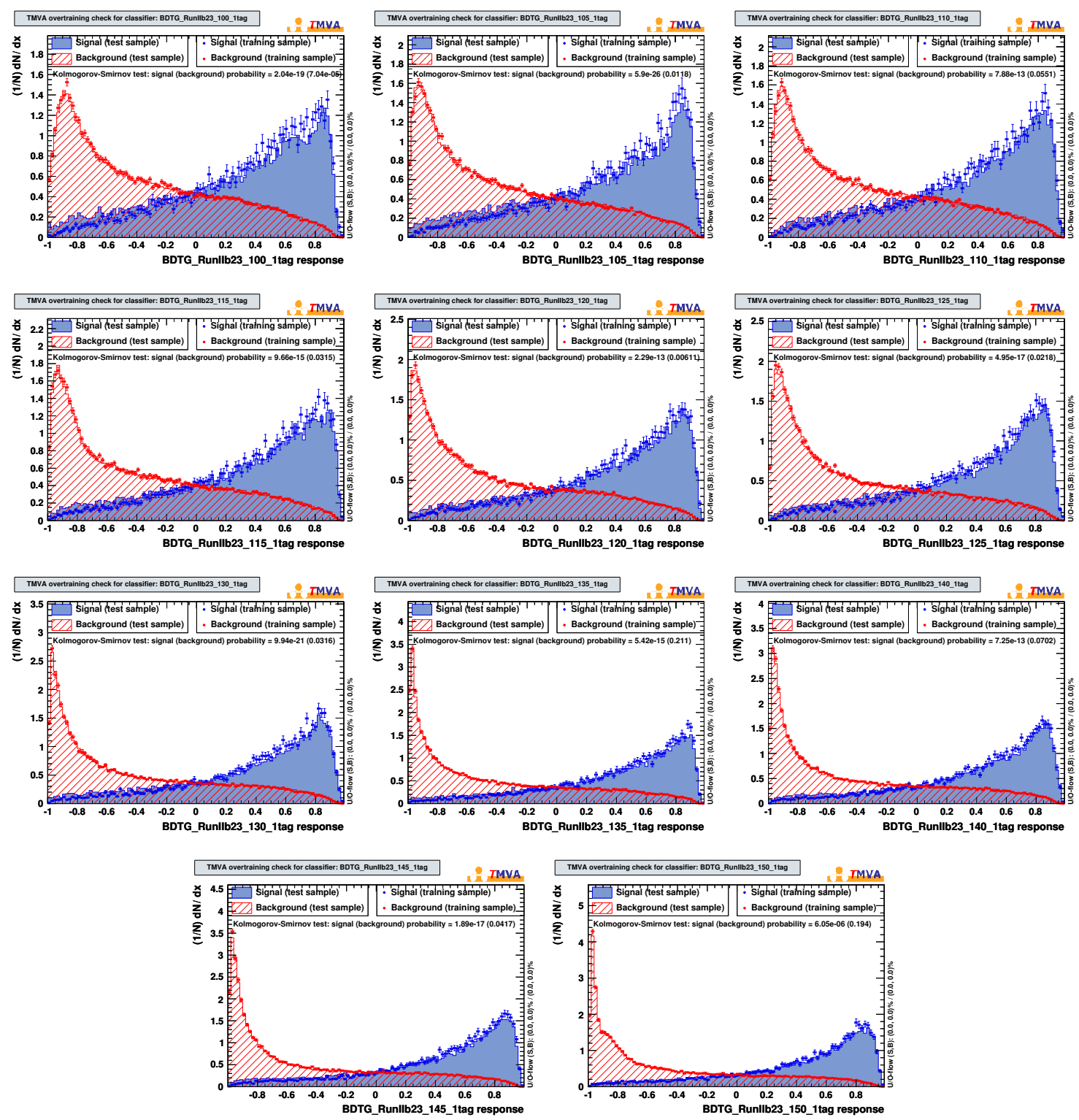

Figure E.16: Overtraining check for the multijet decision tree output. Decision trees are trained for different Higgs masses $(100 \mathrm{GeV}$ in first plot increasing in $5 \mathrm{GeV}$ increments) in the 1-tag sample Run IIb2-3 dataset. 

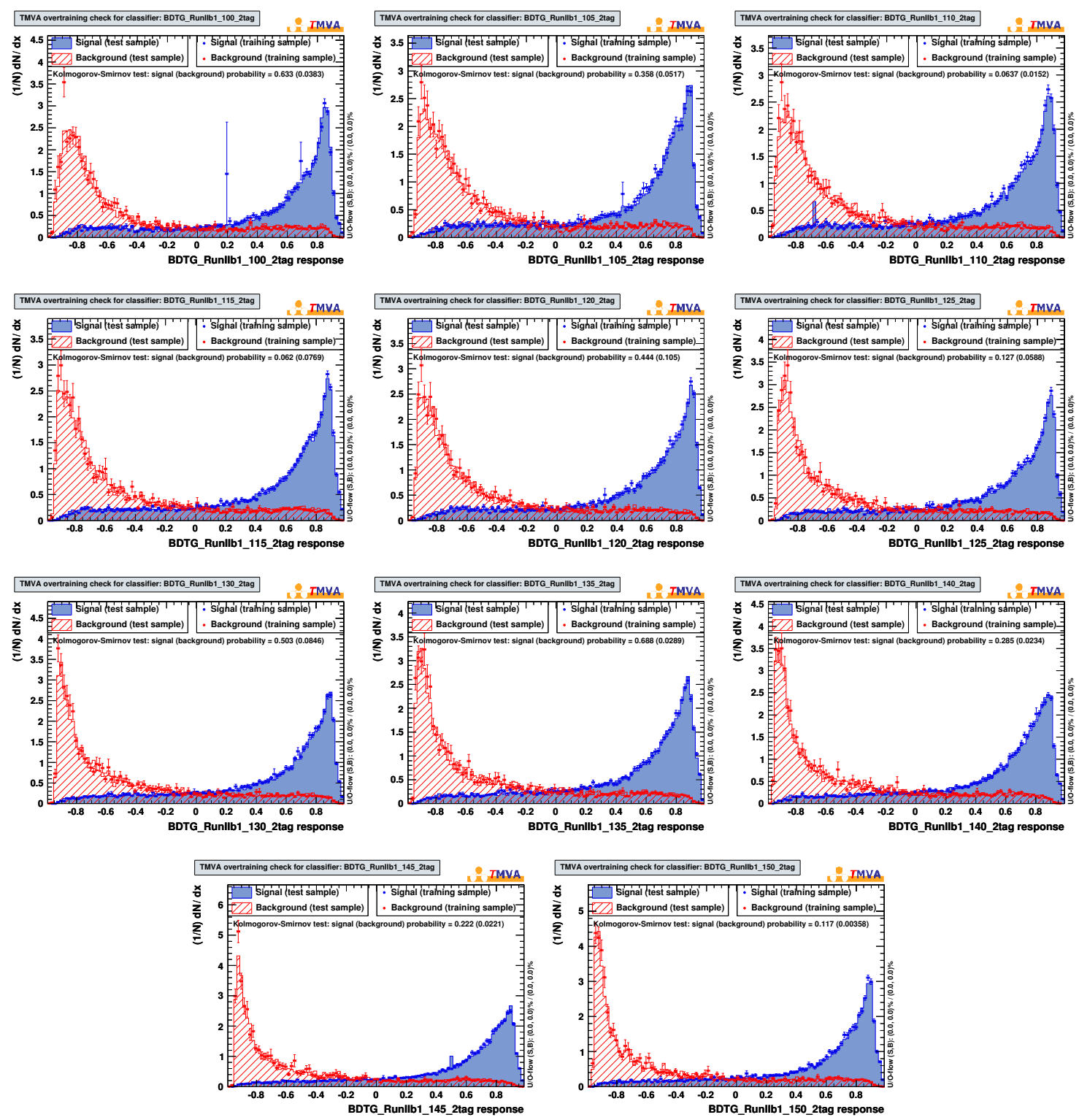

Figure E.17: Overtraining check for the multijet decision tree output. Decision trees are trained for different Higgs masses $(100 \mathrm{GeV}$ in first plot increasing in $5 \mathrm{GeV}$ increments) in the 2-tag sample Run IIb1 dataset. 

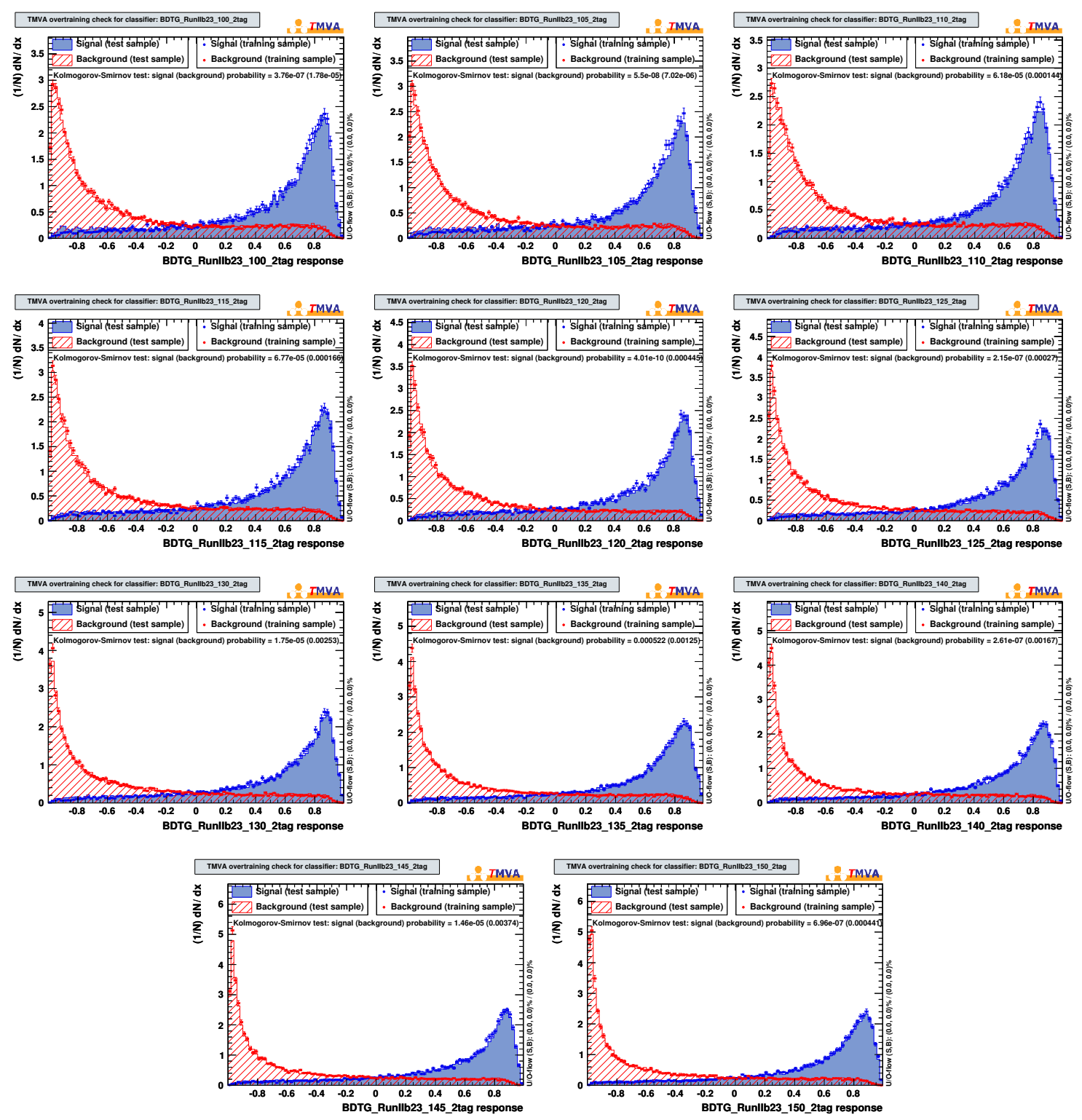

Figure E.18: Overtraining check for the multijet decision tree output. Decision trees are trained for different Higgs masses $(100 \mathrm{GeV}$ in first plot increasing in $5 \mathrm{GeV}$ increments) in the 2-tag sample Run IIb2-3 dataset. 
Appendix F

Physics Decision Trees 

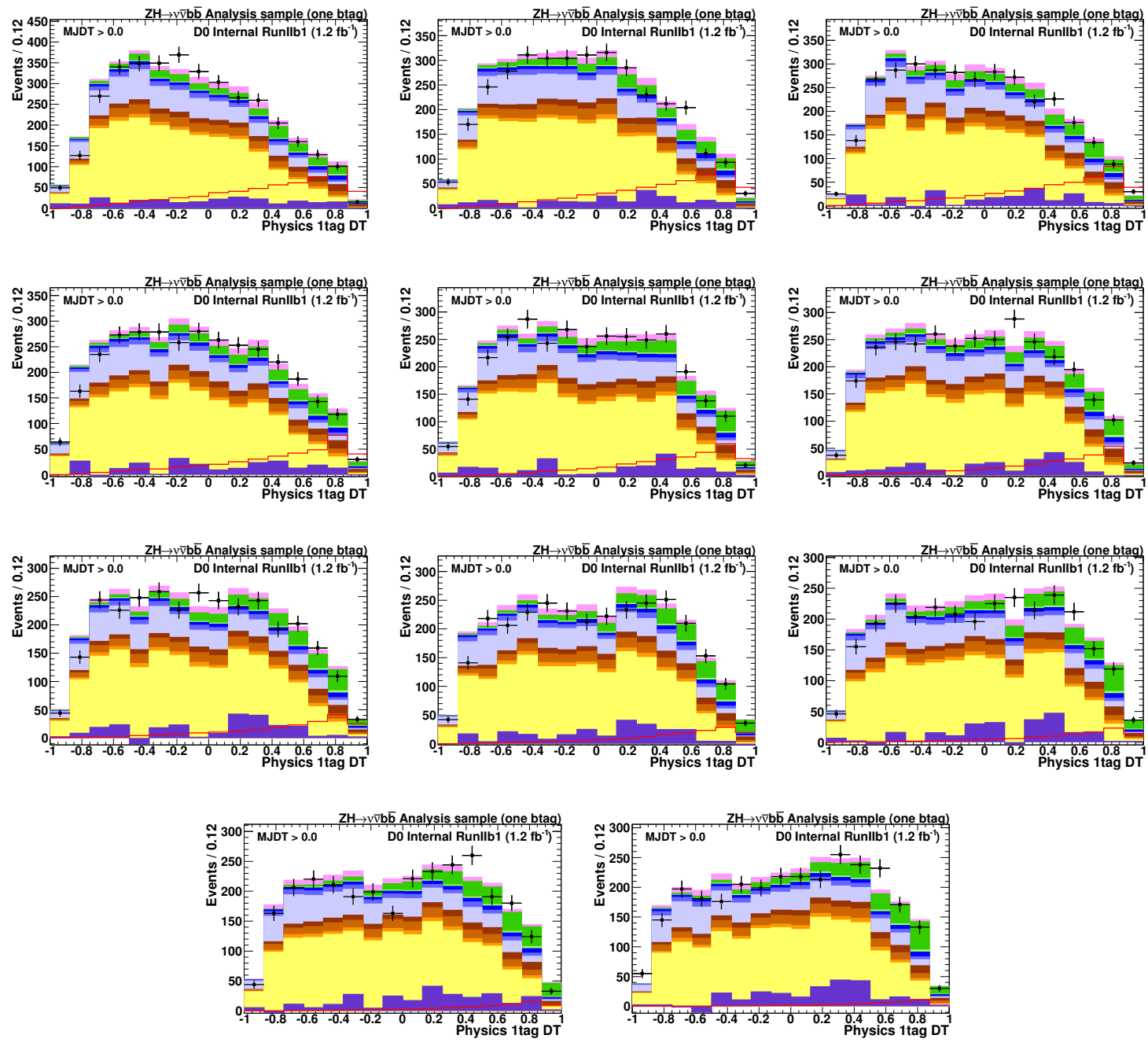

Figure F.1: Physics DT distribution for Higgs mass $100 \mathrm{GeV}-150 \mathrm{GeV}$ (in $5 \mathrm{GeV}$ s) in the Run IIb1 single tag sample. The multijet DT output is required to be greater than 0.0 . 

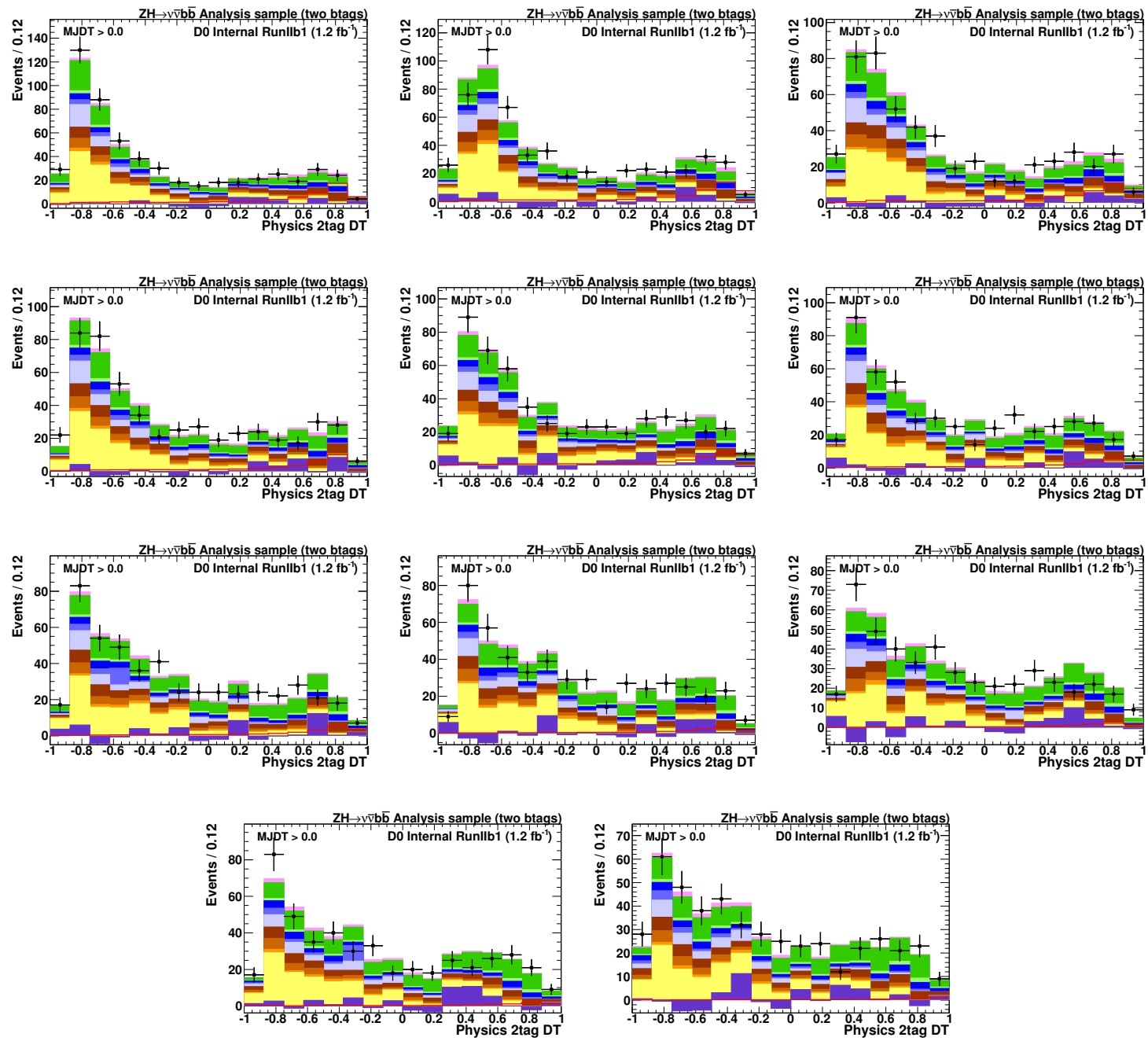

Figure F.2: Physics DT distribution for Higgs mass $100 \mathrm{GeV}-150 \mathrm{GeV}$ (in $5 \mathrm{GeV}$ s) in the Run IIb1 double tag sample. The multijet DT output is required to be greater than 0.0 . 

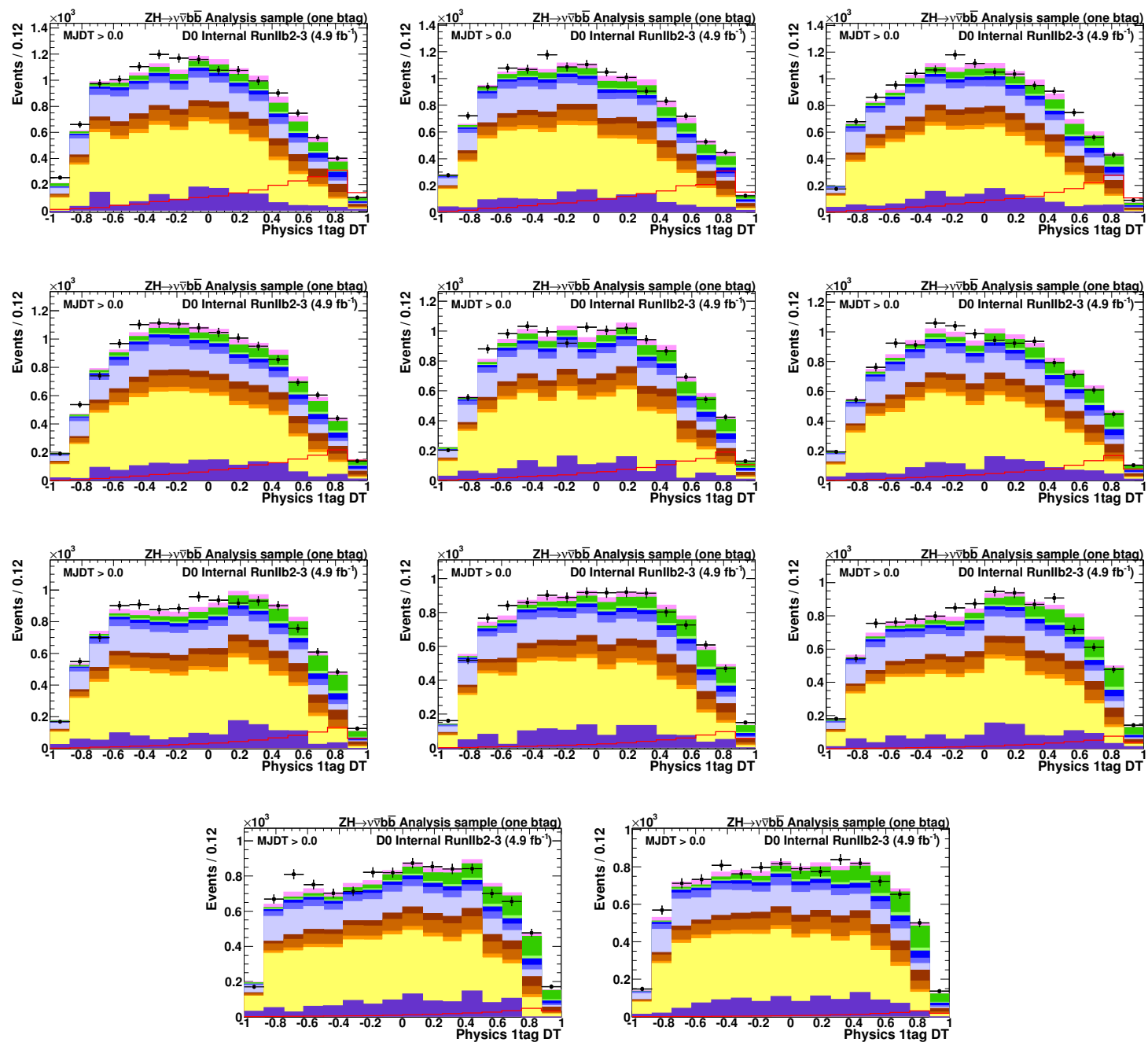

Figure F.3: Physics DT distribution for Higgs mass $100 \mathrm{GeV}-150 \mathrm{GeV}$ (in $5 \mathrm{GeV}$ s) in the Run IIb2-3 single tag sample. The multijet DT output is required to be greater than 0.0 . 

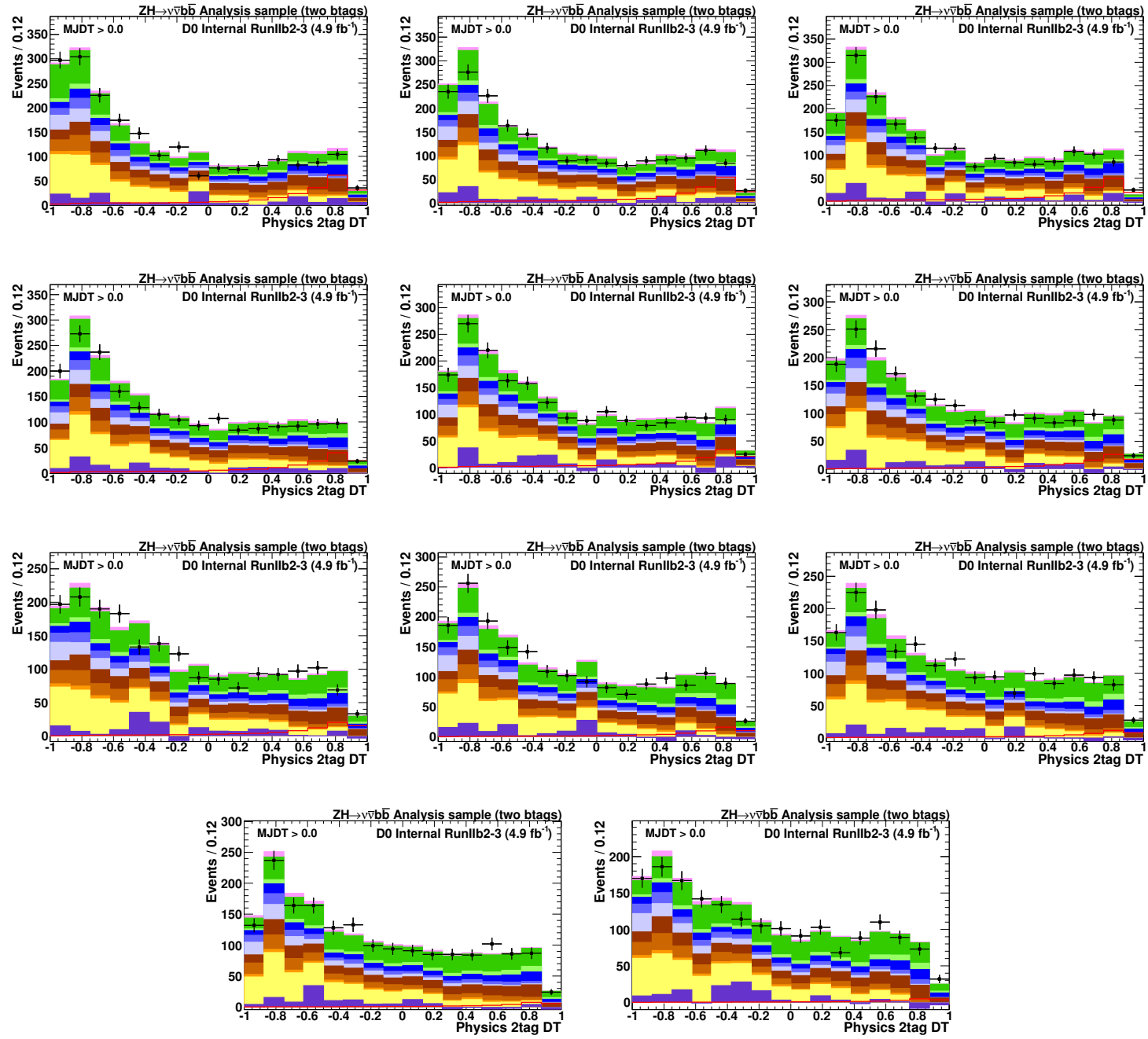

Figure F.4: Physics DT distribution for Higgs mass $100 \mathrm{GeV}-150 \mathrm{GeV}$ (in $5 \mathrm{GeV}$ s) in the Run IIb2-3 double tag sample. The multijet DT output is required to be greater than 0.0 . 
Appendix G

Additional Run IIb1 Multijet

Decision Tree Plots 

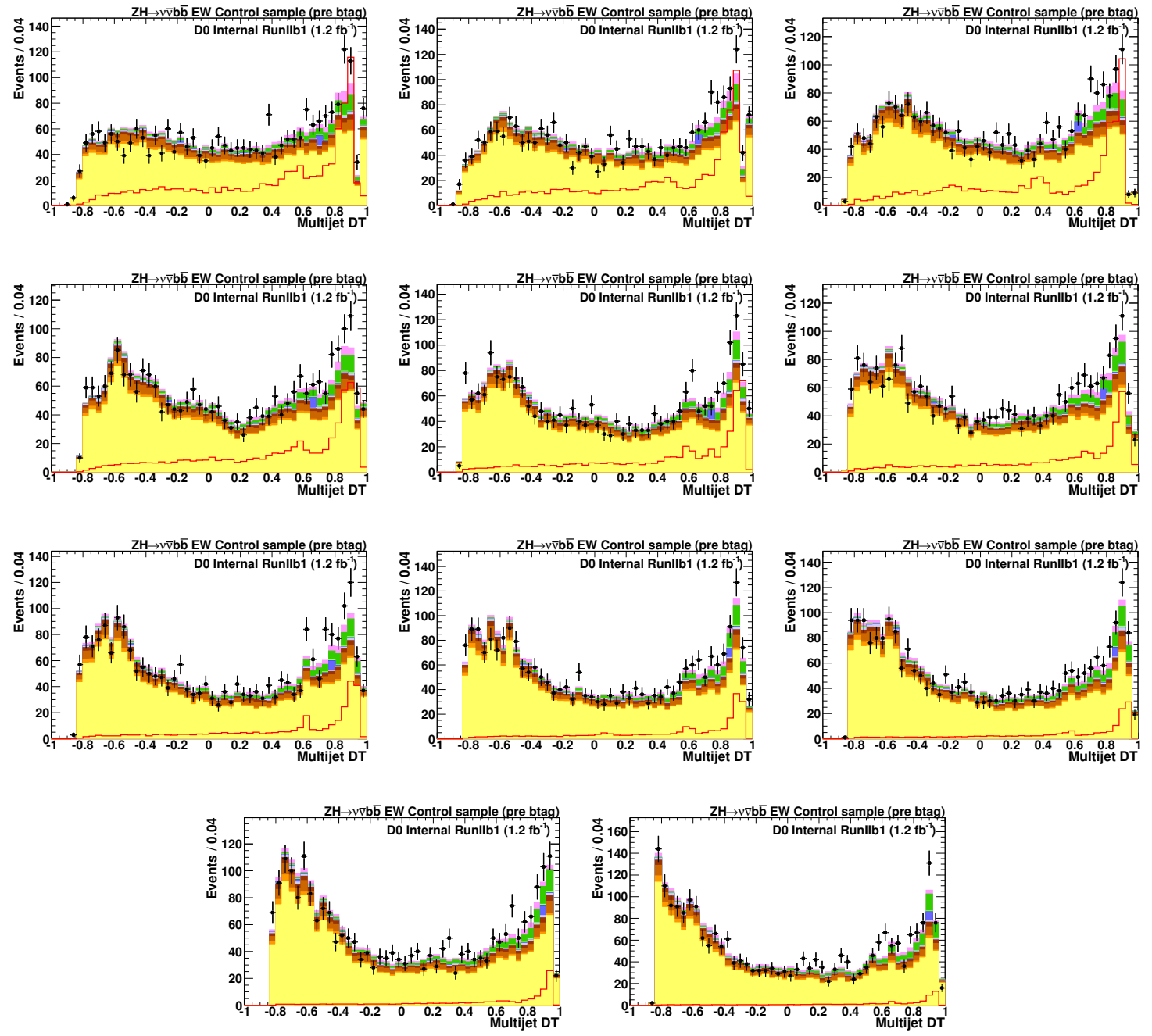

Figure G.1: Multijet decision tree output for pre b-tagged events in the electroweak control sample. Decision trees are trained for different Higgs masses, $100 \mathrm{GeV}$ (top left) to $150 \mathrm{GeV}$ (bottom right) in $5 \mathrm{GeV}$ increments, in the pre-tag sample Run IIb1 dataset. 

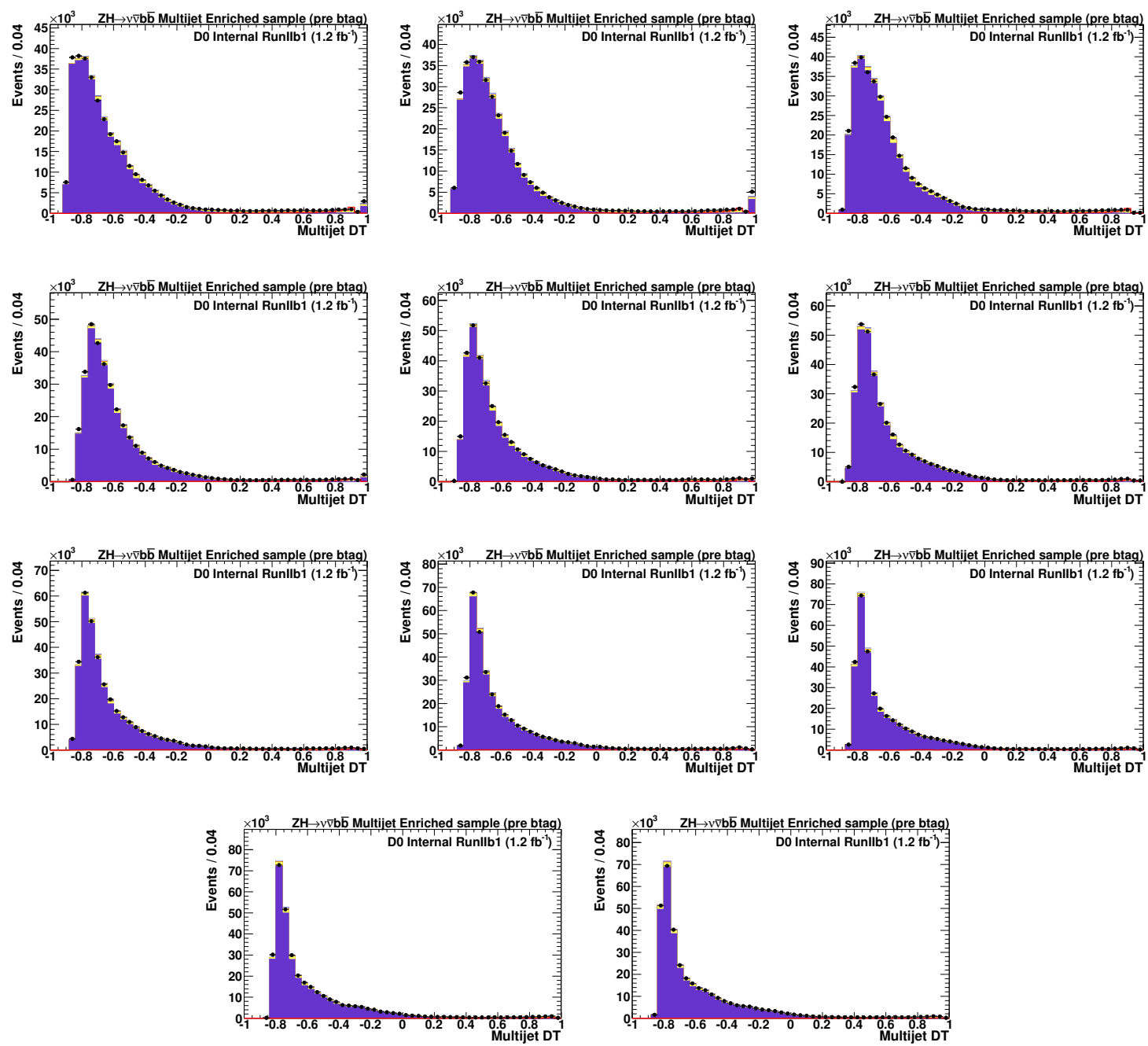

Figure G.2: Multijet decision tree output for pre b-tagged events in the multi-jet control sample. Decision trees are trained for different Higgs masses, $100 \mathrm{GeV}$ (top left) to $150 \mathrm{GeV}$ (bottom right) in $5 \mathrm{GeV}$ increments, in the pre-tag sample Run IIb1 dataset. 

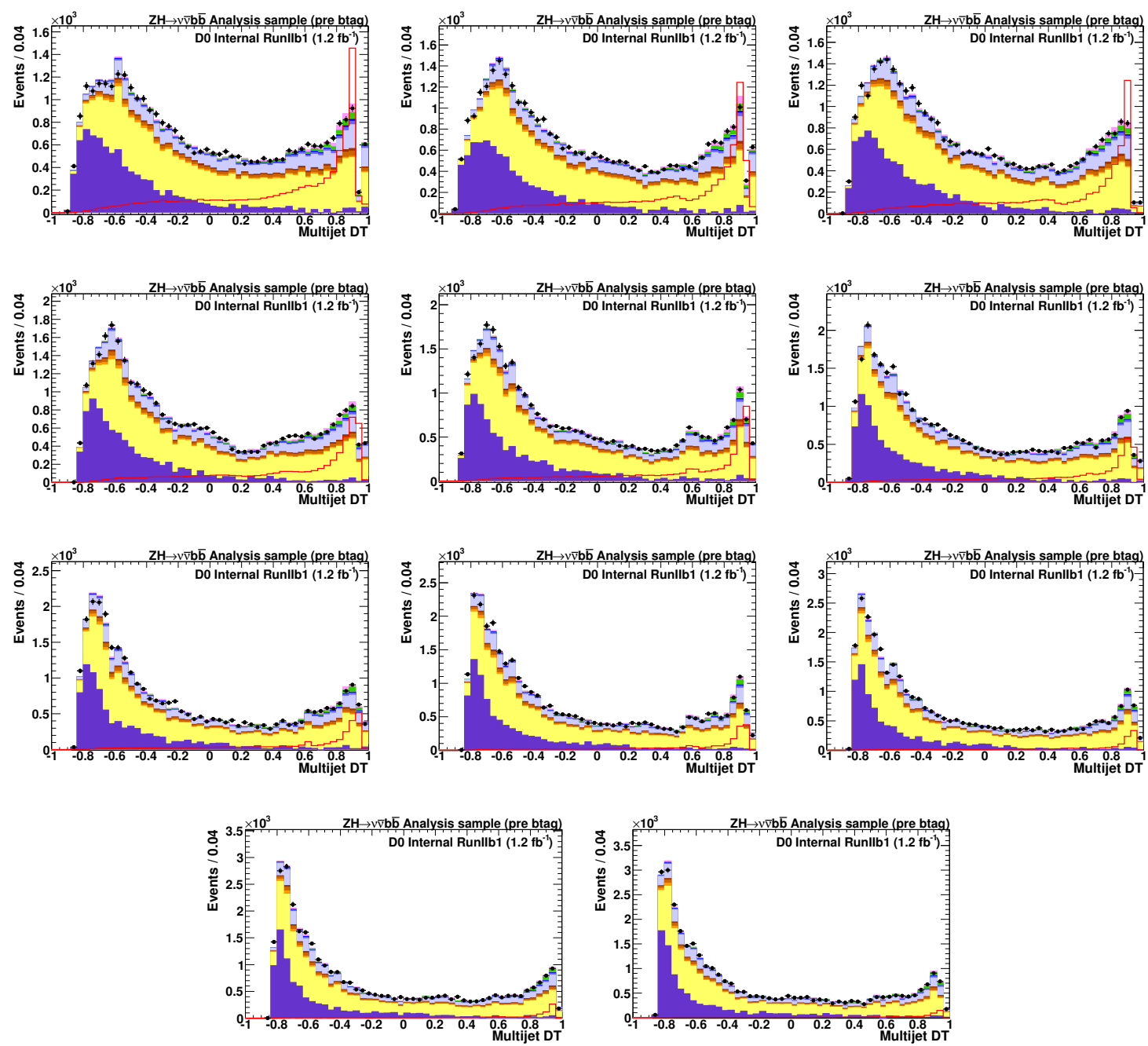

Figure G.3: Multijet decision tree output for pre b-tagged events. Decision trees are trained for different Higgs masses, $100 \mathrm{GeV}$ (top left) to $150 \mathrm{GeV}$ (bottom right) in 5 $\mathrm{GeV}$ increments, in the pre-tag sample Run IIb1 dataset. 

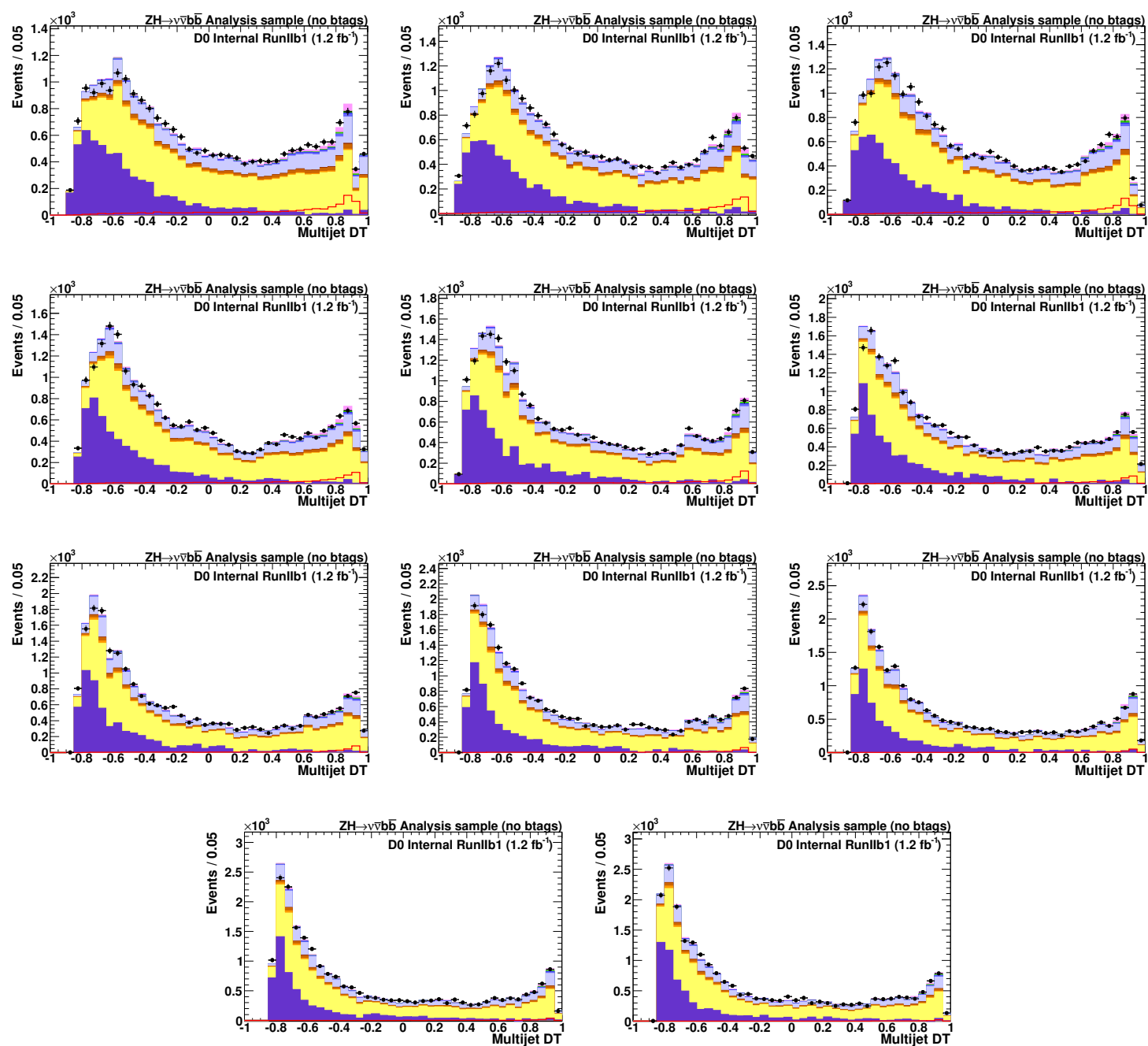

Figure G.4: Multijet decision tree output for zero tagged events. Decision trees are trained for different Higgs masses, $100 \mathrm{GeV}$ (top left) to $150 \mathrm{GeV}$ (bottom right) in 5 $\mathrm{GeV}$ increments, in the pre-tag sample Run IIb1 dataset. 

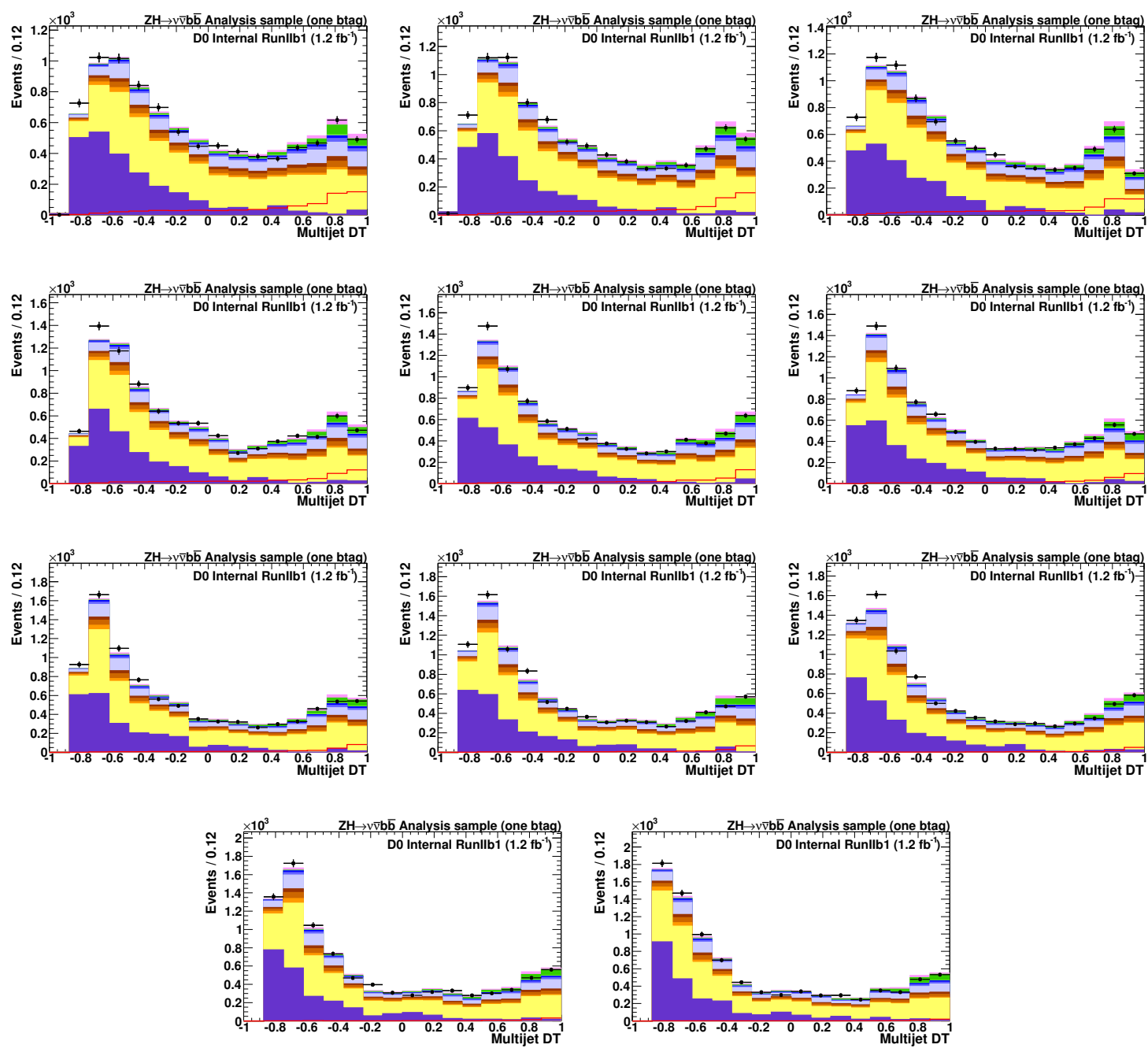

Figure G.5: Multijet decision tree output for single tagged events. Decision trees are trained for different Higgs masses, $100 \mathrm{GeV}$ (top left) to $150 \mathrm{GeV}$ (bottom right) in 5 $\mathrm{GeV}$ increments, in the pre-tag sample Run IIb1 dataset. 

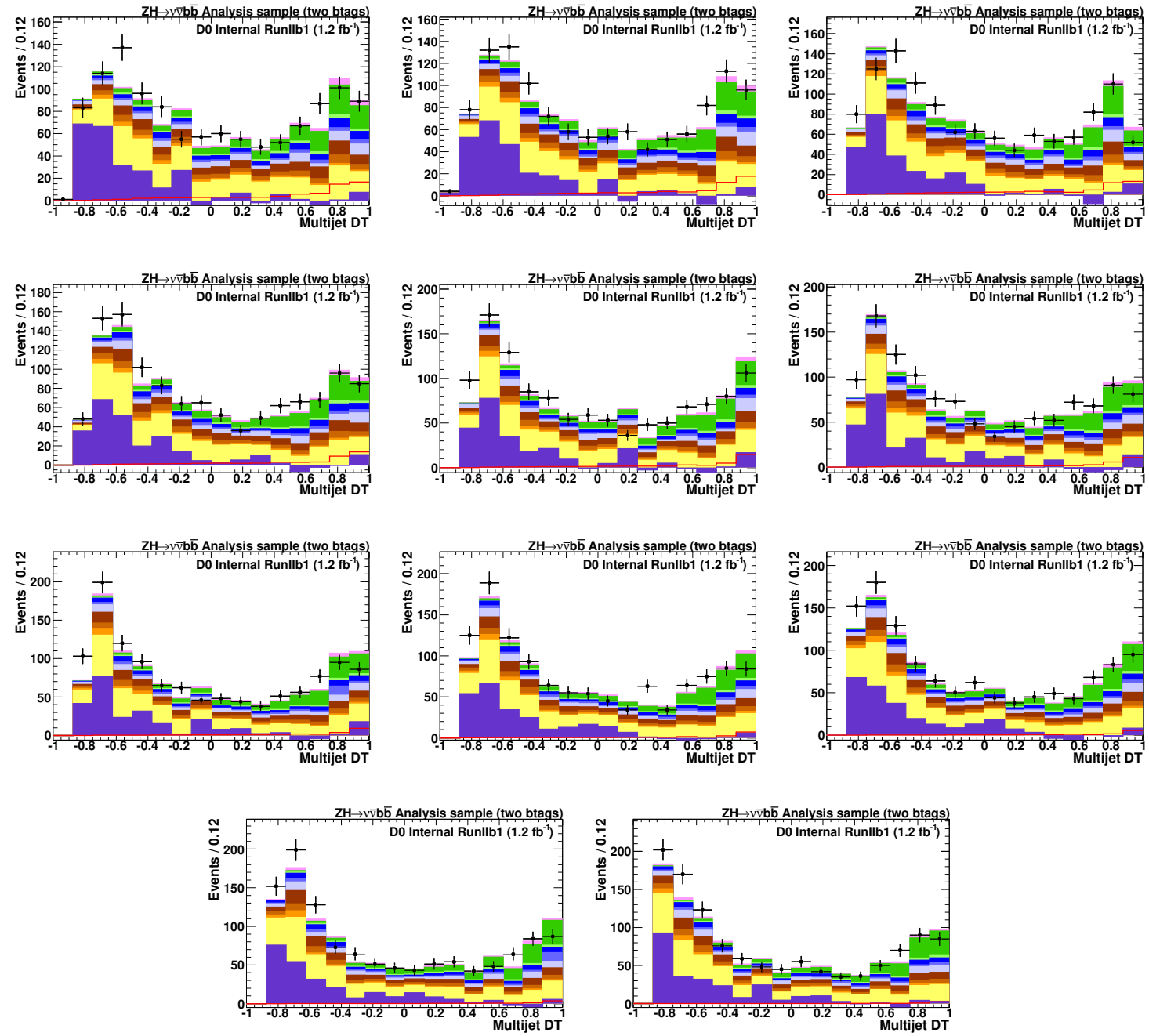

Figure G.6: Multijet decision tree output for double tagged events. Decision trees are trained for different Higgs masses, $100 \mathrm{GeV}$ (top left) to $150 \mathrm{GeV}$ (bottom right) in $5 \mathrm{GeV}$ increments, in the pre-tag sample Run IIb1 dataset. 


\section{G.1 Additional Run IIb1 Physics Decision Tree}

\section{Plots}
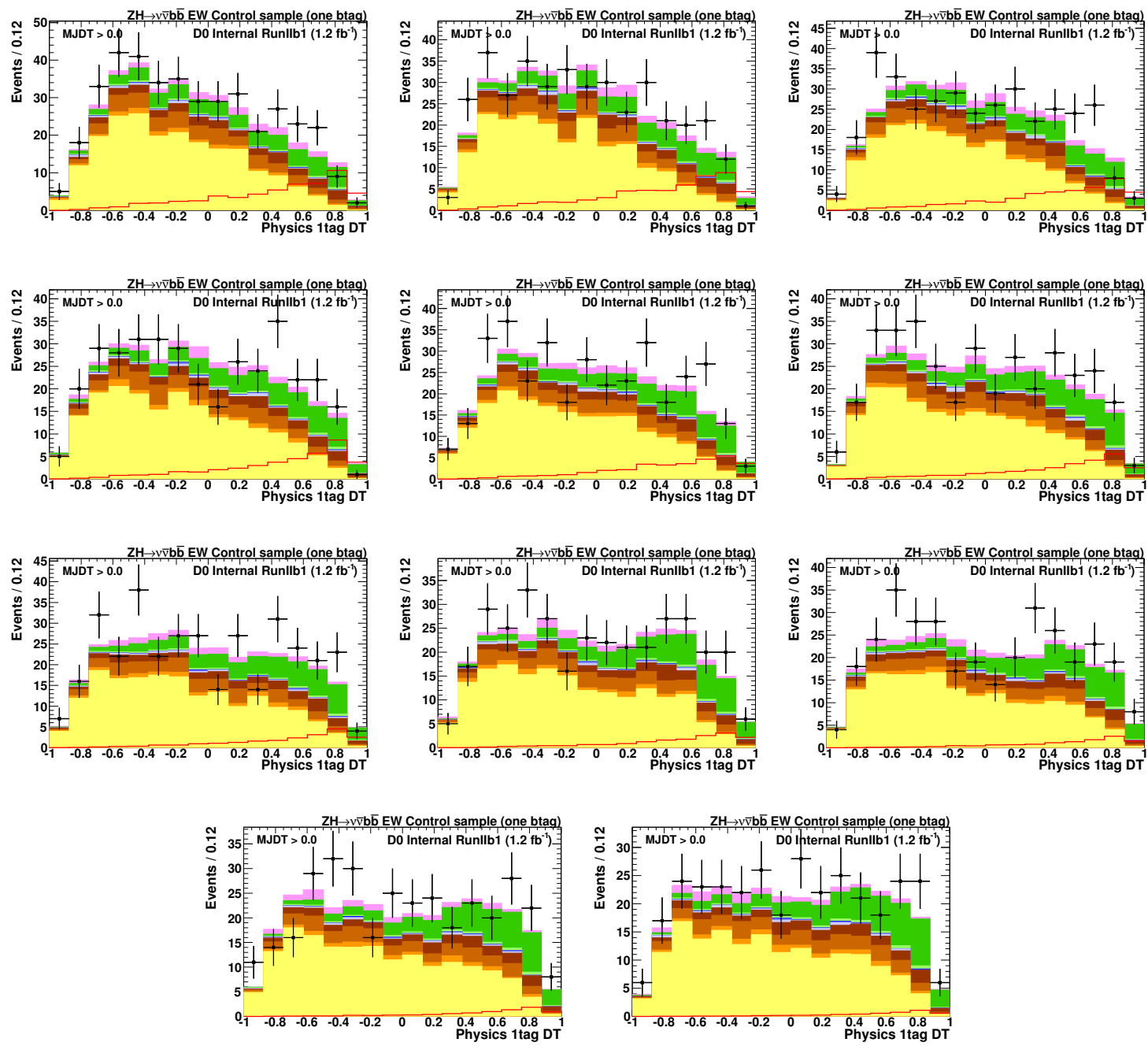

Figure G.7: Physics decision tree output for single tagged events in the electroweak control sample after the MJ-DT cut. Decision trees are trained for different Higgs masses, $100 \mathrm{GeV}$ (top left) to $150 \mathrm{GeV}$ (bottom right) in $5 \mathrm{GeV}$ increments, in the 1 tag sample Run IIb1 dataset. 

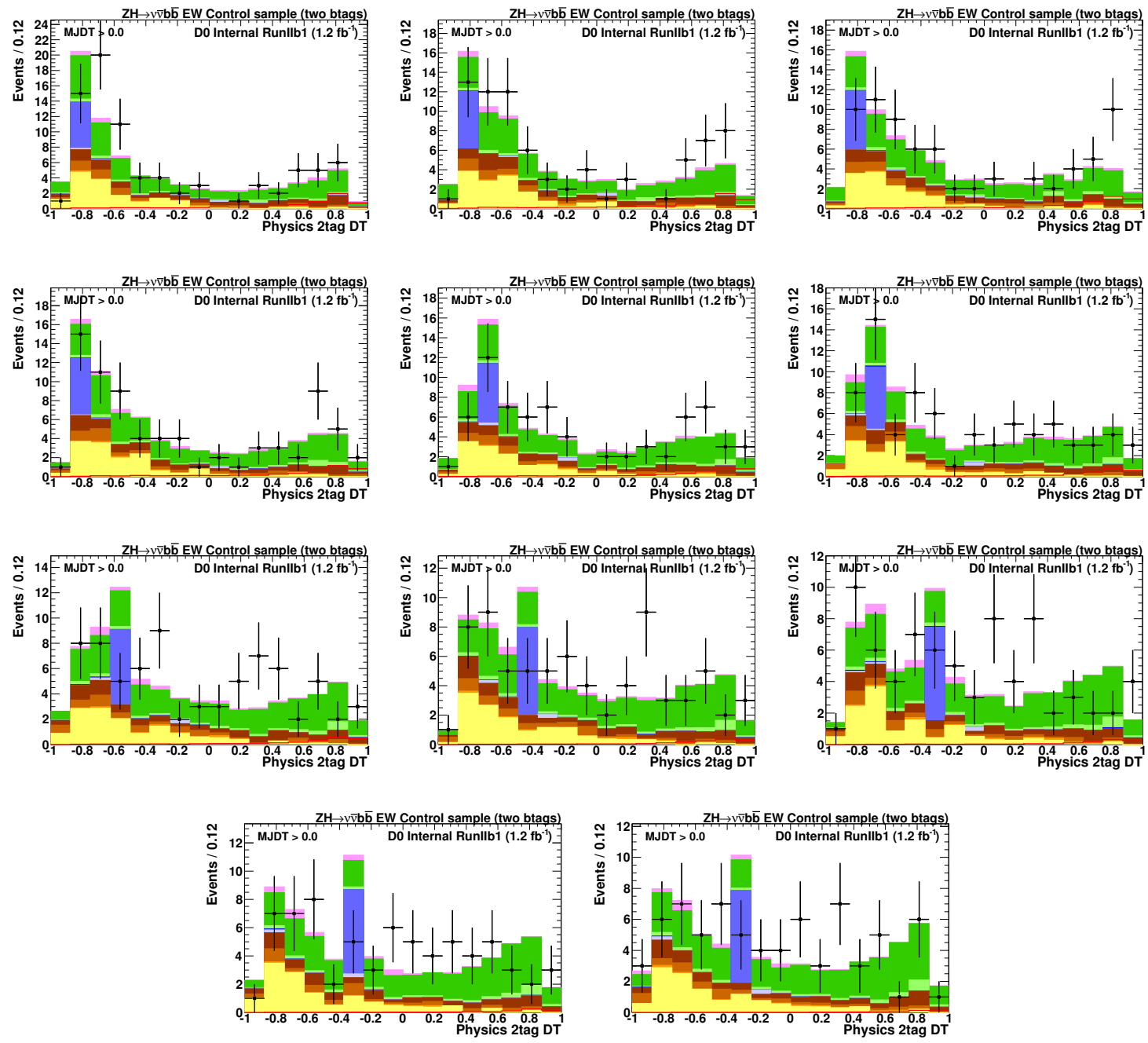

Figure G.8: Physics decision tree output for double tagged events in the electroweak control sample after the MJ-DT cut. Decision trees are trained for different Higgs masses, $100 \mathrm{GeV}$ (top left) to $150 \mathrm{GeV}$ (bottom right) in $5 \mathrm{GeV}$ increments, in the 2tag sample Run IIb1 dataset. 

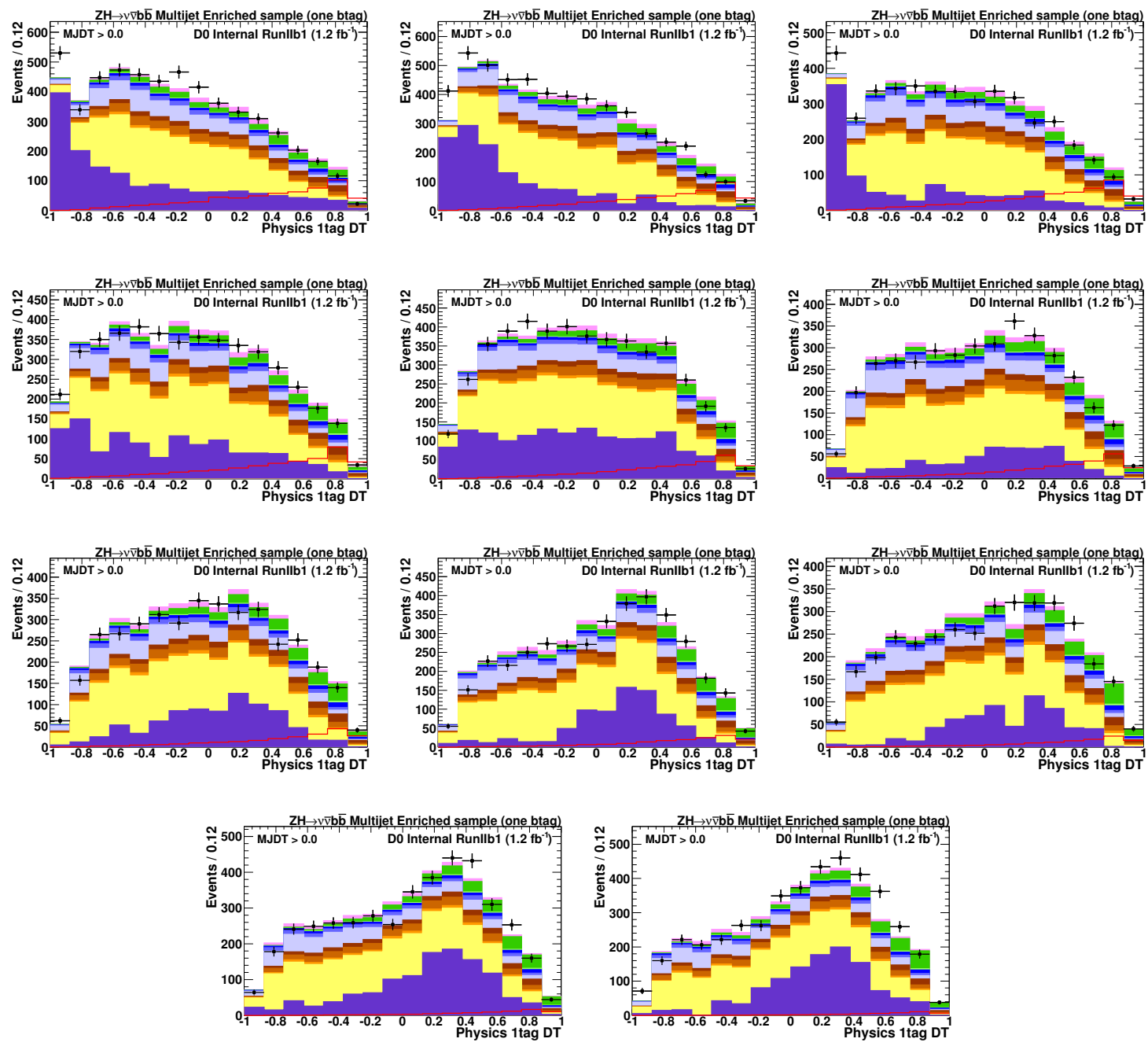

Figure G.9: Physics decision tree output for single tagged events in the multi-jet control sample after the MJ-DT cut. Decision trees are trained for different Higgs masses, $100 \mathrm{GeV}$ (top left) to $150 \mathrm{GeV}$ (bottom right) in $5 \mathrm{GeV}$ increments, in the 1tag sample Run IIb1 dataset. 

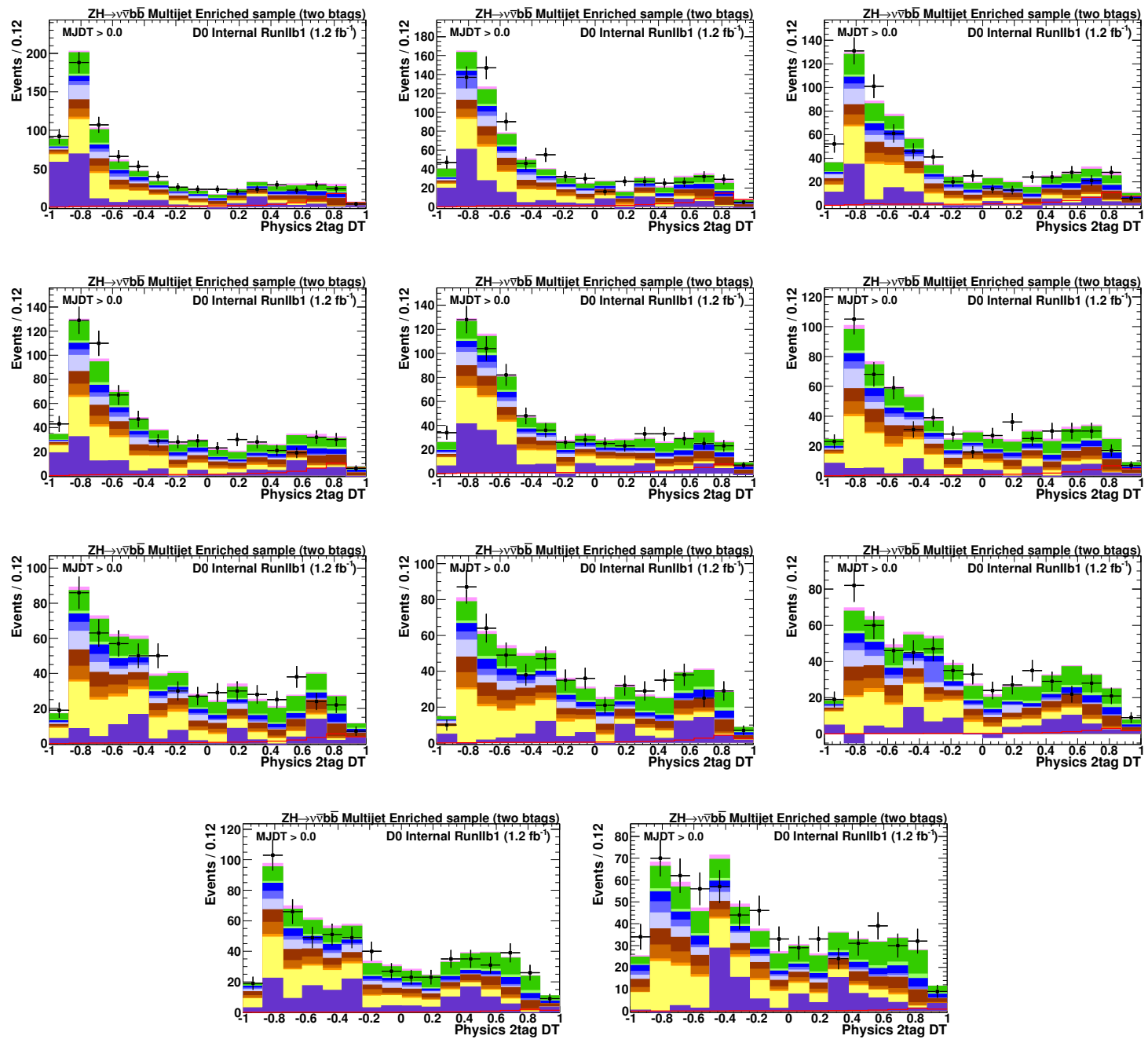

Figure G.10: Physics decision tree output for double tagged events in the multi-jet control sample after the MJ-DT cut. Decision trees are trained for different Higgs masses, $100 \mathrm{GeV}$ (top left) to $150 \mathrm{GeV}$ (bottom right) in $5 \mathrm{GeV}$ increments, in the 2 tag sample Run IIb1 dataset. 
Appendix $\mathbf{H}$

Additional Run IIb2-3 Multijet

Decision Tree Plots 

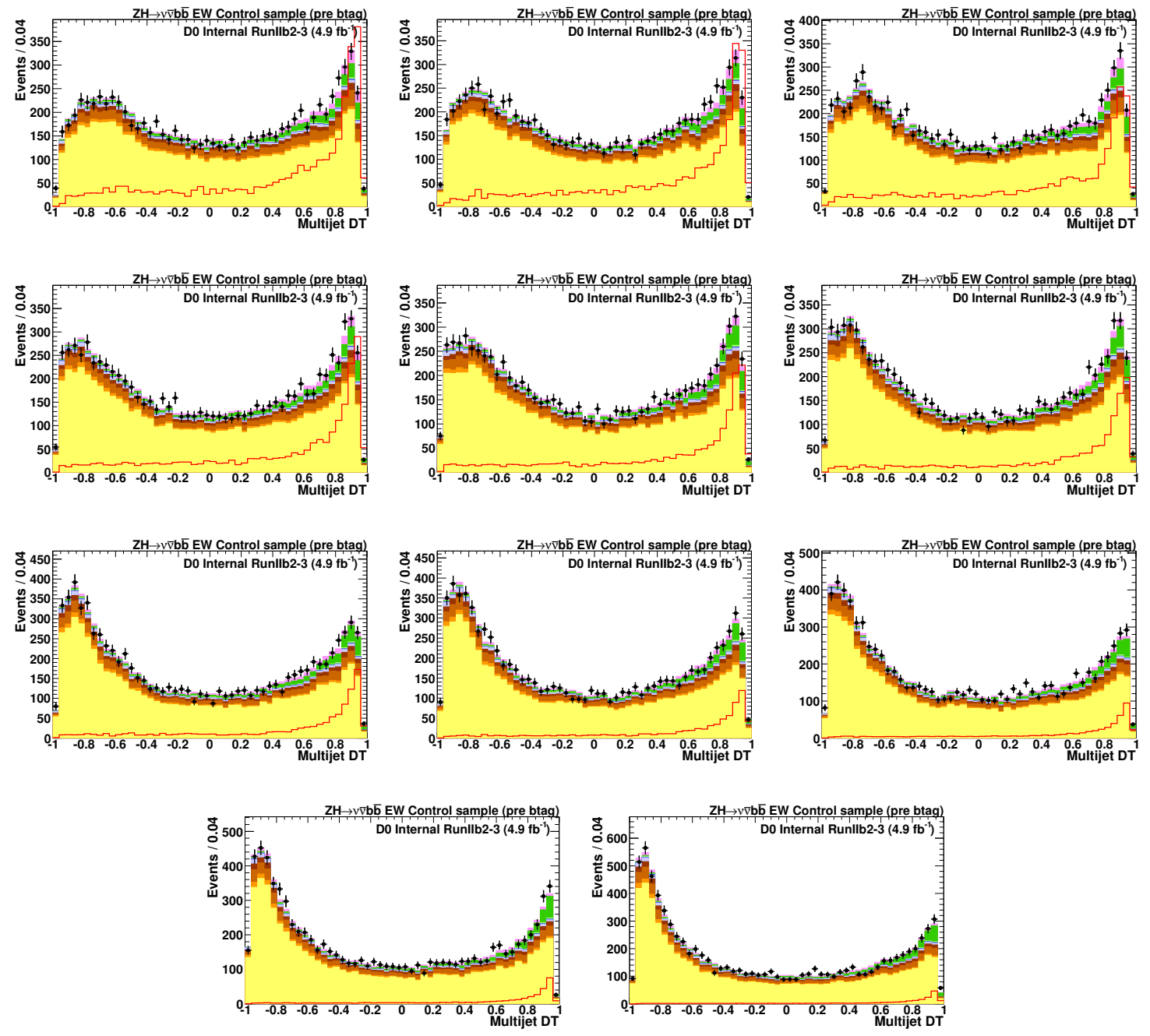

Figure H.1: Multijet decision tree output for pre b-tagged events in the electroweak control sample. Decision trees are trained for different Higgs masses, $100 \mathrm{GeV}$ (top left) to $150 \mathrm{GeV}$ (bottom right) in $5 \mathrm{GeV}$ increments, in the pre-tag sample Run IIb2-3 dataset. 

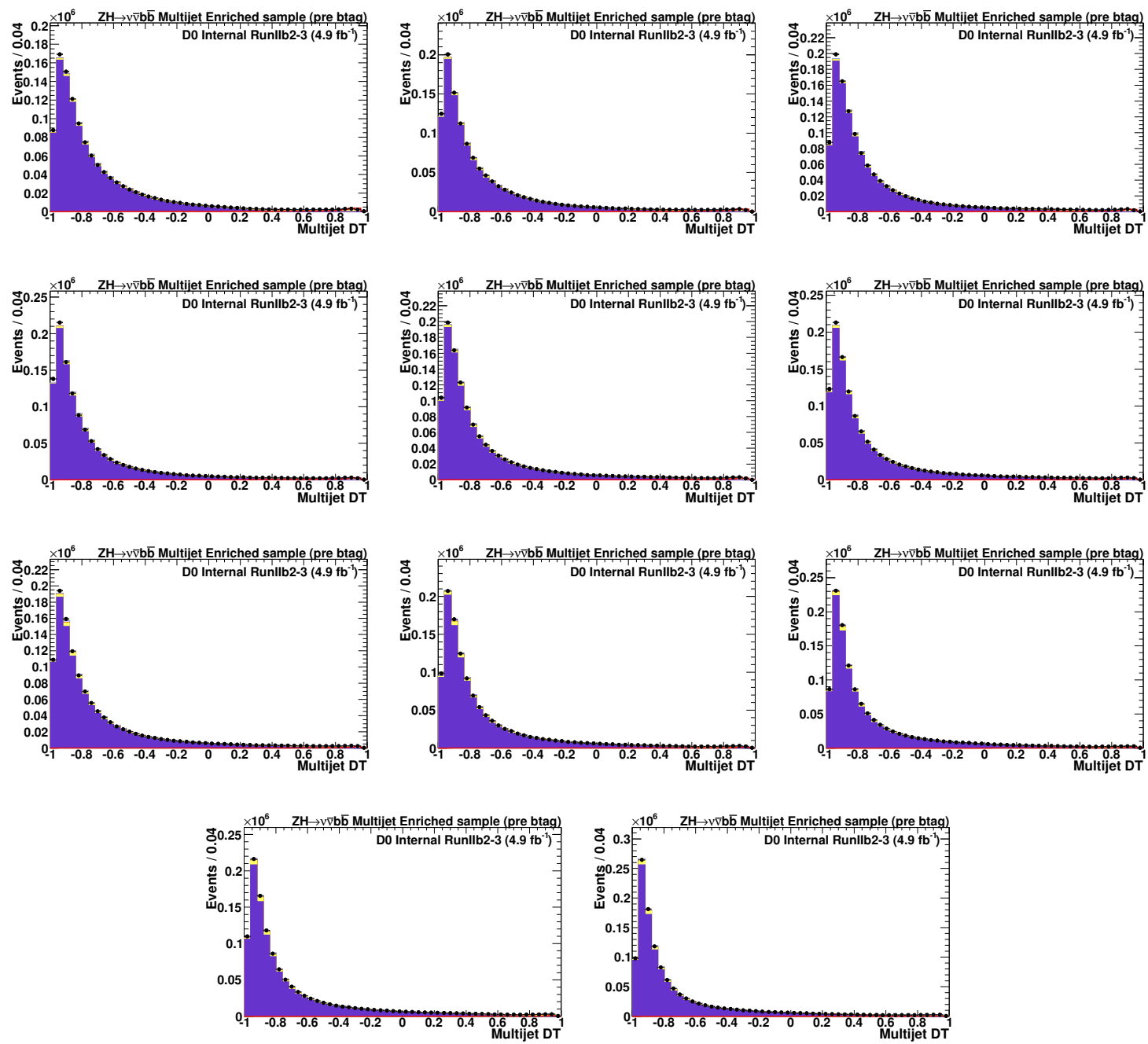

Figure H.2: Multijet decision tree output for pre b-tagged events in the multi-jet control sample. Decision trees are trained for different Higgs masses, $100 \mathrm{GeV}$ (top left) to $150 \mathrm{GeV}$ (bottom right) in $5 \mathrm{GeV}$ increments, in the pre-tag sample Run IIb2-3 dataset. 

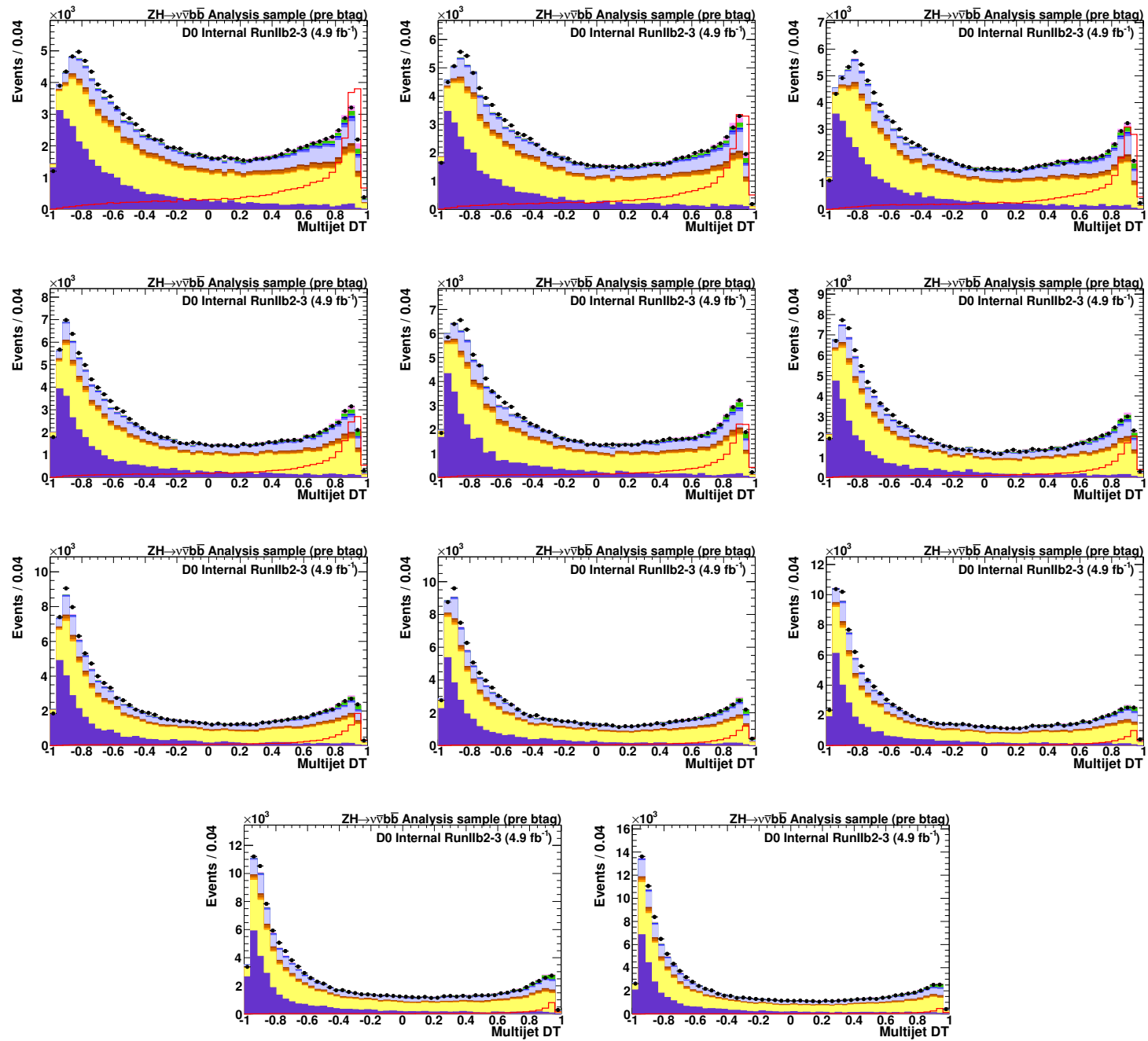

Figure H.3: Multijet decision tree output for pre b-tagged events. Decision trees are trained for different Higgs masses, $100 \mathrm{GeV}$ (top left) to $150 \mathrm{GeV}$ (bottom right) in 5 GeV increments, in the pre-tag sample Run IIb2-3 dataset. 

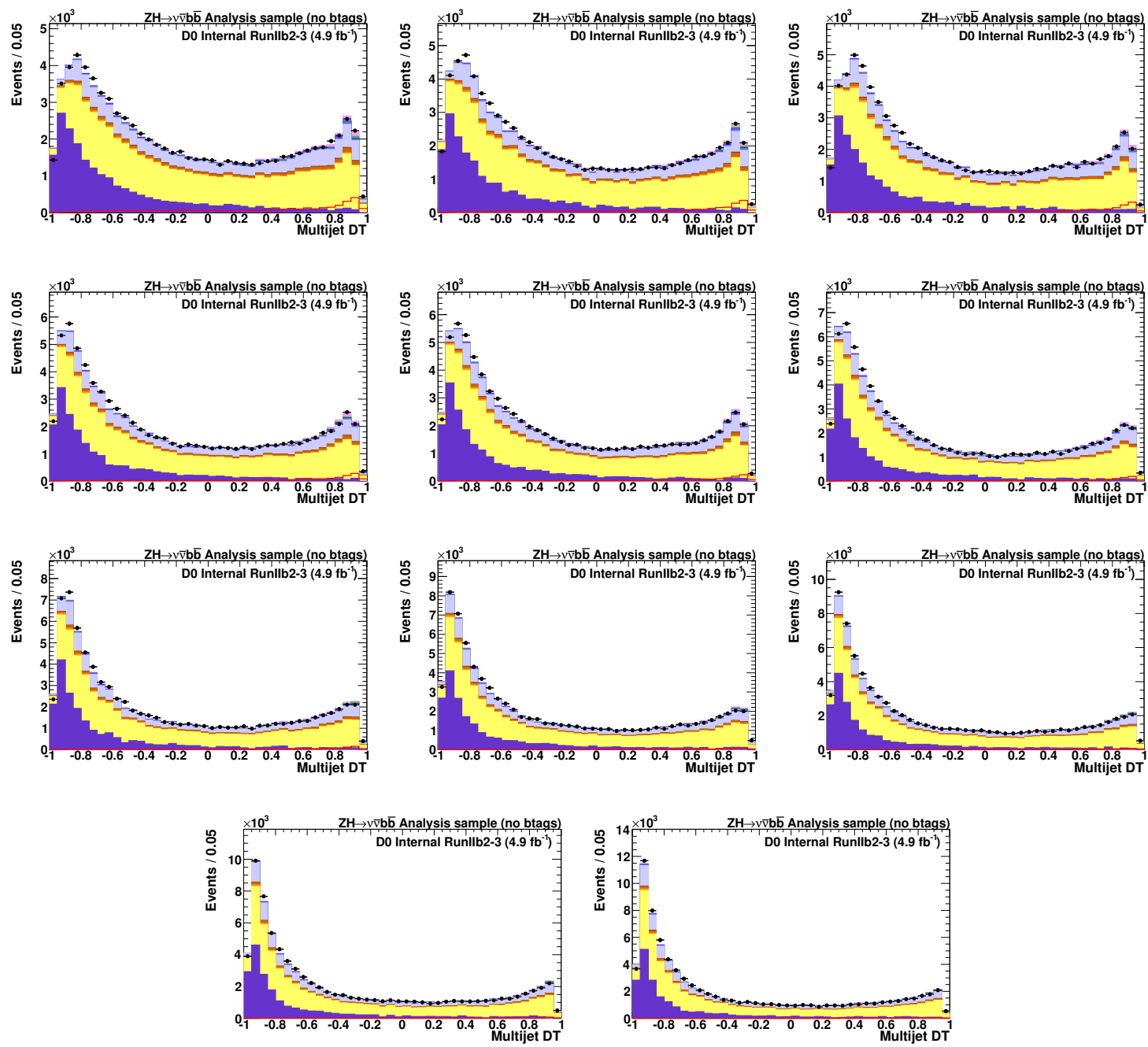

Figure H.4: Multijet decision tree output for zero tagged events. Decision trees are trained for different Higgs masses, $100 \mathrm{GeV}$ (top left) to $150 \mathrm{GeV}$ (bottom right) in 5 GeV increments, in the pre-tag sample Run IIb2-3 dataset. 

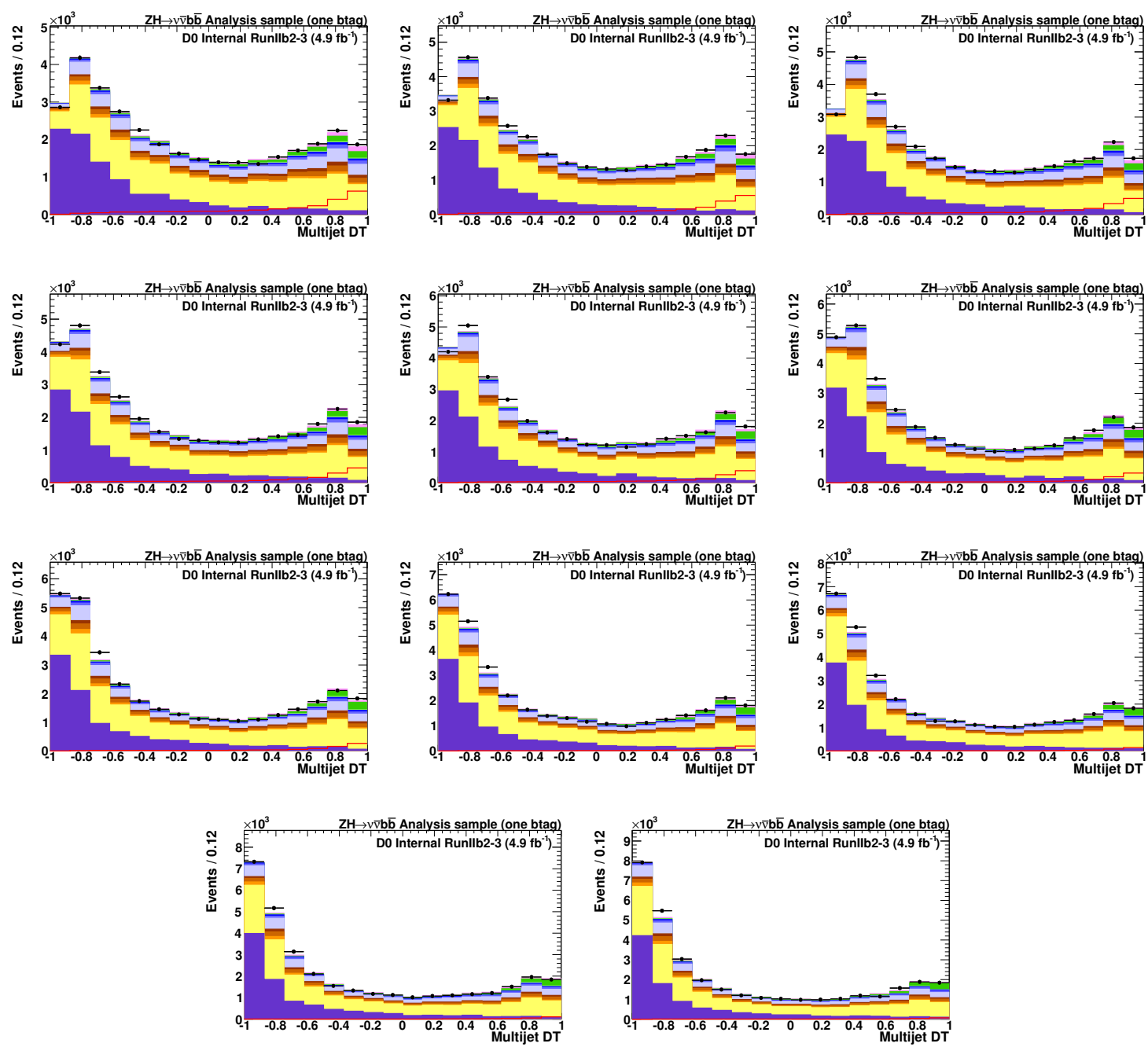

Figure H.5: Multijet decision tree output for single tagged events. Decision trees are trained for different Higgs masses, $100 \mathrm{GeV}$ (top left) to $150 \mathrm{GeV}$ (bottom right) in 5 GeV increments, in the pre-tag sample Run IIb2-3 dataset. 

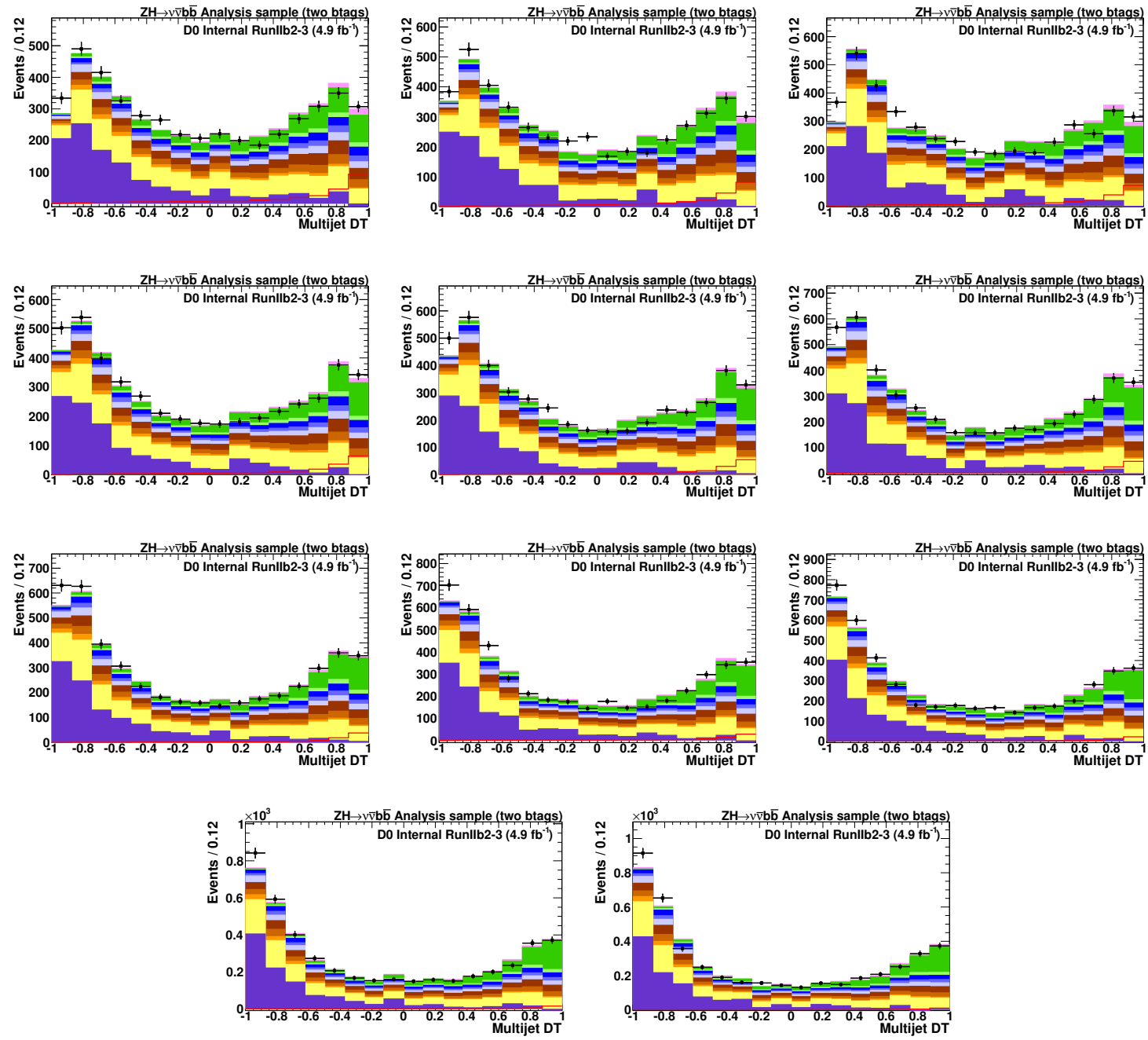

Figure H.6: Multijet decision tree output for double tagged events. Decision trees are trained for different Higgs masses, $100 \mathrm{GeV}$ (top left) to $150 \mathrm{GeV}$ (bottom right) in $5 \mathrm{GeV}$ increments, in the pre-tag sample Run IIb2-3 dataset. 


\section{H.1 Additional Run IIb2-3 Physics Decision Tree}

\section{Plots}
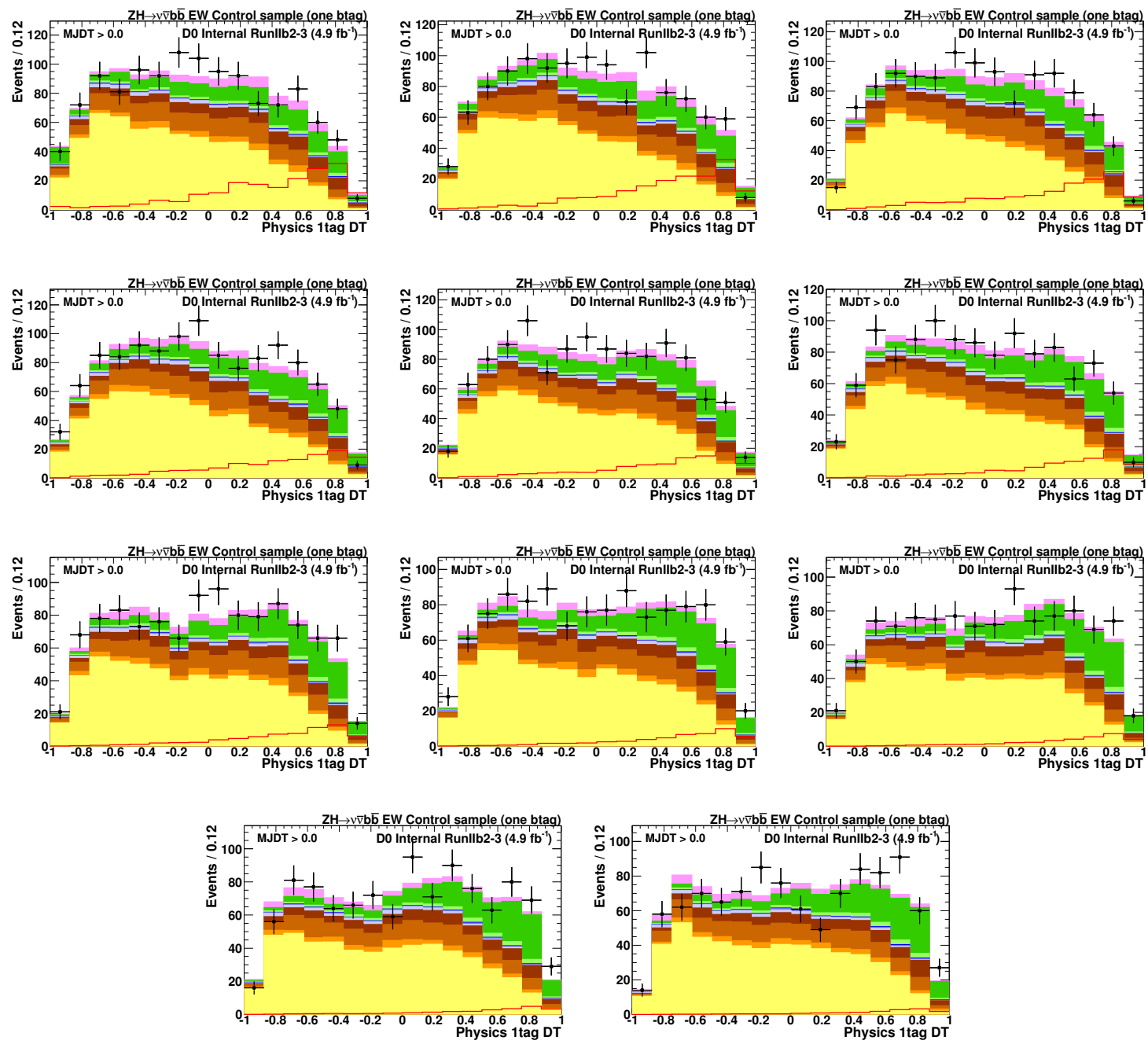

Figure H.7: Physics decision tree output for single tagged events in the electroweak control sample after the MJ-DT cut. Decision trees are trained for different Higgs masses, $100 \mathrm{GeV}$ (top left) to $150 \mathrm{GeV}$ (bottom right) in $5 \mathrm{GeV}$ increments, in the 1tag sample Run IIb2-3 dataset. 

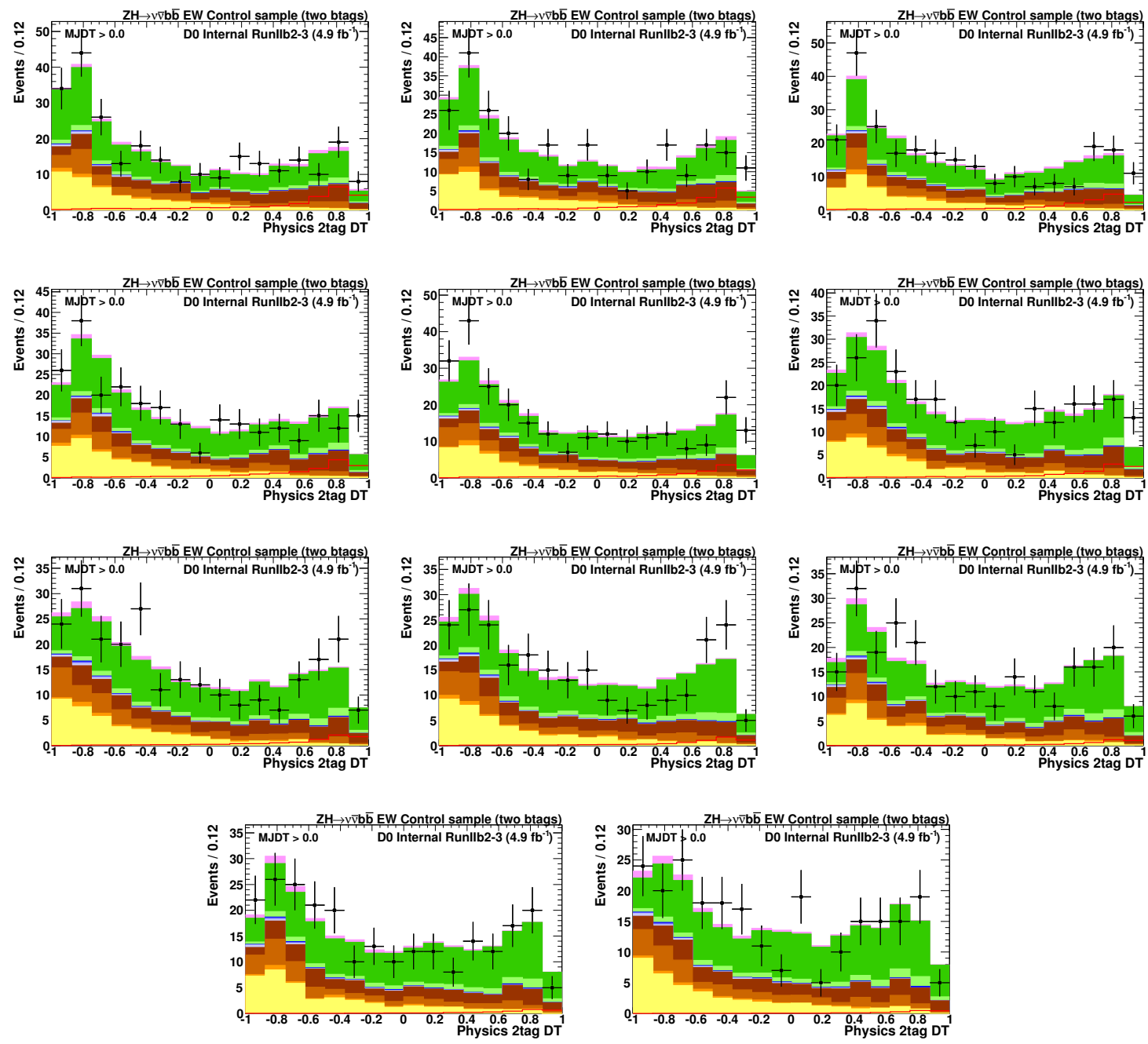

Figure H.8: Physics decision tree output for double tagged events in the electroweak control sample after the MJ-DT cut. Decision trees are trained for different Higgs masses, $100 \mathrm{GeV}$ (top left) to $150 \mathrm{GeV}$ (bottom right) in $5 \mathrm{GeV}$ increments, in the 2 tag sample Run IIb2-3 dataset. 

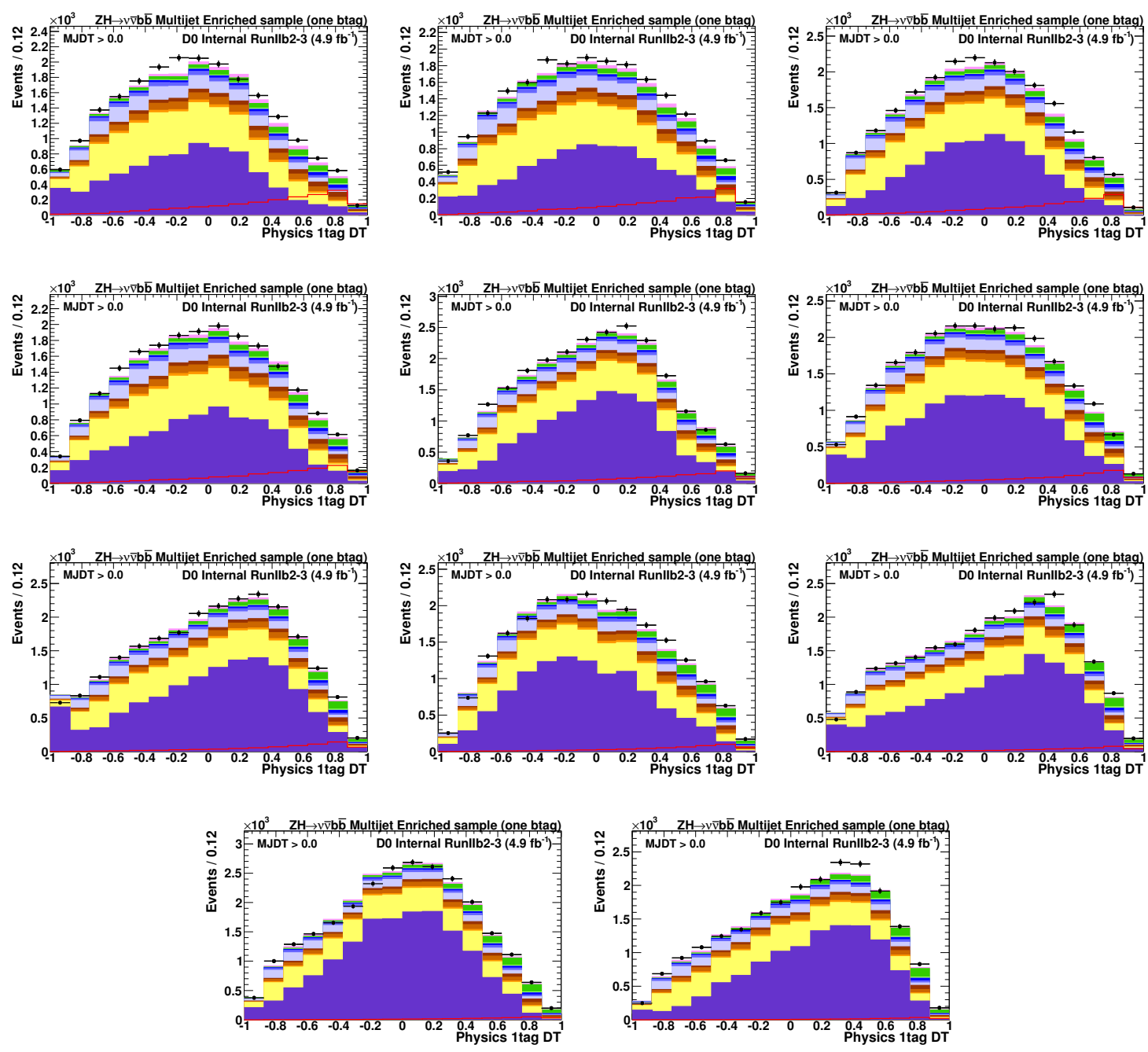

Figure H.9: Physics decision tree output for single tagged events in the multi-jet control sample after the MJ-DT cut. Decision trees are trained for different Higgs masses, $100 \mathrm{GeV}$ (top left) to $150 \mathrm{GeV}$ (bottom right) in $5 \mathrm{GeV}$ increments, in the 1tag sample Run IIb2-3 dataset. 

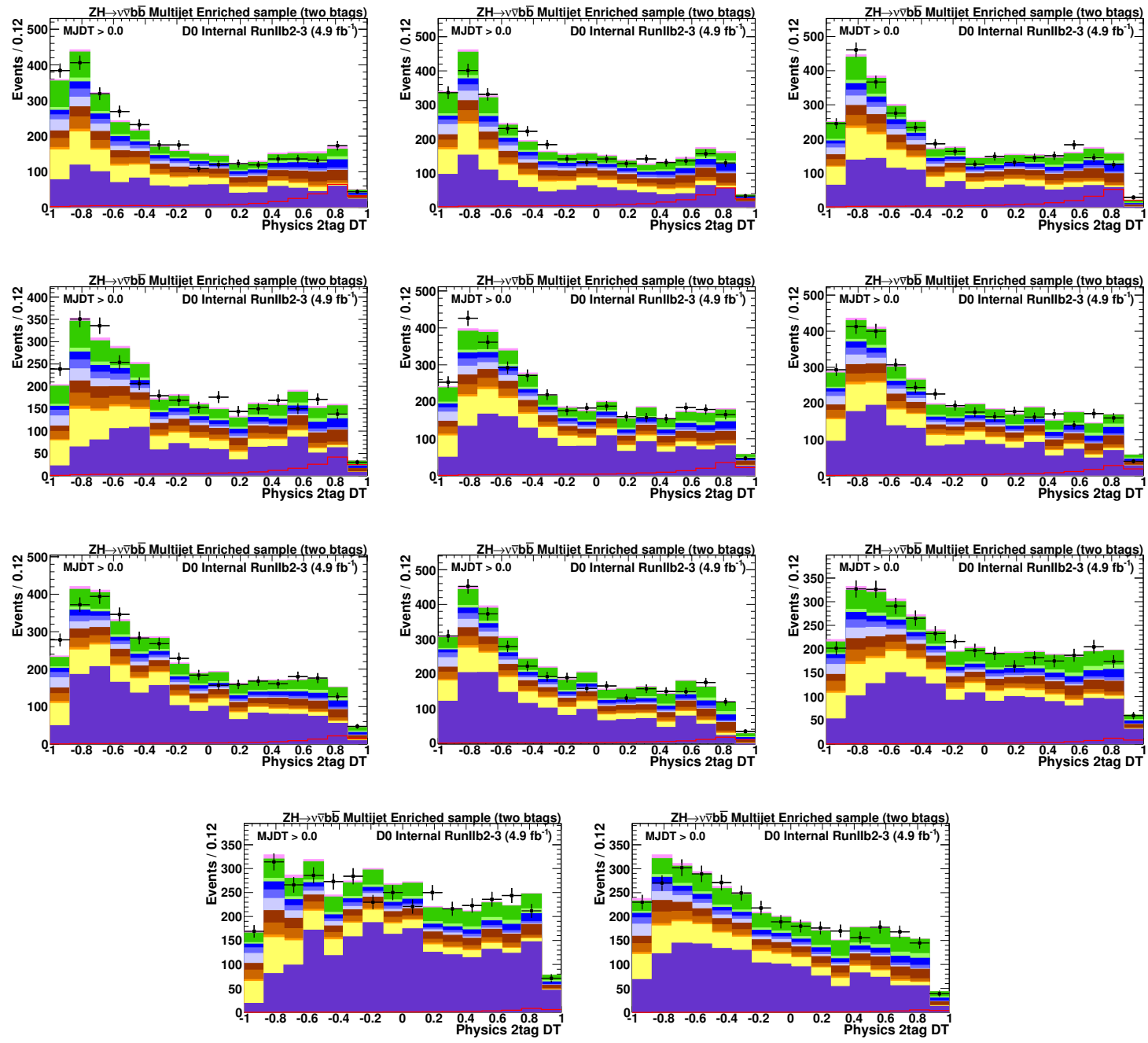

Figure H.10: Physics decision tree output for double tagged events in the multi-jet control sample after the MJ-DT cut. Decision trees are trained for different Higgs masses, $100 \mathrm{GeV}$ (top left) to $150 \mathrm{GeV}$ (bottom right) in $5 \mathrm{GeV}$ increments, in the 2 tag sample Run IIb2-3 dataset. 
Appendix I

Shape Systematics with respect to the BDT for RunIIb1 single tag sample 

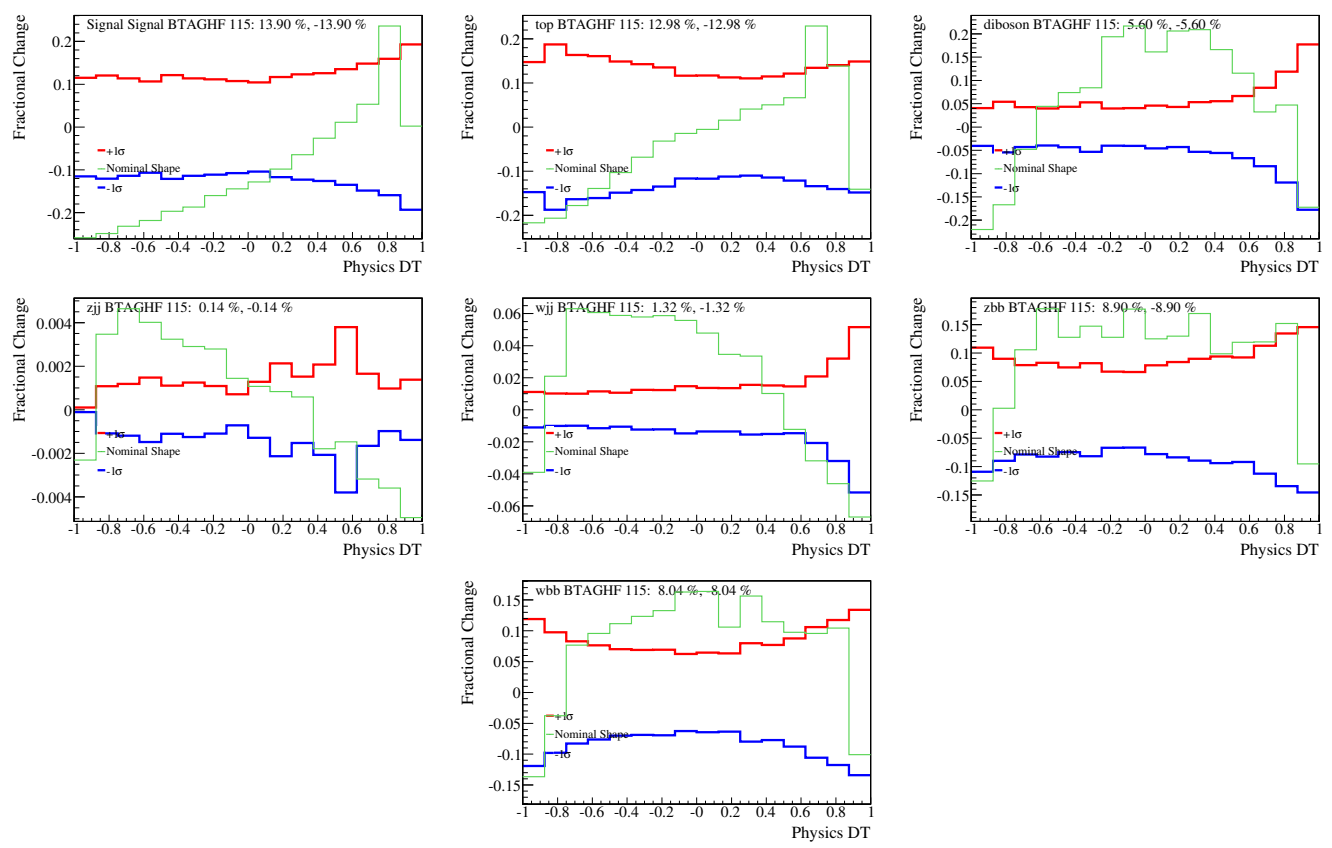

Figure I.1: Heavy flavor b-Tagging rate relative shape dependent systematic errors with respect to the nominal boosted decision tree discriminant in RunIIb1 in the one tag sample for the signal and background samples (as specified in the top left of each plot). The nominal shape for the given sample is shown in green and \pm $1 \sigma$ fractional change in red/blue. 

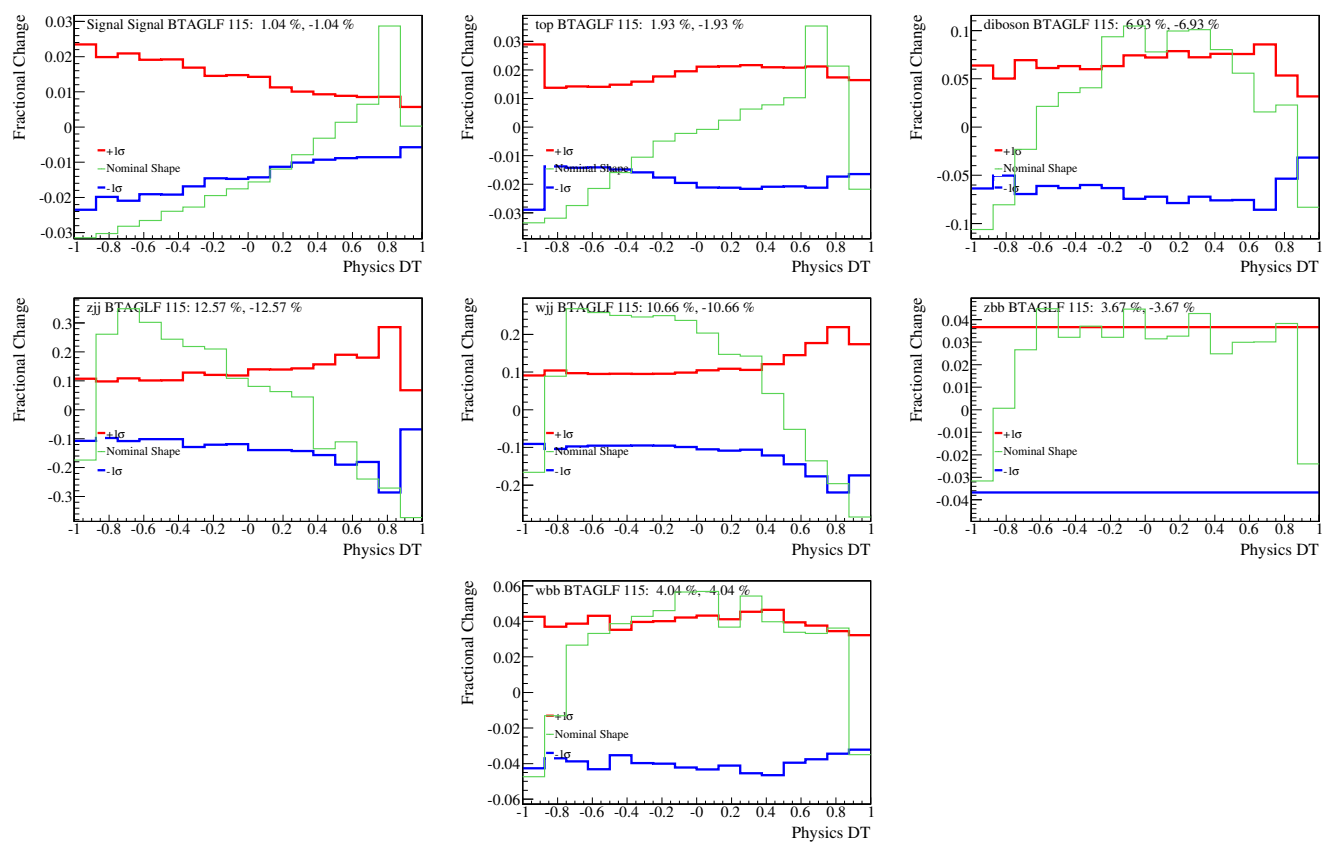

Figure I.2: Light flavor b-Tagging rate relative shape dependent systematic errors with respect to the nominal boosted decision tree discriminant in RunIIb1 in the one tag sample for the signal and background samples (as specified in the top left of each plot). The nominal shape for the given sample is shown in green and $\pm 1 \sigma$ fractional change in red/blue. 

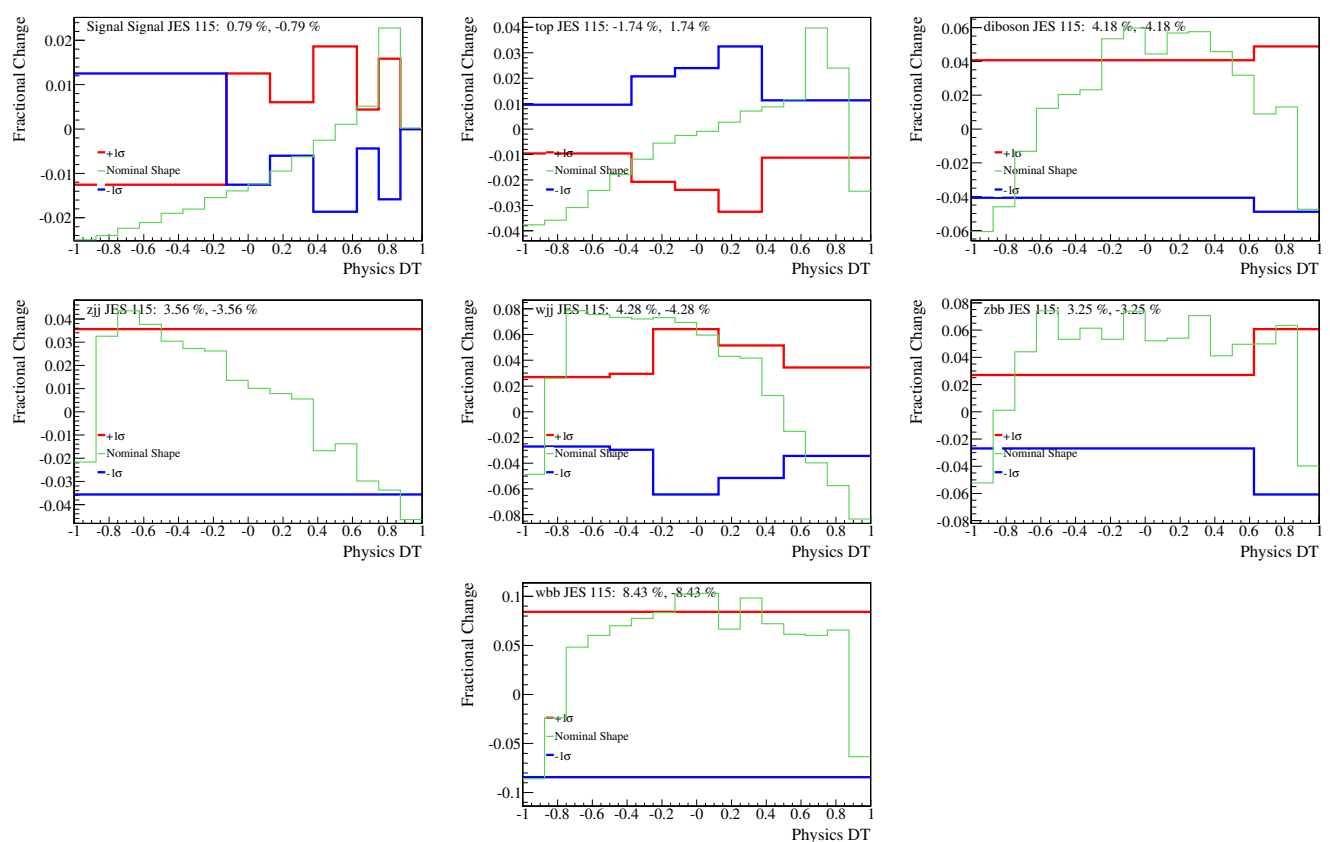

Figure I.3: Jet energy scale relative shape dependent systematic errors with respect to the nominal boosted decision tree discriminant in RunIIb1 in the one tag sample for the signal and background samples (as specified in the top left of each plot). The nominal shape for the given sample is shown in green and $\pm 1 \sigma$ fractional change in red/blue. 

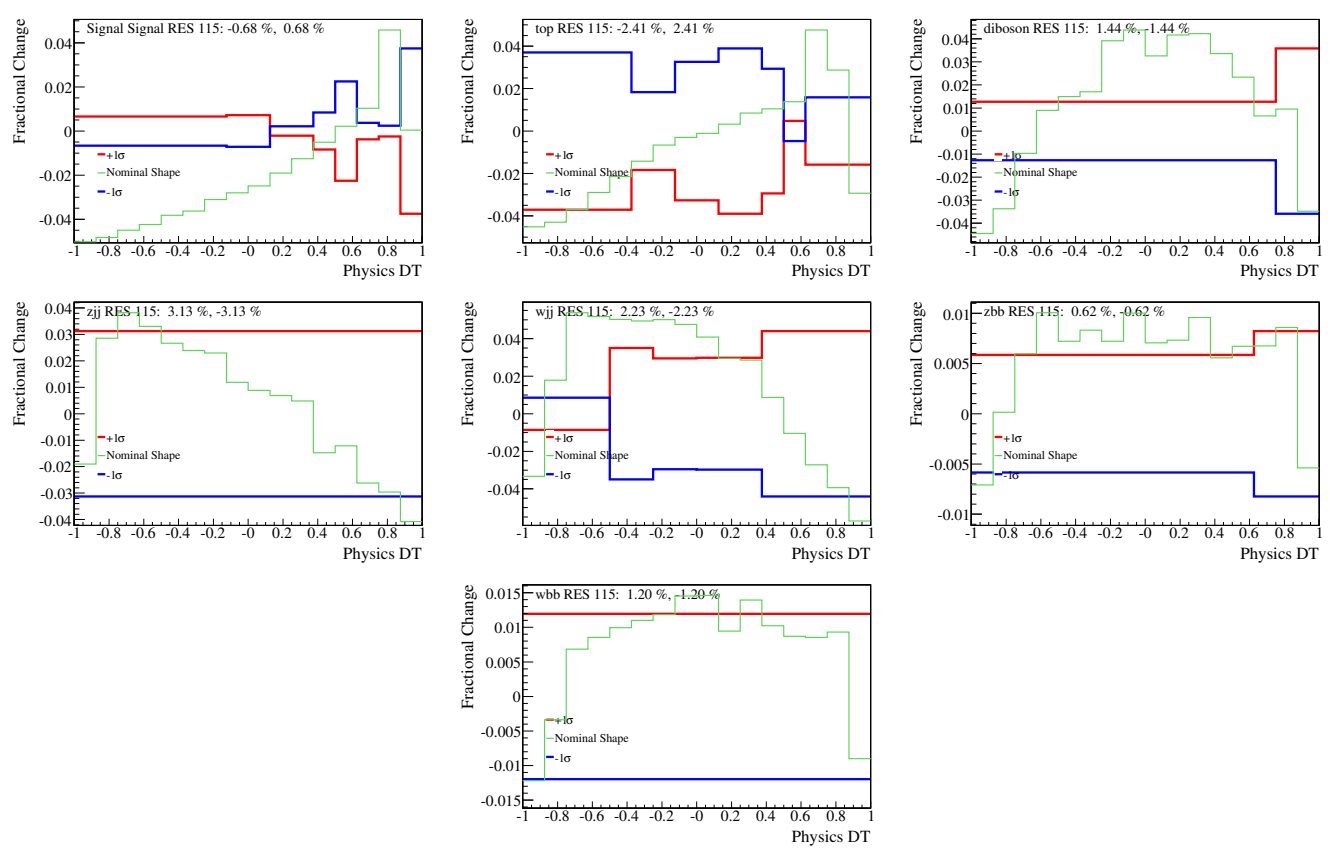

Figure I.4: Jet energy resolution relative shape dependent systematic errors with respect to the nominal boosted decision tree discriminant in RunIIb1 in the one tag sample for the signal and background samples (as specified in the top left of each plot). The nominal shape for the given sample is shown in green and $\pm 1 \sigma$ fractional change in red/blue. 

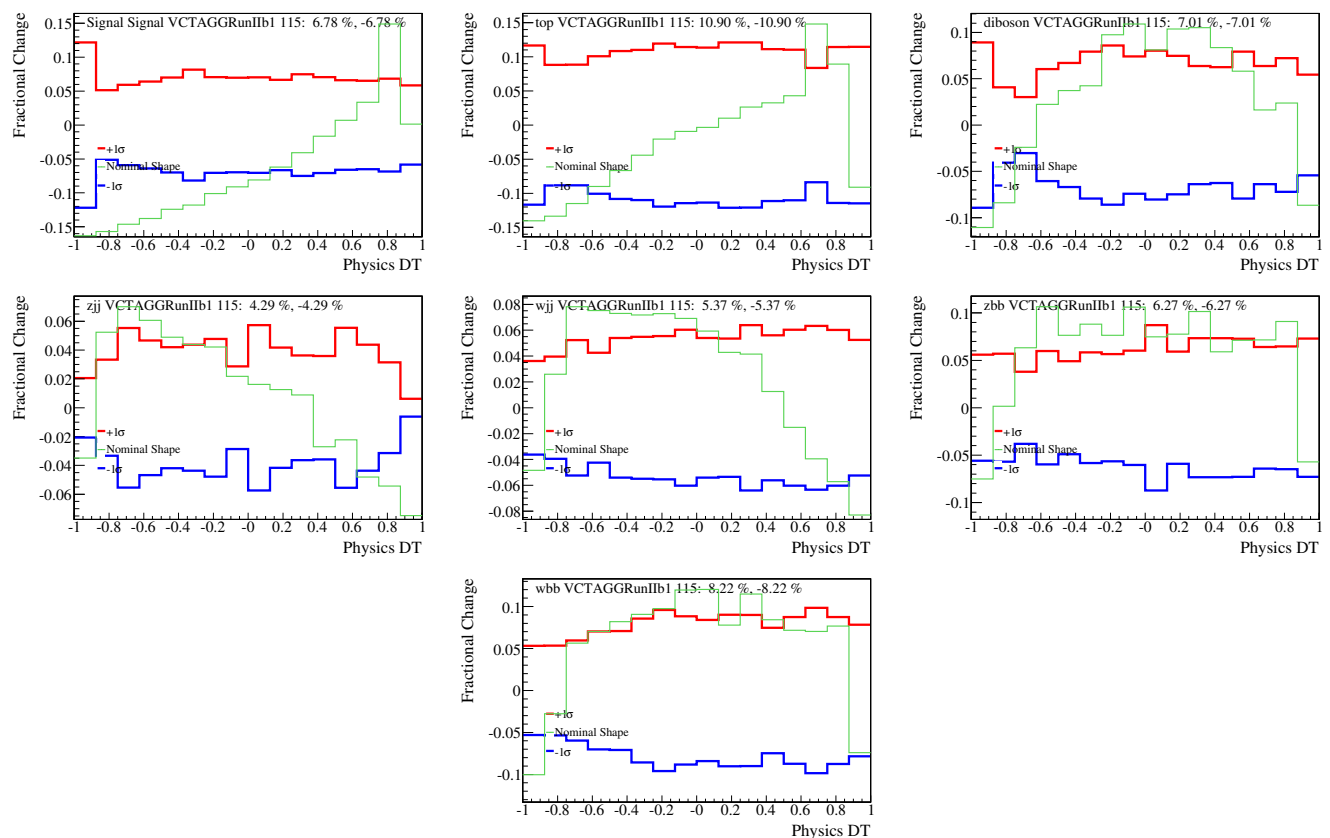

Figure I.5: Direct taggability and Vertex Confirmation relative shape dependent systematic errors with respect to the nominal boosted decision tree discriminant in RunIIb1 in the one tag sample for the signal and background samples (as specified in the top left of each plot). The nominal shape for the given sample is shown in green and $\pm 1 \sigma$ fractional change in red/blue. 

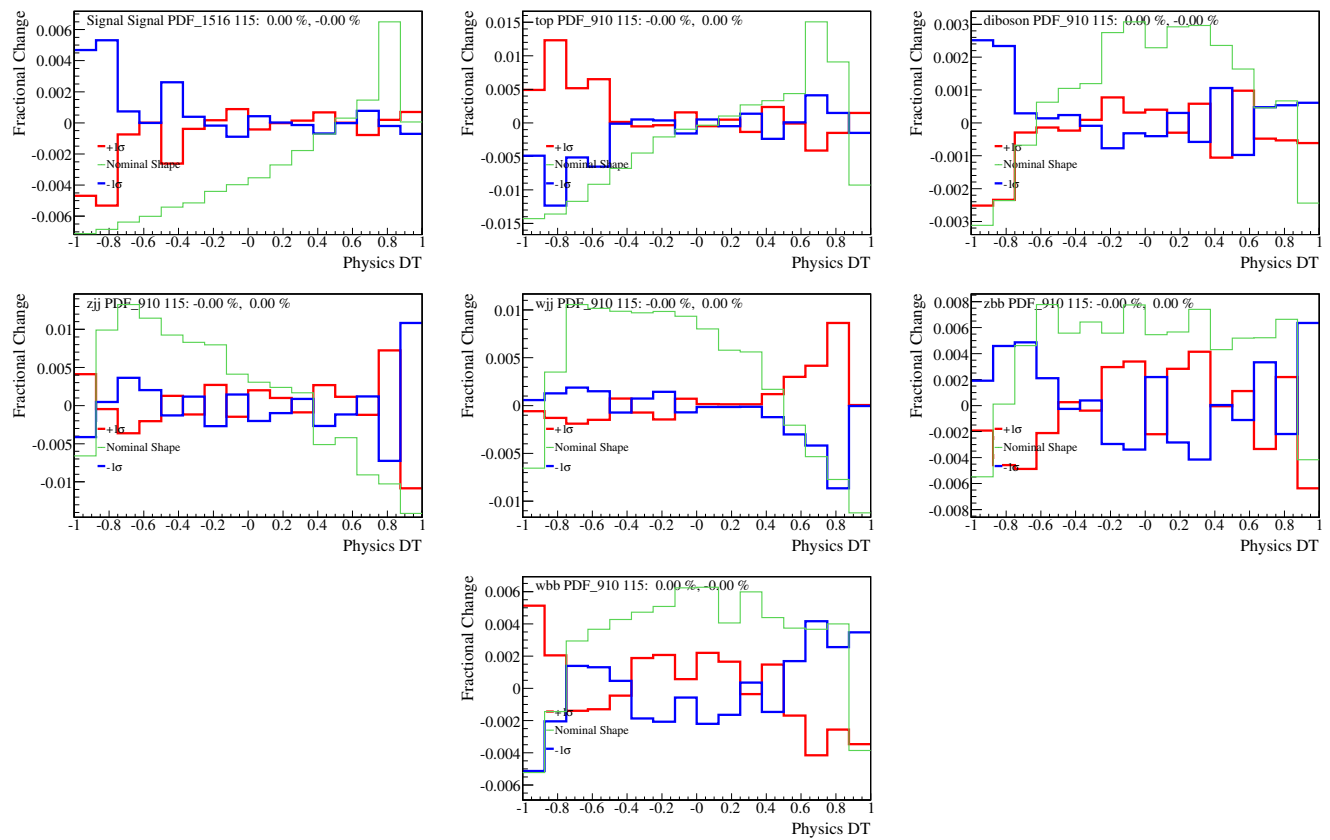

Figure I.6: PDF relative shape dependent systematic errors with respect to the nominal boosted decision tree discriminant in RunIIb1 in the one tag sample for the signal and background samples (as specified in the top left of each plot). (4 of 4 . The nominal shape for the given sample is shown in green and $\pm 1 \sigma$ fractional change in red/blue.
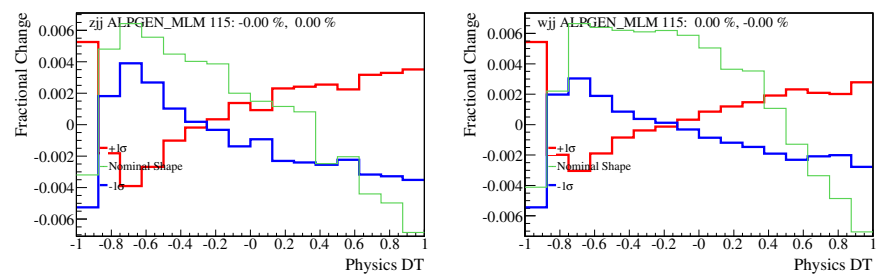

Figure I.7: ALPGEN MLM relative shape dependent systematic errors with respect to the nominal boosted decision tree discriminant in RunIIb1 in the one tag sample for the signal and background samples (as specified in the top left of each plot). The nominal shape for the given sample is shown in green and $\pm 1 \sigma$ fractional change in red/blue. 

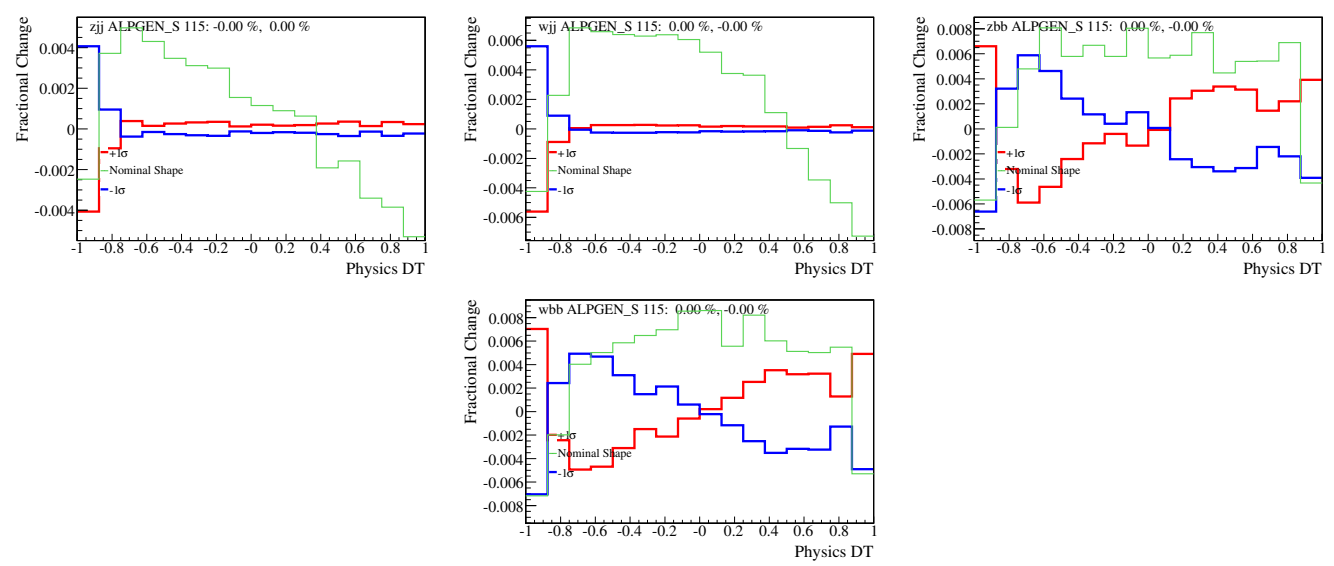

Figure I.8: ALPGEN Scale relative shape dependent systematic errors with respect to the nominal boosted decision tree discriminant in RunIIb1 in the one tag sample for the signal and background samples (as specified in the top left of each plot). The nominal shape for the given sample is shown in green and $\pm 1 \sigma$ fractional change in red/blue.
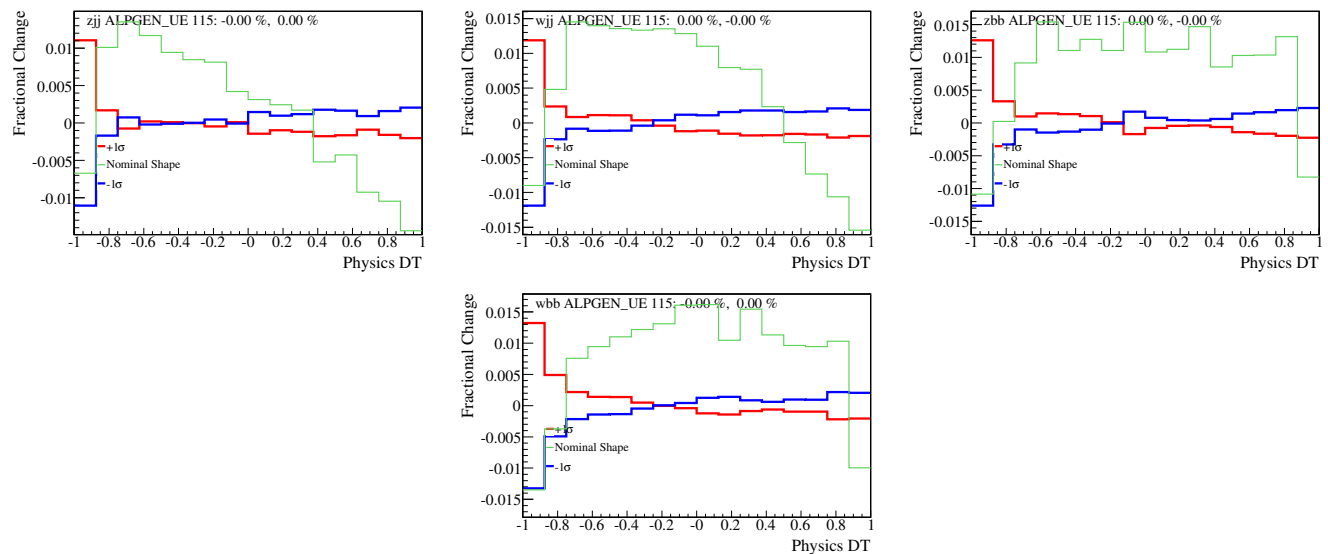

Physics DT

Figure I.9: ALPGEN Underlying Event relative shape dependent systematic errors with respect to the nominal boosted decision tree discriminant in RunIIb1 in the one tag sample for the signal and background samples (as specified in the top left of each plot). The nominal shape for the given sample is shown in green and \pm $1 \sigma$ fractional change in red/blue. 

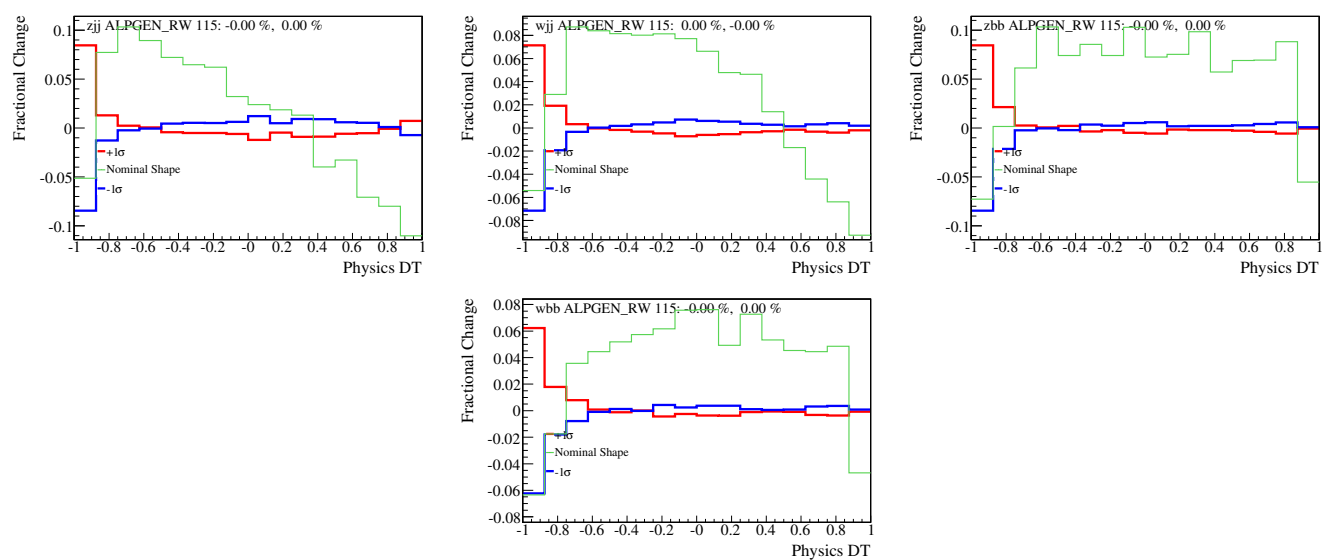

Physics DT

Figure I.10: ALPGEN Angular Reweighting relative shape dependent systematic errors with respect to the nominal boosted decision tree discriminant in RunIIb1 in the one tag sample for the signal and background samples (as specified in the top left of each plot). The nominal shape for the given sample is shown in green and $\pm 1 \sigma$ fractional change in red/blue.
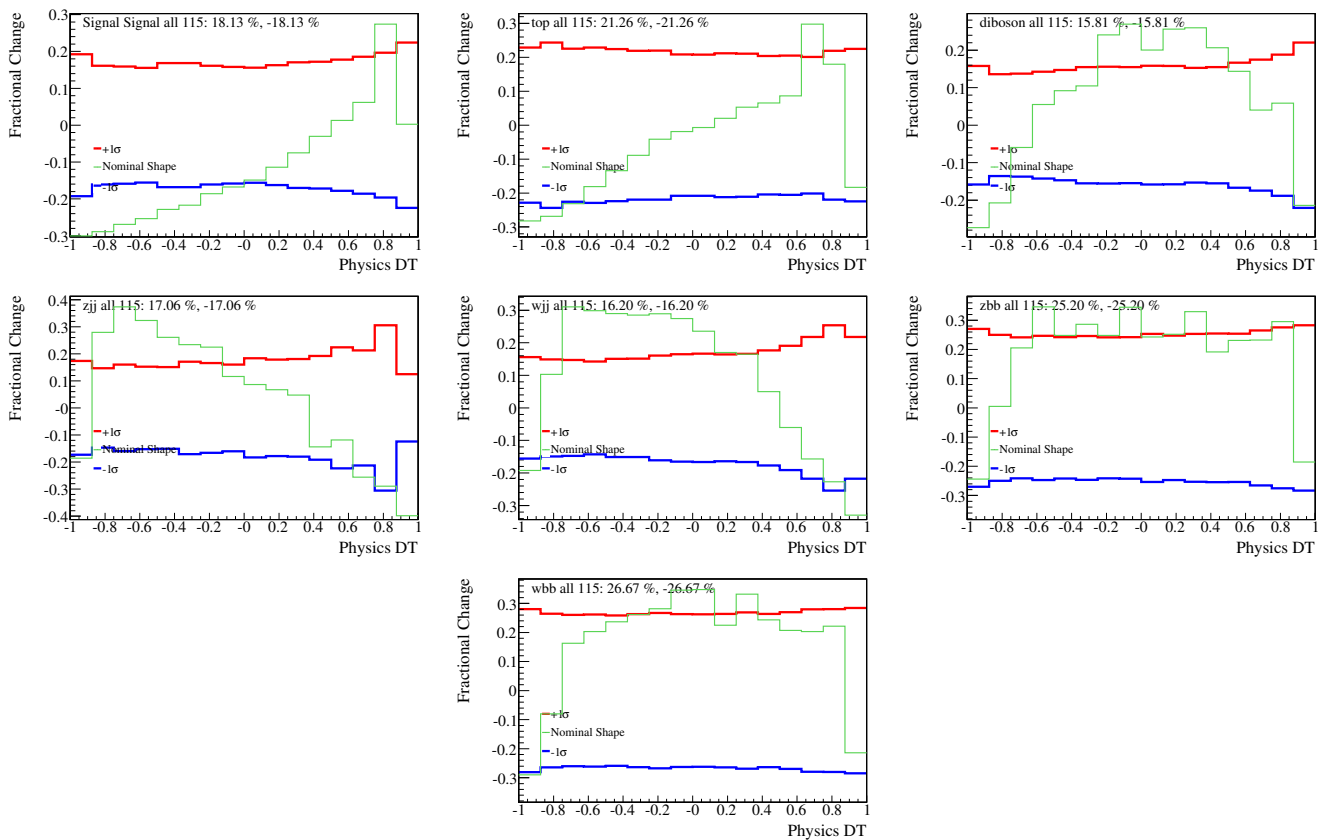

Figure I.11: All systematic errors with respect to the nominal boosted decision tree discriminant in RunIIb1 in the one tag sample for the signal and background samples (as specified in the top left of each plot). The nominal shape for the given sample is shown in green and $\pm 1 \sigma$ fractional change in red/blue. 
Appendix J

Shape Systematics with respect to the BDT for RunIIb1 double tag sample 

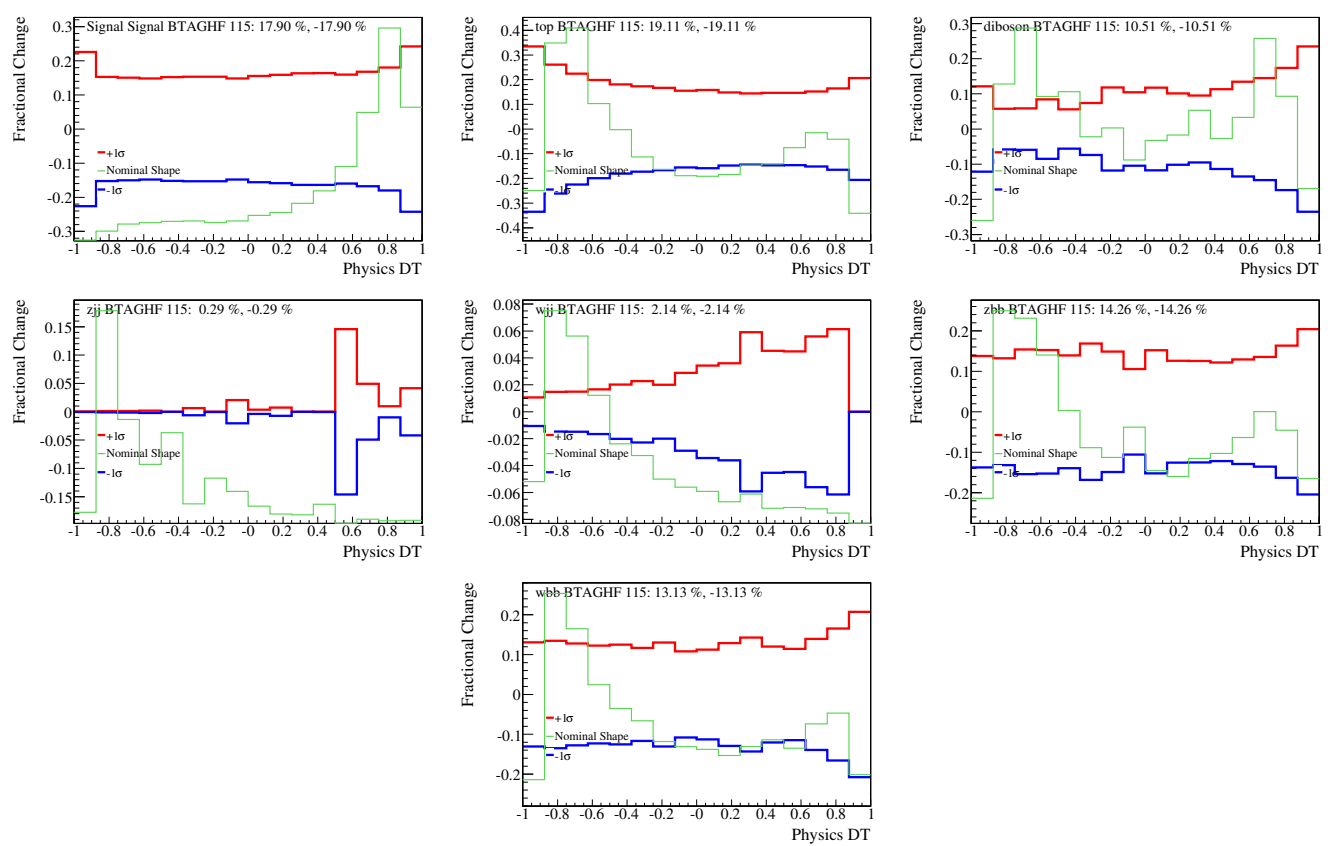

Figure J.1: Heavy flavor b-Tagging rate relative shape dependent systematic errors with respect to the nominal boosted decision tree discriminant in RunIIb1 in the two tag sample for the signal and background samples (as specified in the top left of each plot). The nominal shape for the given sample is shown in green and \pm $1 \sigma$ fractional change in red/blue. 

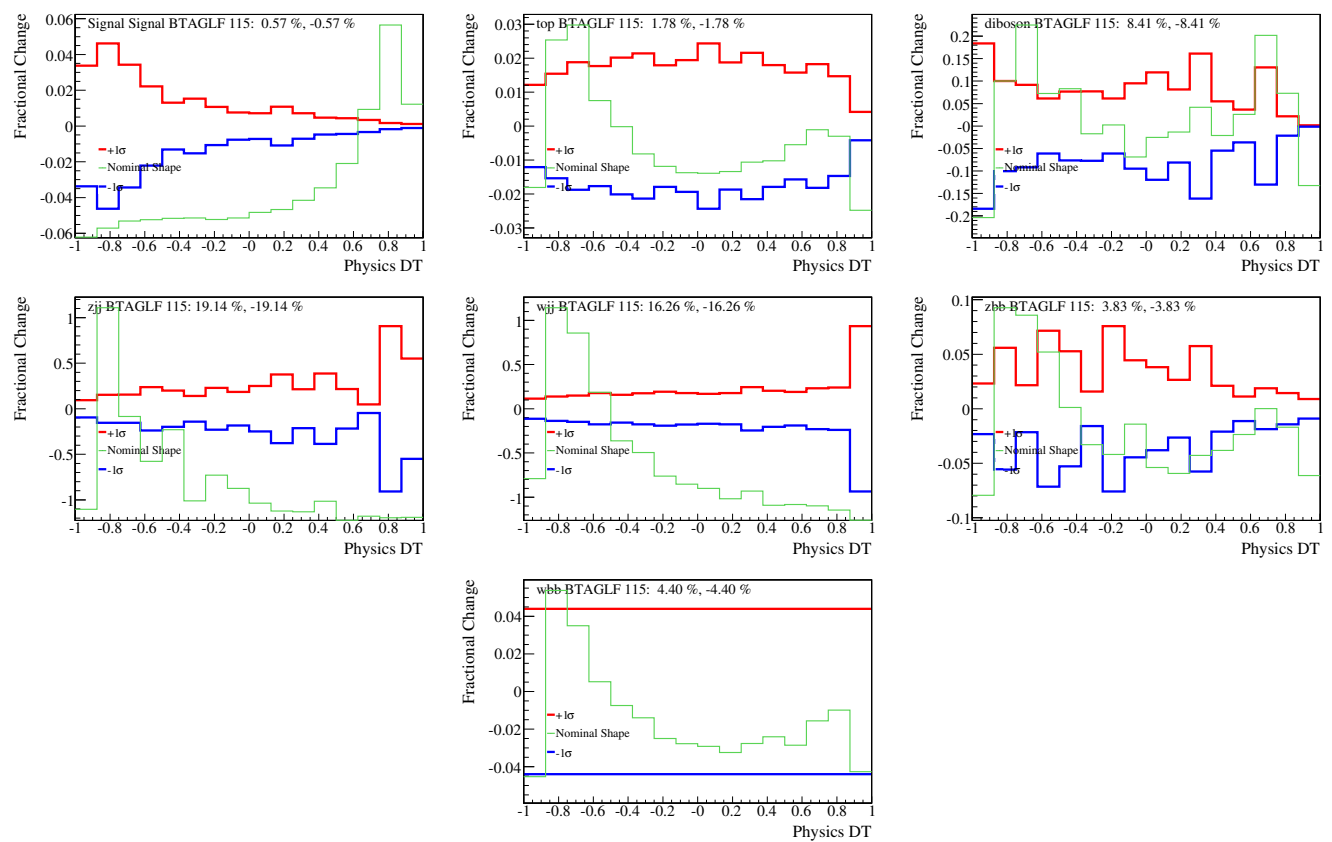

Figure J.2: Light flavor b-Tagging rate relative shape dependent systematic errors with respect to the nominal boosted decision tree discriminant in RunIIb1 in the two tag sample for the signal and background samples (as specified in the top left of each plot). The nominal shape for the given sample is shown in green and \pm $1 \sigma$ fractional change in red/blue. 

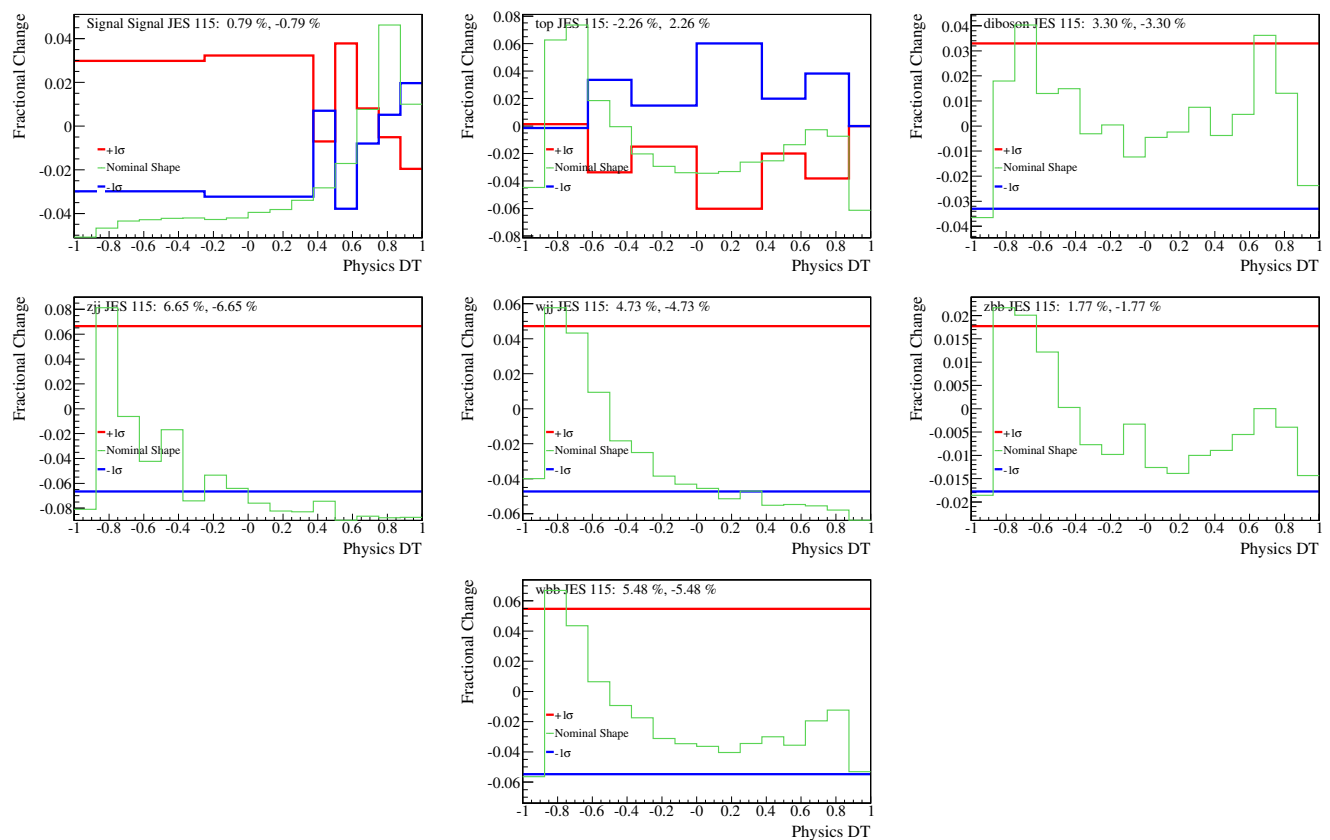

Figure J.3: Jet energy scale relative shape dependent systematic errors with respect to the nominal boosted decision tree discriminant in RunIIb1 in the two tag sample for the signal and background samples (as specified in the top left of each plot). The nominal shape for the given sample is shown in green and $\pm 1 \sigma$ fractional change in red/blue. 

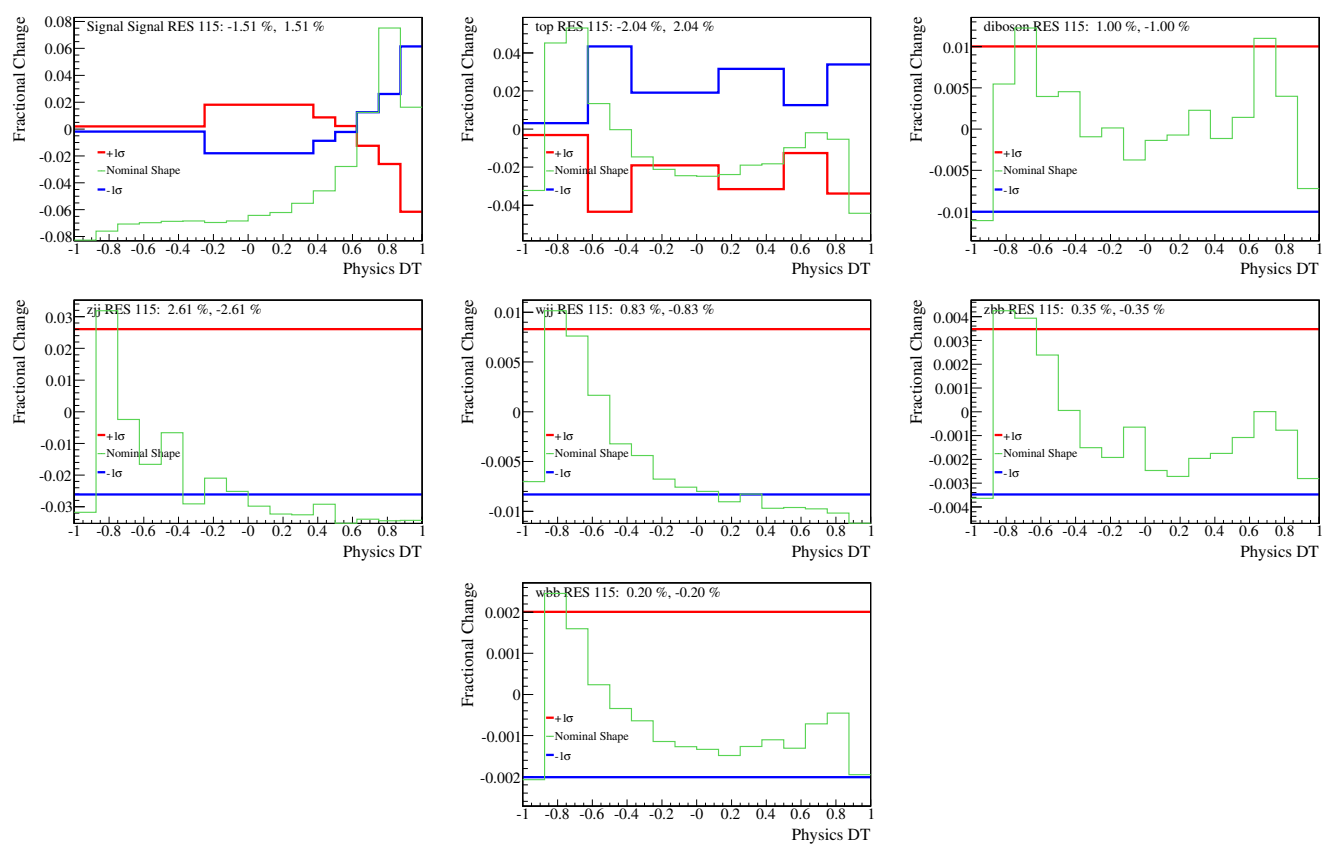

Figure J.4: Jet energy resolution relative shape dependent systematic errors with respect to the nominal boosted decision tree discriminant in RunIIb1 in the two tag sample for the signal and background samples (as specified in the top left of each plot). The nominal shape for the given sample is shown in green and \pm $1 \sigma$ fractional change in red/blue. 

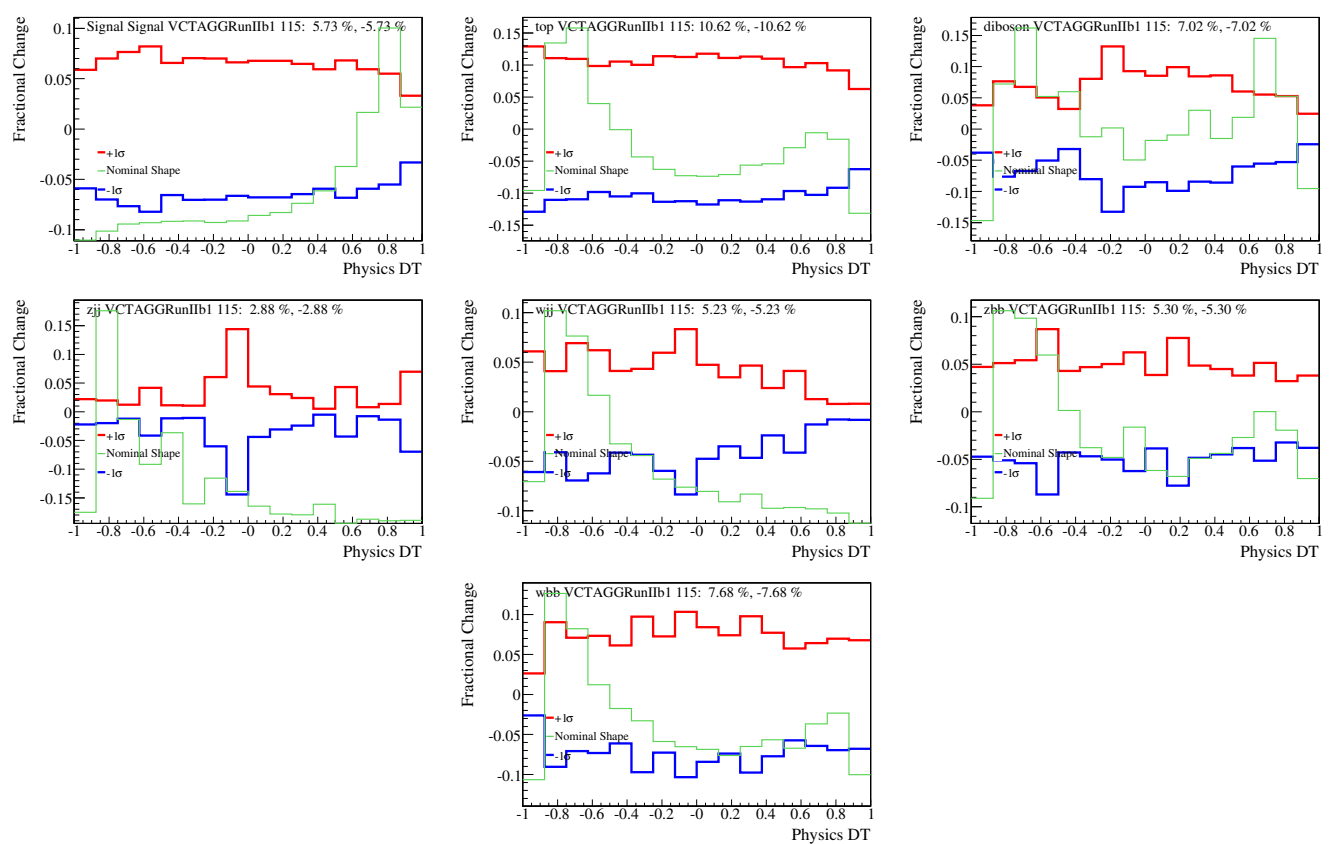

Figure J.5: Direct taggability and Vertex Confirmation relative shape dependent systematic errors with respect to the nominal boosted decision tree discriminant in RunIIb1 in the two tag sample for the signal and background samples (as specified in the top left of each plot). The nominal shape for the given sample is shown in green and $\pm 1 \sigma$ fractional change in red/blue. 

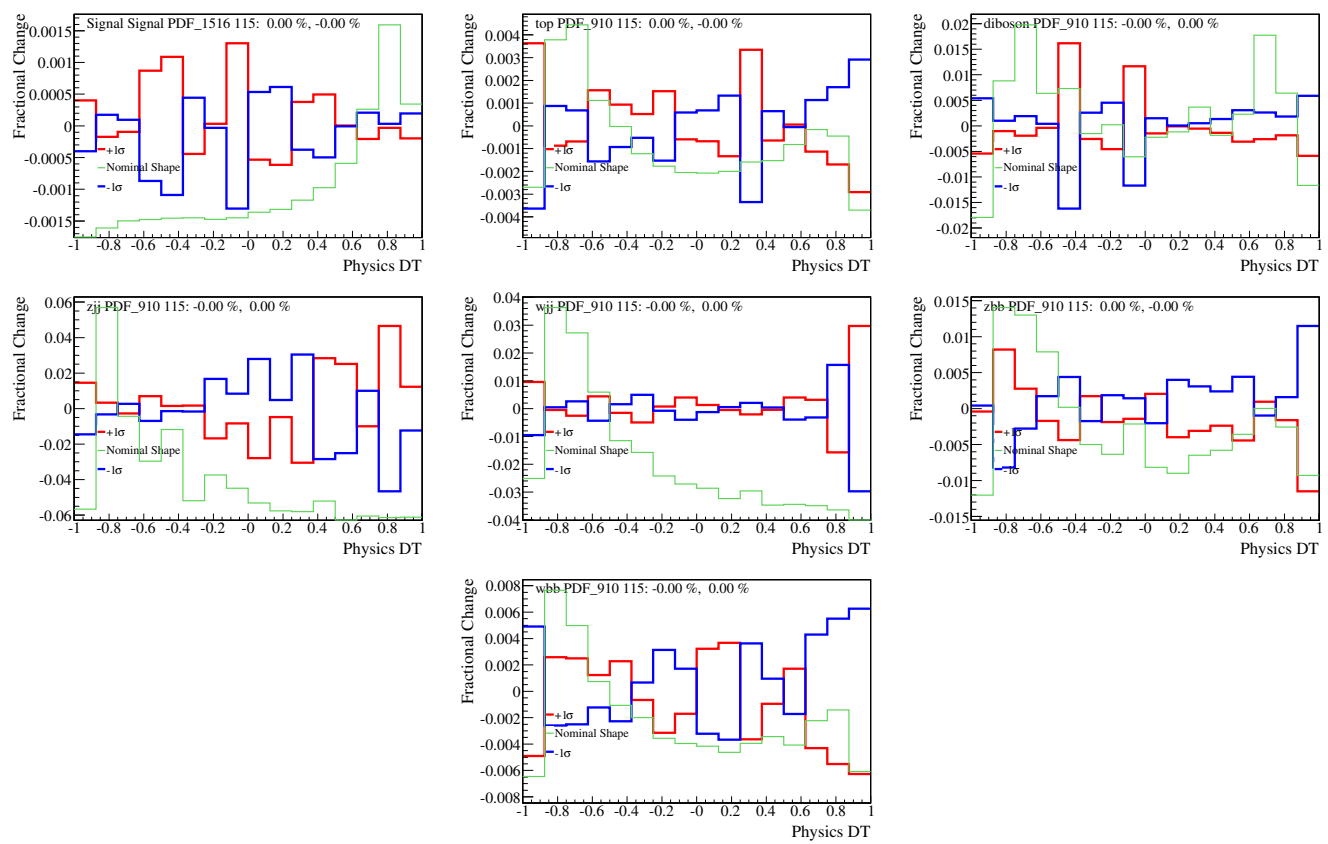

Figure J.6: PDF relative shape dependent systematic errors with respect to the nominal boosted decision tree discriminant in RunIIb1 in the two tag sample for the signal and background samples (as specified in the top left of each plot). (4 of 4. The nominal shape for the given sample is shown in green and $\pm 1 \sigma$ fractional change in red/blue.
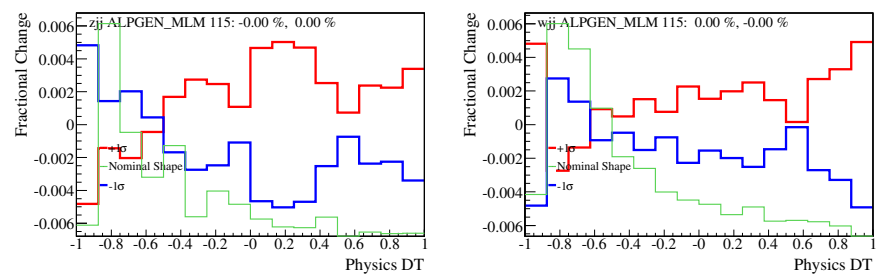

Figure J.7: ALPGEN MLM relative shape dependent systematic errors with respect to the nominal boosted decision tree discriminant in RunIIb1 in the two tag sample for the signal and background samples (as specified in the top left of each plot). The nominal shape for the given sample is shown in green and $\pm 1 \sigma$ fractional change in red/blue. 

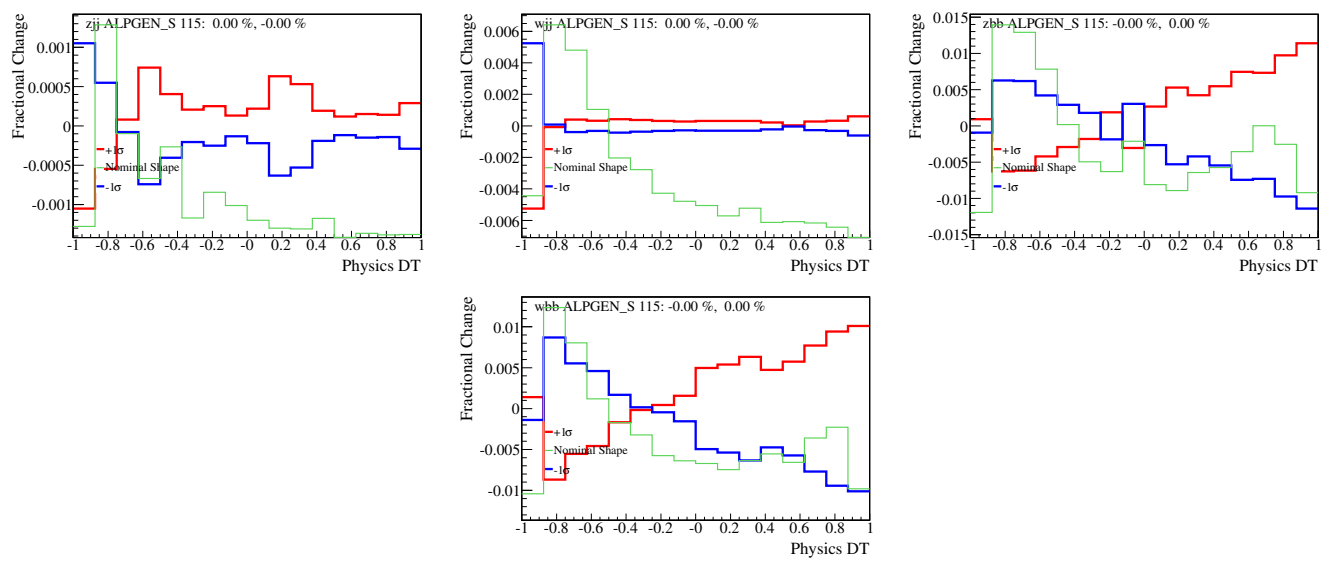

Figure J.8: ALPGEN Scale relative shape dependent systematic errors with respect to the nominal boosted decision tree discriminant in RunIIb1 in the two tag sample for the signal and background samples (as specified in the top left of each plot). The nominal shape for the given sample is shown in green and $\pm 1 \sigma$ fractional change in red/blue.
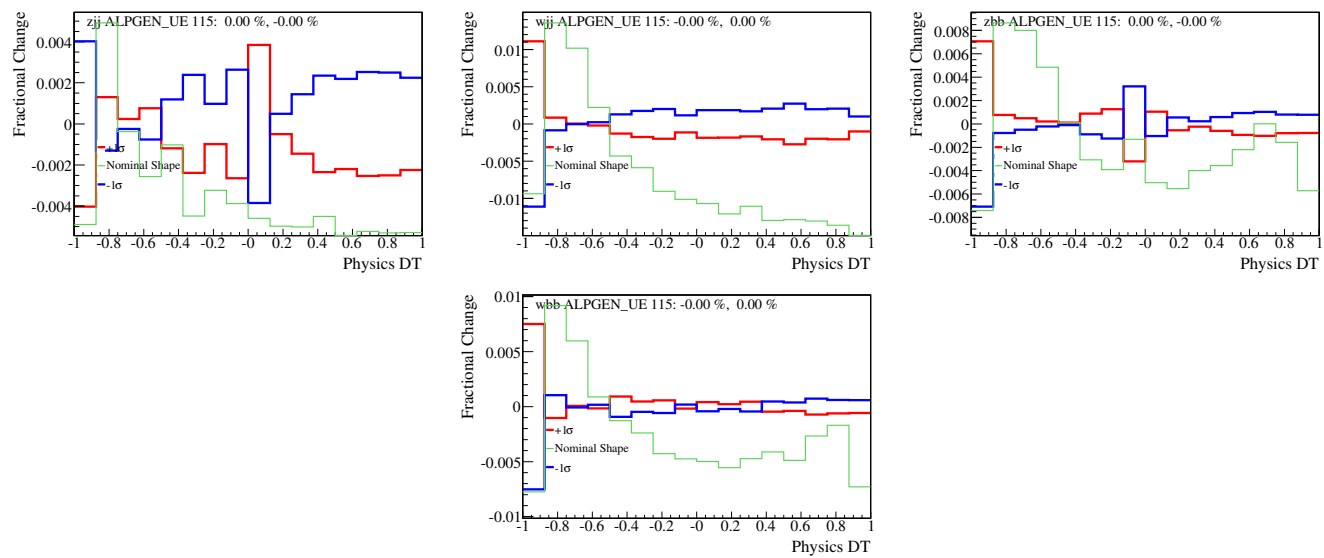

Figure J.9: ALPGEN Underlying Event relative shape dependent systematic errors with respect to the nominal boosted decision tree discriminant in RunIIb1 in the two tag sample for the signal and background samples (as specified in the top left of each plot). The nominal shape for the given sample is shown in green and \pm $1 \sigma$ fractional change in red/blue. 

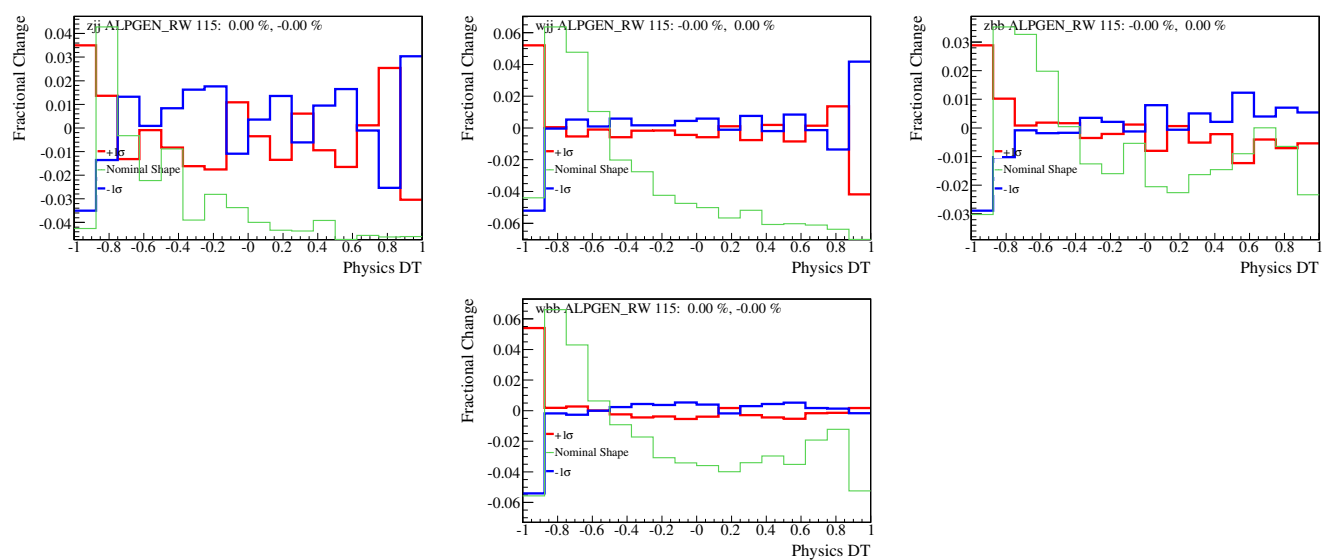

Physics DT

Figure J.10: ALPGEN Angular Reweighting relative shape dependent systematic errors with respect to the nominal boosted decision tree discriminant in RunIIb1 in the two tag sample for the signal and background samples (as specified in the top left of each plot). The nominal shape for the given sample is shown in green and $\pm 1 \sigma$ fractional change in red/blue.
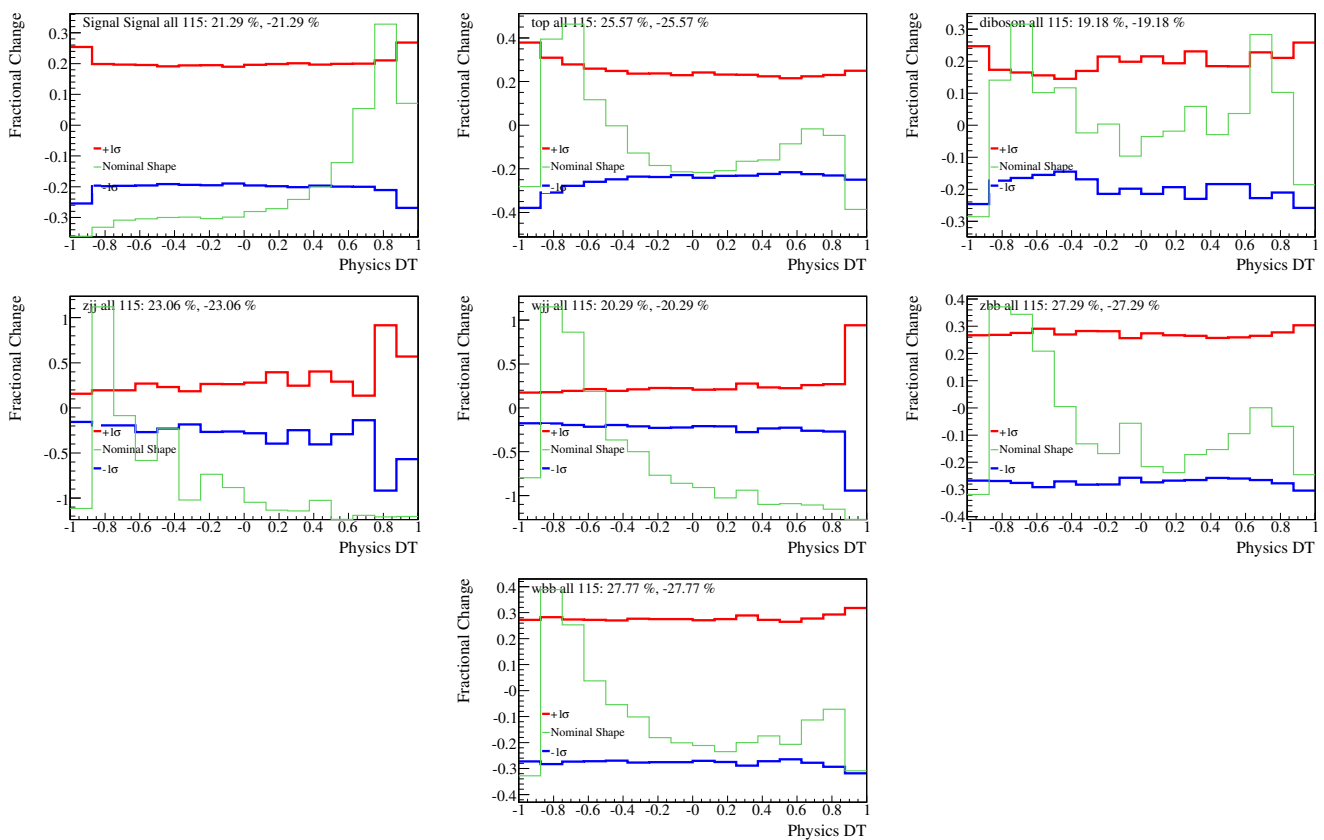

Figure J.11: All systematic errors with respect to the nominal boosted decision tree discriminant in RunIIb1 in the two tag sample for the signal and background samples (as specified in the top left of each plot). The nominal shape for the given sample is shown in green and $\pm 1 \sigma$ fractional change in red/blue. 
Appendix K

Shape Systematics with respect to the BDT for RunIIb23 single tag sample 

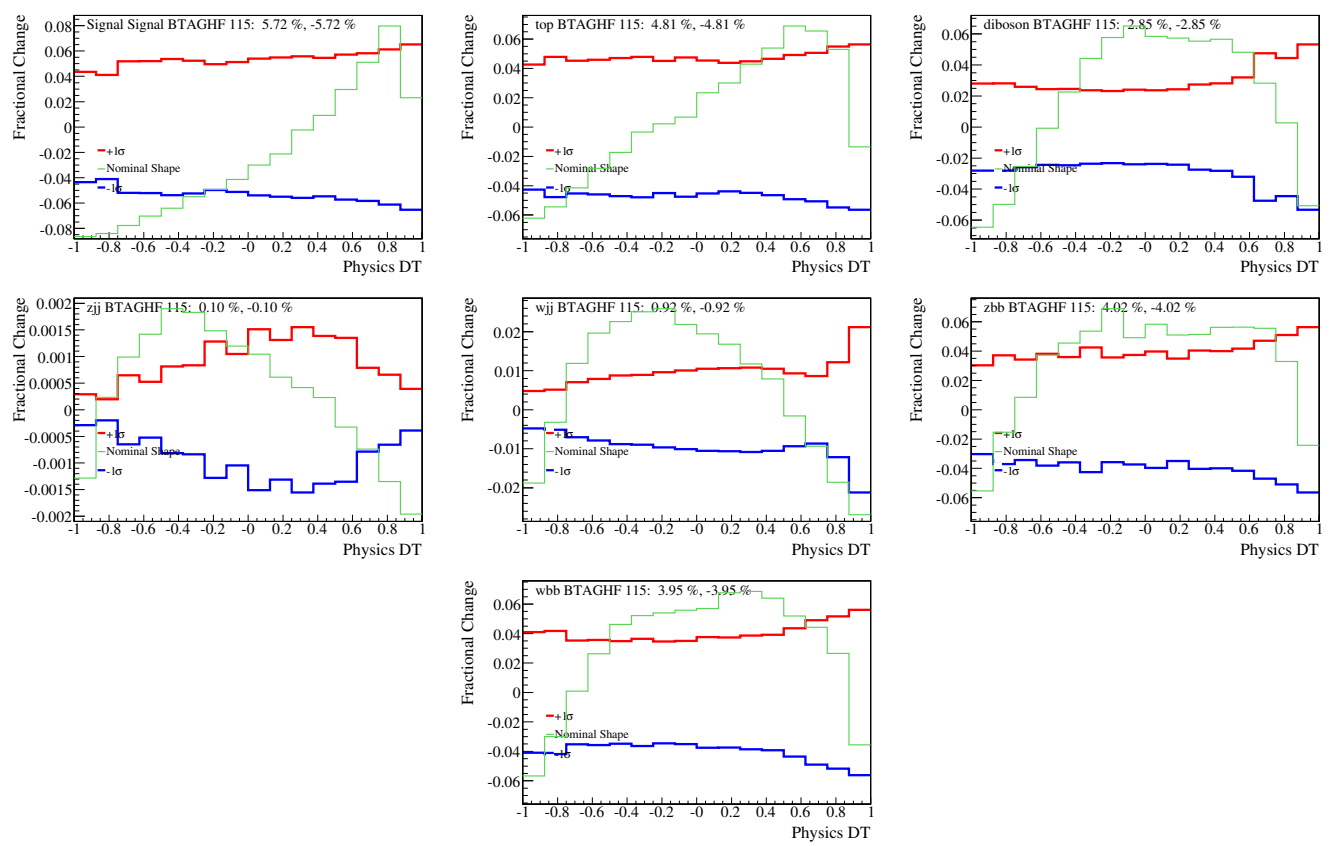

Figure K.1: Heavy flavor b-Tagging rate relative shape dependent systematic errors with respect to the nominal boosted decision tree discriminant in RunIIb2-3 in the one tag sample for the signal and background samples (as specified in the top left of each plot). The nominal shape for the given sample is shown in green and $\pm 1 \sigma$ fractional change in red/blue. 

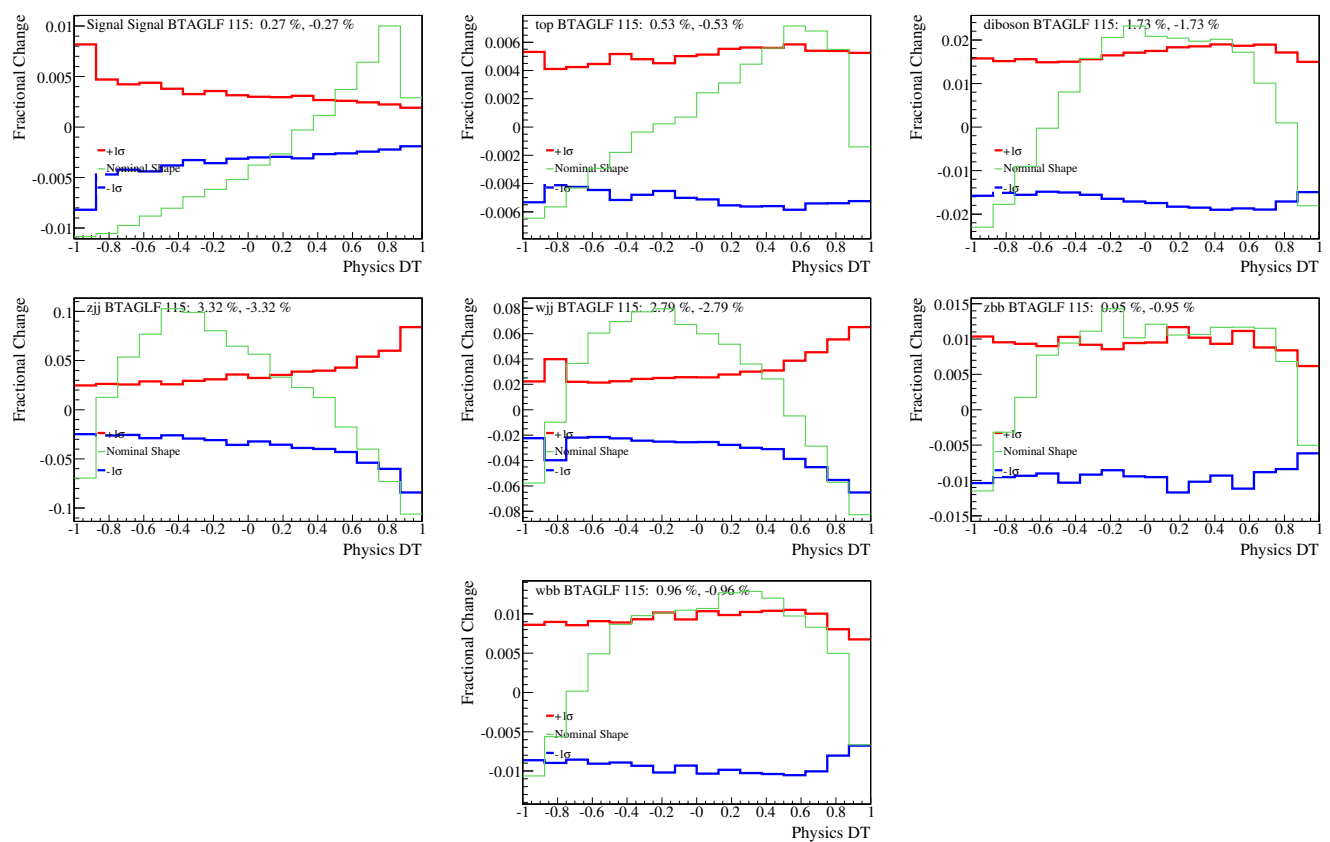

Figure K.2: Light flavor b-Tagging rate relative shape dependent systematic errors with respect to the nominal boosted decision tree discriminant in RunIIb2-3 in the one tag sample for the signal and background samples (as specified in the top left of each plot). The nominal shape for the given sample is shown in green and $\pm 1 \sigma$ fractional change in red/blue. 

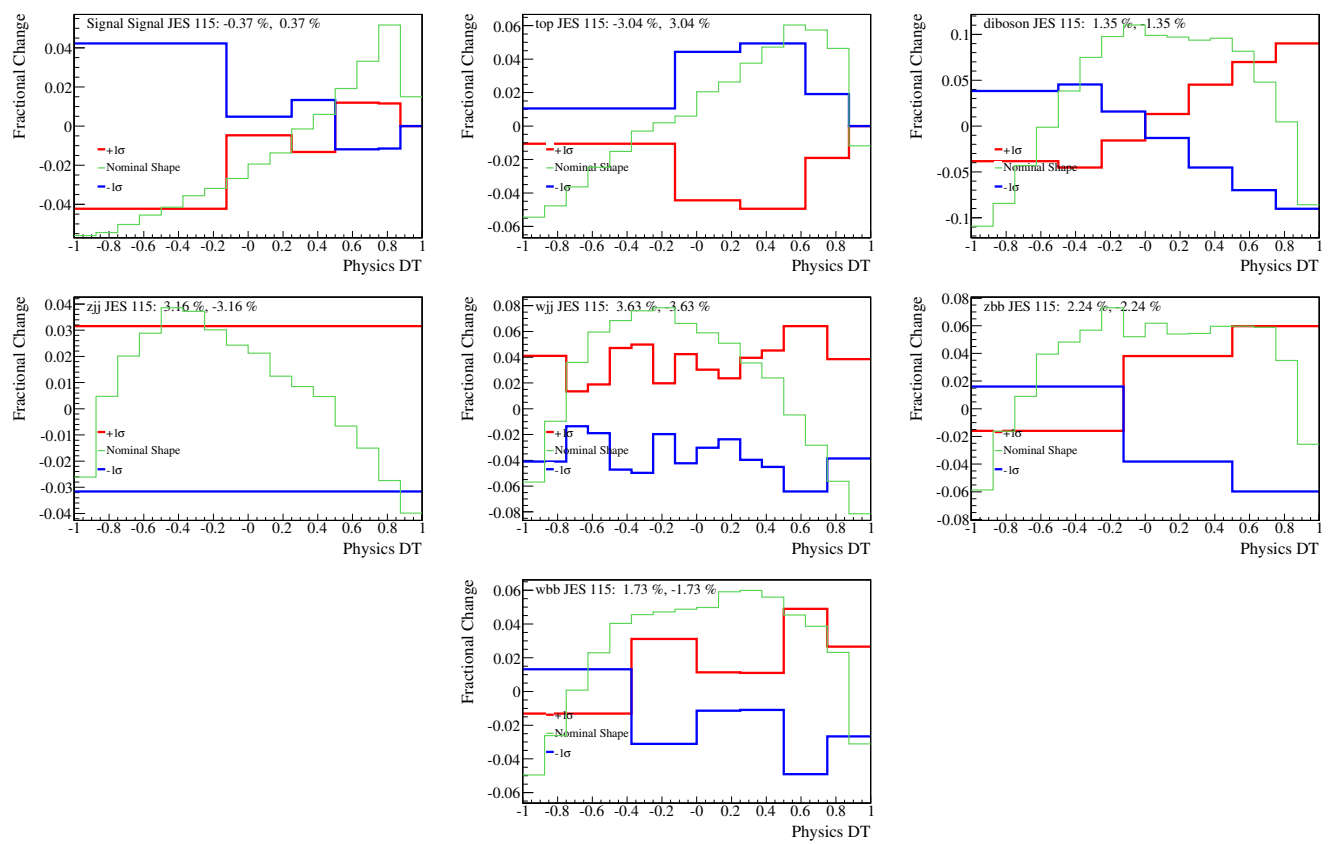

Figure K.3: Jet energy scale relative shape dependent systematic errors with respect to the nominal boosted decision tree discriminant in RunIIb2-3 in the one tag sample for the signal and background samples (as specified in the top left of each plot). The nominal shape for the given sample is shown in green and $\pm 1 \sigma$ fractional change in red/blue. 

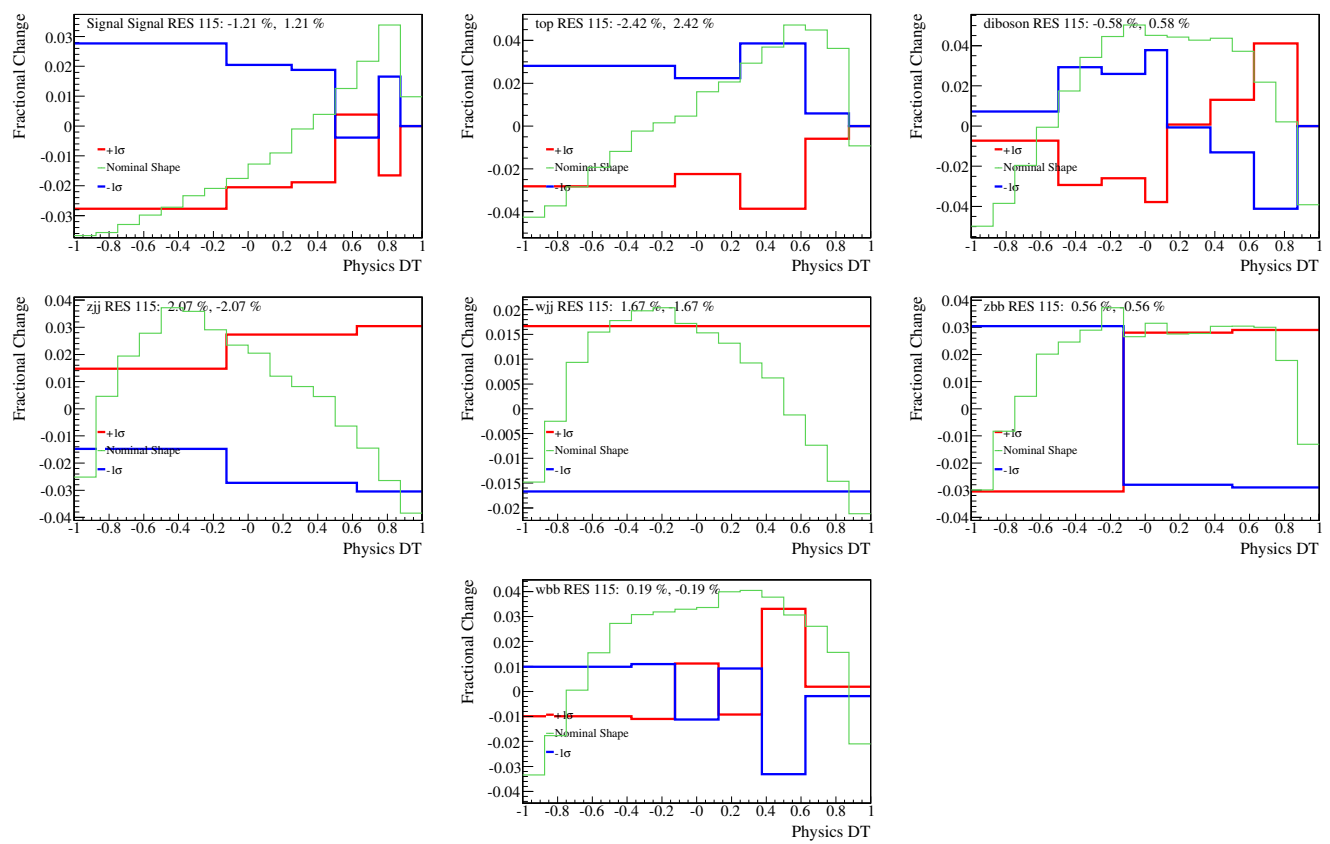

Figure K.4: Jet energy resolution relative shape dependent systematic errors with respect to the nominal boosted decision tree discriminant in RunIIb2-3 in the one tag sample for the signal and background samples (as specified in the top left of each plot). The nominal shape for the given sample is shown in green and \pm $1 \sigma$ fractional change in red/blue. 

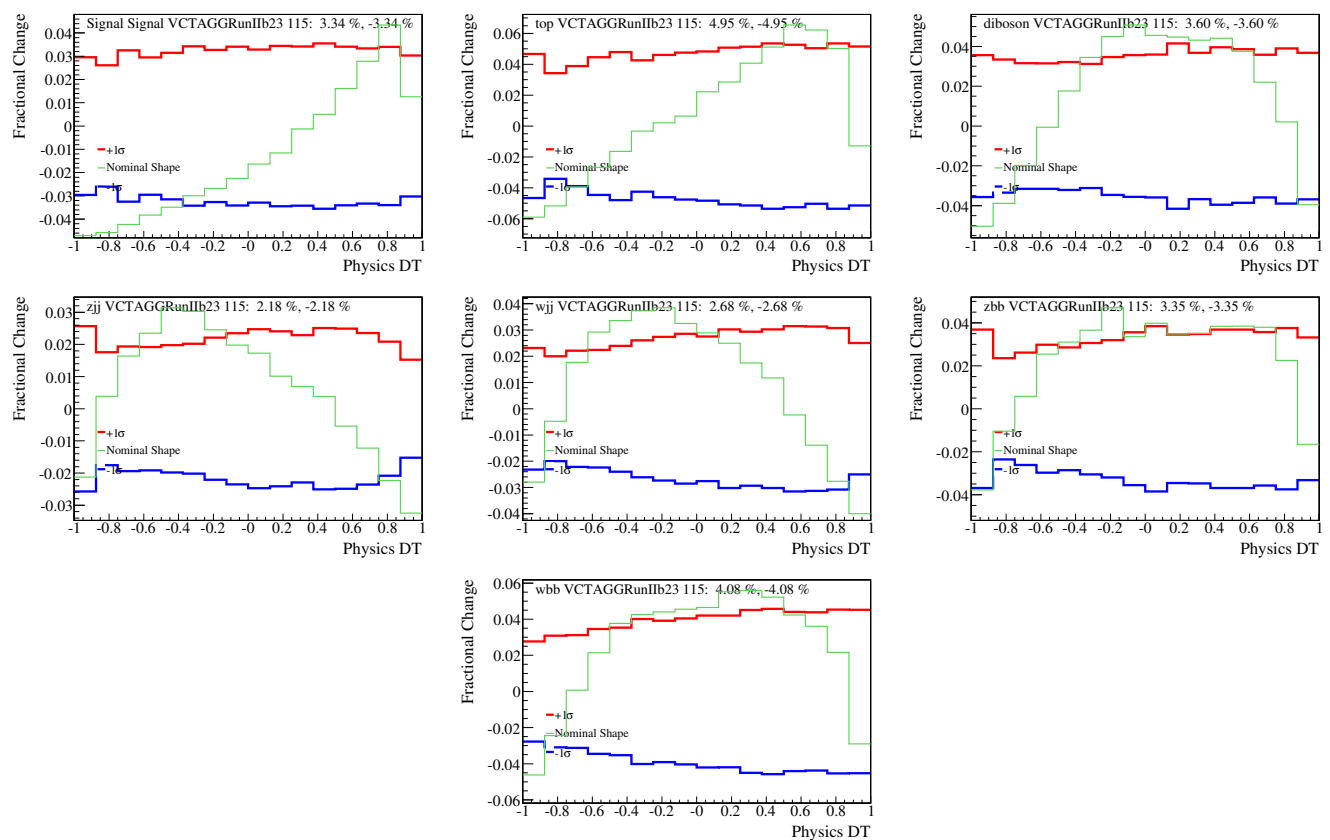

Figure K.5: Direct taggability and Vertex Confirmation relative shape dependent systematic errors with respect to the nominal boosted decision tree discriminant in RunIIb2-3 in the one tag sample for the signal and background samples (as specified in the top left of each plot). The nominal shape for the given sample is shown in green and $\pm 1 \sigma$ fractional change in red/blue. 

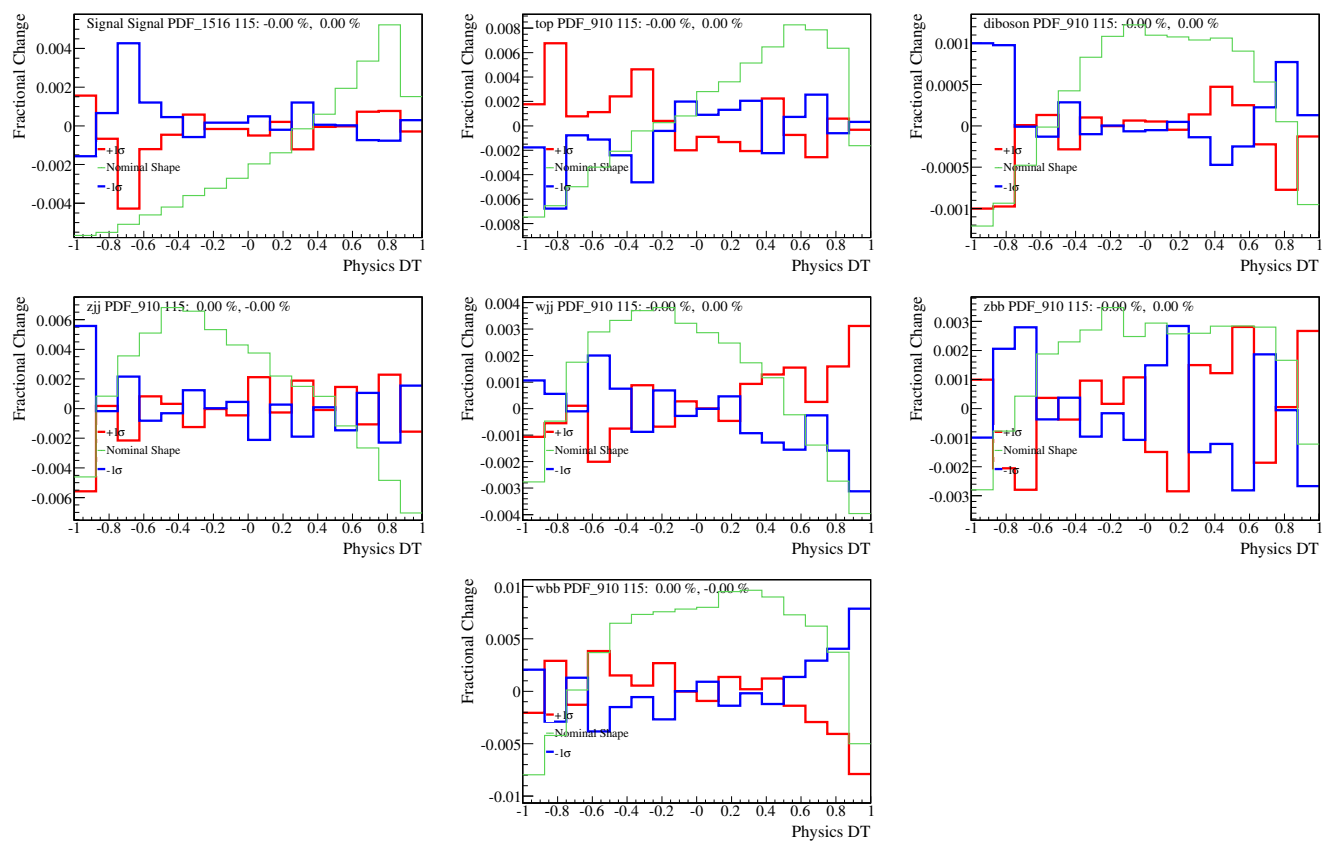

Figure K.6: PDF relative shape dependent systematic errors with respect to the nominal boosted decision tree discriminant in RunIIb2-3 in the one tag sample for the signal and background samples (as specified in the top left of each plot). (4 of 4. The nominal shape for the given sample is shown in green and $\pm 1 \sigma$ fractional change in red/blue.
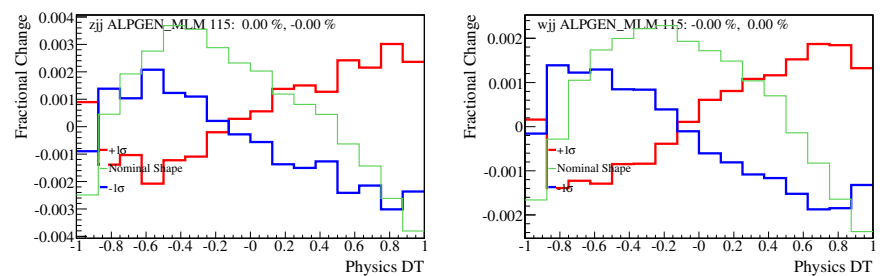

Figure K.7: ALPGEN MLM relative shape dependent systematic errors with respect to the nominal boosted decision tree discriminant in RunIIb2-3 in the one tag sample for the signal and background samples (as specified in the top left of each plot). The nominal shape for the given sample is shown in green and $\pm 1 \sigma$ fractional change in red/blue. 

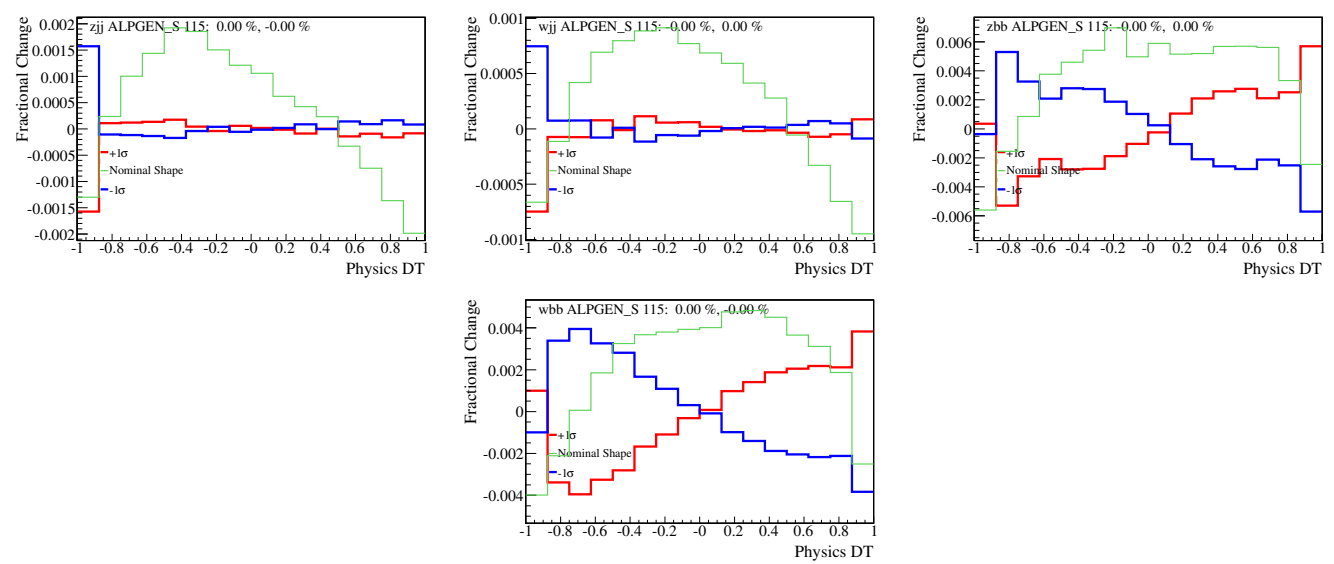

Figure K.8: ALPGEN Scale relative shape dependent systematic errors with respect to the nominal boosted decision tree discriminant in RunIIb2-3 in the one tag sample for the signal and background samples (as specified in the top left of each plot). The nominal shape for the given sample is shown in green and $\pm 1 \sigma$ fractional change in red/blue.
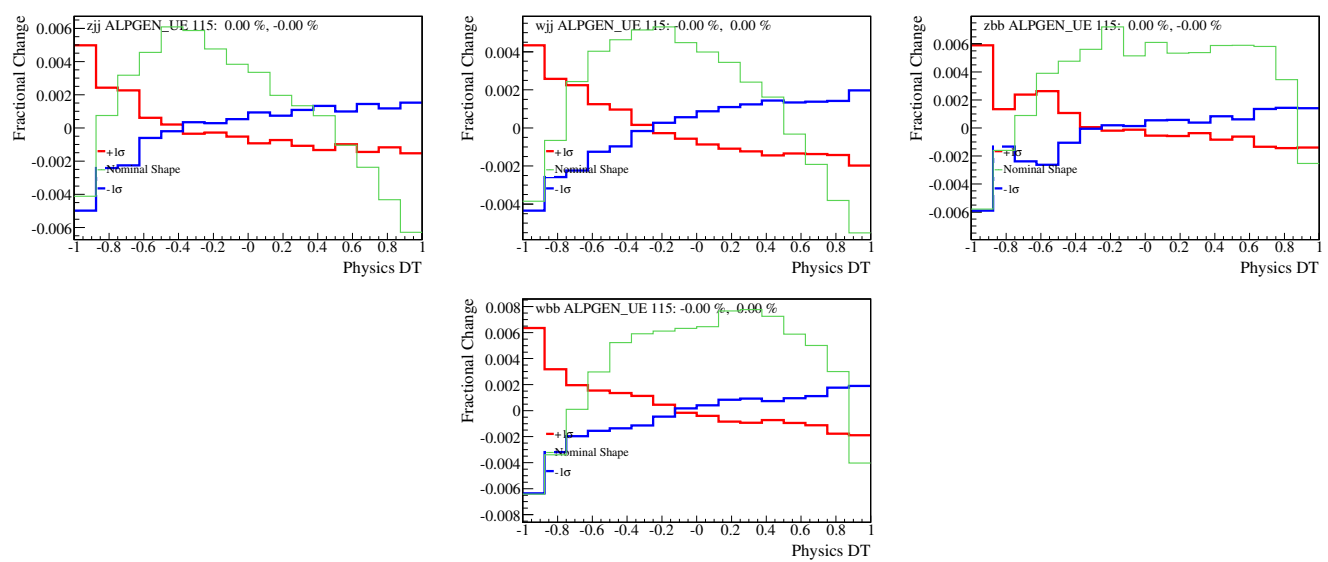

Figure K.9: ALPGEN Underlying Event relative shape dependent systematic errors with respect to the nominal boosted decision tree discriminant in RunIIb2-3 in the one tag sample for the signal and background samples (as specified in the top left of each plot). The nominal shape for the given sample is shown in green and $\pm 1 \sigma$ fractional change in red/blue. 

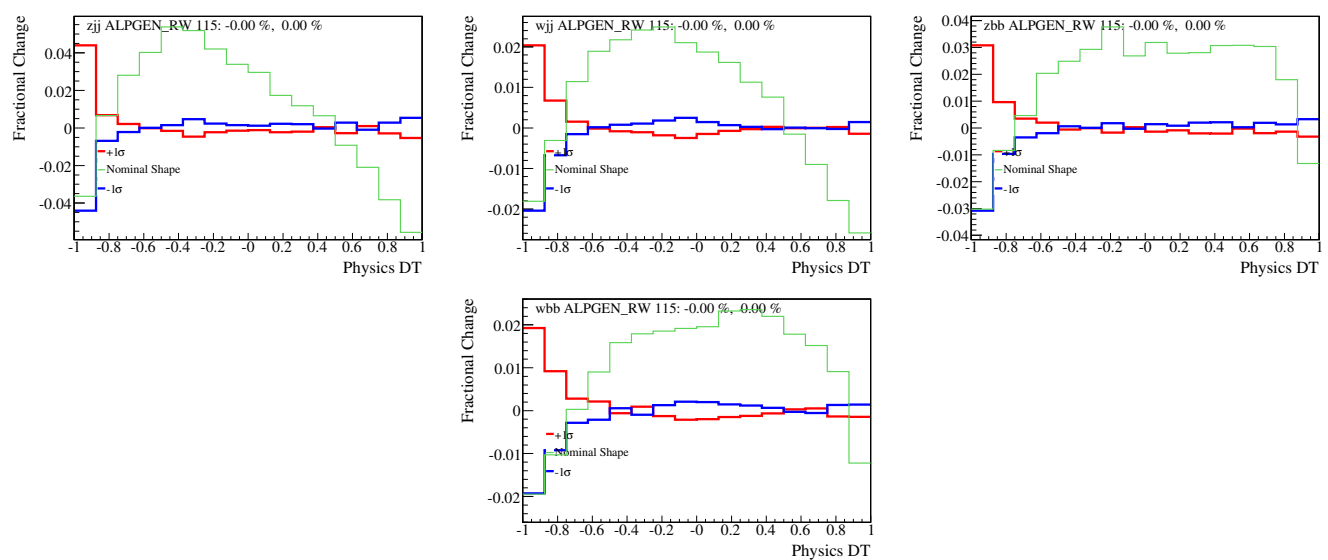

Physics DT

Figure K.10: ALPGEN Angular Reweighting relative shape dependent systematic errors with respect to the nominal boosted decision tree discriminant in RunIIb2-3 in the one tag sample for the signal and background samples (as specified in the top left of each plot). The nominal shape for the given sample is shown in green and $\pm 1 \sigma$ fractional change in red/blue.
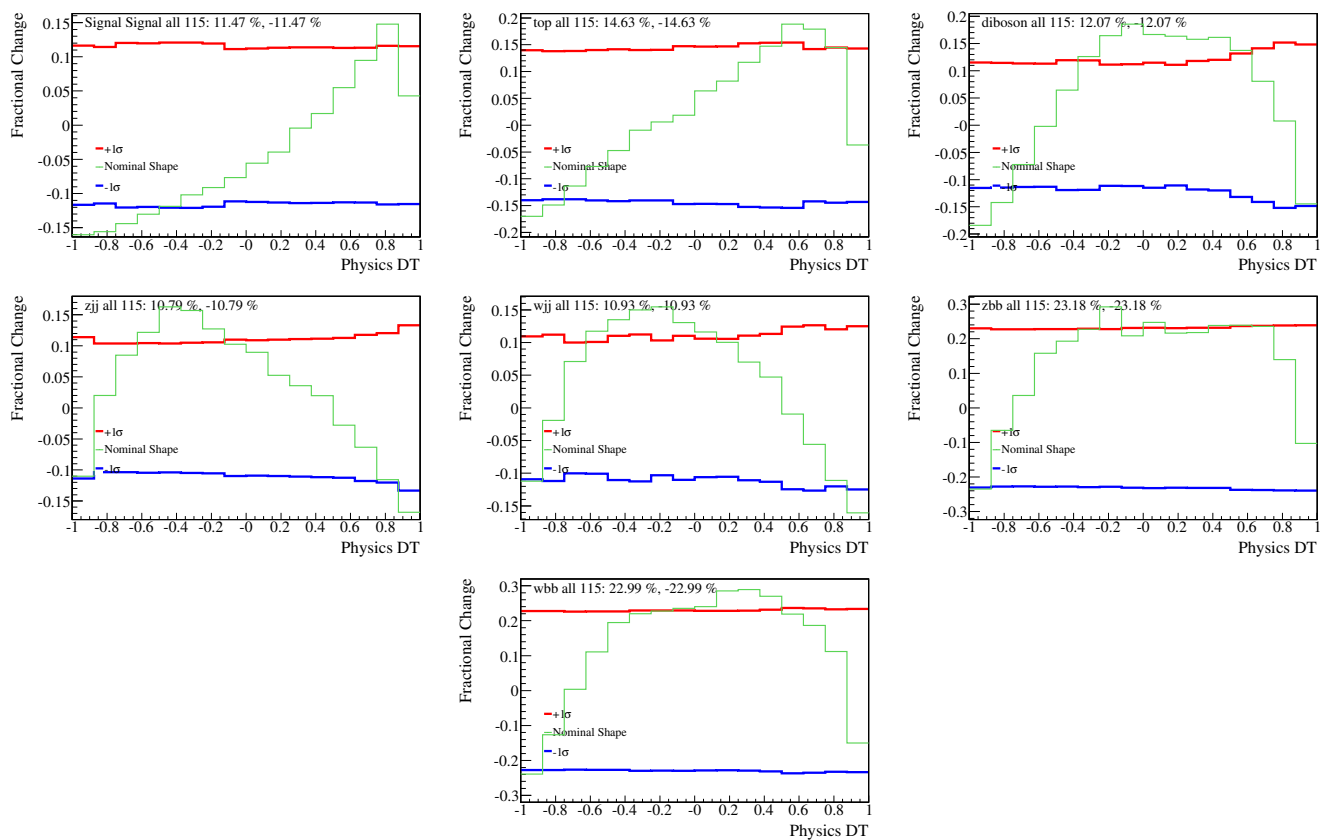

Figure K.11: All systematic errors with respect to the nominal boosted decision tree discriminant in RunIIb2-3 in the one tag sample for the signal and background samples (as specified in the top left of each plot). The nominal shape for the given sample is shown in green and $\pm 1 \sigma$ fractional change in red/blue. 
Appendix L

Shape Systematics with respect to the BDT for RunIIb23 double tag sample 

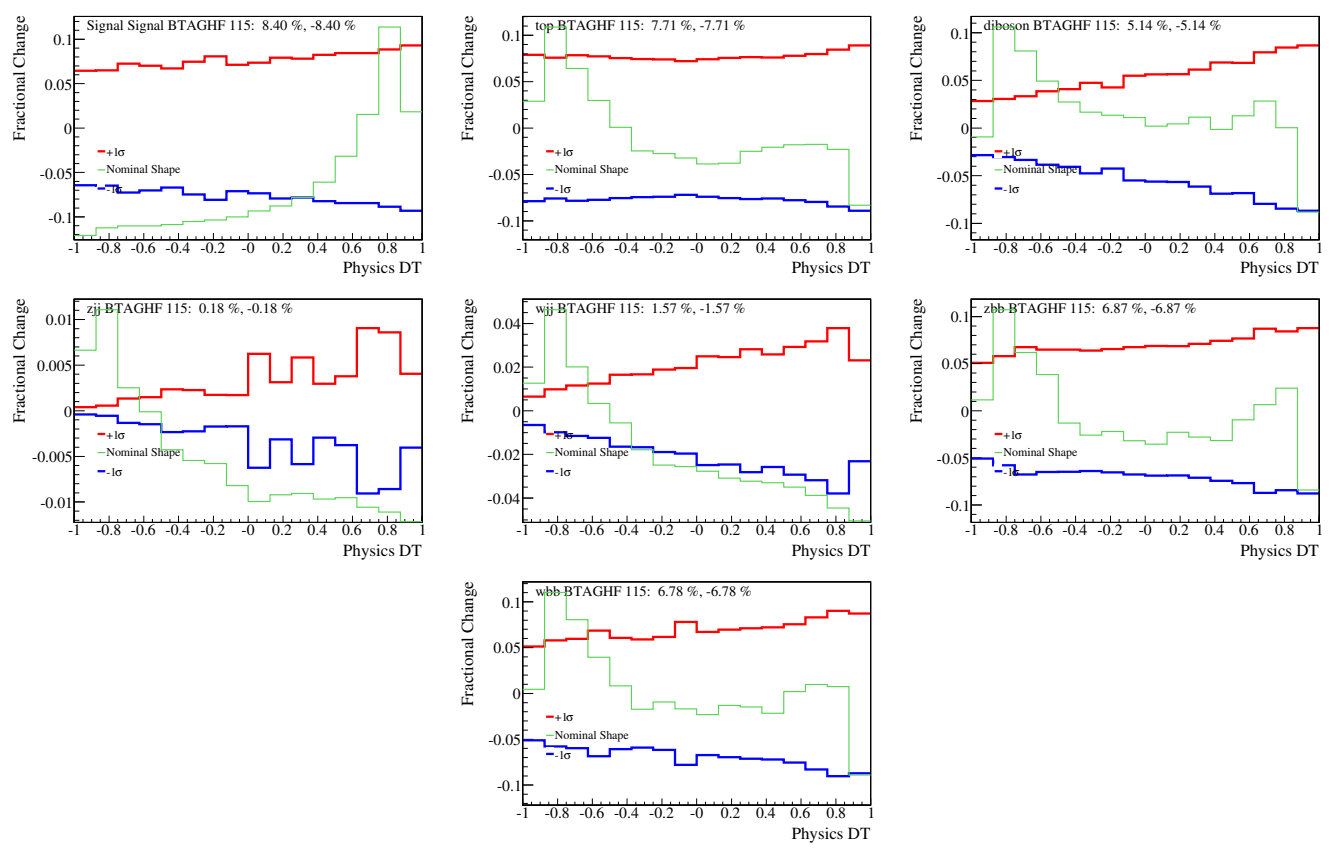

Figure L.1: Heavy flavor b-Tagging rate relative shape dependent systematic errors with respect to the nominal boosted decision tree discriminant in RunIIb2-3 in the two tag sample for the signal and background samples (as specified in the top left of each plot). The nominal shape for the given sample is shown in green and $\pm 1 \sigma$ fractional change in red/blue. 

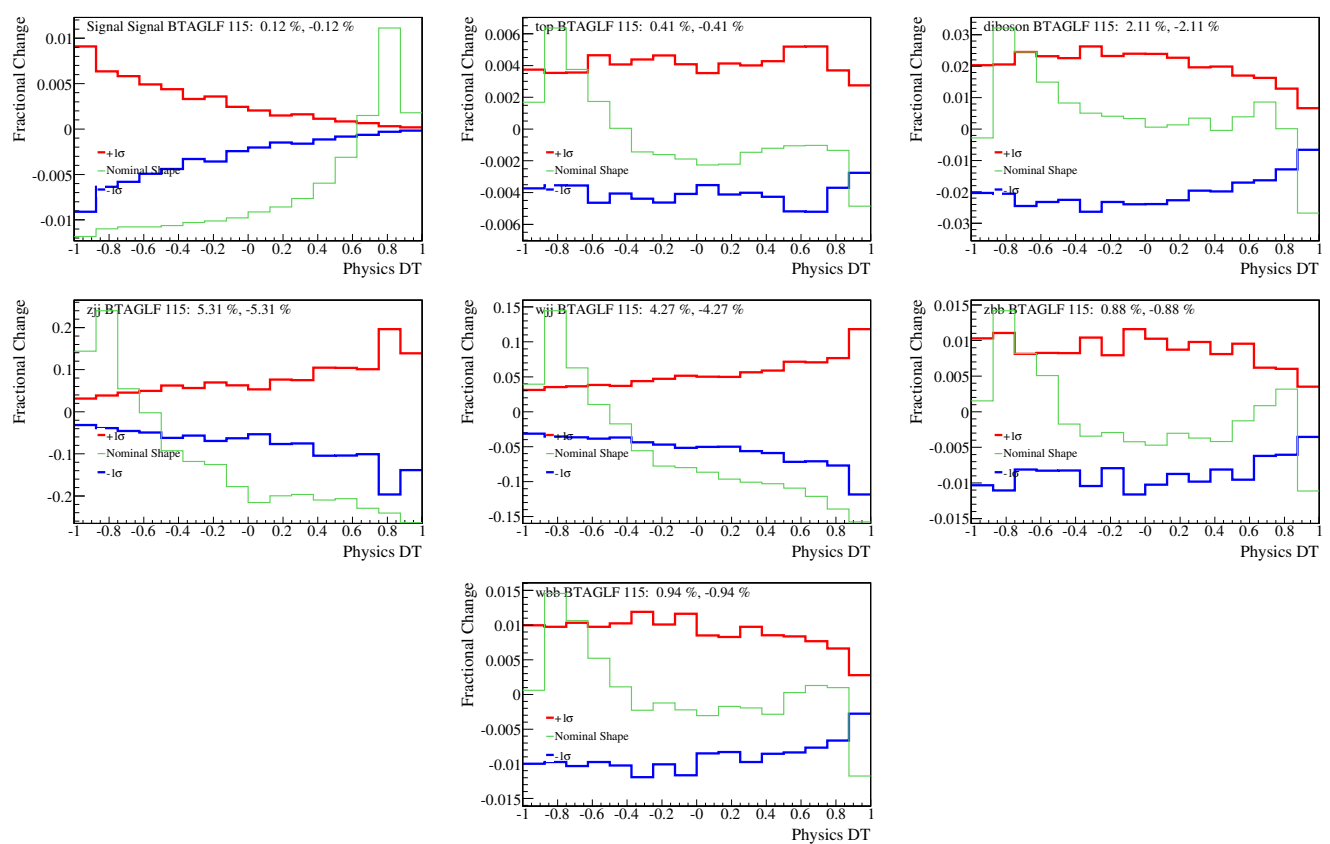

Figure L.2: Light flavor b-Tagging rate relative shape dependent systematic errors with respect to the nominal boosted decision tree discriminant in RunIIb2-3 in the two tag sample for the signal and background samples (as specified in the top left of each plot). The nominal shape for the given sample is shown in green and $\pm 1 \sigma$ fractional change in red/blue. 

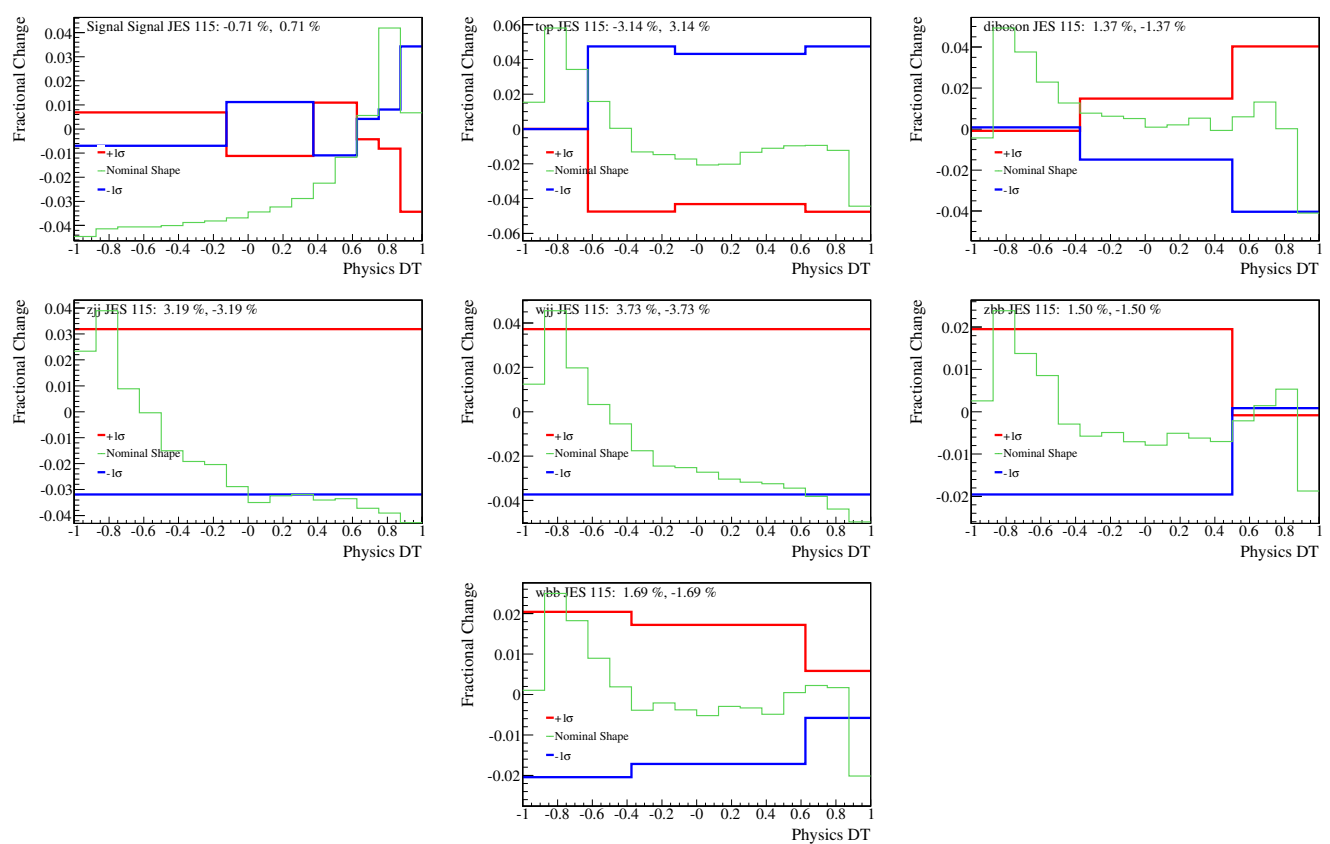

Figure L.3: Jet energy scale relative shape dependent systematic errors with respect to the nominal boosted decision tree discriminant in RunIIb2-3 in the two tag sample for the signal and background samples (as specified in the top left of each plot). The nominal shape for the given sample is shown in green and $\pm 1 \sigma$ fractional change in red/blue. 

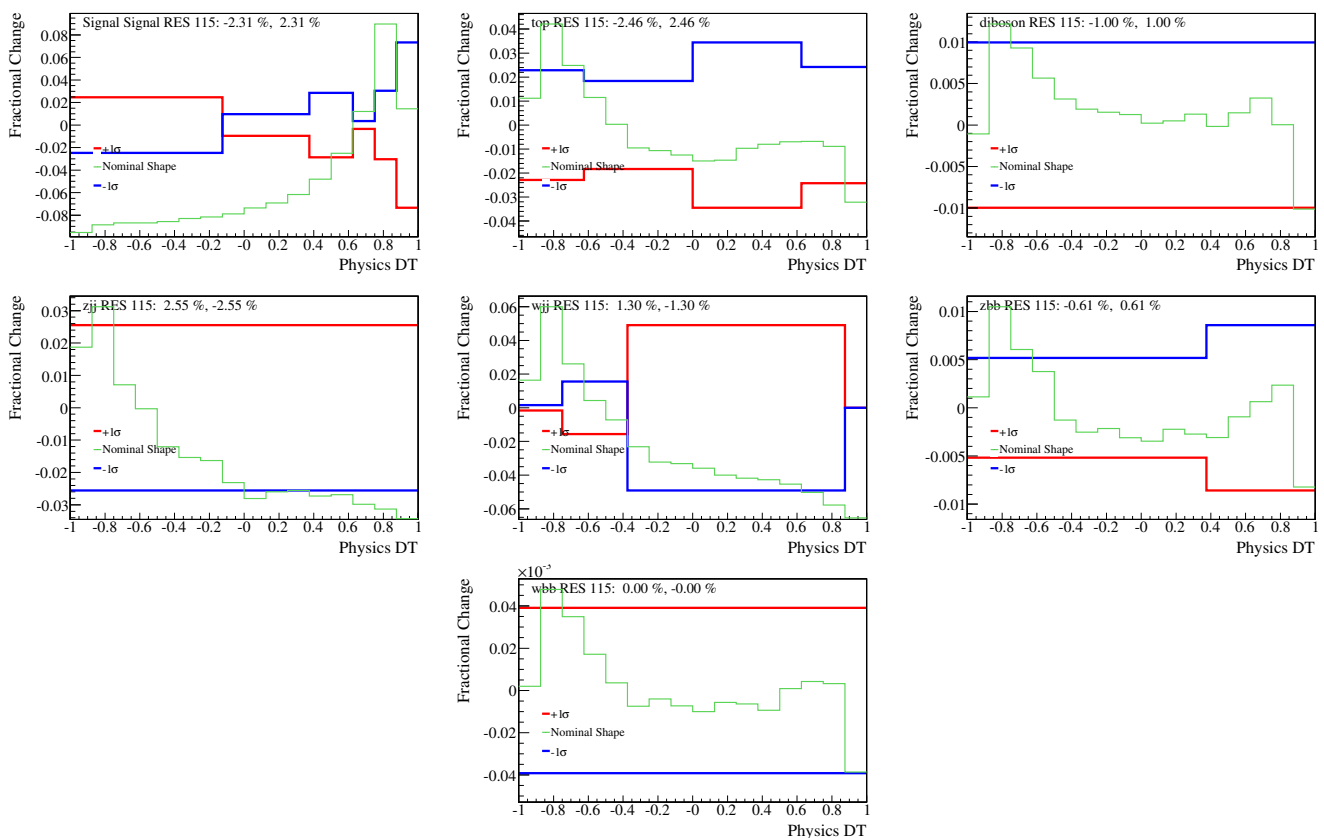

Figure L.4: Jet energy resolution relative shape dependent systematic errors with respect to the nominal boosted decision tree discriminant in RunIIb2-3 in the two tag sample for the signal and background samples (as specified in the top left of each plot). The nominal shape for the given sample is shown in green and \pm $1 \sigma$ fractional change in red/blue. 

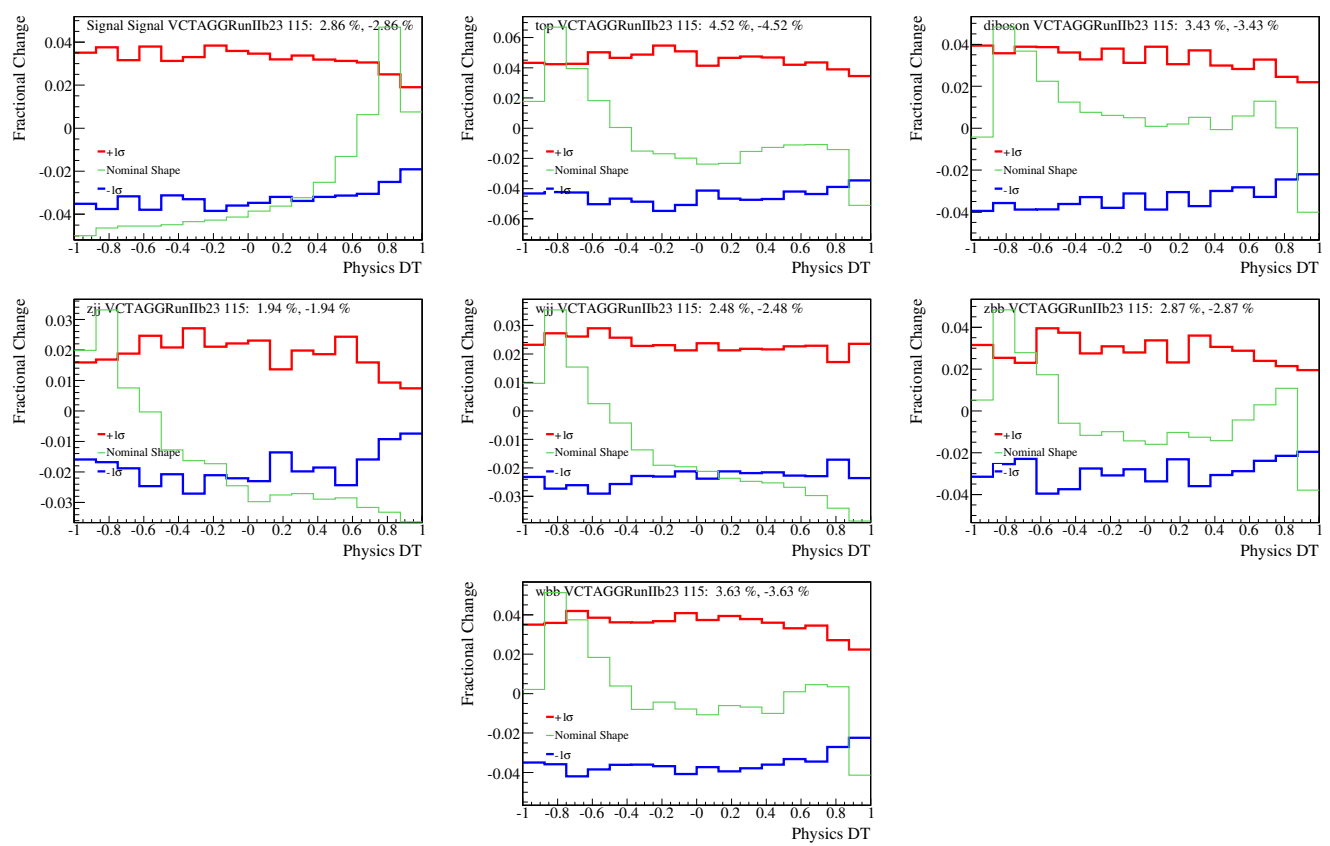

Figure L.5: Direct taggability and Vertex Confirmation relative shape dependent systematic errors with respect to the nominal boosted decision tree discriminant in RunIIb2-3 in the two tag sample for the signal and background samples (as specified in the top left of each plot). The nominal shape for the given sample is shown in green and $\pm 1 \sigma$ fractional change in red/blue.
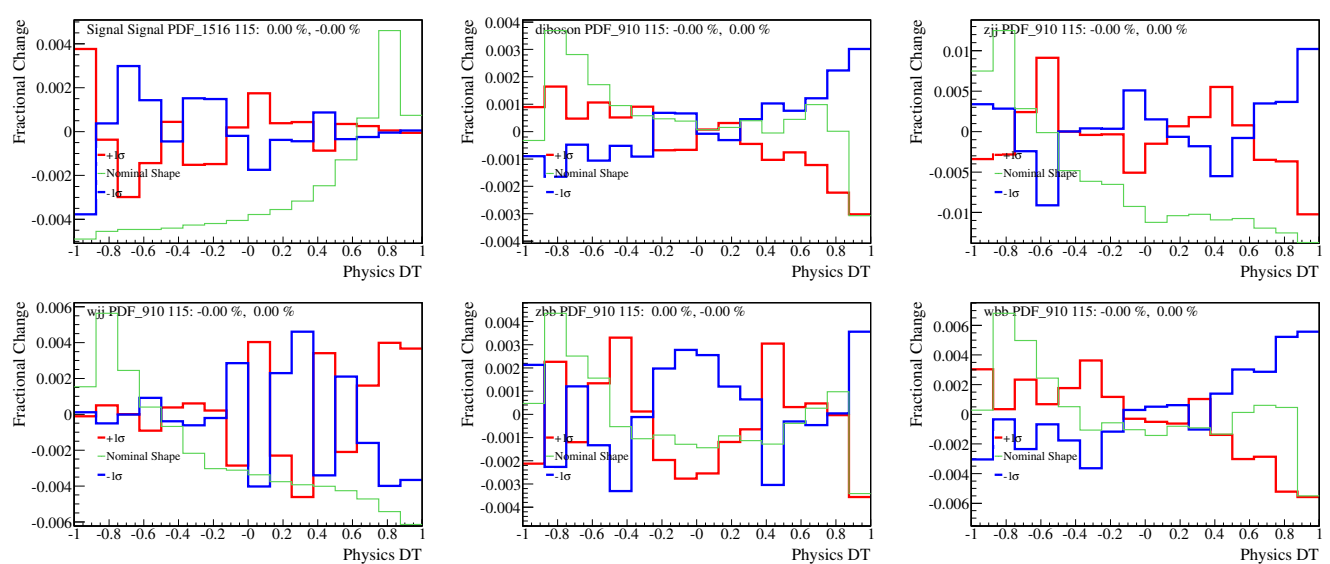

Figure L.6: PDF relative shape dependent systematic errors with respect to the nominal boosted decision tree discriminant in RunIIb2-3 in the two tag sample for the signal and background samples (as specified in the top left of each plot). (4 of 4 . The nominal shape for the given sample is shown in green and $\pm 1 \sigma$ fractional change in red/blue. 

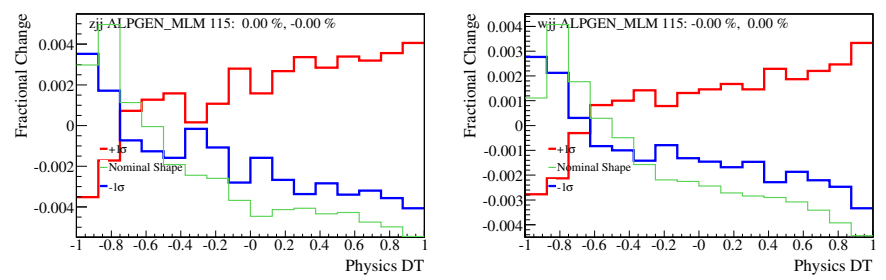

Figure L.7: ALPGEN MLM relative shape dependent systematic errors with respect to the nominal boosted decision tree discriminant in RunIIb2-3 in the two tag sample for the signal and background samples (as specified in the top left of each plot). The nominal shape for the given sample is shown in green and $\pm 1 \sigma$ fractional change in red/blue.
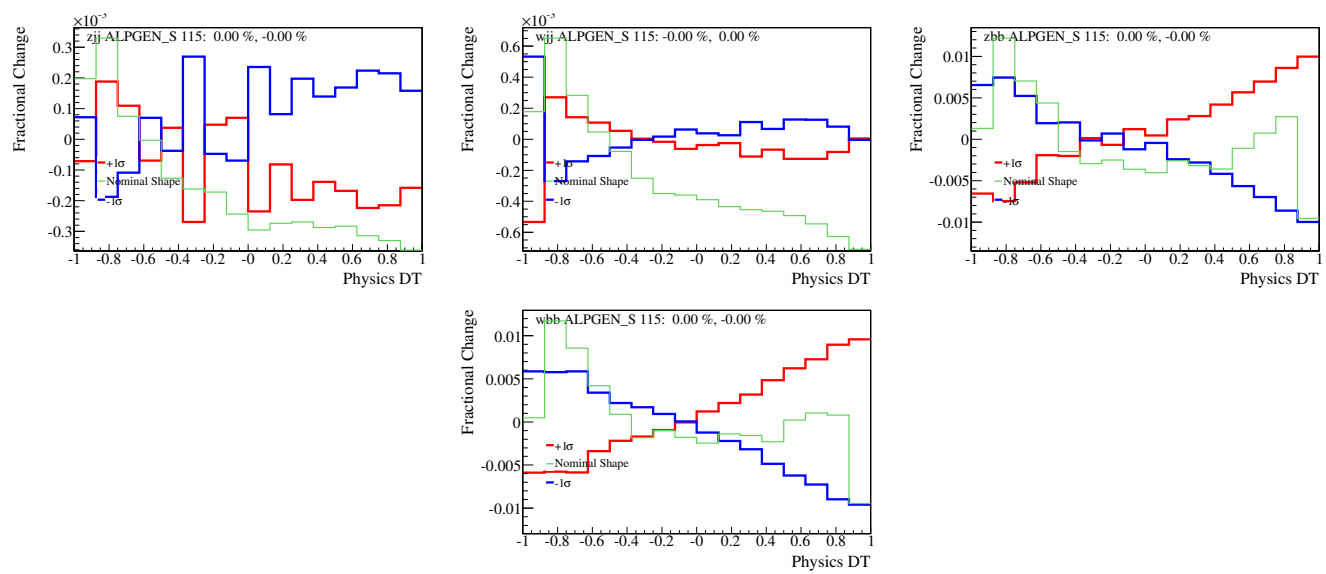

Figure L.8: ALPGEN Scale relative shape dependent systematic errors with respect to the nominal boosted decision tree discriminant in RunIIb2-3 in the two tag sample for the signal and background samples (as specified in the top left of each plot). The nominal shape for the given sample is shown in green and $\pm 1 \sigma$ fractional change in red/blue. 

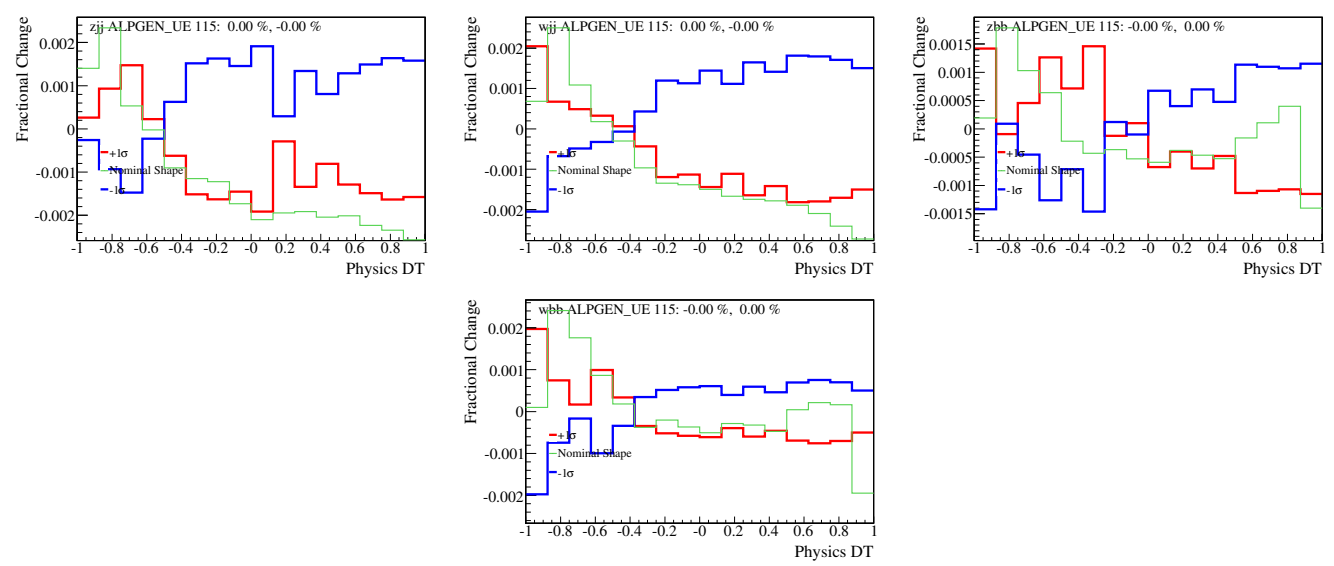

Figure L.9: ALPGEN Underlying Event relative shape dependent systematic errors with respect to the nominal boosted decision tree discriminant in RunIIb2-3 in the two tag sample for the signal and background samples (as specified in the top left of each plot). The nominal shape for the given sample is shown in green and $\pm 1 \sigma$ fractional change in red/blue.
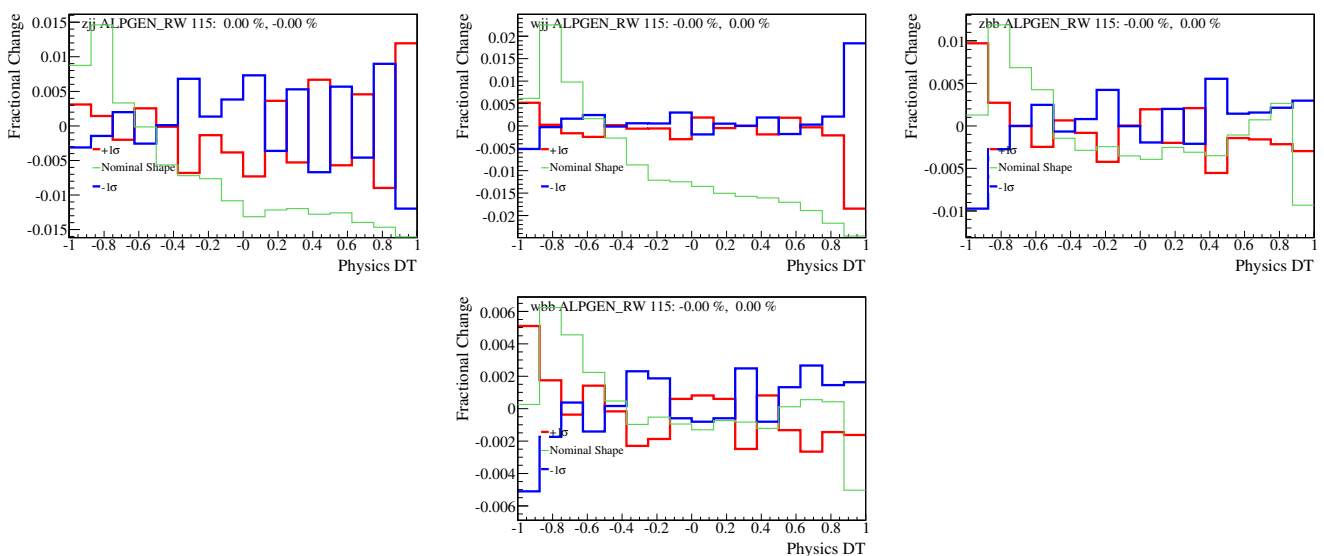

Figure L.10: ALPGEN Angular Reweighting relative shape dependent systematic errors with respect to the nominal boosted decision tree discriminant in RunIIb2-3 in the two tag sample for the signal and background samples (as specified in the top left of each plot). The nominal shape for the given sample is shown in green and $\pm 1 \sigma$ fractional change in red/blue. 

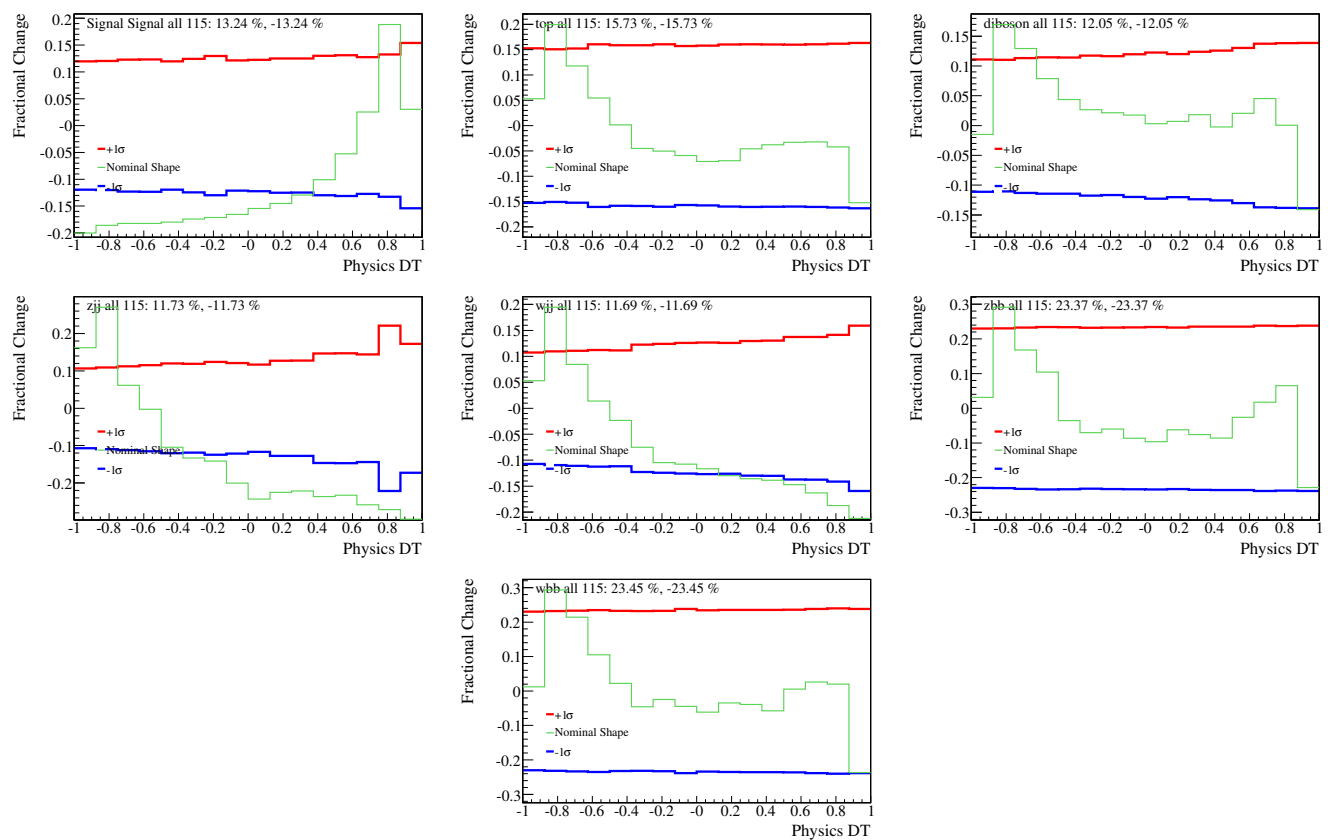

Figure L.11: All systematic errors with respect to the nominal boosted decision tree discriminant in RunIIb2-3 in the two tag sample for the signal and background samples (as specified in the top left of each plot). The nominal shape for the given sample is shown in green and $\pm 1 \sigma$ fractional change in red/blue. 


\section{Bibliography}

[1] V. Abazov et al. (DØ Collaboration), " $Z Z \rightarrow \ell^{-} \ell^{+} \nu \bar{\nu}$ Production in $p \bar{p}$ Collisions at $\sqrt{s}=1.96$ TeV", Phys. Rev. D 78, 072002 (2008), arXiv.org:0808.0269.

[2] V. Abazov et al. (DØ Collaboration), "Observation of ZZ Production in $p \bar{p}$ Collisions at $\sqrt{s}=1.96 \mathrm{TeV}$ ", Phys. Rev. Lett. 101, 171803 (2008), arXiv.org:0808.0703.

[3] V. Abazov et al. (DØ Collaboration), "Search for the Standard Model Higgs Boson in the $Z H \rightarrow \nu \bar{\nu} b \bar{b}$ Channel in $5.2 \mathrm{fb}^{-1}$ of $p \bar{p}$ Collisions at $\sqrt{s}=1.96 \mathrm{TeV}$ ", Phys. Rev. Lett. 104, 071801 (2010), arXiv.org:0912.5285.

[4] S. Abachi et al. (DØ Collaboration), "Search for High Mass Top Quark Production in $p \bar{p}$ Collisions at $\sqrt{s}=1.8 \mathrm{TeV}$ ", Physical Review Letters 74 (13): $24222426(1995)$

[5] F. Abe et al. (CDF Collaboration), "Observation of Top Quark Production in $p \bar{p}$ Collisions with the Collider Detector at Fermilab", Physical Review Letters 74 (14): 26262631 (1995)

[6] V. M. Abazov et al. (DØ Collaboration), "Evidence for an Anomalous Like-Sign Dimuon Charge Asymmetry", Phys. Rev. D 82, 032001, (2010). arXiv.org:1005.2757

V. M. Abazov et al. (DØ Collaboration), "Evidence for an Anomalous LikeSign Dimuon Charge Asymmetry", Phys. Rev. Lett. 105, 081801 (2010). arXiv.org:1007.0395 
[7] F. Halzen, A.D. Martin, "Quarks and Leptons: An Introductory Course in Modern Particle Physics", John Wiley \& Sons, 1984.

[8] D.J. Griffiths, "Introduction to Elementary Particles", John Wiley \& Sons, 1987.

[9] M.E. Peskin, D.V. Schroeder, "An Introduction to Quantum Field Theory", Perseus Books Publishing, 1995.

[10] T. Cheng, L. Li, "Gauge Theory of Elementary Particle Physics", Clarendon Press, 1983.

[11] P. Higgs, "Broken symmetries, massless particlees and gauge fields", Phys. Rev. Lett. 12, 132-133 (1964).

[12] P. Higgs, "Spontaneous Symmetry Breakdown without Massless Bosons", Phys. Rev. 145, 1156 (1966).

[13] R. Brout, F. Englert, "Broken Symmetry and the Mass of Gauge Vector Mesons", Phys. Rev. Lett. 13, 321 (1964).

[14] G. Guralnik, C. R. Hagen, T. W. Kibble, "Global Conservation Laws and Massless Particles" Phys. Rev. Lett. 13, 585 (1964).

[15] LEPEW Working Group.

[16] Gfitter Group.

[17] R. Barate et al. [LEP Working Group for Higgs boson searches], Phys. Lett. B 565, 61 (2003).

[18] The CDF, DØ Collaborations, the TEVNPHWG Working Group, "Combined CDF and DØ Upper Limits on Standard Model Higgs Boson Production with up to $6.7 \mathrm{fb}^{-1}$ of Data", FERMILAB-CONF-10-257-E, arXiv:1007.4587. 
[19] The CDF, DØ Collaborations, the TEVNPHWG Working Group, "Combined CDF and DØ Upper Limits on Standard Model Higgs Boson Production with up to $8.2 \mathrm{fb}^{-1}$ of Data", FERMILAB-CONF-11-044-E, arXiv:1103.3233.

[20] Fermilab's Accelerator Division.

[21] J. Thompson, "Introduction to Colliding Beams at Fermilab", FERMILABTM-1909.

[22] Fermilab Accelerator Details: The $\mathrm{H}^{-}$Source and Multiturn Injection.

[23] Fermilab Accelerator Details: The Antiproton Source.

[24] Fermilab Accelerator Details: The Main Injector.

[25] Fermilab Beams Division, "Run II Parameter List".

[26] S. Abachi et al. (D0 Collaboration), "The DØ Determination", Nucl. Instrum. Methods Phys. Res A 338, 185 (1994). V.M. Abazov et al. (D0 Collaboration), "The Upgraded D $\varnothing$ Detector", Nucl. Instrum. and Methods A 565, 463-537 (2006), physics/0507191.

[27] R. Angstadt et al., "The Layer 0 Inner Silicon Detector of the DØ Experiment", Nucl. Instrum. and Methods A 622, 298-310 (2010), arXiv.org:0911.2522.

[28] S.N. Ahmed et al., "The DØ Silicon Microstrip Tracker", Nucl. Instrum. and Methods A 634, 8-46 (2011), arXiv.org:1005.0801.

[29] T.L. Edwards et al., "Determination of the effective inelastic $p \bar{p}$ cross-section for the DØ Run II luminosity measurement". FERMILAB-TM-2278-E.

[30] M. Abolins et al., "The Run IIb Trigger Upgrade for the D $\varnothing$ Experiment" IEEE Trans Nucl. Sci. 51, 340 (2004). 
[31] T. Sjöstrand, S. Mrenna and P. Skands, JHEP 0605, 026 (2006);

[32] J. Pumplin et al., JHEP 0207, 012 (2002); D. Stump et al., JHEP 0310, 046 (2003).

[33] M.L. Mangano et al., JHEP 0307, 001 (2003);

[34] E. Boos et al. (CompHEP Collaboration), Nucl. Instrum. Methods in Phys. Res. A 534, 250 (2004).

[35] J. Campbell, K. Ellis, Monte Carlo for FeMtobarn Processes

[36] "SHERPA 1. $\alpha$, a proof-of-concept version", T. Gleisberg et al., JHEP02 056, 25 (2004)

[37] DØ Collaboration, V. M. Abazov et al., Phys. Lett. B 669, 278 (2008).

DØ Collaboration, V. M. Abazov et al., Phys. Lett. B 678, 45 (2009).

[38] S. Höche et al., "Matching Parton Showers and Matrix Elements", in Proceedings of the Workshop on the Implications of HERA for LHC Physics, ed. A. De Roeck and H. Jung (CERN, Geneva, 2005), p288.

[39] D. Gillberg "Heavy Flavour Removal and Determination of Weighting Factors for ALPGEN $W+$ jets Monte Carlo", DØ Note 5129.

[40] T. Bolton M. Shamim, "Generator Level Reweighting of Z boson $p_{T}$ ", DØ Note 5565 .

[41] G. Hesketh, "Generator Level Re-weighting of the Inclusive $\mathrm{W} p_{T}$ Distribution", DØ Note 5786.

[42] Kalman, R. E., "A New Approach to Linear Filtering and Prediction Problems", Transaction of the ASMEJournal of Basic Engineering, pp. 35-45 (March 1960). 
[43] A. Khanov, "HTF: histogramming method for finding tracks. The algorithm description", DØ Note 3778.

[44] Mark R.J. Williams, "Observation and Measurements of L=1 Orbitally Excited B Mesons", FERMILAB-THESIS-2008-84.

[45] G. Borissov, "Ordering a Chaos or... Technical Details of AA Tracking".

[46] G. Hesketh, "Central Track Extrapolation through the DØ Detector", DØ Note 4079.

[47] H. Greenlee, "The DØ Kalman Track Fit", DØ Note 4303.

[48] A. Schwartzman, C. Tully, "Primary Vertex Reconstruction by Means of Adaptive Vertex Fitting", DØ Note 4918.

[49] A. Schwartzman, M. Narain, "Probabilistic Primary Vertex Selection", DØ Note 4042.

[50] P. Calfayan et al., "Muon Identification Certification for p17 data", DØ Note 5157.

[51] O. Brandta et al., "Muon Identification Certification for the Summer 2009 Extended Dataset (Run IIb-1 and -2)", DØ Note 6025.

[52] M. Arthaud et al., "Muon Momentum Oversmearing for p20 Data", DØ Note 5449.

[53] F. Fleuret et al., "The DØ Soft Electron Reconstruction Package SEMReco", DØ Note 3872.

[54] F. Fleuret, "The DØ Electron/Photon Analysis Package EMAnalyze", DØ Note 3888. 
[55] EMID Variables webpage

[56] D. Chapin el al., "Measurement of $Z \rightarrow e^{+} e^{-}$and $W \rightarrow e^{ \pm} \nu_{e}$ Production Cross Sections Using One Tight Central Electron", DØ Note 4897.

[57] T. Andeen et al., "Electron Level Energy Correction in DØ Reconstruction p14 pass 2", DØ Note 5104.

[58] M. Vesterinen, "An Improved Energy Calibration and Monte Carlo Oversmearing for Electromagnetic Clusters", DØ Note 6040.

[59] D. Chakraborty, et al., "Reconstruction of $\tau$ leptons in hadronic final states at DØ in Run 2", DØ Note 4210.

[60] M. Arov et al., A-C. Le Bihan, S. Nelson, C. Nöeding, A. Patwa, Y. Pogorelov, S. Protopopescua, " $\tau$ identification with neural networks for pass2 data", DØ Note 4773.

[61] R. Madar, F. Couderc, "Tau lepton identification using MVAoptimizations, CPS detector and bID tools", DØ Note 6061.

[62] C. Galea, S. de Jong, S. Protopopescu, "Tau Energy Correction in Monte Carlo", DØ Note 5303.

[63] C. Galea el al., "Tau energy corrections for data and MC in p17", DØ Note 5533.

[64] C. Galea, S. de Jong, " $\left(\sigma(p \bar{p} \rightarrow Z) \cdot \operatorname{Br}\left(Z \rightarrow \tau^{+} \tau^{-}\right)\right.$measurement with $1 \mathrm{fb}^{-1}$, DØ Note 5492.

[65] E. Busato, B. Andrieu, "Jet Algorithms in the DØ Run II Software: Description and Users Guide", DØ Note 4457.

[66] A. Harel, "Jet ID Optimization", DØ Note 4919. 
[67] B. Abbott, et al. (DØ Collaboration), "Determination of the Absolute Jet Energy Scale in the D $\varnothing$ Calorimeters", Nucl. Instrum. Methods in Phys. Res. Sect. A 424, 352 (1999), hep-ex/9805009.

[68] . D. Gillberg el al., "Measuring Jet Response Using the Missing $E_{T}$ Projection Fraction Method in $\gamma+$ jet Events", DØ Note 4571 (2004).

[69] K. DeVauhan el al., "Jet Energy Scale Determination for DØ Run IIb (final p20 version)", DØ Note 5801.

[70] N. Makovec and J.-F. Grivaz, "Shifting, Smearing and Removing Simulated Jets", DØ Note 4914(2005)

C. Ochando, presentation at the D $\varnothing$ p17 Higgs Workshop, May 17-18, 2006,

[71] T. Guillemin, presentation during DØ 2010 collaboration meeting, May 12, 2010

[72] E. Berger, ed., "Research directions for the decade", Snowmass. Singapore: World Scientific, June 25 - July 13, 1990.

[73] K. Ackerstaff et al. (The OPAL Collaboration), "Search for Anomalous Production of Di-lepton Events with Missing Transverse Momentum in $e^{+} e^{-}$Collisions at sqrt(s) = 161 and 172 GeV", Eur. Phys. J C4 47 (1998).

[74] V. M. Abazov et al. (DØ Collaboration), "Observation of $Z Z$ production in $p \bar{p}$ collisions at $\sqrt{s}=1.96 \mathrm{TeV}$ ", Phys. Rev. Lett. 101, 171803 (2008), arXiv.org:0808.0703.

[75] V. M. Abazov et al. (DØ Collaboration), " $Z Z \rightarrow \ell^{-} \ell^{+} \nu \bar{\nu}$ Production in $p \bar{p}$ Collisions at $\sqrt{s}=1.96$ TeV", Phys. Rev. D 78, 072002 (2008), arXiv.org:0808.0269.

[76] V. M. Abazov et al. (DØ Collaboration), "Precise Study of the $Z / \gamma^{*}$-Boson Transverse Momentum Distribution in $p \bar{p}$ Collisions Using a Novel Technique", Phys. Rev. Lett. 106, 122001 (2011), arXiv.org:1010.0262. 
[77] A. Hoecker et al., TMVA, Tolkit for Multivariate Analysis with ROOT.

[78] I. Narsky, "StatPatternRecognition: A C++ Package for Statistical Analysis of High Energy Physics Data", arXiv:physics/0507143, Website.

[79] R.E. Schapire, "The strenght of weak learnability", Machine Learning 5 197-227 (1990).

[80] Y. Freud, "Boosting a weak learning algorithm by majority", (1995) Inform. and Comput. 121 256-285 (1995).

[81] T. Scanlon, "b-Tagging and the Search for Neutral Supersymmetric Higgs Bosons at DØ", FERMILAB-THESIS-2006-43, 2006

[82] V. Abazov, et al., "b-Jet Identification in the D $\varnothing$ Experiment", Nucl. Instrum. Methods in Phys. Res. Sect. A 609, 250 (2009), arXiv.org:1002.4224.

[83] S. Greder, I. Ripp-Baudot, "B-tagging with combined multivariate techniques", DØ Note 5936.

[84] G.J. Feldman and R.D. Cousins, Phys. ReV. D 57, 3873 (1998).

[85] J. Linnemann, M. Paterno and H. B. Prosper, "Calculating Confidence Limits", DØ Note 4491.

[86] R.T. Cox, Am. J. Phys. 14, 1 (1946).

[87] W. Fisher, "Collie: A Confidence Level Limit Evaluator", DØ Note 5595.

[88] W. Fisher, "Systematics and limit calculations", FERMILAB-TM-2386-E (2006).

[89] T. Andeen et. al., FERMILAB-TM-2365-E (2006). 
[90] C. Ochando, S. Calvet, J.-F. Grivaz Presentation in D $\varnothing$ V+jets workshop, May 20, 2008

[91] S. Muanza, Presentation in D $\varnothing$ V+jets meeting, February 5, 2008

[92] W. Fisher, J. Haley, D. Price, "Studies of Alpgen parameters, corrections and associated uncertainties" DØ Note 5966.

[93] J. Haley, presentation in V+jets workshop, September 28, 2009.

[94] U. Baur, T. Han, J. Ohnemus, "QCD Corrections and Anomalous Couplings in $Z \gamma$ Production at Hadron Colliders", Phys. Rev. D 57, 2823 (1998).

[95] A. Askew, A. Lyon, G. Pawloski, "Measurement of the Charge Signed Photon-

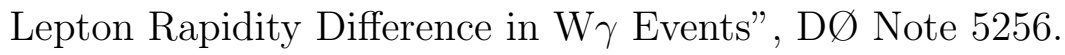

[96] The Particle Data Group.

[97] J. M. Campbell, R.K. Ellis, "Update on vector pair production at hadron colliders", Phys. Rev. D 60, 113006 (1999).

[98] M. Carena et al., "Report of the Tevatron Higgs Working Group", arXiv:hep-ph/0010338; CDF and DØ Collaborations, "Results of the Tevatron Higgs Sensitivity Study", FERMILAB-PUB-03/320-E (2003).

[99] C. Ochando, presentation in JES meeting, May 15, 2008

[100] T. Millet, A. Duperrin, P. Verdier, S. Muanza, "Jets and $\not_{T}$ triggers for the New Phenomena group in the V14 and v15 triggers lists", DØ Note 5120, (2006).

[101] F. Lacroix, C. Ochando, A. Duperrin, P. Verdier, "Higgs and New Phenomena Jets $+E_{T}$ triggers: L3 design and commissioning in v15 Run IIb trigger list", DØ Note 5282 (2006). 
[102] V. Abazov et al. (DØ Collaboration), "Search for $W H$ associated production in $5.3 \mathrm{fb}^{-1}$ of $p \bar{p}$ collisions at the Fermilab Tevatron", Phys. Lett. B 698, 6 (2011), arXiv.org:1012.0874.

[103] Y. Enari, Private Communication

[104] K. Peters, presentation in Higgs meeting, May 22nd, 2008

[105] J. Gallicchio and M. D. Schwartz, Phys. Rev. Lett. 105, 022001 (2010).

[106] J. Bystricky et al., "Algorithms and Architecture for the L1 Calorimeter Trigger at D $\varnothing$ Run IIb" IEEE Trans Nucl. Sci. 51, 351 (2004)

[107] M. Abolins et al., "Design and Implementation of the New DØ Level-1 Calorimeter Trigger" Nucl. Instrum. Methods in Phys. Res. Sect. A 584, 75-97 (2008), arXiv.org:0709.3750

[108] The ATLAS Level-1 Trigger Group "ATLAS Level-1 Trigger Technical Design Report" 